\title{
EXPERIMENT DATA REPORT FOR SEMISCALE MOD-1 \\ TEST S-06-4 (LOFT COUNTERPART TEST)
}

\author{
ROBERT L. GILLINS KENNETH E. SACKETT \\ CHERYL E. COPPIN
}

December 1977

EG\&G Idaho, Inc.

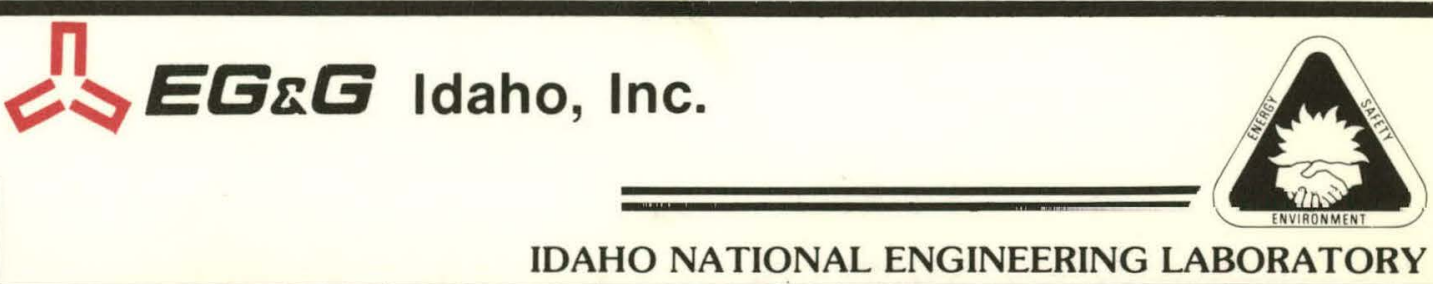

\section{DEPARTMENT OF ENERGY}




\section{DISCLAIMER}

This report was prepared as an account of work sponsored by an agency of the United States Government. Neither the United States Government nor any agency Thereof, nor any of their employees, makes any warranty, express or implied, or assumes any legal liability or responsibility for the accuracy, completeness, or usefulness of any information, apparatus, product, or process disclosed, or represents that its use would not infringe privately owned rights. Reference herein to any specific commercial product, process, or service by trade name, trademark, manufacturer, or otherwise does not necessarily constitute or imply its endorsement, recommendation, or favoring by the United States Government or any agency thereof. The views and opinions of authors expressed herein do not necessarily state or reflect those of the United States Government or any agency thereof. 


\section{DISCLAIMER}

Portions of this document may be illegible in electronic image products. Images are produced from the best available original document. 
Printed in the United States of America

$$
\text { Available from }
$$

National Technical Information Service

U.S. Department of Commerce

5285 Port Royal Road

Springfield, Virginia 22161

Price: Printed Copy $\$ 10.75$; Microfiche $\$ 3.00$

\section{NOTICE}

This report was prepared as an account of work sponsored by the United States Government. Neither the United States nor the Department of Energy, nor any of their employees, nor any of their contractors, subcontractors, or their employees, makes any warranty, express or implied, or assumes any legal liability or responsibility for the accuracy, completeness or usefulness of any information, apparatus, product or process disclosed, or represents that its use would not infring privately owned rights.

"The NRC will make available data tapes and operational computer codes on research programs dealing with postulated loss-of-coolant accidents in light water reactors. Persons requesting this information must reimburse the NRC contractors for their expenses in preparing copies of the data tapes and the operational computer codes. Requests should be submitted to the Research Applications Branch, Office of Nuclear Regulatory Research, Nuclear Regulatory Commission, Washington, D.C. 20555." 
TREE-NUREG-1124

EXPERIMENT DATA REPORT FOR SEMISCALE MOD-1

TEST S-06-4

(LOFT COUNTERPART TEST)

Approved:
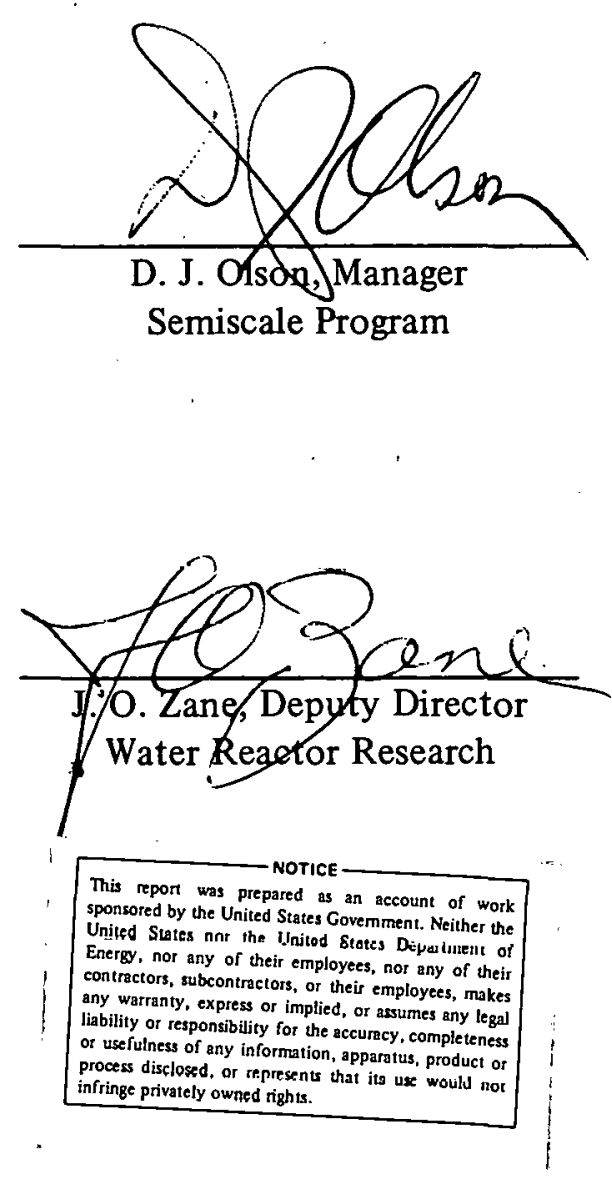
EXPERIMENT DATA REPORT FOR SEMISCALE MOD-1

TEST S-06-4

(LOFT COUNTERPART TEST)

by

Robert L. Gillins

Kenneth E. Sackett

Cheryl E. Coppin

EG\&G IDAHO, INC.

December 1977

PREPARED FOR THE

U.S. NÚCLEAR REGULATORY COMMISSION

AND THE

DEPARTMENT OF ENERGY

IDAHO OPERATIONS OFFICE

UNDER CONTRACT NO. EY-76-C-07-1570 


\begin{abstract}
Recorded test data are presented for Test S-06-4 of the Semiscale Mod-1 LOFT counterpart test series. These tests are among several Semiscale Mod-1 experiments conducted to investigate the thermal and hydraulic phenomena accompanying a hypothesized loss-of-coolant accident in a pressurized water reactor (PWR) system.

Test S-06-4 was conducted from initial conditions of $15653 \mathrm{kPa}$ and $564 \mathrm{~K}$ to investigate the response of the Semiscale Mod-1 system to a depressurization and reflood transient following a simulated double-ended offset shear of the broken loop cold leg piping. During the test, cooling water was injected into the cold leg of the intact loop to simulate emergency core coolant injection in a PWR. The heater rods in the electrically heated core were operated at an axial peak power density which was $100 \%$ of the maximum peak power density $(52.5 \mathrm{~kW} / \mathrm{m})$.
\end{abstract}

The purpose of this report is to make available the uninterpreted data from Test S-06-4 for future data analysis and test reporting activities. The data, presented in the form of graphs in engineering units, have been analyzed only to the extent necessary to ensure that they are reasonable and consistent. 


\section{SUMMARY}

Test S-06-4 was performed as part of the Mod-1 portion of the Semiscale Program conducted by EG\&G Idaho, Inc. for the Unites States Government. This test was part of the LOFT counterpart test series (Test Series 6) performed to investigate the response of the Mod-1 system to specific variations in the peak power densities of the heater rods to assist the LOFT Program in the planning of the first LOFT nuclear test series. The test objective specific to Test S-06-4 was to assist the LOFT Program in assessing possible core response effects when the LOFT systcm, operating at $100 \%$ core power, is subjected to a postulated loss-of-coolant accident (LOCA). Hardware configuration and test parametcrs were selected to yield a system response that simulates the expected response of the LOFT nuclear facility during the first nuclear test series.

Test S-06-4 utilized the Semiscale Mod-1 system equipped with a pressure vessel with a 40-rod electrically heated core; an intact loop with pump, steam generator, and pressurizer; a broken loop with simulated pump, simulated steam generator; simulated reflood bypass lines, LOFT counterpart nozzles, and rupture assemblies; and a pressure suppression system with header, pressure suppression tank, and heated steam supply system. High and low pressure coolant injection pumps and a coolant injection accumulator were provided for the intact loop. For Test S-06-4, four heater rods were intentionally unpowered to simulate LOFT passive rod locations, and the power in the four central heater rods was increased to produce a peaked power profile.

The test was conducted from initial conditions of $15653 \mathrm{kPa}$ and $564 \mathrm{~K}$ (at the intact loop cold leg vessel inlet) with a simulated full size (200\%) double-ended offset shear of the broken loop cold leg piping at an initial core power level of $1.33 \mathrm{MW}$ and an initial core inlet flow rate of $9.55 \mathrm{l} / \mathrm{s}$. The instantaneous offset shear of the broken loop cold leg piping was simulated by simultaneous (within $10 \mathrm{~ms}$ ) actuation of the rupture assemblies. After initiation of blowdown, power to the heated core was reduced to simulate the predicted heat flux response of nuclear fuel rods during a LOCA. Blowdown was accompanied by simulated cmergency core coolant injection into the cold leg piping of the intact loop.

Test S-06-4 was generally conducted as specified. Conditions which did not conform to the specified test configuration were considered acceptable for analysis purposes within the test objectives. The instrumentation used generally functioned as intended. Of 225 measurements taken, 216 produced usable data. 


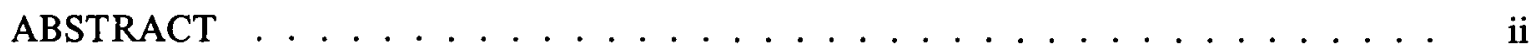

SUMMARY $\ldots \ldots \ldots \ldots \ldots \ldots \ldots \ldots \ldots \ldots \ldots \ldots \ldots$ iii

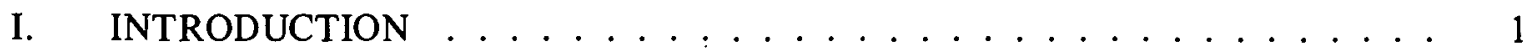

II. SYSTEM, PROCEDURES, CONDITIONS, AND

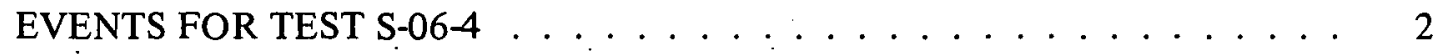

1. SYSTEM CONFIGURATION AND

TEST PROCEDURES . . . . . . . . . . . . . . . . . 2

2. INITIAL TEST CONDITIONS AND

SEQUENCE OI EVENTS $\ldots \ldots \ldots \ldots \ldots \ldots \ldots$

III. DATA PRESENTATION $\ldots \ldots \ldots \ldots \ldots \ldots \ldots$

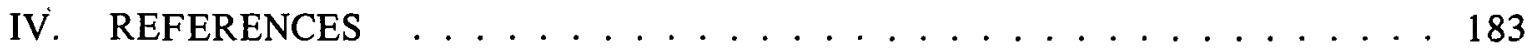

APPENDIX A - DATA ACQUISITION SYSTEM CAPABILITIES $\ldots \ldots \ldots$

APPENDIX B - POSTTEST ADJUSTMENTS TO DATA FROM

SEMISCALE MOD-1 TEST S-06-4 . . . . . . . . . . . . . . . . . 189

1. PRESSURE MEASUREMENTS $\ldots \ldots \ldots \ldots \ldots \ldots \ldots$

2. DIFHERENIIIAL PRESSURE MEASUREMENTS $\ldots \ldots \ldots \ldots . \ldots 192$

3. MOMENTUM FLUX MEASUREMENTS . . . . . . . . . . . . . . . . 194

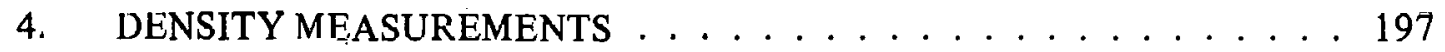

APPENDIX C - SELECTED DATA WITH ESTIMATED TOTAL

ERROR BANDS FROM SEMISCALE MOD-1 TEST S-06-4 . . . . . . . . . 201

\section{FIGURES}

1. Semiscale Mod-1 system for cold leg break

configuration - isometric $\ldots \ldots \ldots \ldots \ldots \ldots$

2. Semiscale Mod-1 system for cold leg break

configuration - schematic $\ldots \ldots \ldots \ldots \ldots \ldots$ 
3. Semiscale Mod-1 system and instrumentation for cold leg break configuration - isometric $\ldots \ldots \ldots \ldots \ldots$

4. Semiscale Mod-1 system and instrumentation for cold leg break configuration - schematic . . . . . . . . . . . . . 12

5. Semiscale Mod-1 pressure vessel - cross section showing instrumentation . . . . . . . . . . . . . . . 13

6. Semiscale Mod-1 pressure vessel - isometric showing instrumentation . . . . . . . . . . . . . . . . 14

7. Semiscale Mod-1 pressure vesscl - penetrations and instrumentation . . . . . . . . . . . . . . . 15

8. Semiscale Mod-1 heated core - plan view . . . . . . . . . . . . 16

9. Fluid temperature in intact loop hot leg (TFU-1 and RBU-2), from -20 to $300 \mathrm{~s}$

10. Fluid temperature in intact loop hot leg (TFU-1 and RBU-2), from -6 to $42 \mathrm{~s} \ldots \ldots \ldots 28$

11. Fluid temperature in intact loop cold leg (TFU-7 and TFU-10), from -20 to $300 \mathrm{~s} \ldots \ldots . \ldots 29$

12. Fluid temperature in intact loop cold leg (TFU-7 and TFU-10), from -6 to $42 \mathrm{~s}$

13. Fluid temperature in in tact loop cold leg

(RBU-14A, TFU-14B, and TFU-15), from -20 to $300 \mathrm{~s} \ldots \ldots 30$

14. Fluid temperature in intact loop cold leg

(RBU-14A, TFU-14B, and TFU-15), from -6 to $42 \mathrm{~s} \ldots \ldots . \ldots 30$

15. Fluid temperature in broken loop, vessel side

(TFB-20, TFB-23, and TFB-24), from -20 to $300 \mathrm{~s} \ldots \ldots . \ldots 31$

16. Fluid temperature in broken loop, vessel side

(1FB-20, TFB-23, and TFB-24), from -6 to $42 \mathrm{~s} \ldots \ldots . \ldots 31$

17. Fluid temperature in broken loop, pump side

(TFB-30, TFB-37, TFB-42, and TFB-43), from -20 to $300 \mathrm{~s} \ldots \ldots 32$

18. Fluid temperature in broken loop, pump side

(TFB-30, TFB-37, TFB-42, and TFB-43), from -6 to $42 \mathrm{~s} \ldots \ldots . . . . . .32$ 
19. Fluid temperature in broken loop, reflood bypass simulators (TFB-RFBC and TFB-RFBH), from -20 to $300 \mathrm{~s}$

20. Fluid temperature in broken loop, reflood bypass simulators (TFB-RFBC and TFB-RFBH), from -6 to 42 s

21. Fluid temperature in inlet annulus (TFV-ANN-4A and TFV-ANN-4M), from -20 to $300 \mathrm{~s} \ldots \ldots . \ldots . . \ldots . . . \ldots 34$

22. Fluid temperature in inlet annulus (TFV-ANN-4A and TFV-ANN-4M), from -6 to $42 \mathrm{~s} \ldots \ldots \ldots$. . . . . . . . . . 34

23. Fluid temperature in downcomer annulus (TFV-ANN-35A, TFV-ANN-35T, and TFV-ANN-70A), from -20 to $300 \mathrm{~s}$

24. Fluid temperature in downcomer annulus (TFV-ANN-35A, TFV-ANN-35T, and TFV-ANN-70A), from -6 to $42 \mathrm{~s}$.

25. Fluid temperature in downcomer annulus (TFV-ANN-115A and TFV-ANN-115M), from -20 to $300 \mathrm{~s}$

26. Fluid temperature in downcomer annulus (TFV-ANN-115A and TFV-ANN-1 15M), from -6 to $42 \mathrm{~s} \ldots \ldots \ldots$

27. Fluid temperature in upper plenum (TFV-UP+13), from -20 to $300 \mathrm{~s}$

28. Fluid temperature in upper plenum (TFV-IIP+13), from -6 to $42 \mathrm{~s} \ldots \ldots \ldots \ldots \ldots \ldots$. . . . . . . . . . . . . . . . . . .

29. Fluid tempcrature in lower plenum (TFV-LP-8, TFV-LP-15, and TFV-LP-22), from -20 to $300 \mathrm{~s} \ldots \ldots . \ldots 38$

30. Fluid temperature in lower plenum (TFV-LP-8, TFV-LP-1 5, and TFV-LP-22), frnm -6 to $42 \mathrm{~s} \ldots \ldots \ldots$

31. Fluid temperature in core inlet (TFV-CORE-IN), from -20 to $300 \mathrm{~s} \ldots \ldots \ldots \ldots$

32. Fluid temperature in core inlet (TFV-CORE-IN), from -6 to $42 \mathrm{~s} \ldots \ldots \ldots \ldots \ldots$

33. Fluid temperature in core, Grid Spacer 5 (TFG-5CD-45), from -20 to $300 \mathrm{~s}$ 
34. Fluid temperature in core, Grid Spacer 5 (TFG-5CD-45),

from -6 to $42 \mathrm{~s} \ldots \ldots \ldots \ldots \ldots \ldots \ldots \ldots \ldots \ldots$

35. Fluid temperature in core, Grid Spacer 6 (TFG-6CD-45

and TFG-6DE-67), from -20 to $300 \mathrm{~s} \ldots \ldots \ldots \ldots$

36. Fluid temperature in core, Grid Spacer 6 (TFG-6CD-45

and TFG-6DE-67), from -6 to $42 \mathrm{~s} \ldots \ldots \ldots \ldots \ldots$. . . . . . . . 41

37. Fluid temperature in core, Grid Spacer 10 (TFG-10AB-45

and TFG-10GH-45), from -20 to $300 \mathrm{~s} \ldots \ldots \ldots \ldots \ldots \ldots . \ldots \ldots$

38. Fluid temperature in core, Grid Spacer 10 (TFG-10AB-45

and TFG-10GH-45), from -6 to $42 \mathrm{~s} \ldots \ldots \ldots \ldots \ldots \ldots$

39. Fluid temperature in intact loop coolant injection line

(TFU-ECC-14), from -20 to $300 \mathrm{~s} \ldots \ldots \ldots \ldots \ldots$

40. Fluid temperature in intact loop coolant injection line

(TFU-ECC-14), from -6 to $42 \mathrm{~s} \ldots \ldots \ldots \ldots \ldots \ldots$

41. Fluid temperature in steam generator, feed water line

(TFU-SGFW), from -20 to $300 \mathrm{~s} \ldots \ldots \ldots \ldots$. . . . . . . . . 44

42. Fluid temperature in steam generator, feedwater line (TFU-SGFW), from -6 to $42 \mathrm{~s} \ldots \ldots \ldots \ldots \ldots$

43. Fluid temperature in steam generator, secondary side

(TFU-SGSD), from -20 to $300 \mathrm{~s} \ldots \ldots \ldots \ldots \ldots \ldots$

44. Fluid temperature in steam generator, secondary side

(TFU-SGSD), from -6 to $42 \mathrm{~s} \ldots \ldots \ldots \ldots \ldots \ldots$

45. Fluid temperature in steam generator, secondary side

(TFU-SG1, TFU-SG3, and TFU-SG4), from -20 to $300 \mathrm{~s} \ldots \ldots \ldots \ldots$

46. Fluid temperature in steam generator, secondary side

(TFU-SG1, TFU-SG3, and TFU-SG4), from -6 to $42 \mathrm{~s} \ldots \ldots \ldots \ldots$

47. Fluid temperature in pressurizer surge line (TFU-PRIZE),

from -20 to $300 \mathrm{~s} \ldots \ldots \ldots \ldots$

48. Fluid temperature in pressurizer surge line (TFU-PRIZE),

from -6 to $42 \mathrm{~s} \ldots \ldots \ldots \ldots \ldots \ldots \ldots \ldots \ldots \ldots$ 
49. Fluid temperature in pressure suppression tank (TF-PSS-33, TF-PSS-63, and TF-PSS-130), from -20 to $300 \mathrm{~s} \ldots \ldots . \ldots 48$

50. Fluid temperature in pressure suppression tank (TF-PSS-33, TF-PSS-63, and TF-PSS-130), from -6 to $42 \mathrm{~s} \ldots \ldots . \ldots . \ldots 48$

51. Material temperature in intact loop (TMU-1T16 and TMU-15T16), from -20 to $300 \mathrm{~s} \ldots \ldots$. . . . . . . . . . . . 49

52. Material temperature in intact loop (TMU-1T16 and TMU-15T16), from -6 to $42 \mathrm{~s}$

53. Material temperature in broken loop (TMB-20B16 and TMB-30B 16), from -20 to $300 \mathrm{~s} \ldots \ldots \ldots 50$

54. Material temperature in broken loop (TMB-20B16 and TMB-30B16), from -6 to $42 \mathrm{~s} \ldots \ldots \ldots \ldots$. . . . . . . . . . . 50

55. Material temperature in vessel. filler (TMV-FI-115A), fiom 20 lu $300 \mathrm{~s}$

56. Material temperature in vessel filler (TMV-FI-1 15A), from -6 to $42 \mathrm{~s} \ldots \ldots \ldots \ldots \ldots \ldots \ldots$

57. Core heater temperature, Rod D-4 (TH-D4-14 and TH-D4-29), from 20 tu $300 \mathrm{~s}$

58. Core heater temperature, Rod D-4 (TH-D4-14 and TH-D4-29), from -6 to $42 \mathrm{~s} \ldots \ldots \ldots \ldots \ldots \ldots$

59. Core heater temperature, Rod D-5 (TH-D5-09, TH-D5-29, and TH-D5-39), from -20 to $300 \mathrm{~s} \ldots \ldots \ldots 5$

60. Core heater temperature, Rod D-5 (TH-D5-09, TH-D5-29, and TH-D5-39), from -6 to $42 \mathrm{~s} \ldots \ldots \ldots \ldots$

61. Core heater temperature, Rod E-4 (TH-E4-09, TH-E4-27, and TH-E4-55), from -20 to $300 \mathrm{~s} \ldots \ldots \ldots \ldots$

62. Core heater temperature, Rod E-4 (TH-E4-09, TH-E4-27, and TH-E4-55), from -6 to $42 \mathrm{~s}$

63. Core heater temperature, Rod E-5 (TH-E5-21 and TH-E5-25), from -20 to 300

64. Core heater temperature, Rod E-5 (TH-E5-21 and TH-E5-25), from -6 to $42 \mathrm{~s}$ 
65. Core heater temperature, Rod A-4 (TH-A4-09),

from -20 to $300 \mathrm{~s} \ldots \ldots \ldots \ldots \ldots \ldots \ldots$

66. Core heater temperature, Rod A-4 (TH-A4-09),

from -6 to $42 \mathrm{~s} \ldots \ldots \ldots \ldots \ldots \ldots \ldots \ldots \ldots \ldots$

67. Core heater temperature, Rod A-5 (TH-A5-29 and TH-A5-45),

from -20 to $300 \mathrm{~s} \ldots \ldots \ldots \ldots \ldots \ldots \ldots$

68. Core heater temperature, Rod A-5 (TH-A5-29 and TH-A5-45),

from -6 to $42 \mathrm{~s} \ldots \ldots \ldots \ldots \ldots \ldots \ldots \ldots \ldots \ldots$

69. Core heater temperature, Rod B-3 (TH-B3-32),

from -20 to $300 \mathrm{~s} \ldots \ldots \ldots \ldots \ldots \ldots \ldots \ldots \ldots$

70. Core heater temperature, Rod B-3 (TH-B3-32),

from -6 to $42 \mathrm{~s} \ldots \ldots \ldots \ldots \ldots \ldots \ldots \ldots \ldots \ldots$

71. Core heater temperature, Rod B-5 (TH-B5-29 and TH-B5-33),

from -20 to $300 \mathrm{~s} \ldots \ldots \ldots \ldots \ldots \ldots \ldots$

72. Core heater temperature, Rod B-5 (TH-B5-29 and TH-B5-33),

from -6 to $42 \mathrm{~s} \ldots \ldots \ldots \ldots \ldots \ldots \ldots \ldots$

73. Core heater temperature, Rod B-6 (TH-B6-29),

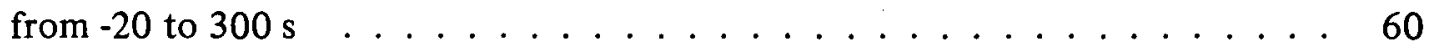

74. Core heater temperature, Rod B-6 (TH-B6-29),

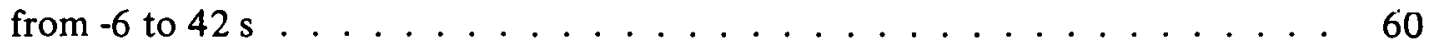

75. Core heater temperature, Rod C-2 (TH-C2-28 and TH-C2-38),

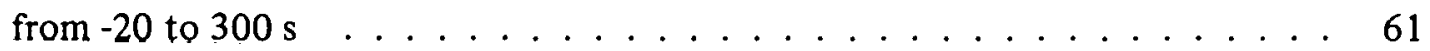

76. Core heater temperature, Rod C-2 (TH-C2-28 and TH-C2-38),

from -6 to $42 \mathrm{~s} \ldots \ldots \ldots \ldots \ldots \ldots \ldots$. . . . . . . . . . . . 61

77. Core heater temperature, Rod C-3 (TH-C3-60),

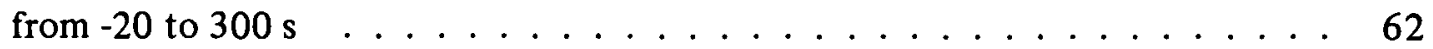

78. Core heater temperature, Rod $\mathrm{C}-3$ (TH-C3-60),

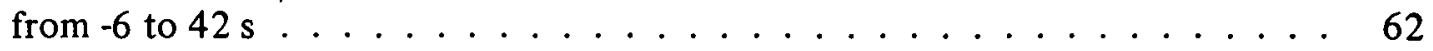

79. Core heater temperaturc, Rod C-4 (TH-C4-20 and TH-C4-26),

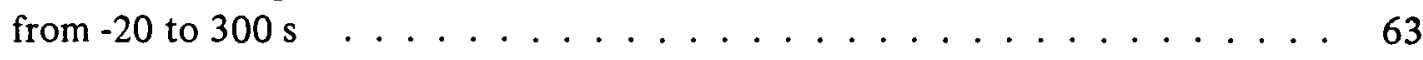


80. Core heater temperature, Rod C-4 (TH-C4-20 and TH-C4-26),

from -6 to $42 \mathrm{~s} \ldots \ldots \ldots \ldots \ldots$

81. Core heater temperature, Rod C-5 (TH-C5-28),

from -20 to $300 \mathrm{~s} \ldots \ldots \ldots \ldots \ldots \ldots$. . . . . . . . . . . . . . . . . .

82. Core heater temperature, Rod C-5 (TH-C5-28),

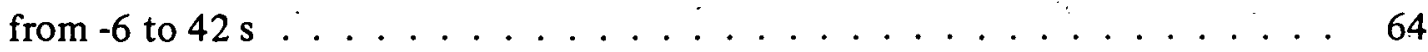

83. Core heater temperature, Rod C-6 (TH-C6-32 and TH-C6-53),

from -20 to $300 \mathrm{~s} \ldots \ldots \ldots 6 . \ldots \ldots \ldots$

84. Core heater temperature, Rod C-6 (TH-C6-32 and TH-C6-53),

from -6 to $42 \mathrm{~s} \ldots \ldots \ldots \ldots \ldots$

85. Core heatcr temperature, Rod C-7 (TH-C. 7-07 and TH-C7-15),

from -200 to $3000 \mathrm{~s} \ldots \ldots \ldots \ldots \ldots$. . . . . . . . . . . . . . . .

86. Core heater temperature, Rod C-7 (TH-C7-07 and TH-C7-15),

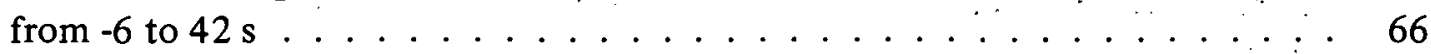

87. Core heater temperature, Rod D-1 (TH-D1-21),

from -20 to $300 \mathrm{~s} \ldots \ldots \ldots \ldots$. . . . . . . . . . . . . . . . .

88. Core heater temperature, Rod D-1 (TH-D1-21),

from -6 to $42 \mathrm{~s} \ldots \ldots \ldots \ldots \ldots$. . . . . . . . . . . . 67

89. Core heater temperature, Rod D-2 (TH-D2-14 and TH-D2-61),

from -20 to $300 \mathrm{~s} \ldots \ldots \ldots \ldots . \ldots \ldots$

90. Core heater temperature, Rod D-2 (TH-D2-14 and TH-D2-61),

from -6 to $42 \mathrm{~s} \ldots \ldots \ldots \ldots$. . . . . . . . . . . . . 68

91. Core heater temperature, Rod D-3 (TH-D3-29, TH-D3-33, and

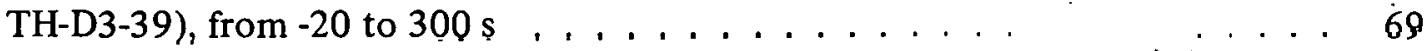

92. Core heater temperature, Rod D-3 (TH-D3-29, TH-D3-33, and

TH-D3-39), from -6 to $42 \mathrm{~s} \ldots \ldots \ldots$. . . . . . . . . . . . . . 69

93. Core heater temperature, Rod D-7 (TH-D7-20),

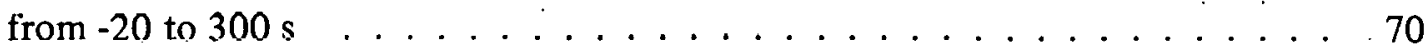

94. Core heater temperature, Rod D-7 (TH-D7-20),

from -6 to $42 \mathrm{~s} \ldots \ldots \ldots \ldots \ldots$. . . . . . . . . . . . . . 70 
95. Core heater temperature, Rod D-8 (TH-D8-57),

from -20 to $300 \mathrm{~s} \ldots \ldots \ldots \ldots$. . . . . . . . . . . . . 71

96. Core heater temperature, Rod D-8 (TH-D8-57),

from -6 to $42 \mathrm{~s} \ldots \ldots \ldots \ldots 71$

97. Core heater temperature, Rod E-1 (TH-E1-26 and TH-E1-33),

from -20 to $300 \mathrm{~s} \ldots \ldots \ldots \ldots$. . . . . . . . . . . . . . . . . . .

98. Core heater temperature, Rod E-1 (TH-E1-26 and TH-E1-33),

from -6 to $42 \mathrm{~s} \ldots \ldots \ldots \ldots \ldots$. . . . . . . . . . . . . . . . . .

99. Core heater temperature, Rod E-3 (TH-E3-05, TH-E3-20, and

TH-E3-24), from -20 to $300 \mathrm{~s} \ldots \ldots \ldots 73$

100. Core heater temperature, Rod E-3 (TH-E3-05, TH-E3-20, and

TH-E3-24), from -6 to $42 \mathrm{~s} \ldots \ldots \ldots 73$

101. Core heater temperature, Rod E-6 (TH-E6-08, TH-E6-28, and

TH-E6-37), from -20 to $300 \mathrm{~s} \ldots \ldots \ldots$. . . . . . . . . . . 74

102. Core heater temperature, Rod E-6 (TH-E6-08, TH-E6-28, and TH-E6-37), from -6 to $42 \mathrm{~s}$

103. Core heater temperature, Rod E-7 (TH-E7-13 and TH-E7-44),

from -20 to $300 \mathrm{~s}$

104. Core heater temperature, Rod E-7 (TH-E7-13 and TH-E7-44),

105. Core heater temperature, Rod E-8 (TH-E8-14, TH-E8-29, and TH-E8-45), from -20 to $300 \mathrm{~s} \ldots \ldots \ldots$. . . . . . . . . . . . . . .

106. Core heater temperature, Rod E-8 (TH-E8-14, TH-E8-29, and TH-E8-45), from -6 to $42 \mathrm{~s}$

107. Core heater temperature, Rod F-3 (TH-F3-06, TH-F3-22, and TH-F3-25), from -20 to $300 \mathrm{~s}$

108. Core heater temperature, Rod F-3 (TH-F3-06, TH-F3-22, and TH-F3-25), from -6 to $42 \mathrm{~s}$

109. Core heater temperature, Rod F-4 (TH-F4-14, TH-F4-29, and TH-F4-44), from -20 to $300 \mathrm{~s}$ 
110. Core heater temperature, Rod F-4 (TH-F4-14, TH-F4-29, and

TH-F4-44), from -6 to 42

111. Core heater temperature, Rod F-6 (TH-F6-08 and TH-F6-28),

112. Core heater temperature, Rod F-6 (TH-F6-08 and TH-F6-28),

from -6 to $42 \mathrm{~s}$

113. Core heater temperature, Rod F-7 (TH-F7-29)

from -20 to $300 \mathrm{~s}$

114. Core heater temperature, Rod F-7 (TH-F7-29)

from -6 to $42 \mathrm{~s}$

115. Core heater temperature, Rod G-3 (TH-G3-13),

from -20 to $300 \mathrm{~s}$

116. Core heater temperature, Rod G-3 (TH-G3-13),

from -6 to 42

117. Core heater temperature, Rod G-4 (TH-G4-29, TH-G4-33, and

TH-G4-38), from -20 to $300 \mathrm{~s}$

118. Core heater temperature, Rod G-4 (TH-G4-29, TH-G4-33, and

TH-G4-38), from -6 to $42 \mathrm{~s}$

119. Core heater temperature, Rod G-5 (TH-G5-14 and TH-G5-24),

from -20 to $300 \mathrm{~s}$

120. Core heater temperature, Rod G-5 (TH-G5-14 and TH-G5-24), from -6 to 42 s

121. Core heater temperature, Rod H-5 (TH-H5-32), from $-2 n$ tn $3 \cap n s$

122. Core heater temperature, Rod H-5 (TH-H5-32), from -6 to $423 \ldots \ldots \ldots$. . . . . . . . . . . . . 84

123. Pressure in intact loop (PU-13 and PU-15L), from -20 to $300 \mathrm{~s}$

124. Pressure in intact loop (PU-13 and PU-15L), from -6 to $42 \mathrm{~s}$ 
125. Pressure in broken loop, vessel side (PB-21, PB-23, and

PB-24), from -20 to $300 \mathrm{~s} \ldots \ldots \ldots \ldots$. . . . . . . . . . 86

126. Pressure in broken loop, vessel side (PB-21, PB-23, and PB-24), from -6 to $42 \mathrm{~s} \ldots \ldots \ldots \ldots \ldots \ldots \ldots$

127. Pressure in broken loop, pump side (PB-42 and $\mathrm{PB}-43$ ), from -20 to $300 \mathrm{~s}$

128. Pressure in broken loop, pump side (PB-42 and PB-43), from -6 to $42 \mathrm{~s} \ldots \ldots \ldots \ldots \ldots \ldots \ldots \ldots$

129. Pressure in broken loop, vessel side (PB-CN1), from -20 to $300 \mathrm{~s}$

130. Pressure in broken loop, vessel side (PB-CN1), from -6 to $42 \mathrm{~s} \ldots \ldots \ldots \ldots \ldots \ldots \ldots \ldots$

131. Pressure in vessel (PV-UP+10 and PV-LP-1 80), from -20 to $300 \mathrm{~s}$

132. Pressure in vessel (PV-UP+10 and PV-LP-1 80), from -6 to $42 \mathrm{~s}$

133. Pressure in intact loop accumulator (PU-ACC1), from -20 to $300 \mathrm{~s} \ldots \ldots \ldots \ldots \ldots$. . . . . . . . . . . 90

134. Pressure in intact loop accumulator (PU-ACC1), from -6 to $42 \mathrm{~s} \ldots \ldots \ldots \ldots \ldots \ldots \ldots \ldots \ldots \ldots$

135. Pressure in steam generator, secondary side (PU-SGSD), from -20 to $300 \mathrm{~s}$

136. Pressure in steam generator, secondary side (PU-SGSD), from -6 to $42 \mathrm{~s}$

137. Pressure in pressurizer (PU-PRIZE), from -20 to $300 \mathrm{~s} \ldots \ldots \ldots \ldots 2$

138. Pressure in pressurizer (PU-PRIZE), from -6 to $42 \mathrm{~s} \ldots \ldots$. . . . . . 92

139. Pressure in pressure suppression tank (P-P.SS), from -20 to $300 \mathrm{~s}$

140. Pressure in pressure suppression tank (P-PSS), from -6 to $42 \mathrm{~s}$ 
141. Differential pressure in intact loop (DPU-UP-3),

from $=20$ to $300 \mathrm{~s}$

142. Differential pressure in intact loop (DPU-UP-3),

from -6 to $42 \mathrm{~s} \ldots \ldots \ldots \ldots \ldots . \ldots \ldots$

143. Differential pressure in intact loop (DPU-3-6),

from -20 to $300 \mathrm{~s}$

1.44. Differential pressure in intact loop (DPU-3-6), from -6 to $42 \mathrm{~s} \ldots \ldots \ldots \ldots$. . . . . . . . . . . . . . . . . . . .

1.45. Differential pressure in in tact loop (DPU-6-SGIP),

from -20 to $300 \mathrm{~s}$

146. Differential pressure in intact loop (DPU-6-SGIP),

from -6 to 42

147. Differential pressure in intact loop (DPU-6-7), from -20 to $300 \mathrm{~s}$

148. Differential pressure in intact loop (DPU-6-7), from -6 to $42 \mathrm{~s}$

149. Differential pressure in intact loop (DPU-SGOP-7), trom -20 to $300 \mathrm{~s}$

150. Differential pressure in intact loop (DPU-SGOP-7),

151. Differential pressure in intact loop (DPU-7-10), from -20 to $300 \mathrm{~s}$

152. Differential pressure in intact loop (DPU-7-10), from -6 to 42

153. Differential pressure in intact loop (DPU-12-10), from -20 to $300 \mathrm{~s}$ 100

154. Differential pressure in intact loop (DPU-12-10), from -6 to $42 \mathrm{~s}$ 100

155. Differential pressure in intact loop (DPU-12-15), from -20 to $300 \mathrm{~s}$ 
156. Differential pressure in intact loop (DPU-12-15), from -6 to $42 \mathrm{~s} \ldots \ldots \ldots \ldots \ldots \ldots \ldots 10 \ldots \ldots \ldots$

157. Differential pressure in intact loop (DPU-15-3), from -20 to $300 \mathrm{~s}$ 102

158. Differential pressure in intact loop (DPU-15-3), from -6 to $42 \mathrm{~s}$

159. Differential pressure in intact loop, low range (DPU-15-3L), from -20 to $300 \mathrm{~s}$ 103

160. Differential pressure in intact loop, low range (DPU-15-3L), from -6 to $42 \mathrm{~s} \ldots \ldots \ldots \ldots \ldots \ldots \ldots \ldots$

161. Differential pressure in intact loop (DPU-15-IANN), from -20 to $300 \mathrm{~s}$ 104

162. Differential pressure in intact loop (DPU-15-IANN), from -6 to $42 \mathrm{~s}$ 104

163. Differential pressure in intact loop (DPU-PRESLL), from -20 to $300 \mathrm{~s}$ 105

164. Differential pressure in intact loop (DPU-PRESLL),

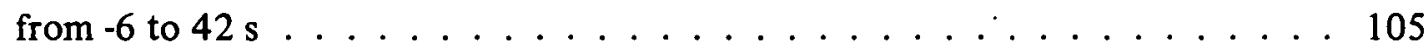

165. Differential pressure in intact loop (DPU-PR-4), from -20 to $300 \mathrm{~s}$ 106

166. Differential pressure in intact loop (DPU-PR-4), from -6 to $42 \mathrm{~s}$ 106

167. Differential pressure in broken loop (DPB-21-IANN), from -20 to $300 \mathrm{~s}$ 107

168. Differential pressure in broken loop (DPB-21-IANN), from -6 to $42 \mathrm{~s}$

169. Differential pressure in broken loop (DPB-21-23), from -20 to $300 \mathrm{~s}$ 108

170. Differential pressure in broken loop (DPB-21-23), from -6 to $42 \mathrm{~s}$ 108 
171. Differential pressure in broken loop (DPB-23-CN1), from -20 to $300 \mathrm{~s} \ldots \ldots \ldots \ldots . \ldots \ldots$

172. Differential pressure in broken loop (DPB-23-CN1), from -6 to $42 \mathrm{~s} \ldots \ldots \ldots \ldots$. . . . . . . . . . . . . . 109

173. Differential pressure in broken loop (DPB-23-24), from -20 to $300 \mathrm{~s} \ldots \ldots \ldots \ldots \ldots$

174. Differential pressure in broken loop (DPB-23-24), from -6 to $42 \mathrm{~s} \ldots \ldots \ldots \ldots \ldots \ldots$

175. Differential pressure in broken loop (DPB-30-36L), from -20 to $300 \mathrm{~s}$

176. Differential pressure in broken loop (DPB-30-36L), from -6 to $42 \mathrm{~s} \ldots \ldots \ldots \ldots \ldots 111 \ldots \ldots \ldots$

177. Differential pressure in broken loop (DPB-30-38), from -20 to $300 \mathrm{~s}$

178. Differential pressure in broken loop (DPB-30-38),

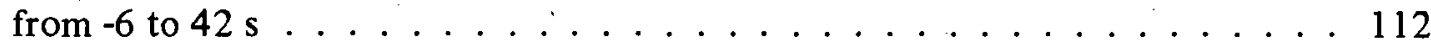

179. Differential pressure in broken loop (DPB-32U-36L), from -20 to $300 \mathrm{~s}$

180. Differential pressure in broken loop (DPB-32U-36L), from -6 to $42 \mathrm{~s} \ldots \ldots \ldots \ldots \ldots \ldots \ldots$. . . . . . . . . . . . 113

181. Differential pressure in broken loop (DPB-38-40), from -20 to $300 \mathrm{~s}$

182. Differential pressure in broken loop (DPB-38-40), from -6 to $12 \mathrm{\varepsilon} \ldots \ldots \ldots \ldots \ldots \ldots$

183. Differential pressure in broken loop (DPB-40-42), fivin -20 lu $300 s \ldots \ldots \ldots \ldots \ldots \ldots$

184. Differential pressure in broken loop (DPB-40-42), from -6 to $42 \mathrm{~s} \ldots \ldots \ldots \ldots \ldots \ldots \ldots$

185. Differential pressure in broken loop (DPB-42-43), from -20 to $300 \mathrm{~s}$ 
186. Differential pressure in broken loop (DPB-42-43), from -6 to $42 \mathrm{~s} \ldots \ldots \ldots \ldots \ldots 16$

187. Differential pressure in vessel (DPV-UP-IANN), from -20 to $300 \mathrm{~s} \ldots \ldots \ldots \ldots \ldots 117$

188. Differential pressure in vessel (DPV-UP-IANN), from -6 to $42 \mathrm{~s} \ldots \ldots \ldots \ldots \ldots 117 \ldots \ldots \ldots$

189. Differential pressure in vessel (DPV-0-9GQ), from -20 to $300 \mathrm{~s} \ldots \ldots \ldots \ldots \ldots \ldots$

190. Differential pressure in vessel (DPV-0-9GQ), from -6 to $42 \mathrm{~s} \ldots \ldots \ldots \ldots \ldots \ldots$. . . . . . . . . . . . 118

191. Differential pressure in vessel (DPV-9-26QQ), from -20 to $300 \mathrm{~s} \ldots \ldots \ldots \ldots \ldots \ldots$

192. Differential pressure in vessel (DPV-9-26QQ), from -6 to $42 \mathrm{~s} \ldots \ldots \ldots \ldots \ldots$. . . . . . . . . . . . . . . . . . . . . . . . . . .

193. Differential pressure in vessel (DPV-26-55QM), from -20 to $300 \dot{s}$

194. Differential pressure in vessel (DPV-26-55QM), from -6 to $42 \mathrm{~s} \ldots \ldots \ldots \ldots$. . . . . . . . . . . . 120

195. Differential pressure in vessel (DPV-55-110MM), from -20 to $300 \mathrm{~s}$

196. Differential pressure in vessel (DPV-55-1 10MM), from -6 to $42 \mathrm{~s} \ldots \ldots \ldots \ldots 121$

197. Differential pressure in vessel (DPV-110-156MQ), from -20 to $300 \mathrm{~s}$

198. Differential pressure in vessel (DPV-1 10-156MQ), from -6 to $42 \mathrm{~s} \ldots \ldots \ldots \ldots 122$

199. Differential pressure in vessel (DPV-156-173QQ), from -20 to $300 \mathrm{~s} \ldots \ldots \ldots \ldots \ldots$. . . . . . . . . . . . . . . . . . . . .

200. Differential pressure in vessel (DPV-156-1 73QQ), from -6 to $42 \mathrm{~s} \ldots \ldots \ldots \ldots \ldots$ 
201. Differential pressure in vessel (DPV-166-192QT),

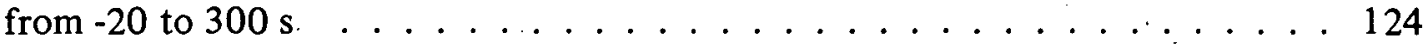

202. Differential pressure in vessel (DPV-166-192QT),

from -6 to $42 \mathrm{~s} \ldots \ldots \ldots \ldots \ldots \ldots \ldots$

203. Differential pressure in vessel. (DPV-173-180QQ),

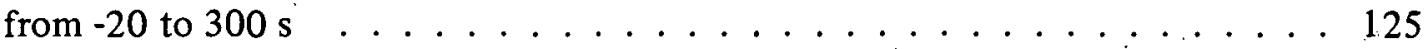

204. Differential pressure in vessel (DPV-173-180QQ),

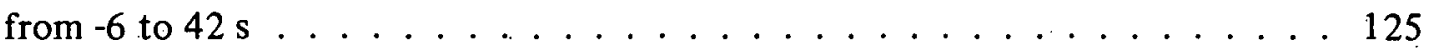

205. Differential pressure in vessel (DPV-LY-UP),

from -20 to $300 \mathrm{~s} \ldots \ldots \ldots \ldots \ldots \ldots \ldots \ldots \ldots \ldots$

206. Differential pressure in vessel (DPV-LP-UP),

from -6 to $42 \mathrm{~s} \ldots \ldots \ldots \ldots \ldots \ldots \ldots \ldots \ldots \ldots \ldots$

207. Differential pressure in intact loop accumulator (DPU-ACC1-TB),

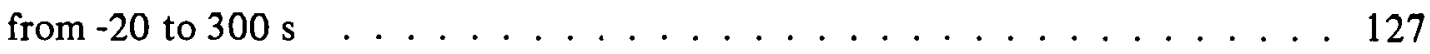

208. Differential pressure in intact loop accumulator (DPU-ACC1-TB),

from -6 to $42 \mathrm{~s} \ldots \ldots \ldots \ldots \ldots \ldots \ldots \ldots \ldots \ldots$

209. Differential pressure across steam generator feedwater orifice

(DPU-SGFW), from -20 to $300 \mathrm{~s} \ldots \ldots \ldots \ldots \ldots$

210. Differential pressure across steam generator feedwater orifice

(DPU-SGFW), from -6 to $42 \mathrm{~s} \ldots \ldots \ldots \ldots \ldots$

211. Differential pressure in steam generator secondary (DPU-SG-SEC),

from $=20$ to $300 \mathrm{~s} \ldots \ldots \ldots \ldots \ldots \ldots \ldots$

212. Differential pressure in steam generator secondary (DPU-SG-SEC),

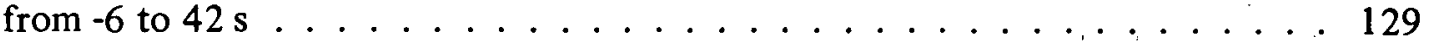

213. Differential pressure across steam generator outlet orifice

(DPU-SG-DISC), from -20 to $300 \mathrm{~s} \ldots \ldots \ldots \ldots \ldots$

214. Differential pressure across steam generator outlet orifice

(DPU-SG-DISC), from -6 to $42 \mathrm{~s} \ldots \ldots \ldots \ldots \ldots \ldots$

215. Volumetric flow in intact loop (FTU-1 and FTU-9),

from -20 to $300 \mathrm{~s}$ 
216. Volumetric flow in intact loop (FTU-1 and FTU-9), from -6 to $42 \mathrm{~s} \ldots \ldots \ldots \ldots \ldots \ldots \ldots$

217. Volumetric flow in intact loop (FTU-13 and FTU-15), from -20 to $300 \mathrm{~s}$

218. Volumetric flow in in tact loop (FTU-13 and FTU-15), from -6 to $42 \mathrm{~s} \ldots \ldots \ldots \ldots . \ldots \ldots 2$

219. Volumetric flow in broken loop (FTB-21), from -20 to $300 \mathrm{~s} \ldots \ldots$. . . . 133

220. Volumetric flow in broken loop (FTB-21), from -6 to $42 \mathrm{~s} \ldots \ldots . \ldots 133$

221. Volumetric flow in broken loop (FTB-30 and FTB-37), from -20 to $300 \mathrm{~s} \ldots \ldots \ldots \ldots \ldots$. . . . . . . . . . . . . . . . . . . . . . .

222. Volumetric flow in broken loop (FTB-30 and FTB-37),

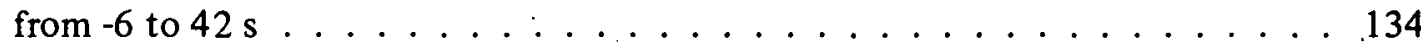

223. Volumetric flow in core entrance (FTV-CORE-IN), from -20 to $300 \mathrm{~s}$

224. Volumetric flow in core entrance (FTV-CORE-IN), from -6 to $42 \mathrm{~s} \ldots \ldots \ldots \ldots \ldots \ldots$. . . . . . . . . . . . . . . . . . . . .

225. Volumetric flow in intact loop high pressure injection line (FTU-HPIS), from -20 to $300 \mathrm{~s}$

226. Volumetric flow in intact loop high pressure injection line (FTU-HPIS), from -6 to $42 \mathrm{~s}$

227. Volumetric flow in intact loop low pressure injection line (FTU-LPIS), from -20 to $300 \mathrm{~s}$

228. Volumetric flow in intact loop low pressure injection line (FTU-LPIS), from -6 to $42 \mathrm{~s}$

229. Volumetric flow in intact loop accumulator discharge line (FTU-ACC1), from -20 to 300

230. Volumetric fluw in intact loop accumulator discharge line (FTU-ACC1), from -6 to $42 \mathrm{~s}$

231. Volumetric flow from pressurizer (FTU-PRIZE), from -20 to $300 \mathrm{~s}$ 
232. Volumetric flow from pressurizer (FTU-PRIZE),

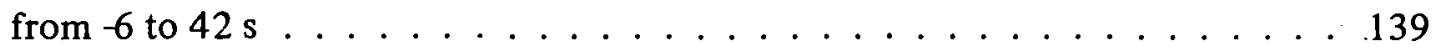

233. Fluid velocity in vessel (FTV-40A and FTV-40M), from -20 to $300 \mathrm{~s} \ldots \ldots \ldots \ldots$. . . . . . . . . . . 140

234. Fluid velocity in vessel (FTV-40A and FTV-40M), from -6 to $42 \mathrm{~s} \ldots \ldots \ldots \ldots \ldots$. . . . . . . . . . . . . . . . . . . . . .

235. Momentum flux in intact loop (FDU-1), from -6 to $42 \mathrm{~s} \ldots \ldots$. . . . . . 141

236. Momentum flux in intact loop (FDU-5), from -6 to $42 \mathrm{~s} \ldots \ldots 141$

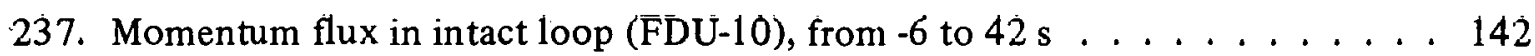

238. Momentum flux in intact loop (FDU-13), from -6 to $42 \mathrm{~s} \ldots \ldots 142$

239. Momentum flux in broken loop (FDB-21), from -6 to $42 \mathrm{~s} \ldots \ldots 143$

240. Momentum flux in broken loop (FDB-23), from -6 to $42 \mathrm{~s} \ldots \ldots 143$

241. Momentum flux in broken loop (FDB-30), from -6 to $42 \mathrm{~s} \ldots \ldots 44$

242. Momentum flux in broken loop (FDB-37), from -6 to $42 \mathrm{~s} \ldots \ldots$. . . . . . 144

243. Momentum flux in broken loop (FDB-42), from -6 to $42 \mathrm{~s} \ldots \ldots 145$

244. Momentum flux in core entrance (FDV-CORE-IN), from -6 to $42 \mathrm{~s} \ldots \ldots \ldots \ldots . \ldots \ldots 145$

245. Density in intact loop (GU-1T and GU-1B), from -20 to $300 \mathrm{~s} \ldots \ldots \ldots \ldots . \ldots \ldots$

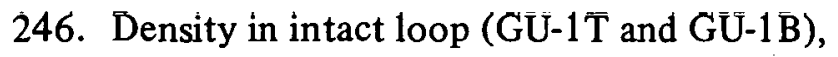

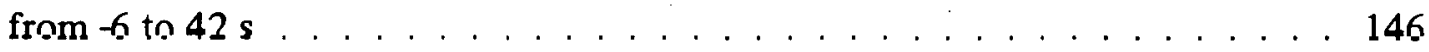

247. Density in intact loop (GU-1C), from -20 to $300 \mathrm{~s} \ldots \ldots \ldots 147$

248. Density in intact loop (GU-1C), from -6 to $42 \mathrm{~s} \ldots \ldots . \ldots 147$

249. Density in intact loop (GU-5VR and GU-10VR), from $-20 \mathrm{tn} 300 \mathrm{~s} \ldots \ldots \ldots \ldots \ldots . \ldots \ldots$

250. Density in intact loop (GU-5VR and GU-10VR), from -6 to $42 \mathrm{~s} \ldots \ldots \ldots \ldots \ldots . \ldots \ldots$ 
251. Density in intact loop (GU-13VR), from -20 to $300 \mathrm{~s} \ldots \ldots 149$

252. Density in intact loop (GU-13VR), from -6 to $42 \mathrm{~s} \ldots \ldots \ldots$. . . . . . 149

253. Density in intact loop (GU-15T and GU-15B),

from -20 to $300 \mathrm{~s} \ldots \ldots \ldots \ldots \ldots$

254. Density in intact loop (GU-15T and GU-15B),

from -6 to $42 \mathrm{~s} \ldots \ldots \ldots \ldots \ldots$

255. Density in intact loop (GU-15C), from -20 to $300 \mathrm{~s} \ldots \ldots \ldots 1$

256. Density in intact loop (GU-15C), from -6 to $42 \mathrm{~s} \ldots \ldots \ldots 151$

257. Density in broken loop (GB-21T and GB-21B),

from -20 to $300 \mathrm{~s} \ldots \ldots \ldots \ldots \ldots \ldots$. . . . . . . . . . . . . . . . . . . . .

258. Density in broken loop (GB-21T and GB-21B), from -6 to $42 \mathrm{~s} \ldots \ldots \ldots \ldots \ldots \ldots$. . . . . . . . . . . . . . . . . .

259. Density in broken loop (GB-21C), from -20 to $300 \mathrm{~s} \ldots \ldots \ldots \ldots$

260. Density in broken loop (GB-21C), from -6 to $42 \mathrm{~s} \ldots \ldots \ldots$

261. Density in broken loop (GB-23VR), from -20 to $300 \mathrm{~s} \ldots \ldots \ldots$

262. Density in broken loop (GB-23VR), from -6 to $42 \mathrm{~s} \ldots \ldots \ldots$

263. Density in broken loop (GB-30T and GB-30B), from -20 to $300 \mathrm{~s} \ldots \ldots \ldots \ldots \ldots$. . . . . . . . . . . . . . . . . 55

264. Density in broken loop (GB-30T and GB-30B), from -6 to $42 \mathrm{~s} \ldots \ldots \ldots \ldots \ldots \ldots \ldots$

265. Density in broken loop (GB-30C), from -20 to $300 \mathrm{~s} \ldots \ldots 156$

266. Density in broken loop (GB-30C), from -6 to $42 \mathrm{~s} \ldots \ldots \ldots$

267. Density in broken loop (GB-37), from -20 to $300 \mathrm{~s} \ldots \ldots \ldots$

268. Density in broken loop (GB-37), from -6 to $42 \mathrm{~s} \ldots \ldots \ldots$

269. Density in broken loop (GB-42VR), from -20 to $300 \mathrm{~s} \ldots \ldots \ldots$

270. Density in broken loop (GB-42VR), from -6 to $42 \mathrm{~s} \ldots \ldots \ldots$ 
271. Density in vessel (GV-COR-150HZ), from -20 to $300 \mathrm{~s} \ldots \ldots \ldots$

272. Density in vessel (GV-COR-150HZ), from -6 to $42 \mathrm{~s} \ldots \ldots$. . . . . . . 159

273. Density in vessel (GV-162/192D), from -20 to $300 \mathrm{~s} \ldots \ldots$. . . . . . 160

274. Density in vessel (GV-162/192D), from -6 to $42 \mathrm{~s} \ldots \ldots \ldots$

275. Density in vessel (GVLP-165HZ and GVLP-172HZ),

from -20 to $300 \mathrm{~s} \ldots \ldots \ldots \ldots \ldots$. . . . . . . . . . . . . . . . . . . .

276. Density in vessel (GVLP-165HZ and GVLP-172HZ),

from -6 to $42 \mathrm{~s} \ldots \ldots \ldots \ldots \ldots$. . . . . . . . . . . . . . . . . . . . . . .

277. Density in pressurizer (GU-PRIZE), from -20 to $300 \mathrm{~s} \ldots \ldots 162$

278. Density in pressurizer (GU-PRIZE), from -6 to $42 \mathrm{~s} \ldots \ldots 162$

279. Mass flow in intact loop (FDU-1, GU-1C), from -6 to $42 \mathrm{~s} \ldots \ldots$. . . . . 163

280. Mass flow in intact loop (FTU-1, GU-1C), from -20 to $300 \mathrm{~s} \ldots \ldots$. . . . . 164

281. Mass flow in intact loop (FTU-1, GU-1 C), from -6 to $42 \mathrm{~s} \ldots \ldots 4$

282. Mass flow in intact loop (FDU-5, GU-5VR), from -6 to $42 \mathrm{~s} \ldots \ldots 165$

283. Mass flow in intact loop (FTU-9, GU-10VR), from -20 to $300 \mathrm{~s} \ldots \ldots 166$

284. Mass flow in intact loop (FTU-9, GU-10VR), from -6 to $42 \mathrm{~s} \ldots \ldots 6$

285. Mass flow in intact loop (FDU-10, GU-10VR), from -6 to $42 \mathrm{~s} \ldots \ldots 7$

286. Mass flow in intact loop (FDU-13, GU-13VR), from -6 to $42 \mathrm{~s} \ldots \ldots 167$

287. Mass flow in intact loop (FTU-13, GU-13VR), from -20 to $300 \mathrm{~s} \ldots 168$

288. Mass flow in intact loop (FTU-13, GU-13VR), from -6 to $42 \mathrm{~s} \ldots 168$

289. Mass flow in in tact loop (FTU-15, GU-15C), from -20 to $300 \mathrm{~s} \ldots \ldots 9$

290. Mass flow in intact loop (FTU-15, GU-15C), from -6 to $42 \mathrm{~s} \ldots \ldots 9$

291. Mass flow in intact loop (FDB-21, GB-21C), from -6 to $42 \mathrm{~s} \ldots \ldots 170$

292. Mass flow in broken loop (FTB-21, GB-21C), from -20 to $300 \mathrm{~s}$. . . . . . 171 
293. Mass flow in broken loop (FTB-21, GB-21C), from -6 to $42 \mathrm{~s} \ldots \ldots$. . . . 171

294. Mass Flow in broken loop (FDB-23, GB-23VR), from -6 to $42 \mathrm{~s} \ldots \ldots$. . . . 172

295. Mass flow in broken loop (FDB-30, GB-30C), from -6 to $42 \mathrm{~s} \ldots \ldots$. . . . . 172

296. Mass flow in broken loop (FTB-30, GB-30C), from -20 to $300 \mathrm{~s} \ldots \ldots$. . . . 173

297. Mass flow in broken loop (FTB-30, GB-30C), from -6 to $42 \mathrm{~s} \ldots \ldots \ldots$

298. Mass flow in broken loop (FDB-37, GB-37), from -6 to $42 \mathrm{~s} \ldots \ldots$. . . . . . 174

299. Mass flow in broken loop (FTB-37, GB-37), from -20 to $300 \mathrm{~s} \ldots \ldots$

300. Mass flow in broken loop (FTB-37, GB-37), from -6 to $42 \mathrm{~s} \ldots \ldots$. . . . 175

301. Mass flow in broken loop (FDB-42, GB-42VR), from -6 to $42 \mathrm{~s} \ldots \ldots 176$

302. Mass flow in vessel (FDV-CORE-IN, GV-COR-150HZ), from -6 to $42 \mathrm{~s} \ldots \ldots \ldots \ldots \ldots$. . . . . . . . . . . . . . . . . . . . . . .

303. Mass flow in vessel (FTV-CORE-IN, GV-COR-150HZ), from -20 to $300 \mathrm{~s} \ldots \ldots \ldots \ldots \ldots$. . . . . . . . . . . . . . . . . . . . .

304. Mass flow in vessel (FTV-CORE-IN, GV-COR-150HZ), from -6 to $42 \mathrm{~s} \ldots \ldots \ldots \ldots \ldots$

305. Mass flow in pressurizer (FTU-PRIZE, GU-PRIZE), from -20 to $300 \mathrm{~s} \ldots \ldots \ldots \ldots \ldots$. . . . . . . . . . . . . . . . . . . .

306. Mass flow in pressurizer (FTU-PRIZE, GU-PRIZE), from -6 to $42 \mathrm{~s} \ldots \ldots \ldots \ldots \ldots \ldots \ldots$. . . . . . . . . . . . . . . . . . . . . .

307. Core heater rod total power (PWRCOR T-1 and PWRCOR T-2), from -20 to $300 \mathrm{~s} \ldots \ldots \ldots \ldots . \ldots \ldots$

308. Core heater rod total power (PWRCOR T-1 and PWRCOR T-2), from -6 to $42 \mathrm{~s} \ldots \ldots \ldots \ldots$. . . . . . . . . . . . . 179

309. Core heater voltage (VOLTCOR-T), from -20 to $300 \mathrm{~s} \ldots \ldots \ldots$

310. Core heater voltage (VOLTCOR-T), from -6 to $42 \mathrm{~s} \ldots \ldots \ldots$

311. Corc heatcr total current (AMPCOR-T), from -20 to $300 \mathrm{~s} \ldots \ldots \ldots 1$

312. Core heater total current (AMPCOR-T), from -6 to $42 \mathrm{~s} \ldots \ldots \ldots 1$ 
313. Primary pump current (PUMPU-CUR), from -20 to $300 \mathrm{~s} \ldots \ldots$. . . . . 182

314. Primary pump current (PUMPU-CUR), from -6 to $42 \mathrm{~s} \ldots \ldots . \ldots 182$

315. Primary pump speed (PUMPU-RPM), from -20 to $300 \mathrm{~s} \ldots \ldots$. . . . . 183

316. Primary pump speed (PUMPU-RPM), from -6 to $42 \mathrm{~s} \ldots \ldots 183$

B-1. Geometry used for processsing of density data obtained from two-beam gamma densitometers . . . . . . . . . . . . . . . . . 199

C-1. Fluid temperature in broken loop (TFB-23) . . . . . . . . . . . 208

C-2. Fluid temperature in downcomer annulus (TFV-ANN-35A) $\ldots \ldots . \ldots 208$

C-3. Fluid temperature in lower plenum (TFV-LP-8) . . . . . . . . . . 209

C-4. Fluid temperature in lower plenum (TFV-LP-15) . . . . . . . . . . . . 209

C-5. Fluid temperature in lower plenum (TFV-LP-22) . . . . . . . . . . 210

C-6. Fluid temperature in core, Grid Spacer 5 (TFG-5CD-45) . . . . . . . . 210

C-7. Fluid temperature in steam generator (TFU-SG3) . . . . . . . . . 211

C-8. Fluid temperature in pressurizer surge line (TFU-PRIZE) . . . . . . . . 211

C.-9. Core heater temperature, Rod E-4 (TH-E4-09) . . . . . . . . . . 212

C-10. Core heater temperature, Rod E-4 (TH-E4-27) . . . . . . . . . . 212

C-1 1. Core heater temperature, Rod E-4 (TH-E4-55) . . . . . . . . . . 213

C-12. Pressure in intact loop, Spool 13 (PU-13) f . . . . . . . . . . 213

C-13. Pressure in broken loop, Spool 23 (PB-23) . . . . . . . . . . . . . 214

C-14. Differential pressure in intact loop (IPU-6-SGIP) . . . . . . . . 214

C-15. Differential pressure in intact loop (DPU-SGOP-7) . . . . . . . . . 215

C-16. Differential pressure in intact loop (DPU-7-10) . . . . . . . . 215

C-17. Differential pressure in intact loop (DPU-12-10) . . . . . . . . 216

C-18. Differential pressure in intact loop (DPU-12-15) . . . . . . . . . 216 
C-19. Volumetric flow in intact loop (FTU-1) . . . . . . . . . . . 217

C-20. Volumetric flow in intact loop (FTU-15) $\ldots \ldots \ldots \ldots \ldots . \ldots 217$

$\mathrm{C}-21$. Volumetric flow in intact loop high pressure injection line

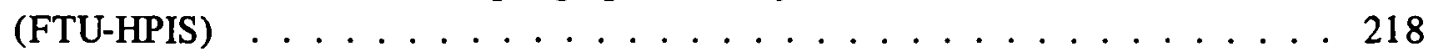

$\mathrm{C}-22$. Volumetric flow in intact loop low pressure injection line

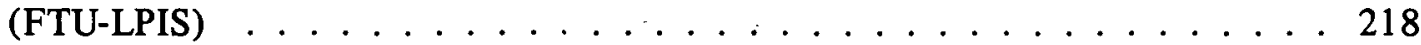

$\mathrm{C}-23$. Volumetric flow in intact loop accumulator discharge line (FTU-ACC1) . . . . . . . . . . . . . . . . . . . . 219

C-24. Fluid velocity in vessel (FTV-40A) f . . . . . . . . . . . . . . . . . 219

C-25. Fluid velocity in vessel (FTV-40M) $\ldots \ldots \ldots \ldots \ldots \ldots$

C-26. Density in intact loop (GU-1T) $\ldots \ldots \ldots \ldots \ldots \ldots \ldots \ldots$

C-27. Density in intact loop (GU-1B) $\ldots \ldots \ldots \ldots \ldots \ldots \ldots \ldots \ldots \ldots$

C-28. Density in intact loop $(\mathrm{GU}-1 \mathrm{C}) \quad \ldots \ldots \ldots \ldots \ldots \ldots \ldots \ldots$

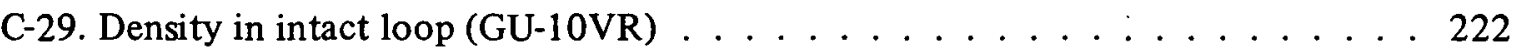

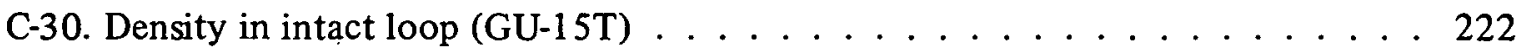

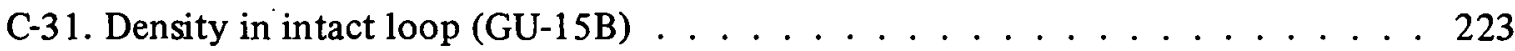

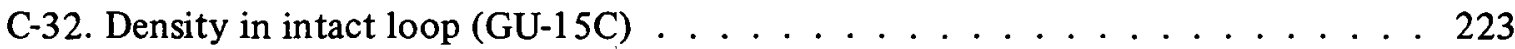

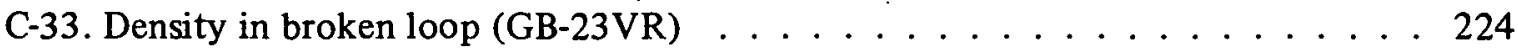

C-34. Density in vessel (GV-COR-150HZ) . . . . . . . . . . . . . . . . 224

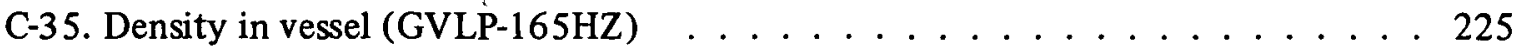

C-36. Density in vessel (GVLP-172HZ) $\ldots \ldots \ldots \ldots \ldots \ldots \ldots$

C-37. Mass flow in in tact loop (FTU-1, GU-1C) $\ldots \ldots \ldots \ldots . \ldots 226$

C-38. Mass flow in intact loop (FDU-5, GU-5VR) $\ldots \ldots \ldots \ldots \ldots \ldots$

C-39. Mass flow in intact loop (FTU-13, GU-13VR) . . . . . . . . . . . . . 227

C-40. Mass flow in intact loop (FTU-15, GU-15C) . . . . . . . . . . . . 227 
C-41. Mass flow in broken loop (FDB-21, GB-21C) . . . . . . . . . 228

C-42. Mass flow in broken loop (FTB-21, GB-21C) . . . . . . . . . . . 228

C-43. Mass flow in vessel (FTV-CORE-IN, GV-COR-150HZ) . . . . . . . . . . . 229

\section{TABLES}

I. Conditions at Blowdown Initiation $\ldots \ldots \ldots \ldots$

II. Primary Coolant Temperature Distribution

Prior to Rupture . . . . . . . . . . . . . . . . . . . 7

III. Water Chemistry Prior to Blowdown ................ 8

IV. Sequence of Events During Test S-06-4 . . . . . . . . . . . . . . 9

V. Data Presentation for Semiscale Mod-1 Test S-06-4 . . . . . . . . 17

B-I. Constants for Pressure Measurement Corrections (Test S-06-4) . . . . . . . . . . . . . . . . . . . . 192

B-II. Constants for Differential Pressure Measurement Corrections (Test S-06-4) . . . . . . . . . . . . . . . . . 194

B-III. Constants for Momentum Flux Measurement Corrections (Test S-06-4) . . . . . . . . . . . . . . . 196

B-IV. Constants for Density Measurement Conversions to Engineering Units (Test S-06-4) f . . . . . . . . . . . . . . . . . 198

C-I. Random Error Variance (Test S-06-4) . . . . . . . . . . . . . . 203

C-II. General Measurement Engineering Error Sources and Error Values (Test S-06-4) . . . . . . . . . . . . . 230

C-III. Time Periods when Flow Regime Errors were Applied

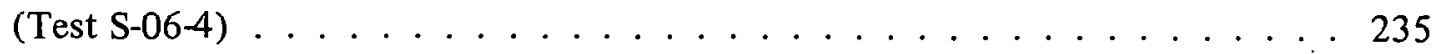




\section{EXPERIMENT DATA REPORT FOR SEMISCALE MOD-1}

\section{TEST S-06-4}

\section{(LOFT COUNTERPART TEST)}

\section{INTRODUCTION}

The Semiscale Mod-1 experiments represent the current phase of the Semiscale Program conducted by EG\&G Idaho, Inc. for the United States Government. The program, which is sponsored by the Nuclear Regulatory Commission through the Department of Energy, is part of the overall program designed to investigate the response of a pressurized water reactor system to a hypothesized loss-of-coolant accident (LOCA). The underlying objectives of the Semiscale Program are to quantify the physical processes controlling system behavior during a LOCA and to provide an experimental data base for assessing reactor safety evaluation models. The Semiscale Mod-1 program has the further objective of providing support to other experimental programs in the form of instrumentation assessment, optimization of test series, selection of test parameters, and evaluation of test results.

Test S-06-4 was conducted August 3.1, 1977 in the Semiscale Mod-1 system as part of the LOFT counterpart test series (Test Series 6), which was designed to obtain thermal-hydraulic response data from blowdown, refill, and reflood transients in a simulated nuclear reactor with a heated core to assist the LOFT Program in the planning of the first LOFT nuclear test series. Test S-06-4 was conducted specifically to assist the LOFT Program in assessing core response effects when the LOFT system, operating at $100 \%$ core power $(52.5 \mathrm{~kW} / \mathrm{m})$, is subjected to a postulated LOCA.

The purpose of this report is to present the test data in an uninterpreted but readily usable form for use by the nuclear community in advance of detailed analysis and interpretation. Section II briefly describes the system configuration, procedures, initial test conditions, and events that are applicable to Test S-06-4; Section III presents the data graphs and provides comments and supporting information necessary for interpretation of the data. A description of the overall Semiscale Program and test series, a more detailed description of the Semiscale Mod-1 system, and a description of the measurement and data processing techniques and uncertainties can be found in Reference 1 . 


\section{SYSTEM, PROCEDURES, CONDITIONS, AND EVENTS FOR TEST S-06-4}

The following system configuration, procedures, initial test conditions, and events are specific to Test S-06-4 as indicated.

\section{SYSTEM CONFIGURATION AND TEST PROCEDURES}

The Semiscale Mod-1 system used for this test consisted of a pressure vessel with internals, including a 40-rod core with 36 electrically heated rods; an intact'loop with steam generator, pump, and pressurizer; a broken loop with simulated steam generator, simulated pump, simulated reflood bypass lines, LOFT counterpart nozzles ${ }^{[a]}$, and two rupture assemblies; a coolant injection accumulator for the intact loop; high and low pressure coolant injection pumps for the intact loop; and a pressure suppression system with a suppression tank, header, and heated steam supply system. Semiscale Mod-1 experimental system configuration information is provided in" Reference 1 . Figures 1 and 2 show the system configuration for Test S-06-4.

For Test S-06-4, the 40-rod electrically heated core was operated at an axial peak power density which was $100 \%$ of the maximum peak power density $(52.5 \mathrm{~kW} / \mathrm{m})$. Four rods (Rods D-4, D-5, E-4, and E-5) were operated at approximately $52.5 \mathrm{~kW} / \mathrm{m}, 32$ rods were operated at approximately $33.2 \mathrm{~kW} / \mathrm{m}$, and 4 rods (Rods $\mathrm{C}-4 ; \mathrm{D}-6, \mathrm{~F}: 2$, and F-5) were unpowered to simulate LOFT passive rod locations. This configuration yielded a peaked power profile which simulates that of LOFT and provides a total core power of approximately $1.33 \mathrm{MW}$.

In preparation for the test, the intact loop acrismularor" was fllled with trieded demineralized water, drained to the specified initial level, and pressurized with nitrogen to $4198 \mathrm{kPa}$. The system was filled with treated demineralized water and vented at strategic points to ensure a liquid-full system. Prior to warmup the system was pressurized to check for leakage, system instrumentation was checked, and transducer readings were initialized. Warmup to initial test conditions was accomplished with the heaters in the vessel core. Heatup of the broken loop piping was accomplished with bypass lines which served to allow circulation through the broken loop. During warmup, the purilication and sampling systems were valved into the primary system to maintain water chemistry requirements and to provide a water sample at system conditions for subsequent analysis. At 56-K-temperature intervals during warmup, detector readings were sampled to allow the integrity of the measurement instrumentation and the operability of the data acquisition system to be checked:

[a] The Semiscale Mod-1 system normally utilizes a break nozzle of the convergingdiverging type referred to as a Henry nozzle. Reference 2 provides an illustration of the LOFT counterpart break nozzle. 


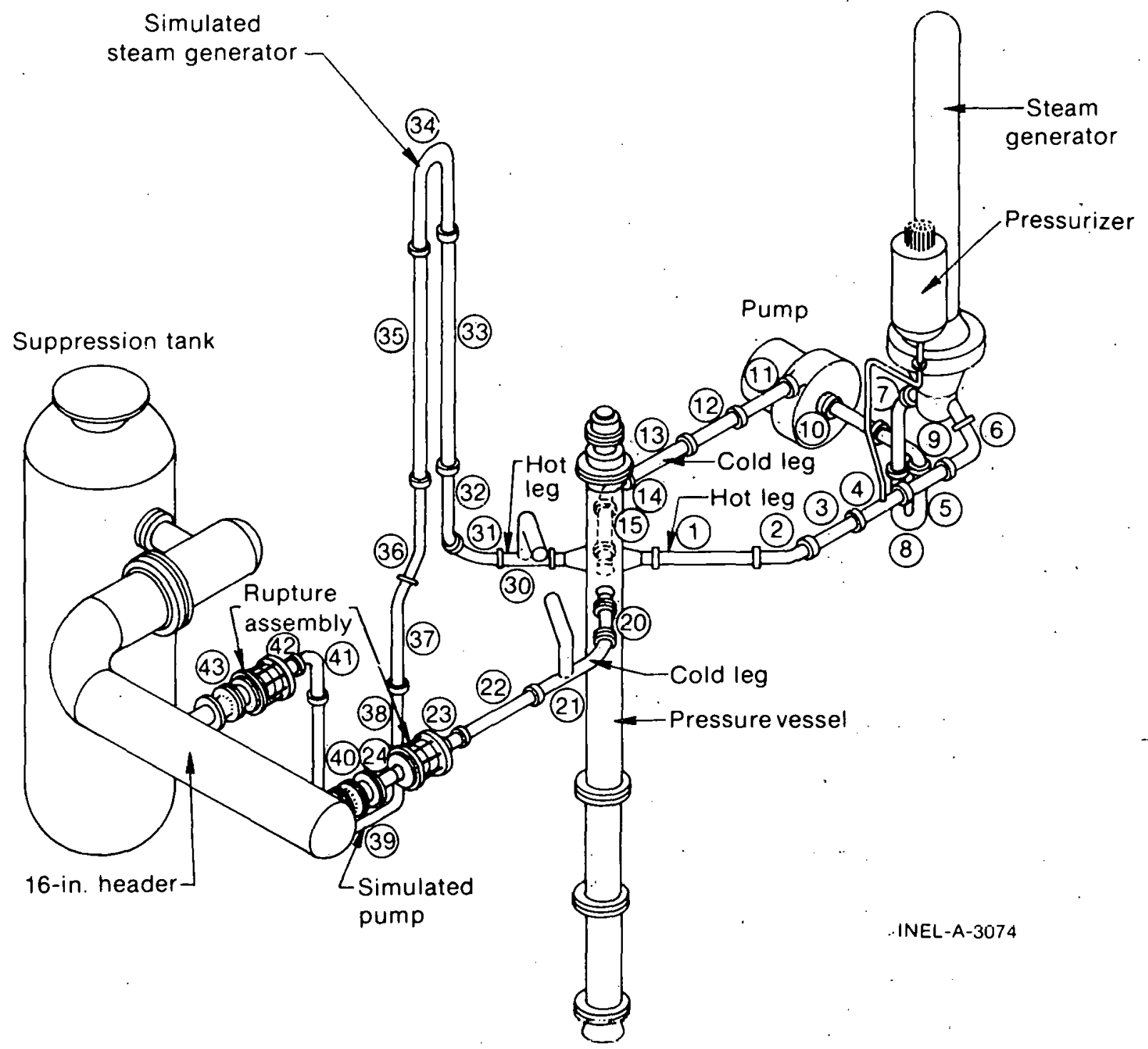

Fig. I Semiscale Mod-1 system for cold leg break configuration -isometric. 


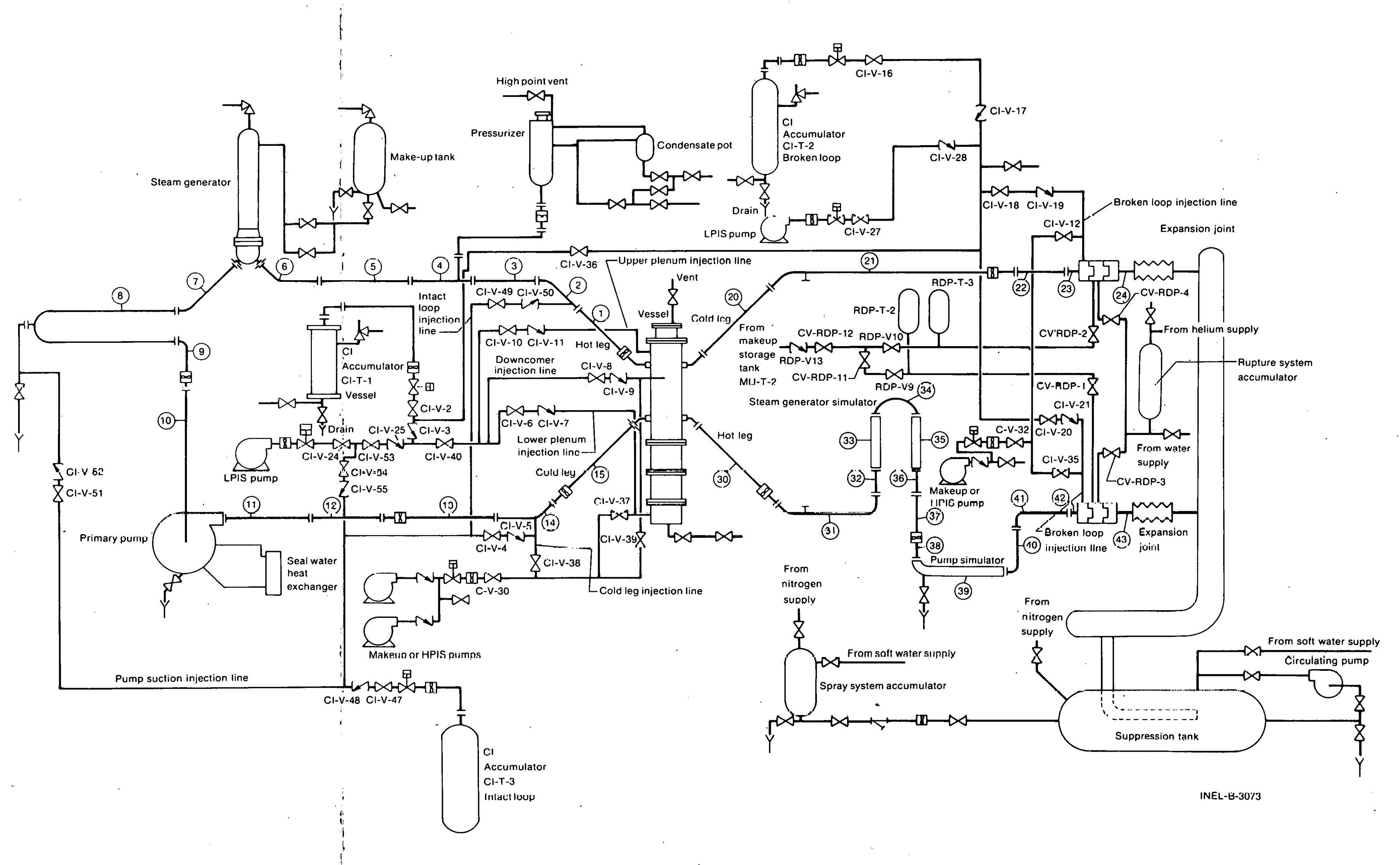

Fig. 2 Semiscale Mod-1 system for cold leg break configuration -schematic. 
Prior to the initial core power level being established, the pressure suppression system was pressurized to $252 \mathrm{kPa}$ with saturated steam from the steam supply system. After the core power was increased to $1328 \mathrm{~kW}$, initial test conditions were held for $3845 \mathrm{~s}$ to establish equilibrium in the system. At the end of this period all auxiliary systems including the bypass lines were isolated to prevent blowdown through those systems.

The system was successfully subjected to a simulated double-ended cold leg break through two rupture assemblies and two LOFT counterpart nozzles, each having a break area of $0.000243 \mathrm{~m}^{2}$. Pressure to operate the rupture assemblies and initiate blowdown was taken from an accumulator system filled with water and pressurized to $15600 \mathrm{kPa}$. Immediately (within $0.02 \mathrm{~s}$ ) after initiation of blowdown, the lines to the accumulator were again isolated. The effluent was ejected from the primary system into the pressure suppression system which was vented to maintain a constant pressure of $252 \mathrm{kPa}$. During the blowdown transient, power to the electrically heated core was automatically controlled to simulate the thermal response of nuclear heated fuel rods. The steam generator feedwater flow and steam control valves were also automatically controlled to aid in the LOFT simulation. Throughout the test, the primary coolant circulation pump speed was maintained at a constant level.

For Test S-06-4, the coolant injection systems were arranged to discharge into the intact loop cold leg injection point (Spool 14). The high pressure coolant injection pump began at blowdown $(0 \mathrm{~s})$ and continued until test termination $(300 \mathrm{~s})$. Coolant injection from the intact loop accumulator started approximately $18 \mathrm{~s}$ after rupture at a system pressure of $4200 \mathrm{kPa}$ and continued to depletion at $68 \mathrm{~s}$ after blowdown. Low pressure coolant injection began $25 \mathrm{~s}$ after blowdown at a system pressure of $1655 \mathrm{kPa}$ and continued until test termination (300 s).

\section{INITIAL TEST CONDITIONS AND SEQUENCE OF EVENTS}

Conditions in the Semiscale Mod-1 system at initiation of blowdown are given in Tables I and II, the primary system water chemistry prior to blowdown is given in Table III, and the sequence of events relative to rupture is given in Table IV. 


\section{TABLE I}

CONDITIONS: AT BLOWDOWN.. INITIATION:

Test S-06-4

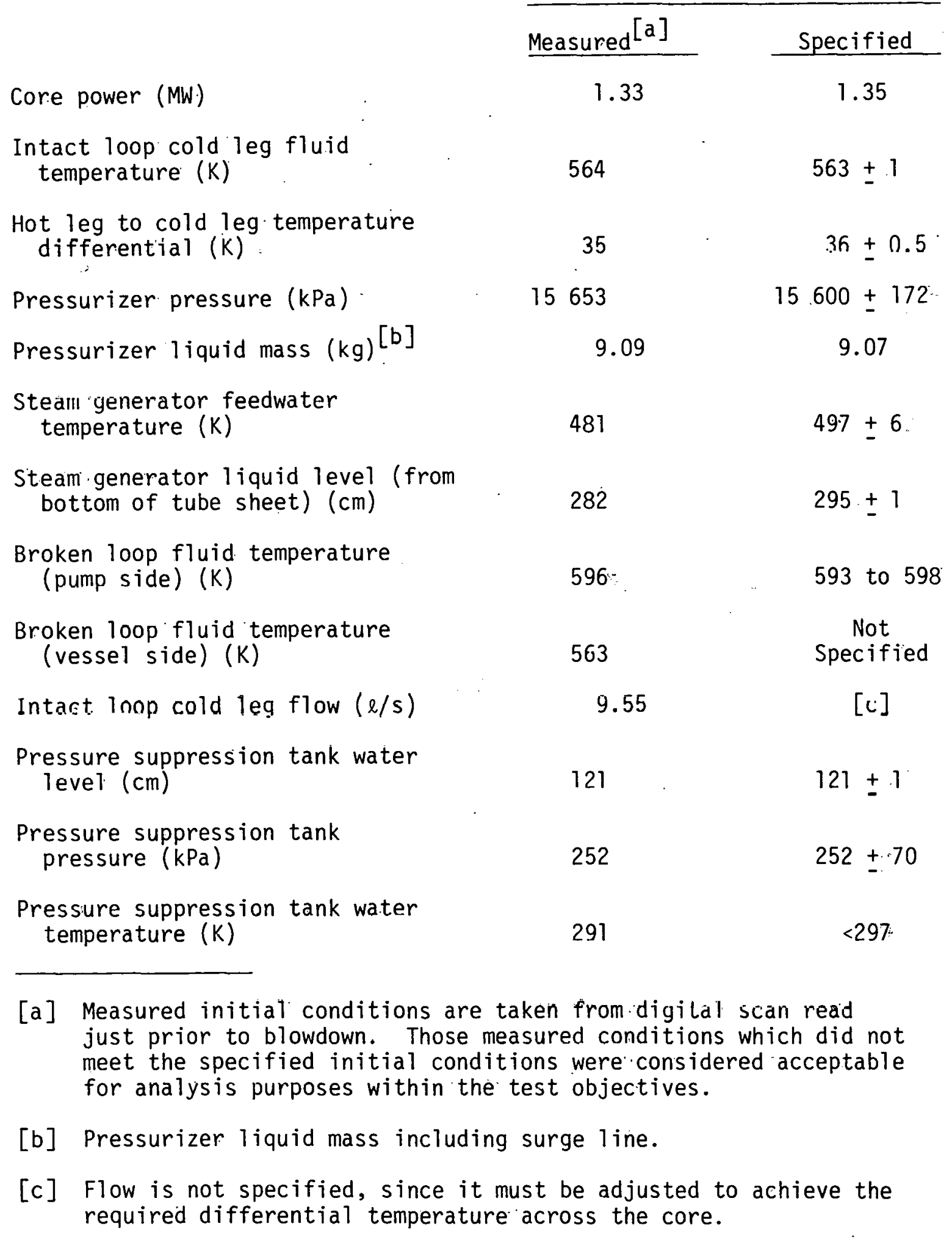


TABLE II

PRIMARY COOLANT TEMPERATURE DISTRIBUTION PRIOR TO RUPTURE[a]

\begin{tabular}{|c|c|c|}
\hline & \multicolumn{2}{|c|}{ Test S-06-4 } \\
\hline & Detector & Temperature (K) \\
\hline $\begin{array}{l}\text { Vessel lower plenum (upper portion } \\
\text { above filler block) }\end{array}$ & TFV-LP-22 & 563 \\
\hline Intact loop hot leg (near vessel) & $\mathrm{RBU}-2$ & 599 \\
\hline Intact loop cold leg (near pump inlet) & TFU-10 & 563 \\
\hline Intact loop cold leg (near vessel) & RBU-74A & 564 \\
\hline Broken loop cold leg (near nozzle) & TFB -23 & 563 \\
\hline Broken loop hot leg (near vessel) & TFB-30 & 596 \\
\hline Broken loop cold leg (near nozzle) & TFB -42 & 593 \\
\hline $\begin{array}{l}\text { Reflood bypass line hot leg (near } \\
\text { end cap) }\end{array}$ & TFB-RFBH & 599 \\
\hline $\begin{array}{l}\text { Reflood bypass line cold leg (near } \\
\text { end cap) }\end{array}$ & TFB-RFBC & 557 \\
\hline
\end{tabular}


TABLE III

WATER CHEMISTRY PRIOR TO BLOWDOWN ${ }^{\text {[a] }}$

\section{Test S-06-4}

$\mathrm{pH}$

9.6

Conductivity $(\mu \mathrm{S} / \mathrm{cm})$

60

Lithium $(\mu \mathrm{g} / \mathrm{m} \ell)$

4.4

Chlorides (ppm)

1.39

Fluorides $(\mu \mathrm{g} / \mathrm{ml})^{[b]}$

$<0.53$

Oxygen $(\mathrm{cc} / \mathrm{l})$

0.3

Total gas $(\mathrm{cc} / \mathrm{l})$

96.3

Suspended solids $(\mu \mathrm{g} / \mathrm{ml})$

1.25

[a] Water sample taken at a system pressure of approximately $15600 \mathrm{kPa}$ and a system temperature of approximately $555 \mathrm{~K}$ (cold leg).

[b] Present analytical methods prevent accurate determination of fluorides at concentrations of less than $0.4 \mu \mathrm{g} / \mathrm{m} \ell$. 


\section{TABLE IV}

SEQUENCE OF EVENTS DURING TEST S-06-4[a]

Time Relative

\section{Event}

Core power level established $-3845$

Bypass lines valved out of system $-2.5$

Blowdown initiated

High pressure injection system pumps started [b]

ECC accumulators valved in

Steam generator feedwater and discharge valves switched to automatic flow control

Core power decay transient started

3

Low pressure injection system pumps started $[\mathrm{b}]$

25

Core power tripped off $[\mathrm{c}]$

[a] A time-controlled sequencer was used to control critical events during the test.

[b] Injection from ECC accumulators and high and low pressure injection system pumps does not start until system pressure drops below accumulator or pump pressure, respectively.

[c] Core power tripped manually at termination of test. 


\section{DATA PRESENTATION}

The data from Semiscale Mod-1 Test S-06-4 are presented with brief comment. Processing analysis has been performed only to the extent necessary to obtain appropriate engineering units and to ensure that the data are reasonable and consistent. In all cases, in converting transducer output to engineering units, a homogeneous fluid was assumed. Further interpretation and analysis should consider that sudden decompression processes such as those occurring during blowdown may have subjected the measurement devices to nonhomogeneous fluid conditions.

The performancc of the system during Test S-06-4 was monitored by 225 detectors. The data obtained were recorded on both digital and analog data acquisition systems. The digital system was used to process the data presented in this report. The digilul data werc recorded at a sample rate of 57.5 points per second. Long-term data ( -20 to $300 \mathrm{~s}$ ) were compressed at a 20 to 1 ratio giving an effective sample ratc of 2.875 points per sccond. Short-term data and plots ( -6 to $42 \mathrm{~s}$ ) were compressed at a 3 to 1 ratio giving an effective sample rate of 19.17 points per second.

The data are presented in some instances in the form of composite graphs to facilitate comparison of the values of given variables at several locations. The scales selected for the graphs do not reflect the obtainable resolution of the data. (The data processing techniques are described further in Reference 1 and Appendix A).

Figures 3 through 8 and Table $V$ provide supporting information for interpretation of the data graphs shown in Figures 9 through 316, and provide relative locations of all detectors used during Test S-06-4. Table V groups the measurements according to measurement type, identifies the specific measurement location and range of the detcctor and actual recording range of the data acquisition system, provides brief s.nmments regarding the data, and references the measurements and comments to the corresponding figure. Figures 9 through 316 present all the blowdown and reflood data obtained: Timé zero on the graphs is the time of rupture initiation. Appendix A provides information explaining the data acquisition system capabilities. Appendix B explains posttest datâ processing for data conversion into engineering units and data adjustments. Presented in Appendix $C$ is an analysis of selected dala which provide a guide to the uncertainty associated with data measurements in the Semiscale Mod-1 system. 


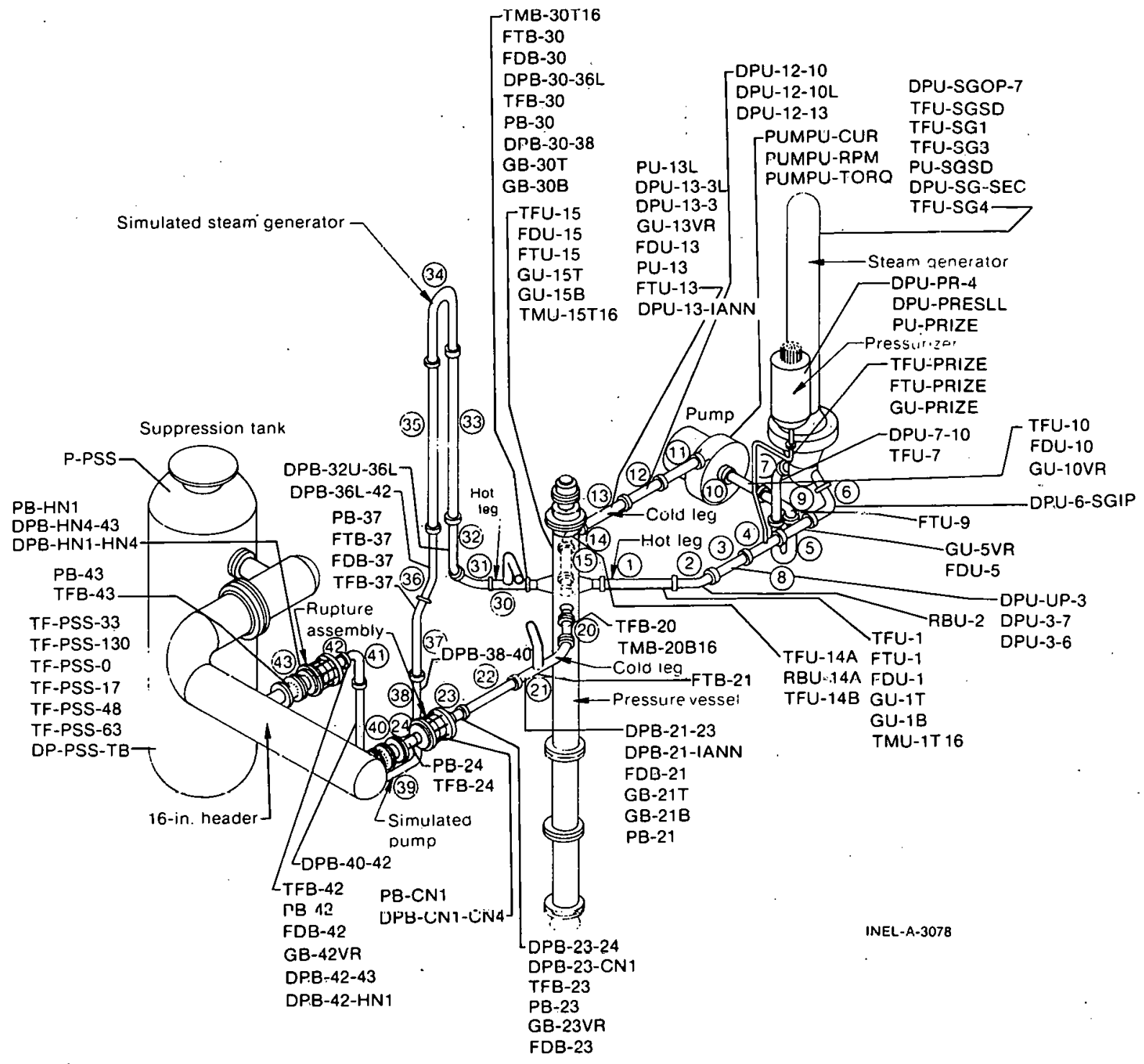

Fig. 3 Semiscale Mod-1 system and instrumentation for cold leg break configuration -- isometric. 


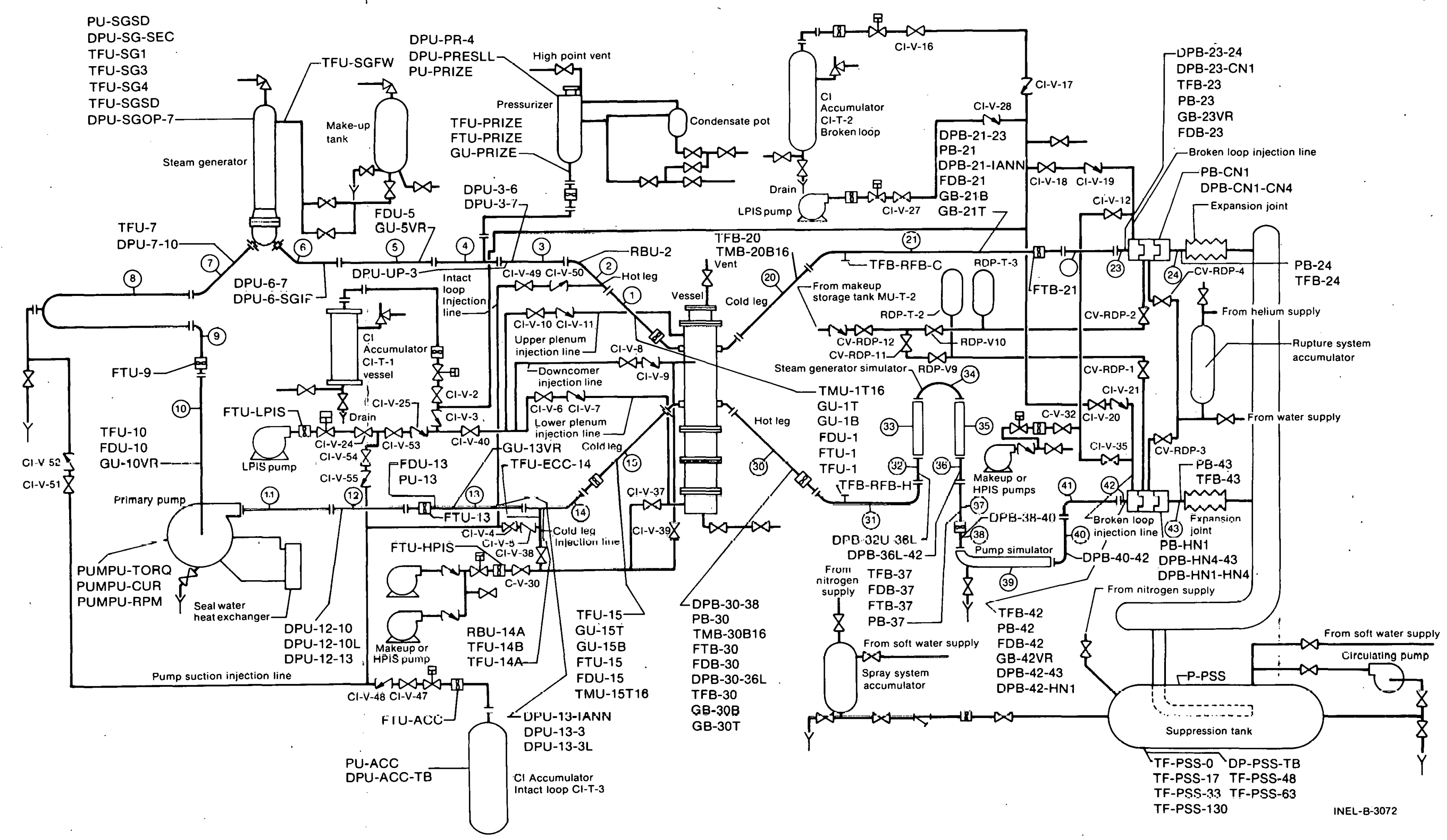

Fig. 4 Semiscale Mod-1 system and instrumentation for cold leg break configuration -- schematic. 


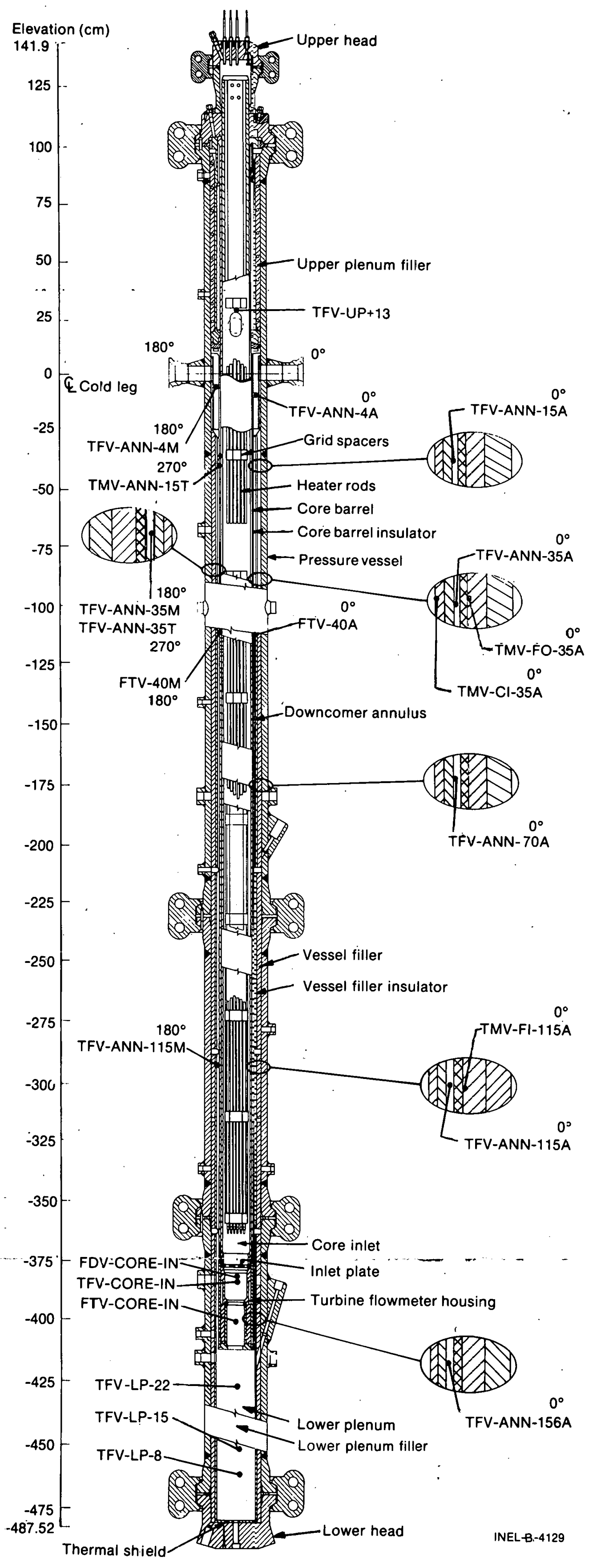

Fig. 5 Semiscale Mod-1 pressure vesse1 -- cross section showing instrumentation. 
Distance from

cold leg \& $(\mathrm{cm})$

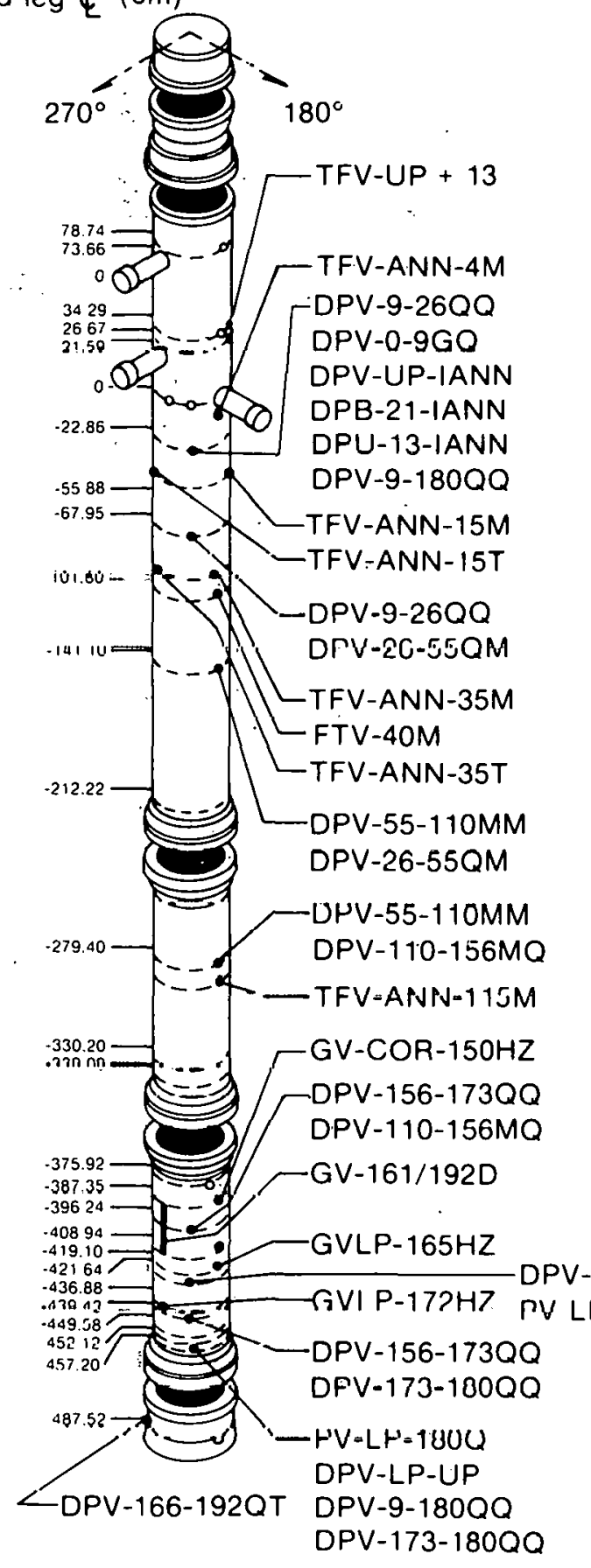

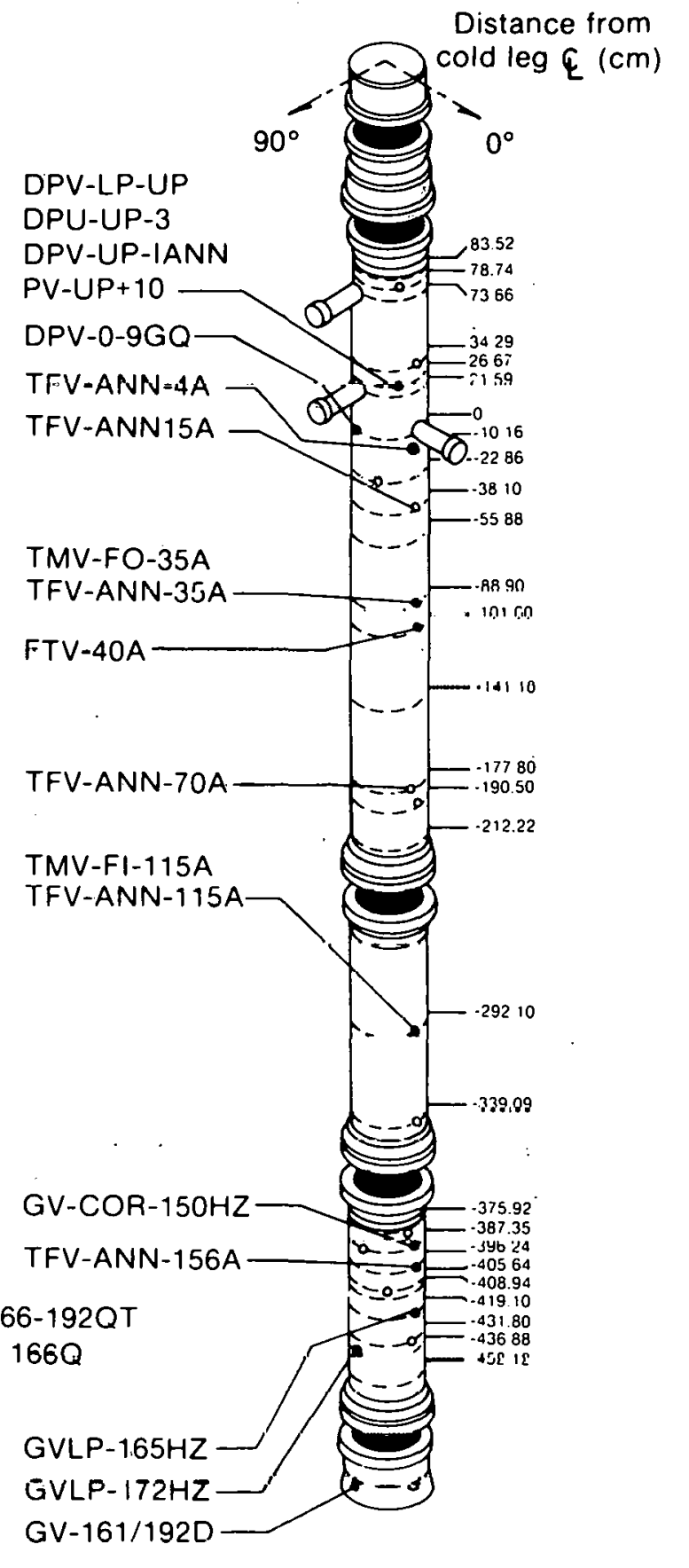

INEL-A-3076

Fig. 6 Semiscale Mod-1 pressure vesse] -- isometric showing instrumentation. 


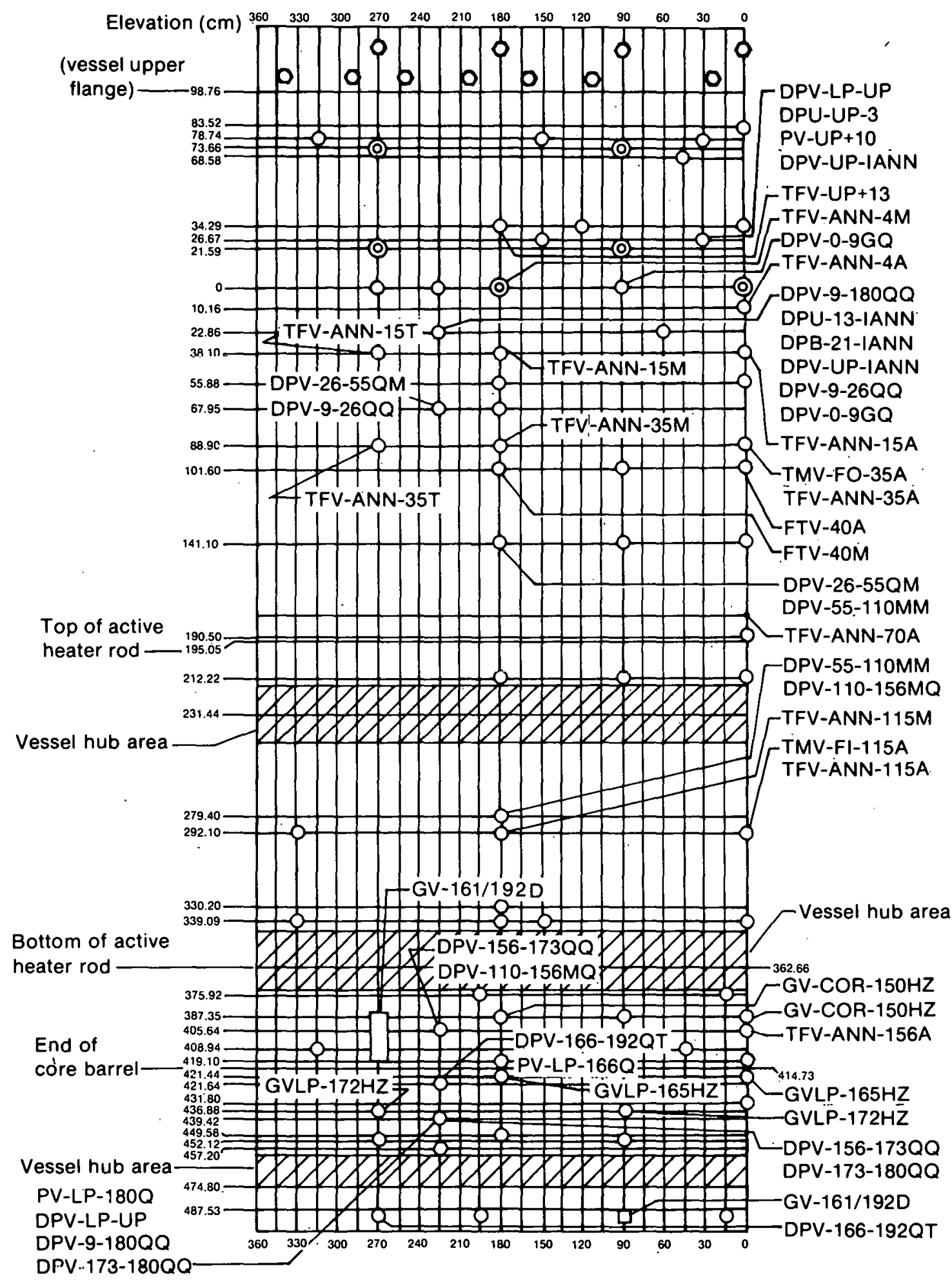

INEL-A-3077

Fig. 7 Semiscale Mod-1 pressure vessel -- penetrations and instrumentation. 
A B $\quad$ C $\quad$ D $\quad E \quad$ F $\quad G \quad H$.

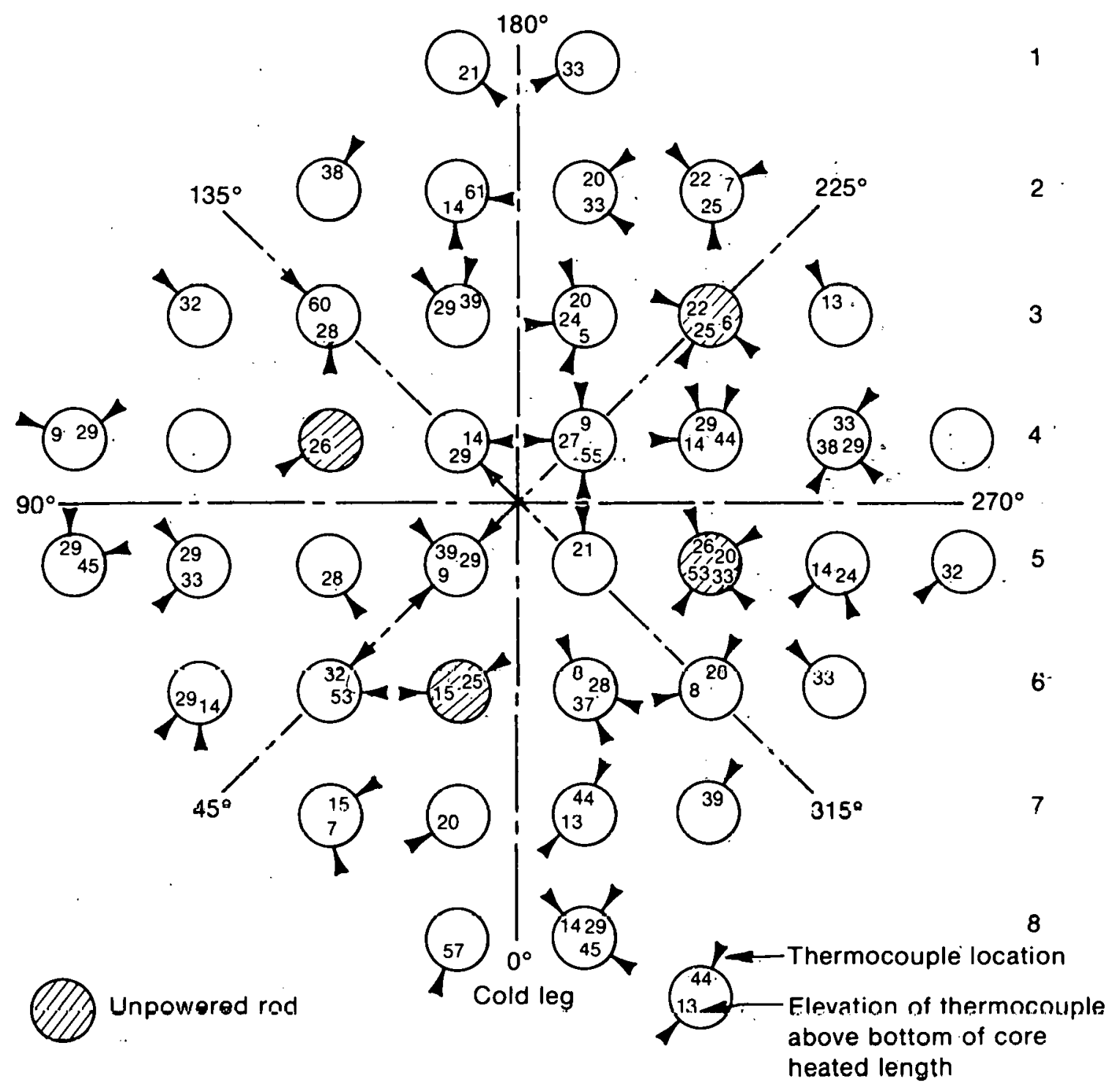

INEL-A-3075

Fig. 8 Semiscale Mod-1 heated core.-- plan view. 
TABLE V

DATA PRESENTATION FOR SEMISCALE MOD-1 TEST S-06-4

\begin{tabular}{|c|c|c|c|c|c|}
\hline \multirow[b]{2}{*}{ Measurement } & \multirow[b]{2}{*}{ Location and Corments $[\mathrm{a}]$} & \multicolumn{2}{|c|}{ Range $^{[\mathrm{a}]}$} & \multirow{3}{*}{ Figure $^{[\mathrm{a}]}$} & \multirow[b]{2}{*}{ Measurement Comments $[\mathrm{b}]$} \\
\hline & & Detector & $\begin{array}{l}\text { Data Acquisition } \\
\text { System }\end{array}$ & & \\
\hline FLUID TEMPERATURE & $\begin{array}{l}\text { Chromel-Alumel thermocouples unless } \\
\text { specified otherwise. }\end{array}$ & & & & \\
\hline Intact loop & & 0 to $1533 \mathrm{~K}$ & 0 to $584 \mathrm{~K}$ & & \\
\hline TFU-I & $\begin{array}{l}\text { Hot leg, Spool 1, } 54 \mathrm{~cm} \text { from vessel } \\
\text { center. }\end{array}$ & & 0 to $820 \mathrm{~K}$ & 9,10 & \\
\hline RBU-2 & $\begin{array}{l}\text { Hot leg, Spool } 2,117 \mathrm{~cm} \text { from vessel } \\
\text { center (platinum resistance bulb). }\end{array}$ & 0 to $811 \mathrm{k}$ & 0 to $811 \mathrm{~K}$ & 9,10 & \\
\hline TFU-7 & $\begin{array}{l}\text { Cold leg, Spool 7, } 624 \mathrm{~cm} \text { from vessel } \\
\text { center. }\end{array}$ & & & 11,12 & \\
\hline TFU-10 & $\begin{array}{l}\text { Cold leg, Spool } 10,367 \mathrm{~cm} \text { from } \\
\text { vessel center. }\end{array}$ & & & 11,12 & \\
\hline$R B U-14 A$ & $\begin{array}{l}\text { Cold leg, Spool } 14,109 \mathrm{~cm} \text { from } \\
\text { vessel center, upstream of cold leg } \\
\text { injection port (platinum resistance } \\
\text { bulb). }\end{array}$ & 0 to $811 \mathrm{k}$ & 0 to $811 \mathrm{k}$ & 13,14 & \\
\hline TFU-14B & $\begin{array}{l}\text { Cold leg. Spool } 14,99 \mathrm{~cm} \text { from } \\
\text { vessel center, downstreami of cold } \\
\text { leg injection port. }\end{array}$ & & & 13,14 & \\
\hline TFU-15 & $\begin{array}{l}\text { Cold leg, Spool } 15,54 \mathrm{~cm} \text { from } \\
\text { vessel center. }\end{array}$ & $\cdot$ & & 13,14 & \\
\hline Broken Loop & & 0 to $1533 \mathrm{~K}$ & 0 to $820 \mathrm{~K}$ & & \\
\hline$T F B-20$ & $\begin{array}{l}\text { Cold leg, Spool } 20,52 \mathrm{~cm} \text { from } \\
\text { vessel center. }\end{array}$ & & & 15,16 & \\
\hline TFB-23 & $\begin{array}{l}\text { Cold leg, Spool } 23,232 \mathrm{~cm} \text { from } \\
\text { vessel center, upstream of vessel- } \\
\text { side nozzle. }\end{array}$ & & & 15,16 & .. \\
\hline TFB-24 & $\begin{array}{l}\text { Cold leg, Spool } 24,264 \mathrm{~cm} \text { from } \\
\text { vessel center, downstream from } \\
\text { vessel-side nozzle. }\end{array}$ & & & 15,16 & . \\
\hline TFB-30 & $\begin{array}{l}\text { Hot leg, Spool } 30,40 \mathrm{~cm} \text { from } \\
\text { vessel center. }\end{array}$ & & & 17,18 & \\
\hline TFB-37 & $\begin{array}{l}\text { Cold leg, Spool } 37,703 \mathrm{~cm} \text { from } \\
\text { vessel center along hot leg, dis- } \\
\text { charge of simulated steam generator. }\end{array}$ & & & 17,18 & $\cdot$ \\
\hline TFB-42 & $\begin{array}{l}\text { Cold leg, Spool } 42,1054 \mathrm{~cm} \text { from } \\
\text { vessel center along hot leg, upstream } \\
\text { of pump-side nozzle. }\end{array}$ & & & $17, .18$ & \\
\hline TFB-13 & $\begin{array}{l}\text { cold leg, Spool } 43,1086 \mathrm{~cm} \text { from } \\
\text { vessel center a long hot leg. }\end{array}$ & & & 17,18 & \\
\hline TFB-RFBC & $\begin{array}{l}\text { Reflood bypass, near end cap, cold } \\
\text { leg side. }\end{array}$ & & & 19,20 & \\
\hline TFB-RFBH & $\begin{array}{l}\text { Reflood bypass, near end cap, hot } \\
\text { leg side. }\end{array}$ & & & 19,20 & \\
\hline Iniet Annulus & $\begin{array}{l}10 \mathrm{~cm} \text { below cold leg centerline, } \\
0.5 \mathrm{~cm} \text { from vessei wall, Type } \\
\text { i. Gii-comstanton themooouplos. }\end{array}$ & 0 to $1033 \mathrm{~K}$ & 0 to $701 \mathrm{~K}$ & & . \\
\hline TFV-ANN-4A & $0^{\circ}$ & & & 21,22 & \\
\hline TFV-ANN-4M & $180^{\circ}$. & & : & 21,22 & \\
\hline Downcomer Annulus & $\begin{array}{l}\text { Centered in anjulus, Type } \mathrm{J} \text { iron- } \\
\text { constantan thermocouples. }\end{array}$ & 0 to $1033 \mathrm{~K}$ & 0 to $701 \mathrm{~K}$ & & \\
\hline TFV-ALN-15T & $\begin{array}{l}38 \mathrm{~cm} \text { below cold leg centerline, } \\
270^{\circ} .\end{array}$ & & & & Thermocouple failed. \\
\hline TFV-ANN-35A & $\begin{array}{l}89 \mathrm{~cm} \text { below cold leg centerline, } \\
0^{\circ} .\end{array}$ & & & 23,24 & \\
\hline IFV-ANN-35T & $\begin{array}{l}89 \mathrm{~cm} \text { below cold leg centerline, } \\
270^{\circ} .\end{array}$ & & & 23,24 & \\
\hline TFY AAM-70A & $\begin{array}{l}178 \mathrm{rm} \text { helom cold leg centerl ine, } \\
0^{\circ} \text {. }\end{array}$ & & - & 23,24 & \\
\hline TFV-ANN-115A & $\begin{array}{l}292 \mathrm{~cm} \text { below cold leg centerline, } \\
0^{\circ} .\end{array}$ & & & 25,26 & \\
\hline TFV-AIN- $115 M$ & $\begin{array}{l}292 \mathrm{~cm} \text { below cold leg centerlíne, } \\
180^{\circ} \text {. }\end{array}$ & & & 25,26 & \\
\hline TFV-ANN-156A & $\begin{array}{l}396 \mathrm{~cm} \text { below cold leg centerline, } \\
0^{\circ} .\end{array}$ & & & & Therroccuuple failed. \\
\hline
\end{tabular}


TABLL V (continued)

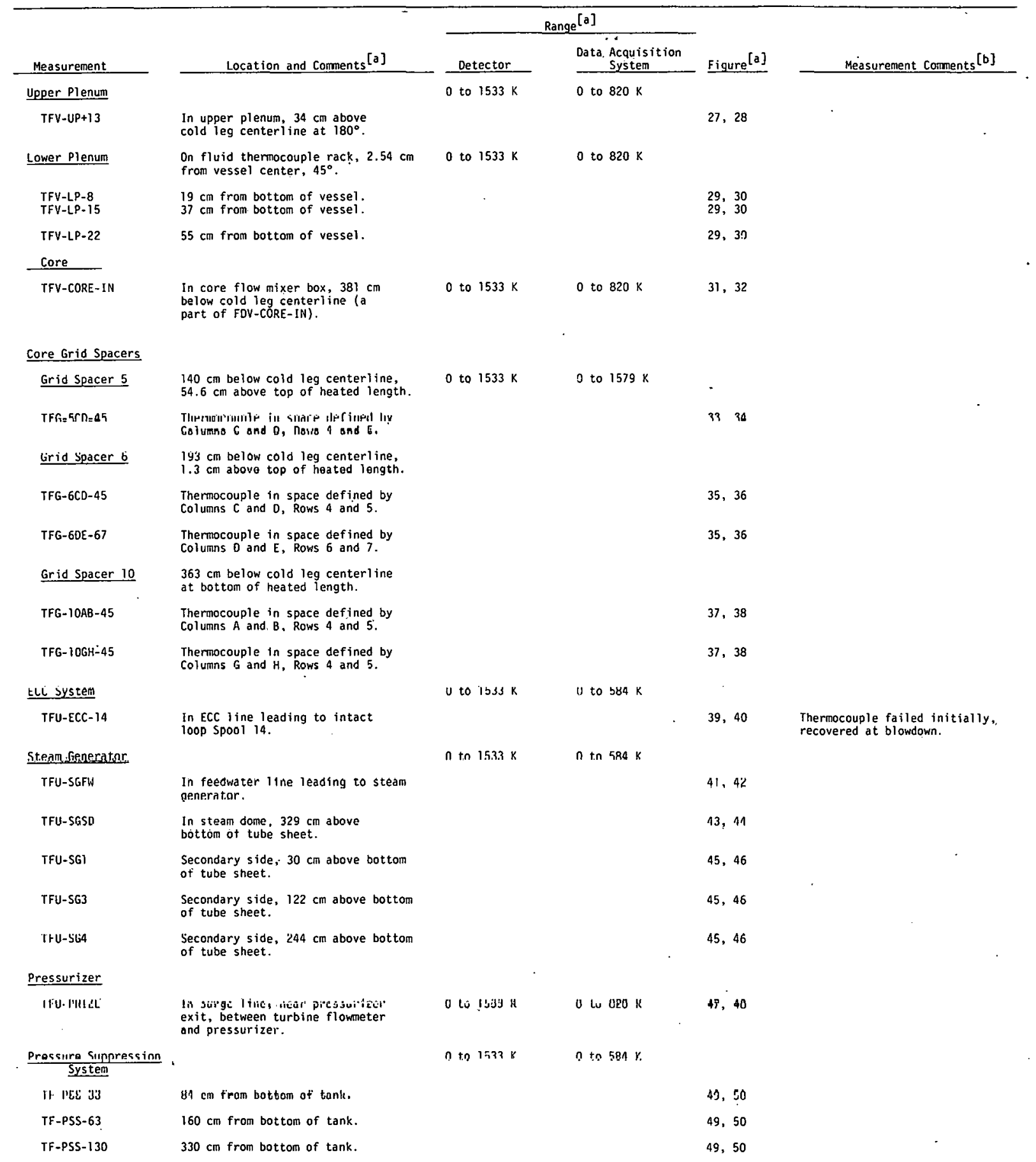


IABLE V (cont inued)

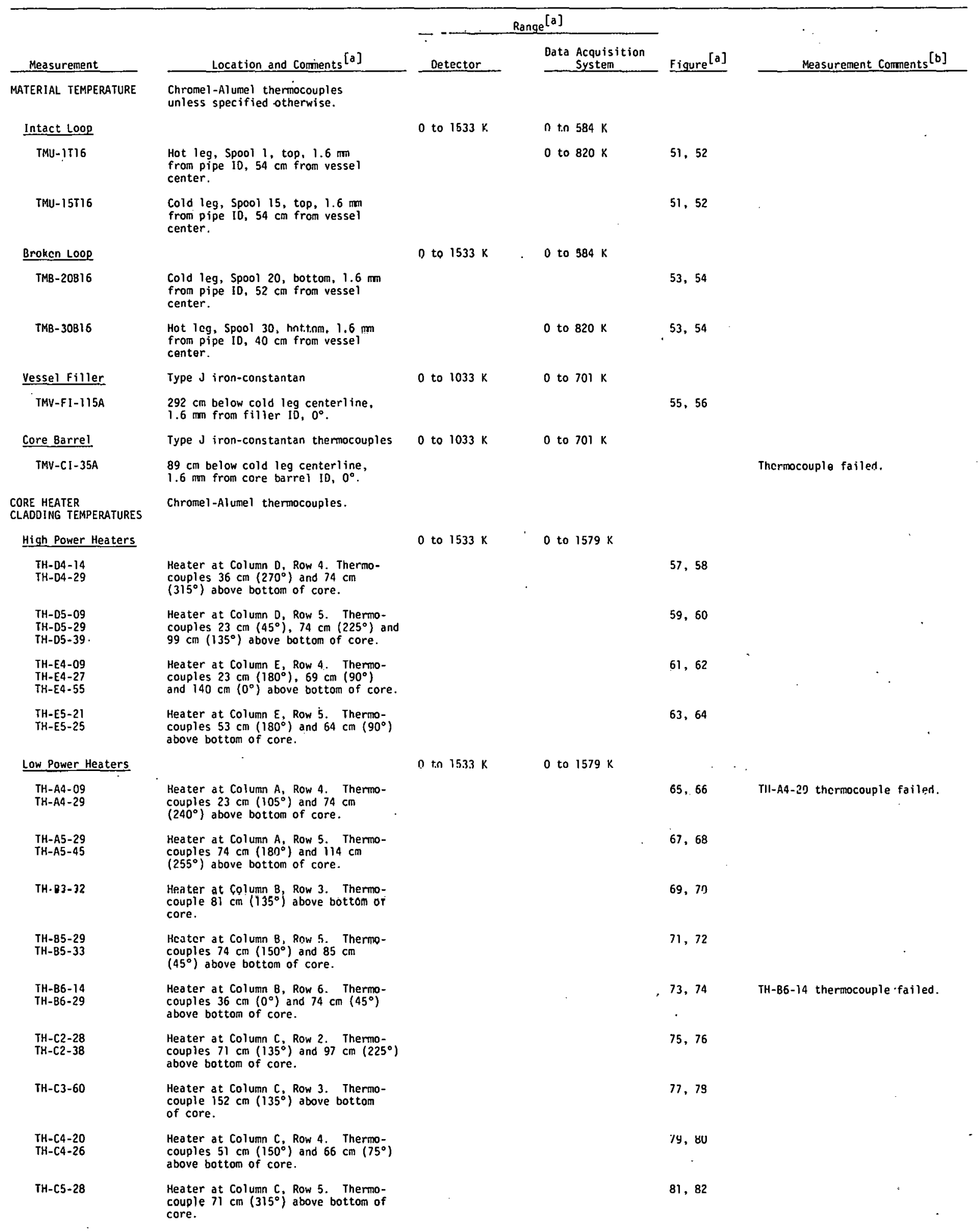


TABLE V (continued)

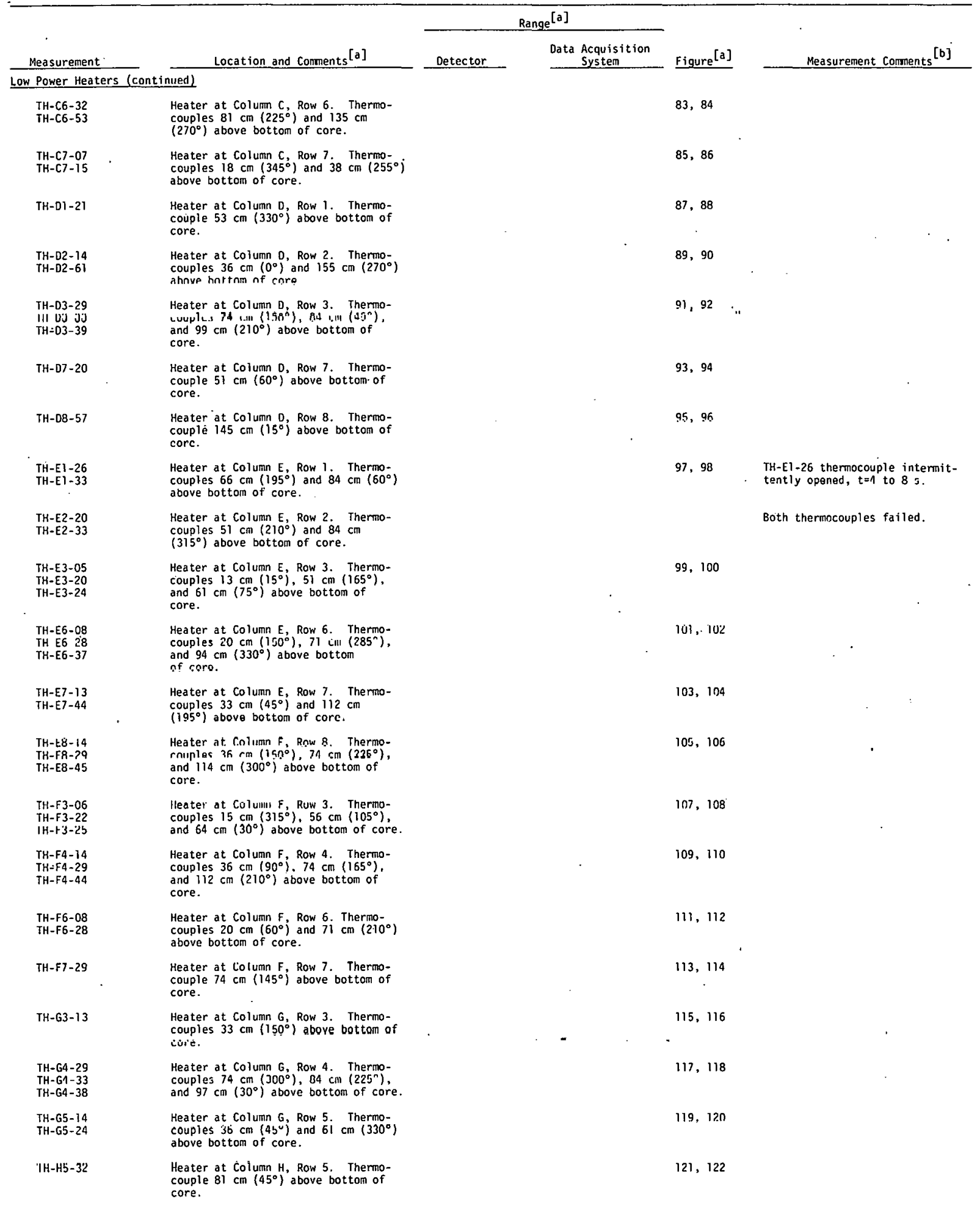




\section{TABLE. V (continued)}

\begin{tabular}{|c|c|c|c|c|}
\hline \multirow[b]{2}{*}{ Measurement } & \multirow[b]{2}{*}{ Location and Comments ${ }^{[a]}$} & \multicolumn{2}{|c|}{ Range $^{[a]}$} & \multirow[b]{2}{*}{ Fiqure ${ }^{[a]}$} \\
\hline & & Detector & $\begin{array}{l}\text { Data Acquisition } \\
\text { System. }\end{array}$ & \\
\hline PRESSURE & * & & & \\
\hline Intact Loop & & $\begin{array}{l}0 \text { to } 20684 \\
\mathrm{kPa}\end{array}$ & & \\
\hline PU-13 & $\begin{array}{l}\text { Cold leg, Spool } 13,138 \mathrm{~cm} \text { from } \\
\text { vessel center. }\end{array}$ & $\cdot$ & 0 to $31992 \mathrm{kPa}$ & 123,124 \\
\hline$P U-15 L$ & $\begin{array}{l}\text { Cold leg, Spool } 15,-55 \mathrm{~cm} \text { from } \\
\text { vessel center, to atmosphere } \\
\text { (low range). }\end{array}$ & 0 to $3447 \mathrm{kPa}$ & 0 to $3561 \mathrm{kPa}$ & 123,124 \\
\hline Broken Loop & & $\begin{array}{l}0 \text { to } 20684 \\
\mathrm{~K} . \mathrm{Pa}\end{array}$ & $\cdot$ & \\
\hline PB-21 & $\begin{array}{l}\text { Cold leg, Spool } 21,112 \mathrm{~cm} \text { from } \\
\text { vessel center. }\end{array}$ & $\cdot$ & 0 to $29931 \mathrm{kPa}$ & 125,126 \\
\hline PB-23 & $\begin{array}{l}\text { Cold leg, Spool } 23,235 \mathrm{~cm} \text { from } \\
\text { vessel center, upstrean of nozzle } \\
\text { (tee off } O P \text { tap). }\end{array}$ & 0 to $17237 \mathrm{kPa}$ & 0 to $21440 \mathrm{kPa}$ & 125,126 \\
\hline PB-24 & $\begin{array}{l}\text { Cold leg, Spool } 24,264 \mathrm{~cm} \text { from } \\
\text { vessel center downstream of } \\
\text { nozzle. }\end{array}$ & & $35612 \mathrm{kPa}$ & 125,126 \\
\hline PB-CN1. & $\begin{array}{l}\text { Vessel-side nozzle, nozzle throat, } \\
245 \mathrm{~cm} \text { from vessel center, (tee off } \\
\text { DP tap). }\end{array}$ & & $33924 \mathrm{kPa}$ & 127,128 \\
\hline PB-42 & $\begin{array}{l}\text { Cold leg, Spool } 42,1057 \mathrm{~cm} \text { from } \\
\text { vessel center along hot leg, up- } \\
\text { stream of pump-side nozzle (tee } \\
\text { off DP tap). }\end{array}$ & 0 to $17237 \mathrm{kPa}$ & 0 to $20569 \mathrm{kPa}$ & 129,130 \\
\hline$P B-43$ & $\begin{array}{l}\text { Cold leg, Spool } 43,1086 \mathrm{~cm} \text { from } \\
\text { vessel center along hot leg, up- } \\
\text { stream of rupture disc's. }\end{array}$ & 0 to $17237 \mathrm{kPa}$ & 0 to $20655 \mathrm{kPa}$ & 129,130 \\
\hline \multicolumn{5}{|l|}{ Vesse1 } \\
\hline$P V-U P+10$ & $\begin{array}{l}\text { in upper plenum, } 25 \mathrm{~cm} \text { above cold } \\
\text { leg centerline, mounted on staridoff, } \\
30^{\circ} \text {. }\end{array}$ & $\begin{array}{l}0 \text { to } 17237 \\
\mathrm{kPa}\end{array}$ & 0 to $21261 \mathrm{kPa}$ & 131,132 \\
\hline PV-LP-180 & $\begin{array}{l}\text { in upper part of lower plenum, } 457 \\
\mathrm{~cm} \text { below cold leg centerl ine, } \\
\text { mounted on standoff, } 225^{\circ} \text {. }\end{array}$ & $\begin{array}{l}0 \text { to } 20684 \\
\mathrm{kPa}\end{array}$ & 0 to $34226 \mathrm{kPa}$ & 131,132 \\
\hline ECC System & & 0 to $6895 \mathrm{kPa}$ & & \\
\hline $\mathrm{PU}-\mathrm{ACCl}$ & In intact loop accumulator. & & 0 to $8629 \mathrm{kPa}$ & 133,134 \\
\hline Steam Generator & . & & & . \\
\hline PU-SGSD & Secondary side steam done. & $\begin{array}{l}0 \text { LU } 20684 \\
K P A\end{array}$ & 0 to $14018 \mathrm{kPa}$ & 135,136 \\
\hline \multicolumn{5}{|l|}{ Pressurizer } \\
\hline PU-PRIZE & Pressurizer steam dome. & $\begin{array}{l}0 \text { to } 17237 \\
\mathrm{KPa}\end{array}$ & 0 to $22329 \mathrm{kPa}$ & 137,138 \\
\hline \multicolumn{5}{|l|}{$\frac{\text { Pressure Suppression }}{\text { Sistcm }}$} \\
\hline P-PSS & Suppression tank top. & $U$ to $345 \mathrm{kPa}$ & 0 to $122 \mathrm{kPd}$ & 139,140 \\
\hline DIFFERENTIAL PKESSUKE & $\begin{array}{l}\text { Elevation differeice between } \\
\text { transducer taps is zero unless } \\
\text { otherwise specified. }\end{array}$ & & & \\
\hline \multicolumn{5}{|l|}{ Intact Loop } \\
\hline DPU-UP-3 & $\begin{array}{l}\text { Upper plenum } 26.7 \mathrm{~cm} \text { above } \\
\text { cold leg centerline at } 30^{\circ} \text { to } \\
\text { hot leg. Spool } 3,158 \mathrm{~cm} \text { from } \\
\text { vessel center. Upper plenum tap } \\
\text { is approximately } 5 \mathrm{~cm} \text { above Spool } \\
3 \text { tap. }\end{array}$ & $\frac{+127 \mathrm{~cm}}{\text { water }}$ & $\pm 16.8 \mathrm{kPa}$ & 141,142 \\
\hline DPU-3-6 & $\begin{array}{l}\text { Hot leg Spool } 3,158 \mathrm{~cm} \text { from vessel } \\
\text { center to hot leg Spool } 6,290 \mathrm{~cm} \\
\text { from vessel center. }\end{array}$ & $\frac{+1270 \mathrm{~cm}}{\text { water }}$ & $\pm 170 \mathrm{kPa}$ & 143,144 \\
\hline OPU-6-SGIP & $\begin{array}{l}\text { Hot leg, Spool } 6,290 \mathrm{~cm} \text { from } \\
\text { vessel center to steam generator } \\
\text { iniet plenum } 368 \mathrm{~cm} \text { from vessel } \\
\text { center. Spool } 6 \text { tap is } 41 \mathrm{~cm} \\
\text { below SGIP tap. }\end{array}$ & $\begin{array}{l} \pm 1270 \mathrm{~cm} \\
\text { water }\end{array}$ & $\pm 169 \mathrm{kPa}$ & 145,146 \\
\hline
\end{tabular}


TABLE V (continued)

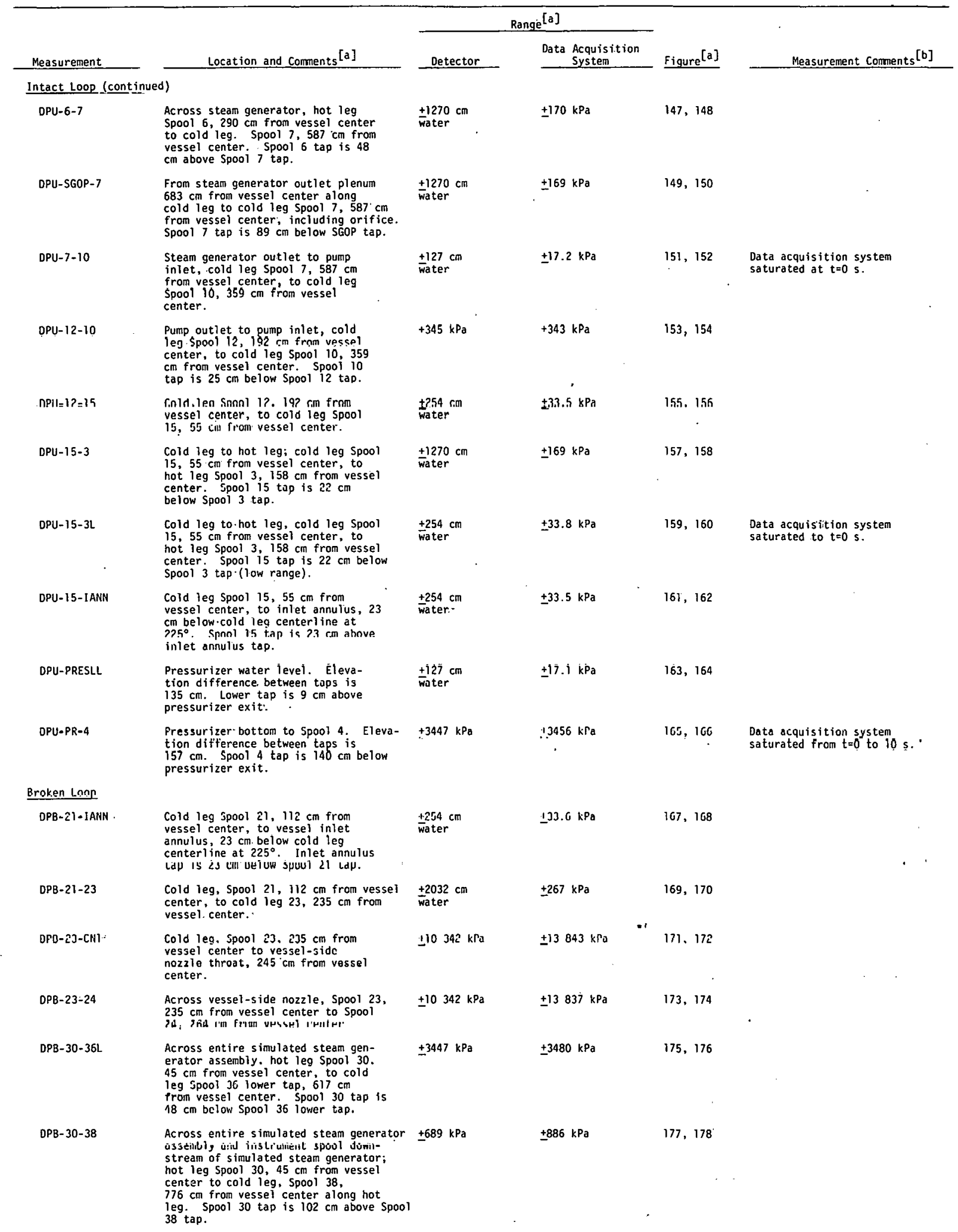


TABLE V (continued)

\begin{tabular}{|c|c|c|c|c|c|c|c|}
\hline \multirow[b]{2}{*}{ Measurement } & \multirow[b]{2}{*}{ Location and Comments $[\mathrm{a}]$} & \multicolumn{3}{|c|}{ Range $^{[a]}$} & \multirow[b]{2}{*}{ Figure $^{[a]}$} & \multirow{2}{*}{\multicolumn{2}{|c|}{ Measurement Comments ${ }^{[\mathrm{b}]}$}} \\
\hline & & Detector & Data & $\begin{array}{l}\text { Acquisition } \\
\text { System }\end{array}$ & & & \\
\hline \multicolumn{8}{|c|}{ Broken Loop (continued) } \\
\hline$D P B-32 U-36 L$ & $\begin{array}{l}\text { Across simulated steam generator } \\
\text { orifice assembly, hot leg Spool } 32 \\
\text { upper tap, } 189 \mathrm{~cm} \text { from' vessel center, } \\
\text { to } 5 p 00136 \text { lower tap, } 617 \mathrm{~cm} \text { from } \\
\text { vessel center. Spool } 32 \text { upper tap } \\
\text { is } 41 \mathrm{~cm} \text { above Spool } 36 \text { lower tap. }\end{array}$ & $\pm 3447 \mathrm{kPa}$ & \pm 3466 & $6 \mathrm{kPa}$ & 179,180 & & . \\
\hline$D P B-38-40$ & $\begin{array}{l}\text { Across simulated pump, cold leg } \\
\text { Spool } 38,776 \mathrm{~cm} \text { from vessel center } \\
\text { along hot leg, to cold leg Spool } 40, \\
929 \text { from vessel center along hot } \\
\text { leg. }\end{array}$ & $\pm 6895 \mathrm{kPa}$ & \pm 9227 & $7 \mathrm{kPa}$ & 181,182 & & \\
\hline DPB- $40-42$ & $\begin{array}{l}\text { Across elbow leading to spool up- } \\
\text { stream of pump-side nozzle. Cold } \\
\text { leg Spool } 40,929 \text { from vessel } \\
\text { center along hot leg, to } 5 p 00142 \text {, } \\
1057 \text { from vessel center along } \\
\text { hot leg. Spool } 40 \text { tap is } 102 \mathrm{~cm} \\
\text { below Spool } 42 \text { tap. }\end{array}$ & $\frac{+127 \mathrm{~cm}}{\text { water }}$ & \pm 16.2 & $2 \mathrm{kPa}$ & 183,184 & $\begin{array}{l}\text { Detector saturated from } \\
\mathrm{t}=0 \text { to } 1 \mathrm{~s} \text {. }\end{array}$ & \\
\hline$D P B=42-43$ & $\begin{array}{l}\text { Across pump-side nozzle cold leg } \\
\text { Spool } 42 \text {, } 1057 \mathrm{~cm} \text { from vessel center } \\
\text { along hot leg, to cold leg, Spool } 43 \\
\text { lo86 cm from vessel center along } \\
\text { hot leg. }\end{array}$ & $\pm 6895 \mathrm{kPa}$ & \pm 9230 & $0 \mathrm{kPa}$ & 185,186 & $\begin{array}{l}\text { Data acquisition system } \\
\text { saturated at } t=0 \text { to } 2 \text { s }\end{array}$ & \\
\hline \multicolumn{8}{|l|}{ Vessel } \\
\hline DPV-UP-IANN & $\begin{array}{l}\text { Upper pienum, } 27 \mathrm{~cm} \text { above cold } \\
\text { leg centerline at } 30^{\circ} \text { to inlet } \\
\text { annulus, } 23 \text { cm below cold leg center- } \\
\text { line at } 225^{\circ} \text {. Elevation difference } \\
\text { between taps is } 48 \mathrm{~cm} \text {. }\end{array}$ & $\frac{+762 \mathrm{~cm}}{\text { water }}$ & \pm 102 & $\mathrm{kPa}$ & 187,188 & & \\
\hline$D P V-0-96 Q$ & $\begin{array}{l}\text { Inlet annulus cold leg centerline at } \\
90^{\circ} \text {, to } 23 \mathrm{~cm} \text { below cold leg center- } \\
\text { line at } 225^{\circ} \text {. Elevation difference } \\
\text { between taps is } 23 \mathrm{~cm} \text {. }\end{array}$ & $\stackrel{+127 \mathrm{~cm}}{\text { water }}$ & \pm 18.4 & $\mathrm{kPa}$ & 189,190 & . & $\because$ \\
\hline DPV-9-260Q & $\begin{array}{l}\text { Inlet annulus, } 23 \mathrm{~cm} \text { below cold leg } \\
\text { centerline at } 225^{\circ} \text {, to downcomer gap, } \\
66 \mathrm{~cm} \text { below cold leg centerline at } \\
225^{\circ} \text {. Elevation difference between } \\
\text { taps is } 43 \mathrm{~cm} \text {. }\end{array}$ & $\frac{+127 \mathrm{~cm}}{\text { water }}$ & \pm 16.8 & $3 \mathrm{kPa}$ & 191,192 & & " \\
\hline OPV-9-180QQ & $\begin{array}{l}\text { Inlet annulus, } 23 \mathrm{~cm} \text { below cold leg } \\
\text { centerline at } 225^{\circ} \text { to lower plenum, } \\
457 \mathrm{~cm} \text { below cold ieg centerline } \\
\text { at } 225^{\circ} \text {. Elevation difference be- } \\
\text { tween taps is } 434 \mathrm{~cm} \text {. }\end{array}$ & $\begin{array}{l}+762 \mathrm{~cm} \\
\text { water }\end{array}$ & \pm 103 & $\mathrm{kPa}$ & . & Detector failed. & \\
\hline $\mathrm{DPV}-26-550 \mathrm{M}$ & $\begin{array}{l}\text { Across part of downcomer, } 66 \mathrm{~cm} \\
\left(225^{\circ}\right) \text {, to } 140 \mathrm{~cm}\left(180^{\circ}\right) \text {, below cold } \\
\text { leg centerl ine. Elevation difference } \\
\text { belween taps is } 74 \mathrm{~cm} \text {. }\end{array}$ & $\begin{array}{l} \pm 127 \mathrm{kPa} \\
\text { water }\end{array}$ & +21.2 & $2 \mathrm{kPa}$ & 193,194 & & $\cdot$ \\
\hline OPV-55-110M9 & $\begin{array}{l}\text { Across part of downcomer, } 140 \mathrm{~cm} \\
\left(180^{\circ}\right) \text {, to } 279 \mathrm{~cm}\left(180^{\circ}\right) \text {, below } \\
\text { cold leg centerl ine. Elevation } \\
\text { difference between taps is } 140 \mathrm{~cm} \text {. }\end{array}$ & $\begin{array}{l}+254 \mathrm{~cm} \\
\text { water }\end{array}$ & $\pm 33.8^{\circ}$ & $\mathrm{kPa}$ & 195,196 & & \\
\hline DPV-110-156MO & $\begin{array}{l}\text { Across part of downcomer, } 279 \mathrm{~cm} \\
\left(100^{\circ}\right), \text { to } 996 \text { chin }\left(225^{\circ}\right), \text { below cold } \\
\text { leg centerl ine. Flevation difference } \\
\text { between taps is } 117 \mathrm{~cm} \text {. }\end{array}$ & $\begin{array}{l}+254 \mathrm{~cm} \\
\text { water }\end{array}$ & \pm 33.7 & $\mathrm{kPa}$ & 197,198 & . & \\
\hline$D P V-156-173 Q Q$ & $\begin{array}{l}\text { Across part of lower plenum, } 346 \mathrm{~cm} \\
\left(225^{\circ}\right) \text {, to } 439 \mathrm{~cm}\left(225^{\circ}\right) \text {, below cold } \\
\text { leg centerline. Elevation difference } \\
\text { between taps is } 43 \mathrm{~cm} \text {. }\end{array}$ & $\begin{array}{l}+127 \mathrm{~cm} \\
\text { water }\end{array}$ & \pm 17.7 & $\mathrm{kPa}$ & 199,200 & & \\
\hline OPV-166-192QT & $\begin{array}{l}\text { Across lower plenum, } 422 \mathrm{~cm}\left(225^{\circ}\right) \\
\text { to } 488 \mathrm{~cm}\left(270^{\circ}\right) \text {, below cold leg center- } \\
\text { line. Elevation difference between } \\
\text { taps is } 66 \mathrm{~cm} \text {. }\end{array}$ & $\begin{array}{l} \pm 127 \mathrm{~cm} \\
\text { water }\end{array}$ & \pm 17.1 & $\mathrm{kPa}$ & 201,202 & & \\
\hline $0 F V-373-10009$ & $\begin{array}{l}\text { Reross part of lower plonum, } 430 \mathrm{~cm} \\
\left(225^{\circ}\right) \text { to } 457 \mathrm{~cm}\left(225^{\circ}\right) \text {, below cold leg } \\
\text { centerline. Elevation difference } \\
\text { between taps is } 18 \mathrm{~cm} \text {. }\end{array}$ & $\begin{array}{l} \pm 51 \mathrm{~cm} \\
\text { water }\end{array}$ & \pm 65 & $r P_{A}$ & $2 m, 2 n 4$ & & \\
\hline DPV-LP-UP & $\begin{array}{l}\text { Lower plenum, } 457 \mathrm{~cm} \text { below cold leg } \\
\text { centerline at } 225^{\circ} \text { to upper plenum, } \\
27 \mathrm{~cm} \text { above cold leg centerline at } \\
30^{\circ} \text { Elevation difference between } \\
\text { taps is } 485 \mathrm{~cm} \text {. }\end{array}$ & $\begin{array}{l}+762 \mathrm{~cm} \\
\text { water }\end{array}$ & \pm 101 & $\mathrm{kPa}$ & 205,206 & & \\
\hline
\end{tabular}


TABLE V (continued)

\begin{tabular}{|c|c|c|c|c|c|c|c|c|}
\hline \multirow[b]{2}{*}{ Measurement } & \multirow[b]{2}{*}{ Location and Comments ${ }^{\text {[a] }}$} & \multicolumn{5}{|c|}{ Range $^{\text {[a] }}$} & \multirow{3}{*}{ Figure $^{[\mathrm{a}]}$} & \multirow[b]{2}{*}{ Measurement Comnents ${ }^{[\mathrm{b}]}$} \\
\hline & & \multicolumn{2}{|l|}{ Detector } & \multicolumn{3}{|c|}{$\begin{array}{l}\text { Data Acquisition } \\
\text { Systen }\end{array}$} & & \\
\hline \multicolumn{8}{|l|}{ ECC SYSTEM } & \\
\hline DPU-ACCI-TB & $\begin{array}{l}\text { Top to bottom of intact loop } \\
\text { accumulator. Elevation difference } \\
\text { between taps is } 274 \mathrm{~cm} \text {. }\end{array}$ & $\begin{array}{l}+762 \mathrm{~cm} \\
\text { water }\end{array}$ & & $\pm 104 k$ & $\mathrm{kPa}$ & & 207,208 & \\
\hline \multicolumn{9}{|l|}{$\underline{\text { Steam Generator }}$} \\
\hline DPU-SGFW & $\begin{array}{l}\text { In steam generator feedwater } \\
\text { line. }\end{array}$ & $\stackrel{+762 \mathrm{~cm}}{\text { water }}$ & & $\stackrel{+762 \mathrm{c}}{\text { water }}$ & $\mathrm{cm}$ & & 209,210 & \\
\hline DPU-SG-SEC & $\begin{array}{l}\text { Secondary side, differential pressure } \\
\text { taps at } 114 \mathrm{~cm} \text { and } 320 \mathrm{~cm} \text { above } \\
\text { bottom of tube sheet. Elevation } \\
\text { difference between taps is } 206 \mathrm{~cm} \text {. }\end{array}$ & $\begin{array}{l}+254 \mathrm{~cm} \\
\text { water }\end{array}$ & & \pm 33.7 & $\mathrm{k} \dot{\mathrm{Pa}}$ & & 211,212 & . \\
\hline DPU-SG-DISC & $\begin{array}{l}\text { Acruss verului i tube, } 160 \mathrm{~cm} \text { down- } \\
\text { stream from steam generator dis- } \\
\text { charge. }\end{array}$ & $\begin{array}{l}\frac{11270}{17 m} \\
\text { water }\end{array}$ & & $1170 \mathrm{k}$ & kra & & 213,214 & $\begin{array}{l}\text { Data acquisition system } \\
\text { saturated at } 1=41 \mathrm{~s} \text {. }\end{array}$ \\
\hline \multicolumn{9}{|l|}{$\frac{\text { Pressure Suppression }}{\text { System }}$} \\
\hline DP-PSS-TB & $\begin{array}{l}\text { Top to bottom of pressure } \\
\text { supprressiun lallk. Elevaliun } \\
\text { difference, between taps } \\
\text { is } 338 \mathrm{~cm} \text {. }\end{array}$ & $\begin{array}{l}+762 \mathrm{~cm} \\
\text { water: }\end{array}$ & & $\pm 103 k$ & $\mathrm{kPa}$ & . & . & Detector failed. \\
\hline VOLUMETRIC FLOW RATE & Turbine flowneter, bidirectional. & . & & & . & & & $\begin{array}{l}\text { Data acquisition system } \\
\text { rallye may unceed laled } \\
\text { detector range; however, } \\
\text { turbine response is } \\
\text { linear to flow rates } \\
\text { well beyond the rated range. }\end{array}$ \\
\hline Intact Loop & 3-in. Schedule 160 pipe. & & & & & & & ${ }^{*}$ \\
\hline ETU-1 & $\begin{array}{l}\text { Hot leg, Spool } 1,42 \mathrm{~cm} \text { from } \\
\text { vessel center. }\end{array}$ & $\begin{array}{l} \pm 1.26 \text { to } \\
\pm 25.2 \mathrm{l} / \mathrm{s}\end{array}$ & & \pm 75.7 & $\ell / \mathrm{s}$ & . & $215,216^{\circ}$ & . \\
\hline FTU-9 & $\begin{array}{l}\text { Cold leg, Spool } 9,393 \mathrm{~cm} \text { from } \\
\text { vesscl center. }\end{array}$ & $\begin{array}{l}+5.05 \text { to } \\
\$ 60.6 \mathrm{k} / \mathrm{c}\end{array}$ & & \pm 75.7 & $\ell / s^{\circ}$ & & 215,216 & . \\
\hline FTU-13 & $\begin{array}{l}\text { Cold leg, Spool 13, } 163 \mathrm{~cm} \text { from } \\
\text { vessel cenler. }\end{array}$ & \pm+1.26 to & & \pm 75.7 & $2 / \mathrm{s}$ & $\therefore$ & 217,218 & $\begin{array}{l}\text { Data acquisition system } \\
\text { saturated at } t=203 \text { and } \\
t=65 \text { to } 685 \text {. }\end{array}$ \\
\hline FTU-15 & $\begin{array}{l}\text { Cold leg, fpool } 15,4 \hat{2} \mathrm{~cm} \text { from } \\
\text { vessel center. }\end{array}$ & $\begin{array}{l}11.26 \text { to } \\
\pm 50.5 \mathrm{l} / \mathrm{s}\end{array}$ & · & 163.1 & $\ell / 9$ & & 217,218 & . \\
\hline Broken Loop & Schedule 160 pipe. & & & & & & . & \\
\hline rTD- 21 & $\begin{array}{l}\text { Culd ley, sposel } \mathrm{gl}, 140 \mathrm{~cm} \text { from } \\
\text { ves3cl ecnters } 3 \text { in. pipo. }\end{array}$ & $\begin{array}{l}11.26 \text { to } \\
\pm 26.3 \mathrm{~B} / \mathrm{c}\end{array}$ & & $175: 7$ & $k / 9$ & & 210,220 & . \\
\hline FIb-30 & 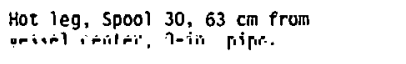 & 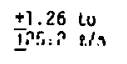 & & \pm 50.5 & $\ell / \mathrm{s}$ & & 221,222 & . \\
\hline FTB-37 & $\begin{array}{l}\text { Cold leg, Spool } 37,739 \mathrm{~cm} \text { from } \\
\text { vessel center along hot leg: } 2 \text {-in. } \\
\text { Pipe. }\end{array}$ & $\begin{array}{l} \pm 1.26 \text { to } \\
\pm 50.5 \mathrm{l} / \mathrm{s}\end{array}$ & & \pm 50.5 & $\ell / \mathrm{s}$ & & 221,222 & . . \\
\hline Core & & & & & & & & \\
\hline FTV-CORE-IN & $\begin{array}{l}\text { Entrance to core, } 401 \mathrm{~cm} \text { below } \\
\text { cold leg centerline. }\end{array}$ & $\begin{array}{l} \pm 1.26 \text { to } \\
\pm 12.6 \mathrm{R} / \mathrm{s}\end{array}$ & & \pm 56.78 & $8 \mathrm{l} / \mathrm{s}$ & & $\ddot{223}, 224$ & r \\
\hline ECC System & .. & & & & & & & \\
\hline FTU-HPIS & $\begin{array}{l}\text { In line imediately after HPIS } \\
\text { pump tór intát loob, } 1 / 2-1 h \text {. } \\
\text { line. }\end{array}$ & $\begin{array}{l}+0.047 \text { to } \\
\pm 0.41 \mathrm{i} / \mathrm{s}\end{array}$ & & \pm 0.126 & $6 \mathrm{2} / \mathrm{s}$ & & 225,226 & - \\
\hline FTU-LPIS & $\begin{array}{l}\text { In line leading from LPIS pump } \\
\text { fur lildel luup, } 1 / 2=1 \text { lI. l lite. }\end{array}$ & $\begin{array}{l}+0.047 \text { to } \\
10.47 \mathrm{i} / \mathrm{s}\end{array}$ & & \pm 0.631 & $17 / 5$ & & 227,228 & $\begin{array}{l}\text { Data acquisition system } \\
\text { salui aled al } l-\varepsilon \sigma \mathrm{s} \text {. }\end{array}$ \\
\hline FTU $A C S$ & $\begin{array}{l}\text { In ling loaring from intart lnnp } \\
\text { accumulatór, } 1-i n ̃ \text { line. }\end{array}$ & $\begin{array}{l}+n 315 \text { th } \\
\Psi_{-3.15}\end{array}$ & & $\pm 44 ?$ & & & ?วq, ว ?ก & . \\
\hline Pressurizer & $1-1 / 2-i n$. turbine & & & & & & & \\
\hline FTII-PRIZE & Surge Line. & $\begin{array}{l} \pm 0.126 \text { to } \\
\pm 1.26 \mathrm{R} / \mathrm{s}\end{array}$ & & $\pm 6.3 \ell$ & $2 / \mathrm{s}$ & & 231.232 & \\
\hline FLUID VELOCITY & Turbine flowmeter, bidirectional. & & & & & & & \\
\hline Anumrnmer & & & & & . & & . & . \\
\hline FTV $-40 A$ & $\begin{array}{l}102 \mathrm{~cm} \text { below cold leg centerline, } \\
0^{\circ} \text {. }\end{array}$ & $\begin{array}{l}+0.762 \text { to } \\
\pm 15.24 \mathrm{~m} / \mathrm{s}\end{array}$ & & \pm 15.24 & $4 \mathrm{~m} / \mathrm{s}$ & & 233,234 & $\begin{array}{l}\text { Data acquisition system } \\
\text { saturated intermittently from } \\
\text { t=26 to } 37 \mathrm{~s} \text {. }\end{array}$ \\
\hline FTV $-40 \mathrm{H}$ & $\begin{array}{l}102 \mathrm{~cm} \text { below cold leg centerline, } \\
180^{\circ} \text {. }\end{array}$ & $\begin{array}{l}+0.762 \text { to } \\
\pm 15.24 \mathrm{~m} / \mathrm{s}\end{array}$ & & \pm 15.24 & $4 \mathrm{~m} / \mathrm{s}$ & & 233,234 & $\begin{array}{l}\text { Data acquisition system. } \\
\text { saturated at } t=29 \text { and } 36 \mathrm{~s} .\end{array}$ \\
\hline
\end{tabular}


TABLE V (continued)

\begin{tabular}{|c|c|c|c|c|c|}
\hline \multirow[b]{2}{*}{ Measurement } & \multirow[b]{2}{*}{ Location and Comments ${ }^{[\mathrm{a}]}$} & \multicolumn{2}{|c|}{ Range $^{[a]}$} & \multirow[b]{2}{*}{$\underline{F_{\text {igure }}{ }^{[a]}}$} & \multirow[b]{2}{*}{ Measurement Comments ${ }^{[\mathrm{b}]}$} \\
\hline & & Detector & $\begin{array}{l}\text { Data Acquisition } \\
\text { System }\end{array}$ & & \\
\hline MOMENTUM FLUX & Orag disc, bidirectional. & & & & $\begin{array}{l}\text { Momentum flux reported only for } \\
-6 \text { to } 425 \text {, except as noted. } \\
\text { Drag disc data may exhibit } \\
\text { significant temperature } \\
\text { dependence. Drag disc data } \\
\text { should be used only for short - } \\
\text { term transient response. }\end{array}$ \\
\hline Intact Loop & 3-in. pipe. & & & & \\
\hline FDU-1 & $\begin{array}{l}\text { Hot leg, Spool } 1,60 \mathrm{~cm} \text { from } \\
\text { vessel center; target size } \\
2.22 \text { cill. }\end{array}$ & $\begin{array}{l} \pm 298 \text { to } \pm 17 \quad 113 \\
\mathrm{~kg} / \mathrm{m}-\mathrm{s}^{2}\end{array}$ & $\begin{array}{l} \pm 56739 \\
\mathrm{~kg} / \mathrm{m}-\mathrm{s}^{2}\end{array}$ & 235 & \\
\hline FDU -5 . & $\begin{array}{l}\text { Hot leg, Spool } 5,256 \mathrm{~cm} \text { from } \\
\text { vessel center; target size } \\
2.54 \mathrm{~cm} \text {. }\end{array}$ & $\frac{ \pm 1.49 \text { to }}{\mathrm{kg} / \mathrm{m}-\mathrm{s}^{2}} \pm 2997$ & $\frac{ \pm 6994}{\mathrm{~kg} / \mathrm{m}-\mathrm{s}^{2}}$ & 236 & \\
\hline FDU-10 & $\begin{array}{l}\text { Cold leg, Spool 10,349 cm from } \\
\text { vessel céiler'; target size } 2.22 \mathrm{~cm} \text {. }\end{array}$ & $\frac{ \pm 298 \text { to } \pm 154800}{\mathrm{~kg} / \mathrm{m}-\mathrm{s}^{2}}$ & $\begin{array}{l} \pm 35 \quad 344 \\
\mathrm{~kg} / \mathrm{m}-\mathrm{s}^{2}\end{array}$ & 237 & \\
\hline FDU-13 & $\begin{array}{l}\text { Cold leg, Spool } 13,138 \mathrm{~cm} \text { from } \\
\text { vessel center; target size } 2.22 \mathrm{~cm} \text {. }\end{array}$ & $\begin{array}{l} \pm 298 \text { to } \pm 22029 \\
\mathrm{~kg} / \mathrm{m}-\mathrm{s}^{2}\end{array}$ & $\begin{array}{l} \pm 28528 \\
\mathrm{~kg} / \mathrm{m}-\mathrm{s}^{2}\end{array}$ & 238 & \\
\hline \multicolumn{6}{|l|}{ Broken Loop } \\
\hline FDB-21 & $\begin{array}{l}\text { Cold leg, Spool } 21,134 \mathrm{~cm} \text { from } \\
\text { vessel center, 3-in. pipe; target } \\
\text { size } 2.22 \mathrm{~cm} \text {. }\end{array}$ & $\frac{ \pm 298 \text { to }}{\mathrm{kg} / \mathrm{m}-\mathrm{s}^{2}} \pm 104915$ & $\frac{+66774}{\mathrm{~kg} / \mathrm{m}-\mathrm{s}^{2}}$ & 239 & \\
\hline FDB-23 & $\begin{array}{l}\text { Cold leg, Spool } 23,238 \mathrm{~cm} \text { from } \\
\text { vessel center, upstream of vessel } \\
\text { side nozzle, } 2 \text {-in. pipe; target } \\
\text { size } 1.03 \mathrm{cml} \text {. }\end{array}$ & $\frac{ \pm 298 \text { to }}{\mathrm{kg} / \mathrm{m}-\mathrm{s}^{2}} \pm 180067$ & $\begin{array}{l} \pm 155319 \\
\mathrm{~kg} / \mathrm{m}-\mathrm{s}^{2}\end{array}$ & 240 & - \\
\hline FDB -30 & $\begin{array}{l}\text { Hot leg, Spool } 30,52 \mathrm{~cm} \text { from } \\
\text { vessel cenler, 3-iil. pipe; target } \\
\text { size } 1.67 \mathrm{~cm} \text {. }\end{array}$ & $\begin{array}{l} \pm 298 \text { to } \\
\mathrm{kg} / \mathrm{m}-\mathrm{s}^{2}\end{array}$ & $\begin{array}{l} \pm 67280 \\
\mathrm{~kg} / \mathrm{m}-\mathrm{s}^{2}\end{array}$ & 241 & $\begin{array}{l}\text { Questionable data, transducer } \\
\text { was monuter harizantally and } \\
\text { and possibly filled with } \\
\text { subcooled water. }\end{array}$ \\
\hline FDB-37 & $\begin{array}{l}\text { Cold leg, 5pool 37, } 725 \mathrm{~cm} \text { from } \\
\text { vessel center along hot leg, steam } \\
\text { generator outlet, vertical pipe, } \\
2 \text {-in. pipe; target size } 1.03 \mathrm{~cm} \text {. }\end{array}$ & $\begin{array}{l} \pm 298 \text { to } \\
\mathrm{kg} / \mathrm{m}-\mathrm{s}^{2}\end{array}$ & $\begin{array}{l} \pm 374272 \\
\mathrm{~kg} / \mathrm{m}-\mathrm{s}^{2}\end{array}$ & 242 & $\begin{array}{l}\text { Questionable data, transducer } \\
\text { was mounted horizontally and } \\
\text { possibly filled with } \\
\text { subcooled water. }\end{array}$ \\
\hline FDB-42 & $\begin{array}{l}\text { Cold leg, spool } 42,1057 \mathrm{~cm} \text { from } \\
\text { vessel center along hot leg, up- } \\
\text { stream of pump-side nozzle, down- } \\
\text { stream of injection point, 2-in. } \\
\text { pipe; target size } 1.03 \mathrm{~cm} \text {. }\end{array}$ & $\begin{array}{l} \pm 298 \text { to } \\
\mathrm{kg} / \mathrm{m}-\mathrm{s}^{2}\end{array}$ & $\begin{array}{l} \pm 165353 \\
\mathrm{~kg} / \mathrm{m}-\mathrm{s}^{2}\end{array}$ & 243 & \\
\hline \multicolumn{6}{|l|}{ Vessel } \\
\hline FDV-CORE-IN & $\begin{array}{l}\text { In core flow mixer box, } 381 \mathrm{~cm} \\
\text { below cold leg centerline; carget } \\
\text { size } 2.54 \mathrm{~cm} \text {. }\end{array}$ & $\frac{+1.44 \text { to }}{\mathrm{kg} / \mathrm{m}-\mathrm{s}^{2}} \pm 2977$ & $\frac{ \pm 1725}{\mathrm{~kg} / \mathrm{m}-\mathrm{s}^{2}}$ & 244 & . \\
\hline \multicolumn{6}{|l|}{ DENSITY } \\
\hline Intact Loop & & $\begin{array}{l}1.6 \text { to } 1600 \\
\mathrm{~kg} / \mathrm{m}^{3}\end{array}$ & $\begin{array}{l}0 \text { to } 1600 \\
\mathrm{~kg} / \mathrm{m}^{3}\end{array}$ & & \\
\hline $\begin{array}{l}\text { CU. IT } \\
\text { GU-1B } \\
\text { GU-IC }\end{array}$ & $\begin{array}{l}\text { Hot log, spon! } 7,77 \text { rom frmm } \\
\text { vessel center. } T \text { (top) ranges } \\
270 \text { to } 360^{\circ} \text {. B (bottom) ranges } \\
30 \text { to } 330^{\circ} \text { C, mathematical } \\
\text { composite of } Y \text { and } B \text {. }\end{array}$ & & & $\begin{array}{l}245,246 \\
247,248\end{array}$ & \\
\hline GU-5VR & $\begin{array}{l}\text { Hot leg, Spool } 5,246 \mathrm{~cm} \text { from } \\
\text { vessel center, vertical. }\end{array}$ & & & 249,250 & \\
\hline GU-10VR & $\begin{array}{l}\text { Cold leg, spool } 10,359 \mathrm{~cm} \text { from } \\
\text { vessel center, vertical. }\end{array}$ & & & 249,250 & \\
\hline GU-13VR & $\begin{array}{l}\text { Cold leg, } 5 p o o l 13,142 \mathrm{~cm} \text { from } \\
\text { vessel center, vertical. }\end{array}$ & 、 & & 251,252 & \\
\hline $\begin{array}{l}\text { GU-15T } \\
\text { GU- } 15 B \\
\text { GU- } 15 C\end{array}$ & $\begin{array}{l}\text { Cold leg, Spool } 15,77 \mathrm{~cm} \text {. from vessel } 1 \\
\text { center. T (top) ranges } 270 \text { to } 360^{\circ} . \\
B \text { (bottom) ranges } 30 \text { to } 33^{\circ} \text { C. } \\
\text { mathematical composite of } T \text { and } B \text {. }\end{array}$ & & - & $\begin{array}{l}253,254 \\
255,256\end{array}$ & . \\
\hline Broken Loop & & $\begin{array}{l}1.6 \text { to } 1600 \\
\mathrm{~kg} / \mathrm{m}^{3}\end{array}$ & $\begin{array}{l}0 \text { to } 1600 \\
\mathrm{~kg} / \mathrm{m}^{3}\end{array}$ & & \\
\hline $\begin{array}{l}G B-21 T \\
G B-21 B \\
G B-21 C\end{array}$ & $\begin{array}{l}\text { Cold leg, Spool } 21,123 \mathrm{~cm} \text { from } \\
\text { vecsel center. I (top) ranges } \\
270 \text { to } 360^{\circ} \text {. B (bottom) ranges } \\
30 \text { to } 330^{\circ} \text { C. mathematical } \\
\text { composite of } T \text { and } B \text {. }\end{array}$ & & & $\begin{array}{l}257, \quad 258 \\
259 ; 260\end{array}$ & . \\
\hline GB-23VR & $\begin{array}{l}\text { Cold leg, spool } 23,235 \mathrm{~cm} \text { from } \\
\text { vessol contar, vertical. }\end{array}$ & & & 261,262 & \\
\hline
\end{tabular}


TABLE V (comtinued)

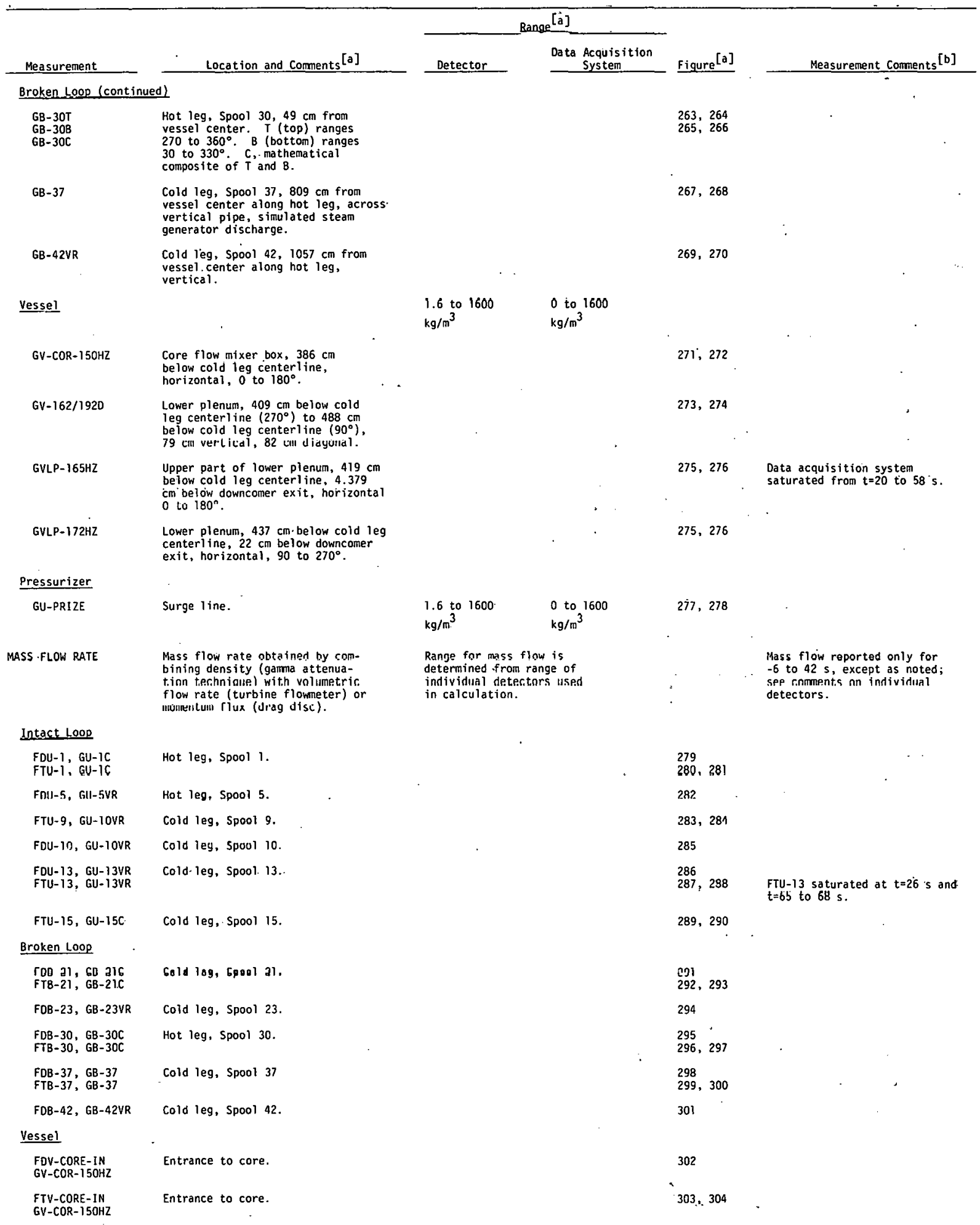


TABLE $\vee$ (continued)

\begin{tabular}{|c|c|c|c|c|c|}
\hline \multirow[b]{2}{*}{ Measurement } & \multirow[b]{2}{*}{ Location and Comments [a] } & \multicolumn{2}{|c|}{ Range [a] } & \multirow[b]{2}{*}{ Figure $^{[\mathrm{a}]}$} & \multirow[b]{2}{*}{ Measurement Comments ${ }^{[\mathrm{b}]}$} \\
\hline & & Detector & $\begin{array}{c}\text { Data Acquisition } \\
\text { System }\end{array}$ & & \\
\hline \multicolumn{6}{|l|}{ Pressurizer } \\
\hline $\begin{array}{l}\text { FTU-PRIZE } \\
\text { GU-PRIZE }\end{array}$ & Pressurizer surge line. & & & 305,306 & \\
\hline CORE CHARACTERISTICS & & & & & - \\
\hline PWRCOR T-1 & Core power. & 0 to $1600 \mathrm{kw}$ & $2119 \mathrm{~kW}$ & 307,308 & \\
\hline PWRCOR T-2 & Core power. & 0 to $1600 \mathrm{kH}$ & $2670 \mathrm{~kW}$ & 307,308 & \\
\hline VOLTCOR-T & Core vol tage. & & 0 to $200 \mathrm{Vdc}$ & 309,310 & \\
\hline AMPCOR-1 & Core current. & 0 to 1000 amps & 0 to $9790 \mathrm{~A}$ & 311,312 & \\
\hline PUMP CHARACTERISTICS & & & & & \\
\hline PUMPU-CUR & Pump current. & & 0 to $44 \mathrm{~A}$ & 313,314 & \\
\hline - PUMPU-RPM & Pump speed. & & 0 to $3600 \mathrm{rpm}$ & 315,316 & \\
\hline
\end{tabular}

[a] Statements at the beginning of a measurement category regarding location and corments, range, and figure apply to all subsequent measurements within the given category uniess specified otherwise.

[b] Detectors which were subjected to overrange conditions during portions of the test were capable of withstanding these conditions without change in operating or measuring characteristics when the physical conditions were again within the detector range. 


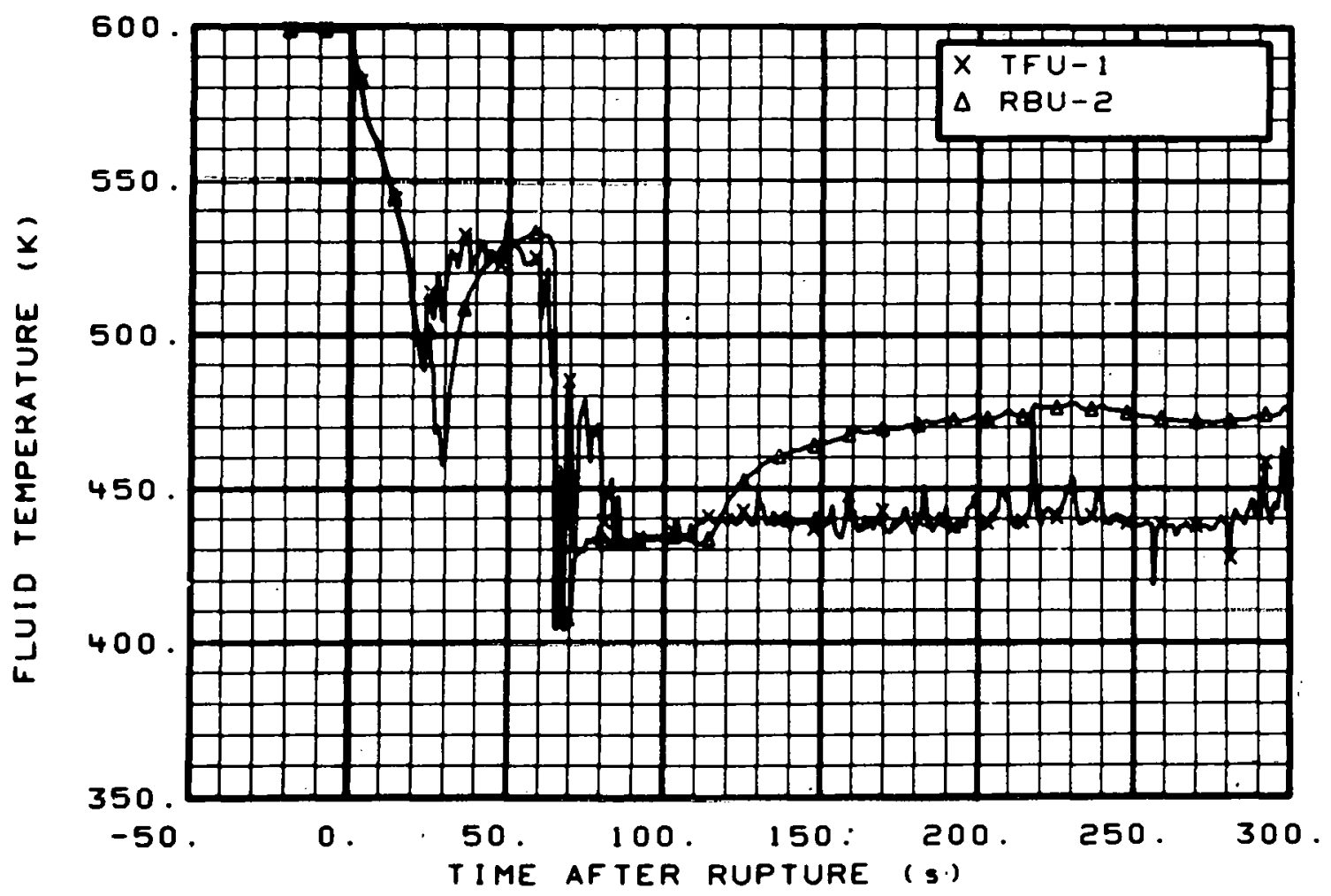

Fig: 9 Fluid temperature in intact loop hot leg (TFU-1 and RBU-2), from -20 to $300 \mathrm{~s}$.

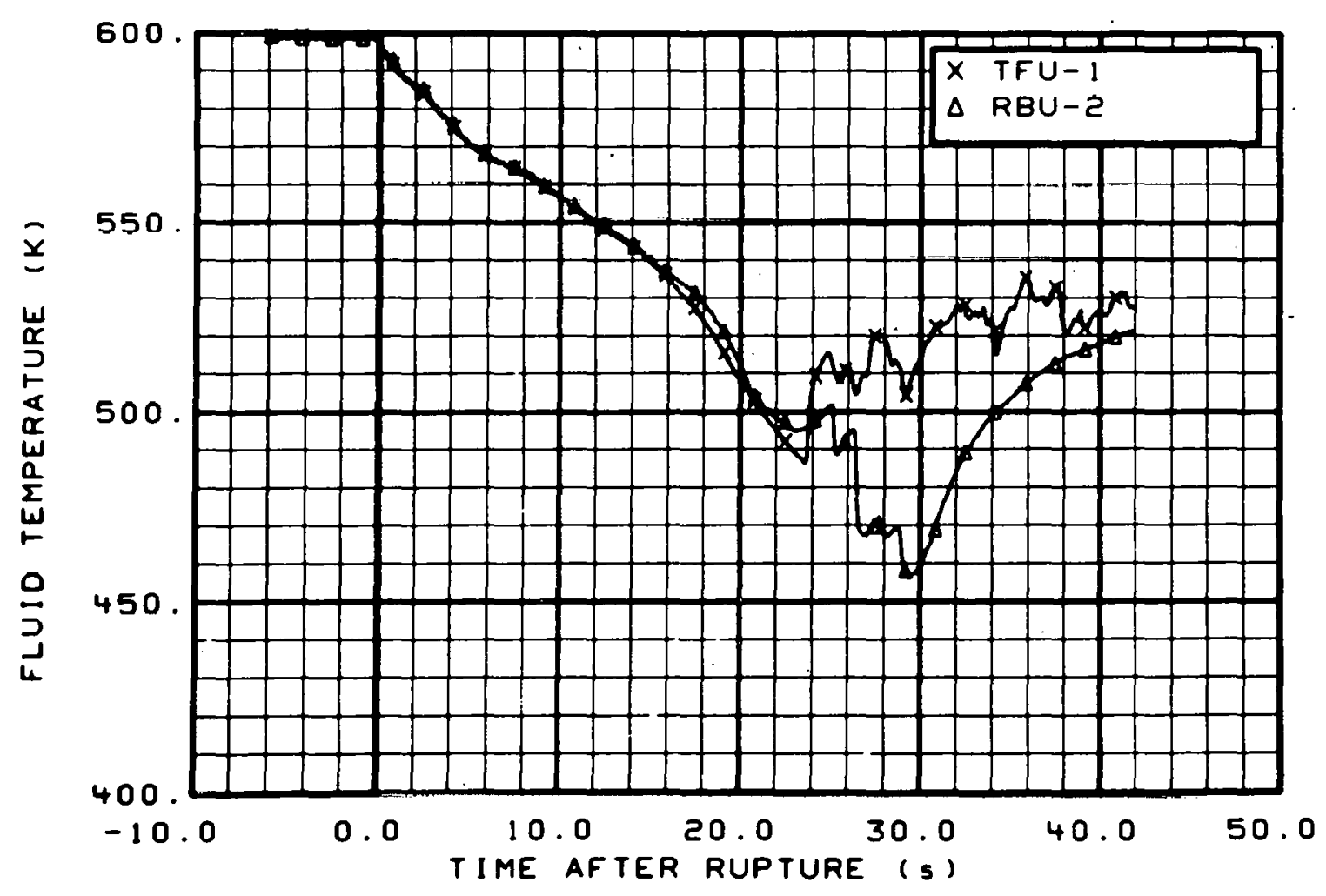

Fig. 10 Fluid temperature in intact loop hot leg (TFU-1 and RBU-2), from -6 to $42 \mathrm{~s}$. 


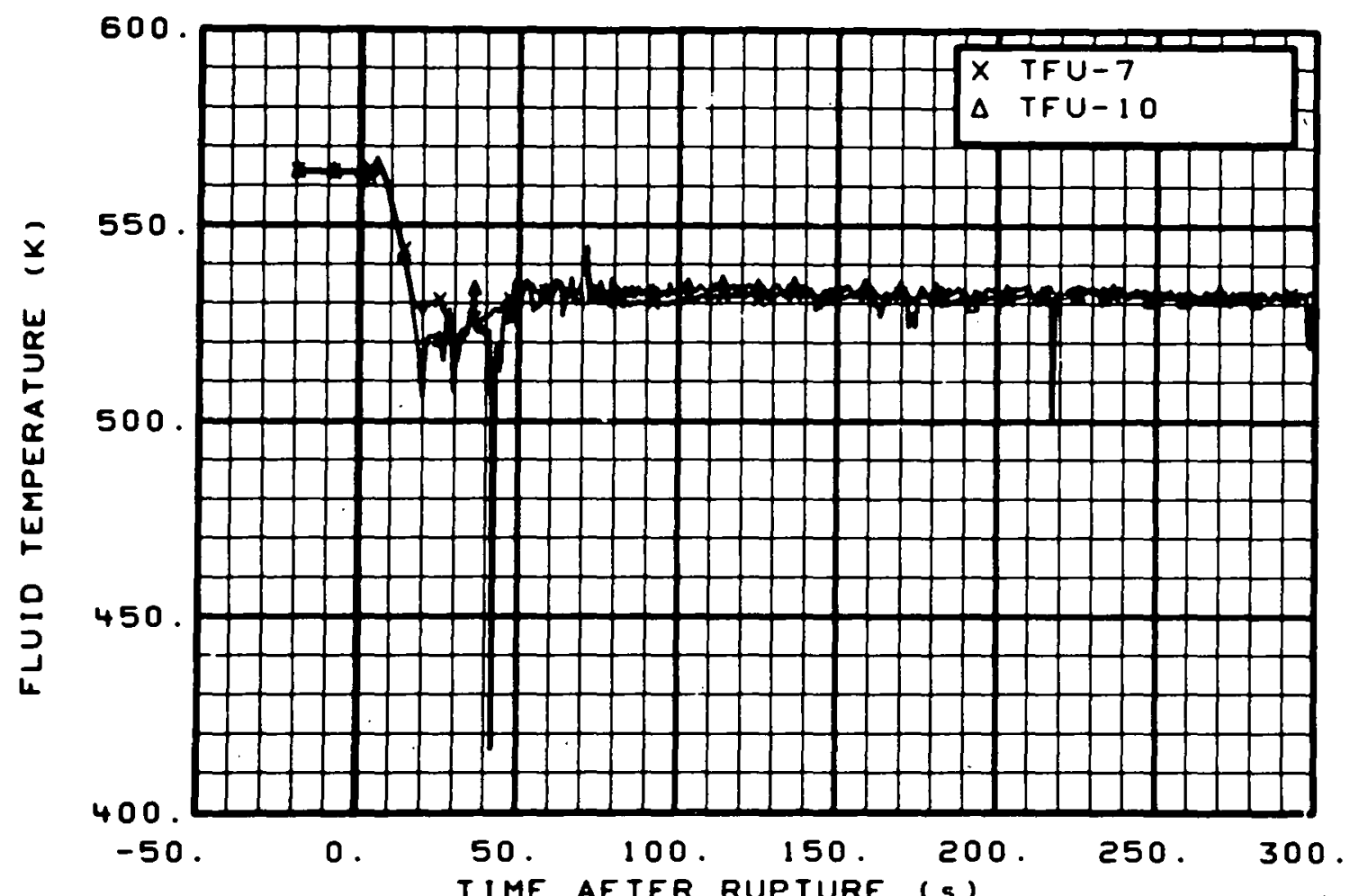

Fig. 11 Fluid temperature in intact loop cold leg (TFU-7 and TFU-10), from -20 to $300 \mathrm{~s}$.

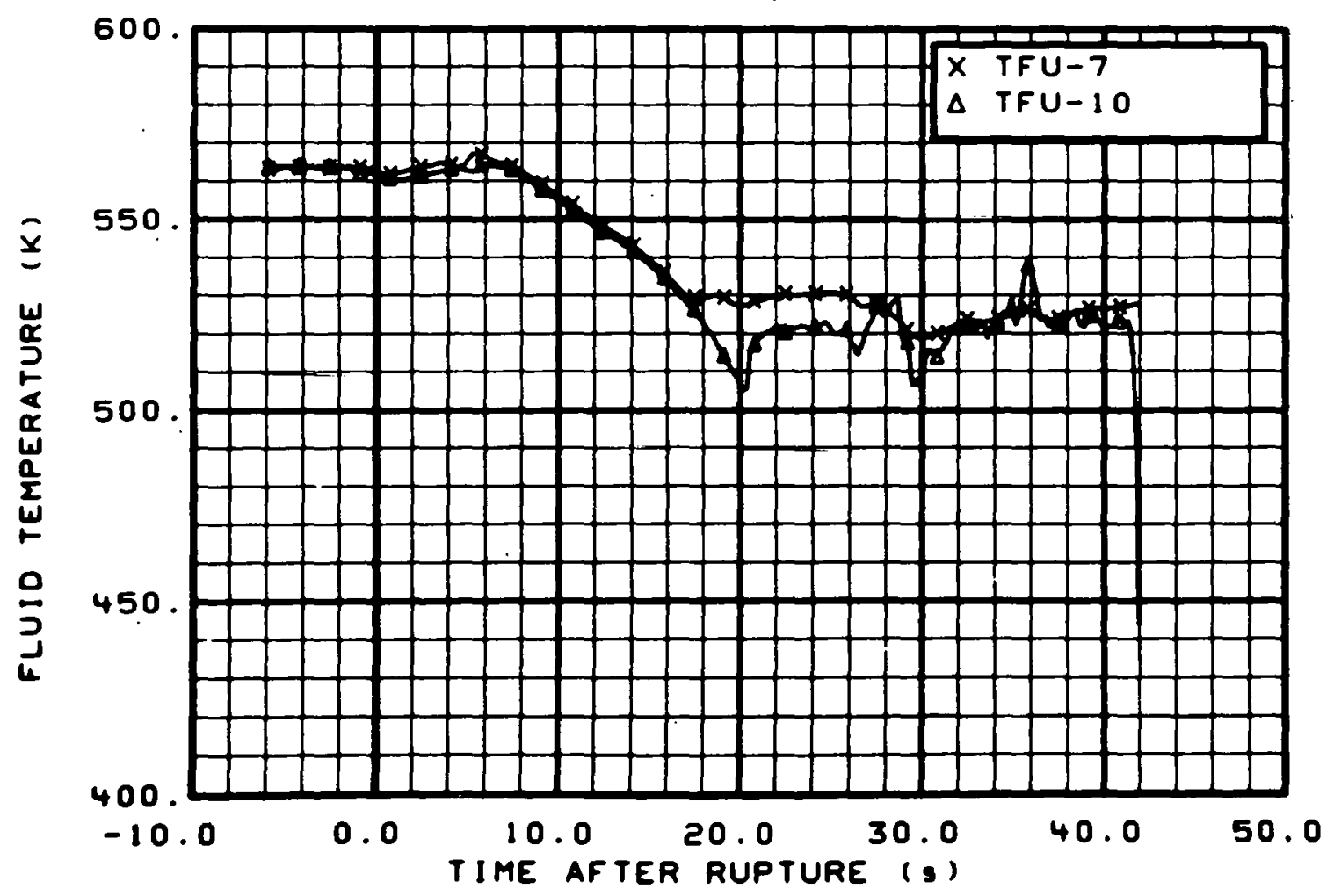

Fig. 12 Fluid temperature in intact loop cold leg (TFU-7 and TFU-10), from -6 to $42 \mathrm{~s}$. 


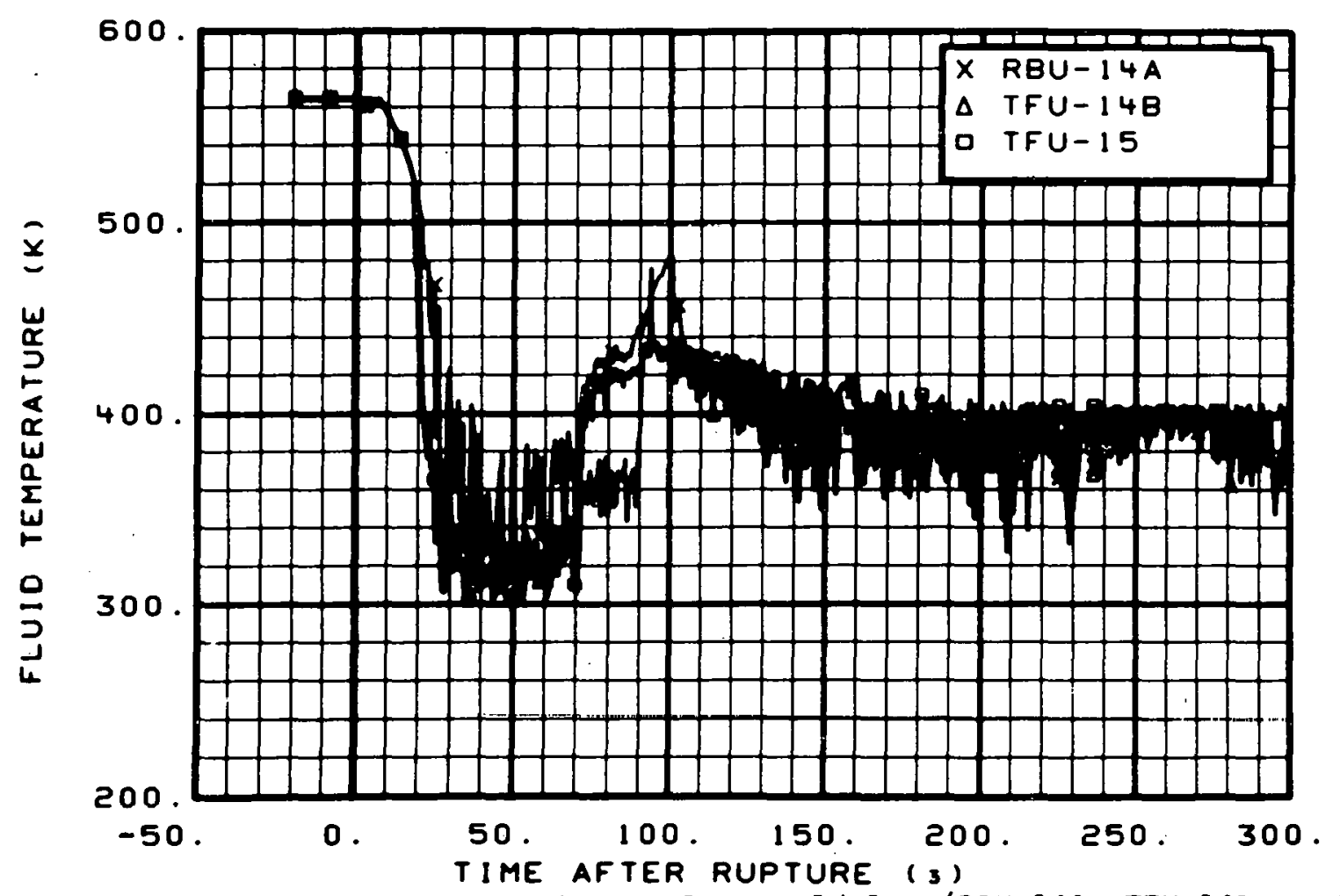

Fig. 13 Fluid temperature in intact loop cold leg (RBU-14A, TFU-14B, and TFU-15), from -20 to $300 \mathrm{~s}$.

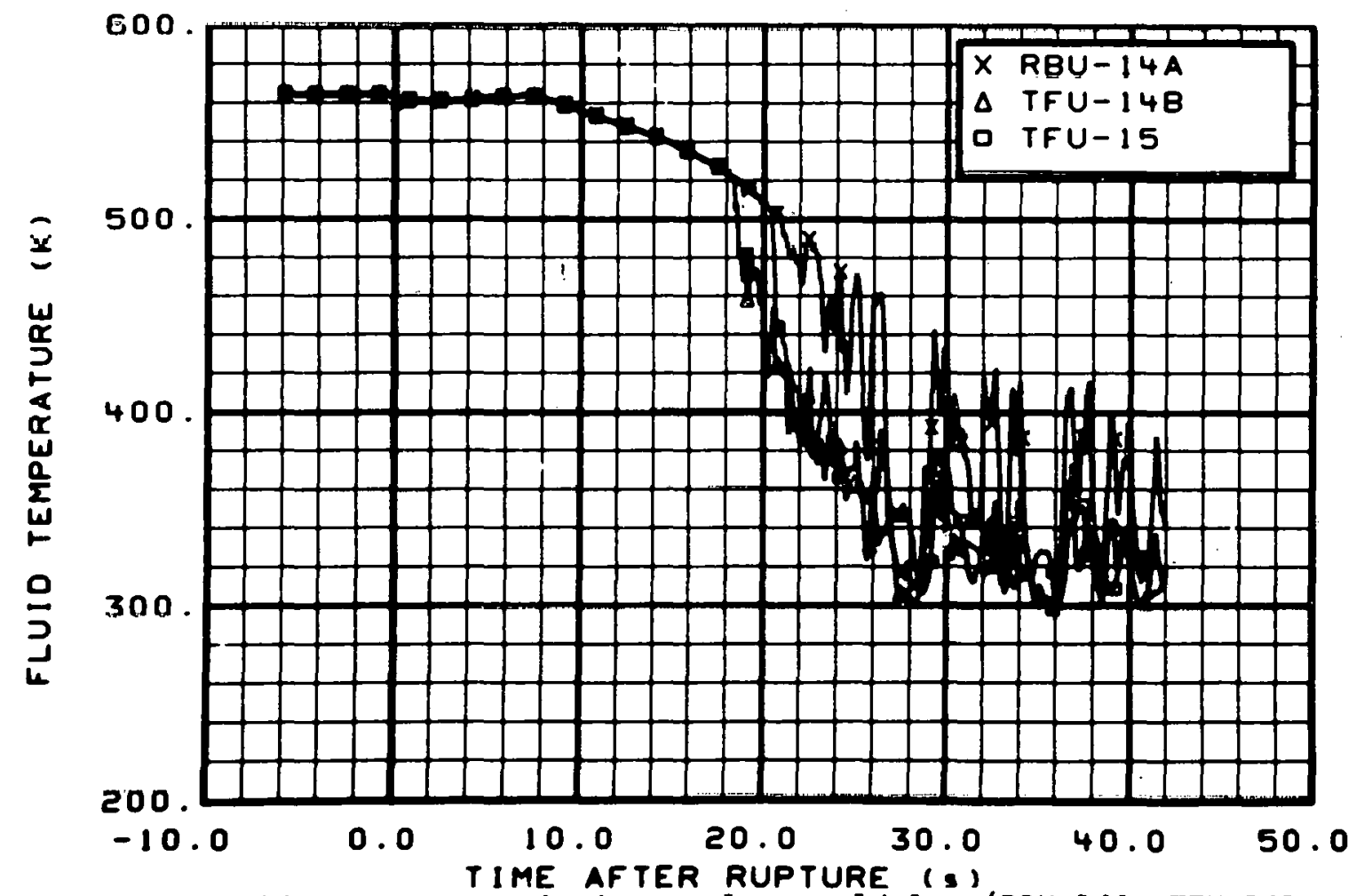

Fig. 14 Fluid temperature in intact loop cold leg (RBU-14A, TFU-14B, and TFU-15), from -6 to $42 \mathrm{~s}$. 


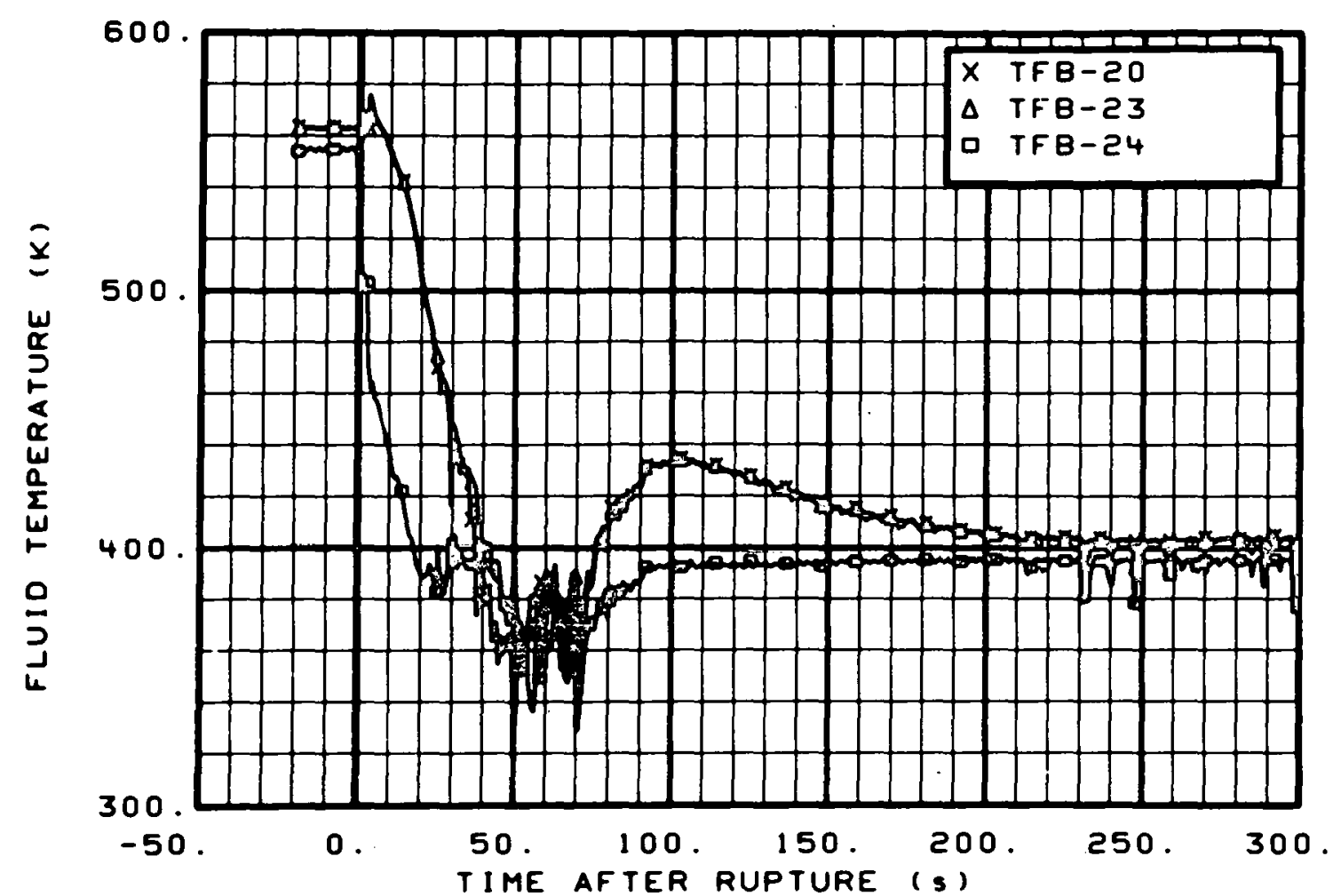

Fig. 15 Fluid temperature in broken loop, vessel side (TFB-20, TFB-23, and TFB-24), from -20 to $300 \mathrm{~s}$.

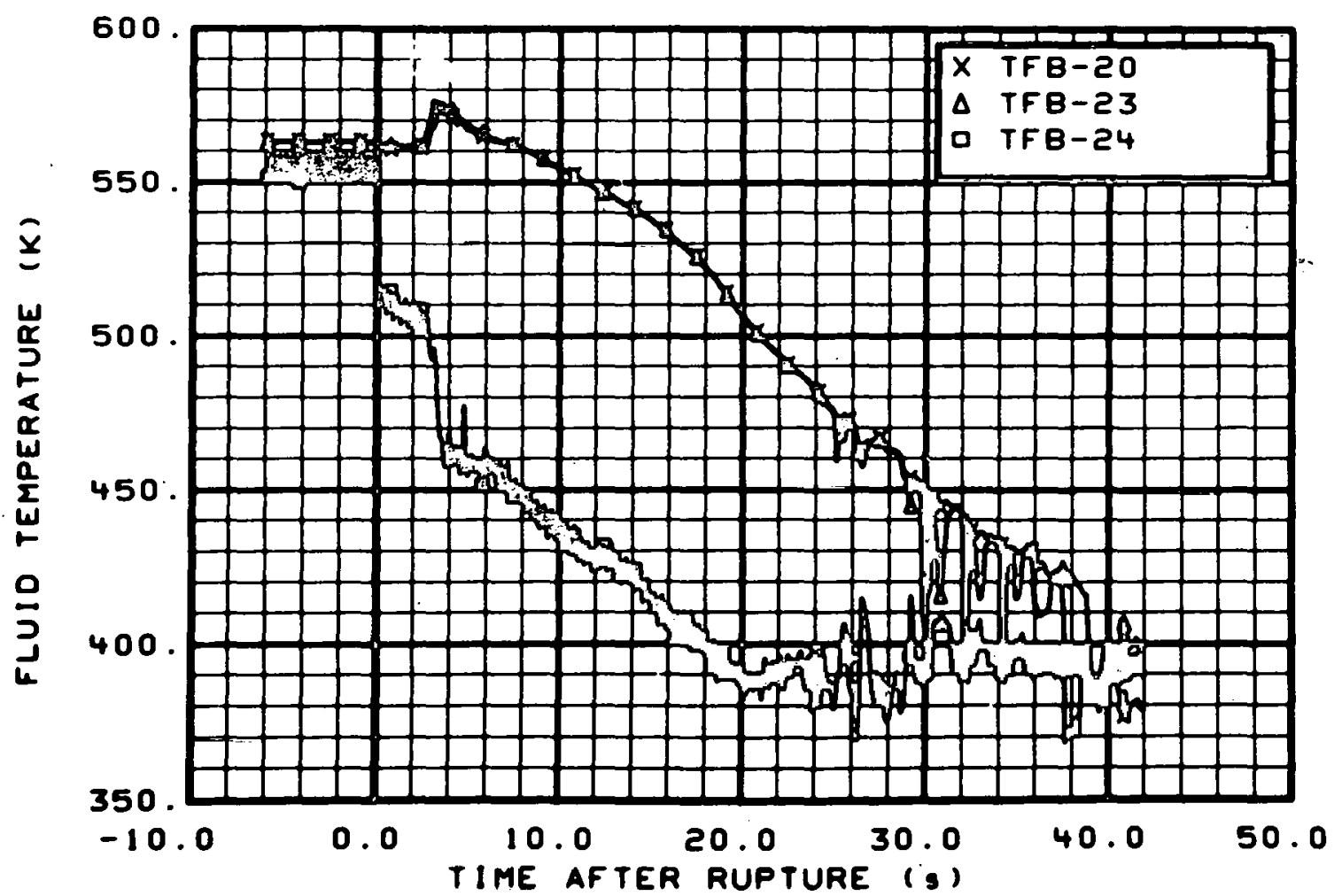

Fig. 16 Fluid temperature in broken loop, vessel side (TFB-20, TFB-23, and TFB-24), from -6 to $42 \mathrm{~s}$. 


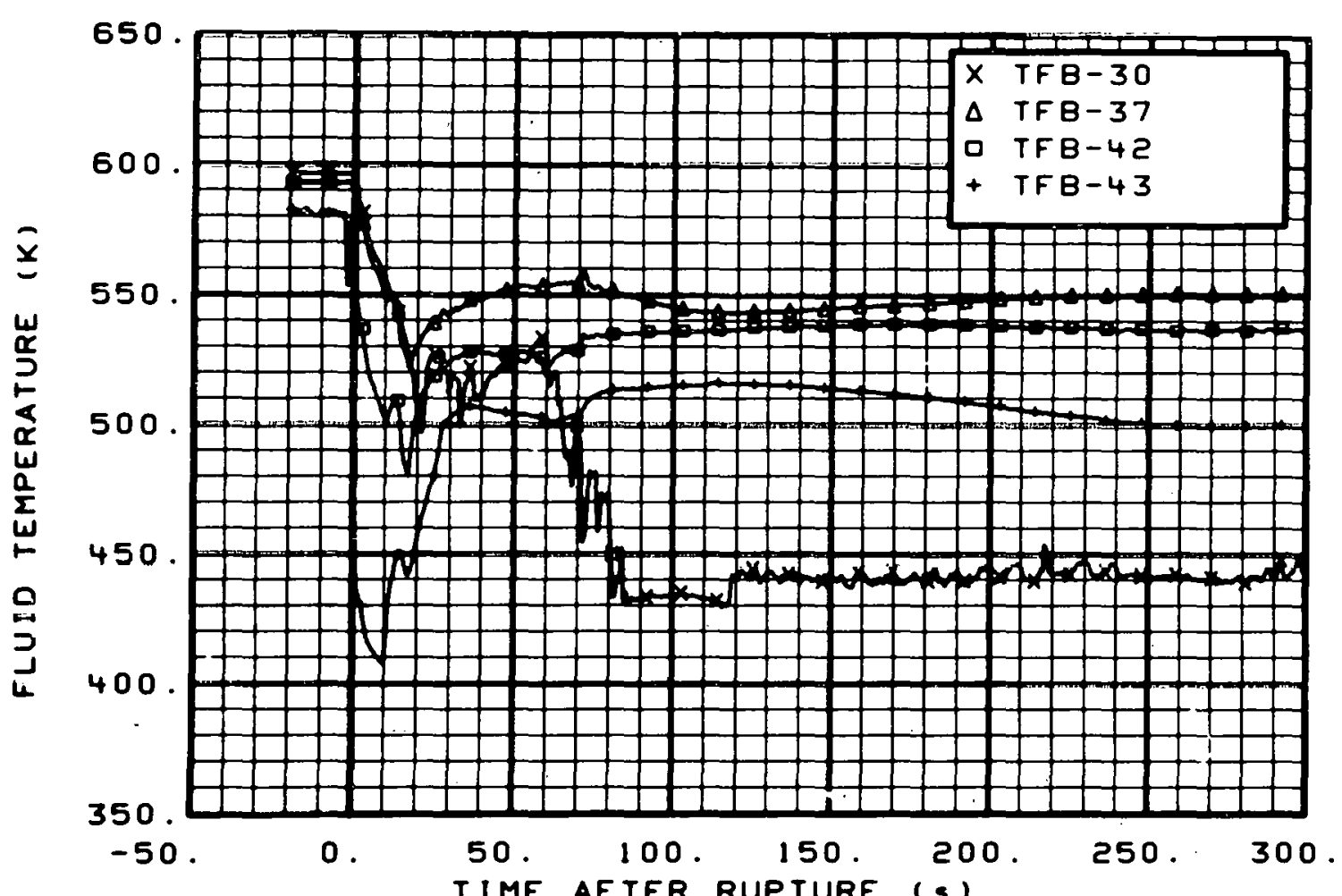

Fig. 17 Fluid temperature in broken loop, pump side (TFB-30, TFB-37, TFB-42, and TFB-43), from -20 to $300 \mathrm{~s}$.

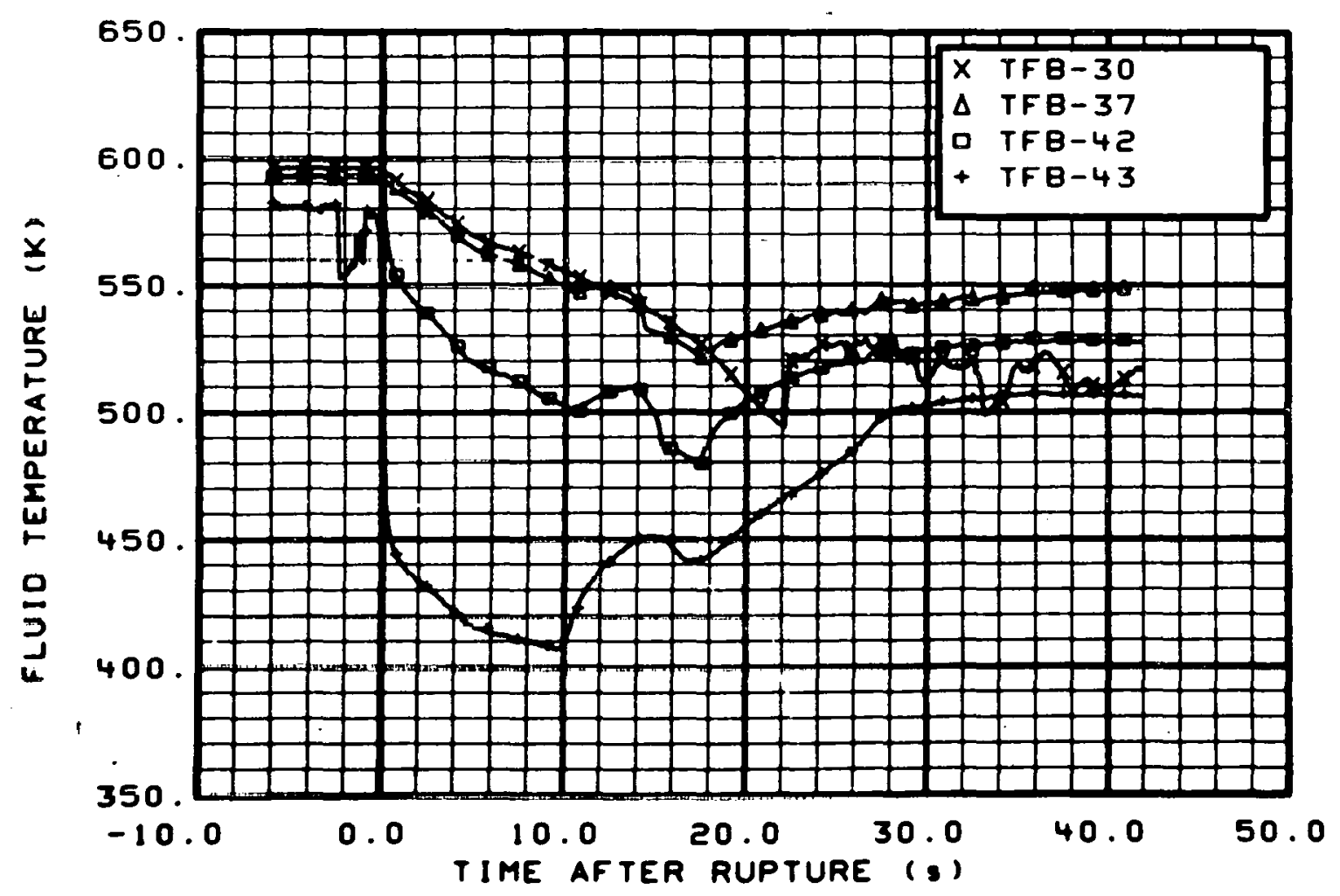

Fig. 18 Fluid temperature in broken loop, pump side (TFB-30, TFB-37, TFB-42, and TFB-43), from -6 to $42 \mathrm{~s}$. 


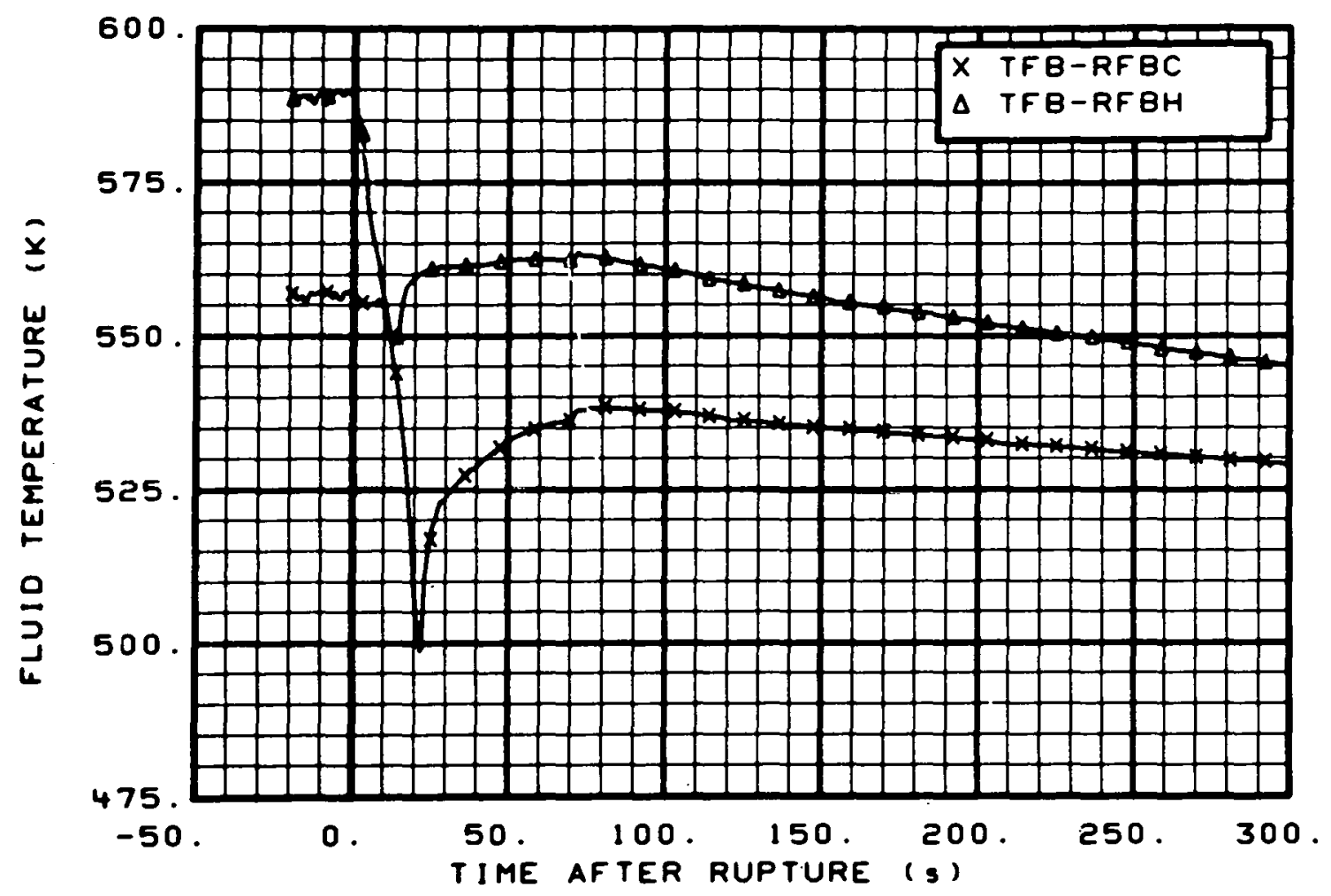

Fig. 19 Fluid temperature in broken loop, reflood bypass simulators (TFB-RFBC and TFB-RFBH), from -20 to $300 \mathrm{~s}$.

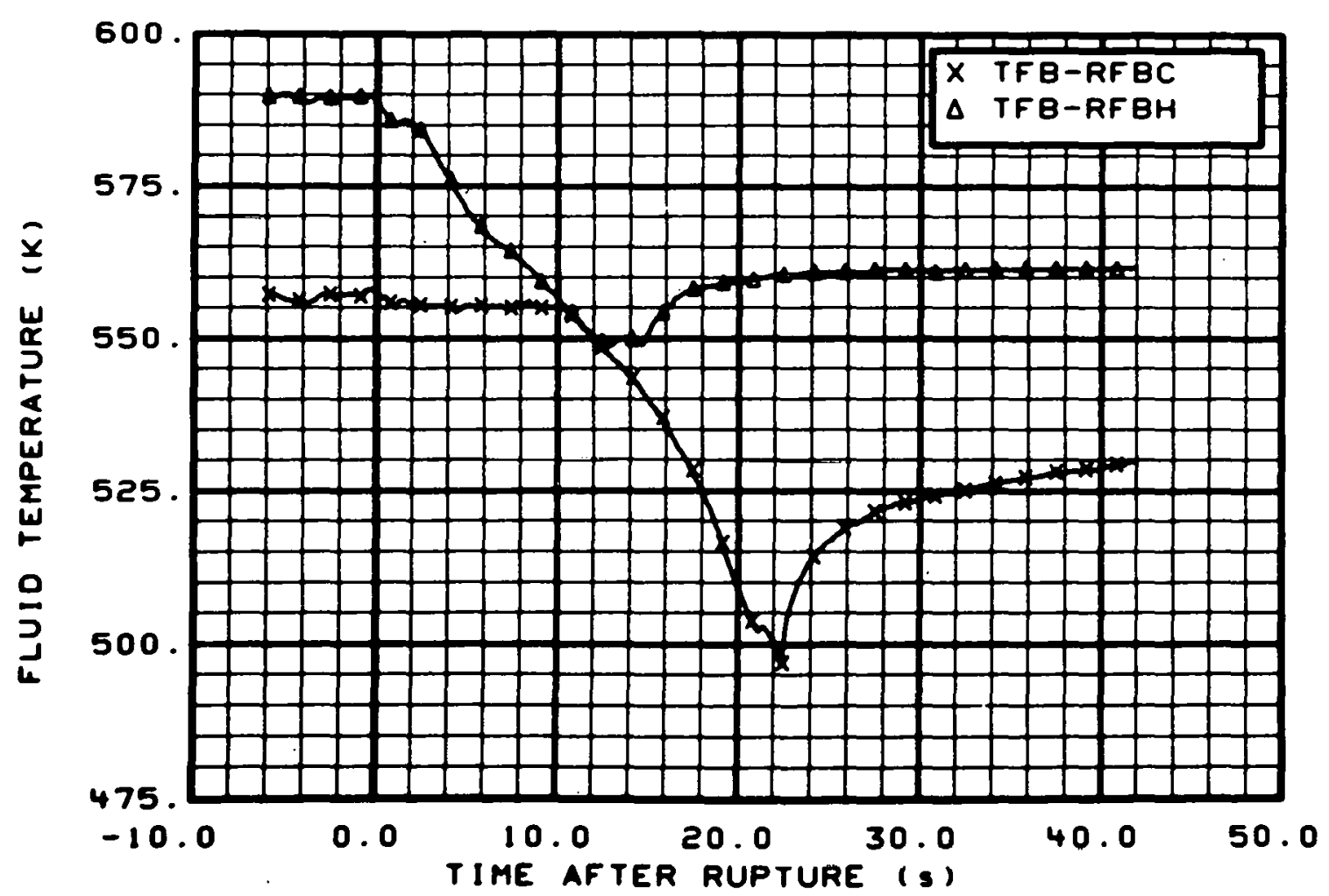

Fig. 20 Fluid temperature in broken loop, reflood bypass simulators (TFB-RFBC and TFB-RFBH), froill -6 to $42 \mathrm{~s}$. 


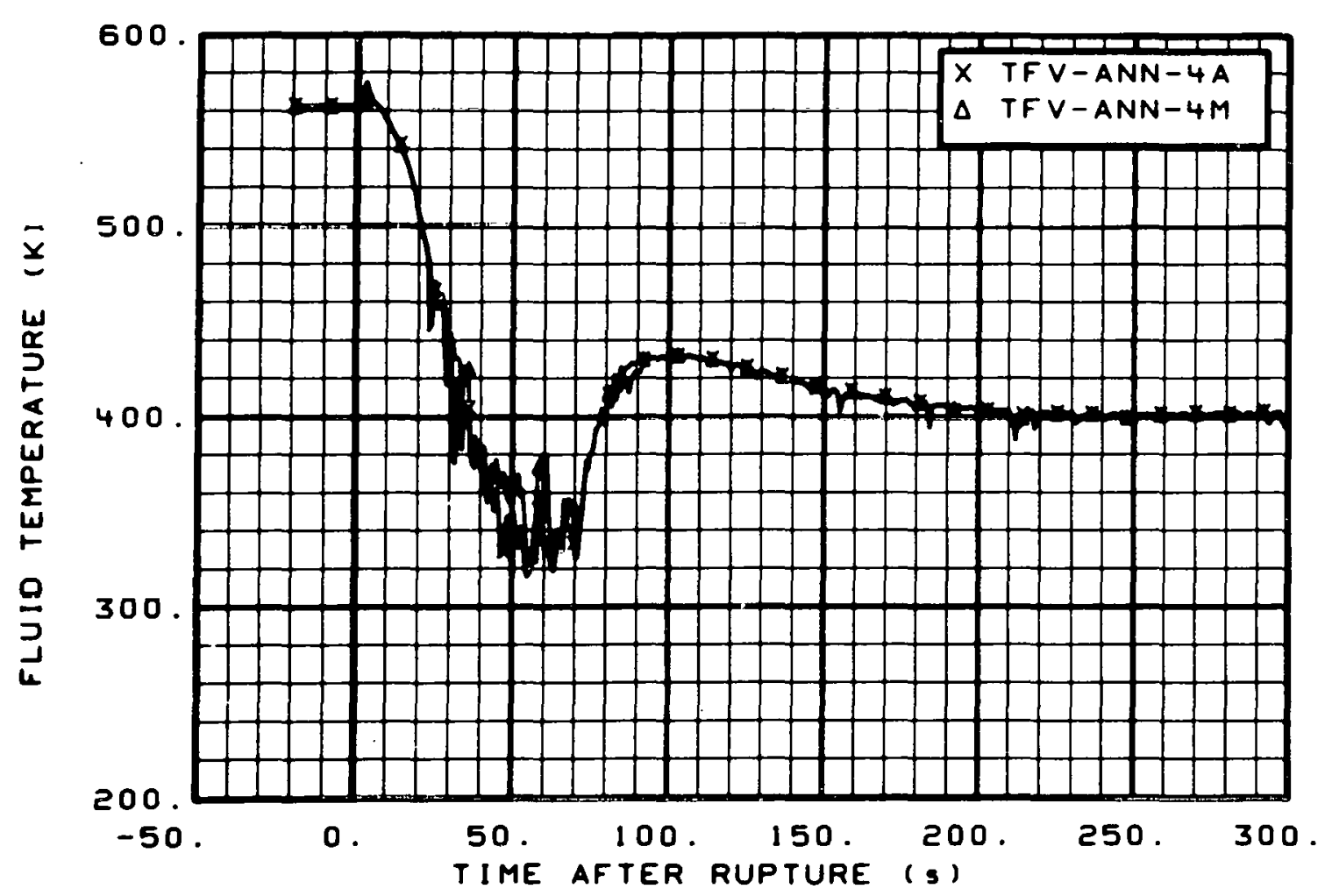

Fig. 21 Fluid temperature in inlet annulus (TFV-ANN-4A and TFV-ANN-4M), from -20 to $300 \mathrm{~s}$.

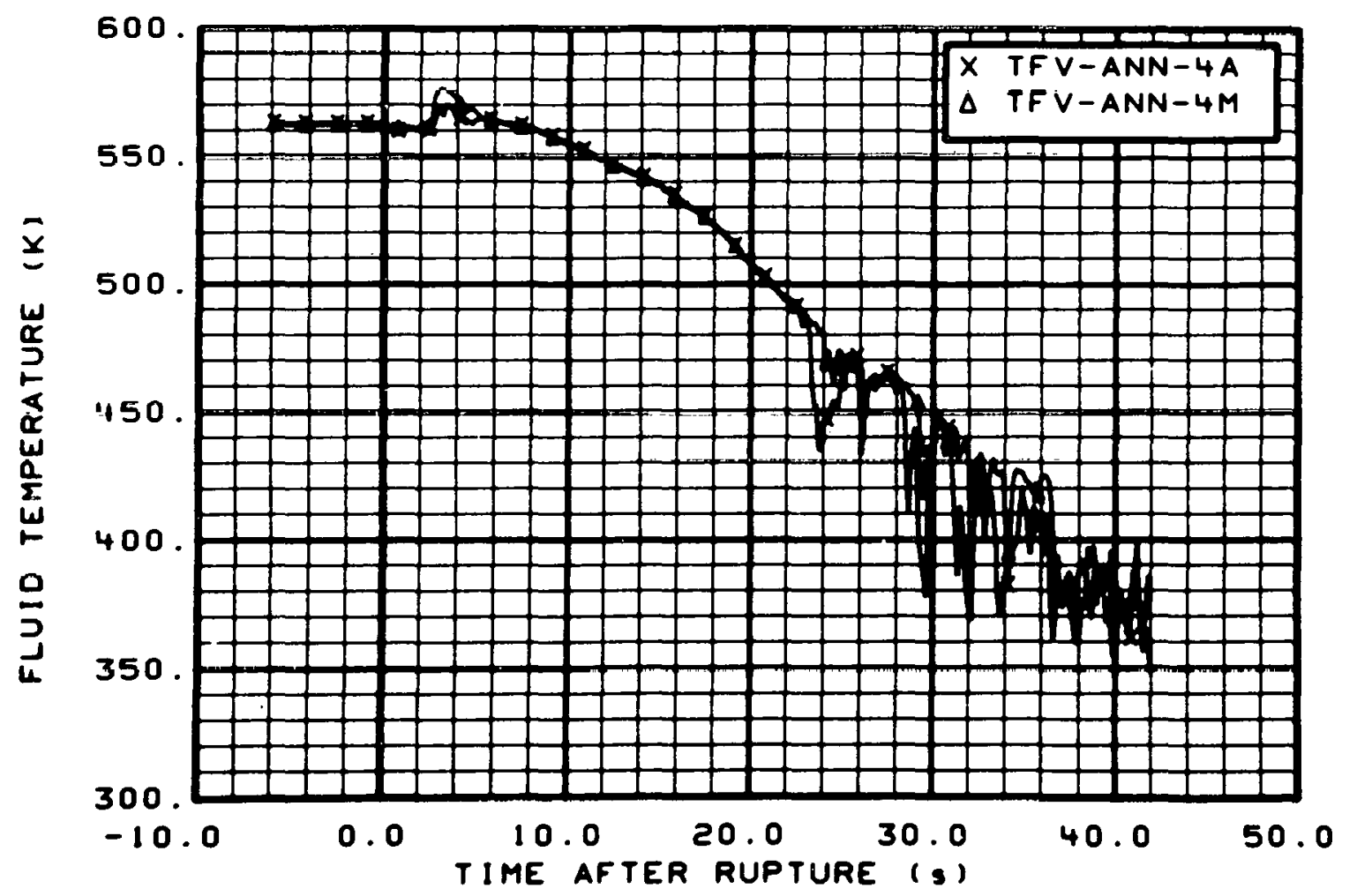

Fig. 22 Fluid temperature in inlet annulus (TFV-ANN-4A and TFV-ANN-4M), from -6 to $42 \mathrm{~s}$. 


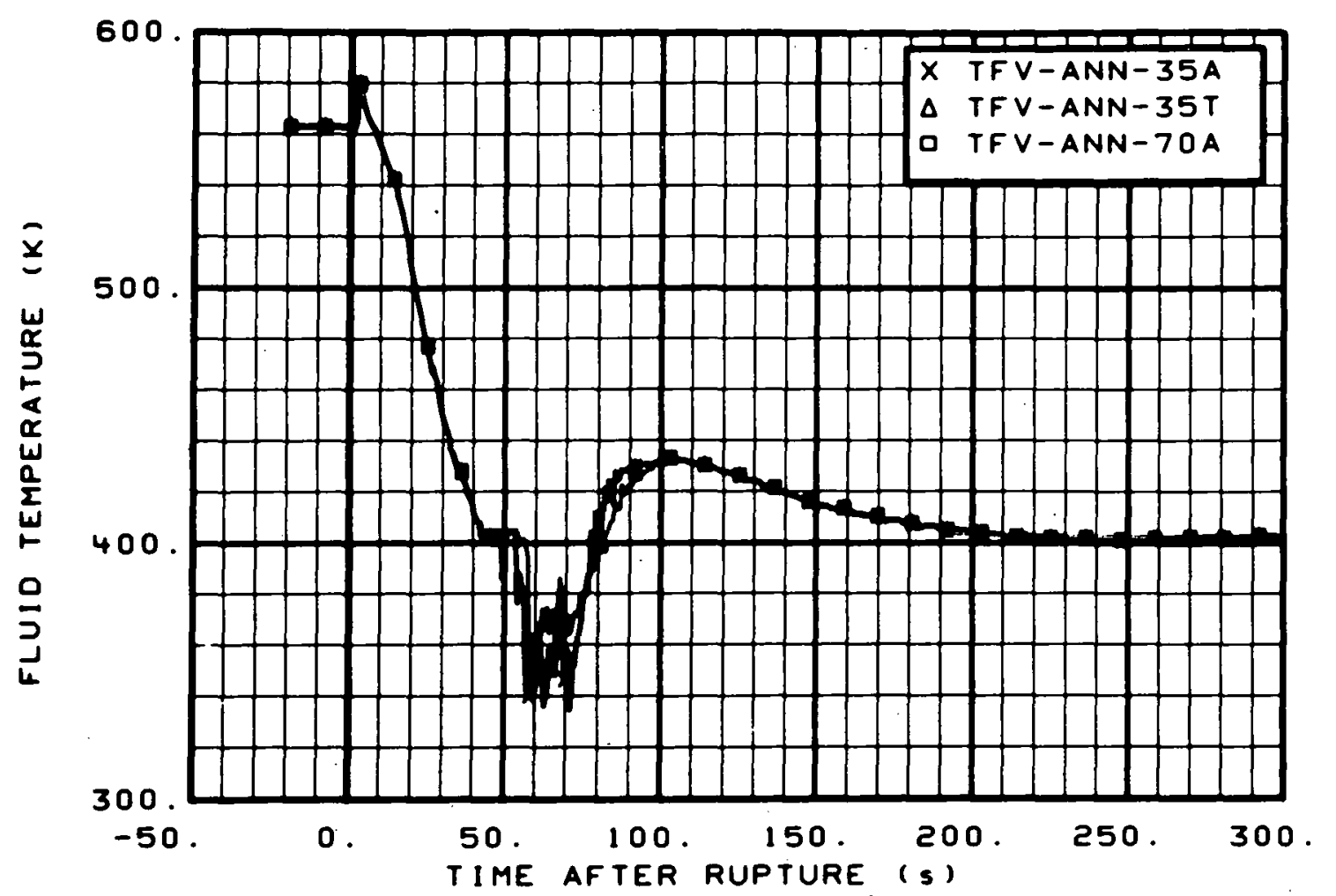

Fig. 23 Fluid temperature in downcomer annulus (TFV-ANN-35A, TFV-ANN-35T, and TFV-ANN-70A), from -20 to $300 \mathrm{~s}$.

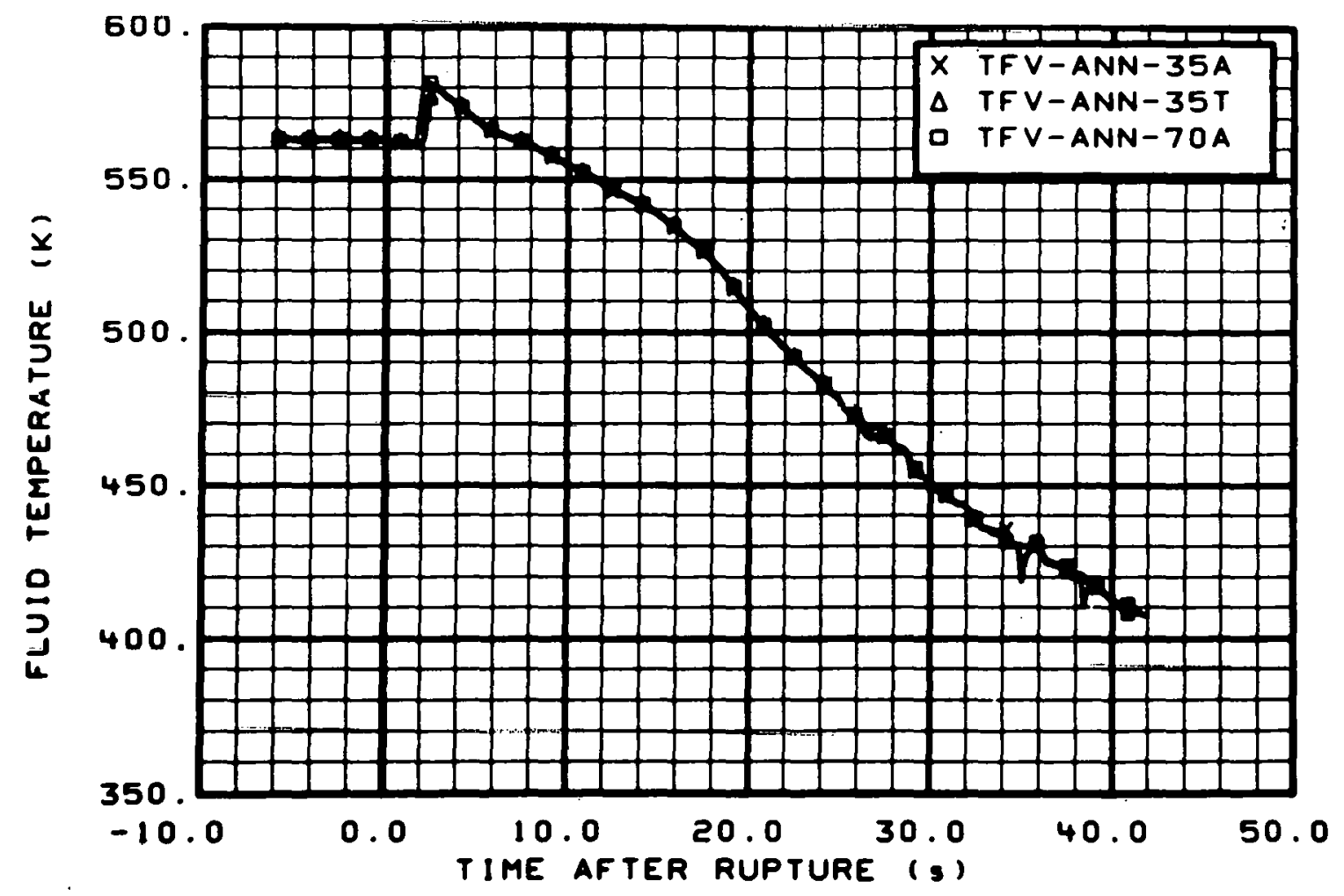

Fig. 24 Fluid temperature in downcomer annulus (TFV-ANN-35A, TFV-ANN-35T, and TFV $-A N N-70 A$ ), from -6 to $42 \mathrm{~s}$. 


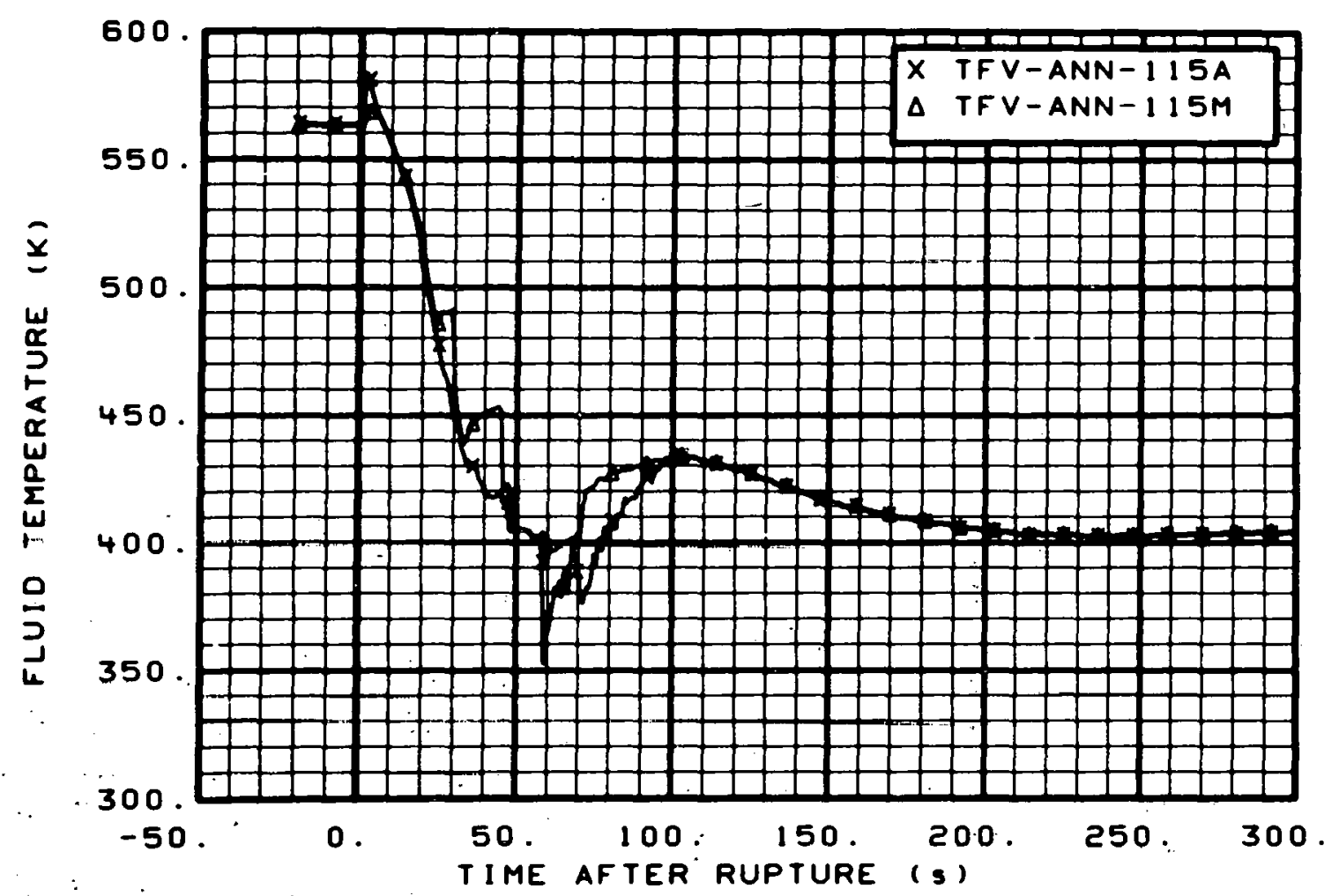

Fig. 25 Fluid temperature in downcomer annulus (TFV-ANN-115A and TFV-ANN-115M), from -20 to $300 \mathrm{~s}$.

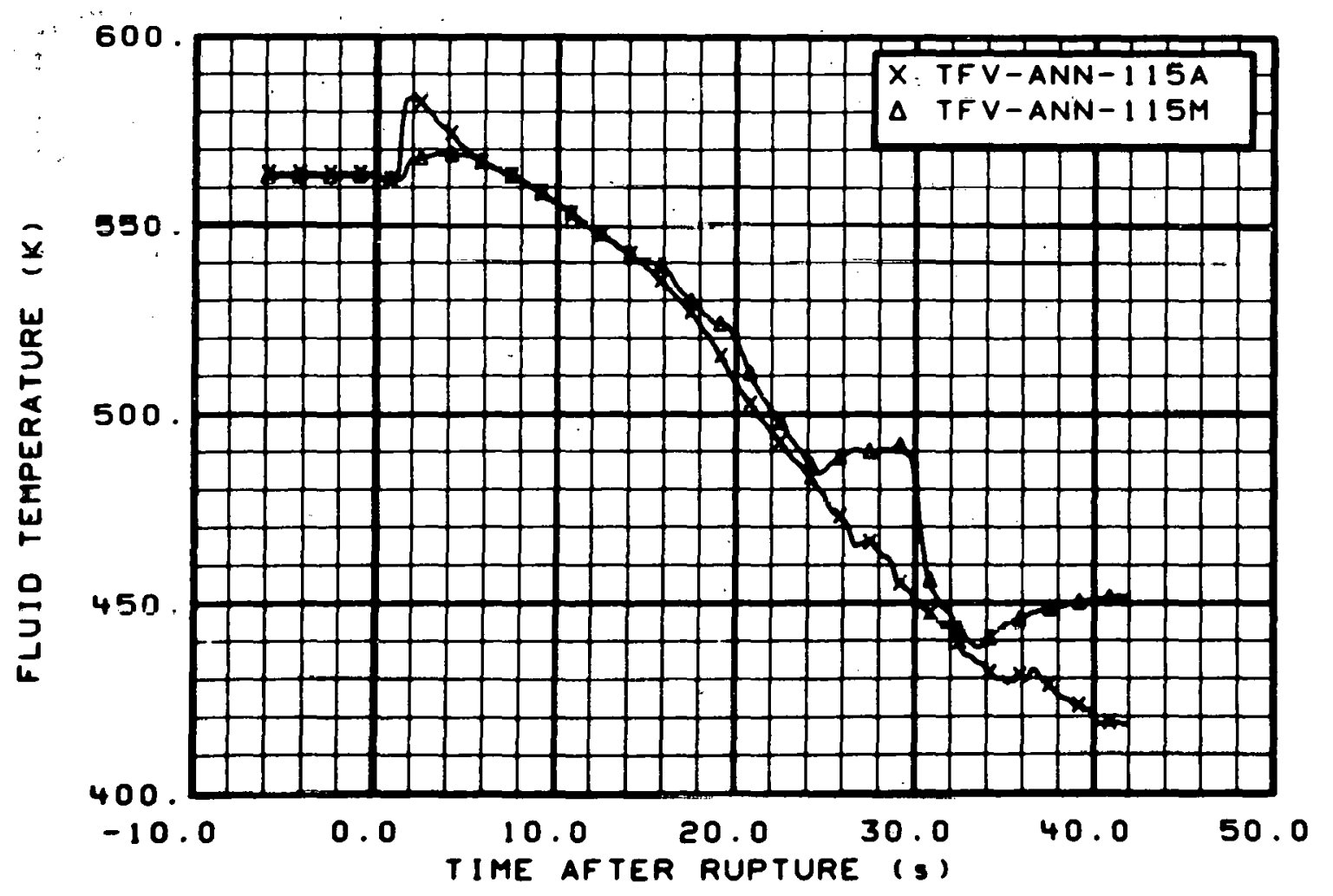

Fig. 26 Fluid temperature in downcomer annulus (TFV-ANN-115A and TFV-ANN-115M), from -6 to $42 \mathrm{~s}$. 


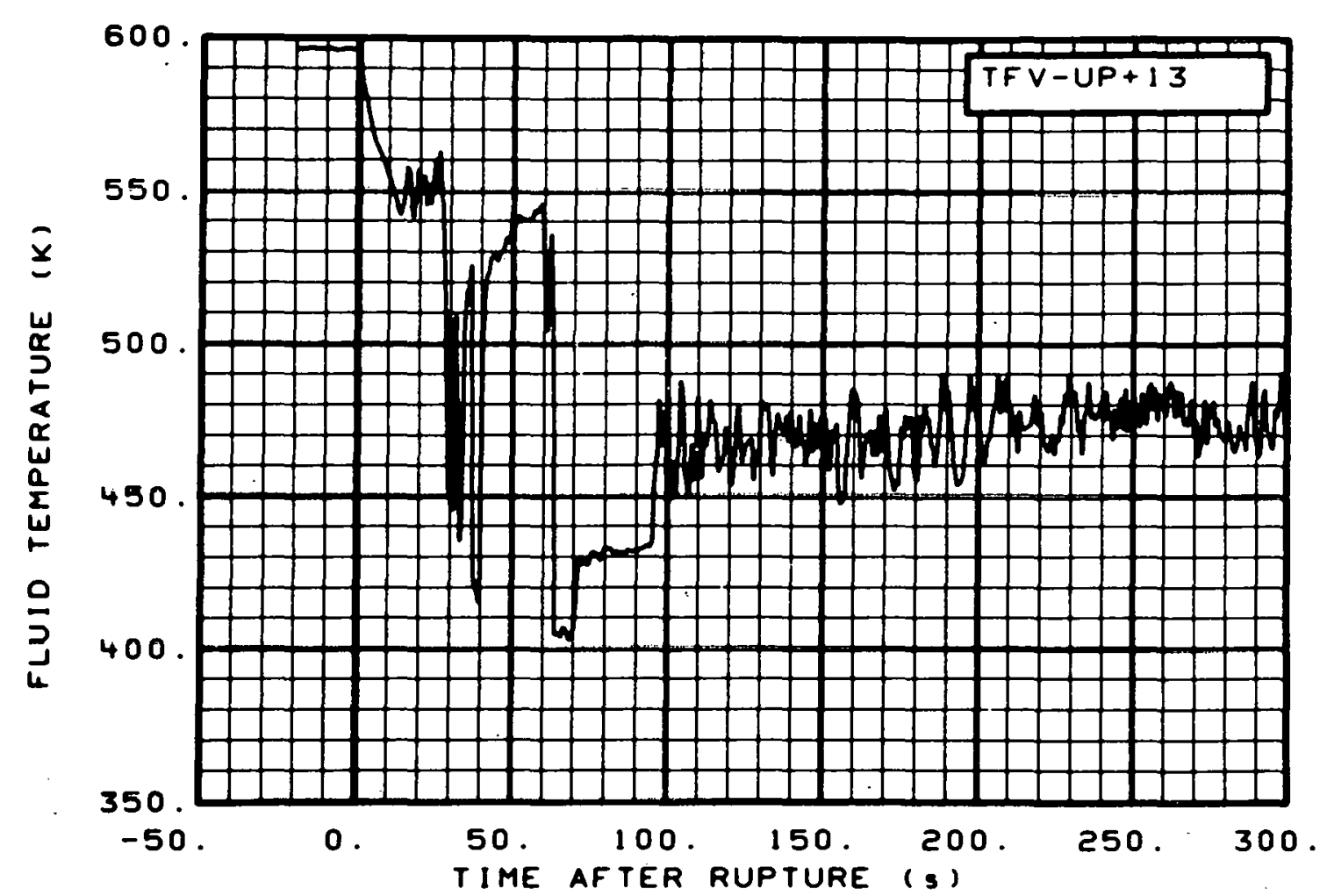

Fig. 27 Fluid temperature in upper plenum (TFV-UP+13), from -20 to $300 \mathrm{~s}$.

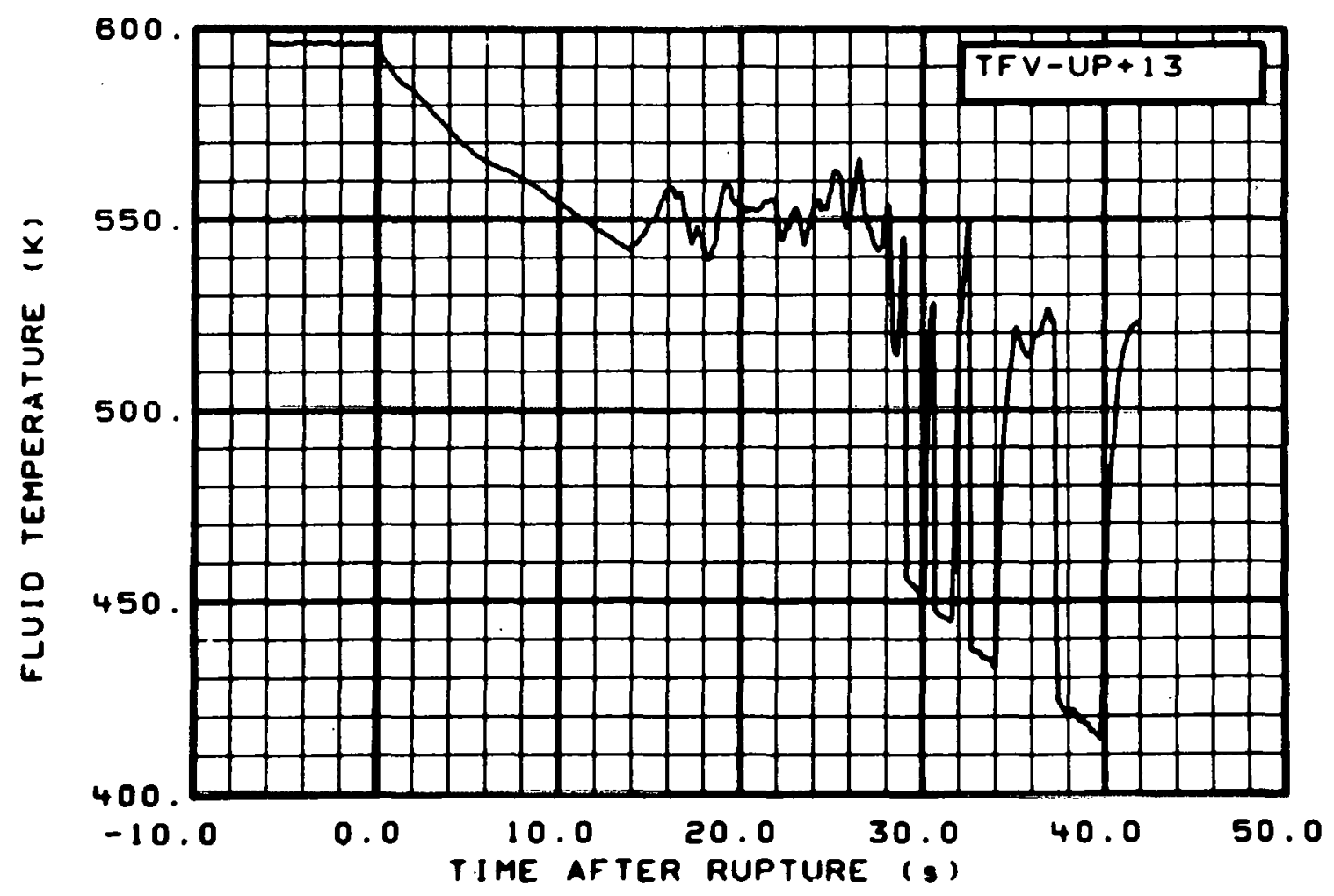

Fig. 28 Fluid temperature in upper plenum $(T F V-U P+13)$, from -6 to $42 \mathrm{~s}$. 


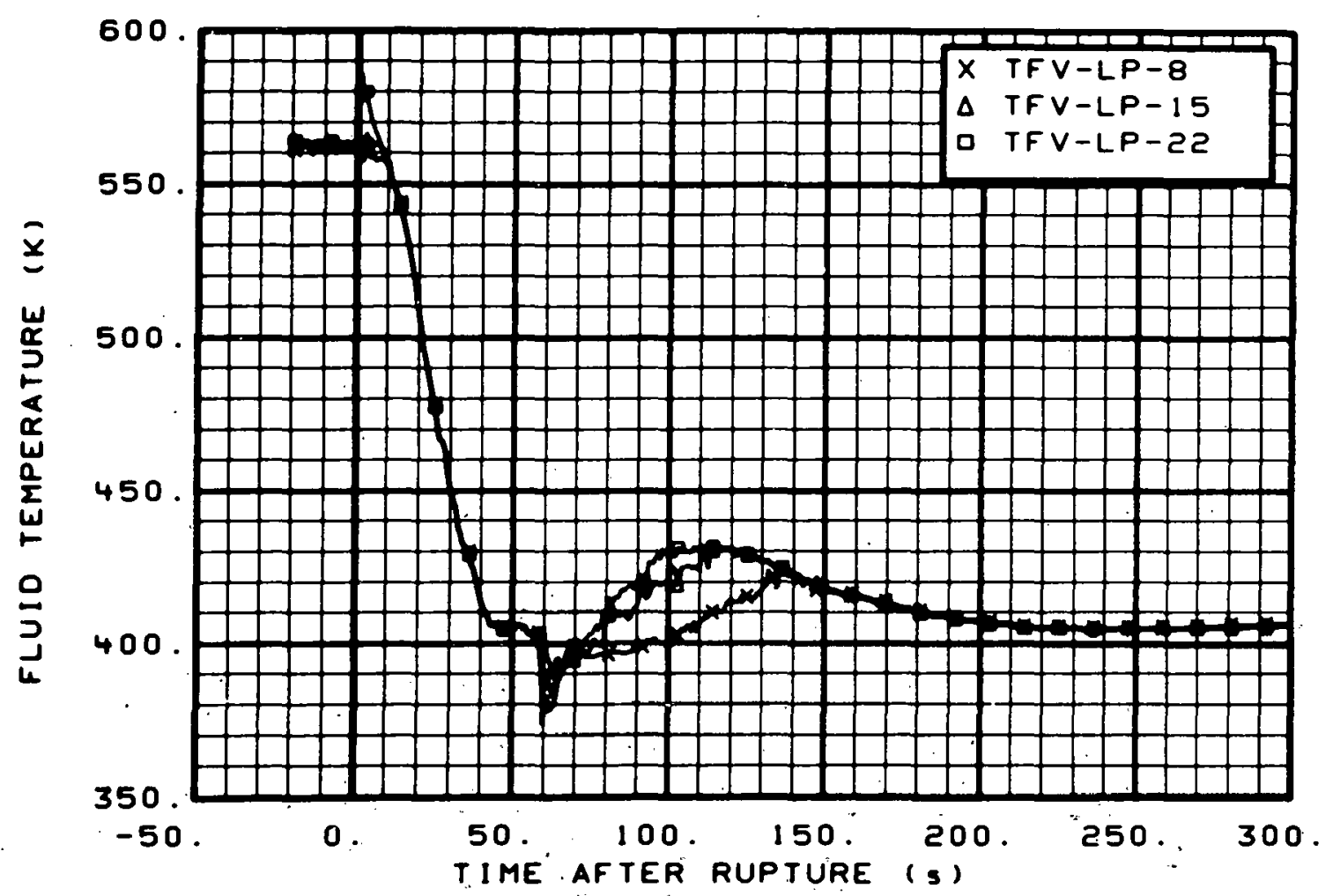

Fig. 29 Fluid temperature in lower plenum (TFV-LP-8, TFV-LP-15, and TFV-LP-22), from -20 to $300 \mathrm{~s}$.

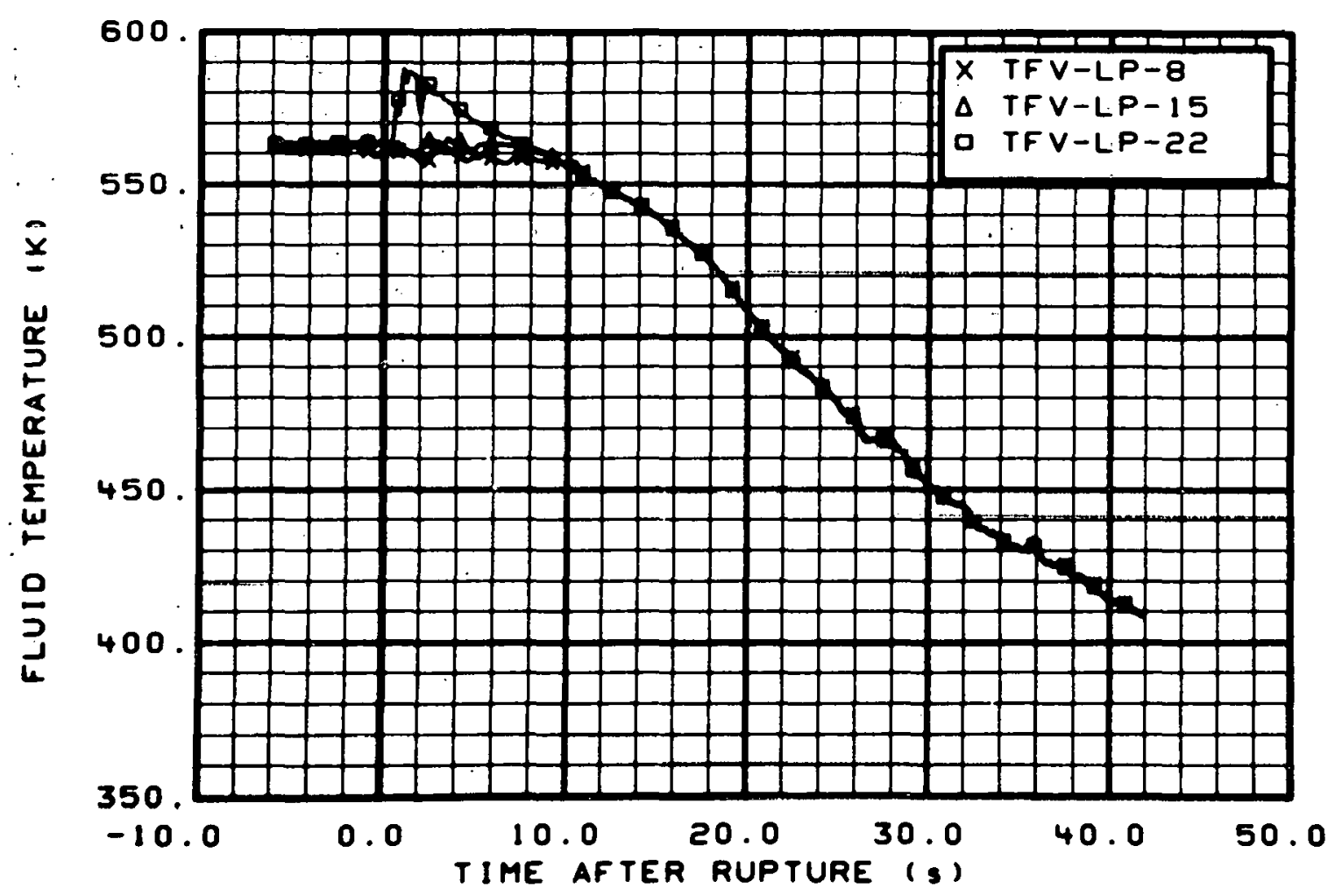

Fig. 30 Fluid temperature in lower plenum (TFV-LP-8, TFV-LP-15, and TFV-LP-22), from -6 to $42 \mathrm{~s}$. 


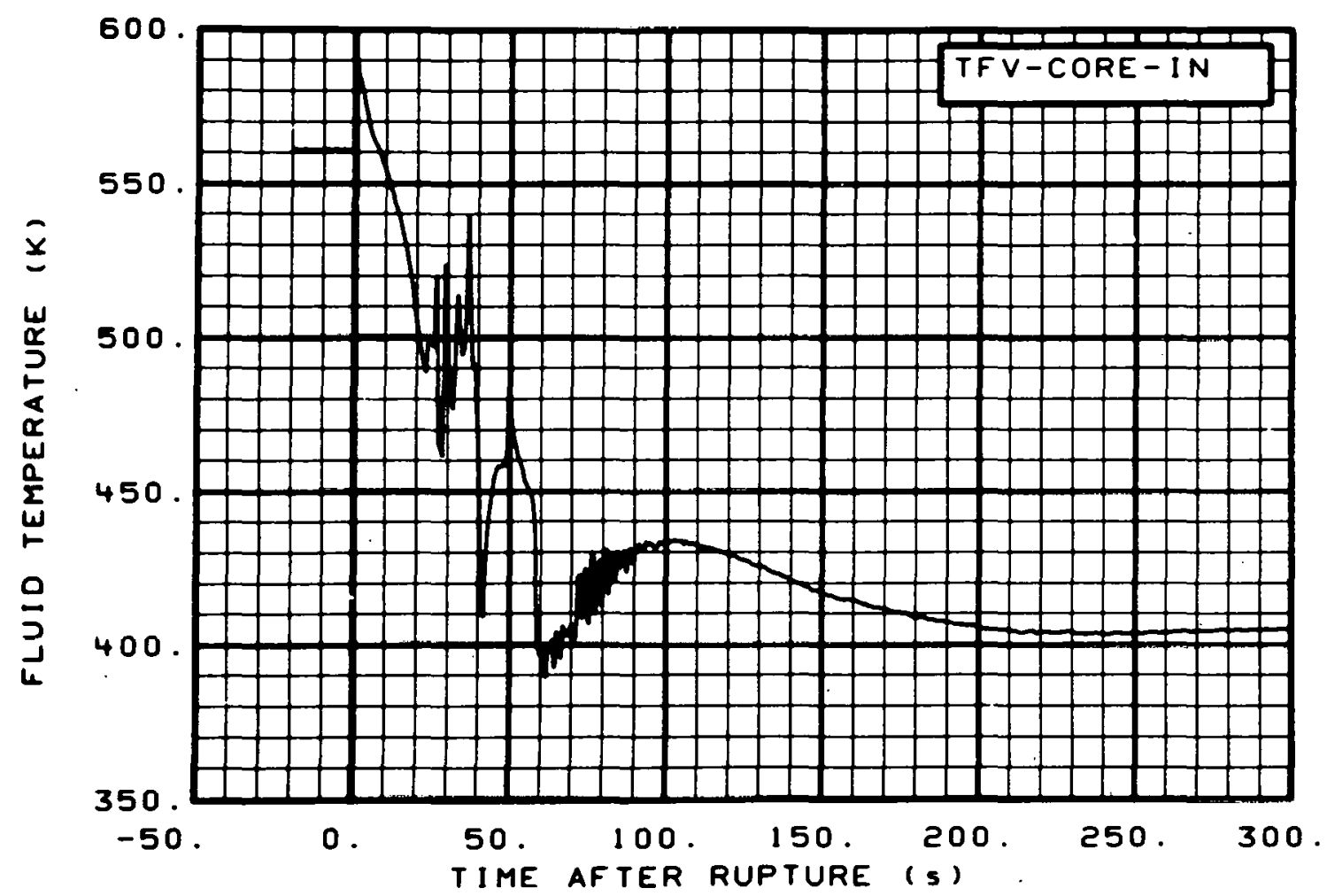

Fig. 31 Fluid temperature in core inlet (TFV-CORE-IN), from -20 to $300 \mathrm{~s}$.

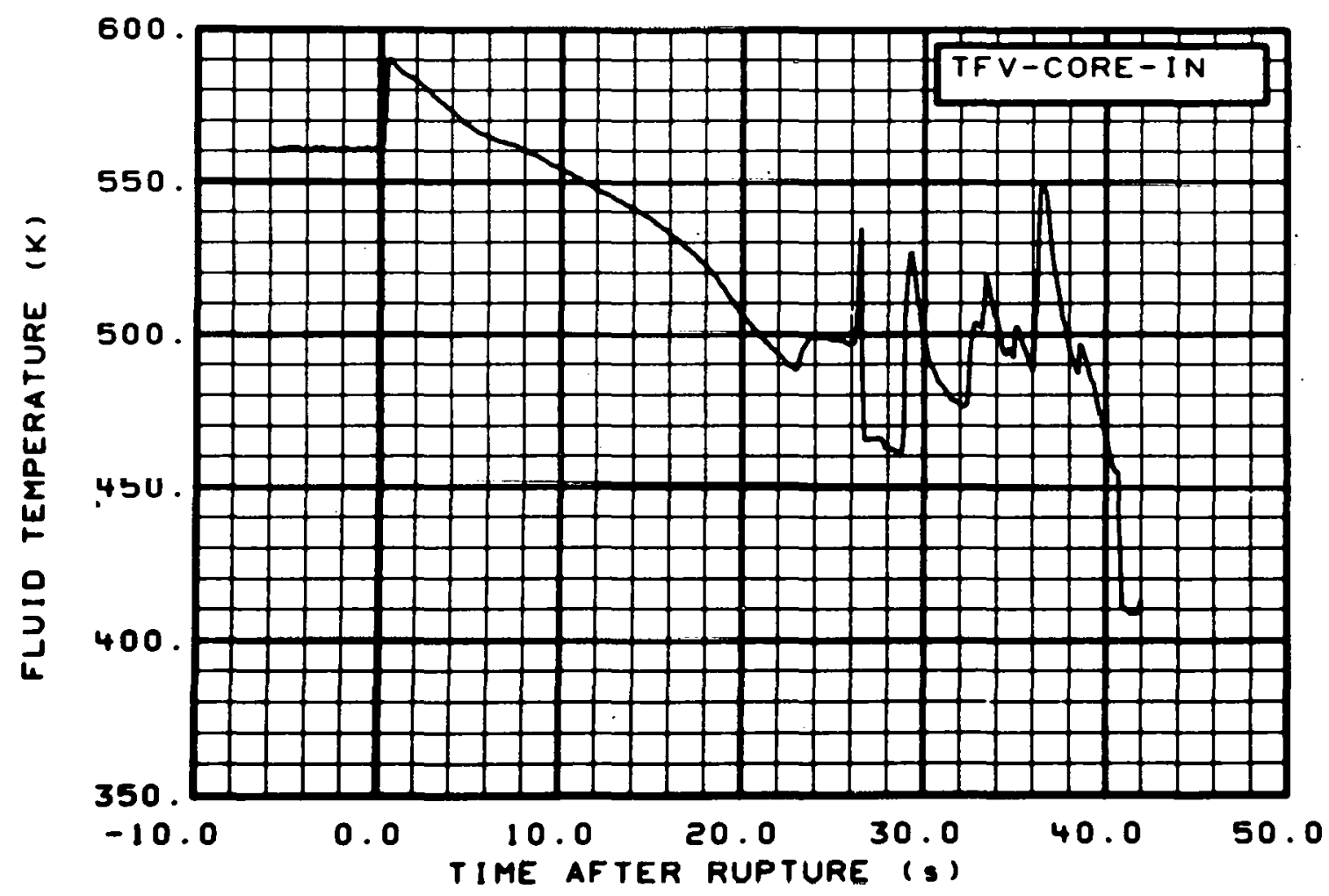

Fig. 32 Fluid temperature in core inlet (TFV-CORE-IN), from -6 to $42 \mathrm{~s}$. 


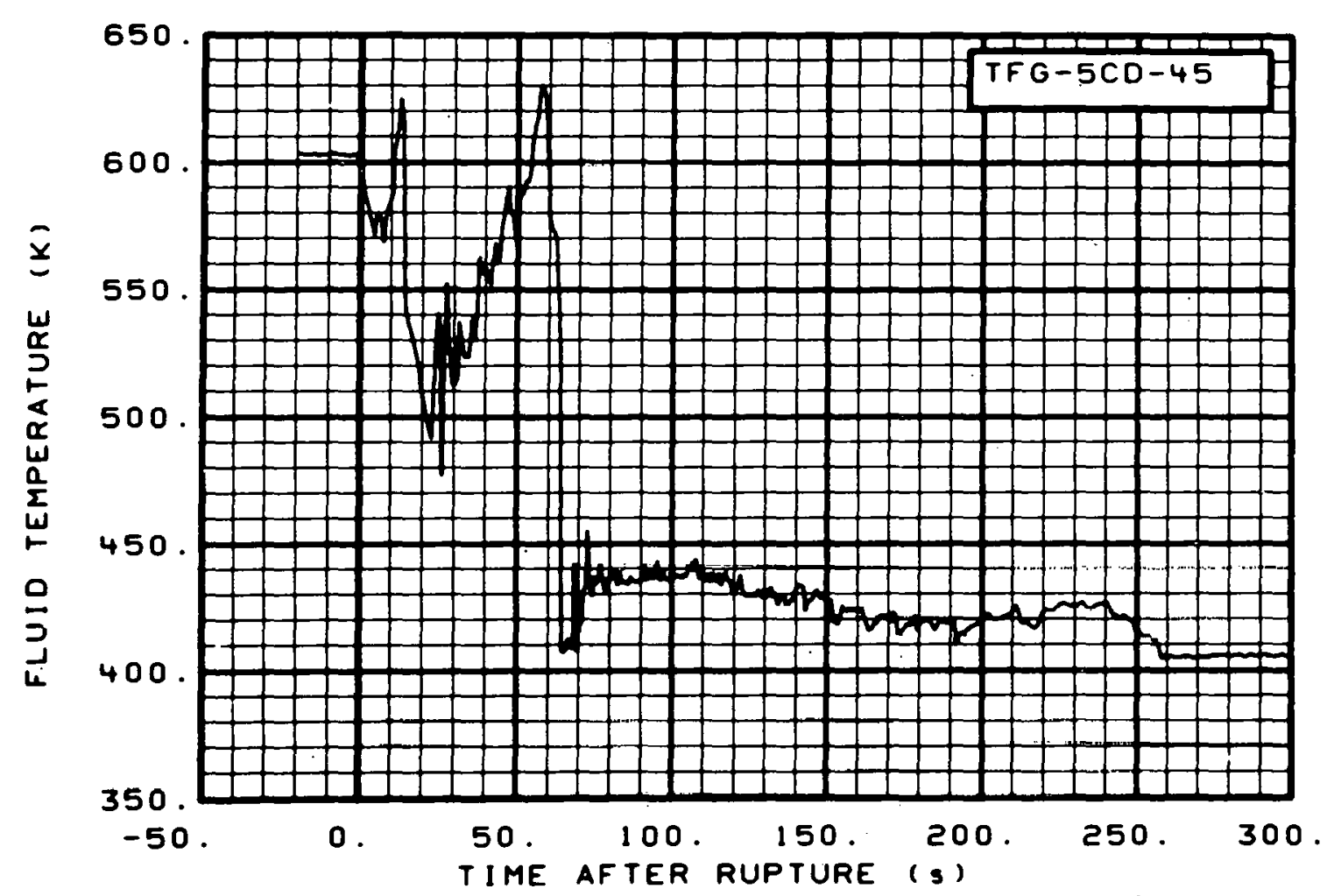

Fig. 33 Fluid temperature in core, Grid Spacer 5 (TFG-5CD-45), from -20 to $300 \mathrm{~s}$.

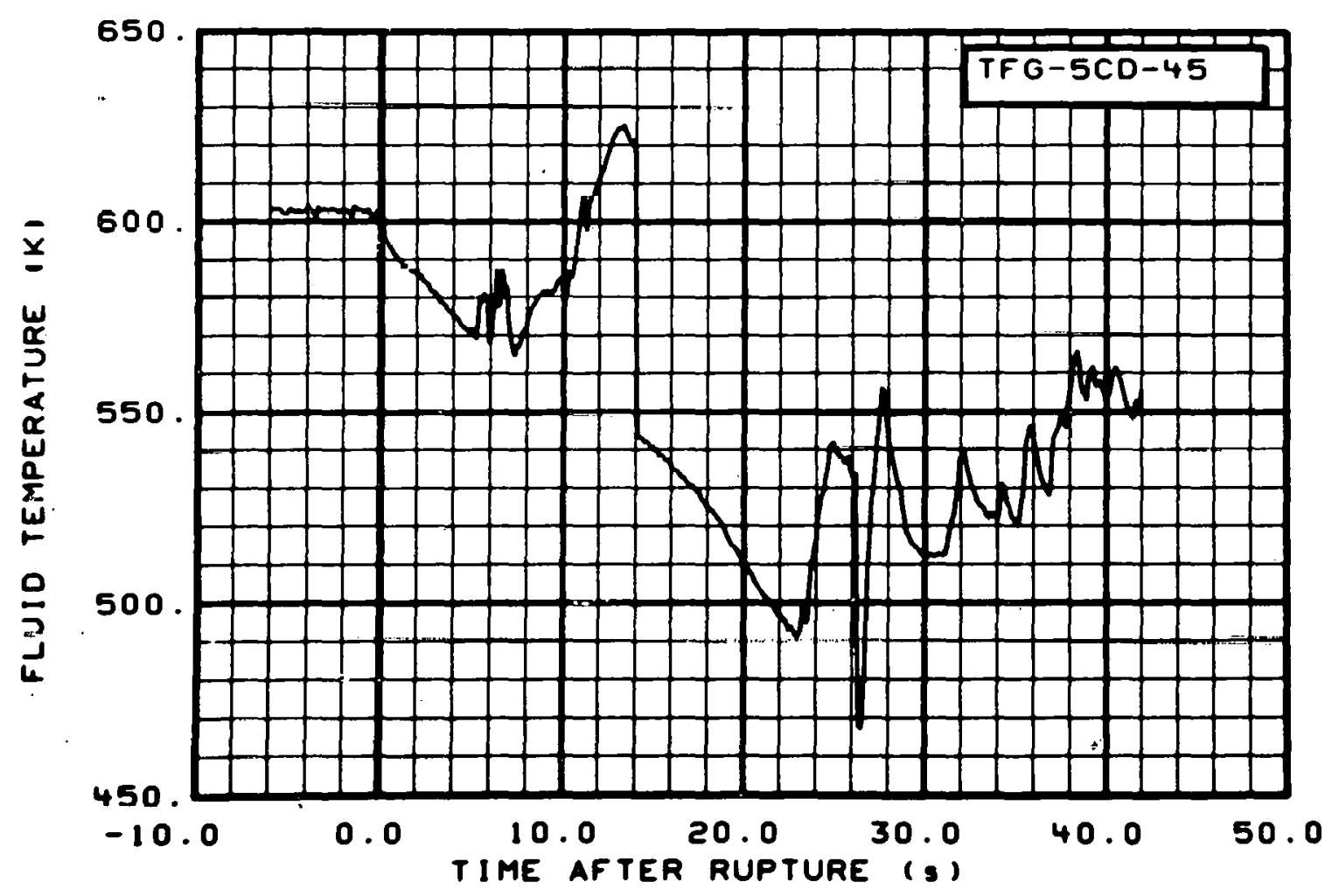

Fig. 34 Fluid temperature in core, Grid Spacer 5 (TFG-5CD-45), from -6 to $42 \mathrm{~s}$. 


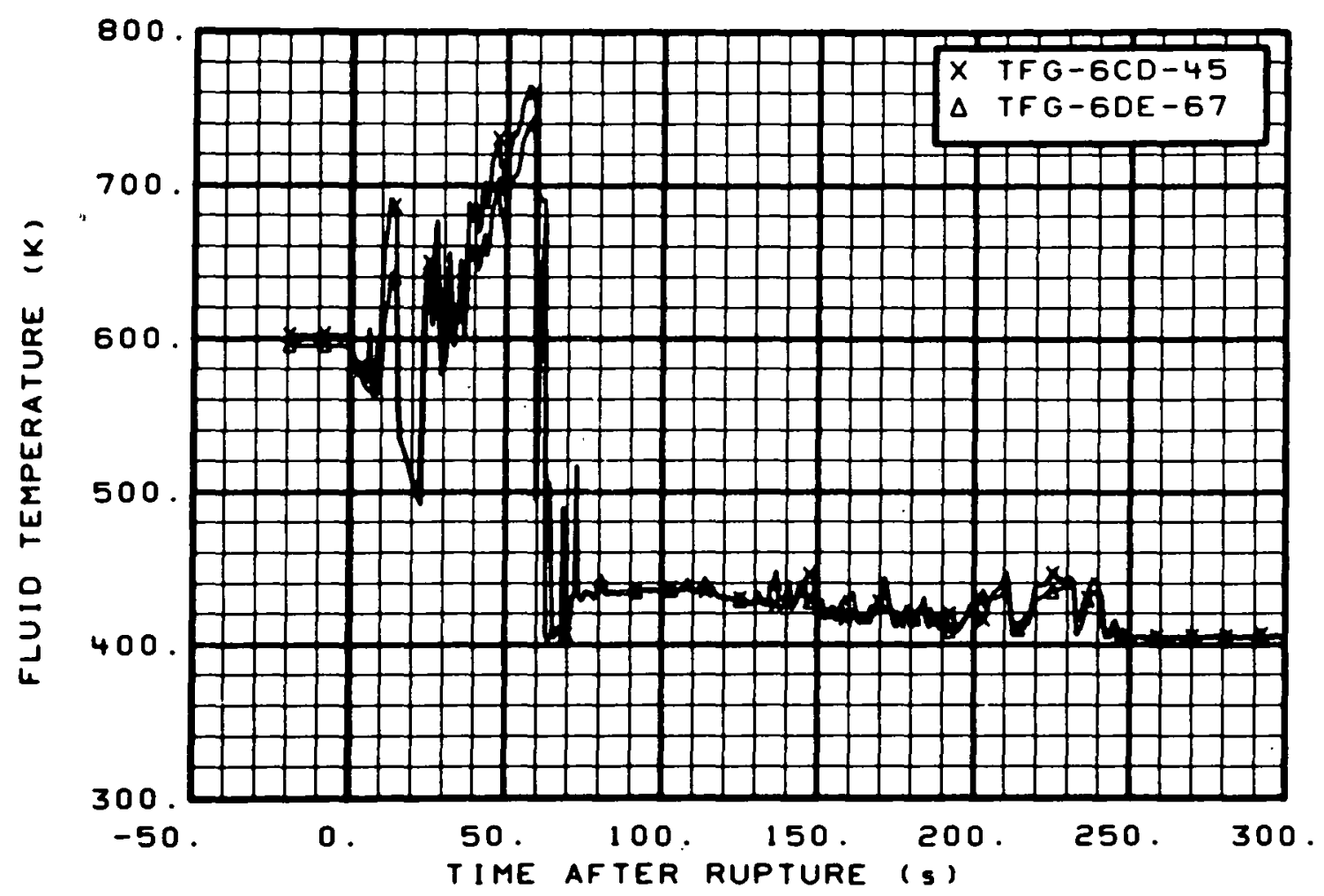

Fig. 35 Fluid temperature in core, Grid Spacer 6 (TFG-6CD-45 and TFG-6DE-67), from -20 to $300 \mathrm{~s}$.

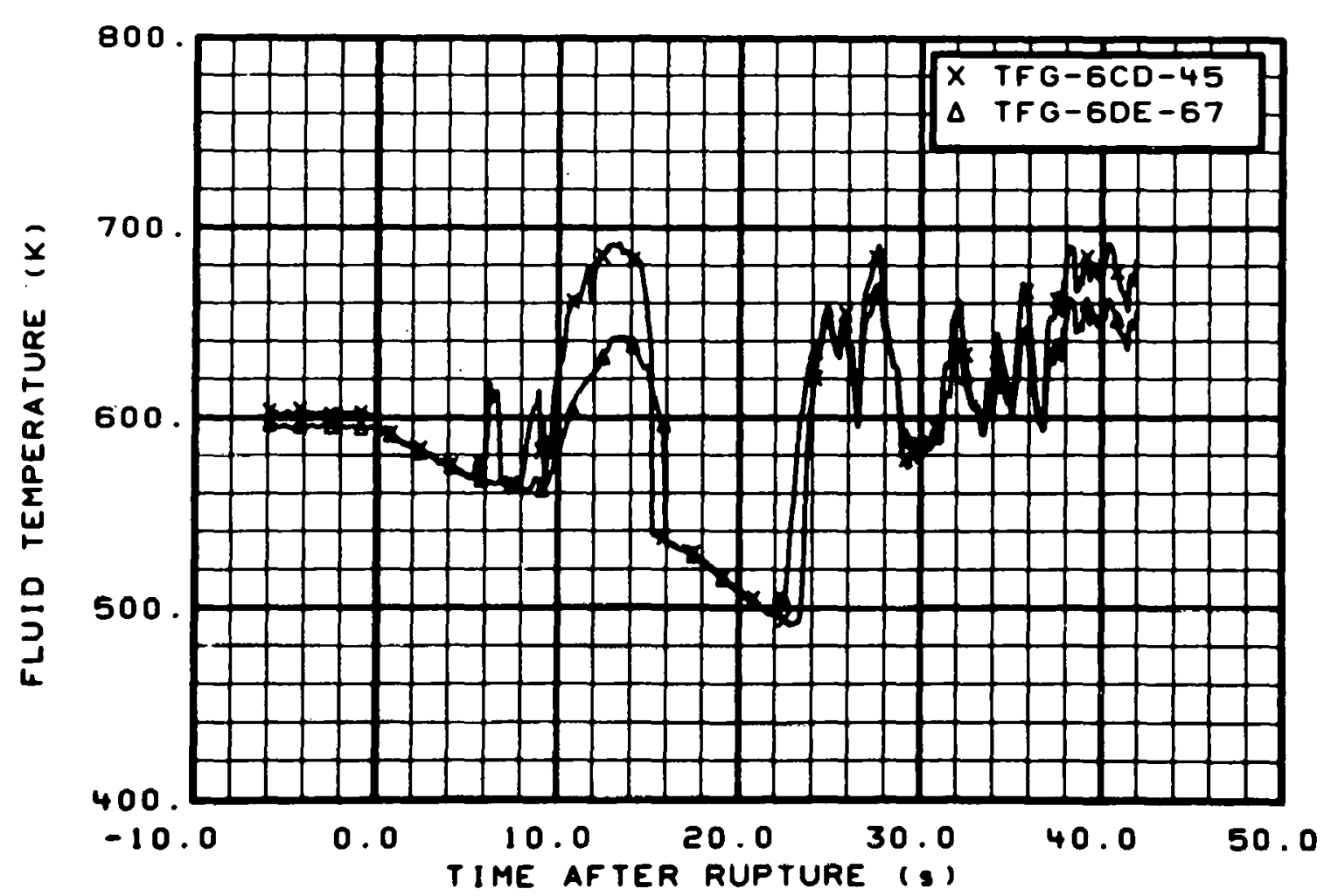

Fig. 36 Fluid temperature in core, Grid Spacer 6 (TFG-6CD-45 and TFG-6DE-67), from -6 to $42 \mathrm{~s}$. 


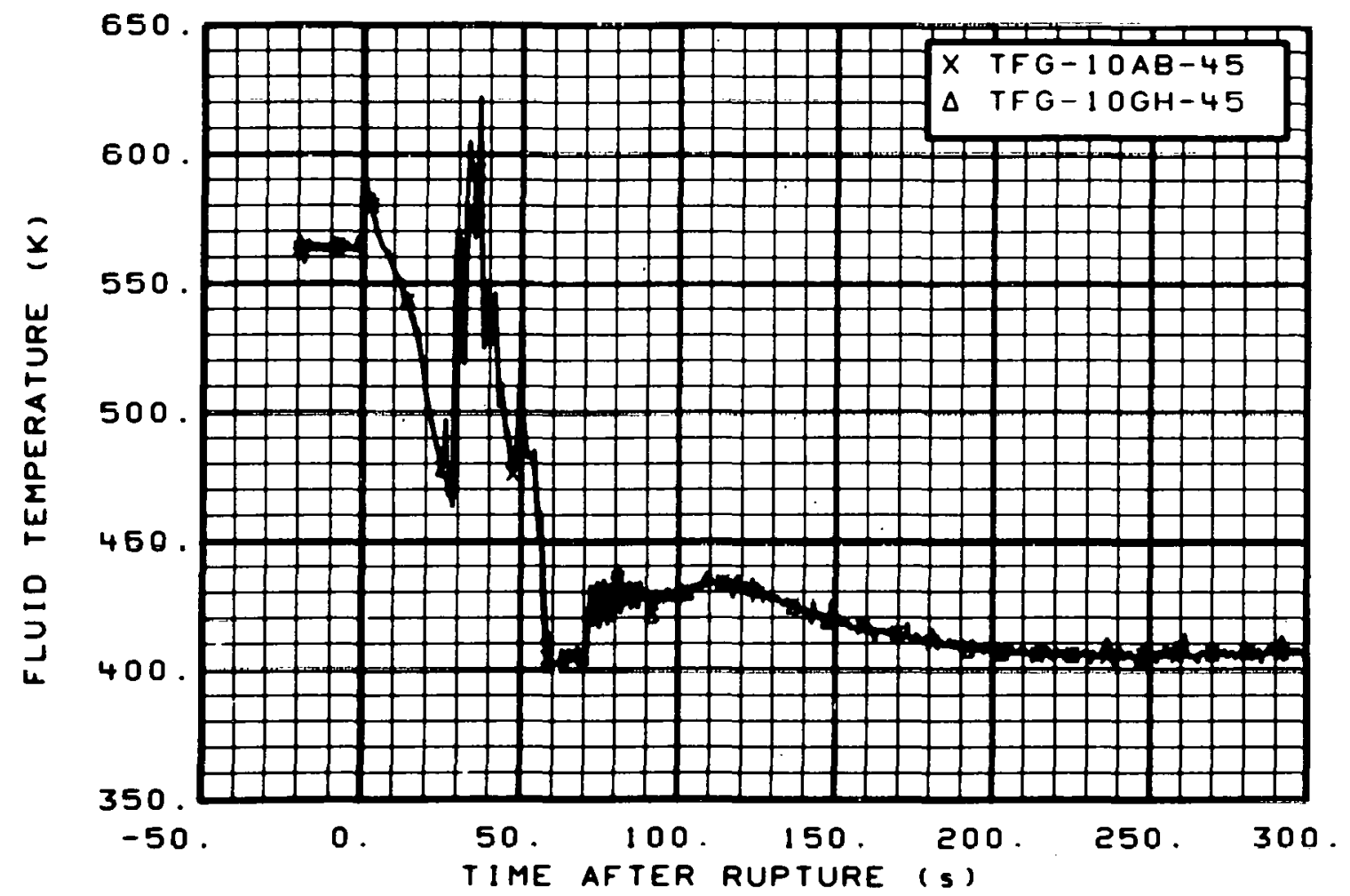

Fig. 37 Fluid temperature in core, Grid Spacer 10 (TFG-10AB-45 and TFG-10GH-45), from -20 to $300 \mathrm{~s}$.

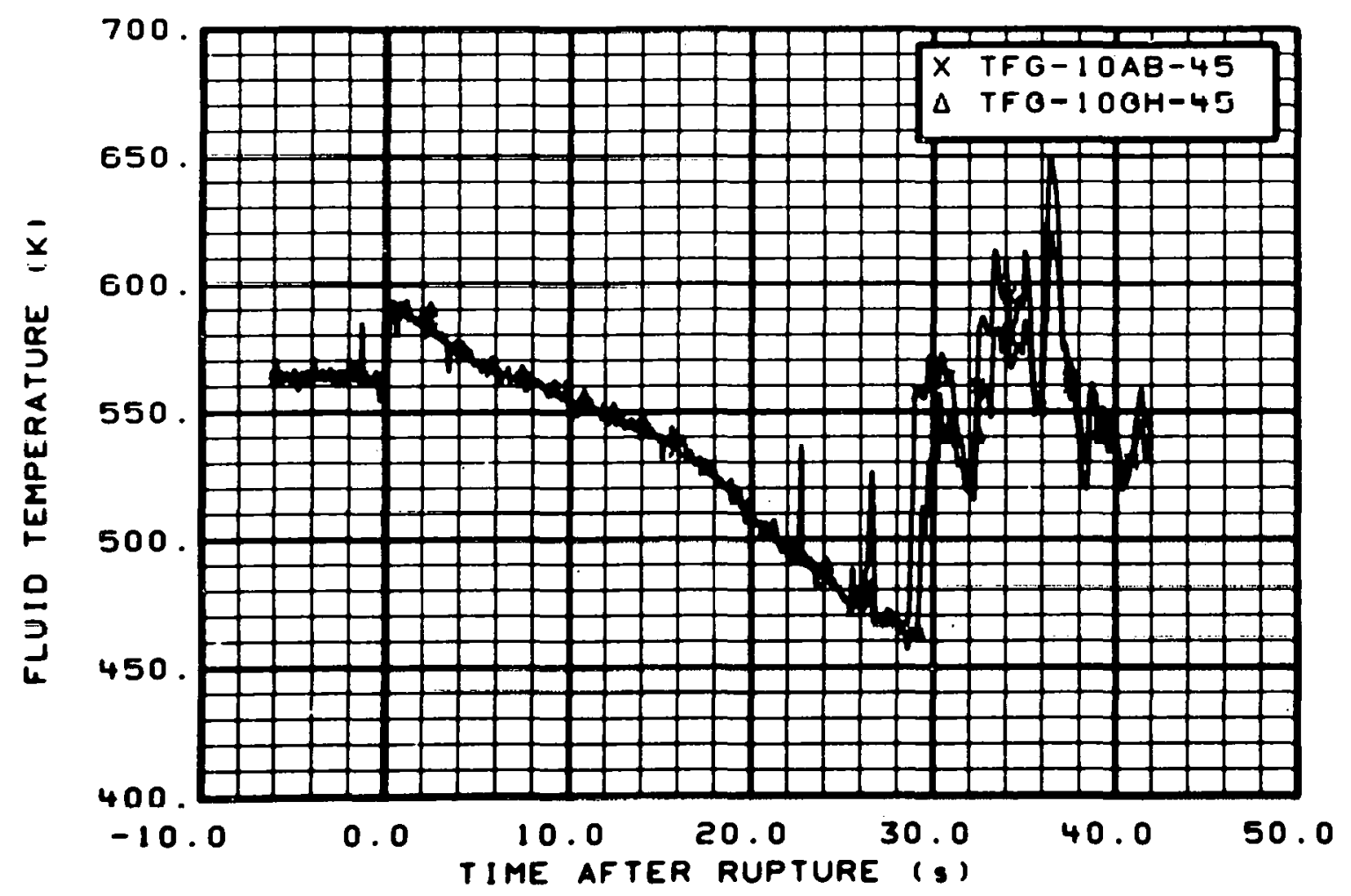

Fig. 38 Fluid temperature in core, Grid Spacer 10 (TFG-10AB-45 and $T F G-10 G H-45)$, from -6 to. $42 \mathrm{~s}$. 


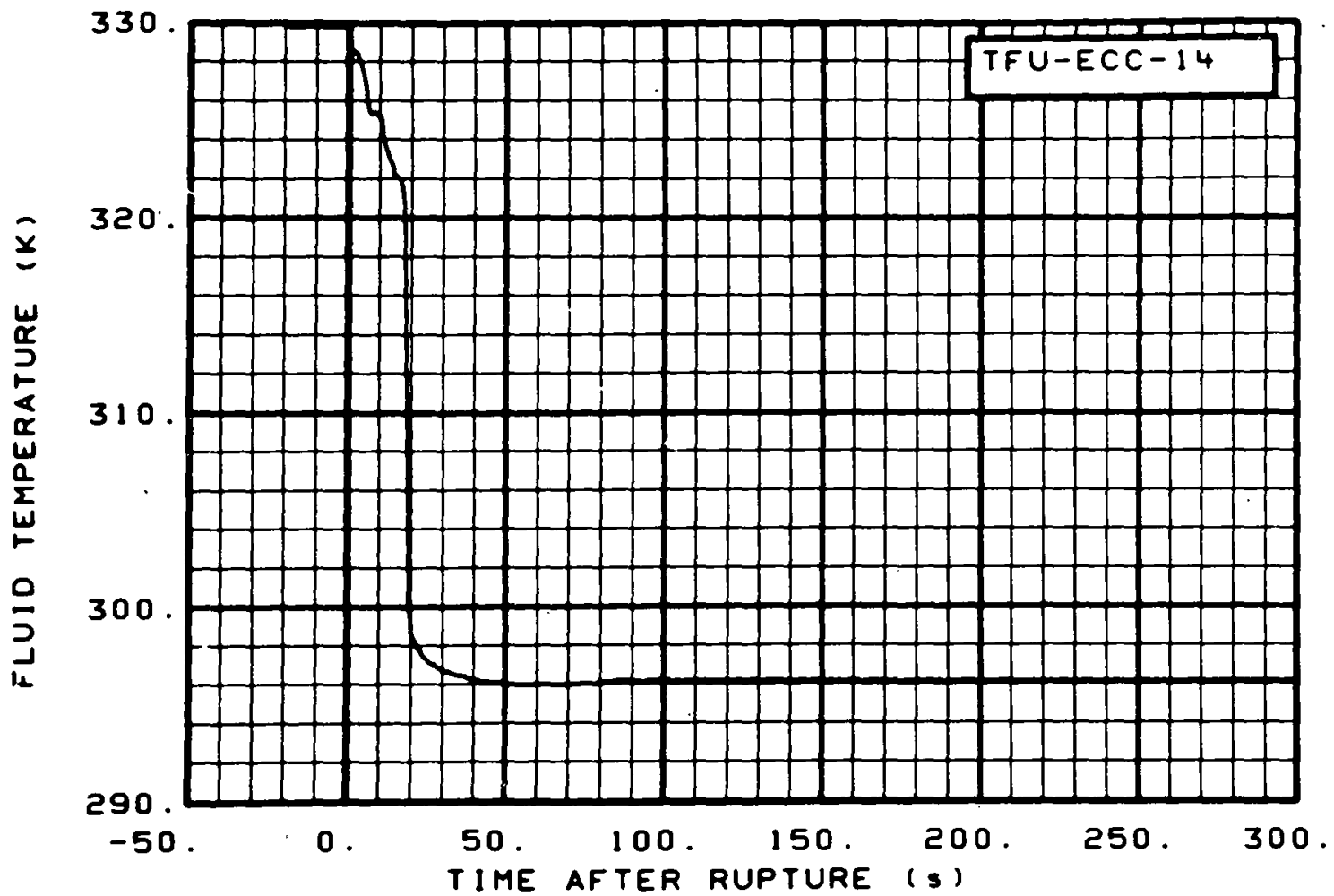

Fig. 39 Fluid temperature in intact loop coolant injection line (TFU-ECC-14), from -20 to $300 \mathrm{~s}$.

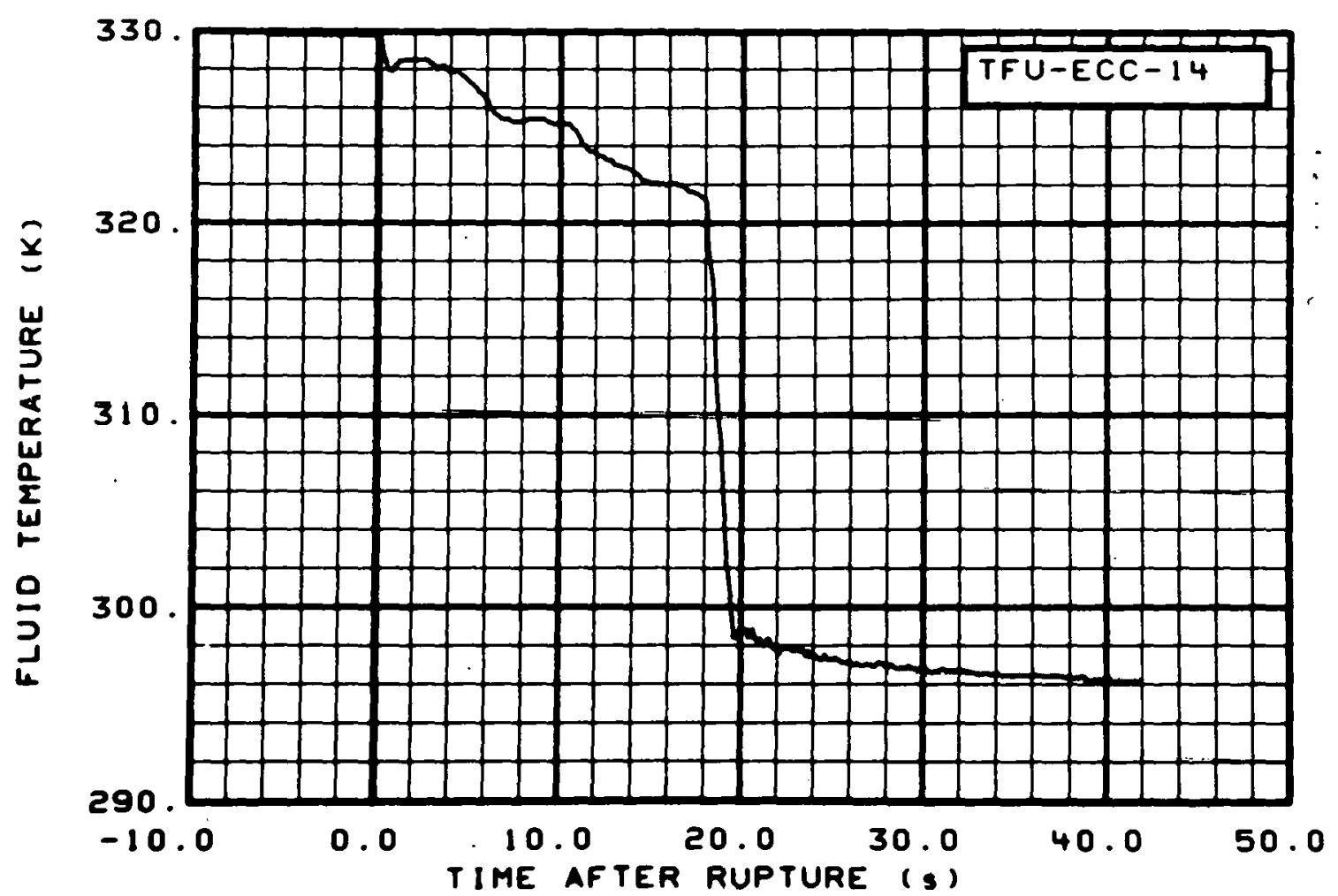

Fig. 40 Fluid temperature in intact loop coolant injection line (TFU-ECC-14), from -6 to $42 \mathrm{~s}$. 


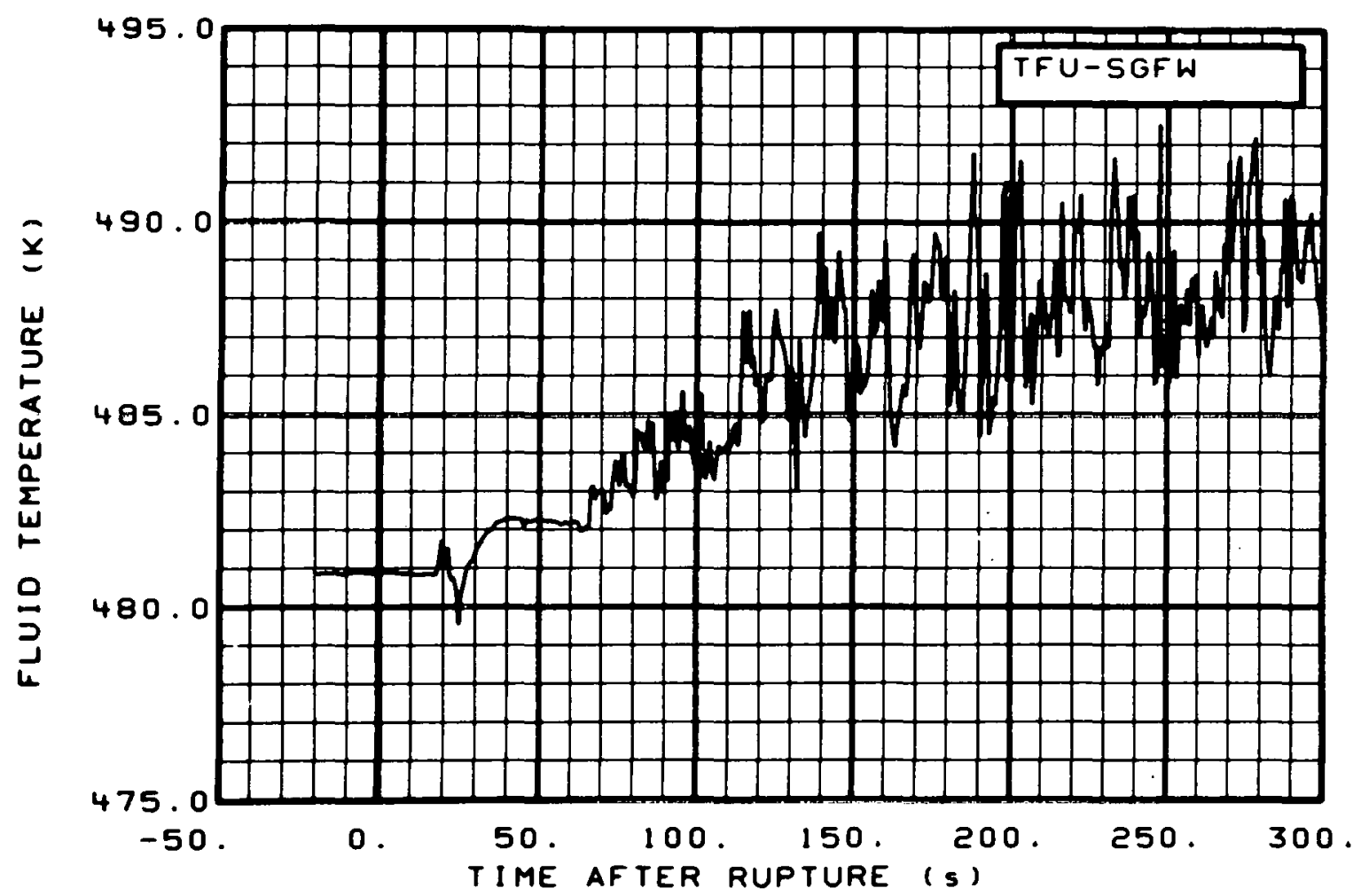

Fig. 41 Fluid temperature in steam generator, feedwater jine (TFU-SGFW), from -20 to $300 \mathrm{~s}$.

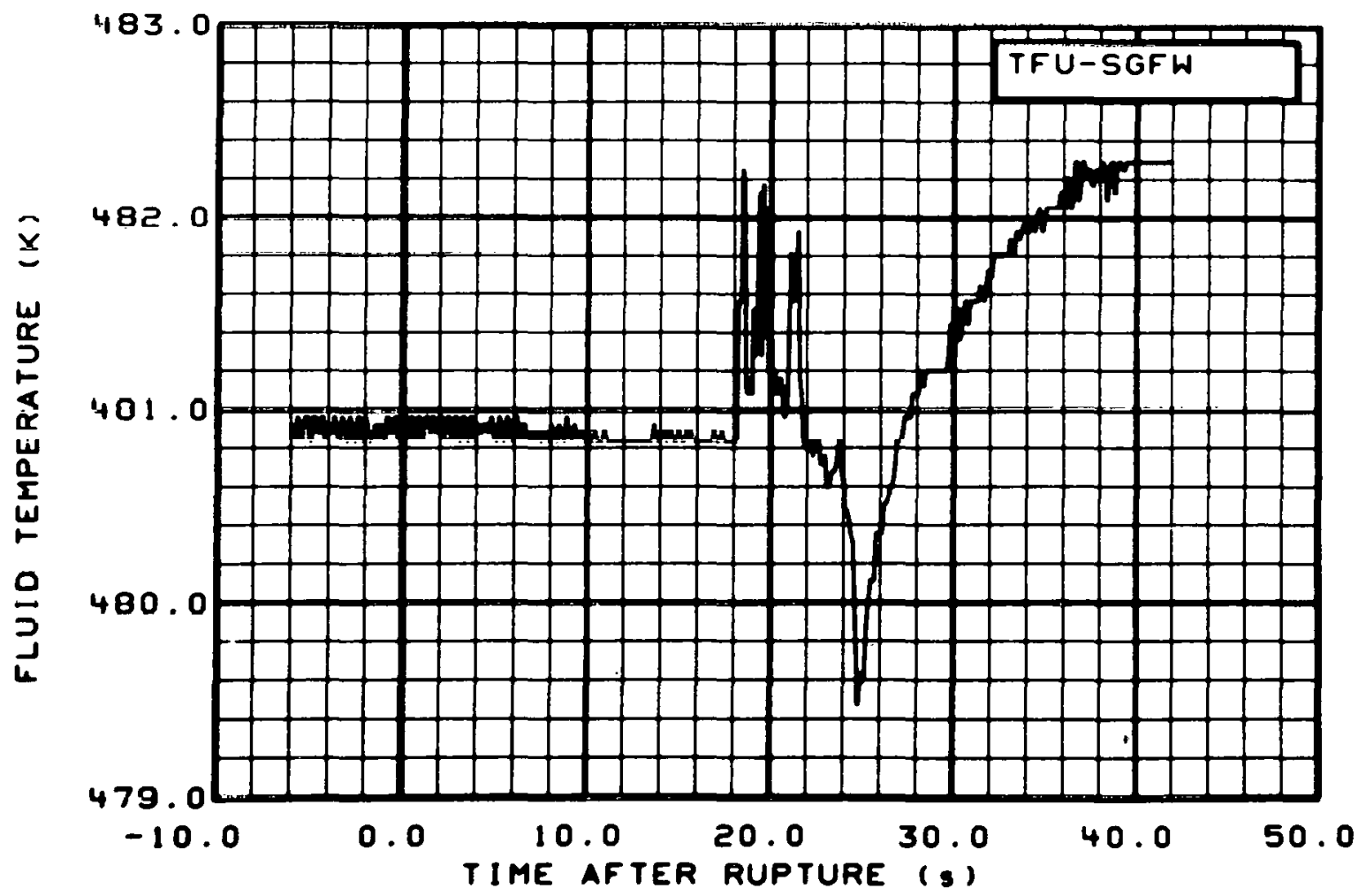

Fig. 42 Fluid temperature in steam generator, feedwater line (TFU-SGFW), from -6 to $42 \mathrm{~s}$. 


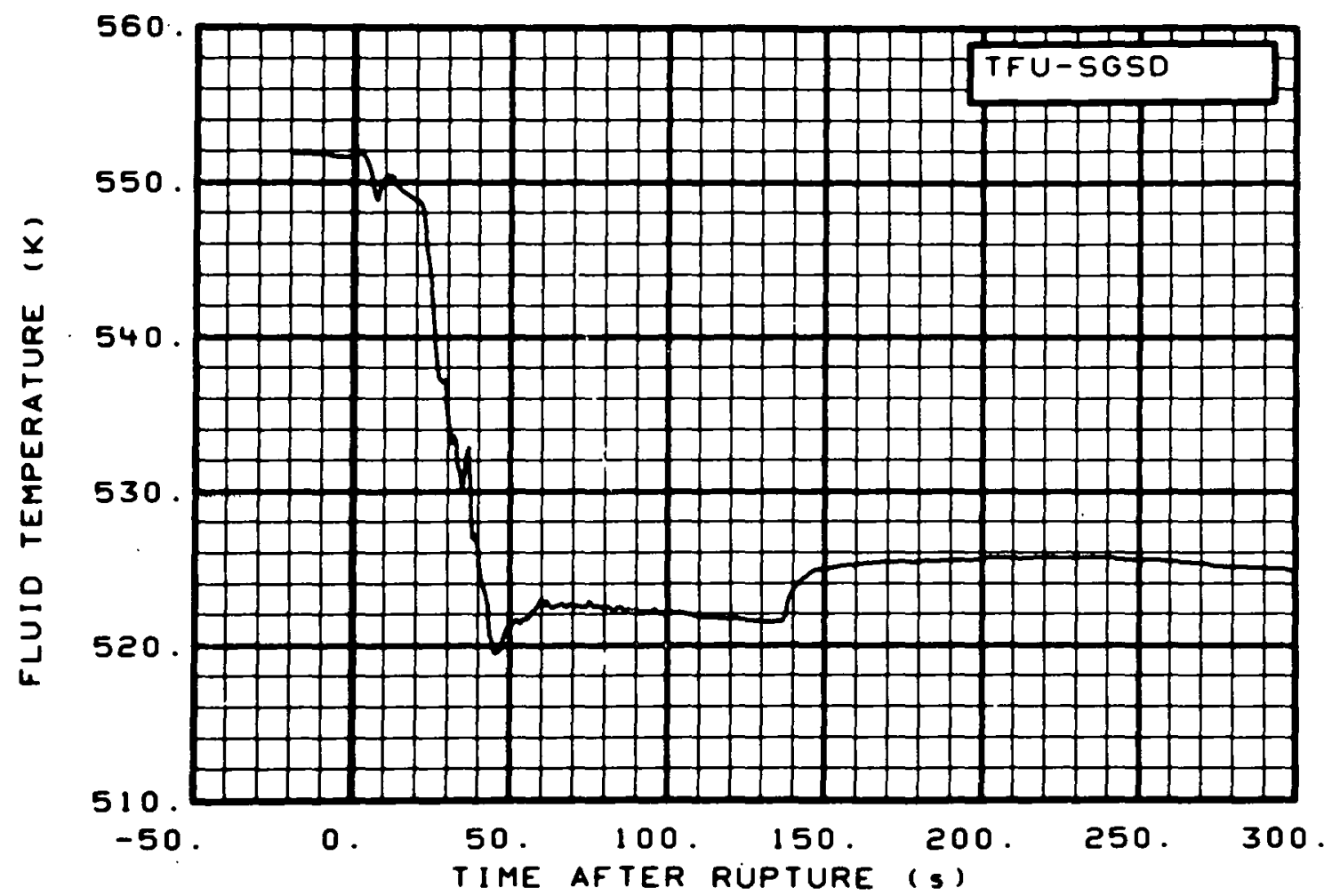

Fig. 43 Fluid temperature in steam generator, secondary side (TFU-SGSD), from -20 to $300 \mathrm{~s}$.

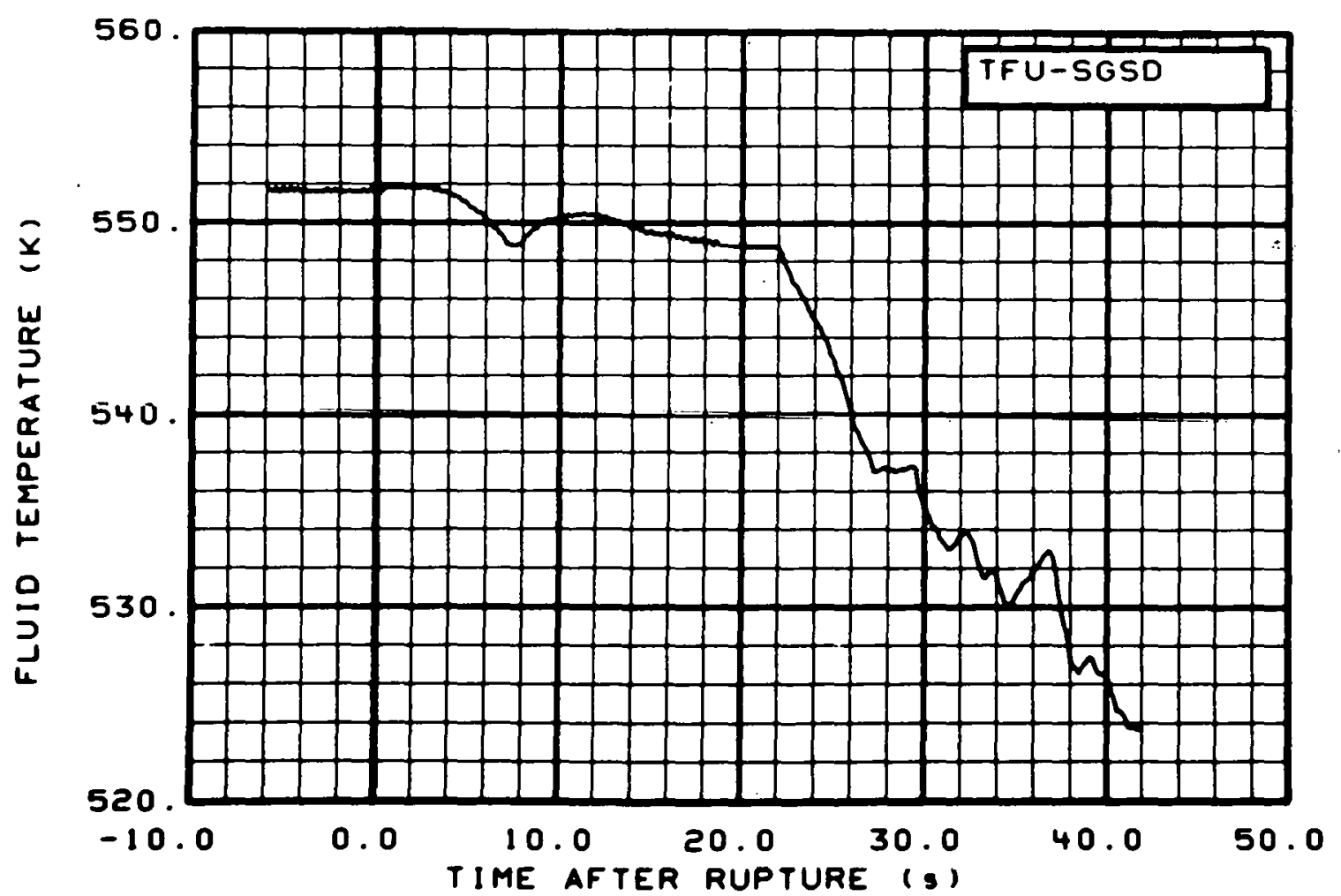

Fig. 44 Fluid temperature in steam generator, secondary side (TFU-SGSD), fruil -6 to $42 \mathrm{~s}$. 


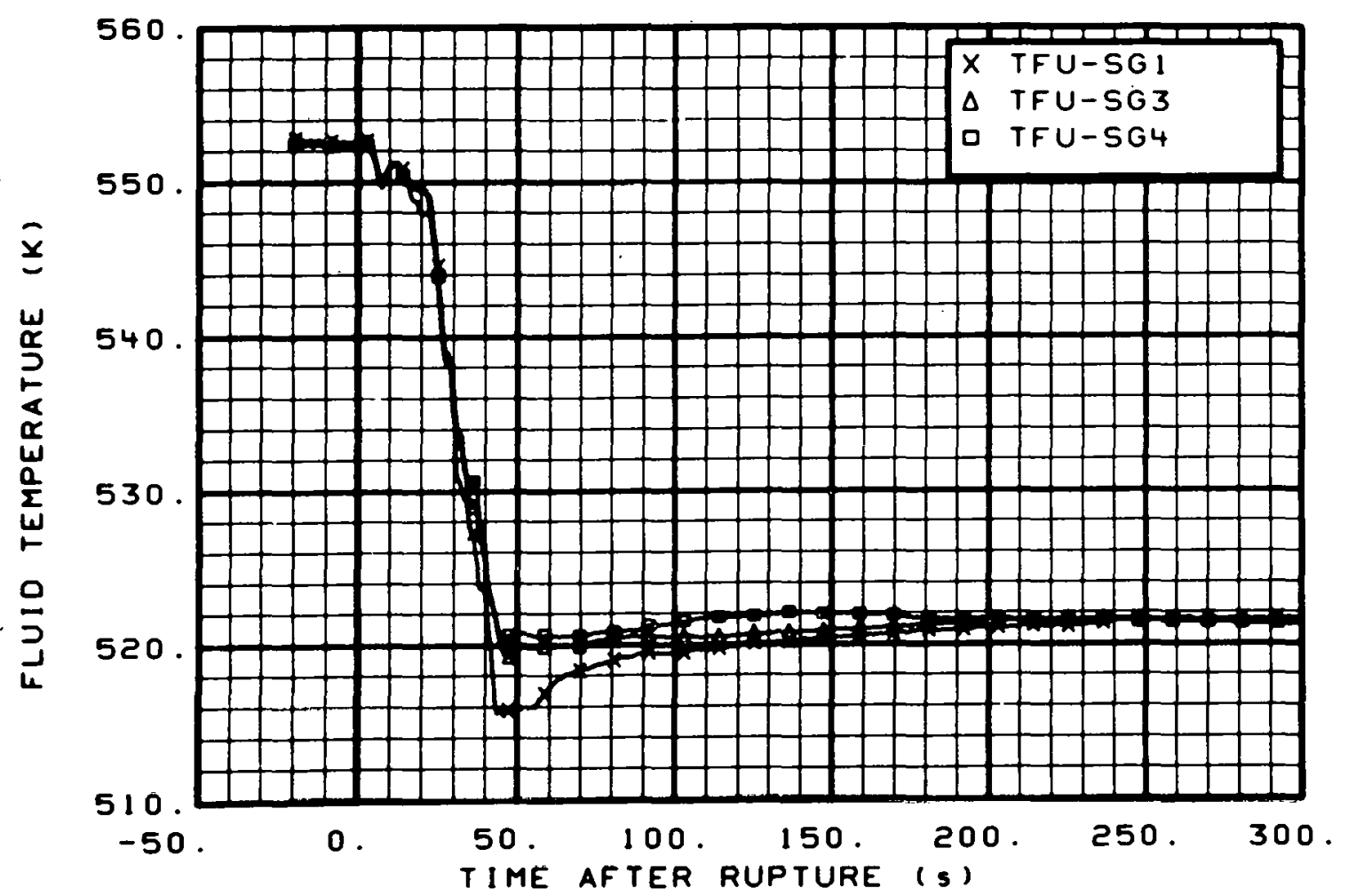

Fig. 45 Fluid temperature in steam generator, secondary side (TFU-SG1, TFU-SG3, and TFU-SG4), -20 to $300 \mathrm{~s}$.

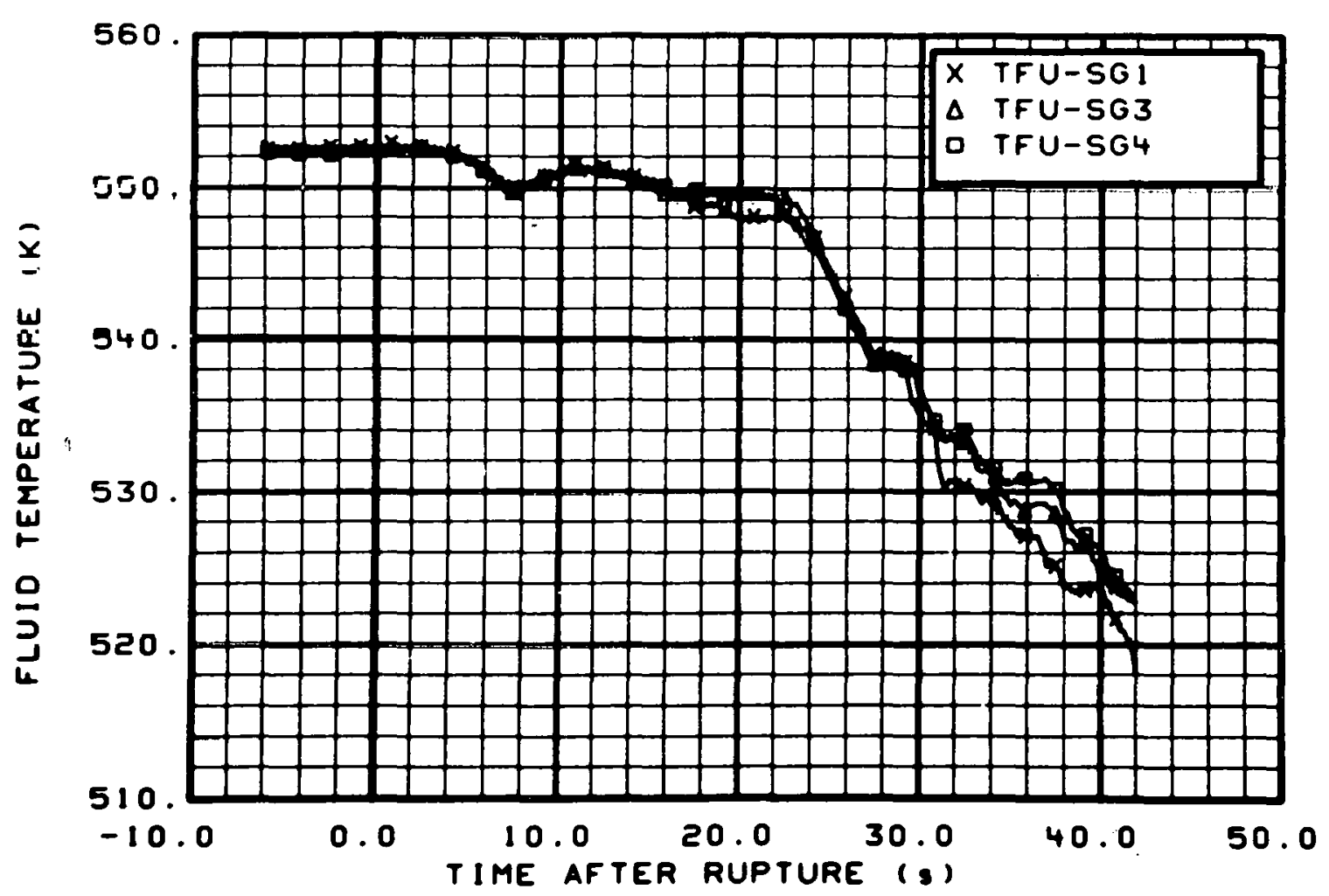

Fig. 46 Fluid temperature in steam generator, secondary side (TFU-SGI, TFU-SG3, and TFU-SG4), from -6 to $42 \mathrm{~s}$. 


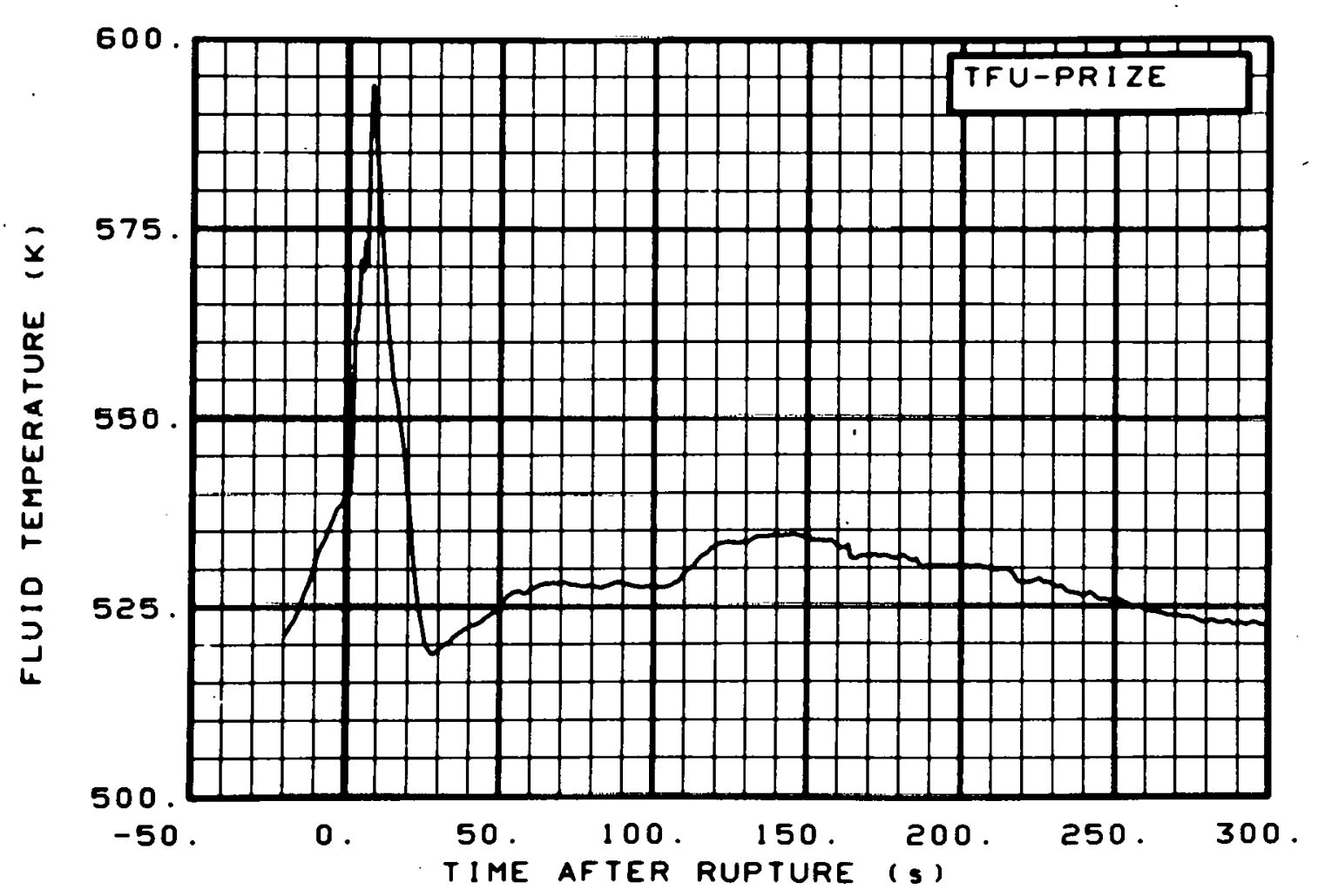

Fig. 47 Fluid temperature in pressurizer surge line (TFU-PRIZE), from : -20 to $300 \mathrm{~s}$.

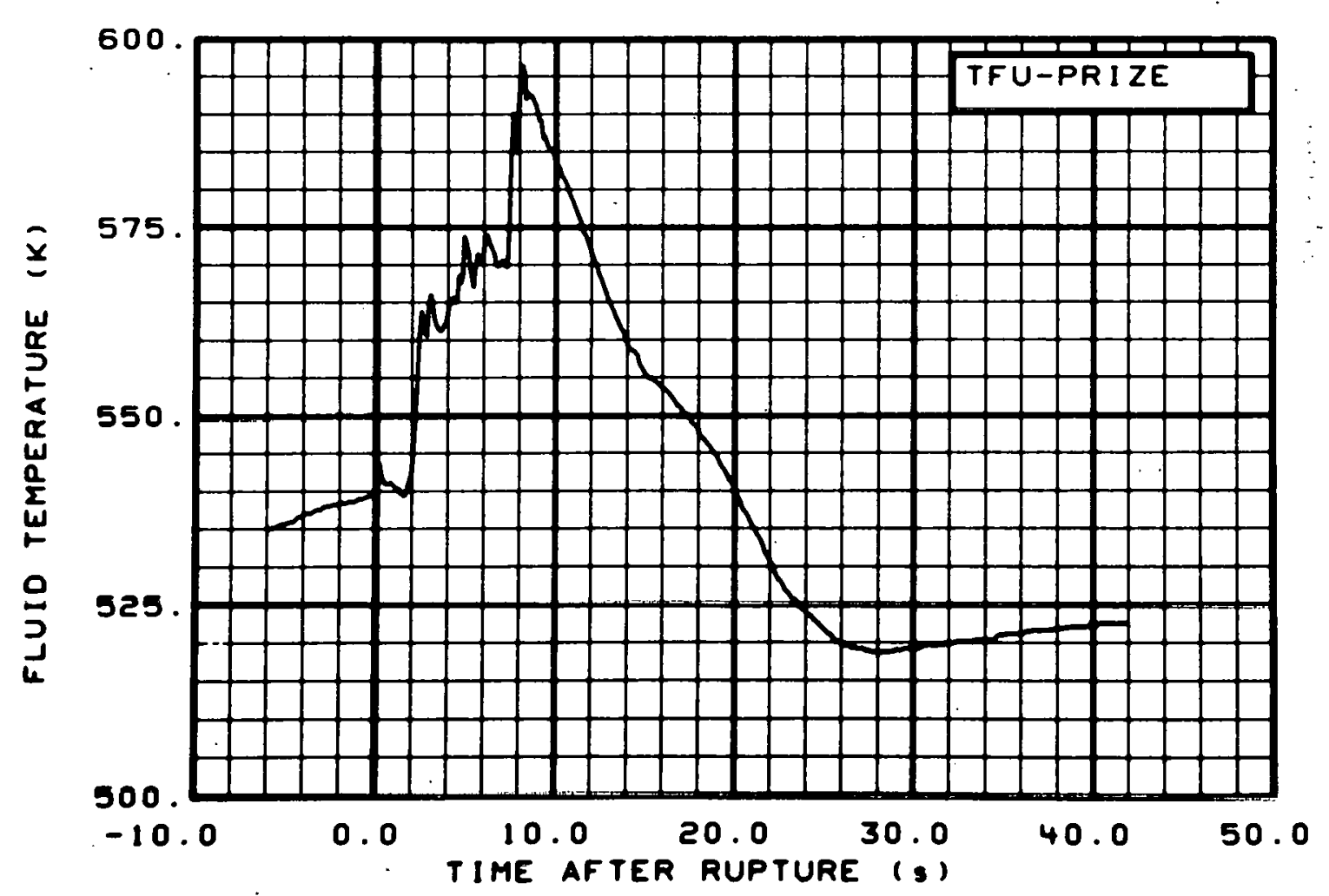

Fig. 48 Fluid temperature in pressurizer surge line (TFU-PRIZE), from -6 to $42 \mathrm{~s}$. 


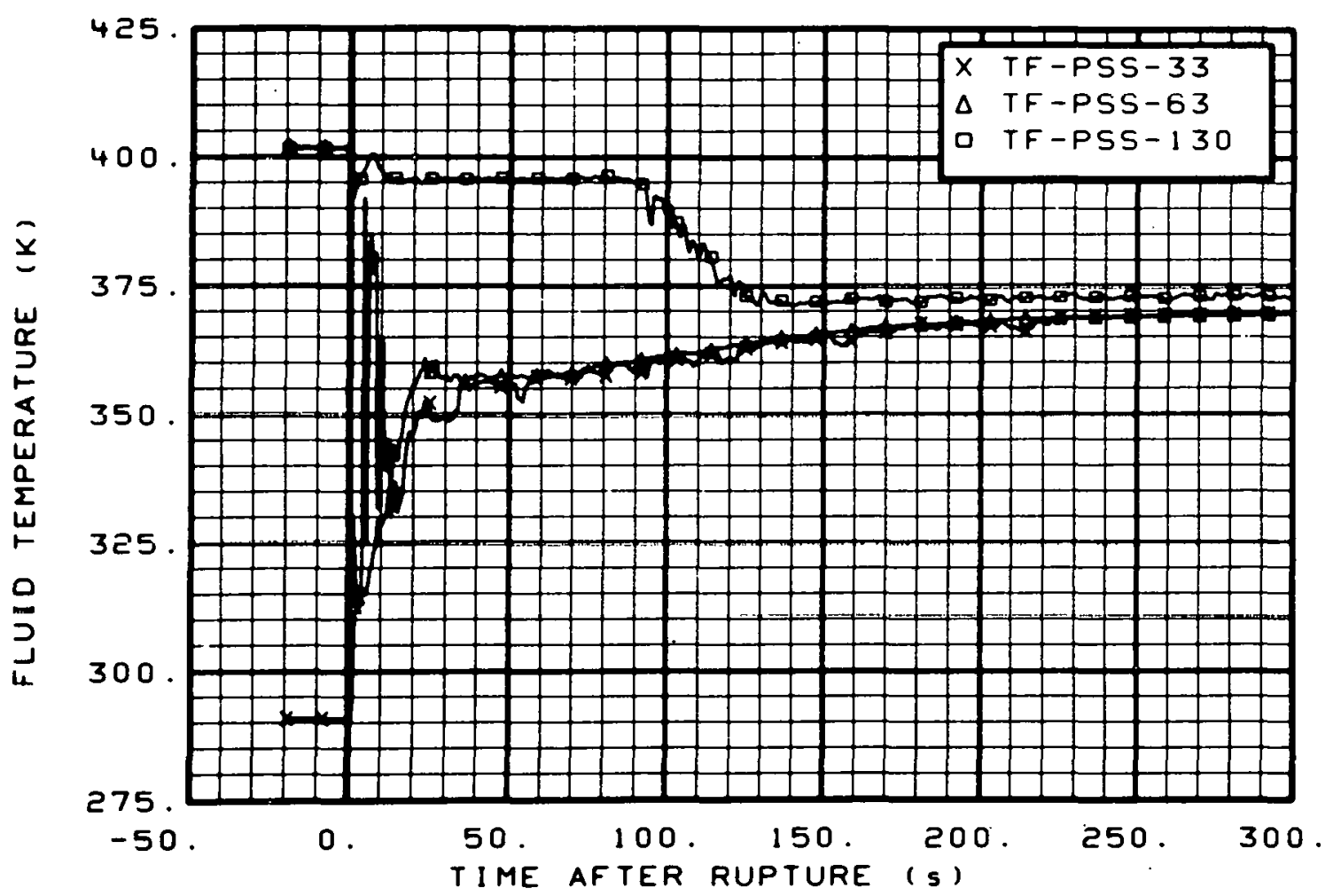

Fig. 49 Fluid temperature in pressure suppression tank (TF-PSS-33, TF-PSS-63, and TF-PSS-130), from -20 to $300 \mathrm{~s}$.

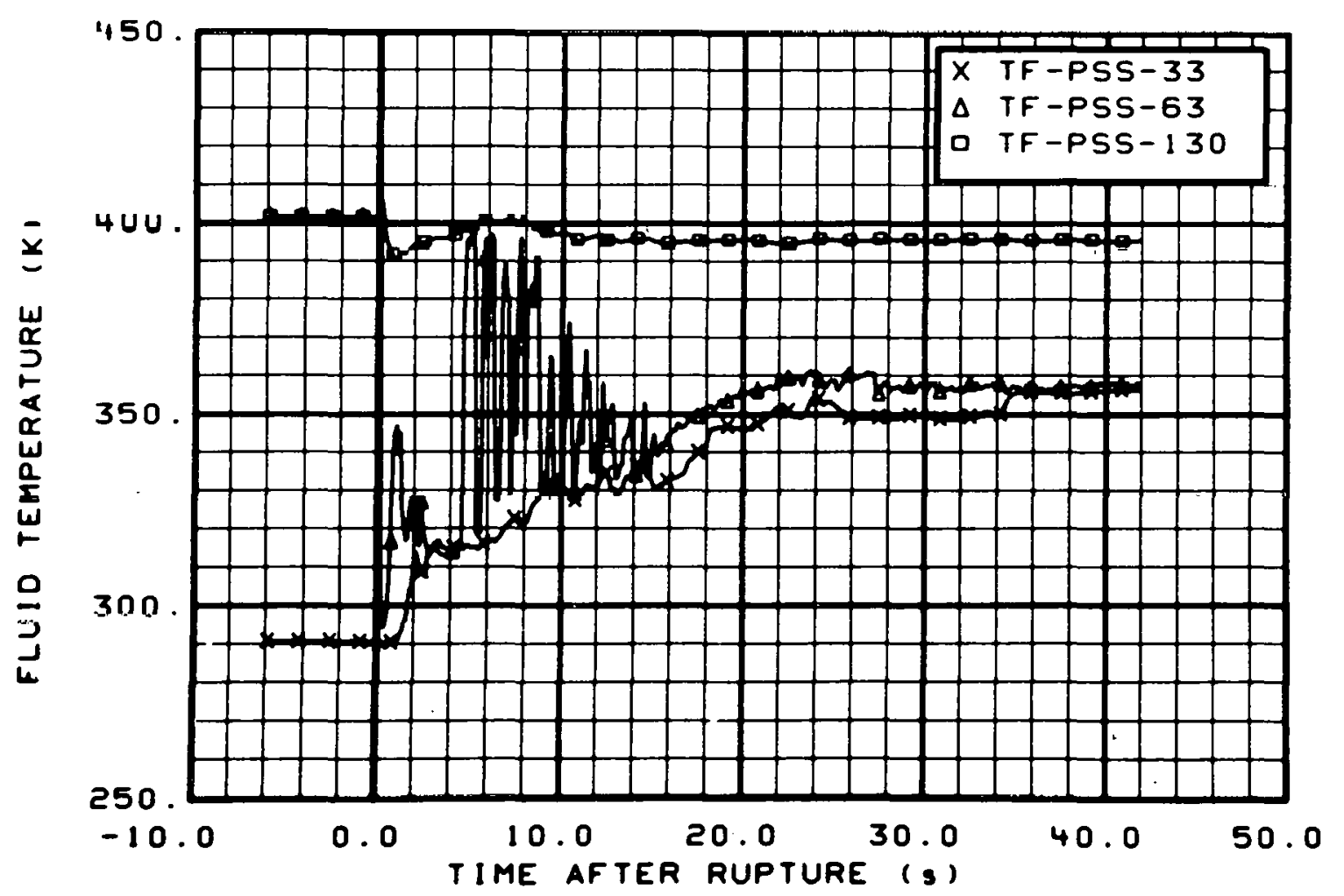

Fig. 50 Fluid temperature in pressure suppression tank (TF-PSS-33, TF-PSS-63, and TF-PSS-130), from -6 to $42 \mathrm{~s}$. 


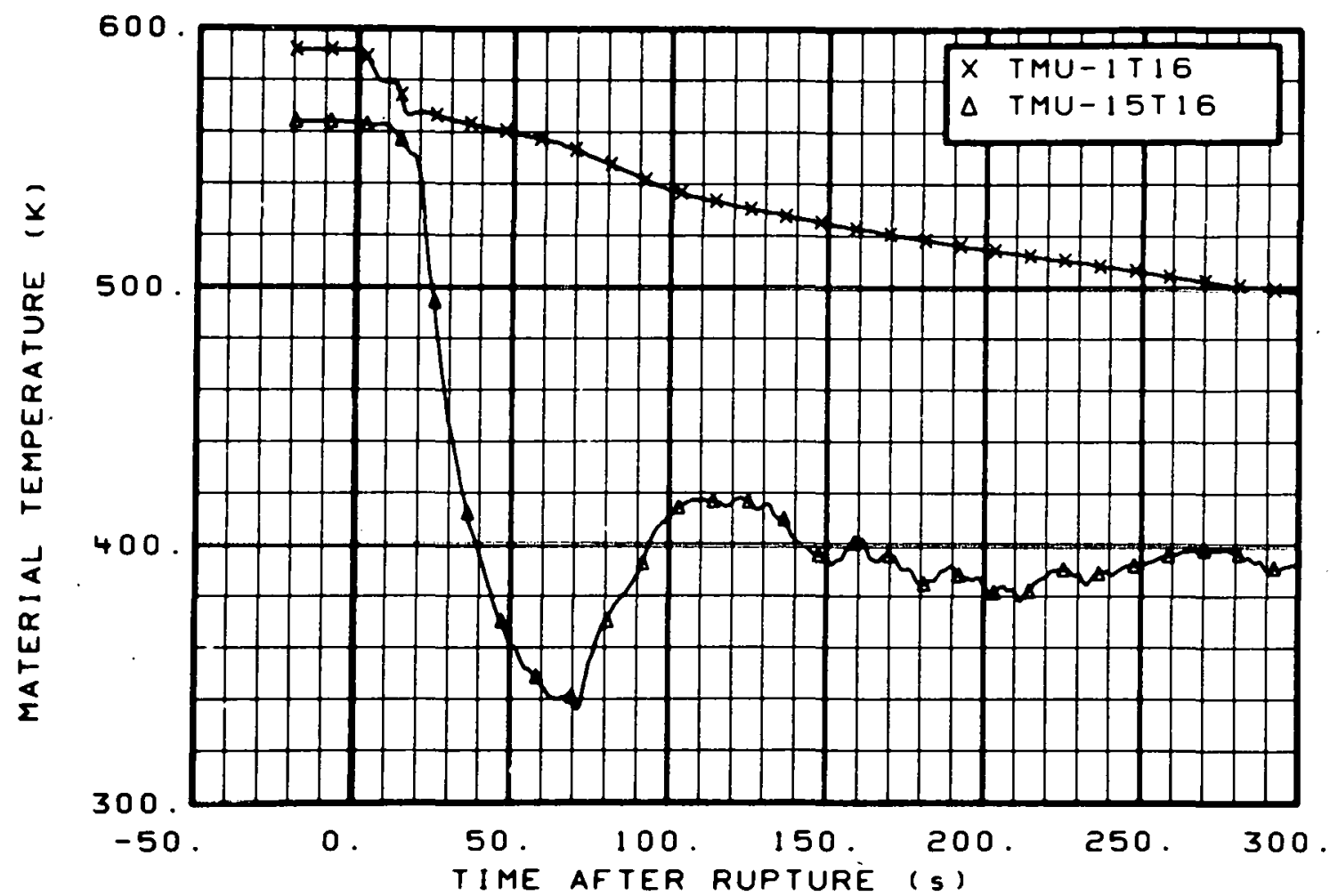

Fig. 51 Material temperature in intact loop (TMU-1T16 and TMU-15T16), from -20 to $300 \mathrm{~s}$.

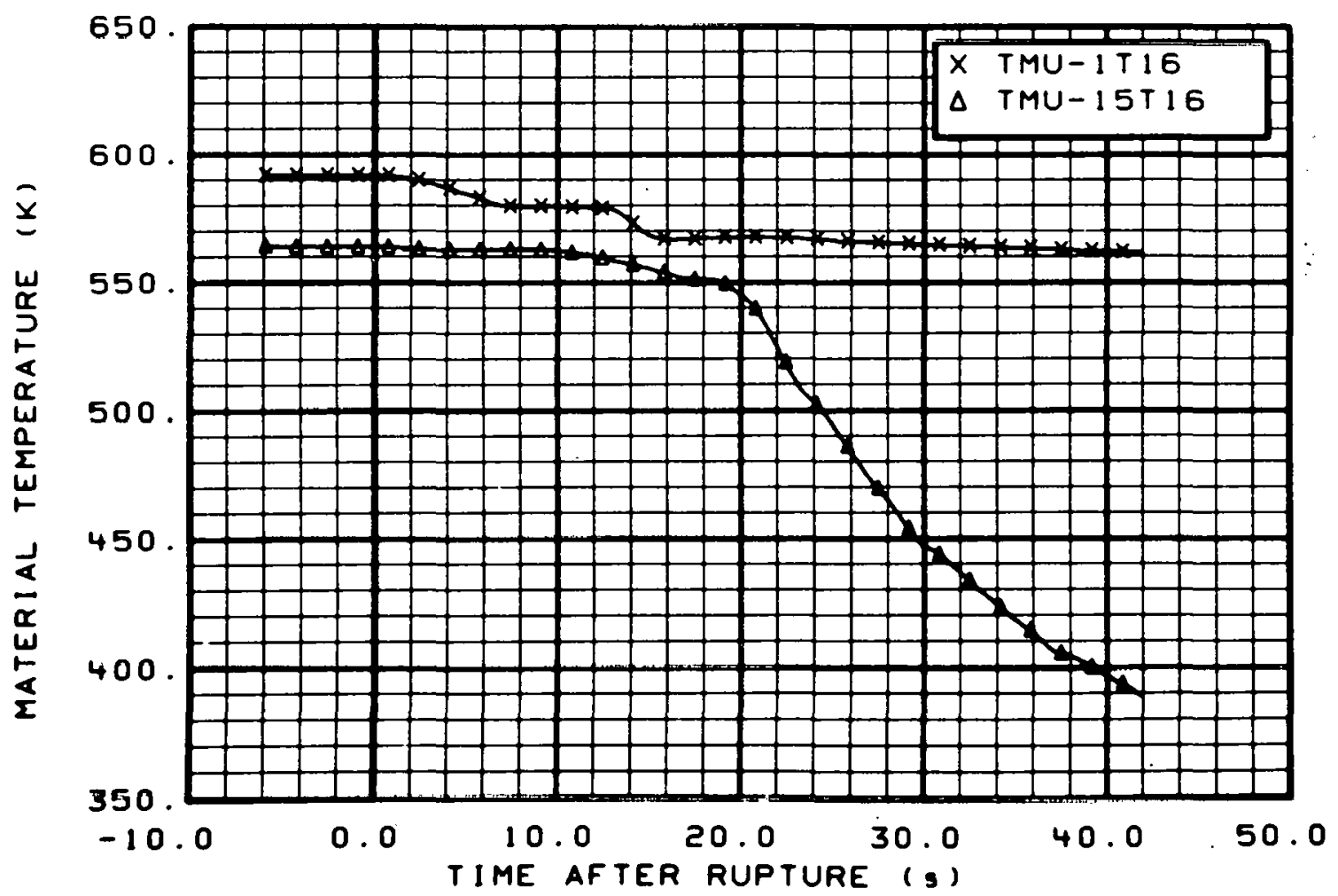

Fig. 52 Material temperature in intact 10op (TMU-1T16 and TMU-15T16), from -6 to $42 \mathrm{~s}$. 


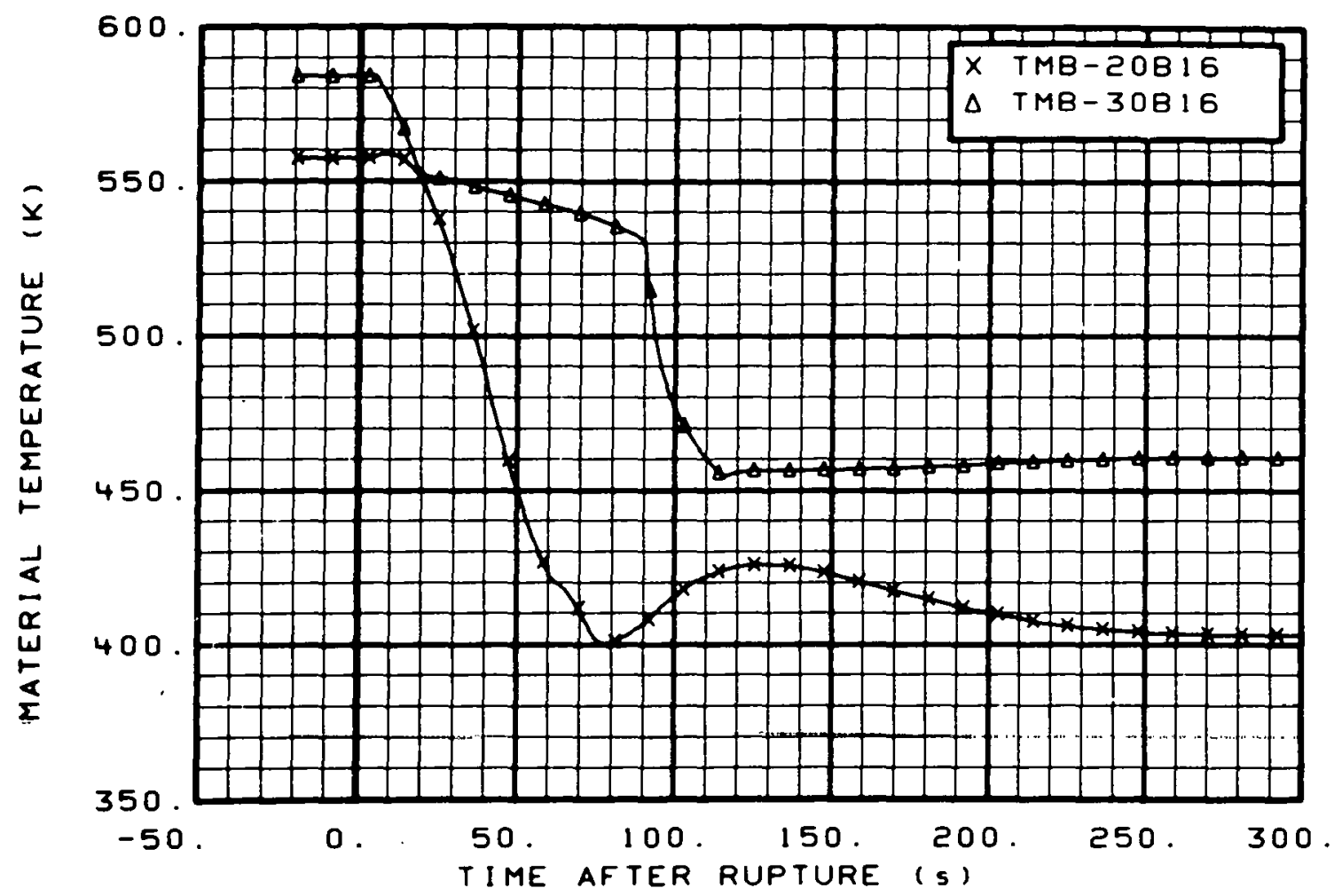

Fig. 53 Material temperature in broken loop (TMB-20B16 and TMB-30B16), from -20 to $300 \mathrm{~s}$.

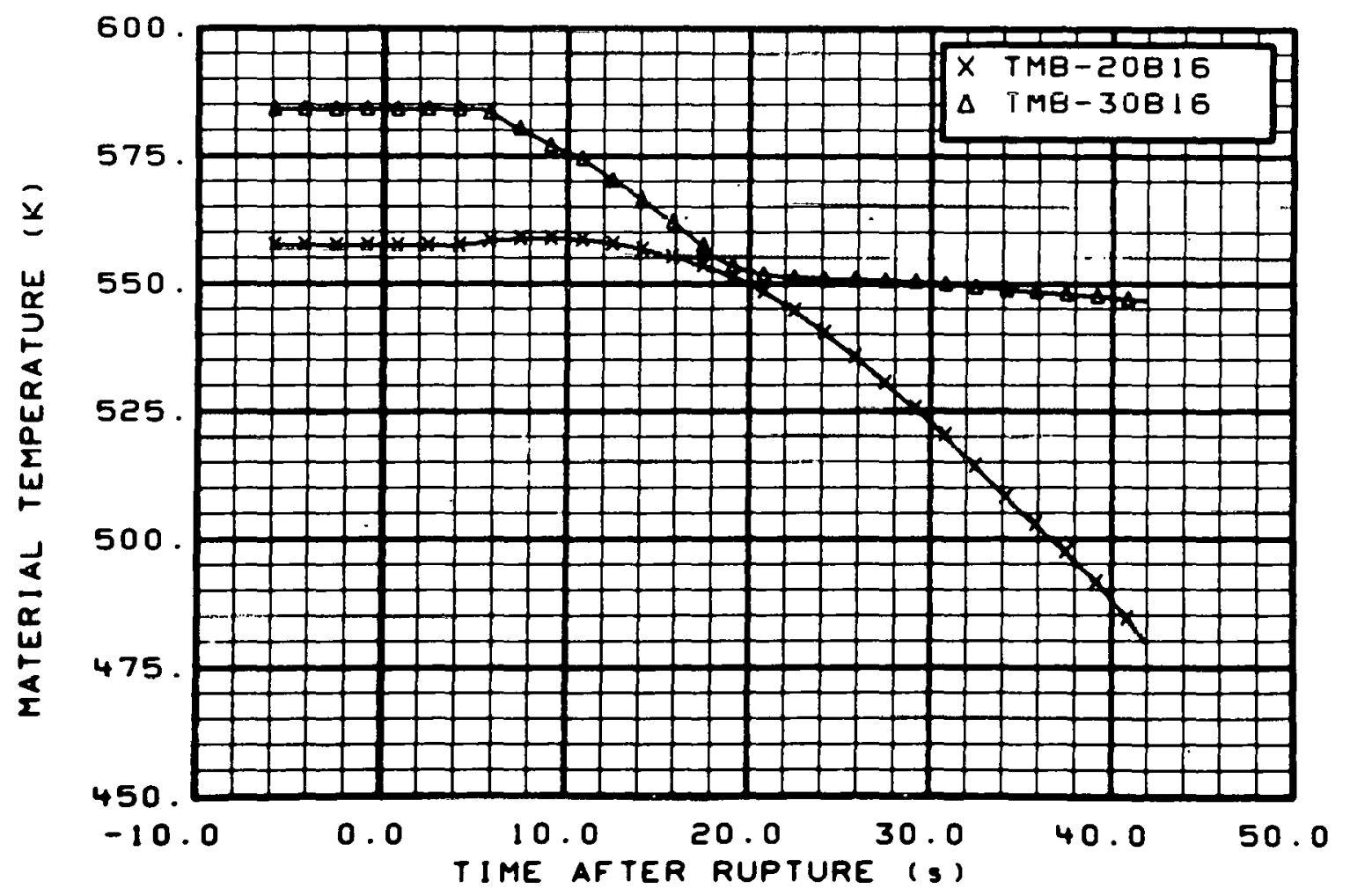

Fig. 54 Material temperature in broken loop (TMB-20B16 and TMB-30B 16), from -6 to $42 \mathrm{~s}$. 


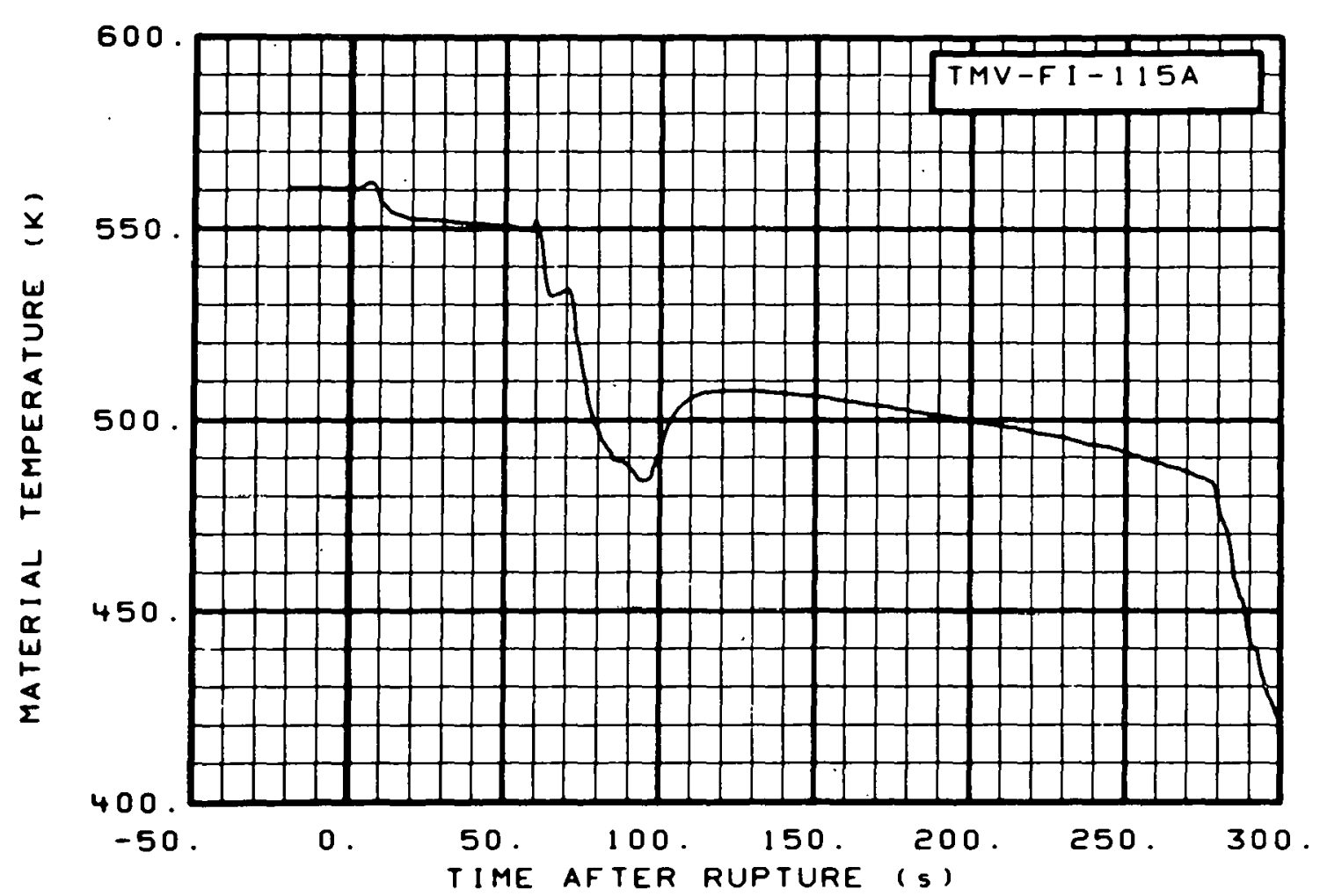

Fig. 55 Material temperature in vessel filler (TMV-FI-115A), from -20 to $300 \mathrm{~s}$.

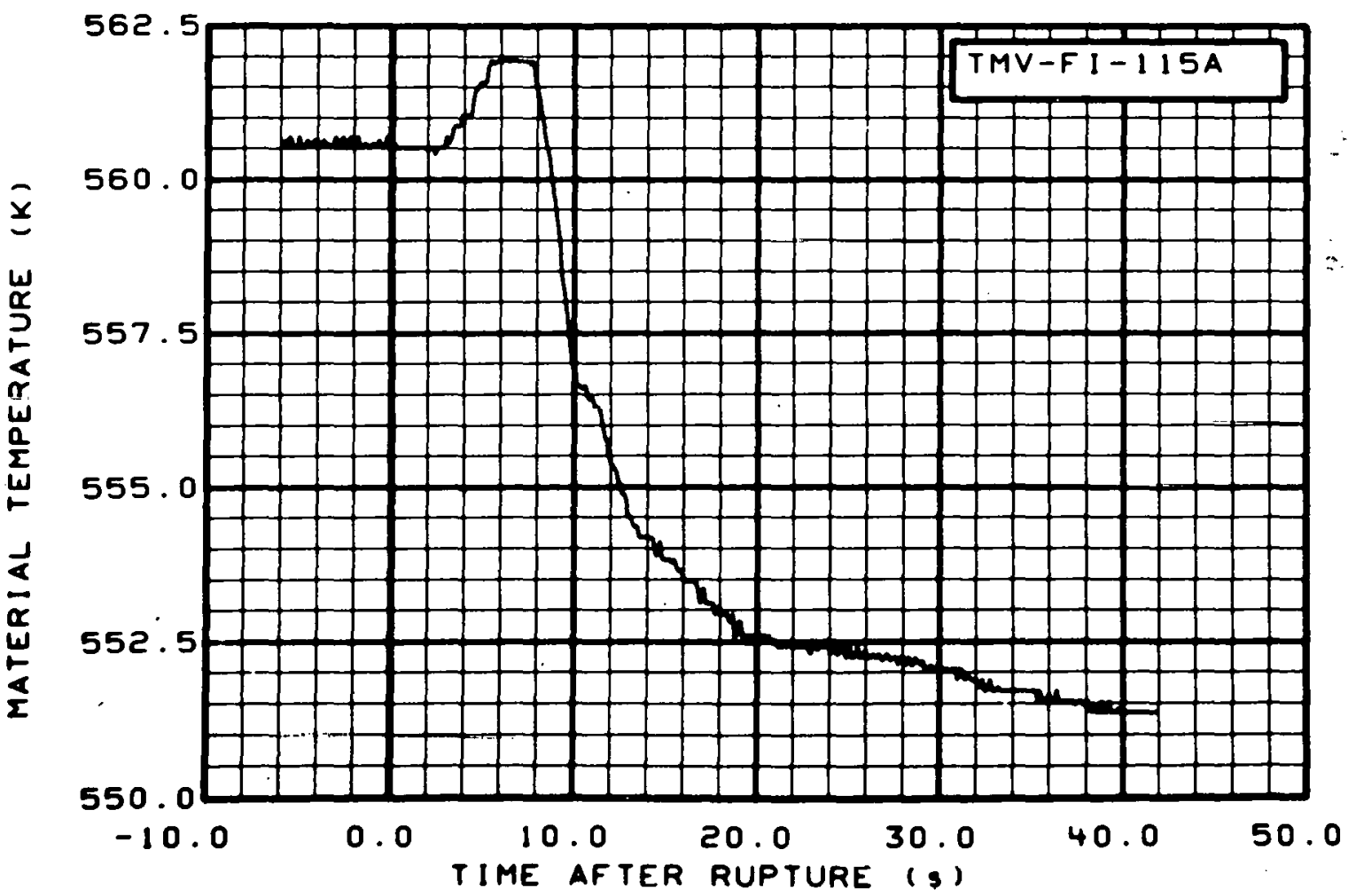

Fig. 56 Material temperature in vessel filler (TMV-FI-115A), from -6 to $42 \mathrm{~s}$. 


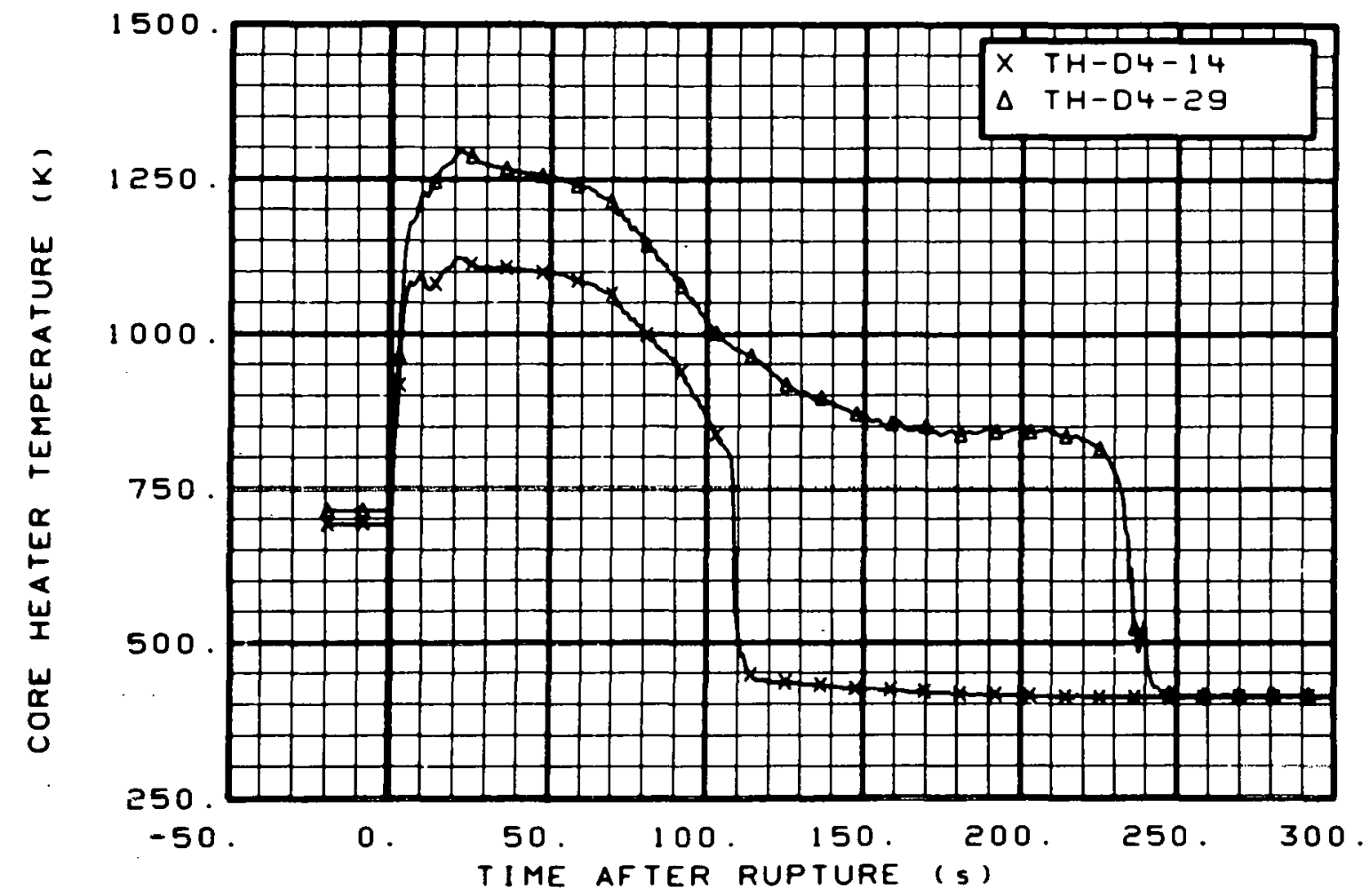

Fig. 57 Core heater temperature, Rod D-4 (TH-D4-14 and TH-D4-29), from -20 to $300 \mathrm{~s}$.

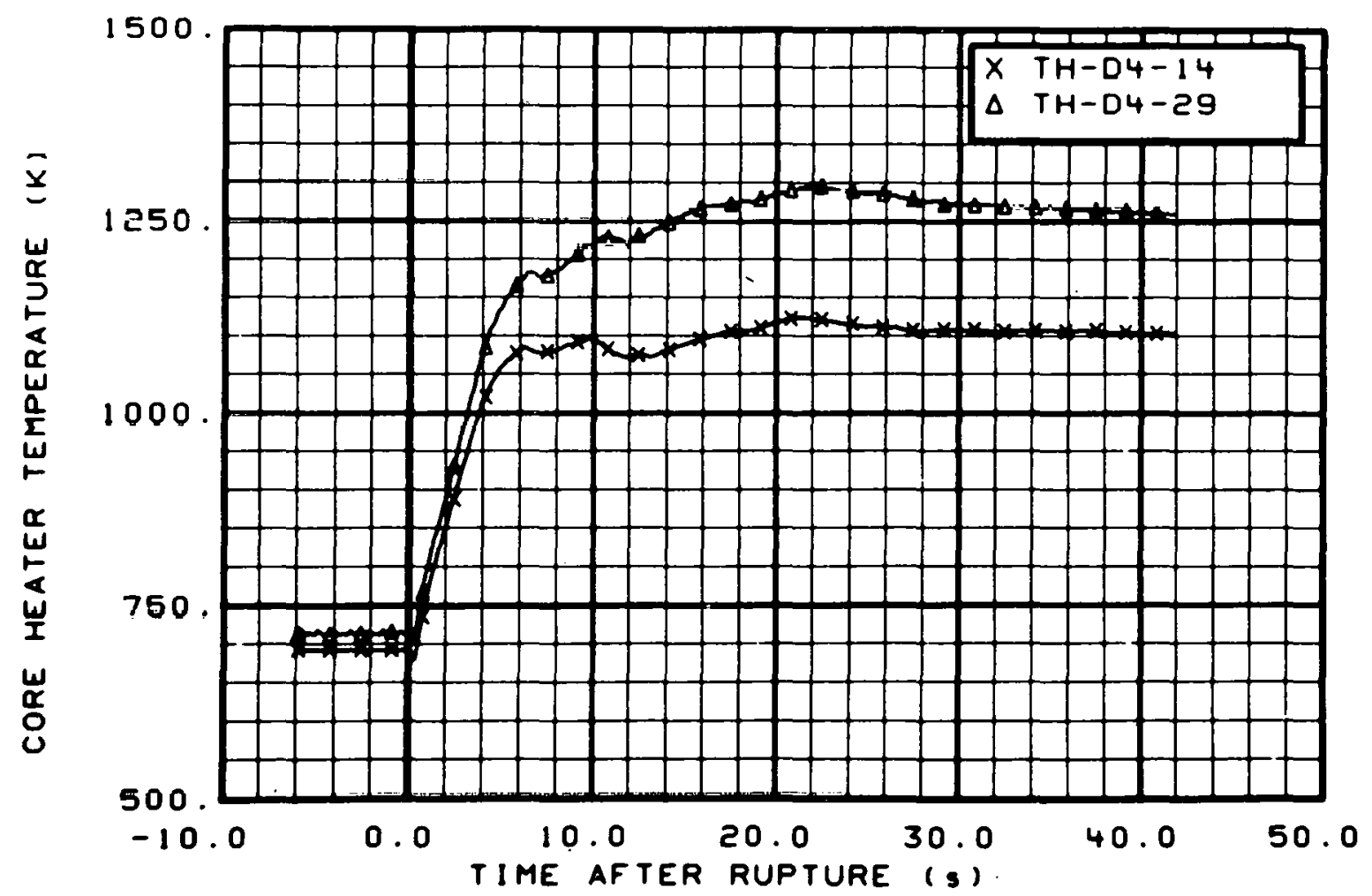

Fig. 58 Core heater temperature, Rod D-4 (TH-D4-14 and TH-D4-29), from -6 to $42 \mathrm{~s}$. 


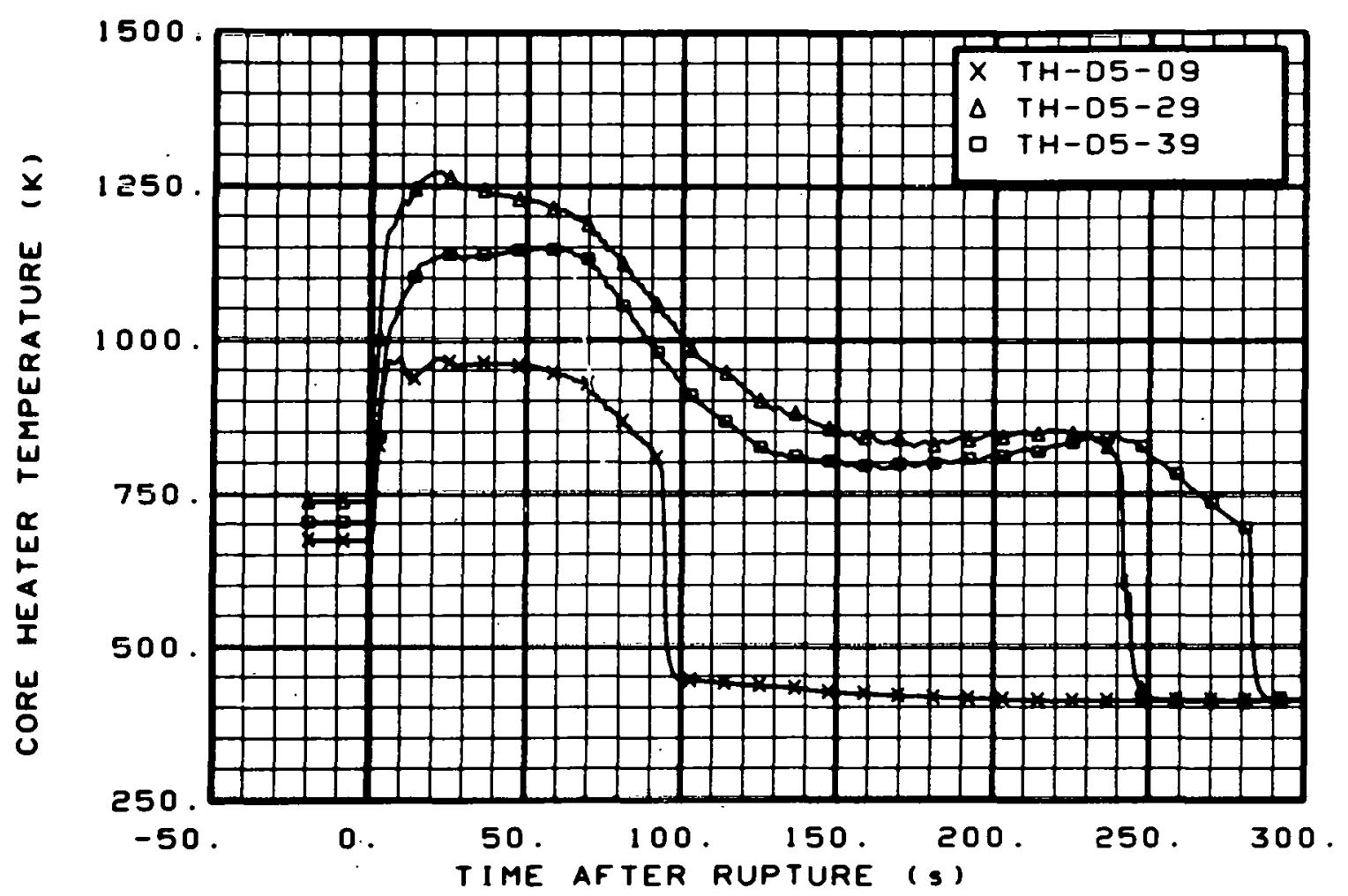

Fig. 59 Core heater temperature, Rod D-5 (TH-D5-09, TH-D5-29, and TH-D5-39), from -20 to $300 \mathrm{~s}$.

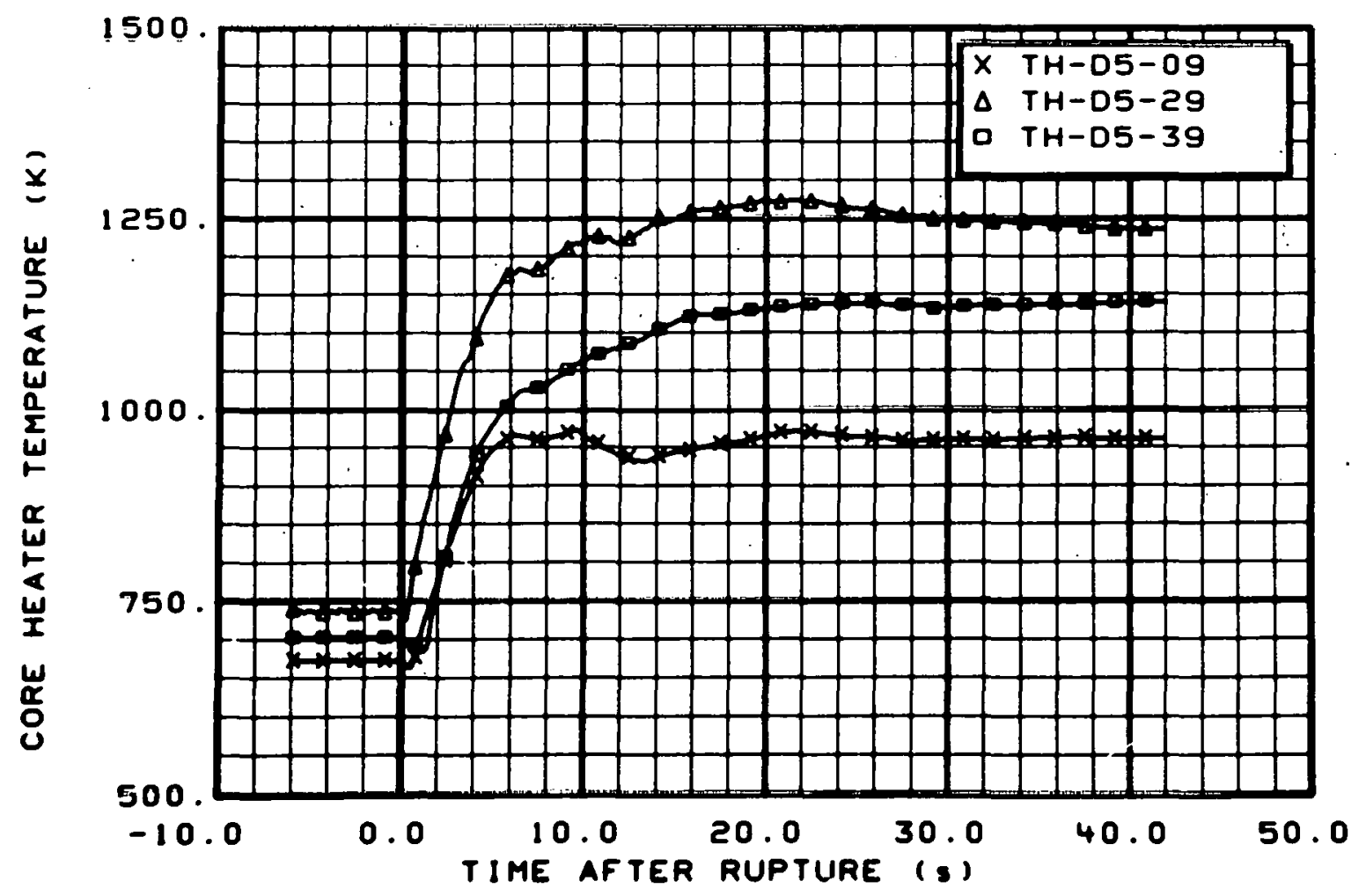

Fig. 60 Core heater temperature, Rod D-5 (TH-D5-09, TH-D5-29, and TH-D5-39), from -6 to $42 \mathrm{~s}$. 


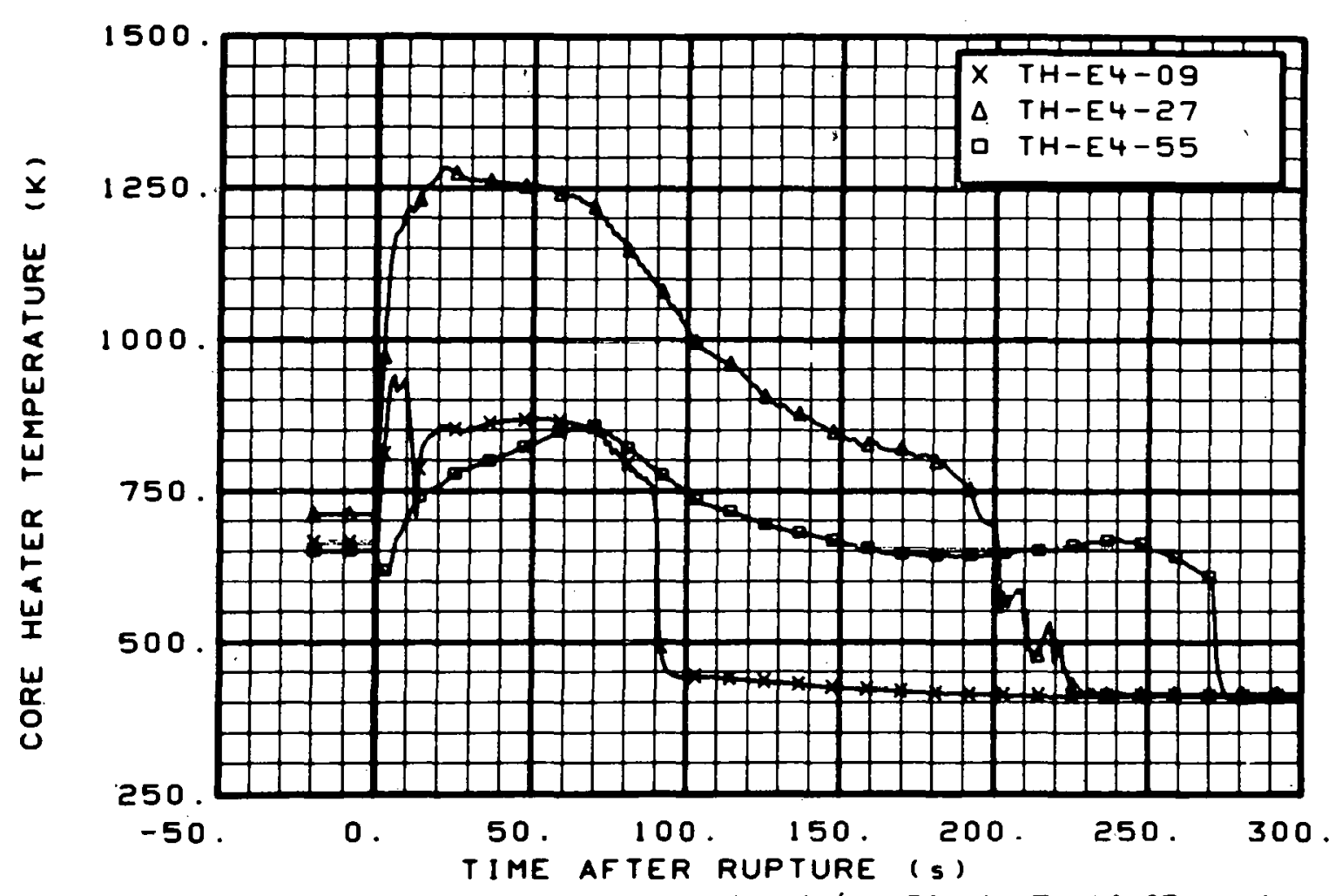

Fig. 61 Core heater temperature, Rod E-4 (TH-E4-09, TH-E4-27, and TH-E4-55), from -20 to $300 \mathrm{~s}$.

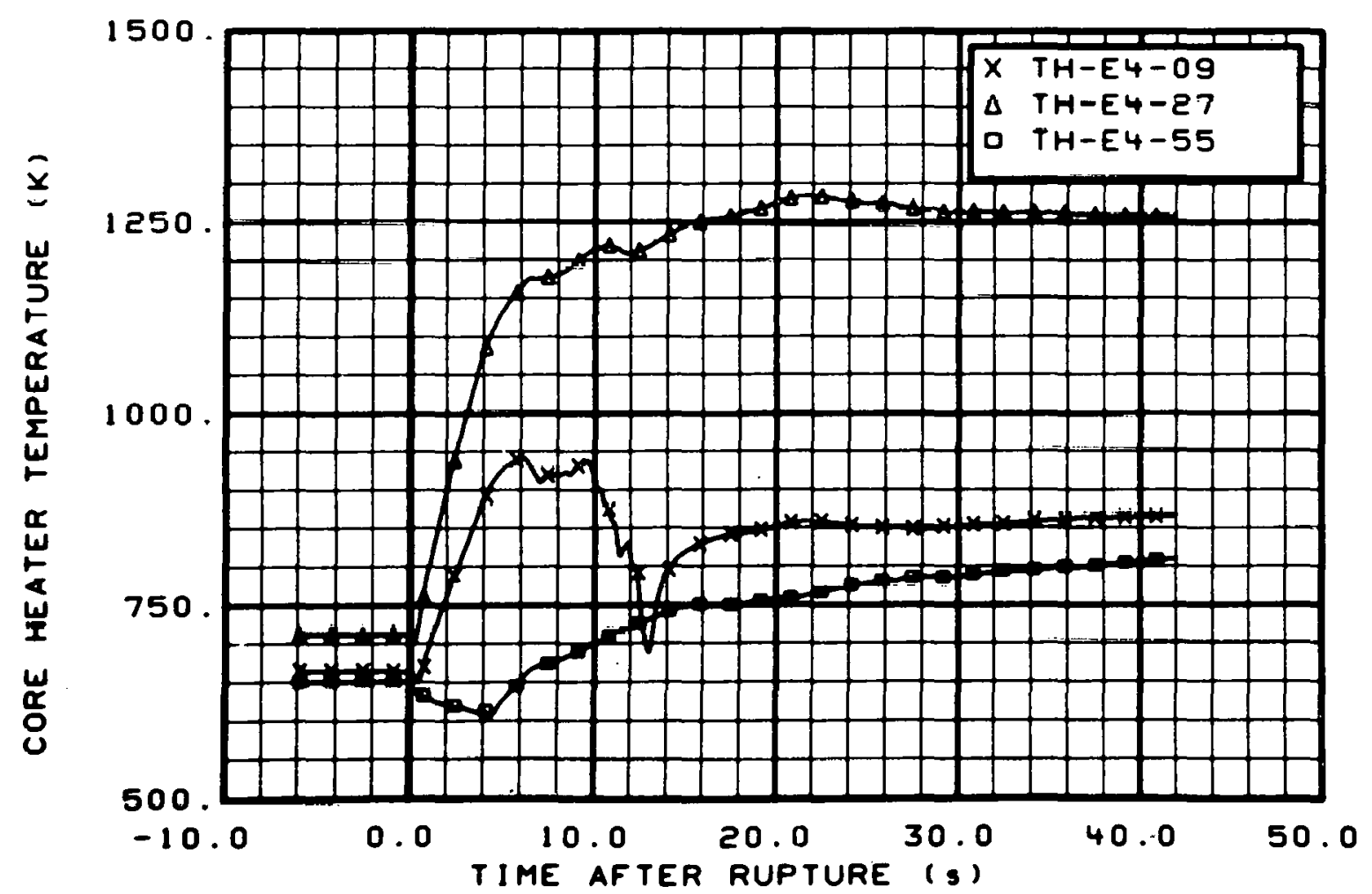

Fig. 62 Core heater temperature, Rod E-4 (TH-E4-09, TH-E4-27, and TH-E4-55), from -6 to $42 \mathrm{~s}$. 


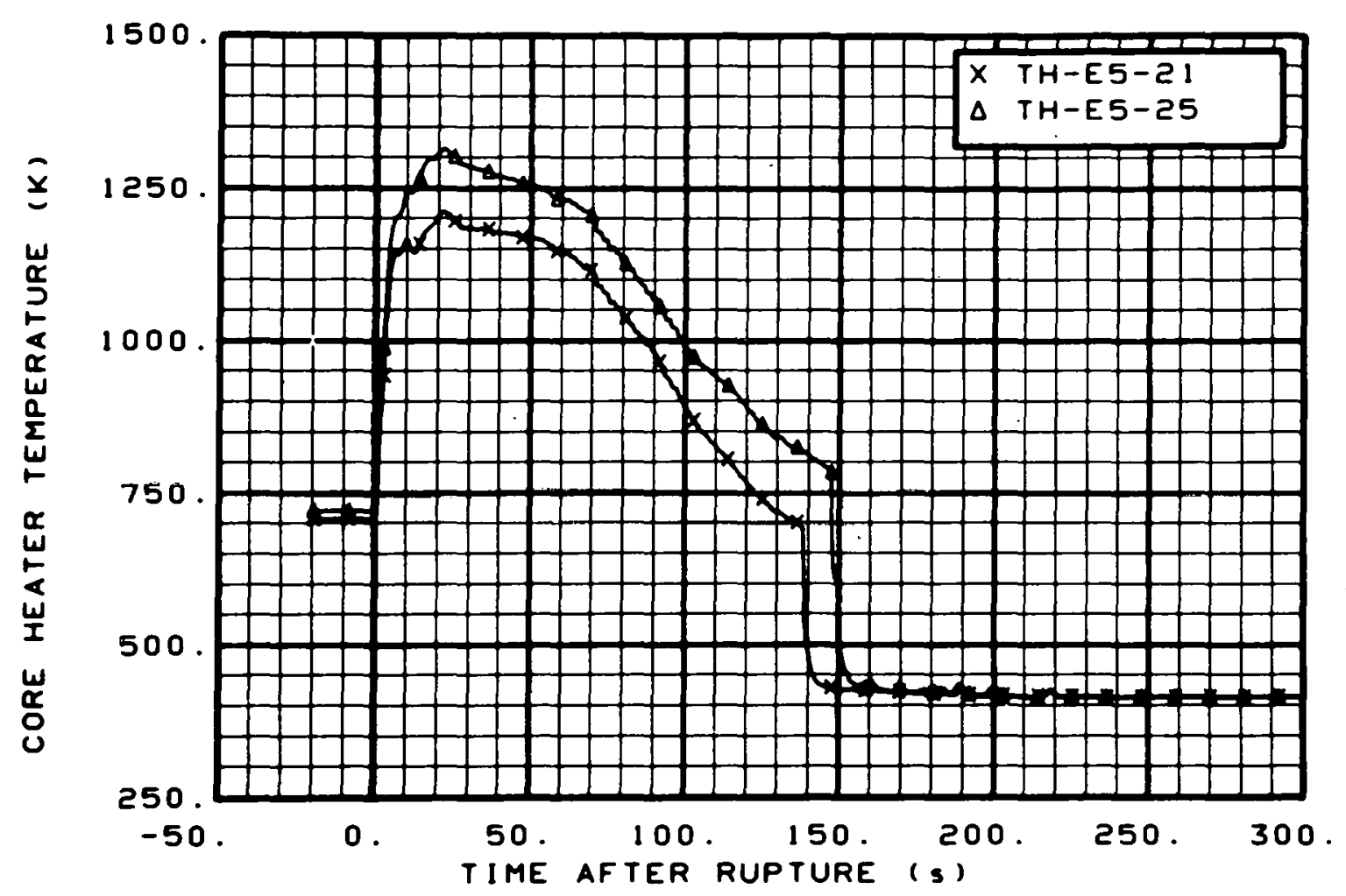

Fig. 63 Core heater temperature, Rod E-5 (TH-E5-21 and TH-E5-25), from -20 to $300 \mathrm{~s}$.

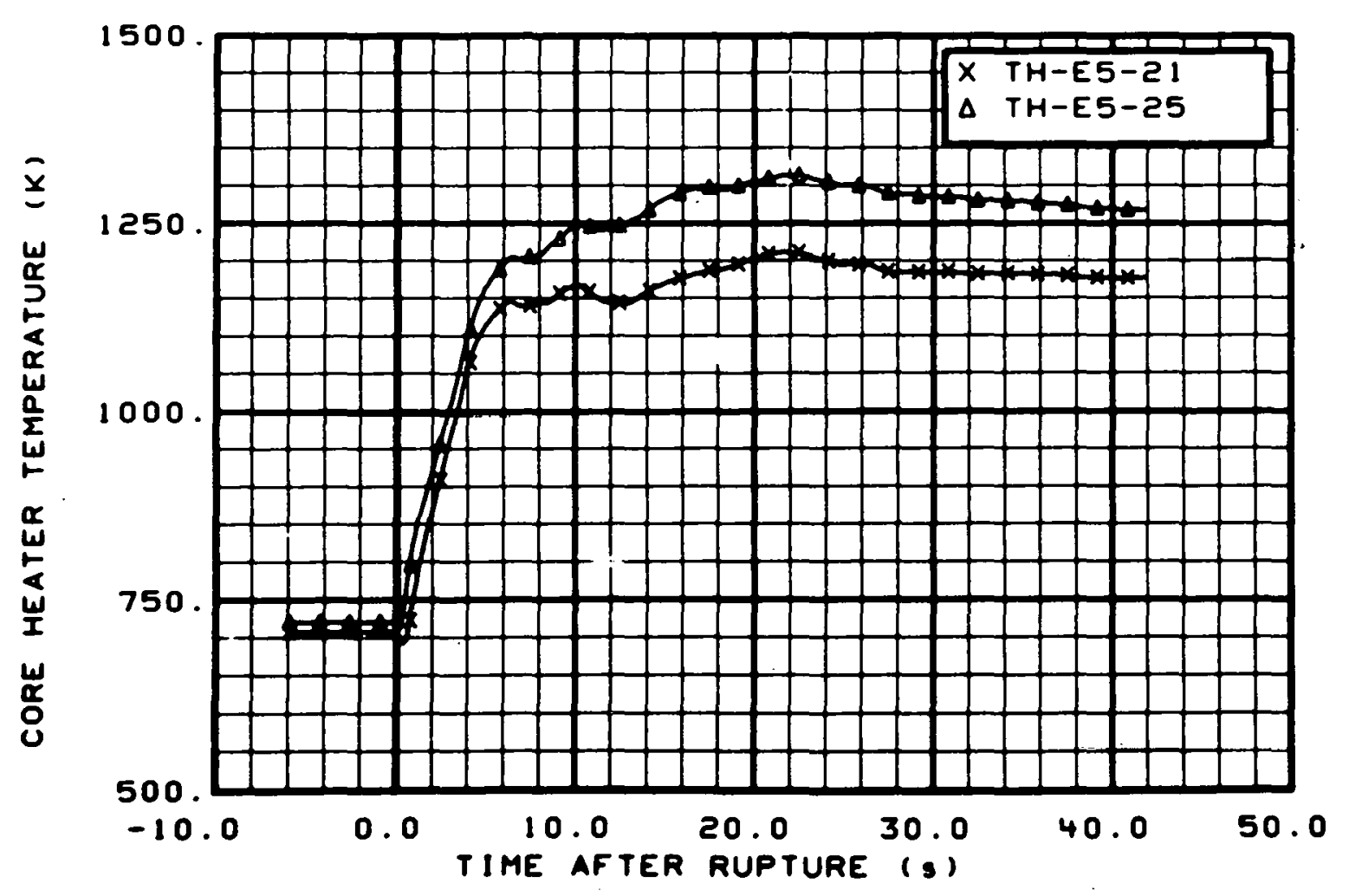

Fig. 64 Core heater temperature, Rod E-5 (TH-E5-21 and TH-E5-25), from -6 to 42 s. 


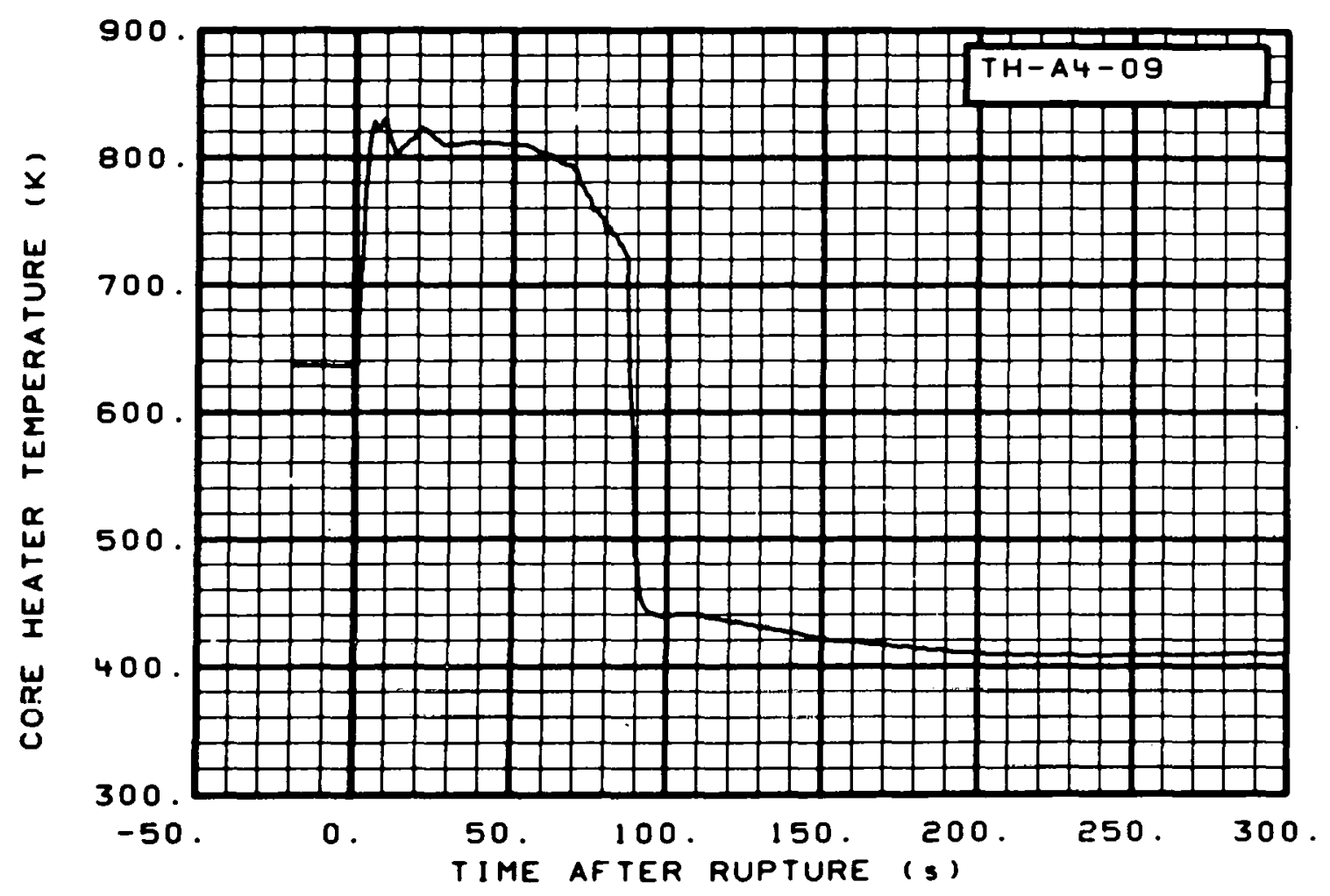

Fig. 65 Core heater temperature, Rod A-4 (TH-A4-09), from -20 to $300 \mathrm{~s}$.

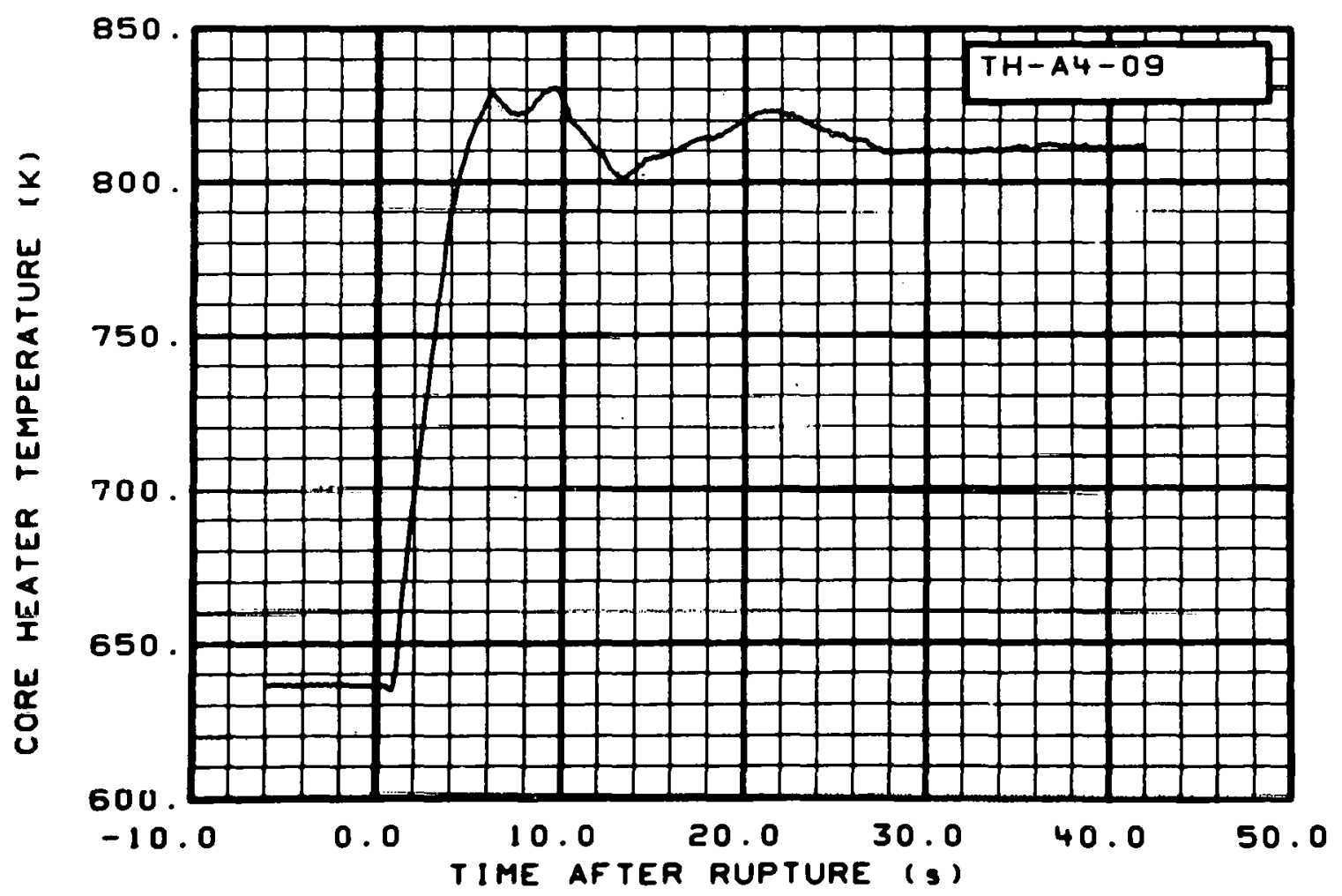

Fig. 66 Core heater temperature, Rod A-4 (TH-A4-09), from -6 to $42 \mathrm{~s}$. 


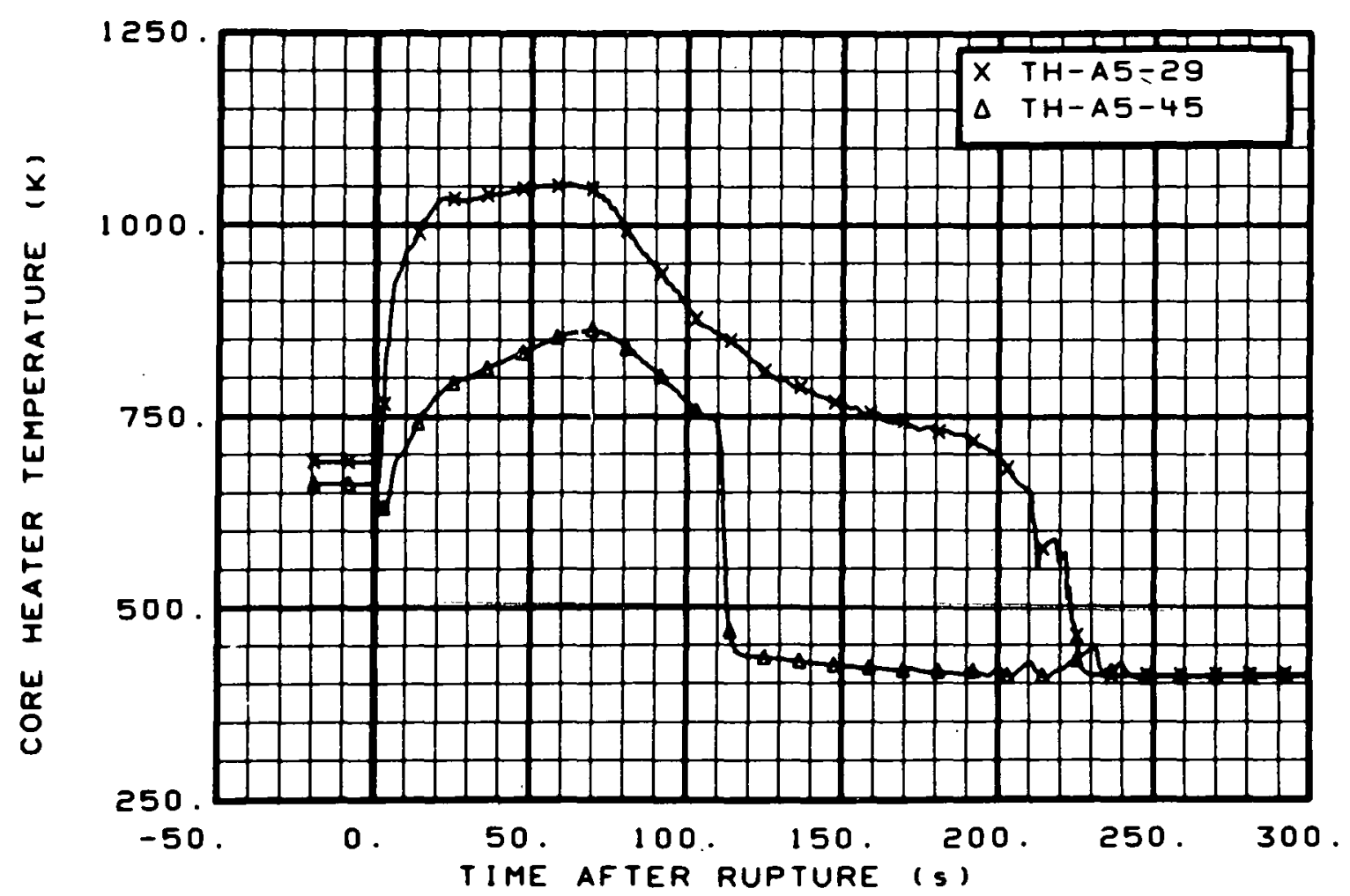

Fig. 67 Core heater temperature, Rod A-5 (TH-A5-29 and TH-A5-45), from -20 to $300 \mathrm{~s}$.

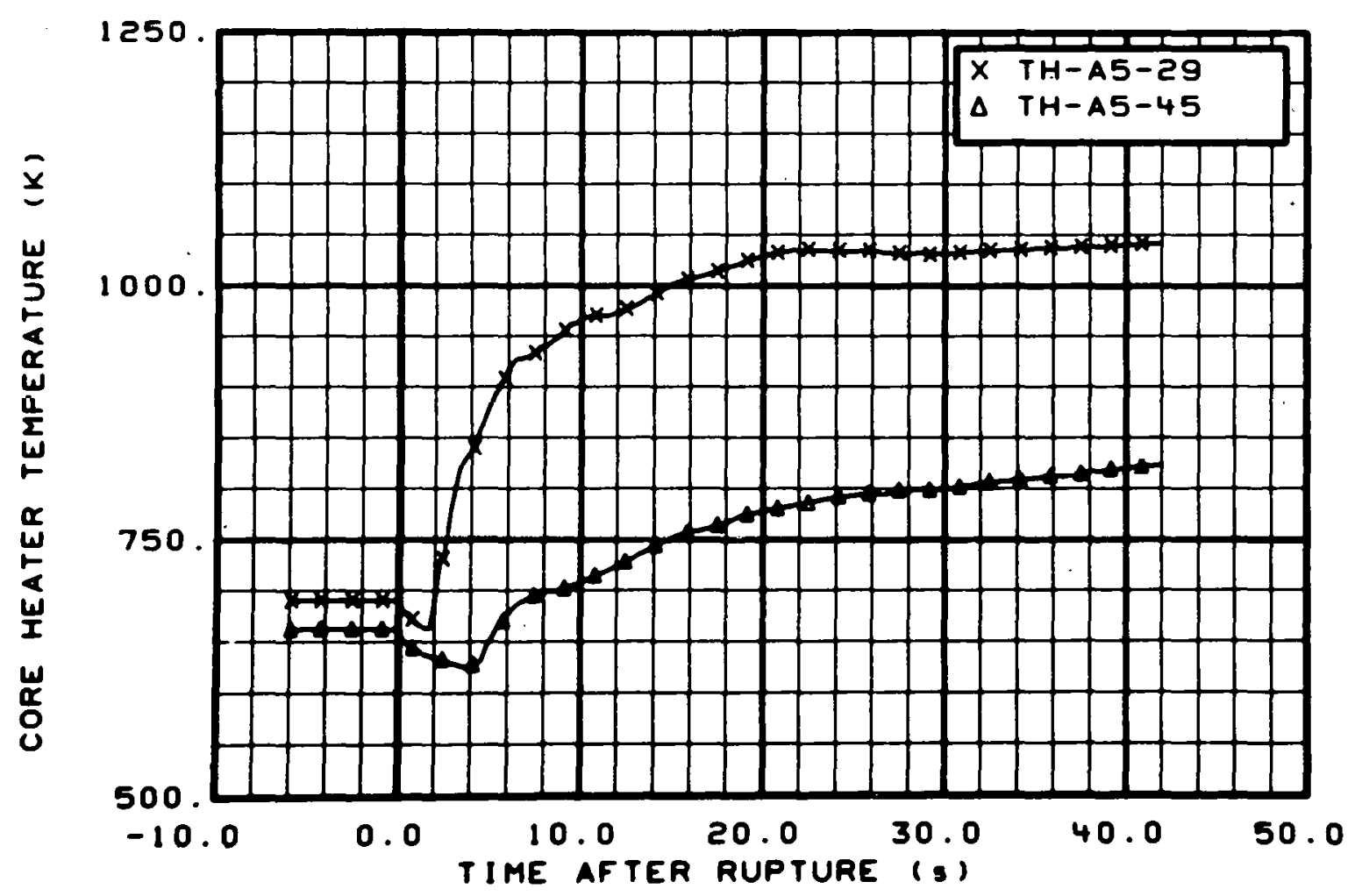

Fig. 68 Core heater temperature, Rod A-5 (TH-A5-29 and TH-A5-45), from -6 to $42 \mathrm{~s}$. 


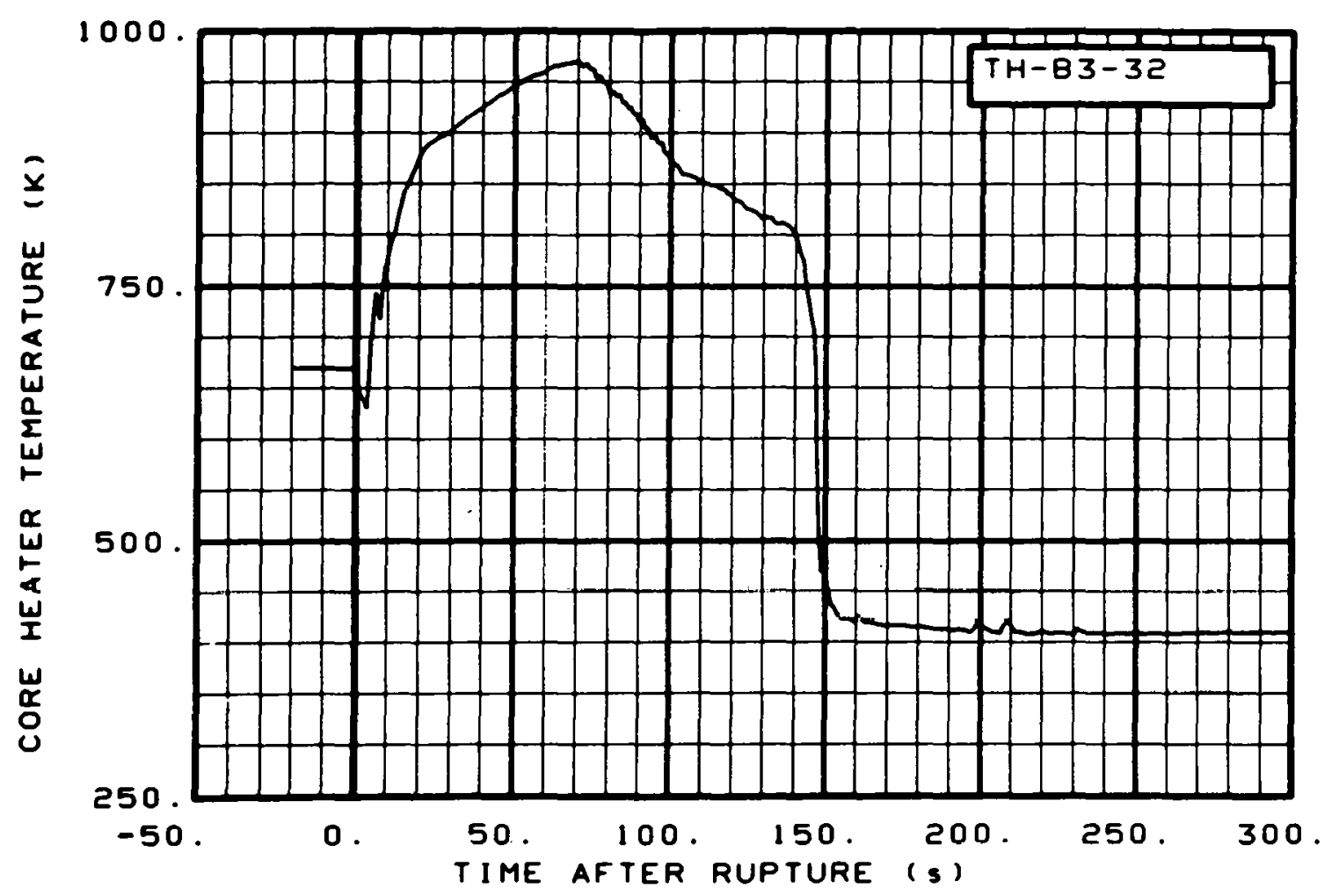

Fig. 69 Core heater temperature, Rod B-3 (TH-B3-32), from -20 to $300 \mathrm{~s}$.

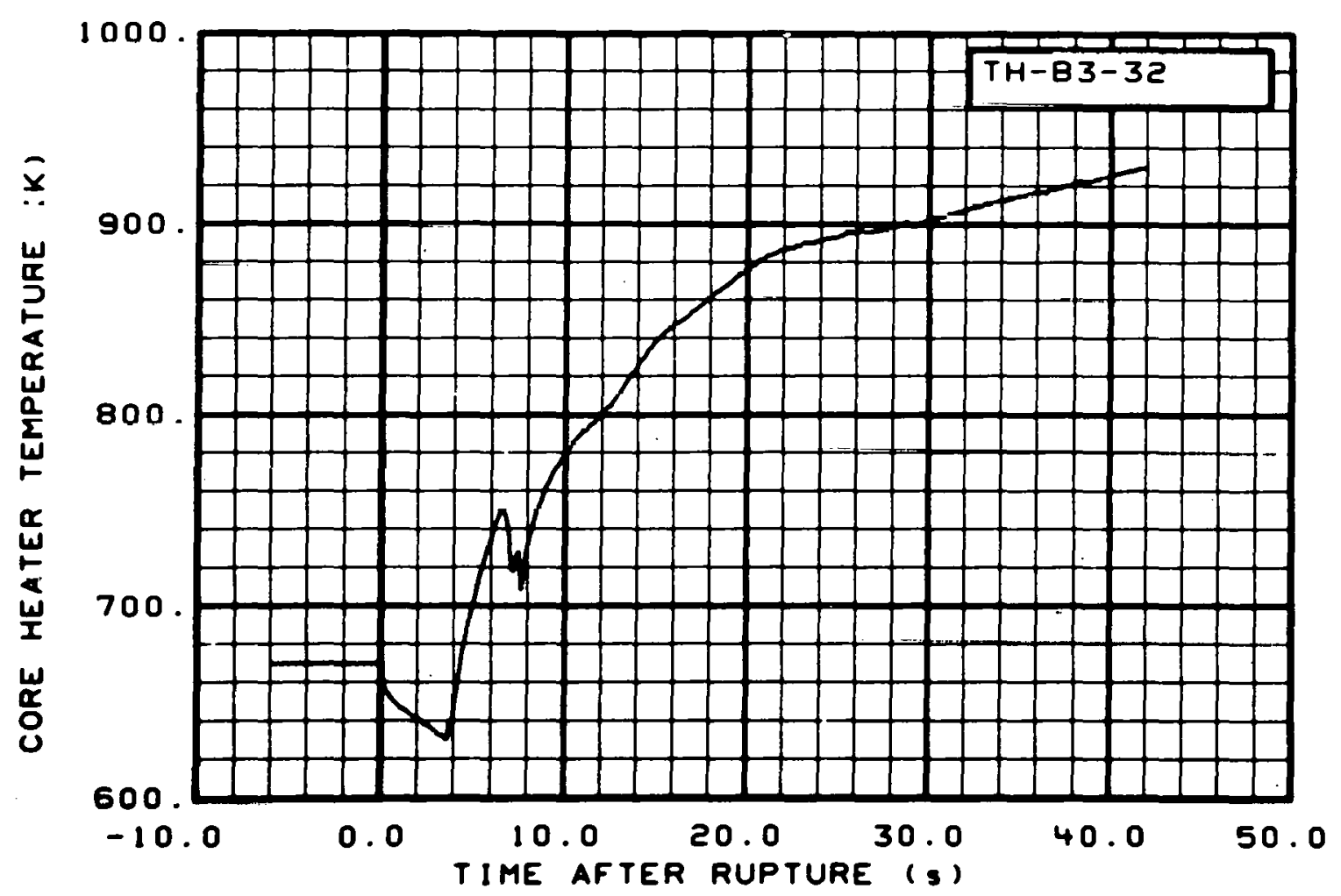

Fig. 70 Core heater temperature, Rod B-3 (TH-B3-32), from -6 to $42 \mathrm{~s}$. 


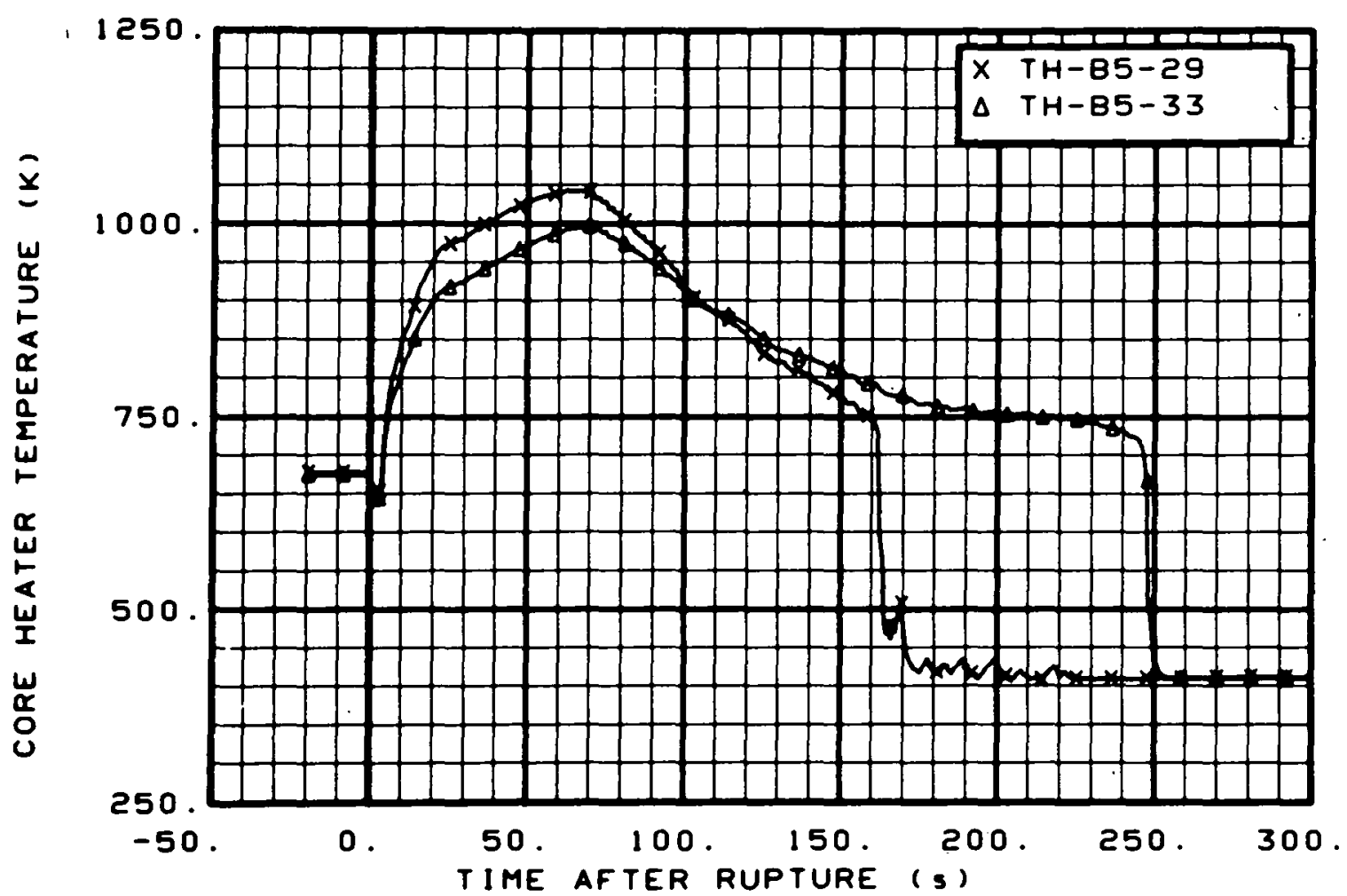

Fig. 71 Core heater temperature, Rod B-5 (TH-B5-29 and TH-B5-33), from -20 to $300 \mathrm{~s}$.

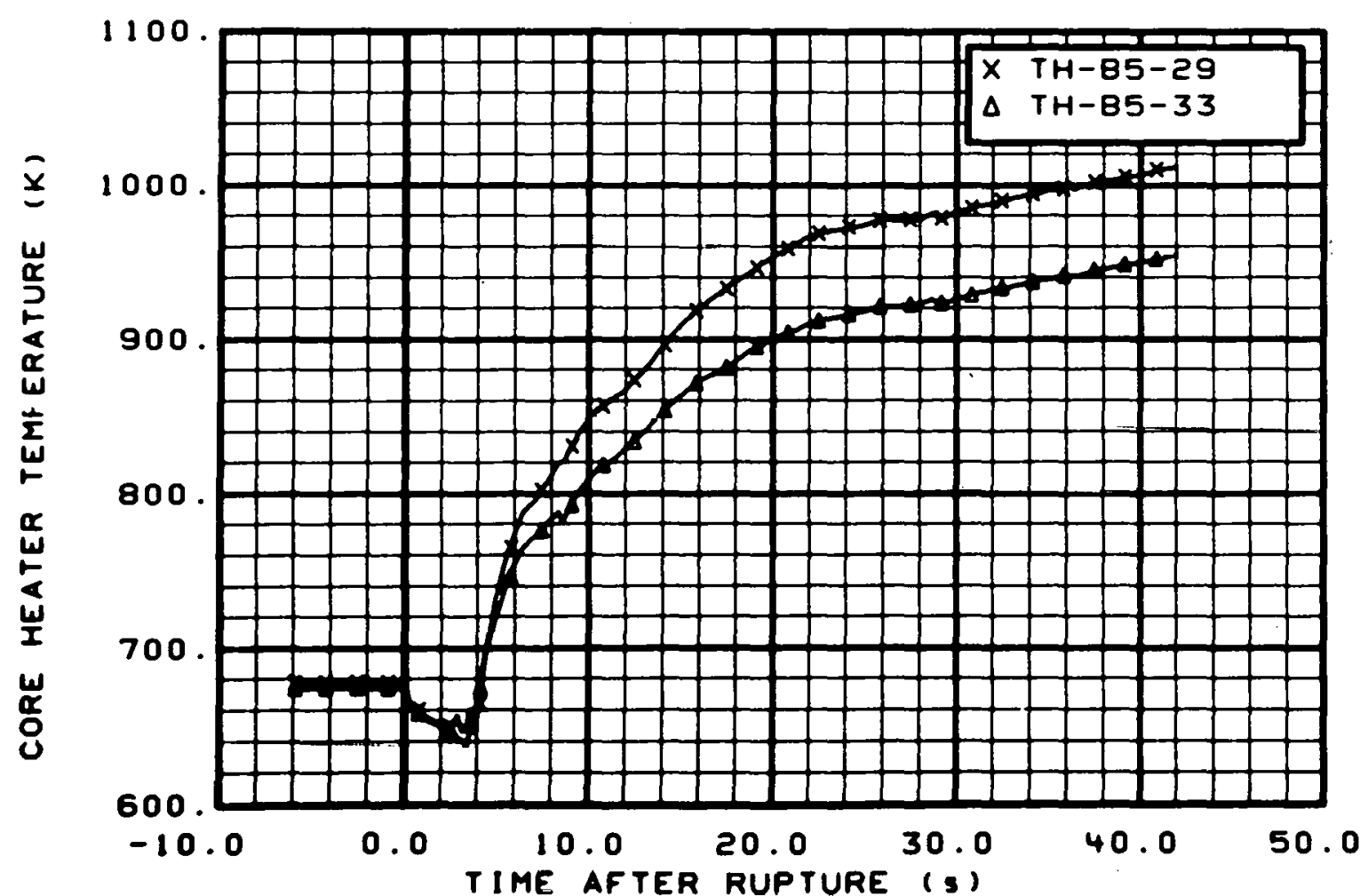

Fig. 72 Core heater temperature, Rod B-5 (TH-B5-29 and TH-B5-33), from -6 to $42 \mathrm{~s}$. 


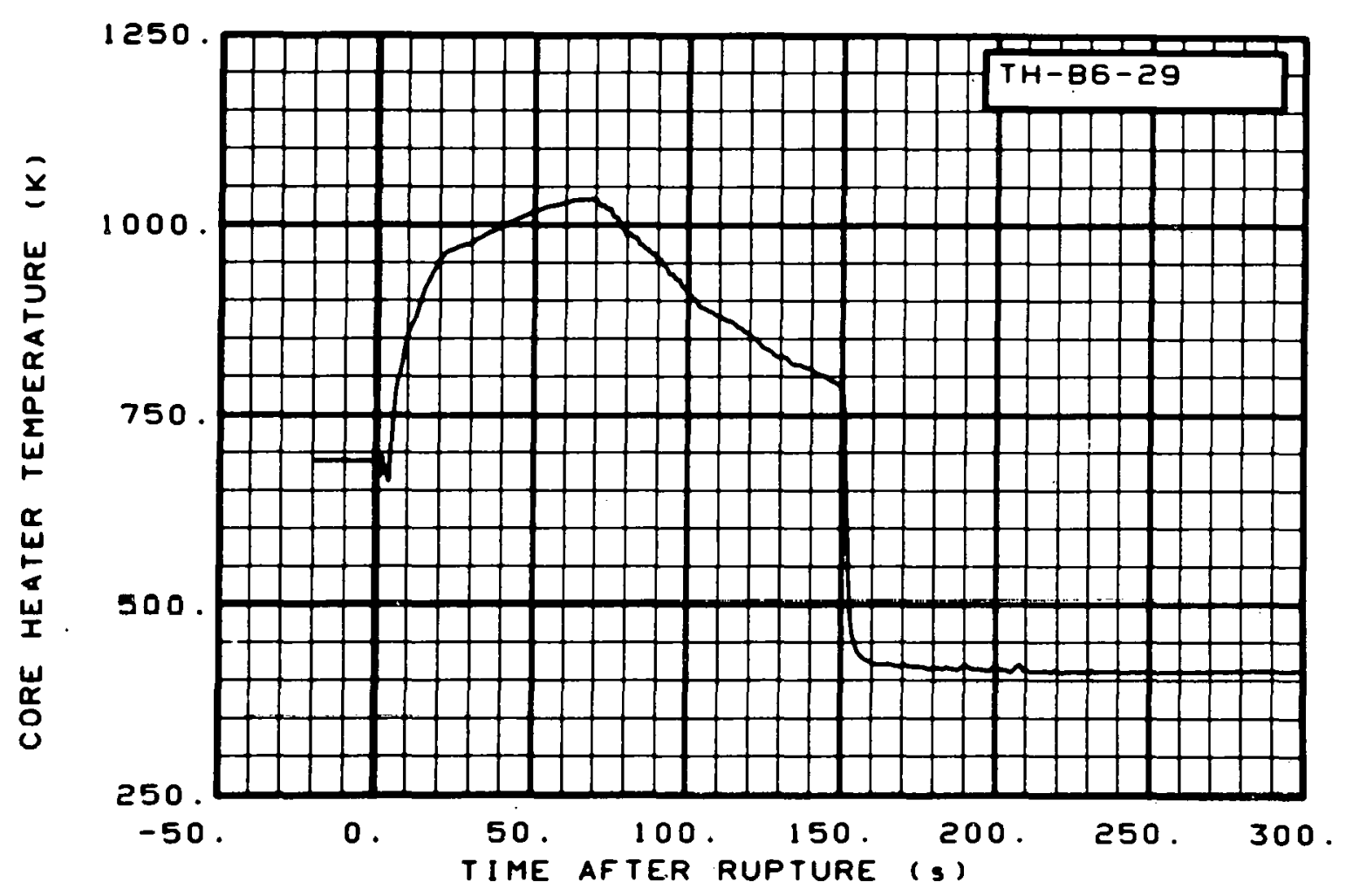

Fig. 73 Core heater temperature, Rod B-6 (TH-B6-29), from -20 to $300 \mathrm{~s}$.

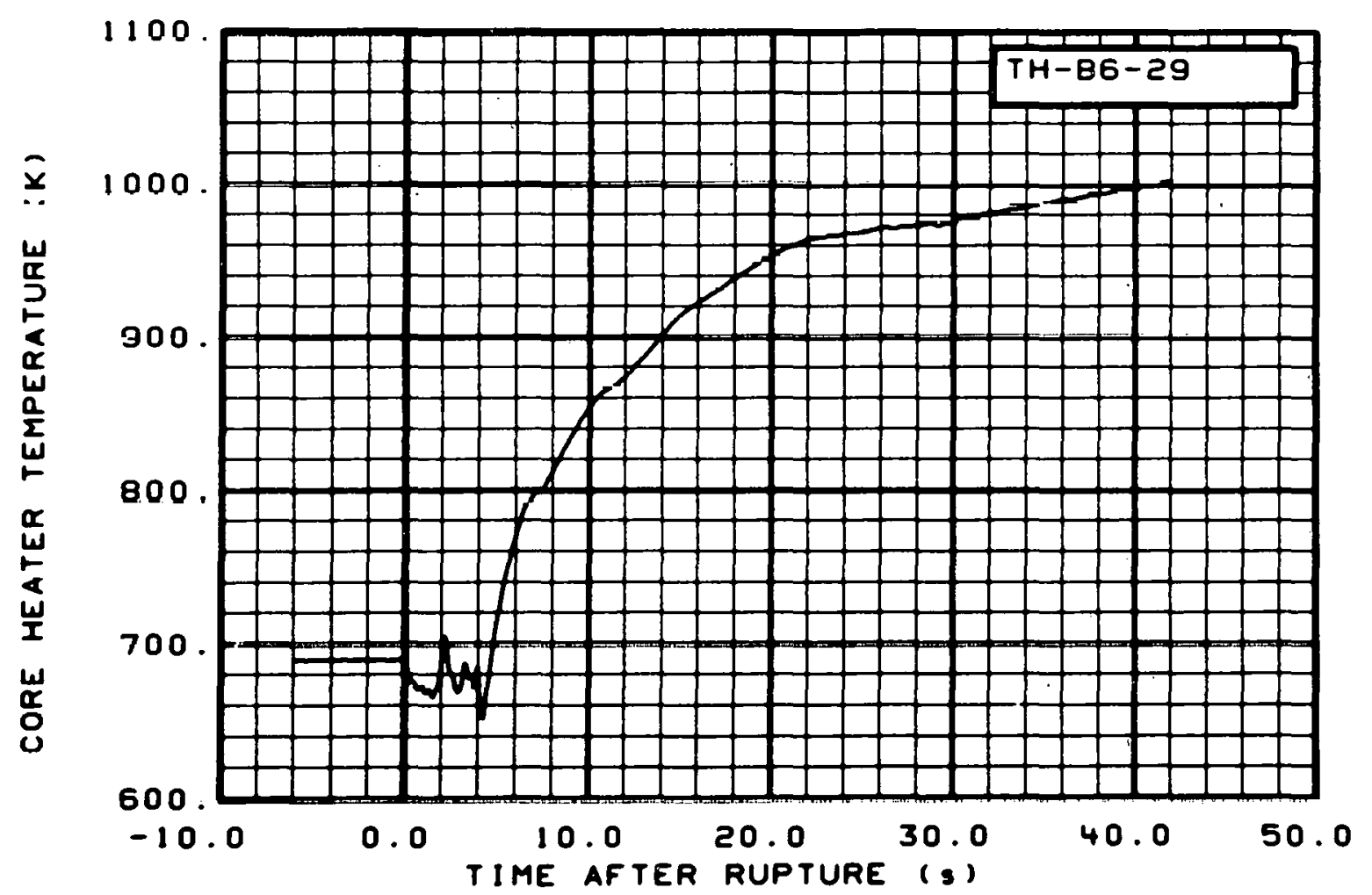

Fig. 74 Core heater temperature, Rod B-6 (TH-B6-29), from -6 to $42 \mathrm{~s}$. 


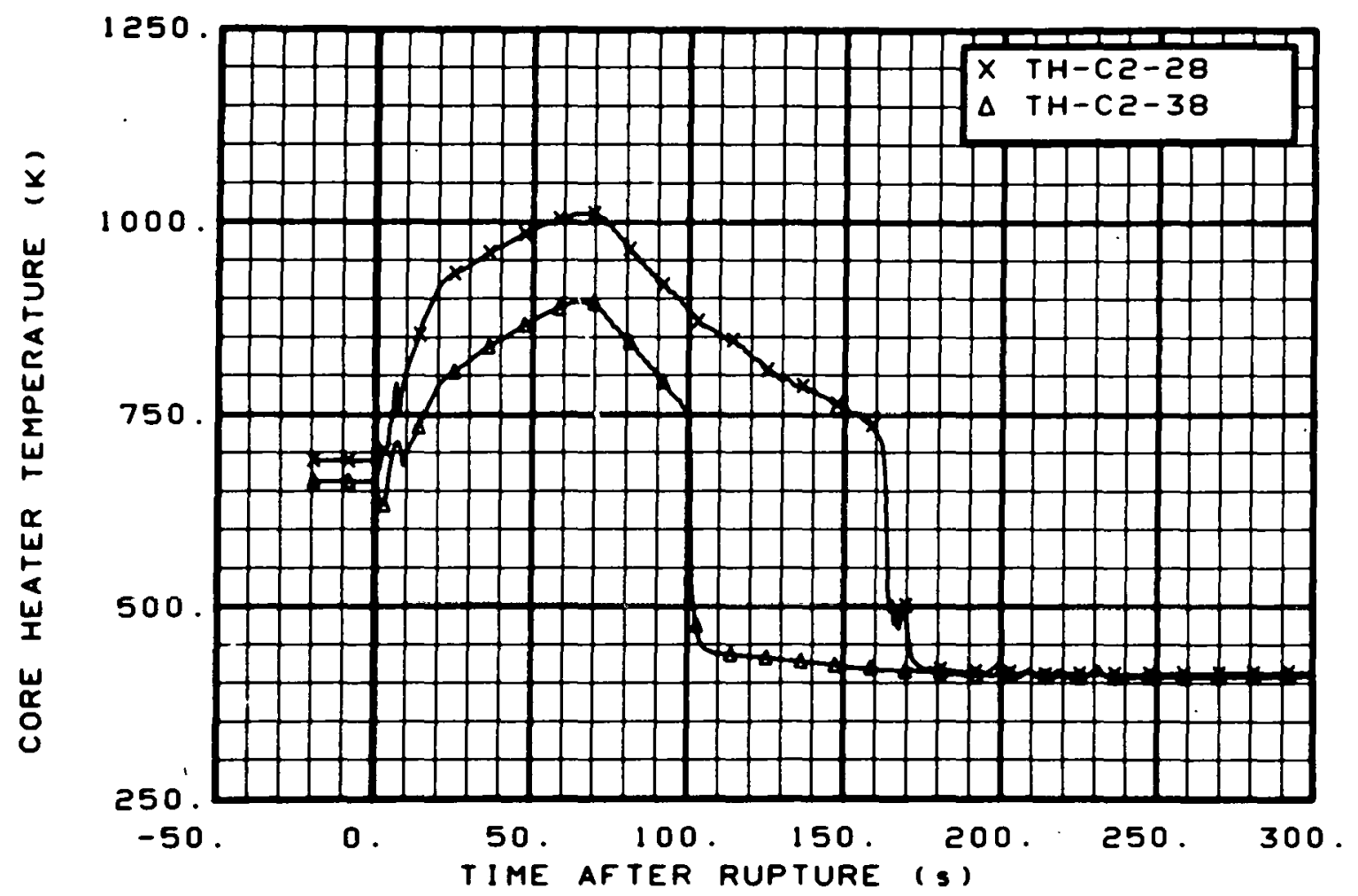

Fig. 75 Core heater temperature, Rod C-2 (TH-C2-28 and TH-C2-38), from -20 to $300 \mathrm{~s}$.

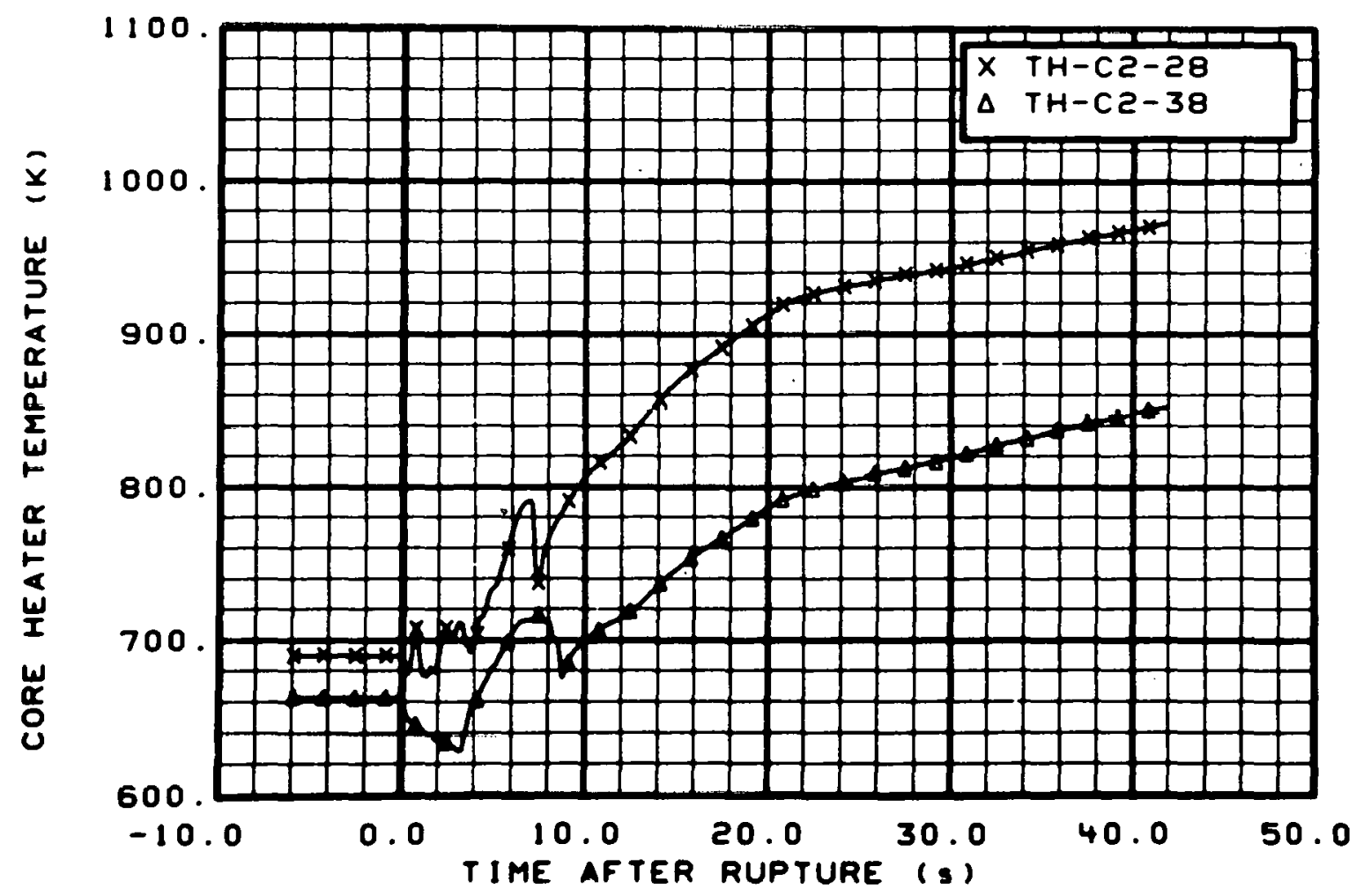

Fig. 76 Core heater temperature, Rod C-2 (TH-C2-28 and TH-C2-38), from -6 to $42 \mathrm{~s}$. 


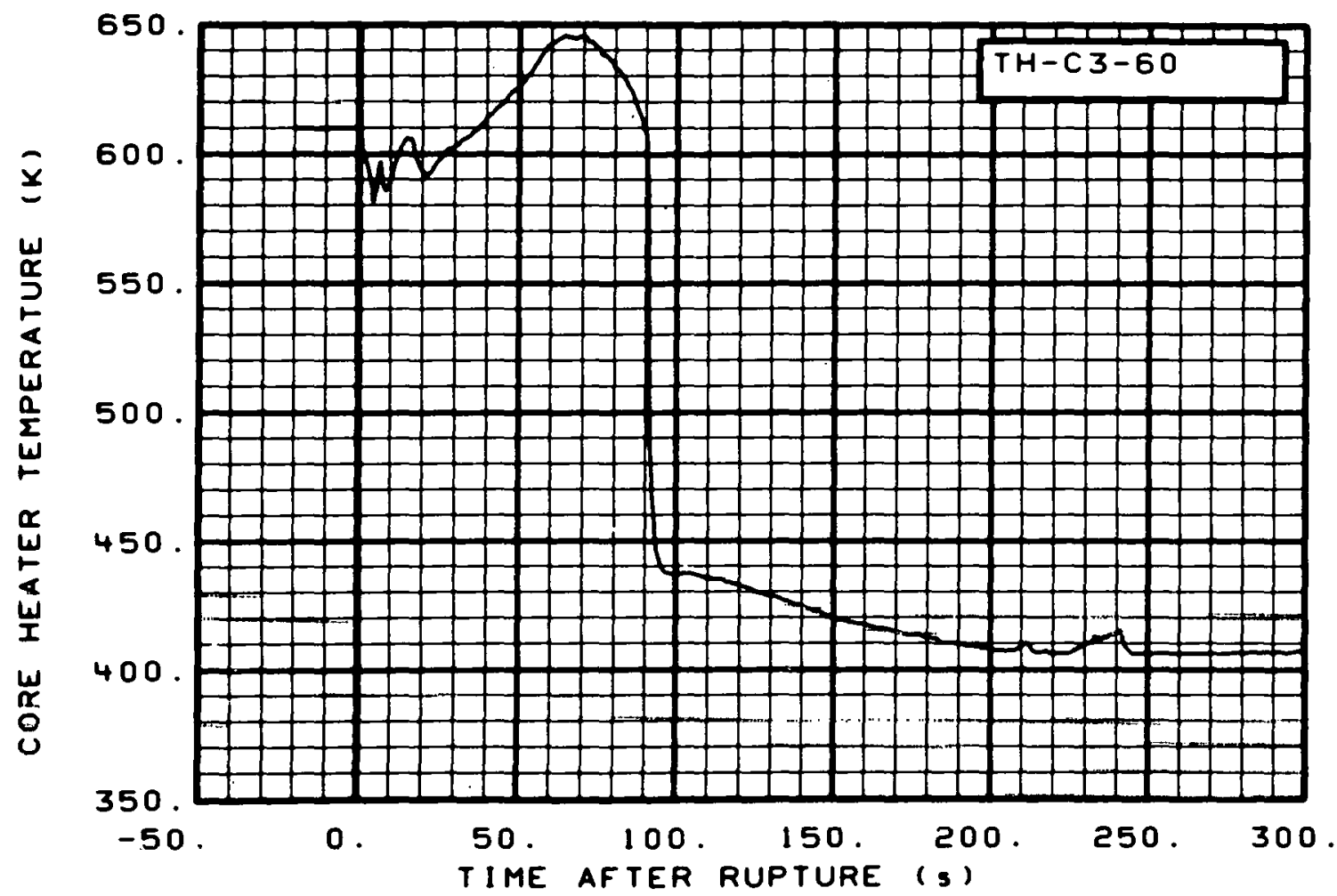

Fig. 77 Core heater temperature, Rod $\mathrm{C}-3(\mathrm{TH}-\mathrm{C} 3-60)$, from -20 to $300 \mathrm{~s}$.

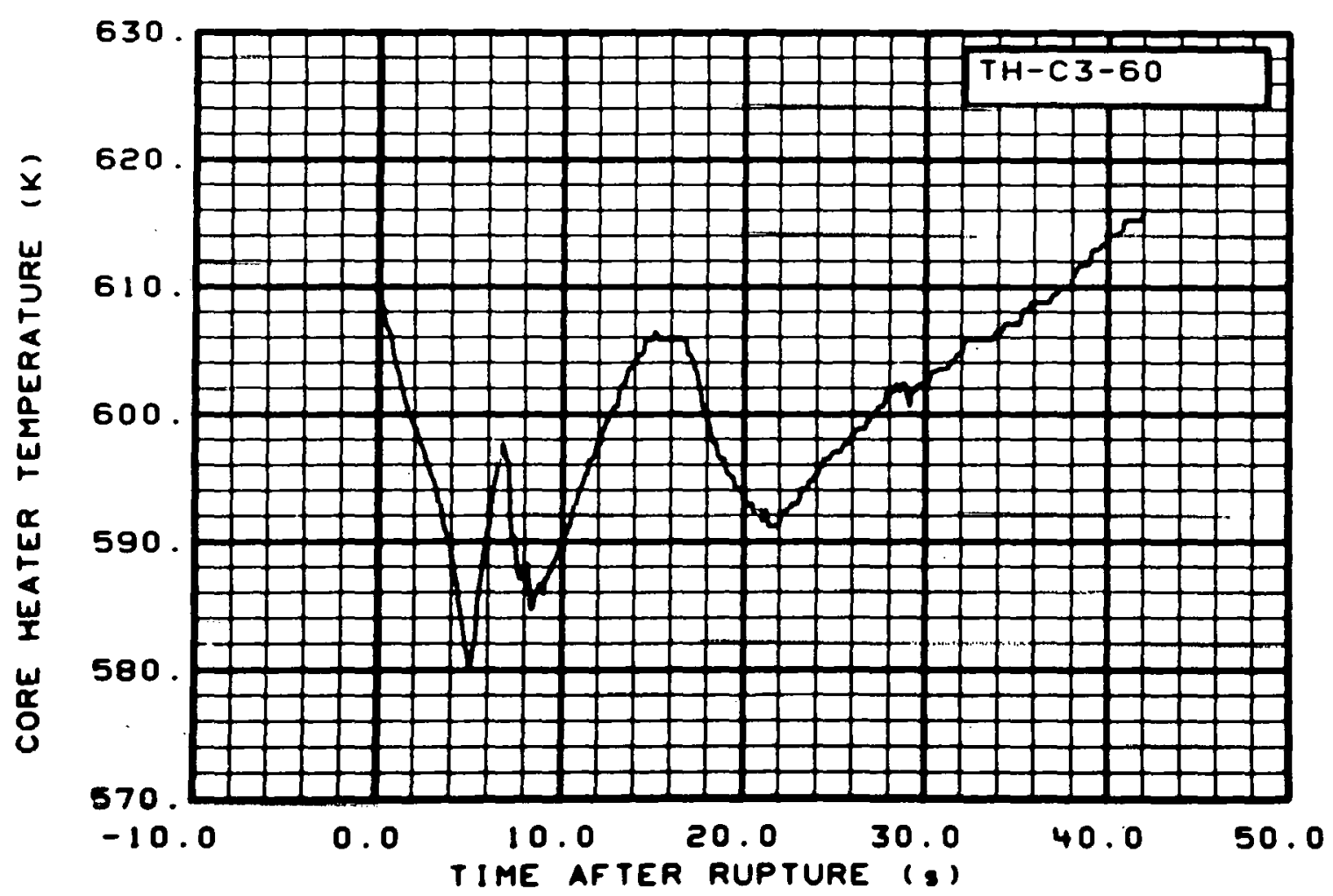

Fig. 78 Core heater temperature, Rod C-3 (TH-C3-60), from -6 to $42 \mathrm{~s}$. 


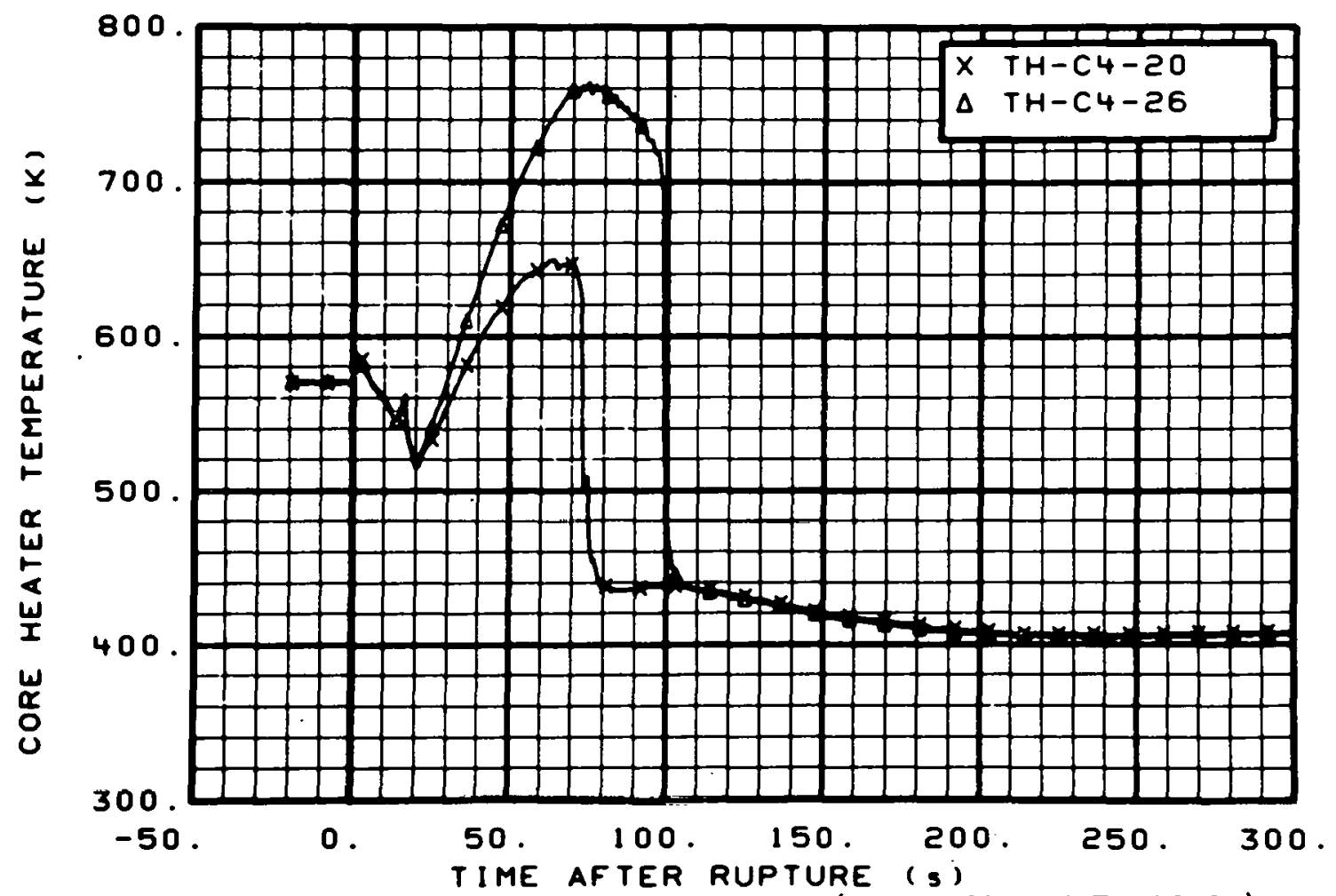

Fig. 79 Core heater temperature, Rod C-4 (TH-C4-20 and TH-C4-26), from -20 to $300 \mathrm{~s}$.

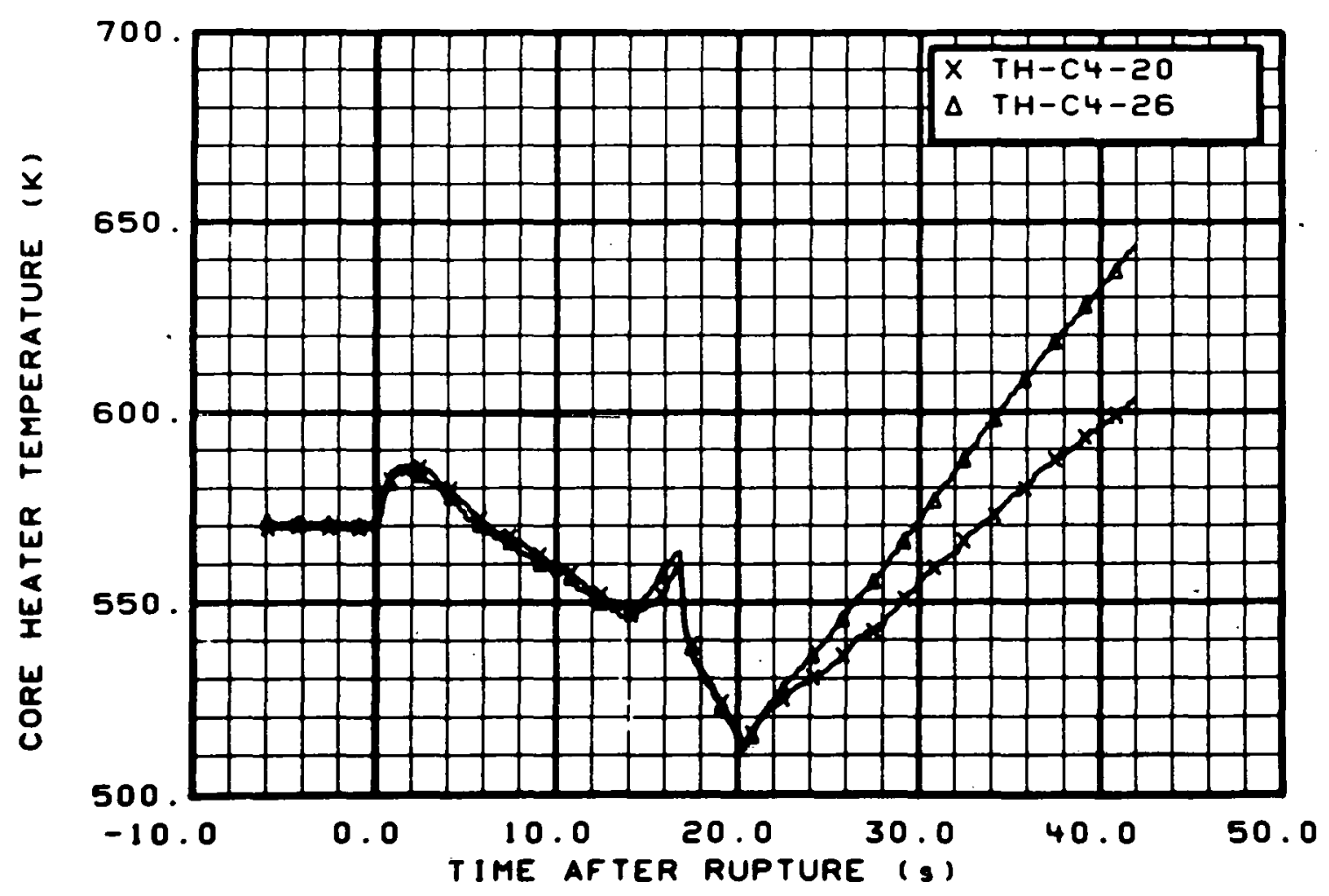

Fig. 80 Core heater temperature, Rod $\mathrm{C}-4$ (TH-C4-20 and $\mathrm{TH}-\mathrm{C} 4-26)$, from -6 to $42 \mathrm{~s}$. 


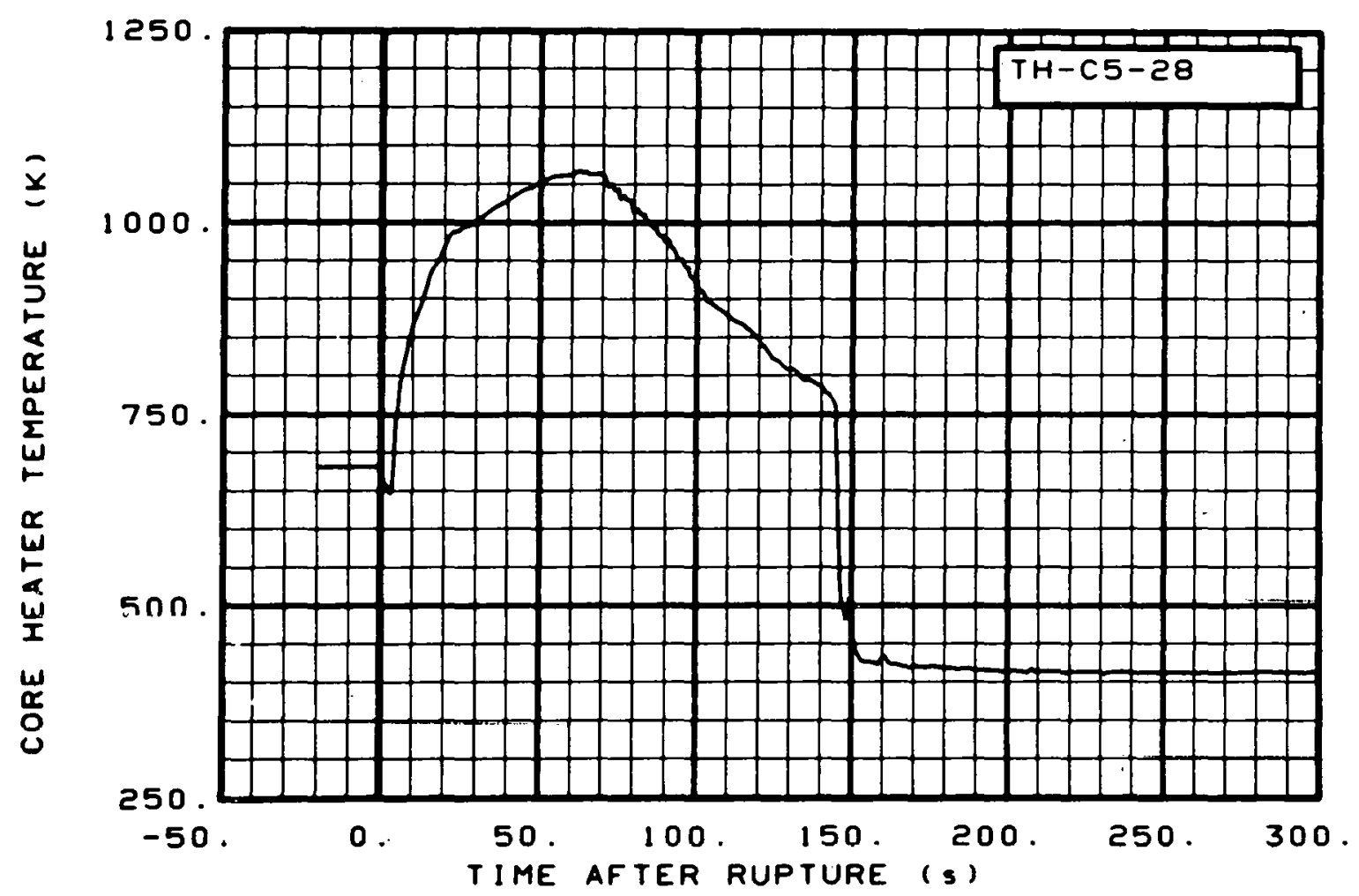

Fig. 81 Core heater temperature, Rod C-5 (TH-C5-28), from -20 to $300 \mathrm{~s}$.

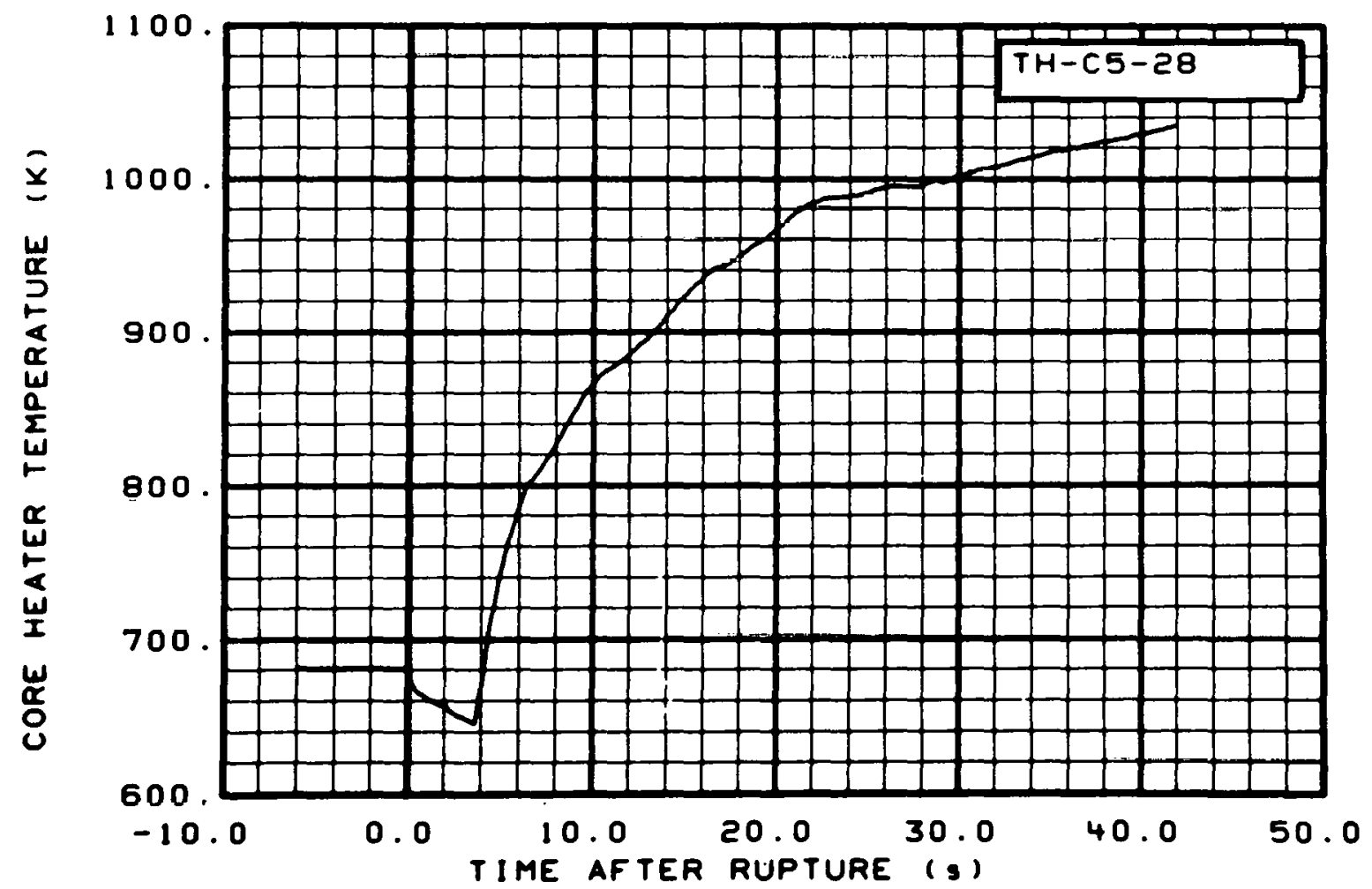

Fig. 82 Core heater temperature, Rod $\mathrm{C}-5$ (TH-C5-28), from -6 to $42 \mathrm{~s}$. 


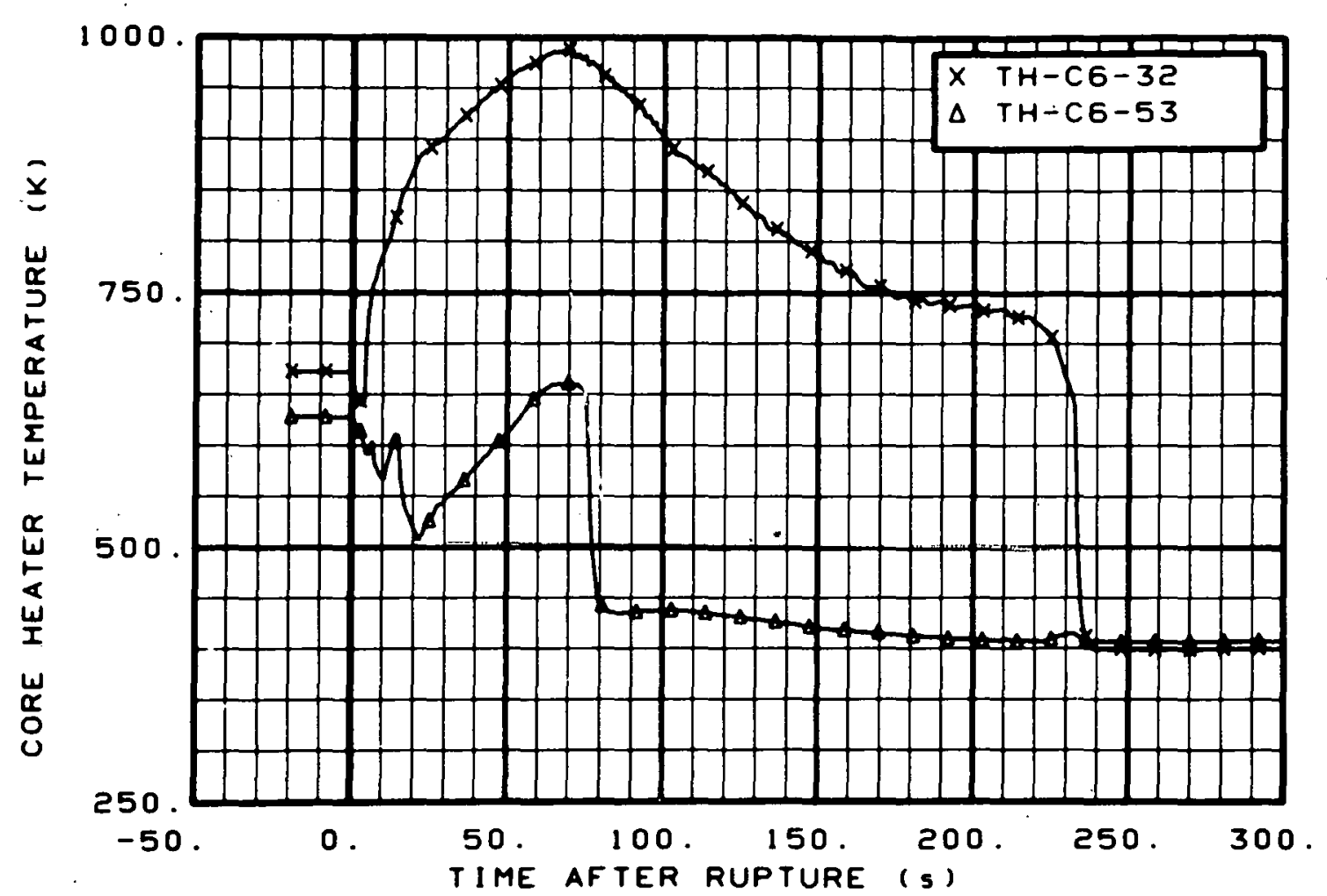

Fig. 83 Core heater temperature, Rod $\mathrm{C}-6$ (TH-C6-32 and TH-C6-53), from -20 to $300 \mathrm{~s}$.

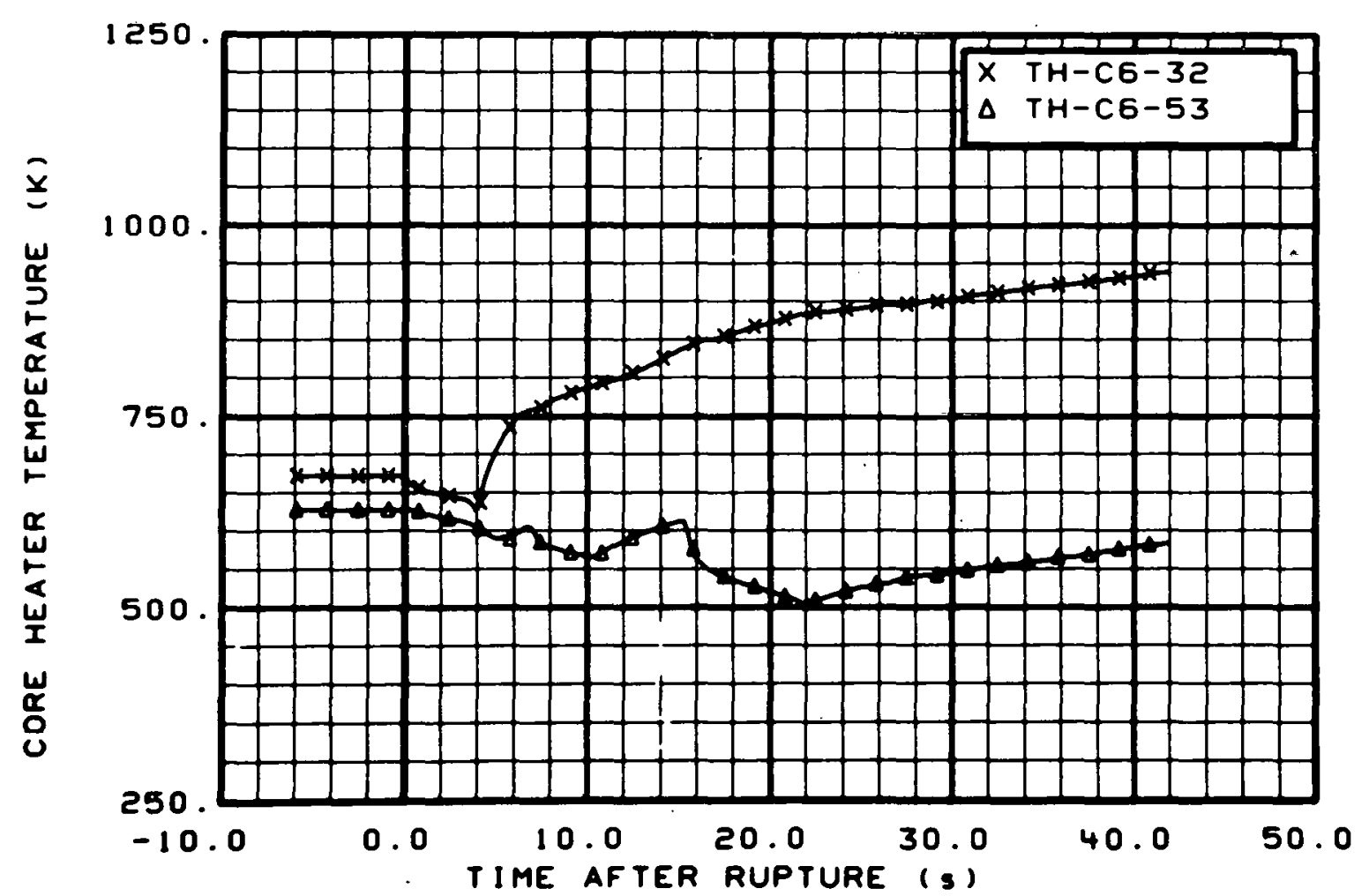

Fig. 84 Core heater temperature, Rod C-6 (TH-C6-32. and TH-C6-53), from -6 to $42 \mathrm{~s}$. 


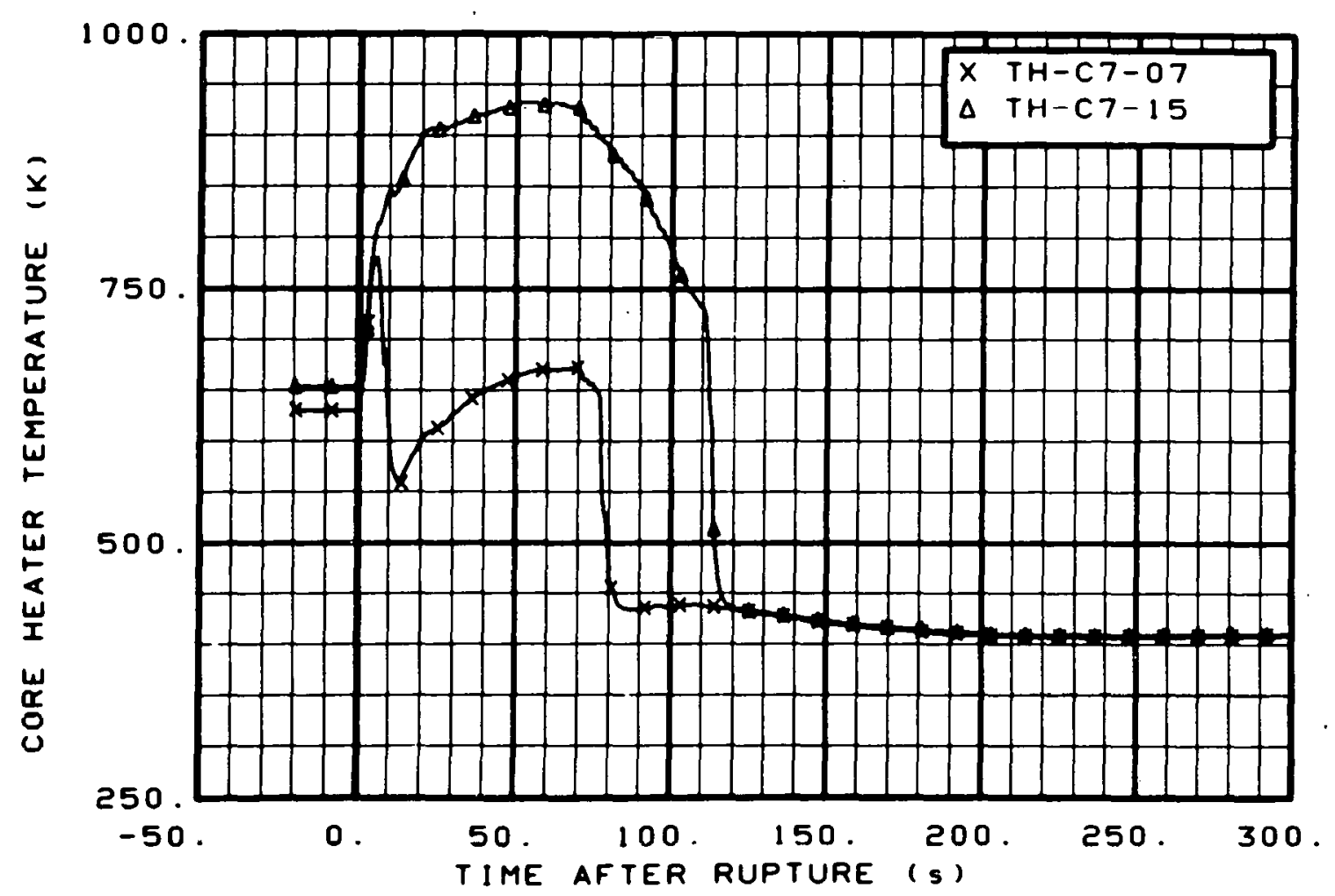

Fig. 85 Core heater temperature, Rod C-7 (TH-C7-07 and TH-C7-15), from -20 to $300 \mathrm{~s}$.

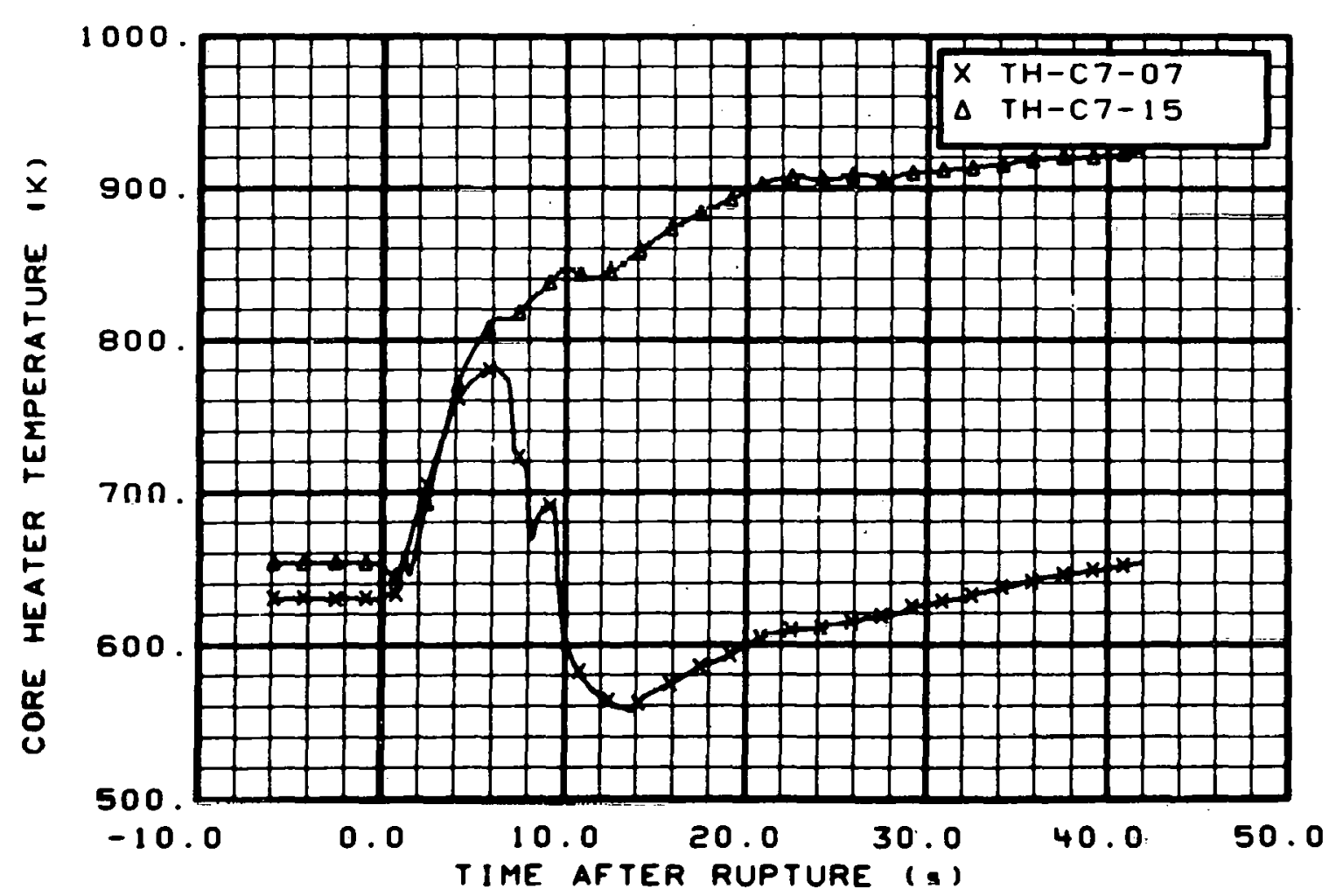

Fig. 86 Core heater temperature, Rod $\mathrm{C}-7$ (TH-C7-07 and $\mathrm{TH}-\mathrm{C} 7-15)$, from -6 to $42 \mathrm{~s}$. 


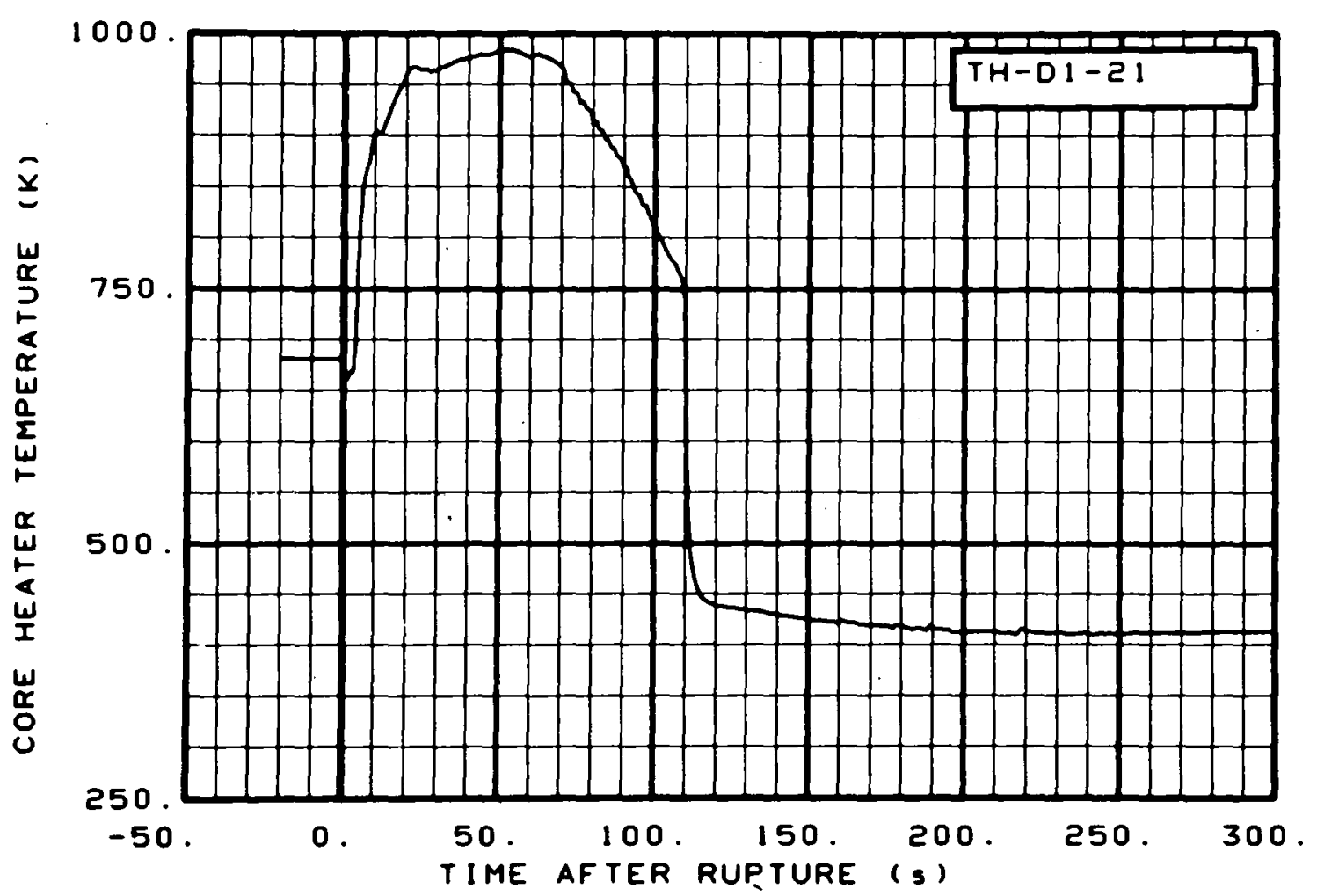

Fig. 87 Core heater temperature, Rod D-1 (TH-D1-21), from -20 to $300 \mathrm{~s}$.

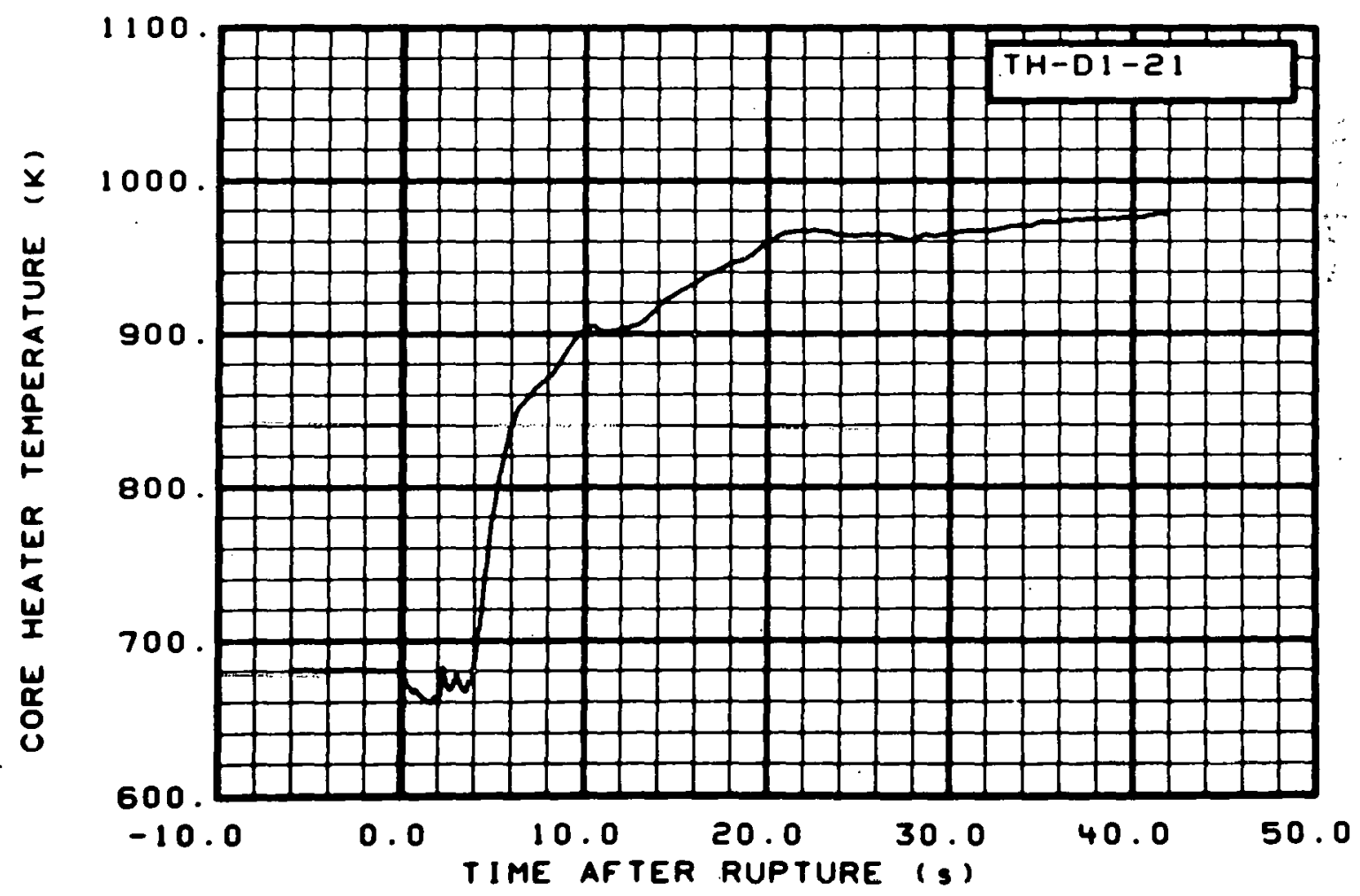

Fig. 88 Core heater temperature, Rod D-1 (TH-D1-21), from -6 to $42 \mathrm{~s}$. 


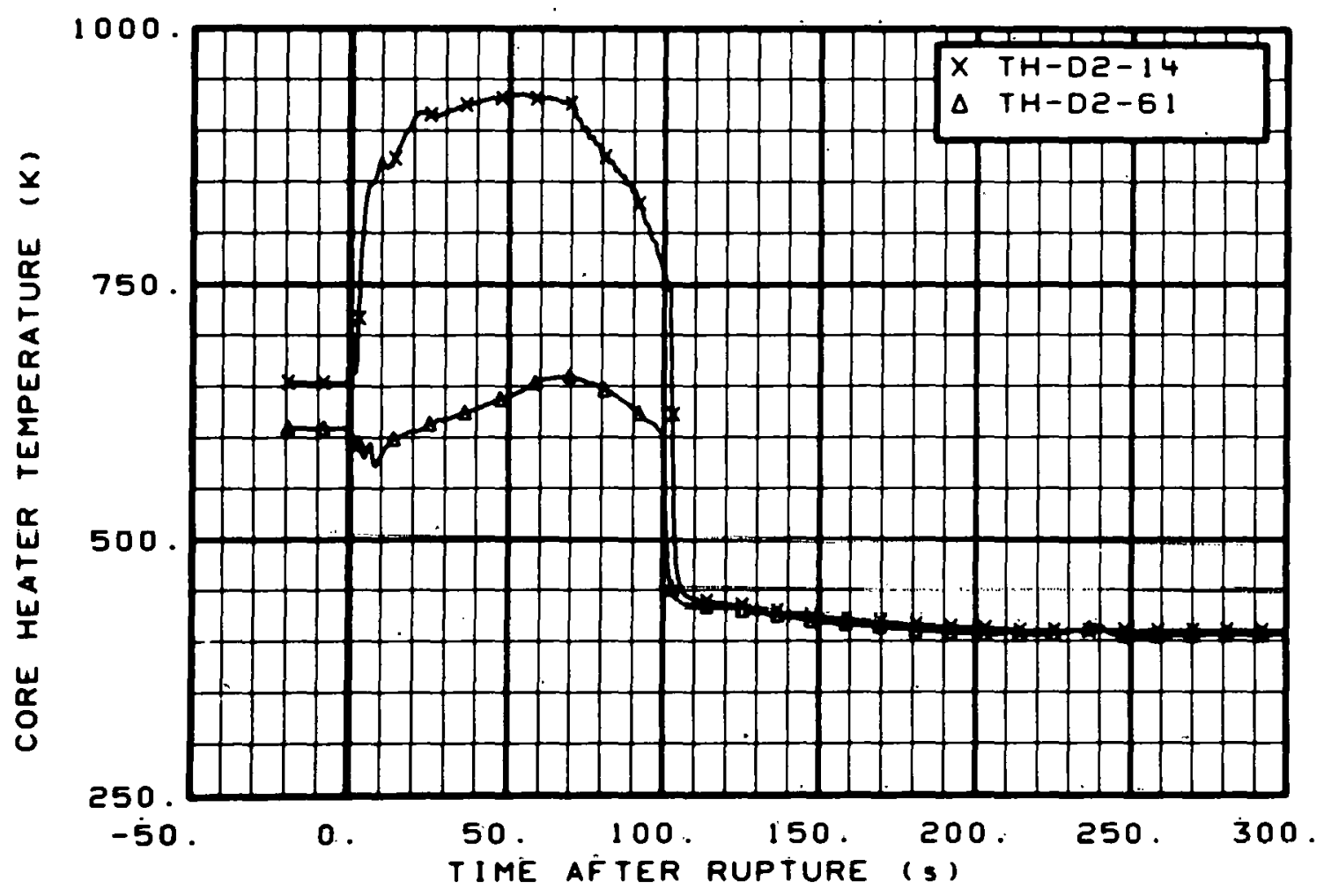

Fig. 89 Core heater temperature, Rod D-2 (TH-D2-14 and TH-D2-61), from -20 to $300 \mathrm{~s}$.

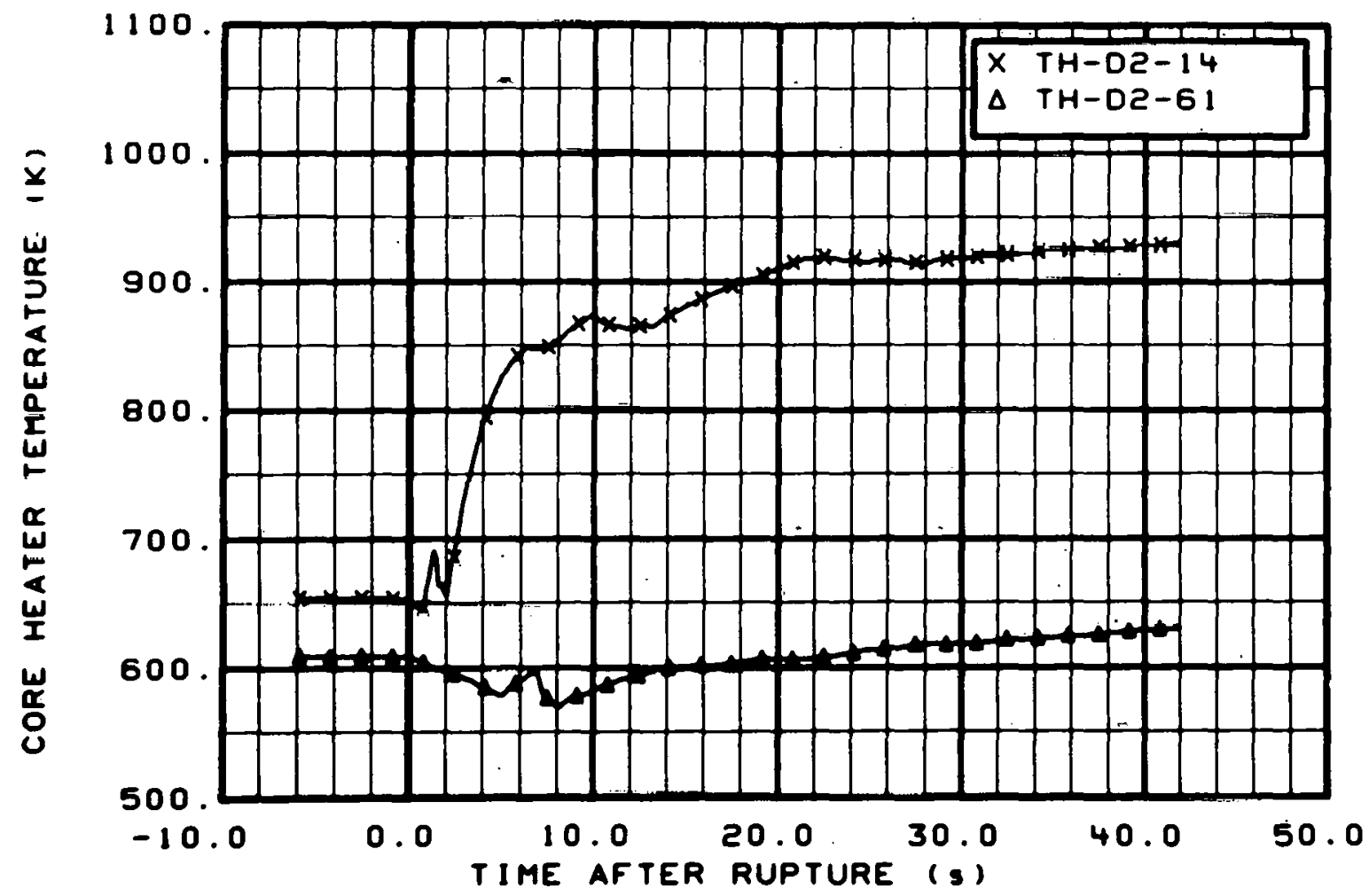

Fig. 90 Core heater temperature, Rod D-2 (TH-D2-14 and TH-D2-61), from -6 to $42 \mathrm{~s}$. 


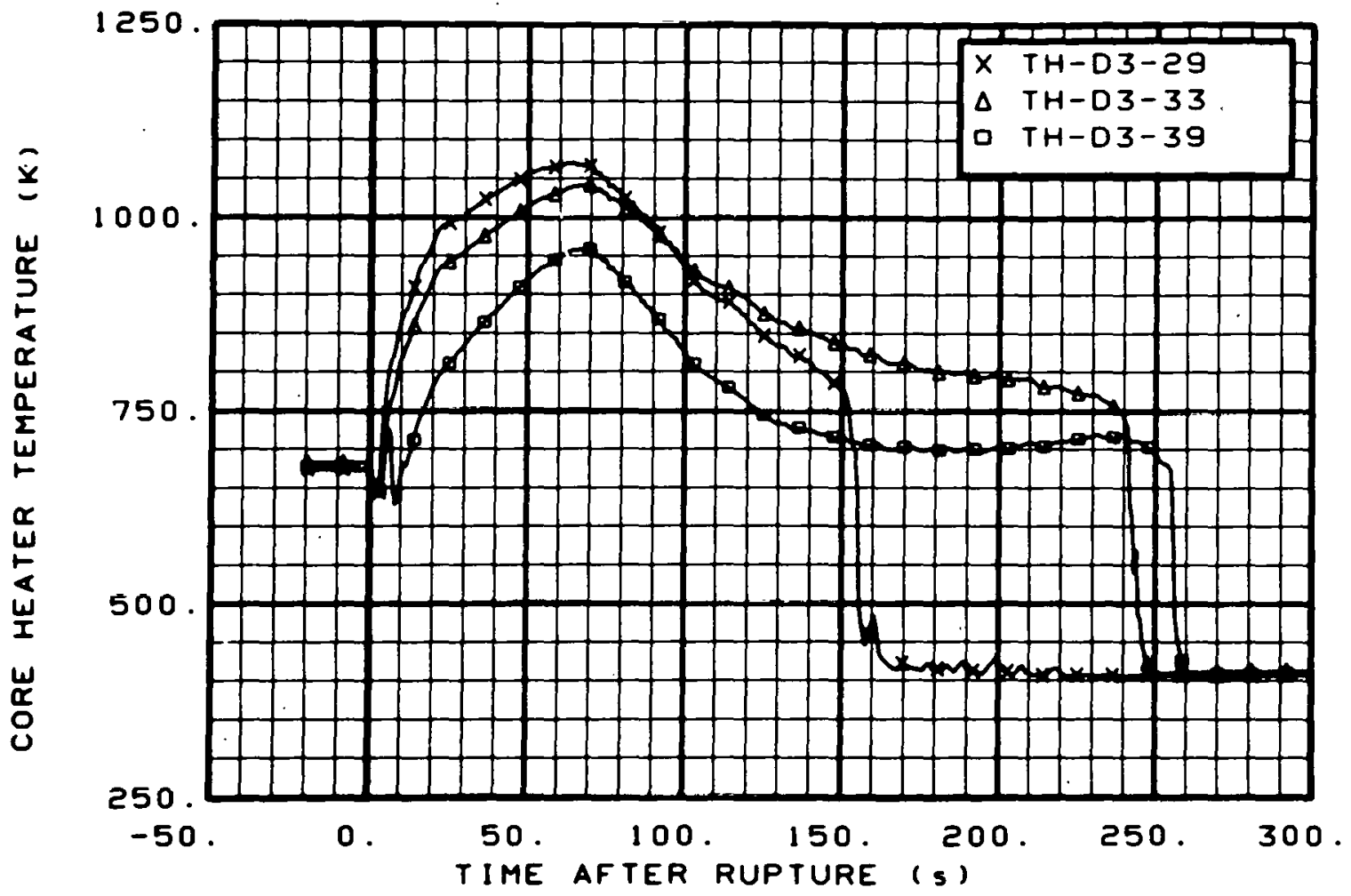

Fig. 91 Core heater temperature, Rod D-3 (TH-D3-29, TH-D3-33, and TH-D3-39.), from -20 to $300 \mathrm{~s}$.

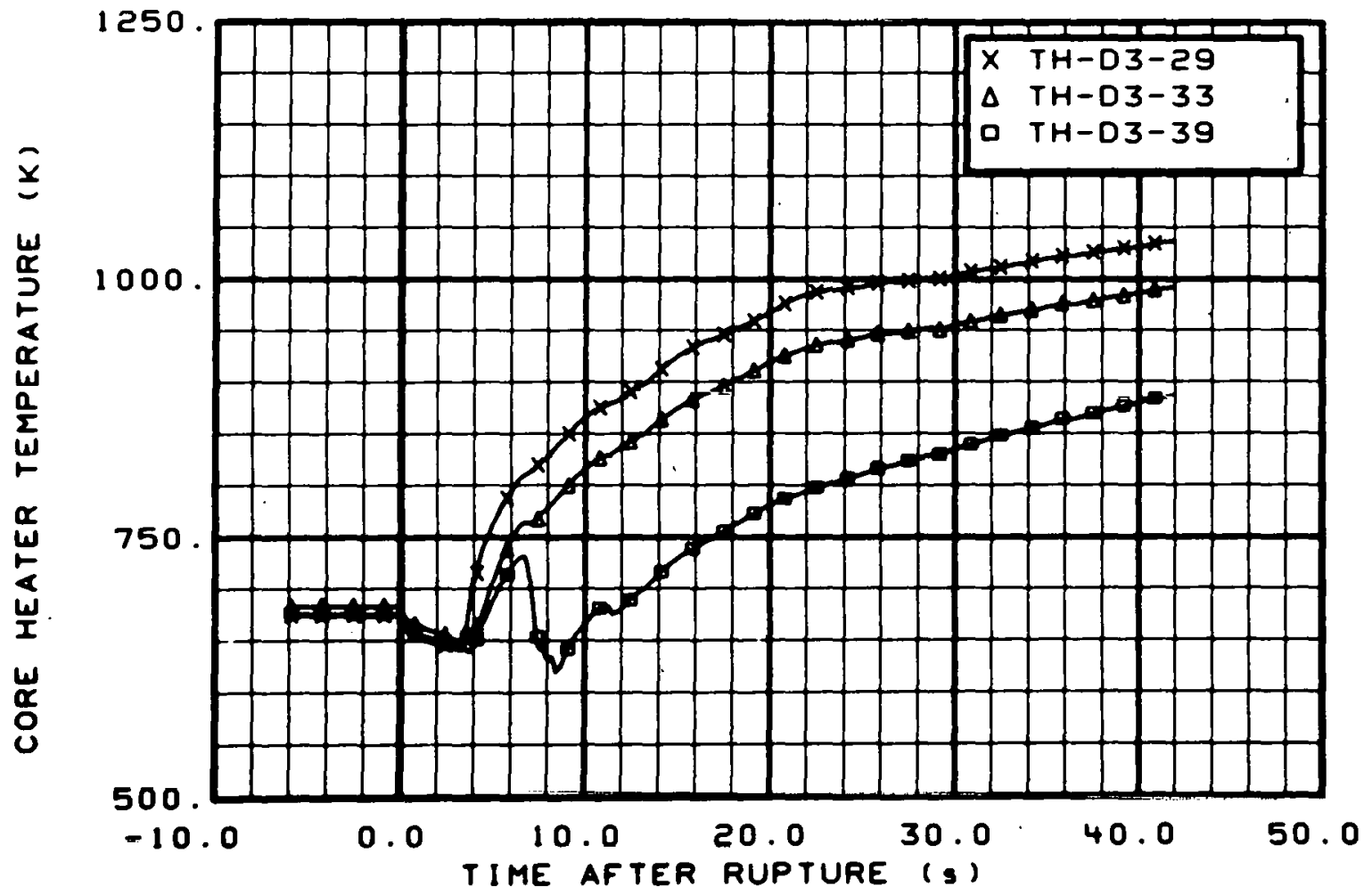

Fig. 92 Core heater temperature, Rod D-3 (TH-D3-29, TH-D3-33, and TH-D3-39), from -6 to $42 \mathrm{~s}$. 


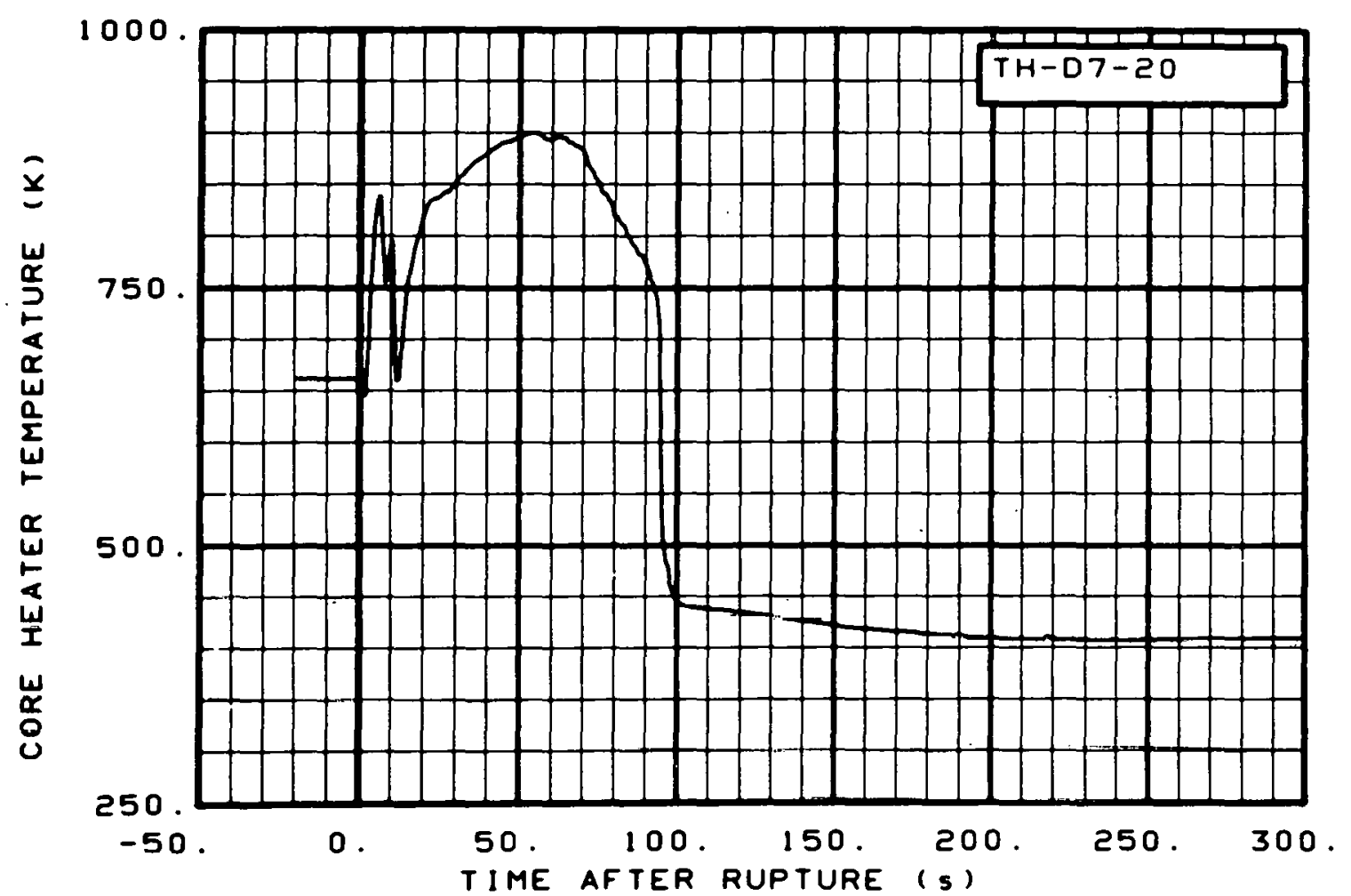

Fig. 93 Core heater temperature, Rod D-7 (TH-D7-20), from -20 to $300 \mathrm{~s}$.

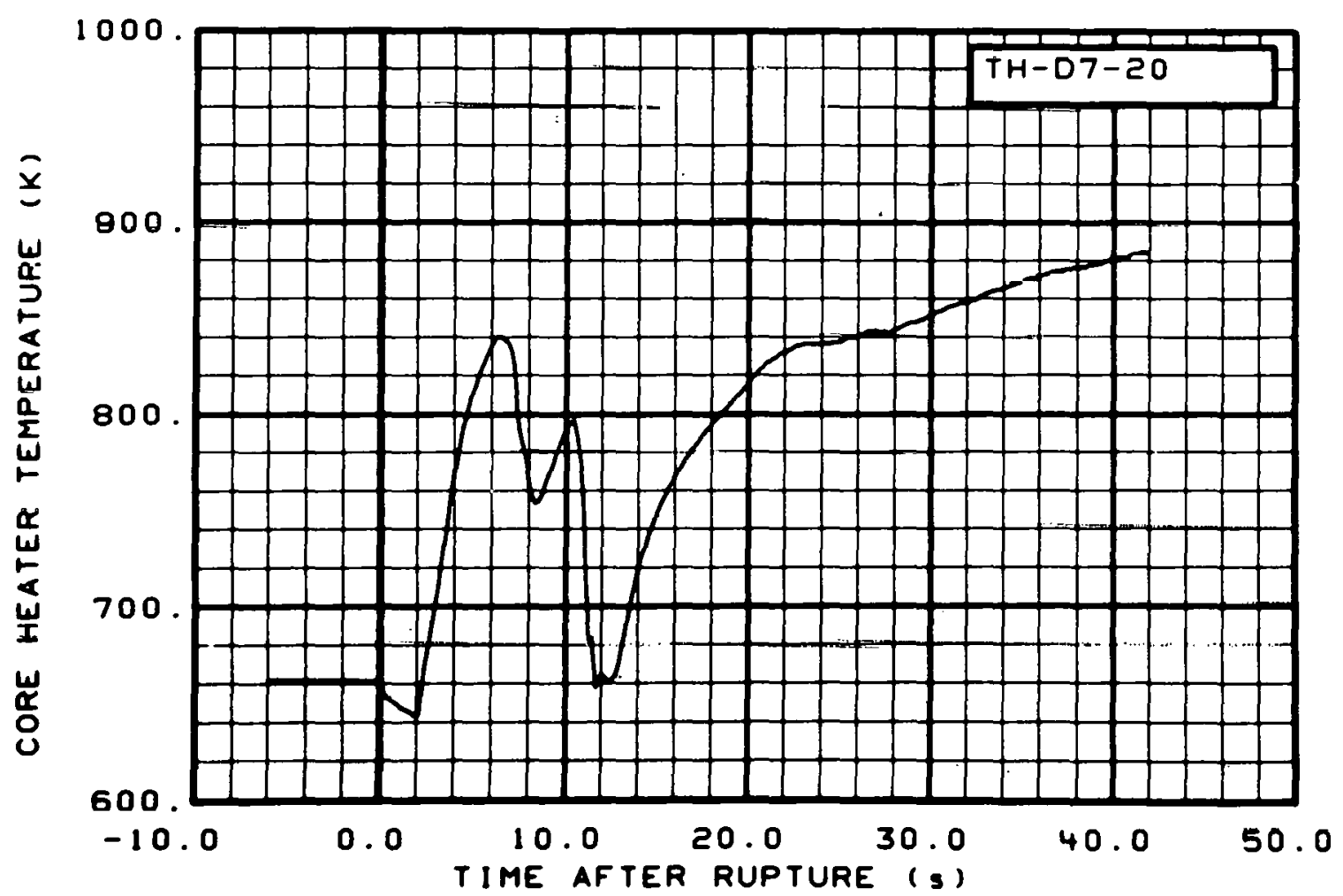

Fig. 94 Core heater temperature, Rod D-7 (TH-D7-20), from -6 to $42 \mathrm{~s}$. 


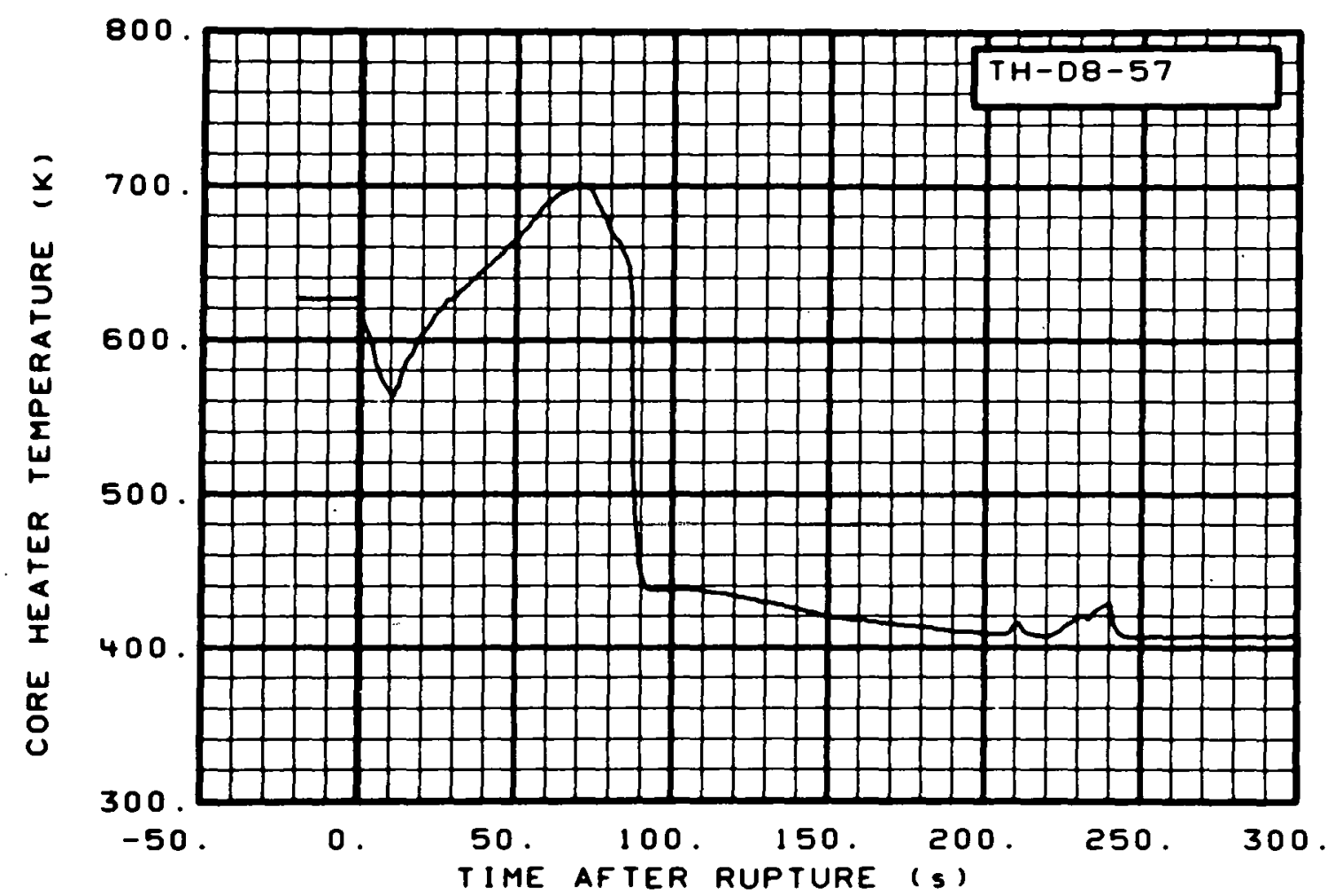

Fig. 95 Core heater temperature, Rod D-8 (TH-D8-57), from -20 to $300 \mathrm{~s}$.

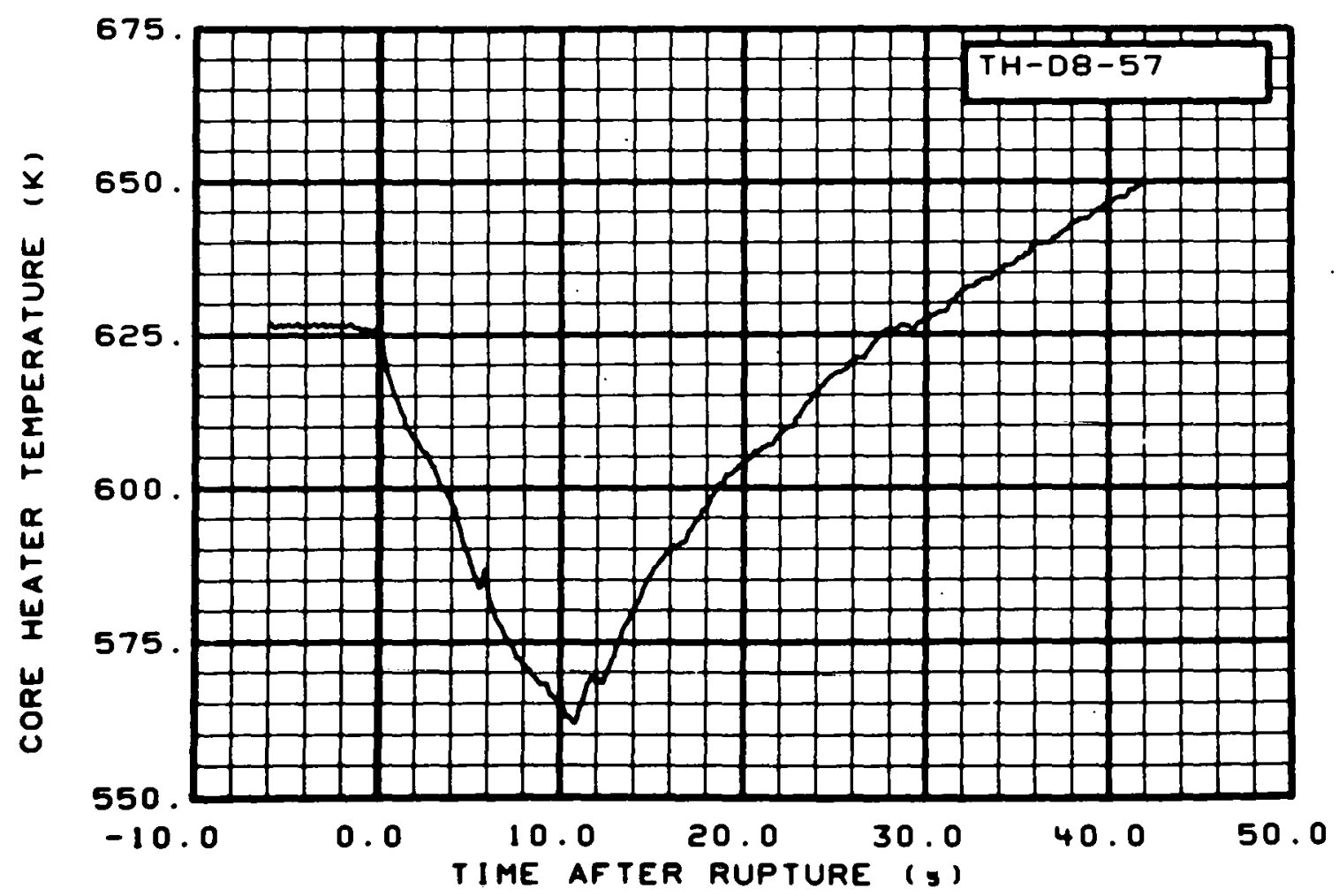

Fig. 96 Core heater temperature, Rod D-8 (TH-D8-57), from -6 to $42 \mathrm{~s}$. 


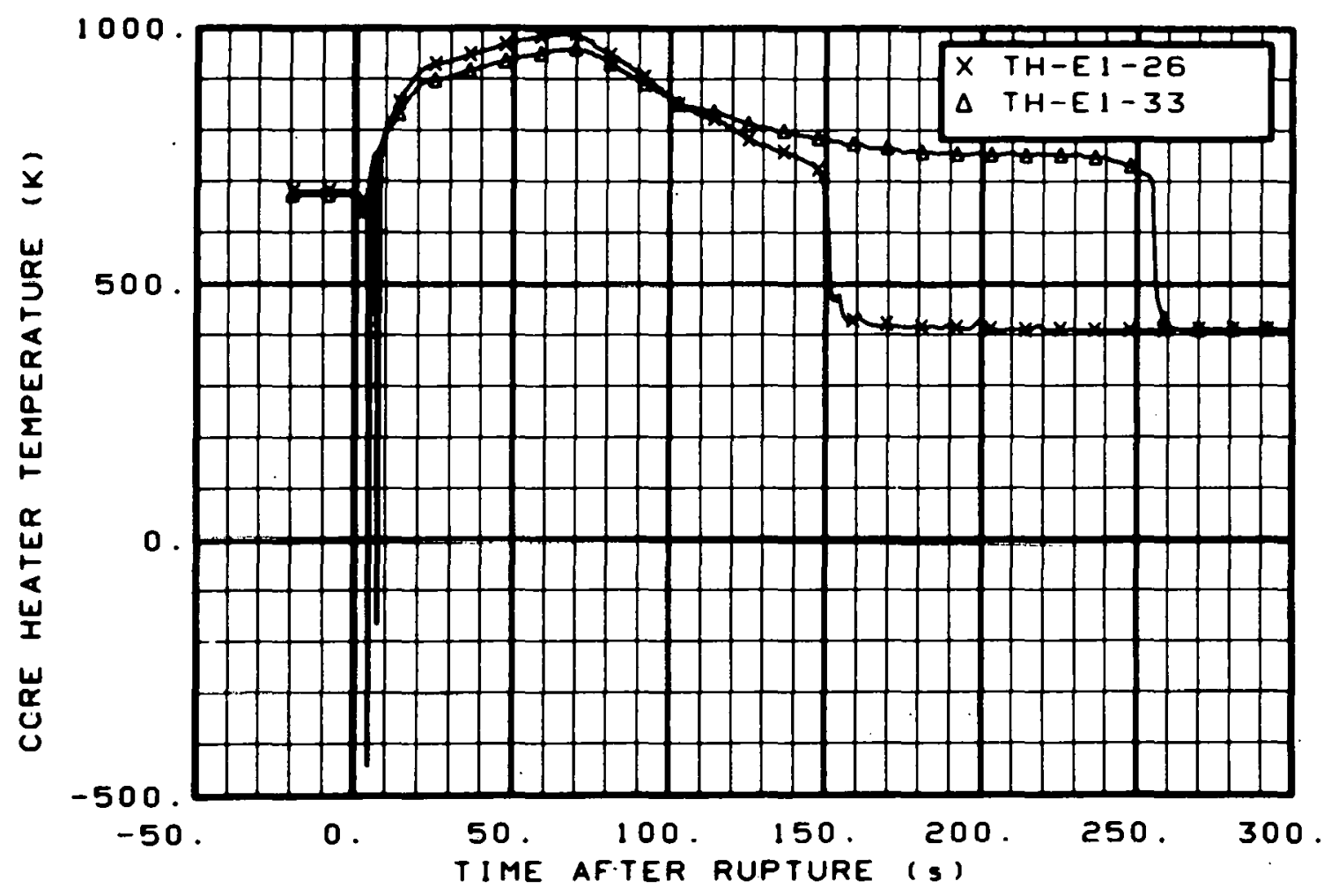

Fig. 97 Core heater temperature, Rod E-1 (TH-El-26 and TH-E]-33), from -20 to $300 \mathrm{~s}$.

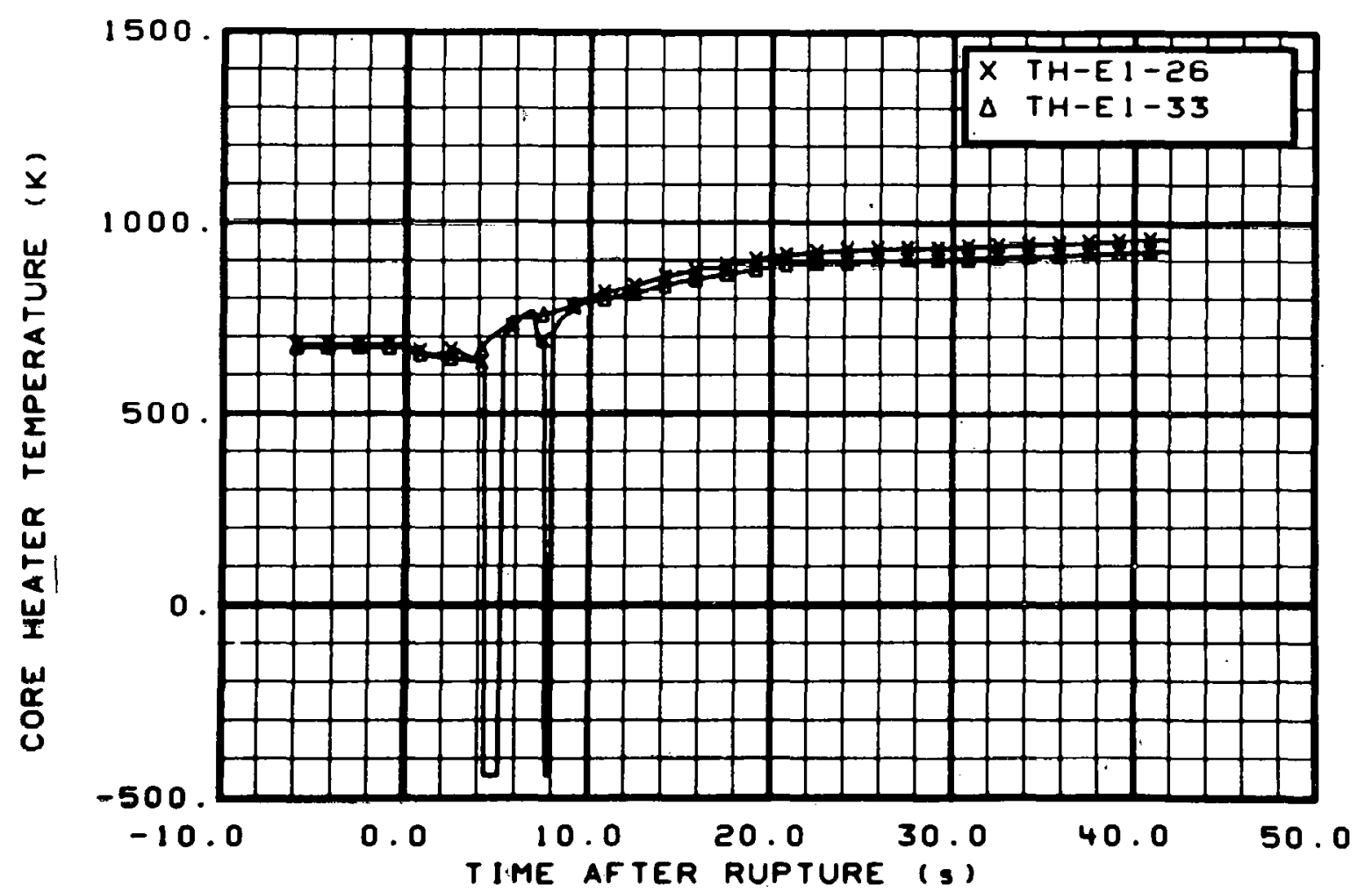

Fig. 98 Core heater temperature, Rod E-1 (TH-ET-26 and TH-ET-33), from -6 to $42 \mathrm{~s}$. 


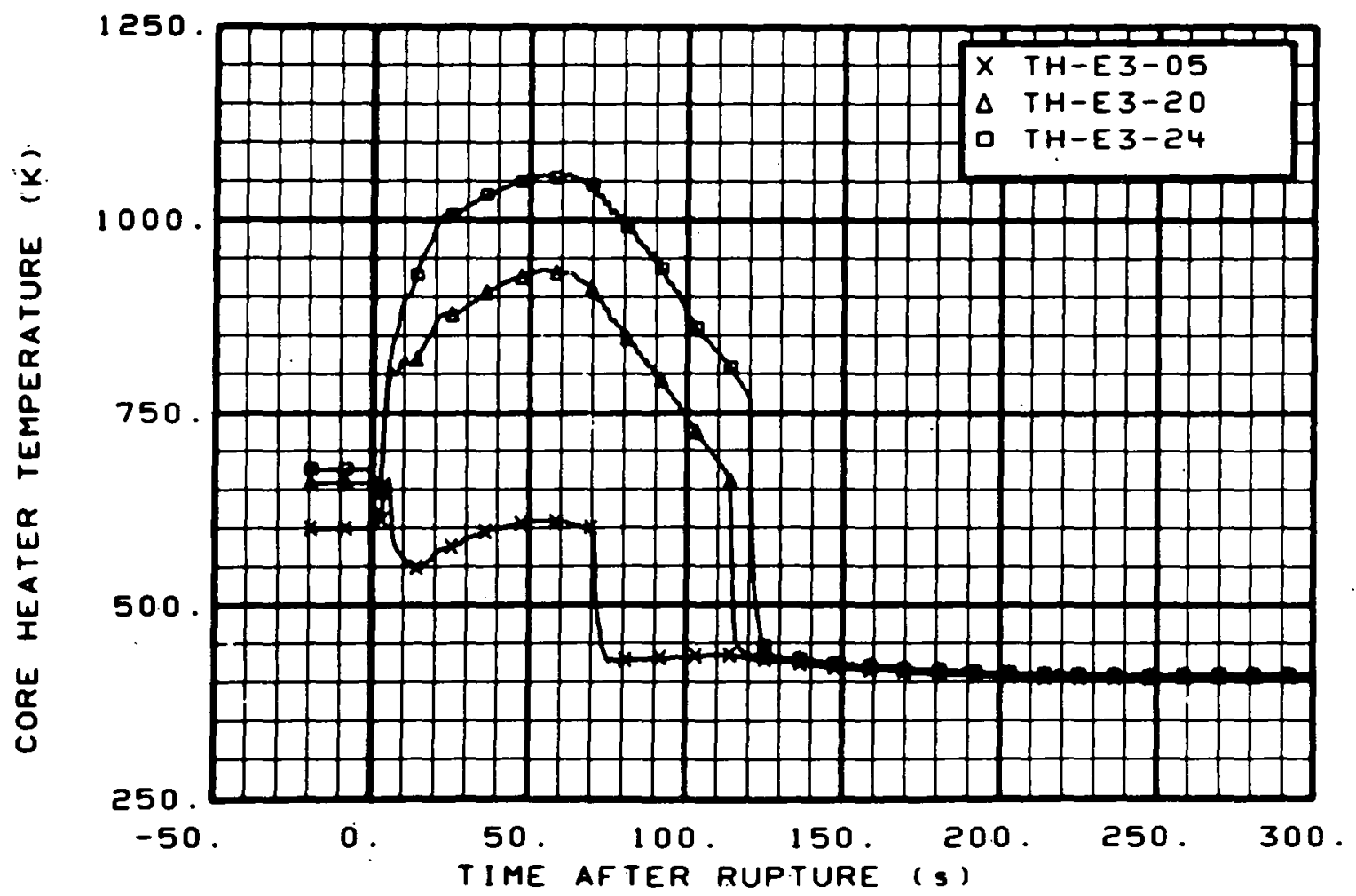

Fig. 99 Core heater temperature, Rod E-3 (TH-E3-05, TH-E3-20, and. TH-E3-24), from -20 to $300 \mathrm{~s}$.

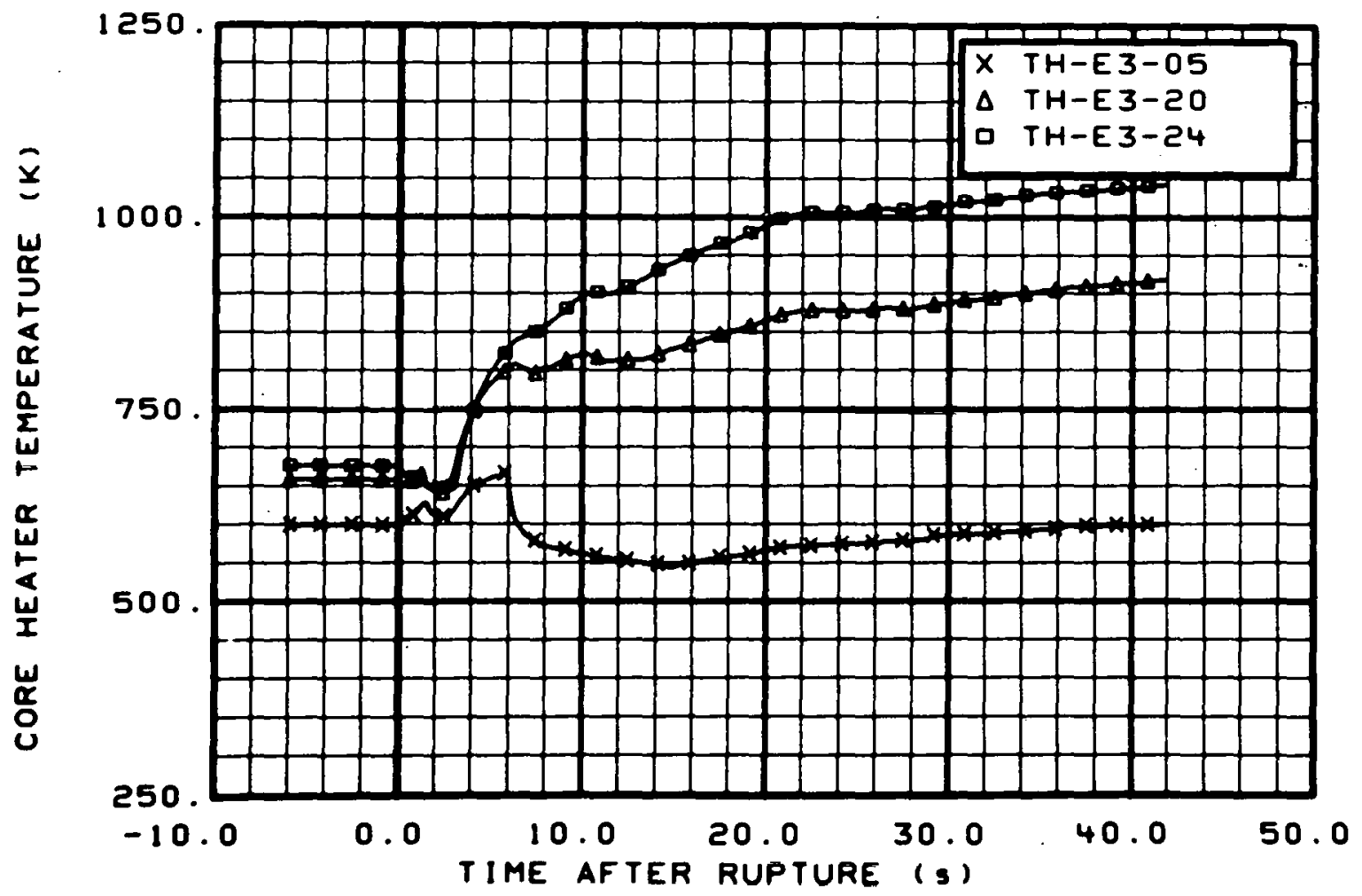

Fig. 100 Core heater temperature, Rod E-3 (TH-E3-05, TH-E3-20, and TH-E3-24), from -6 to $42 \mathrm{~s}$. 


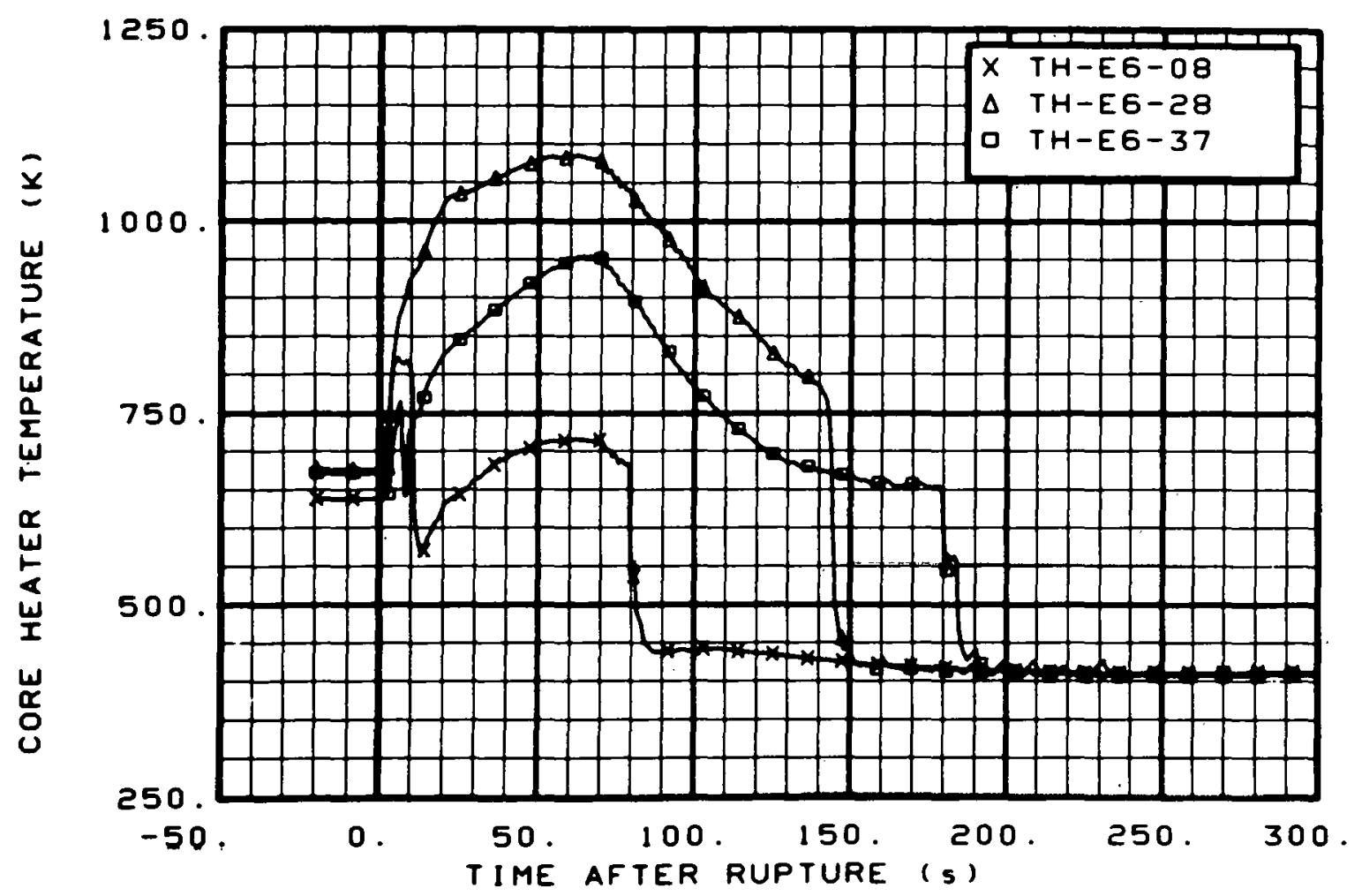

Fig. 101 Core heater temperature, Rod E-6 (TH-E6-08, TH-E6-28, and TH-E6-37), from -20 to $300 \mathrm{~s}$.

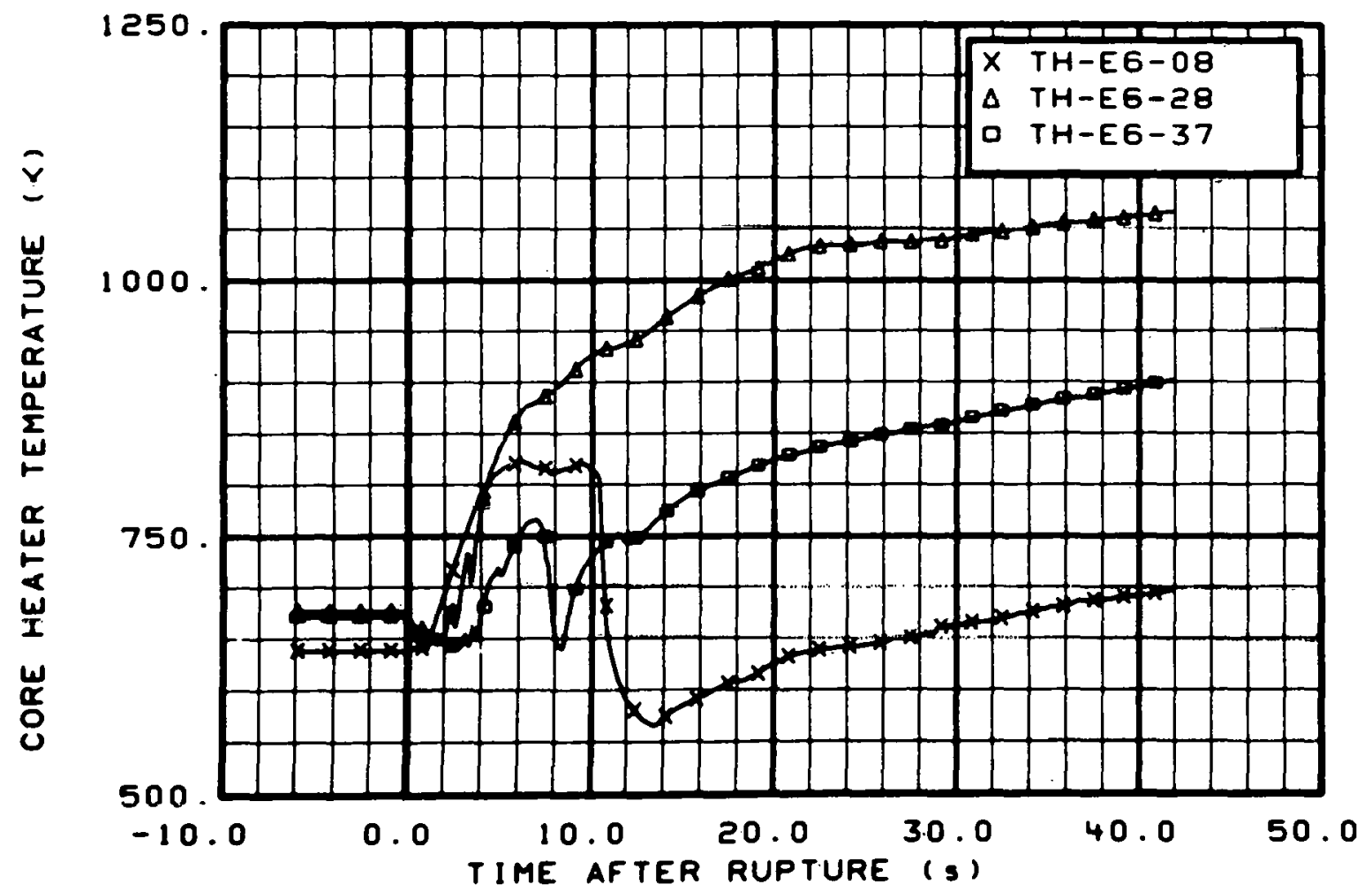

Fig. 102 Core heater temperature, Rod E-6 (TH-E6-08, TH-E6-28, and TH-E6-37), from -6 to $42 \mathrm{~s}$. 


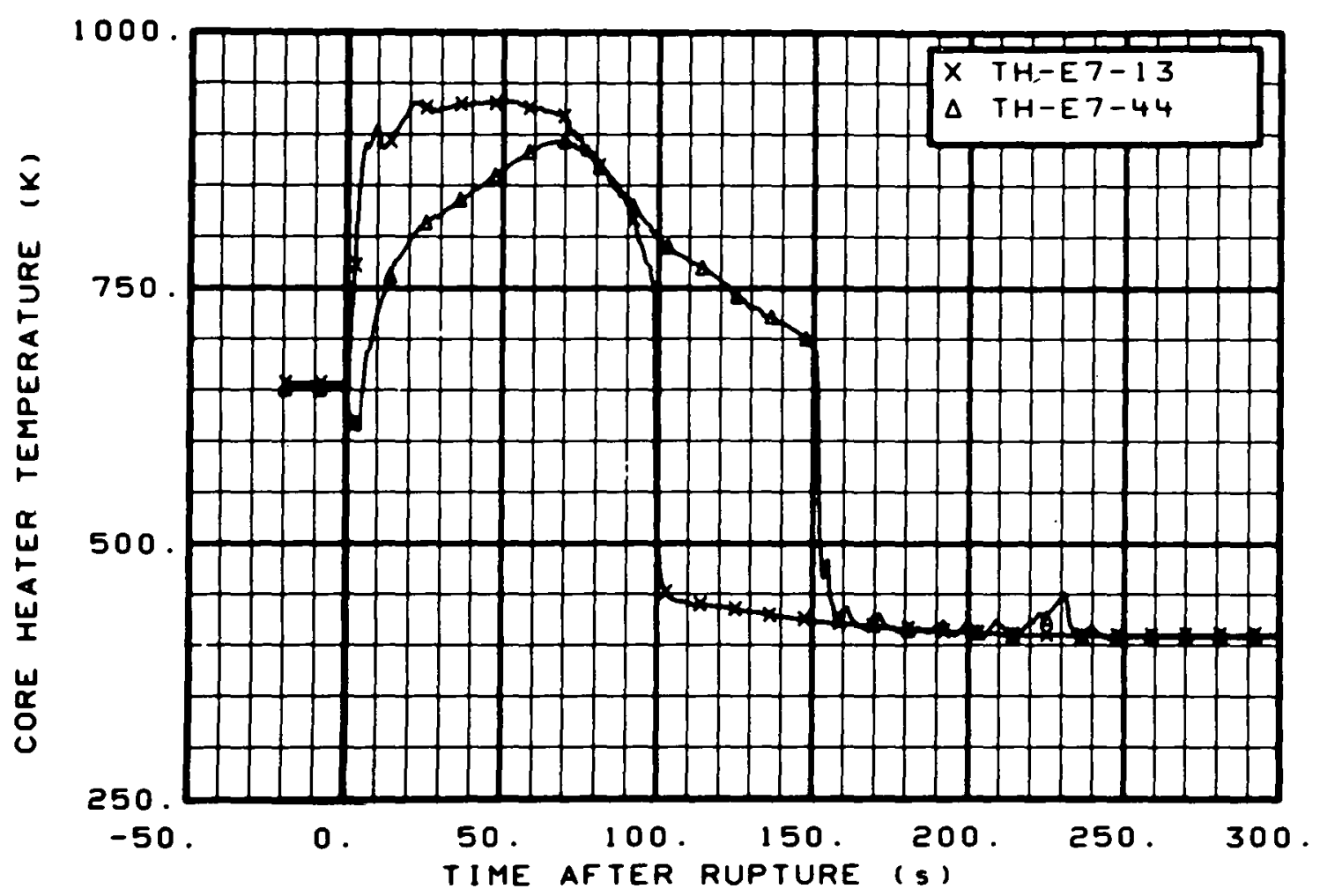

Fig. 103 Core heater temperature, Rod E-7 (TH-E7-13 and TH-E7-44), from -20 to $300 \mathrm{~s}$.

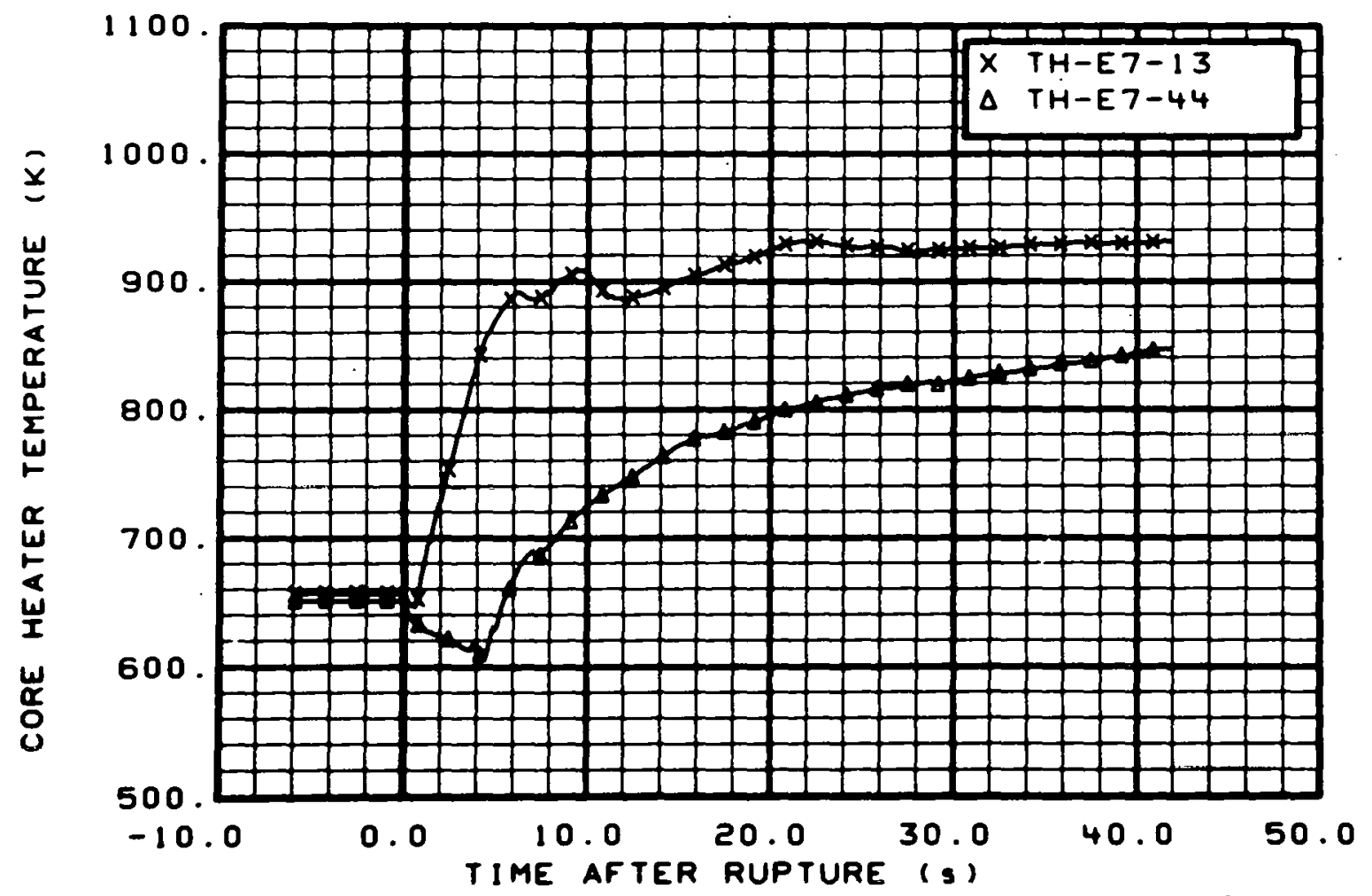

Fig. 104 Core heater temperature, Rod E-7 (TH-E7-13 and TH-E7-44), from -6 to $42 \mathrm{~s}$. 


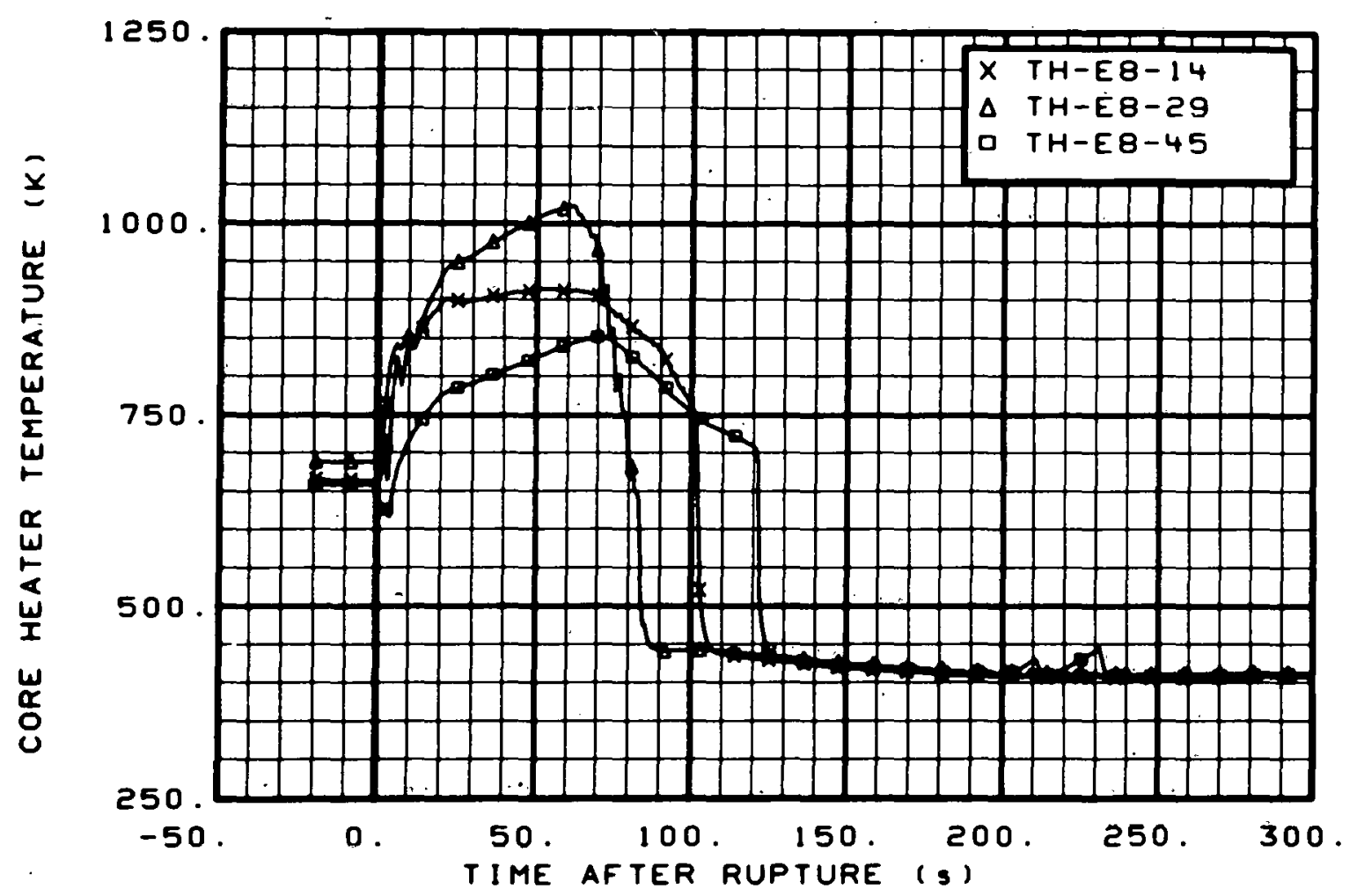

Fig. 105 Core heater temperature, Rod E-8 (TH-E8-14, TH-E8-29, and TH-E8-45), from -20 to $300 \mathrm{~s}$.

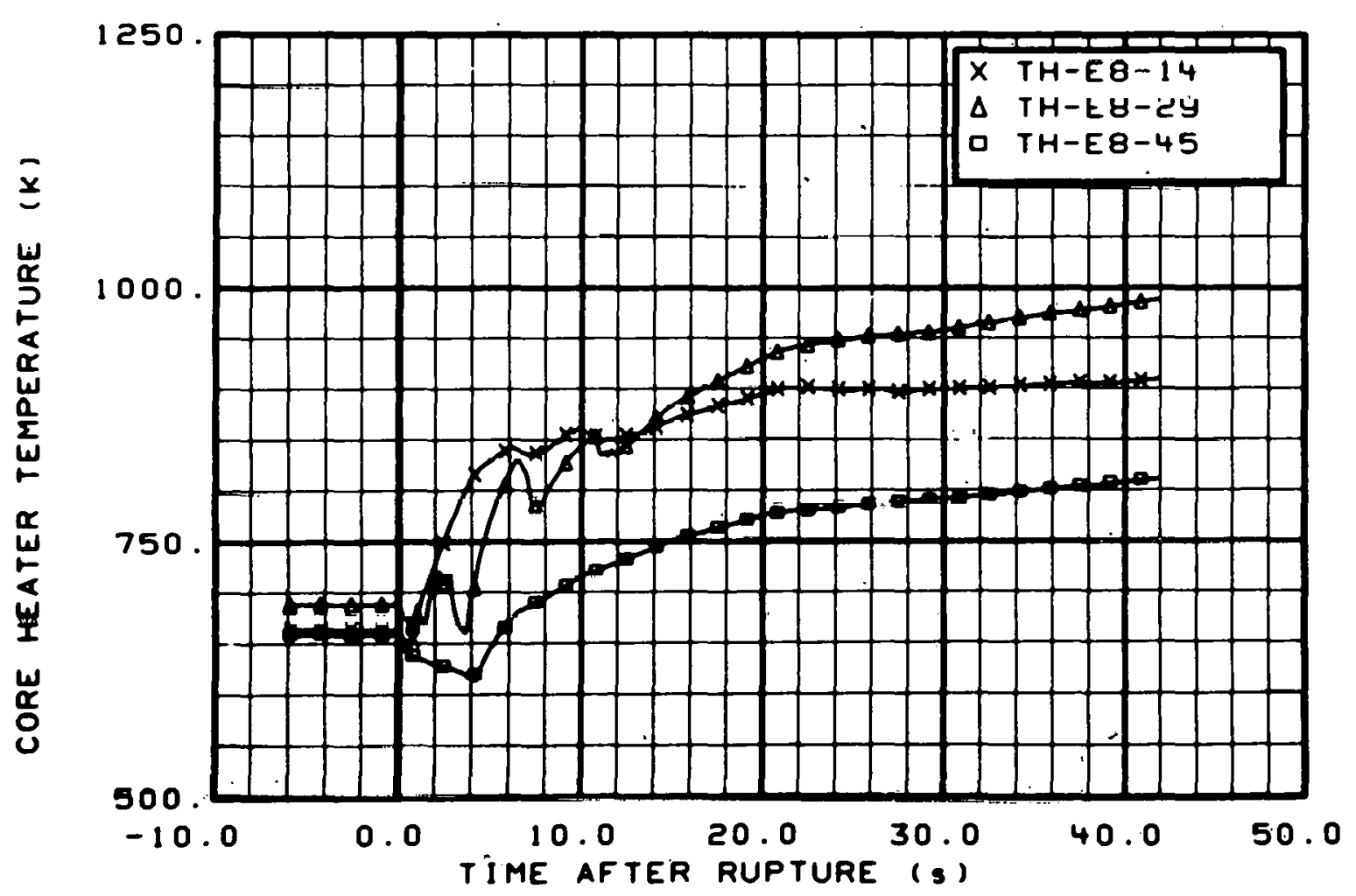

Fig. 106 Core heater temperature, Rod E-8 (TH-E8-14, TH-E8-29, and TH-E8-45), from -6 to $42 \mathrm{~s}$. 


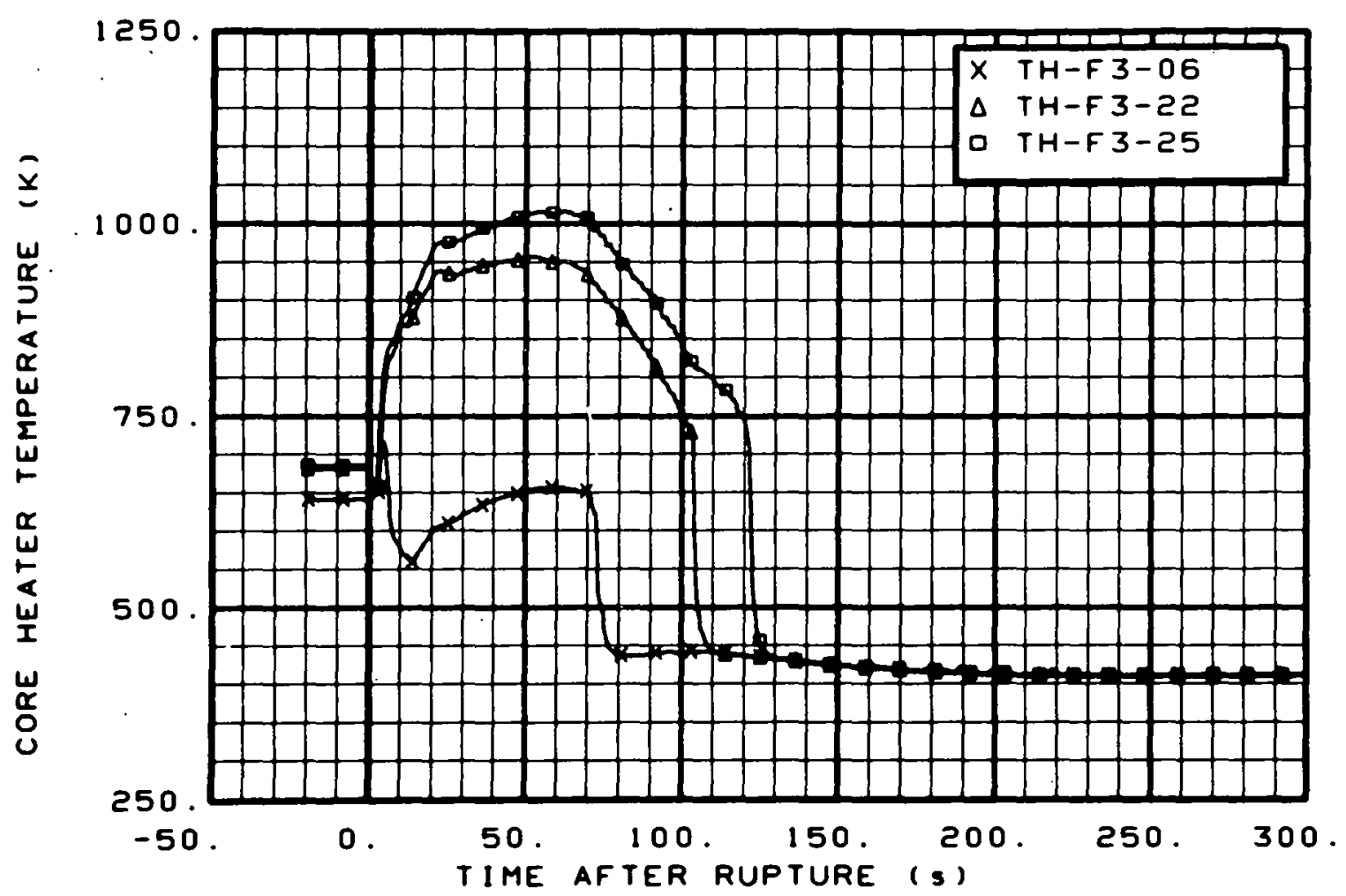

Fig. 107 Core heater temperature, Rod F-3 (TH-F3-06, TH-F3-22, and TH-F3-25), from -20 to $300 \mathrm{~s}$.

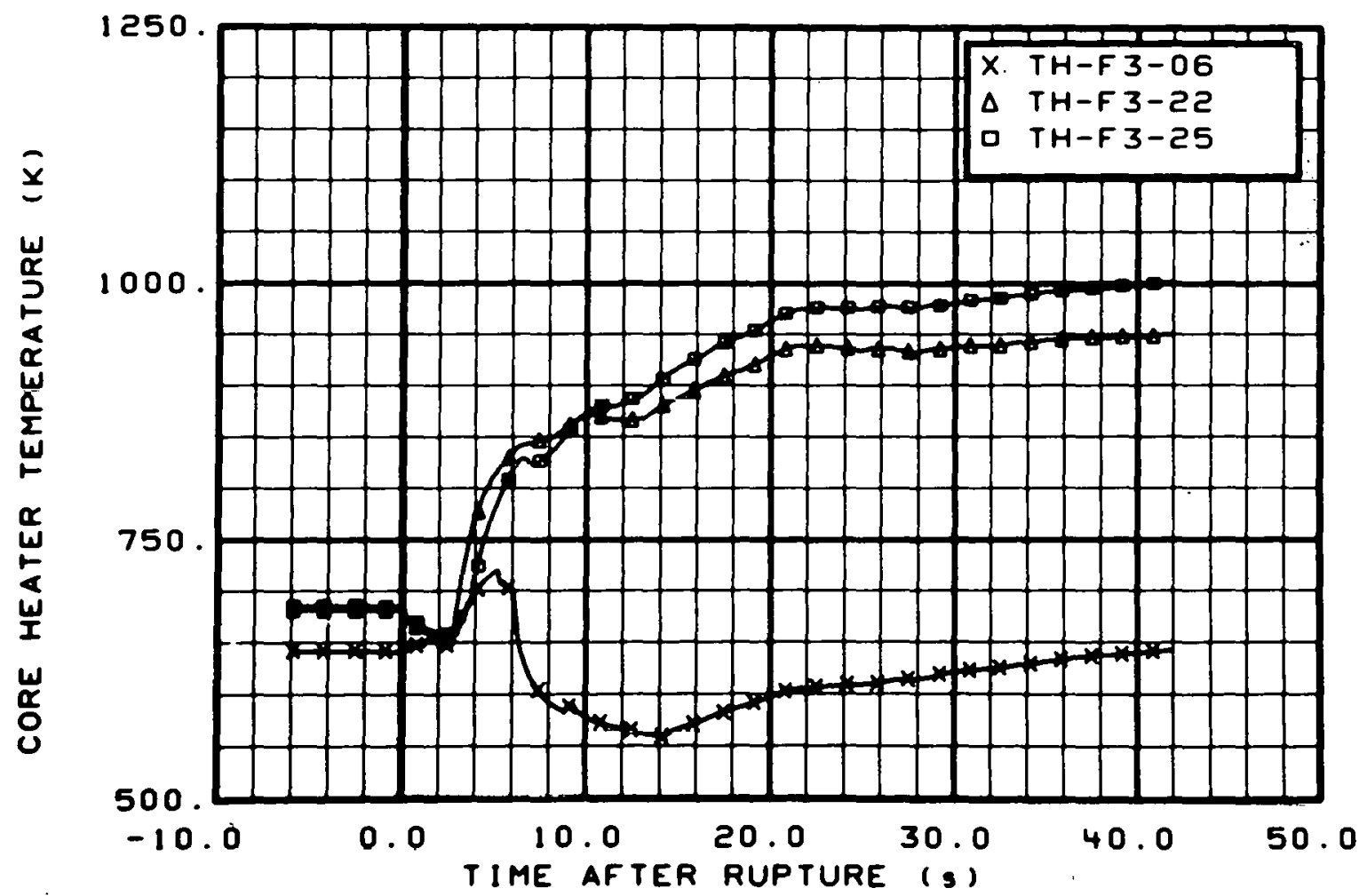

Fig. 108 Core heater temperature, Rod F-3 (TH-F3-06, TH-F3-22, and T.H- $+3-25$ ), from -6 to $42 \mathrm{~s}$. 


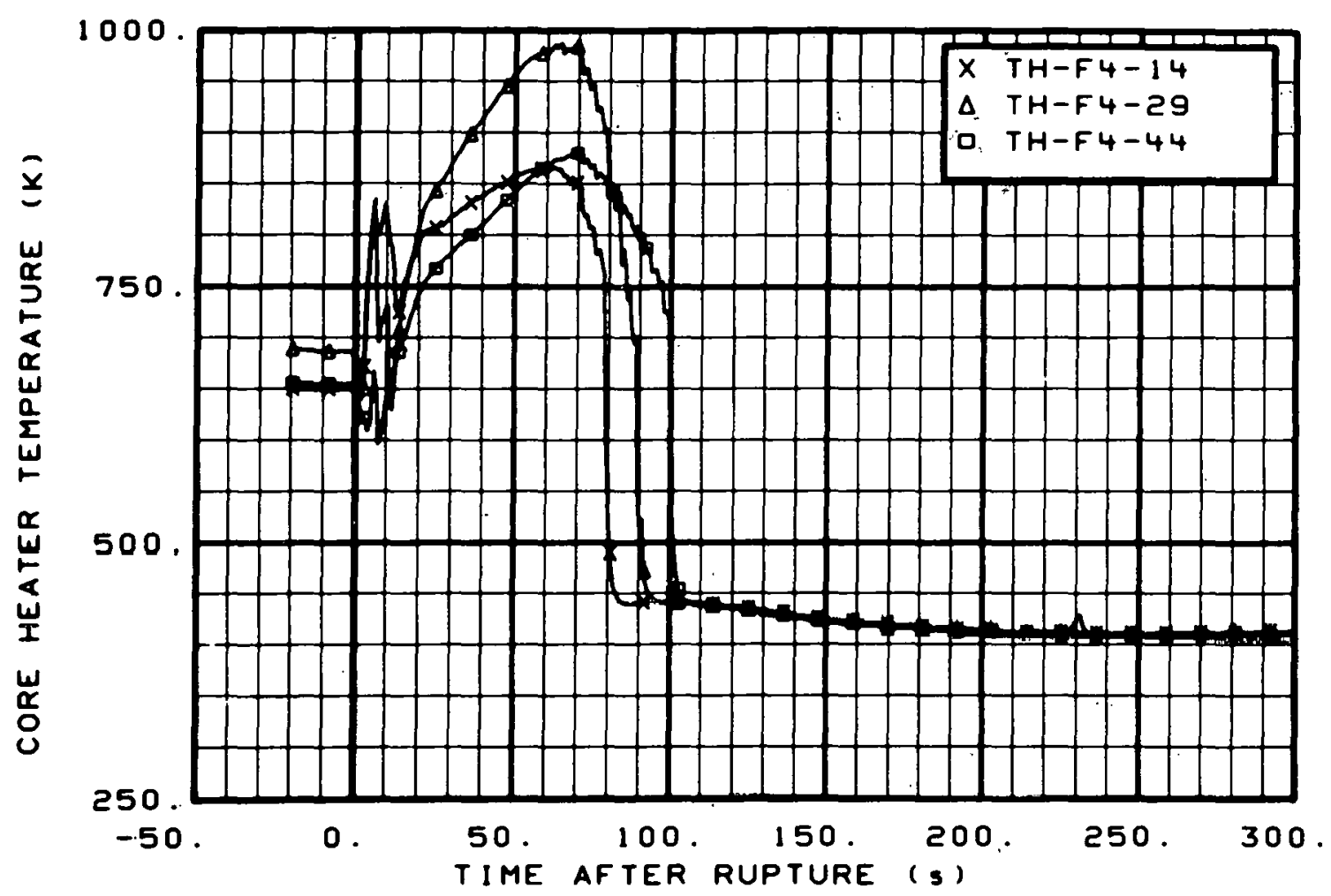

Fig. 109 Core heater temperature, Rod F-4 (TH-F4-14, TH-F4-29, and TH-F4-44), from -20 to $300 \mathrm{~s}$.

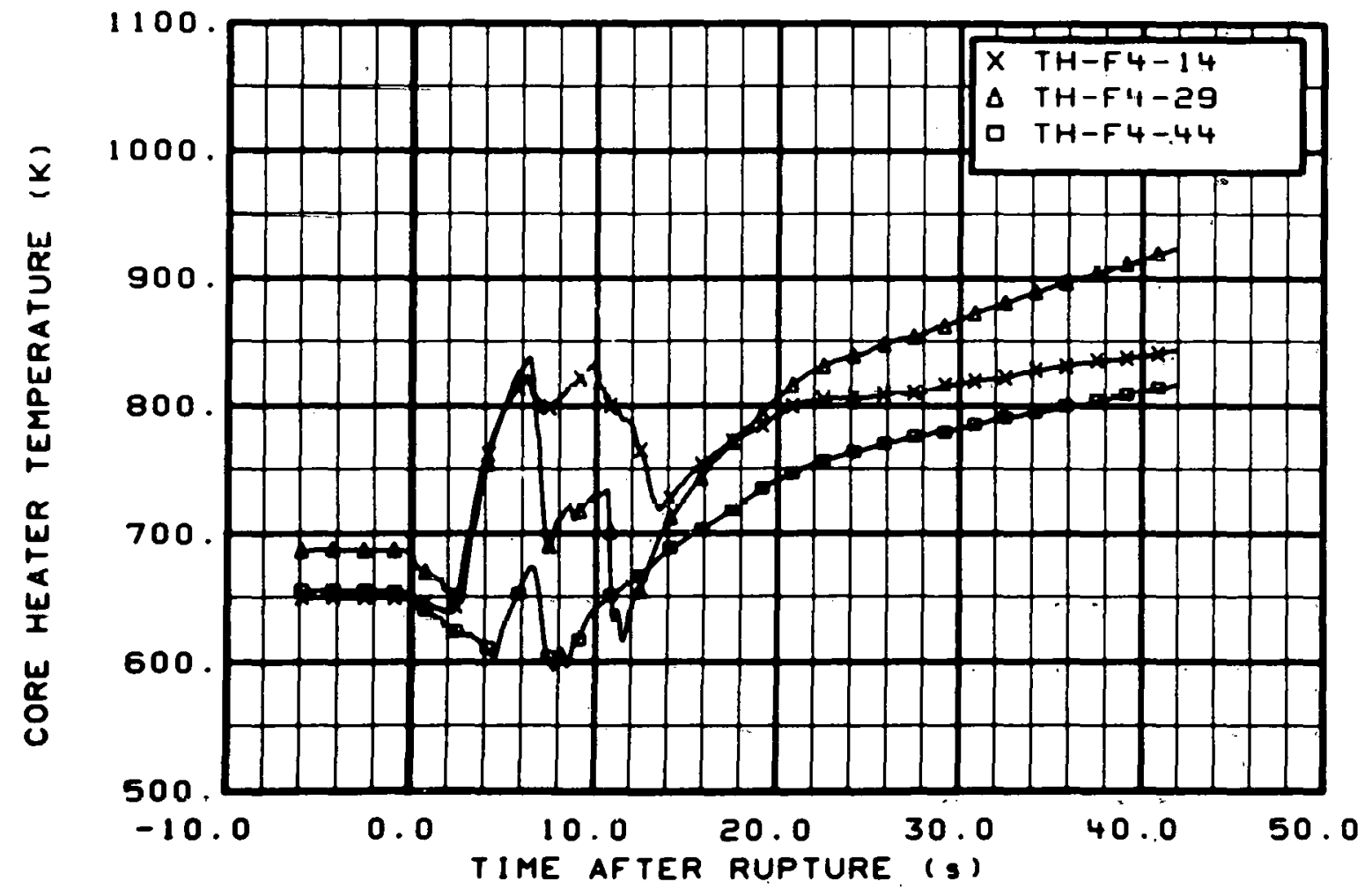

Fig. 110 Core heater temperature, Rod F-4 (TH-F4-14, TH-F4-29, and TH-F-44), from -6 to $42 \mathrm{~s}$. 


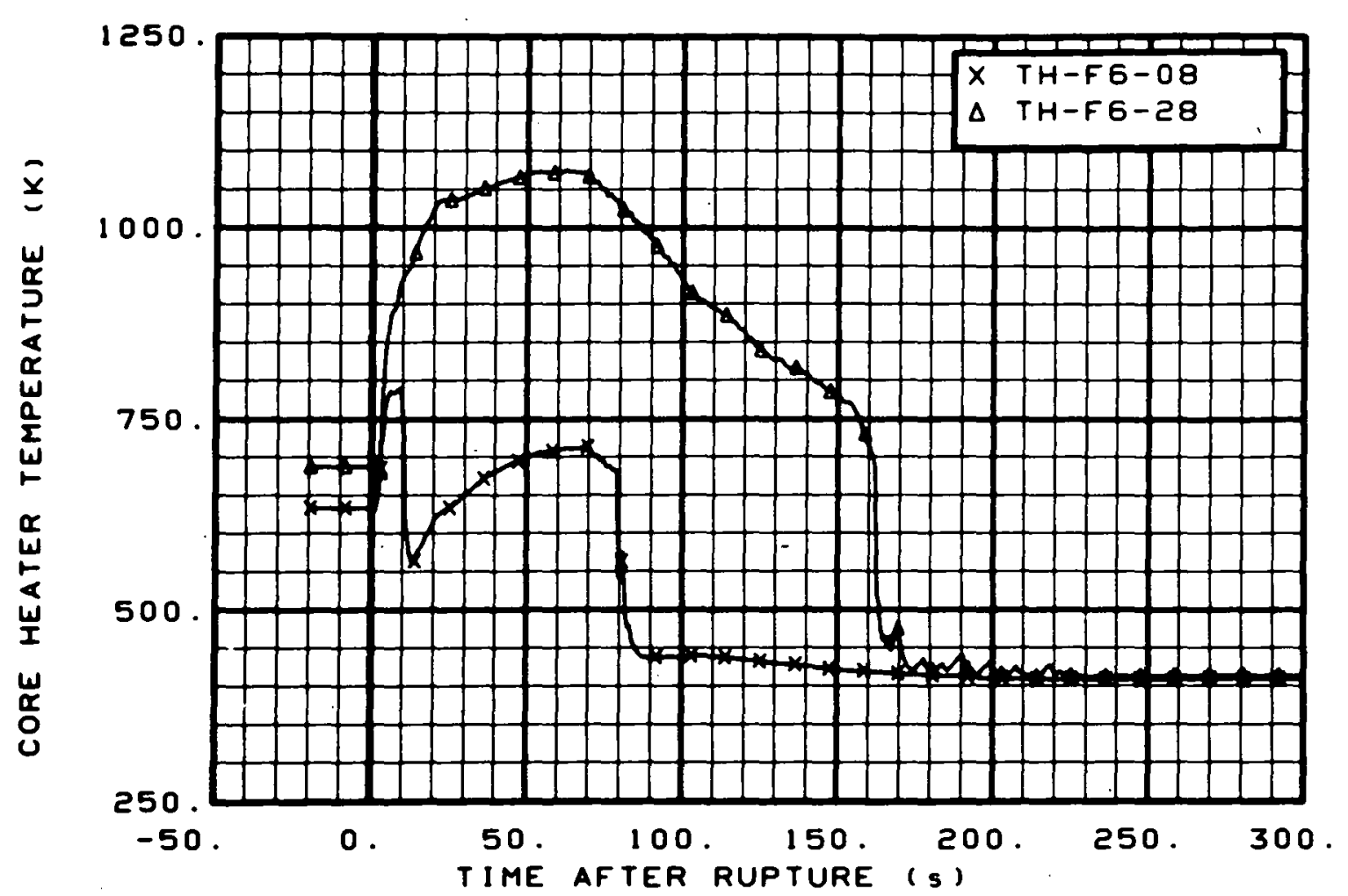

Fig. 111 Core heater temperature, Rod F-6 (TH-F6-08 and TH-F6-28), from -20 to $300 \mathrm{~s}$.

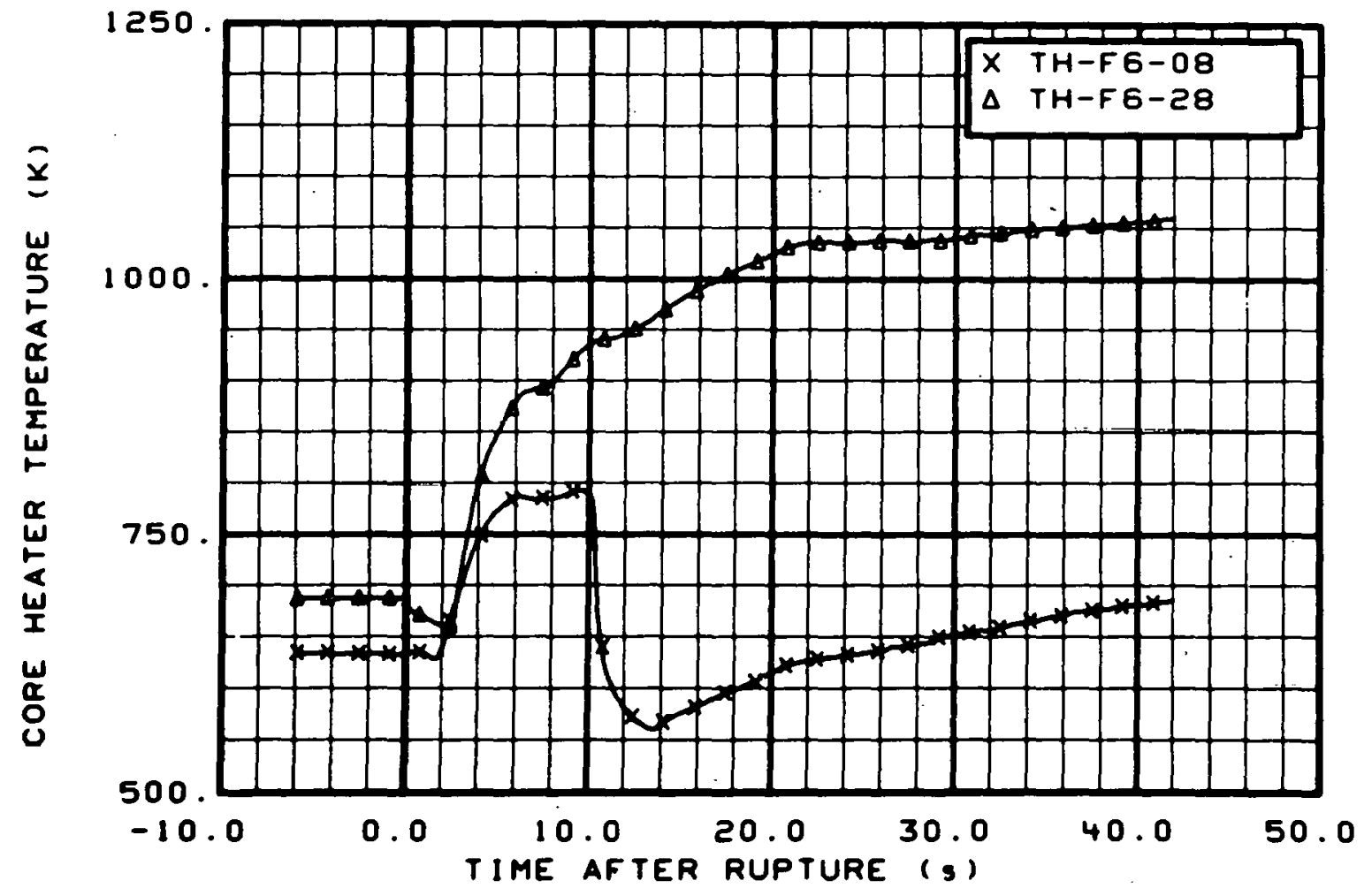

Fig. 112 Core heater temperature, Rod F-6 (TH-F6-08 and TH-F6-28), from -6 to $42 \mathrm{~s}$. 


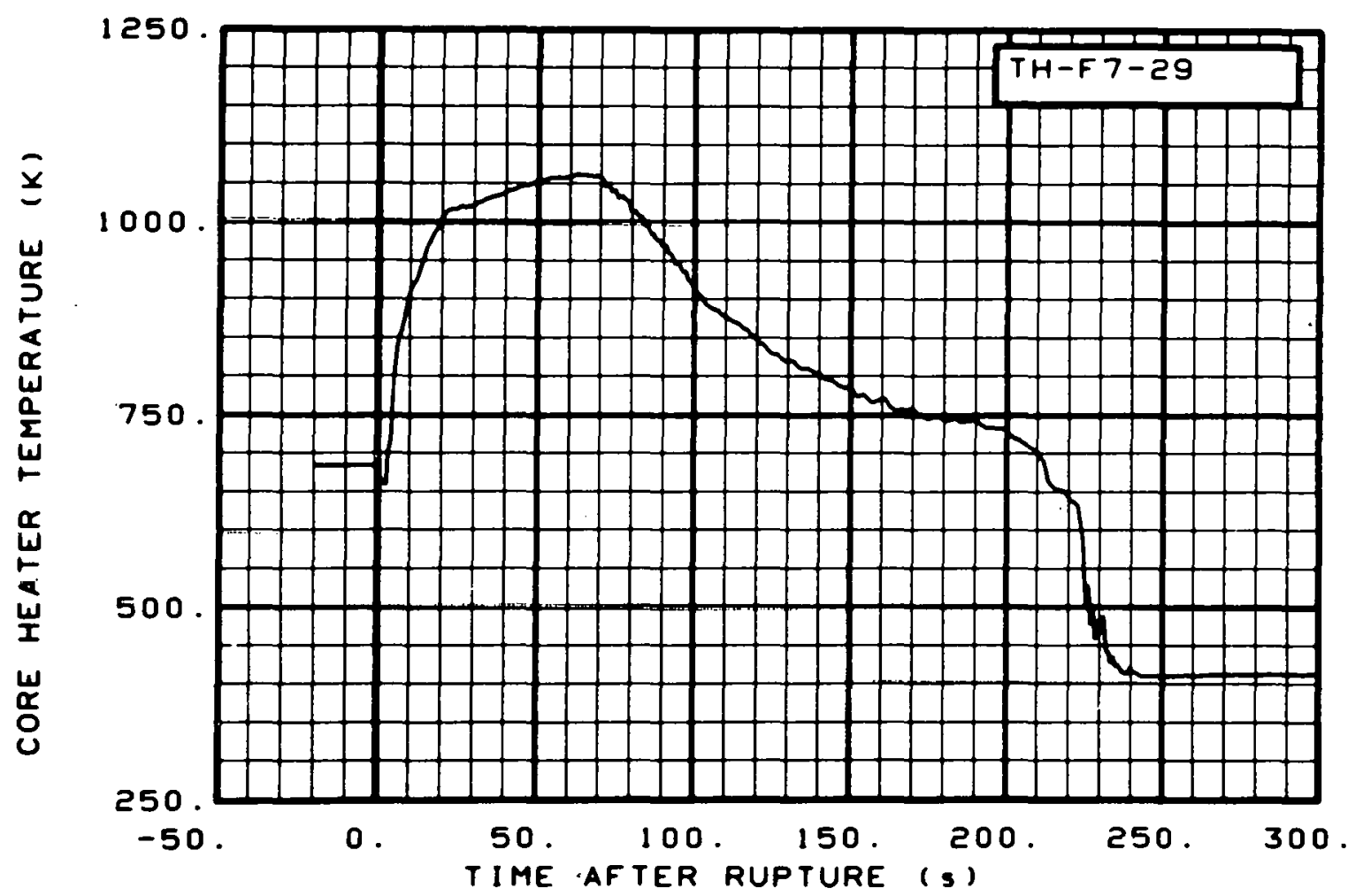

Fig. 113 Core heater temperature, Rod F-7 (TH-F7-29), from -20 to $300 \mathrm{~s}$.

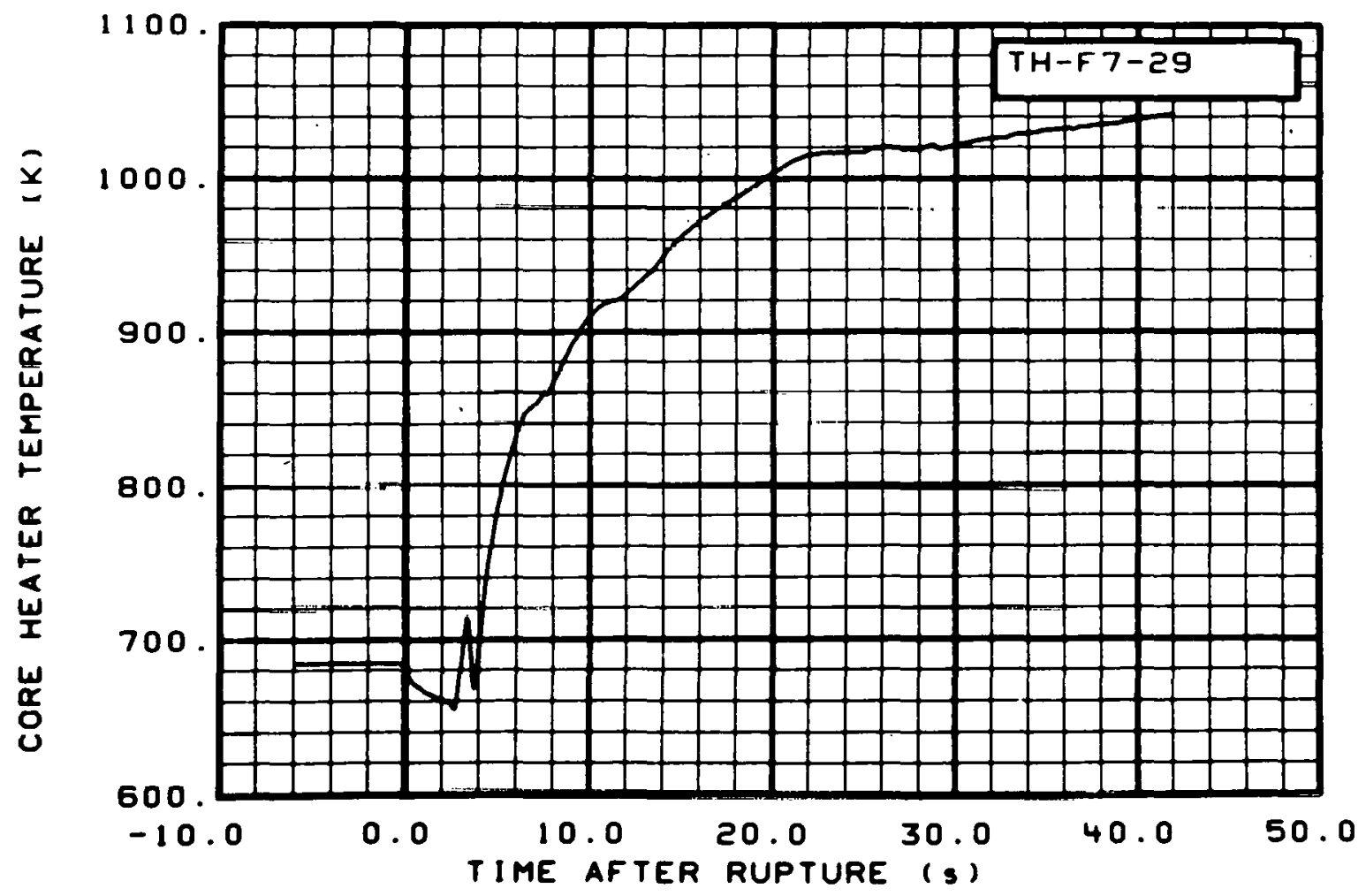

Fig. 114 Core heater temperature, Rod F-7 (TH-F7-29), from -6 to $42 \mathrm{~s}$. 


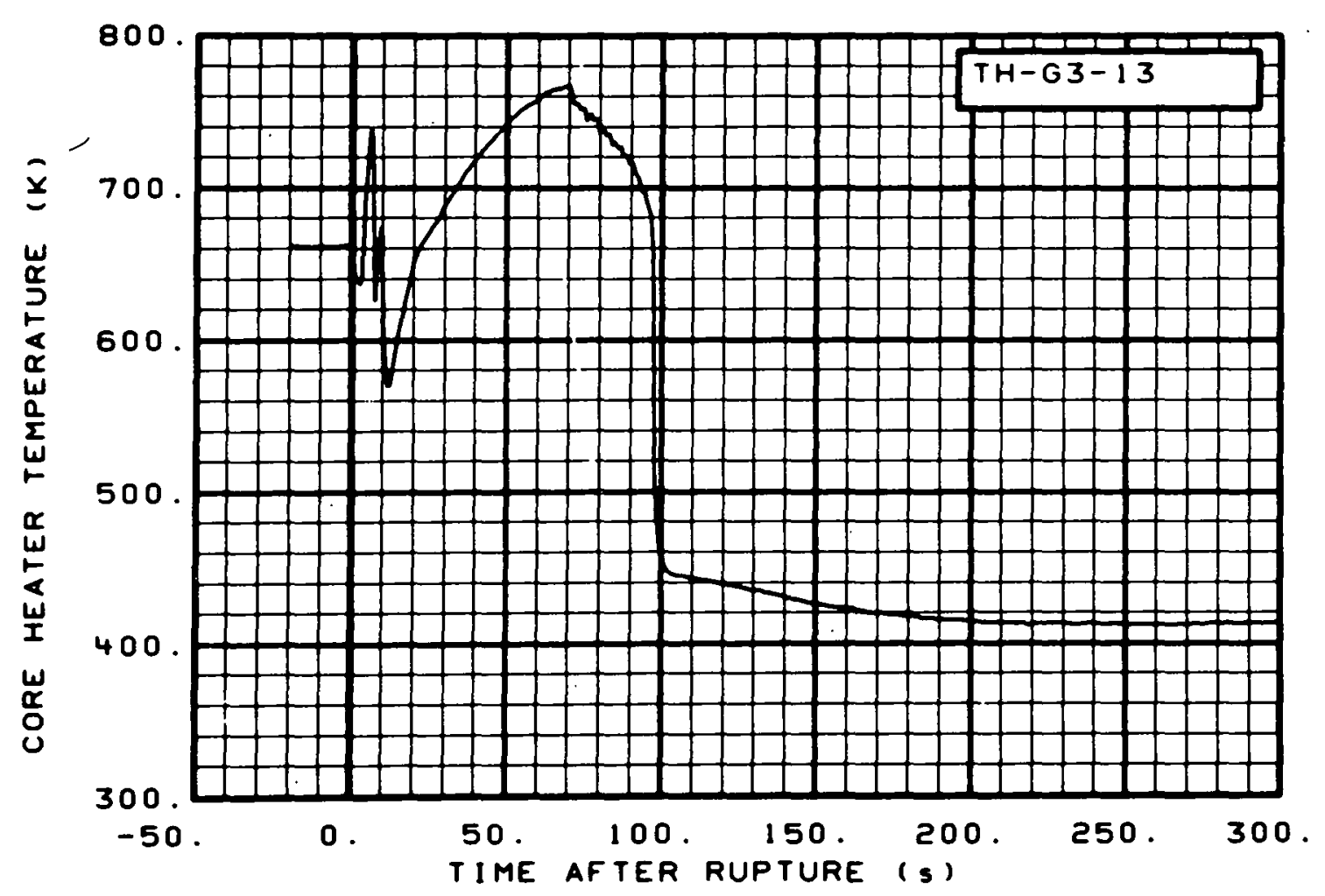

Fig. 115 Core heater temperature, Rod G-3 (TH-G3-13), from -20 to $300 \mathrm{~s}$.

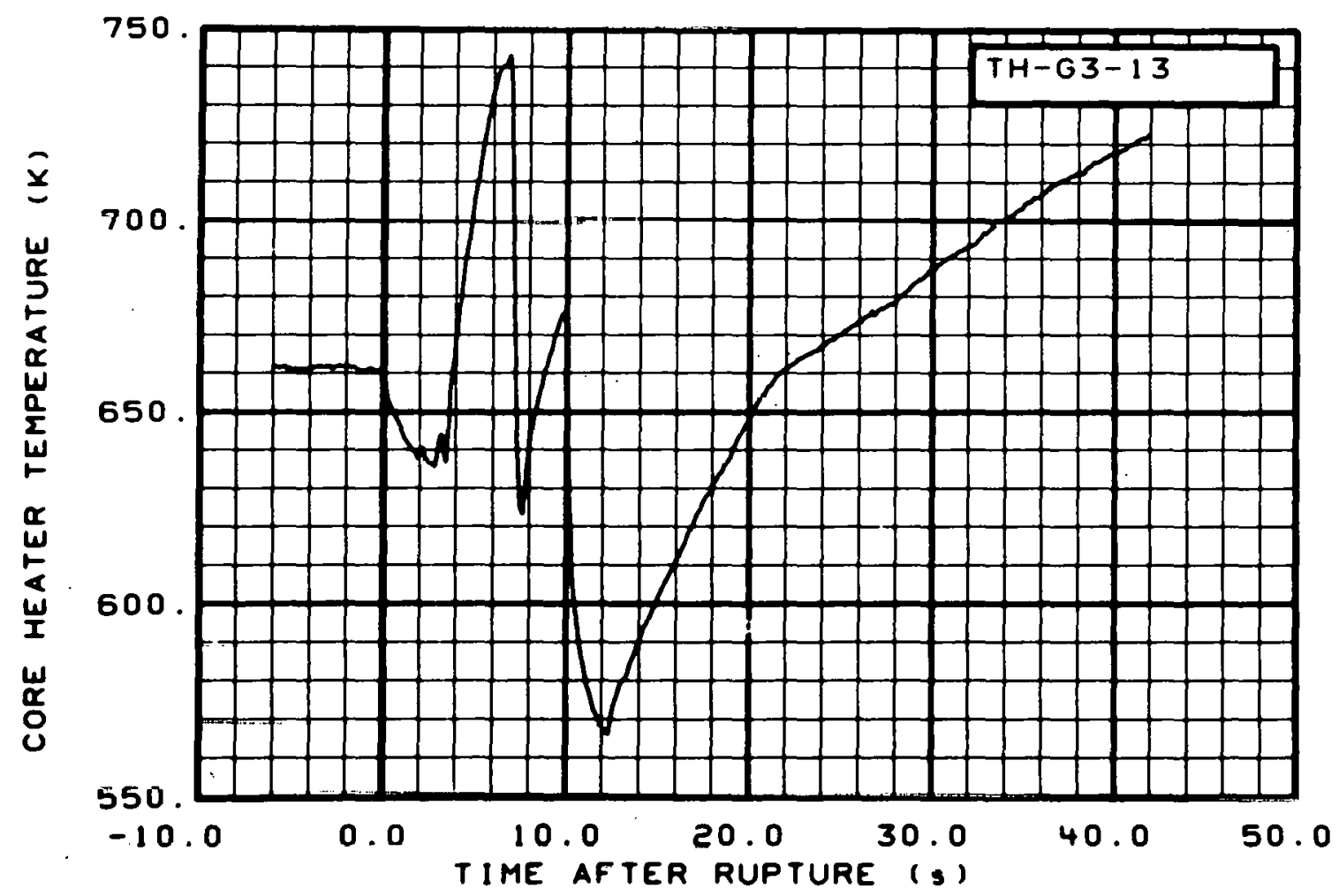

Fig. 116 Core heater temperature, Rod G-3 (TH-G3-13), from -6 to $42 \mathrm{~s}$. 


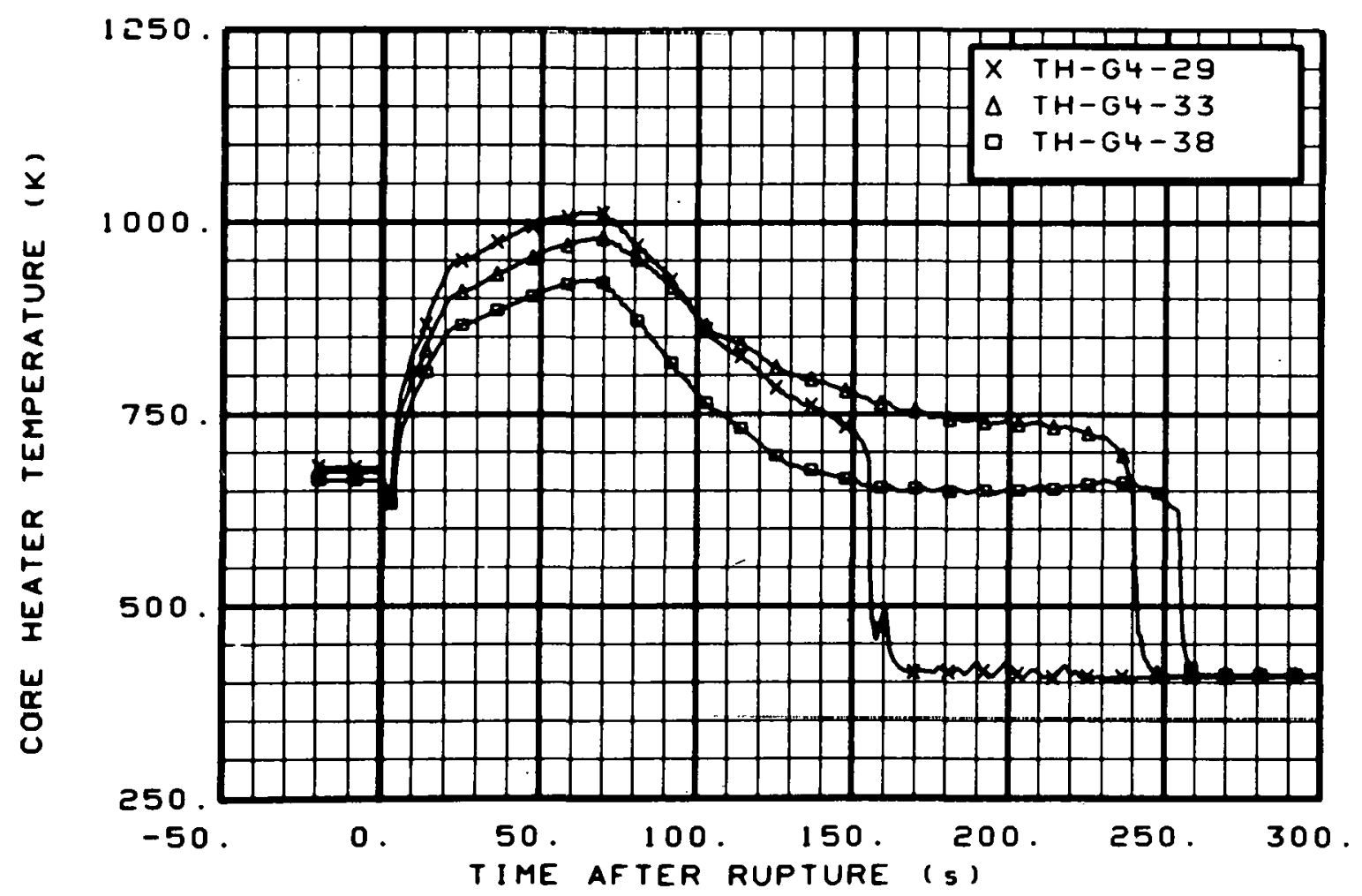

Fig. 117 Core heater temperature, Rod G-4 (TH-G4-29, TH-G4-33, and $\mathrm{TH}-\mathrm{G} 4-38$ ), from -20 to $300 \mathrm{~s}$.

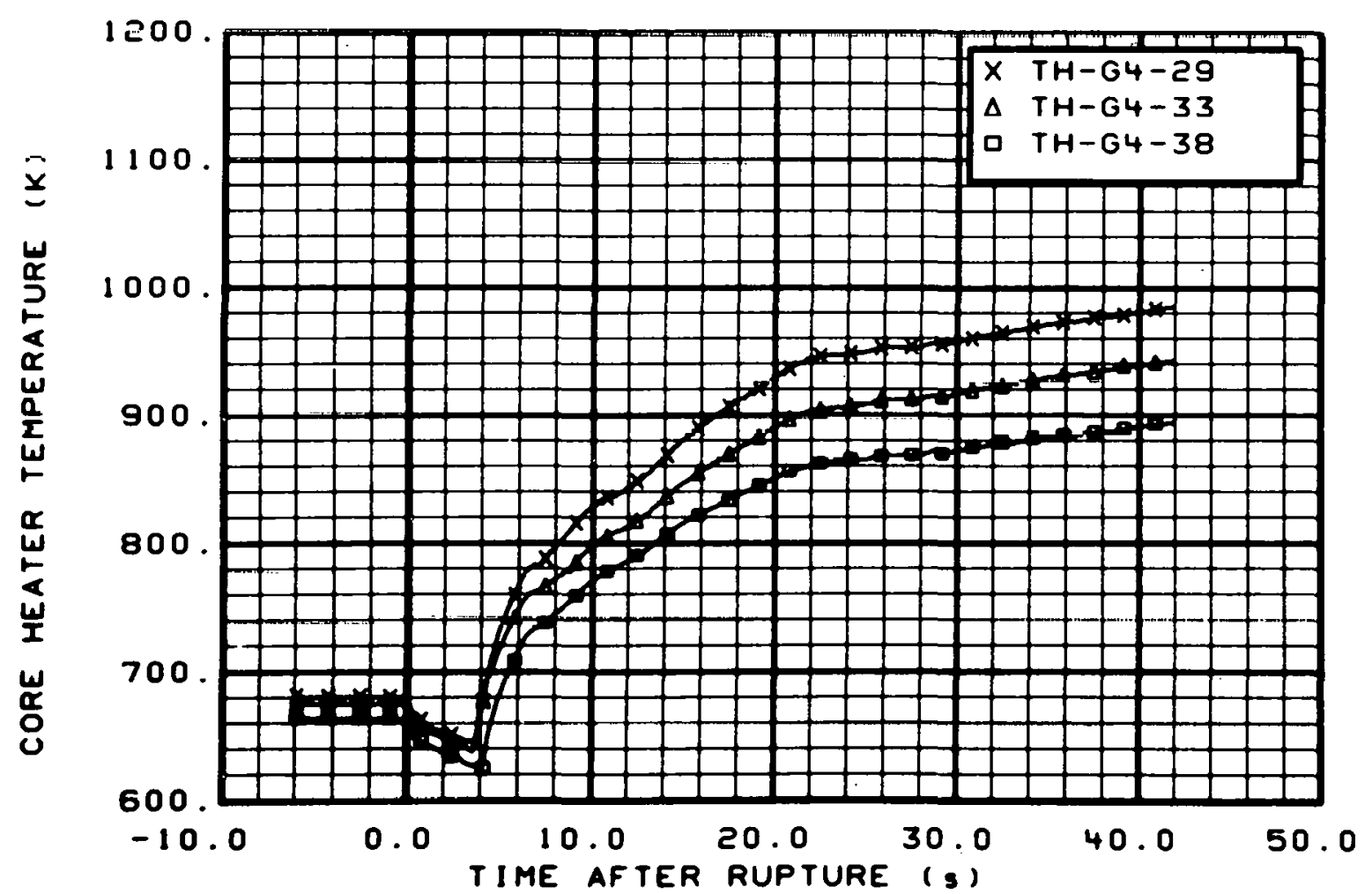

Fig. 118 Core heater temperature, Rod G-4 (TH-G4-29, TH-G4-33, and TH-G4-38), from -6 to $42 \mathrm{~s}$. 


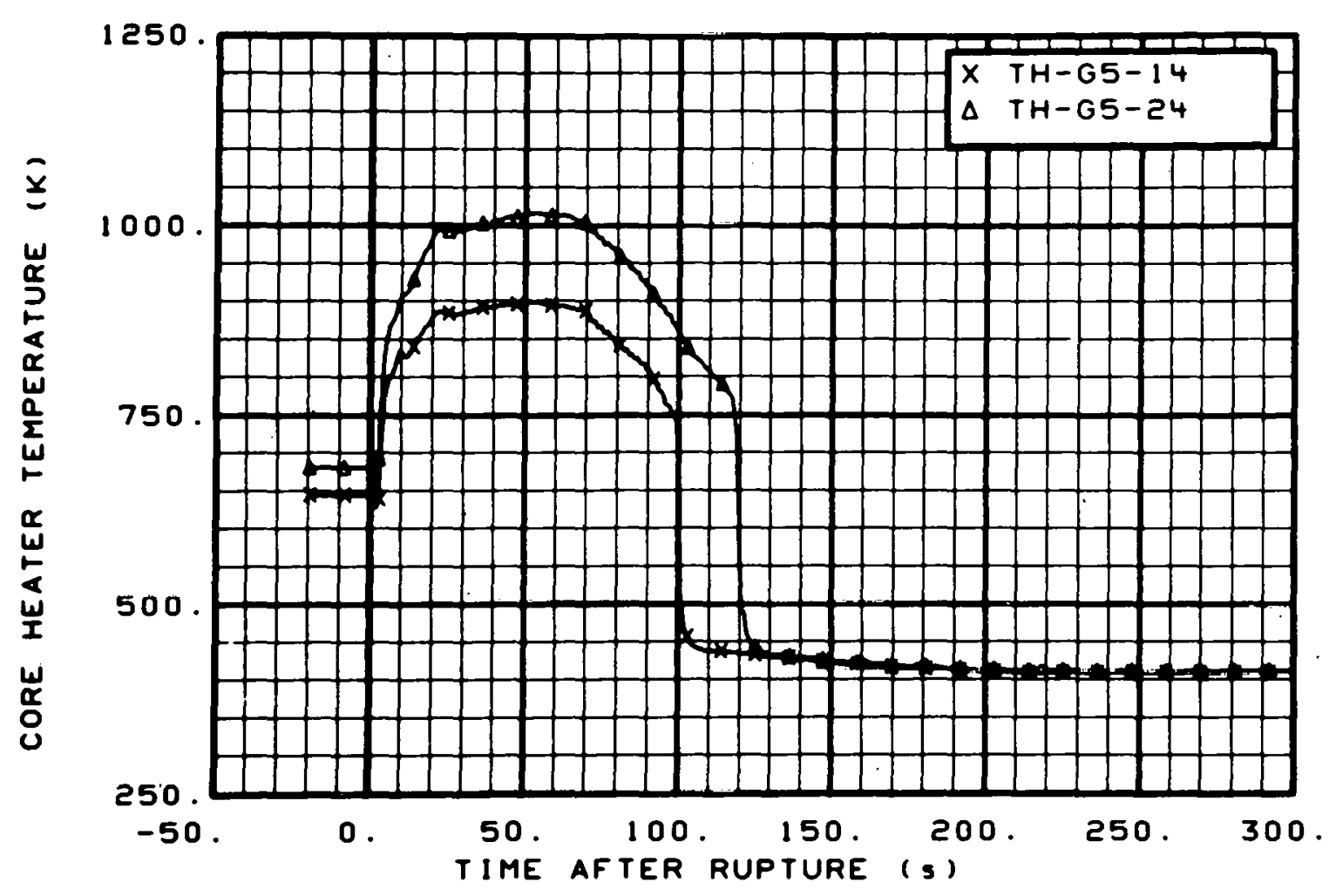

Fig. 119 Core heater temperature, Rod G-5 (TH-G5-14 and TH-G5-24), from -20 to $300 \mathrm{~s}$.

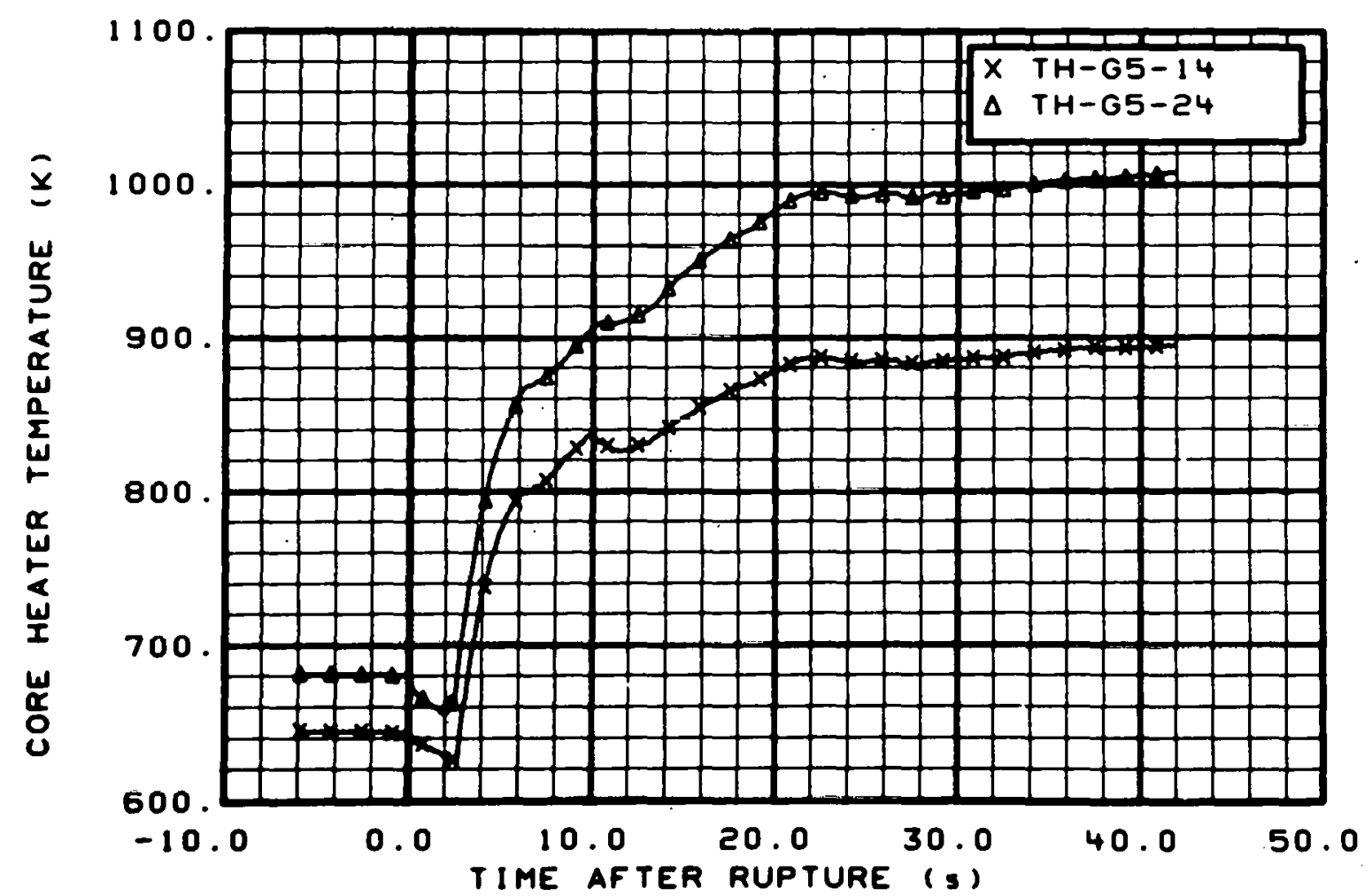

Fig. 120 Core heater temperature, Rod G-5 (TH-G5-14 and TH-G5-24), from -6 to $42 \mathrm{~s}$. 


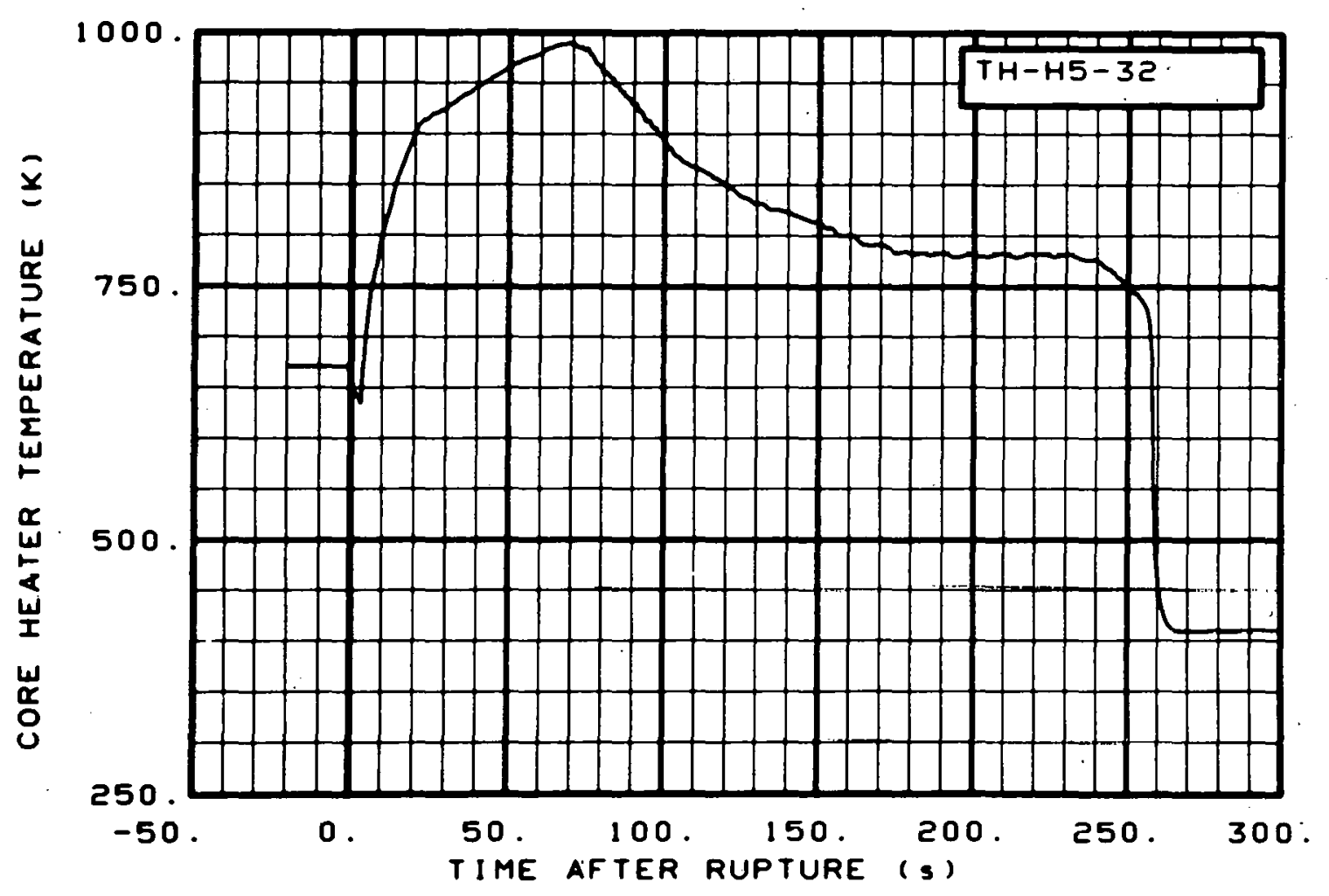

Fig. 121 Core heater temperature, Rod H-5 (TH-H5-32), from -20 to $300 \mathrm{~s}$.

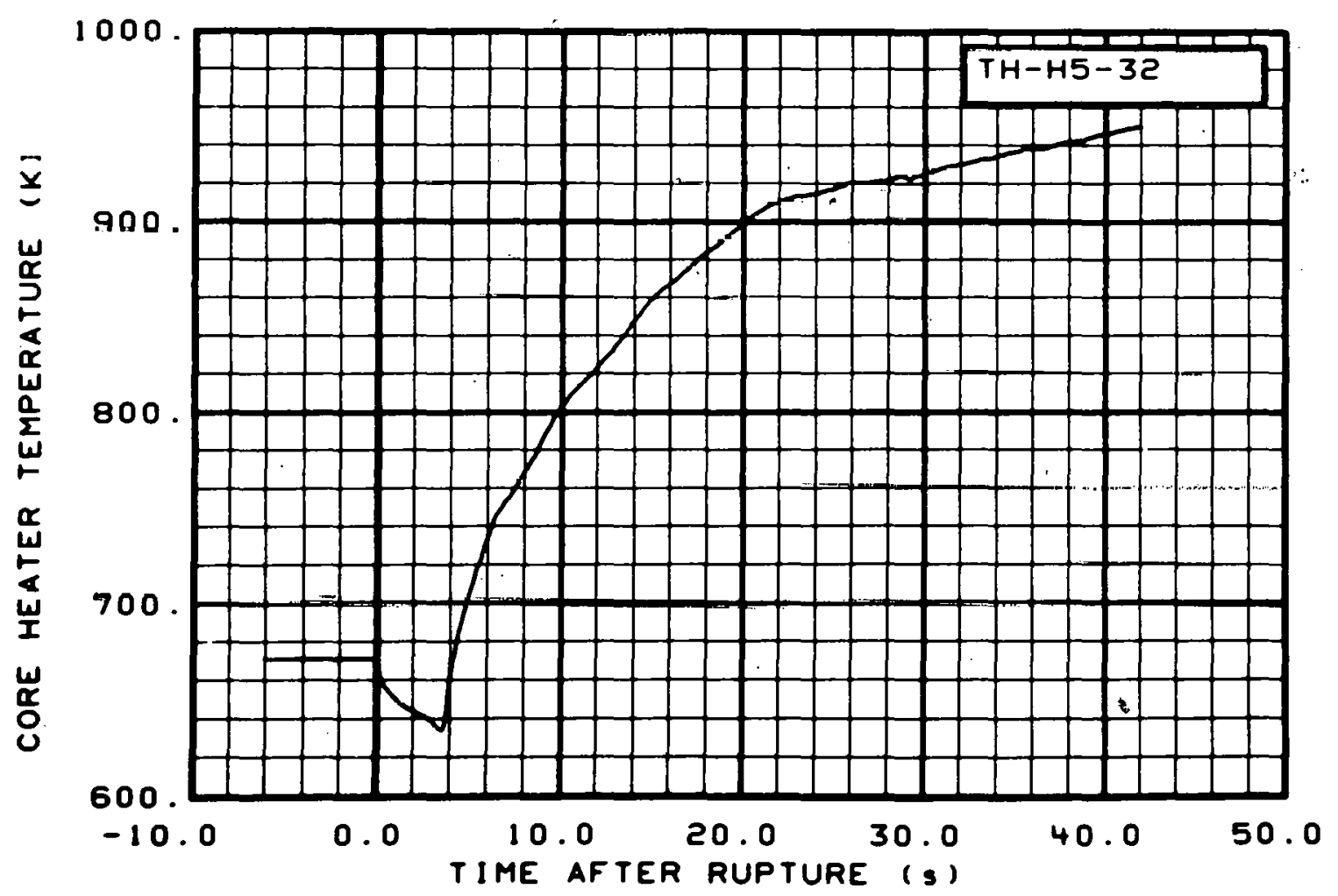

Fig. 122 Core heater temperature, Rod H-5 (TH-H5-32), from -6 to $42 \mathrm{~s}$. 


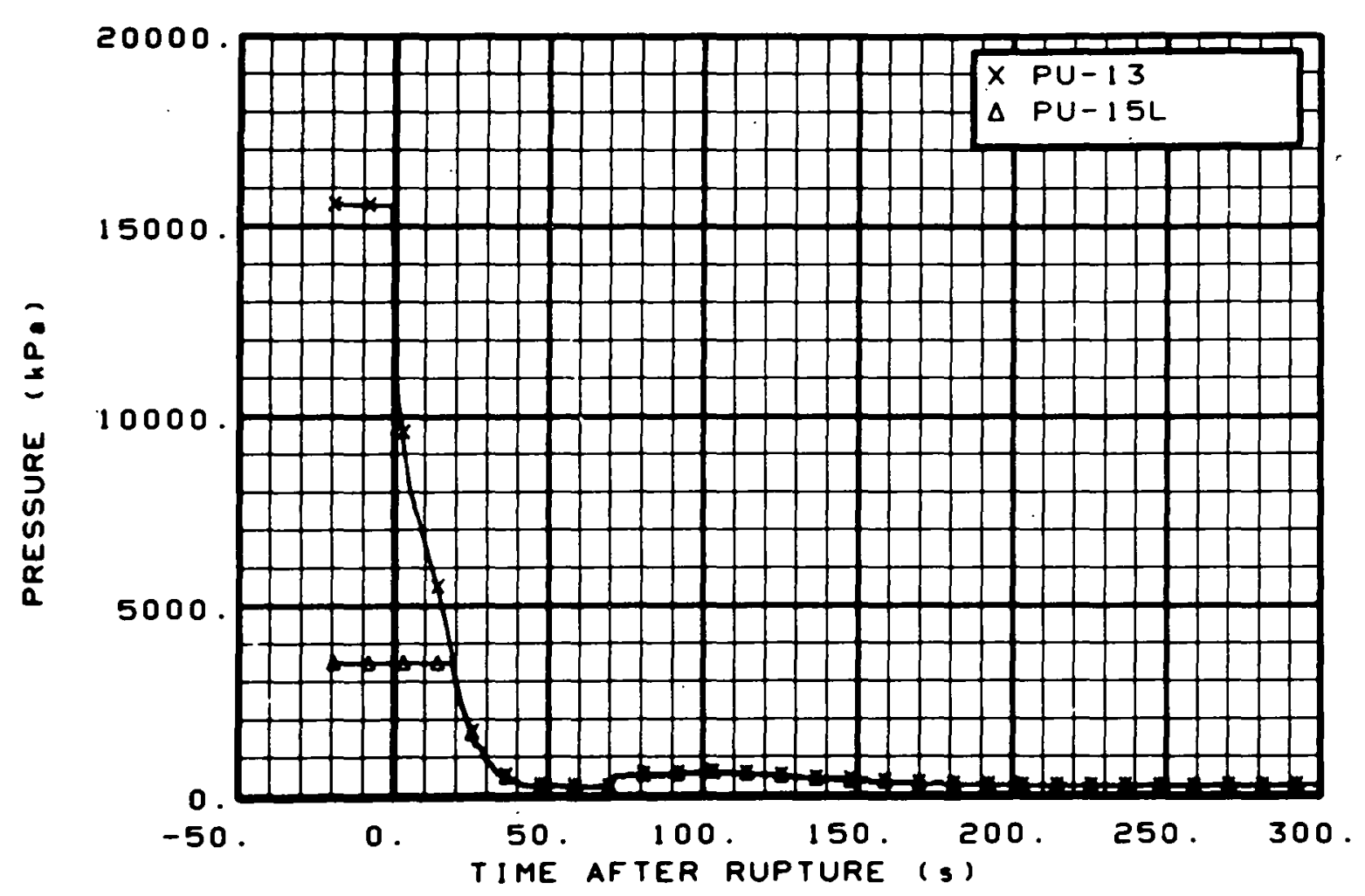

Fig. 123 Pressure in intact loop (PU-13 and PU-15L), from .20 to $300 \mathrm{~s}$.

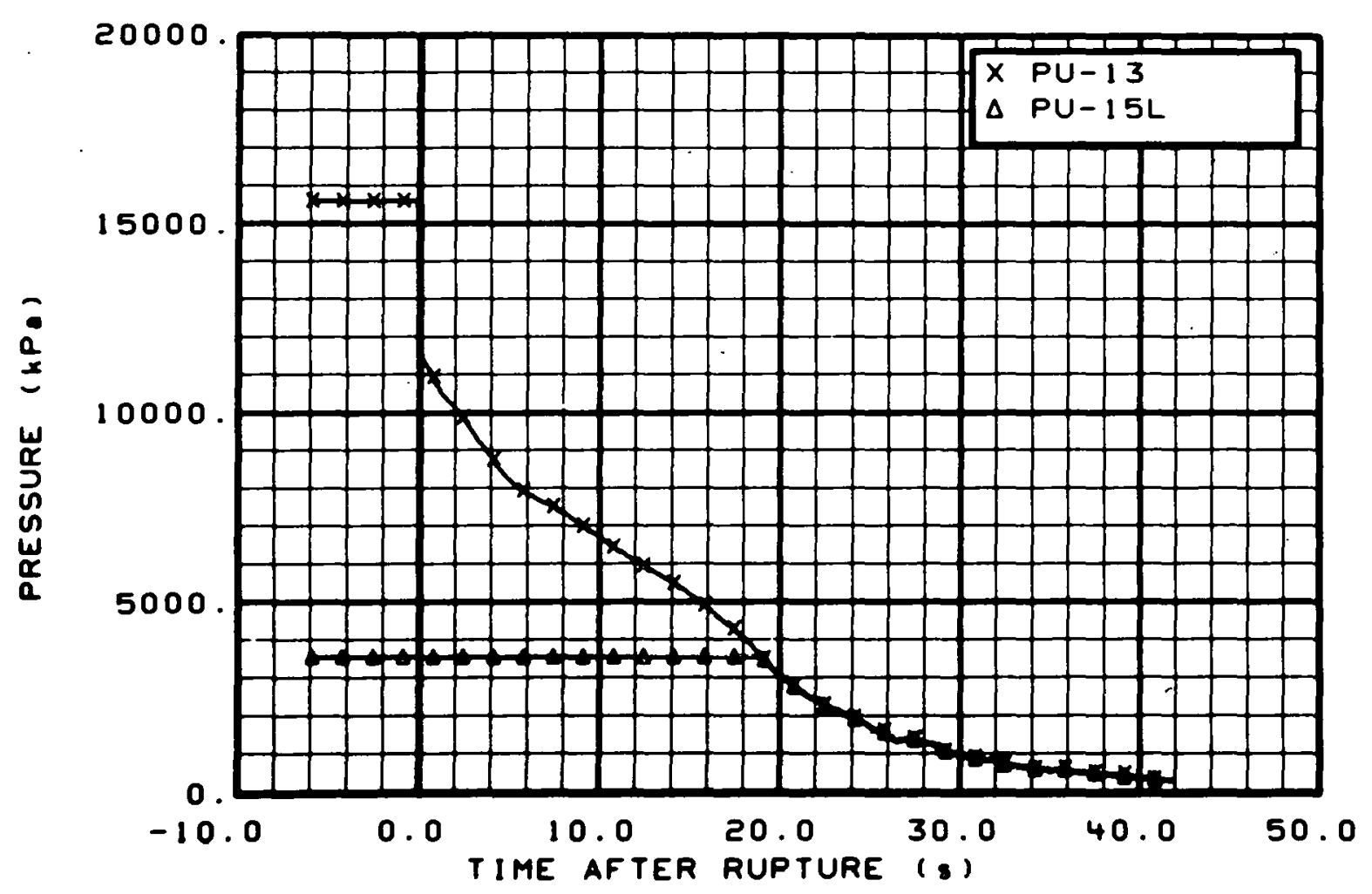

Fig. 124 Pressure in intact loop (PU-13 and PU-15L), from -6 to $42 \mathrm{~s}$. 


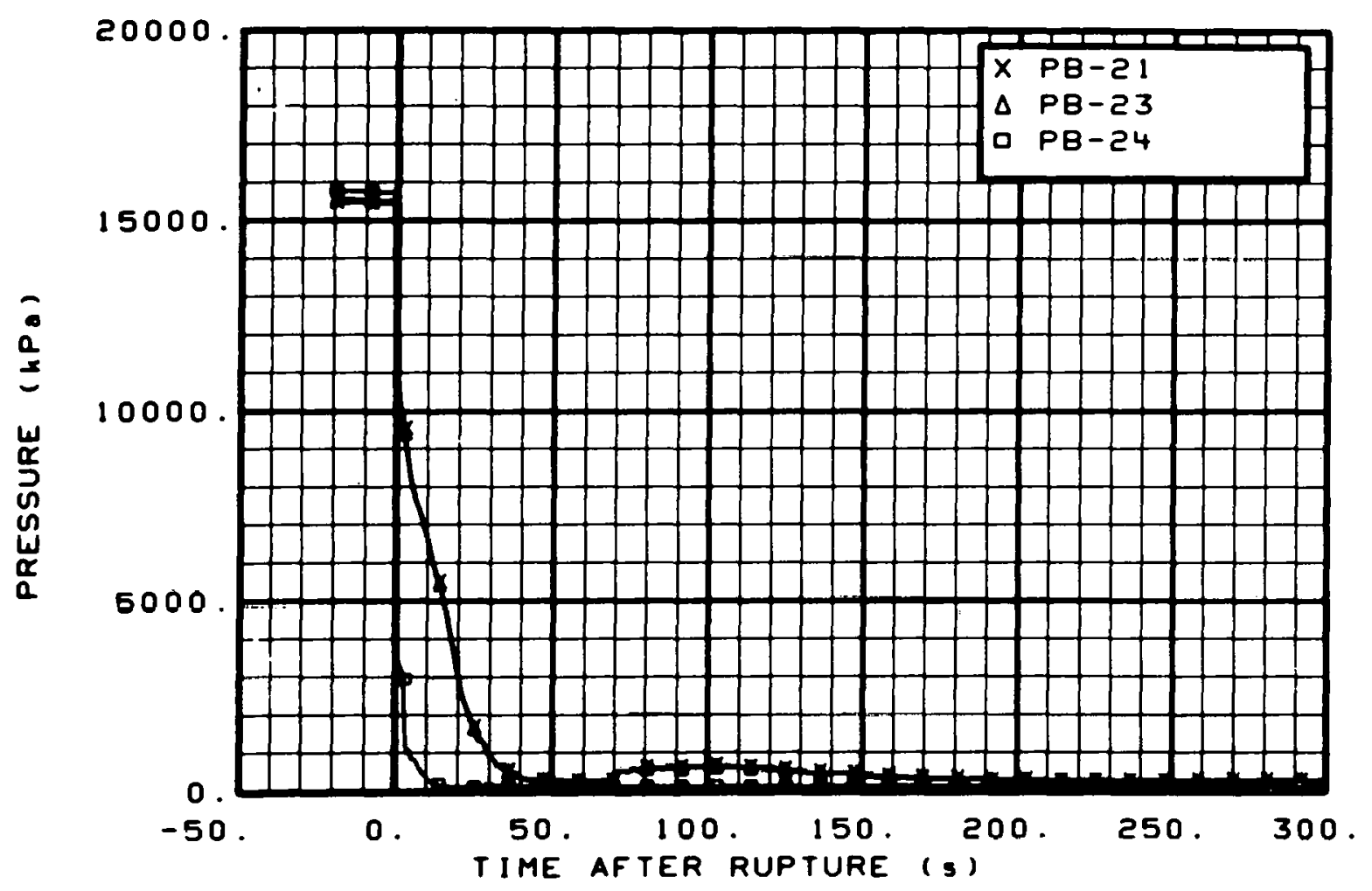

Fig. 125 Pressure in broken loop, vessel side (PB-21, PB-23, and PB-24), from -20 to $300 \mathrm{~s}$.

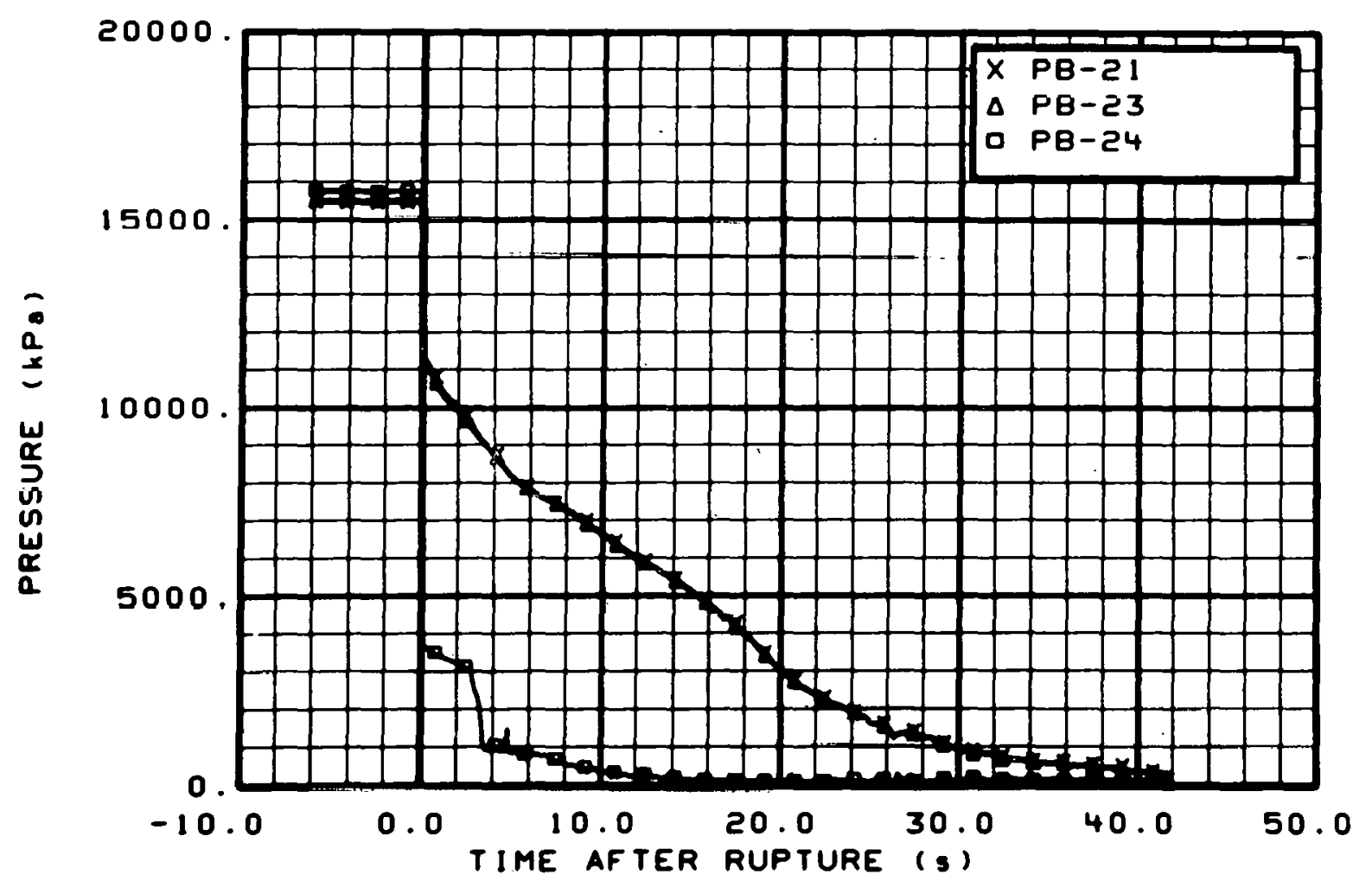

Fig. 126 Pressure in broken 10op, vessel side (PB-21, PB-23, and PB-24), from -6 to $42 \mathrm{~s}$. 


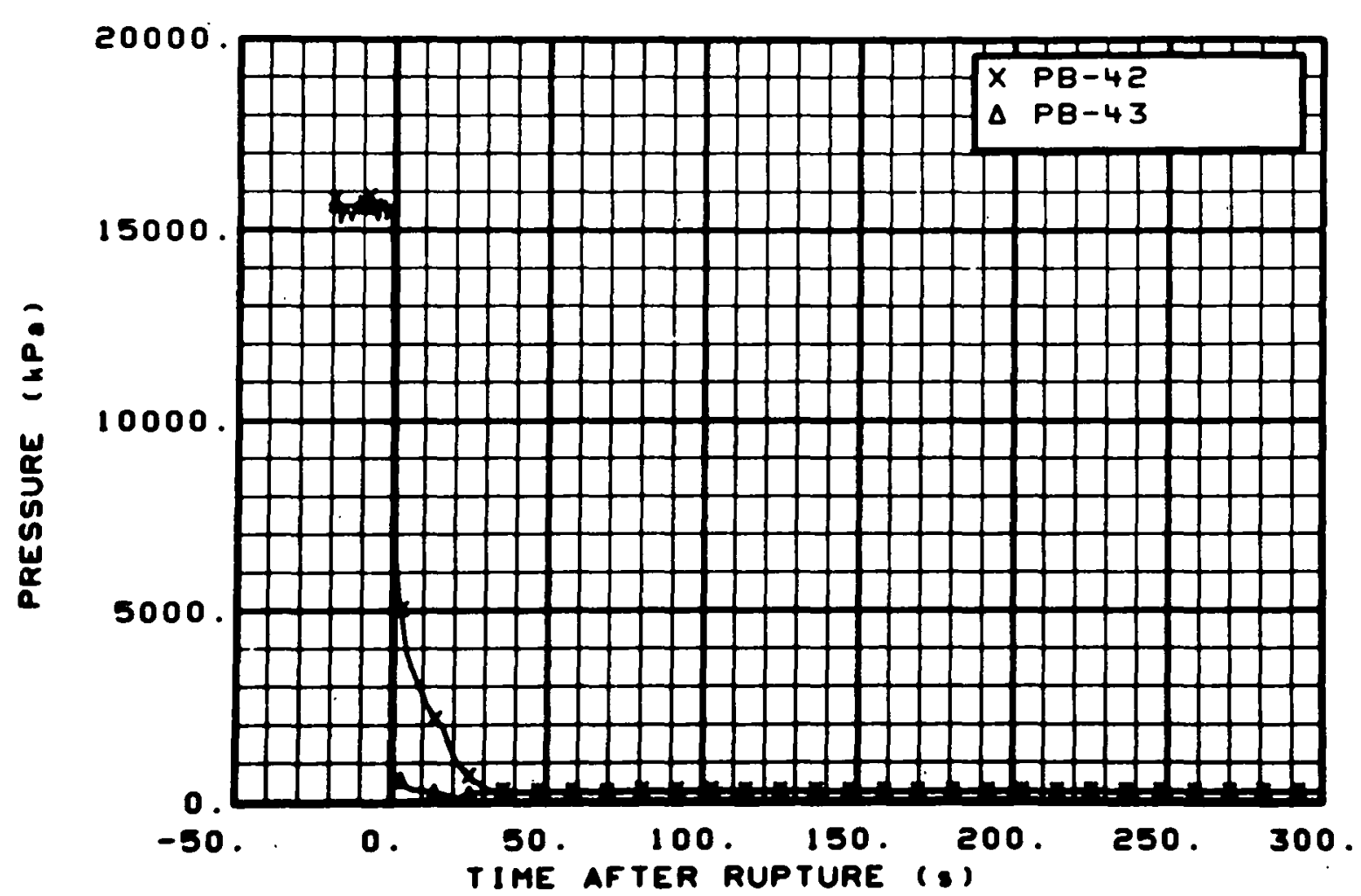

Fig. 127 Pressure in broken loop, pump side (PB-42 and PB-43), from -20 to $300 \mathrm{~s}$.

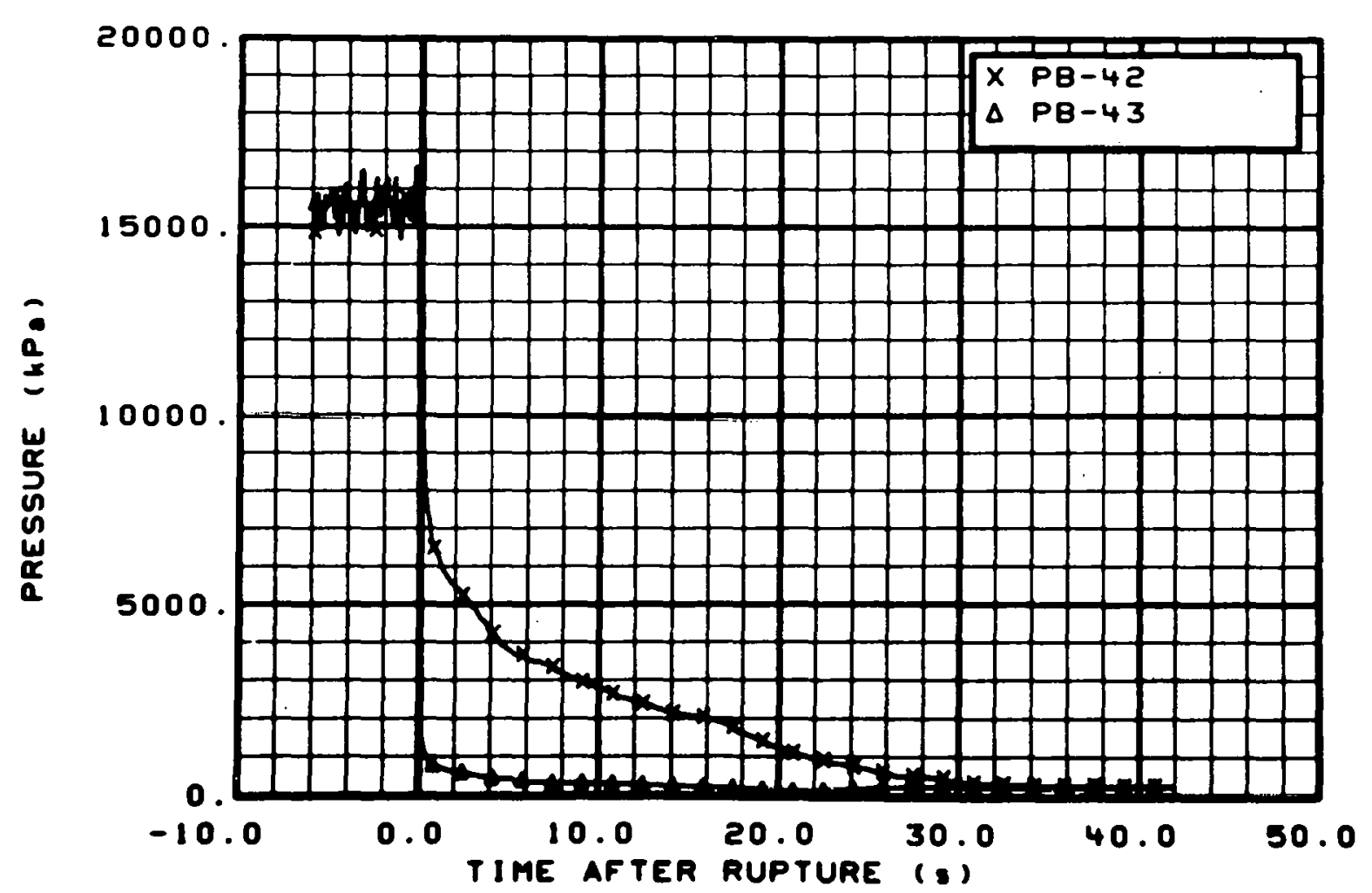

Fig. 128 Pressure in broken loop, pump side (PB-42 and PB-43), from -6 to $42 \mathrm{~s}$. 


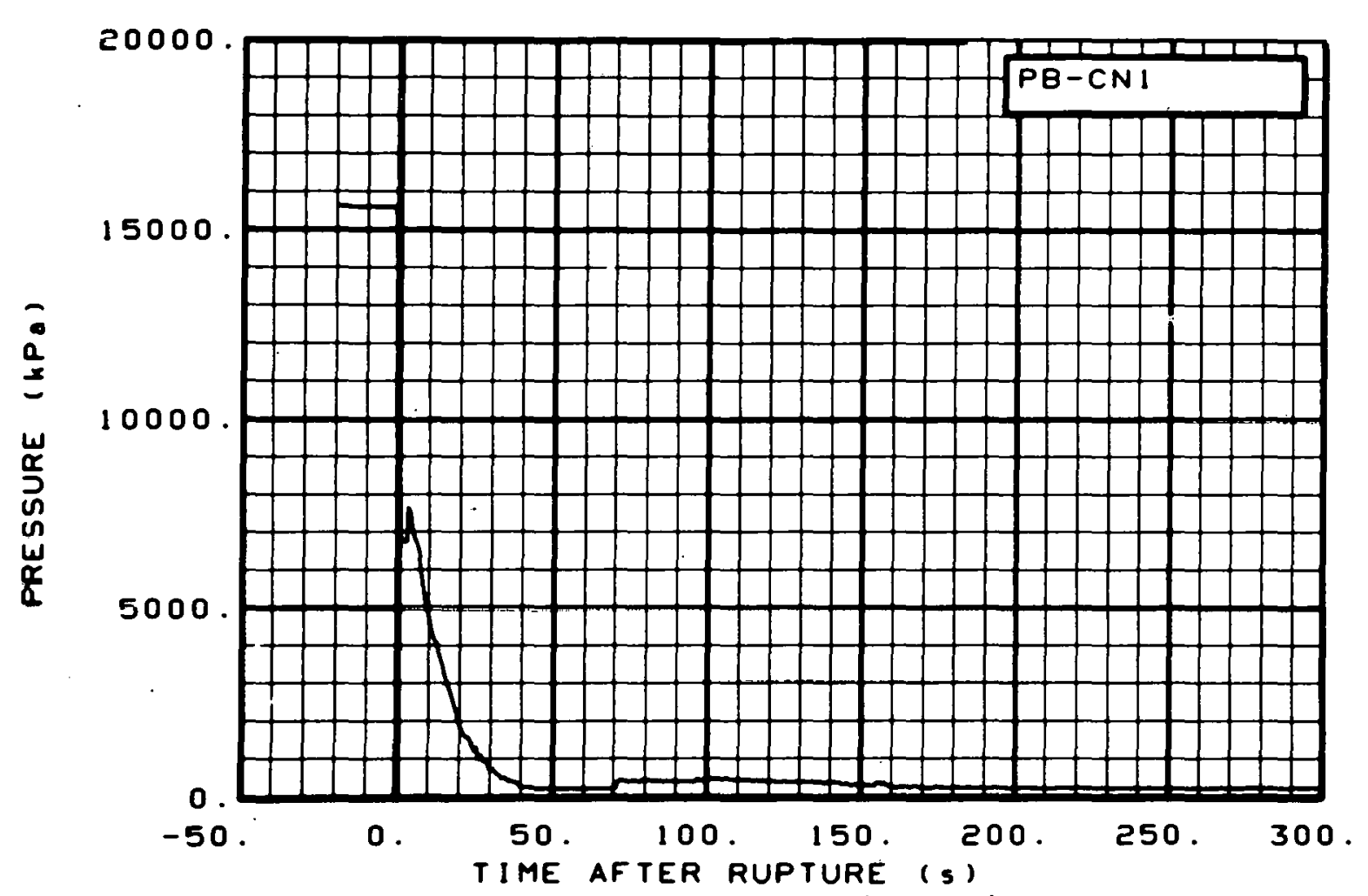

Fig. 129 Pressure in broken loop, vessel side (PB-CN1), from -20 to $300 \mathrm{~s}$.

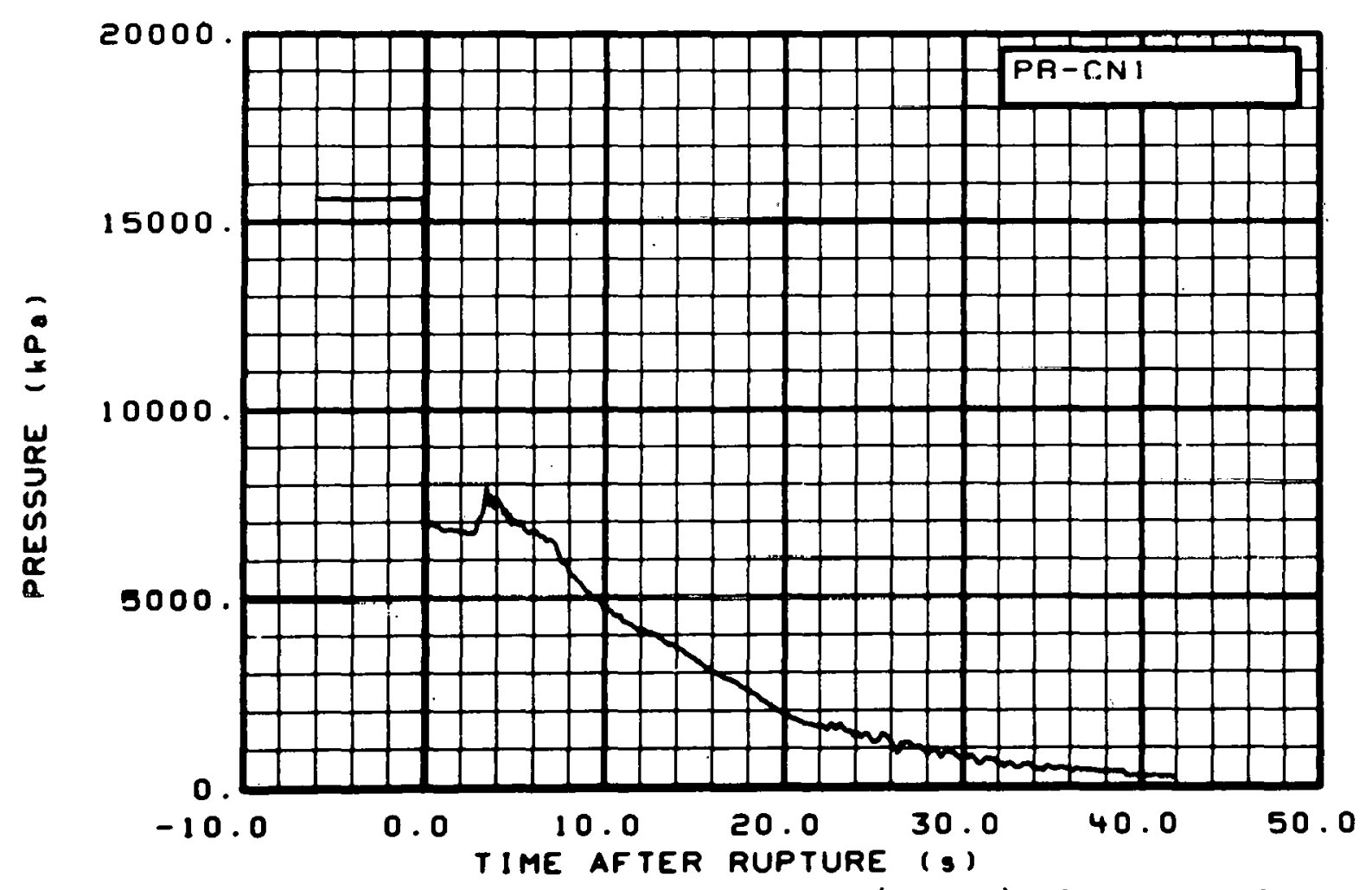

Fig. 130 Pressure in broken loop, vessel side (PB-CN1), from -6 to $42 \mathrm{~s}$. 


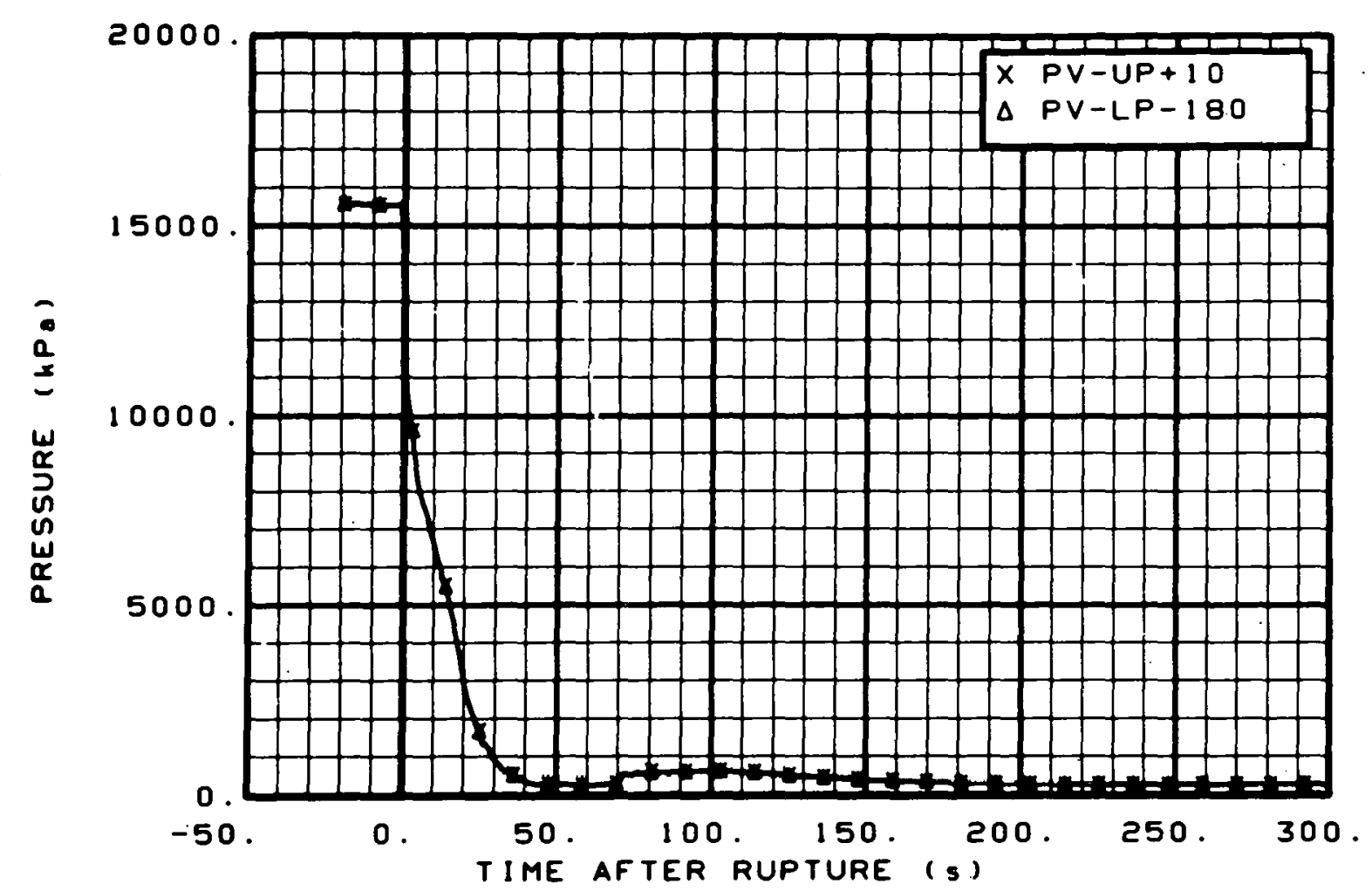

Fig. 131 Pressure in vessel (PV-UP+10 and PV-LP-180), from -20 to $300 \mathrm{~s}$.

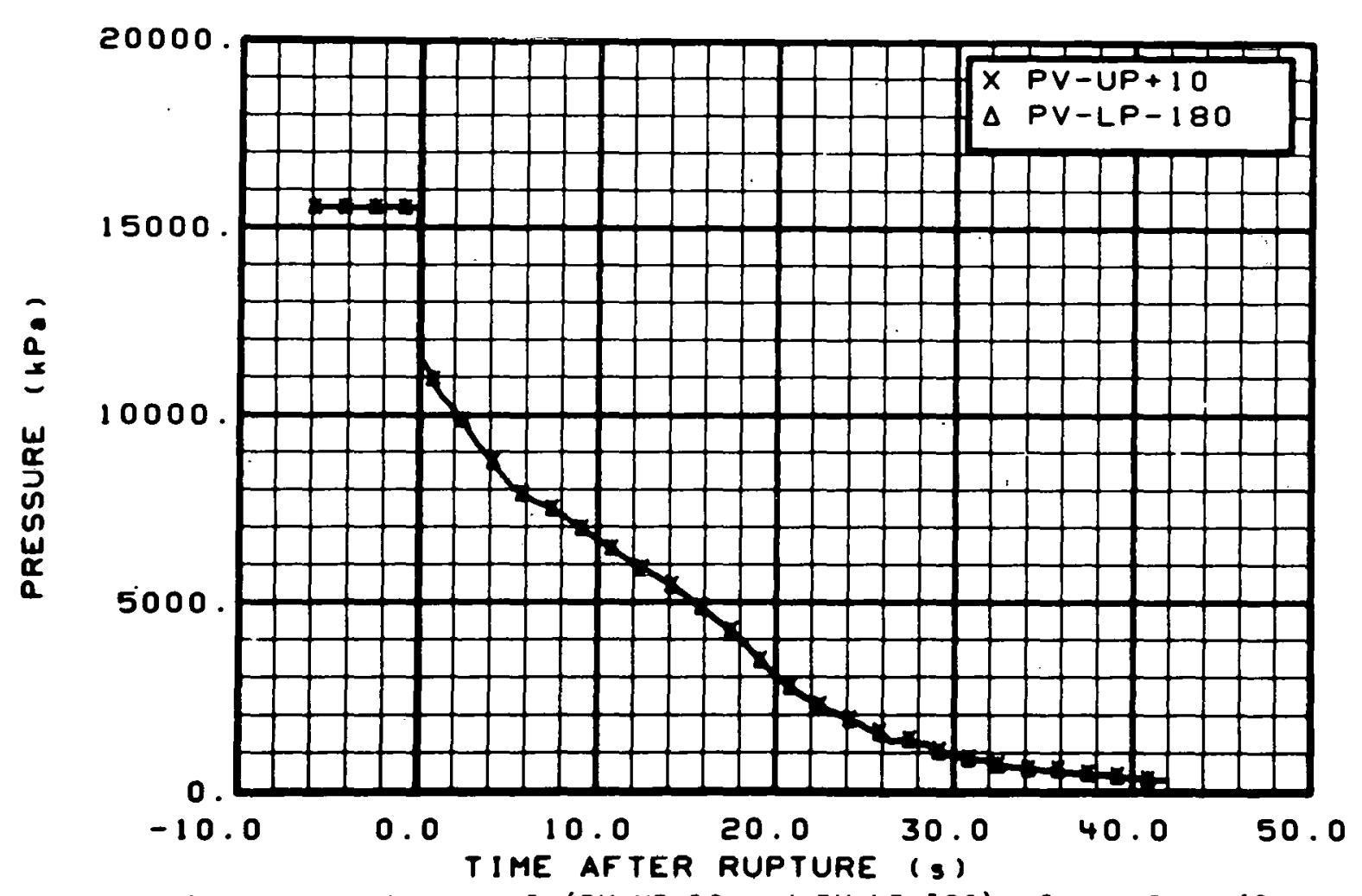

Fig. 132 Pressure in vessel (PV-UP+10 and PV-LP-180), from -6 to $42 \mathrm{~s}$. 


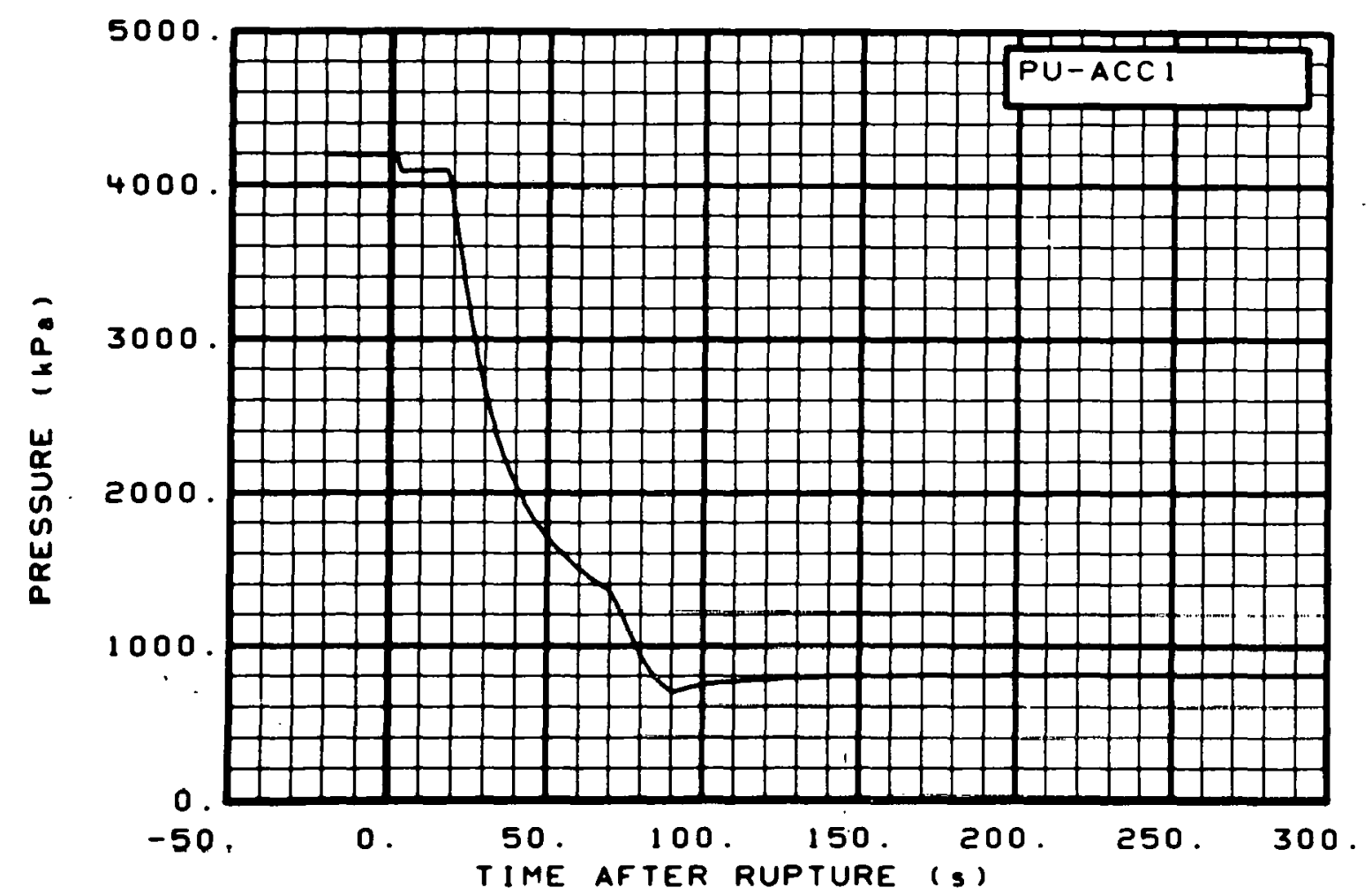

Fig. 133 Pressure in intact loop accumulator (PU-ACC1), from -20 to $300 \mathrm{~s}$.

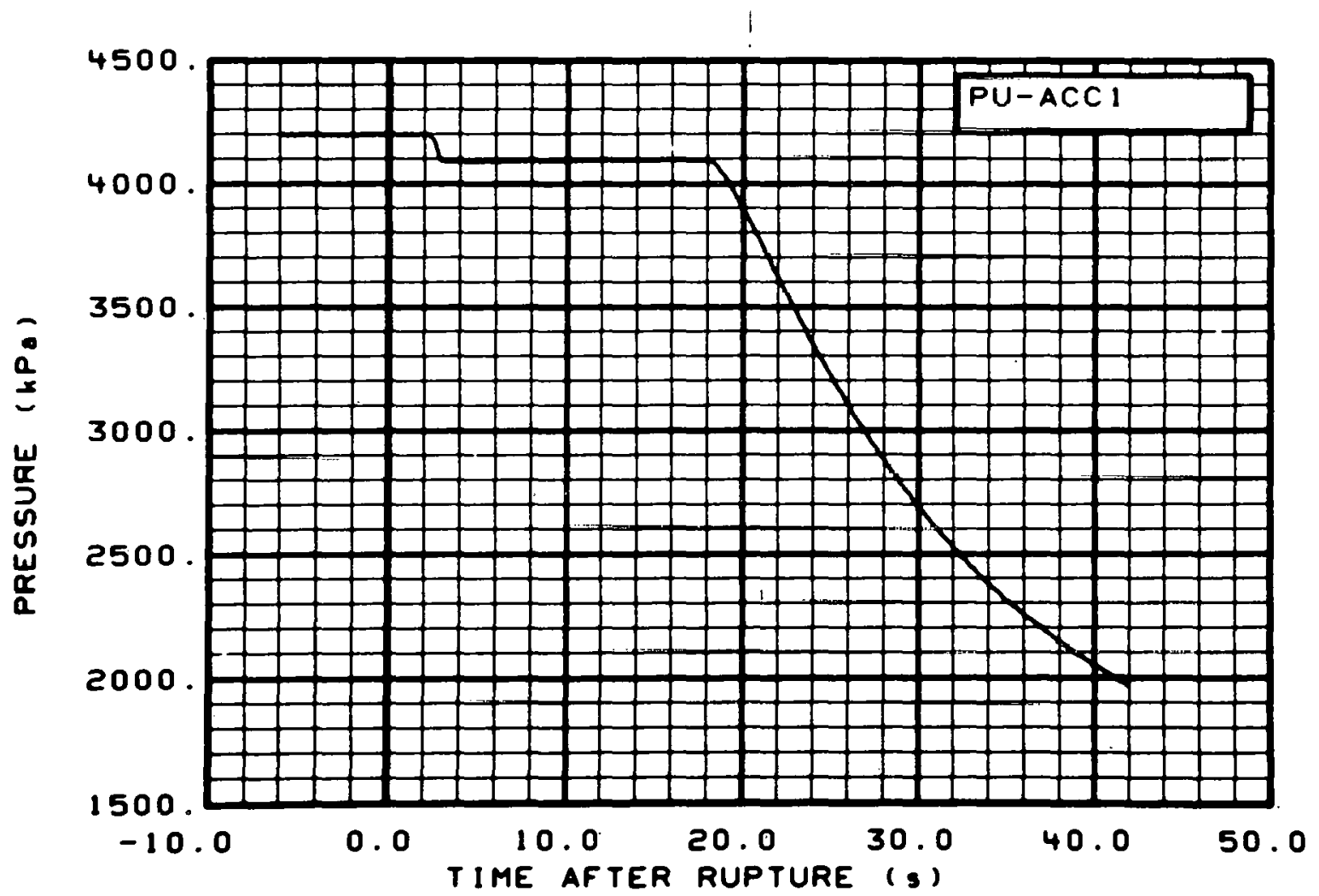

Fig. 134 Pressure in intact loop accumulator (PU-ACC1), from -6 to $42 \mathrm{~s}$. 


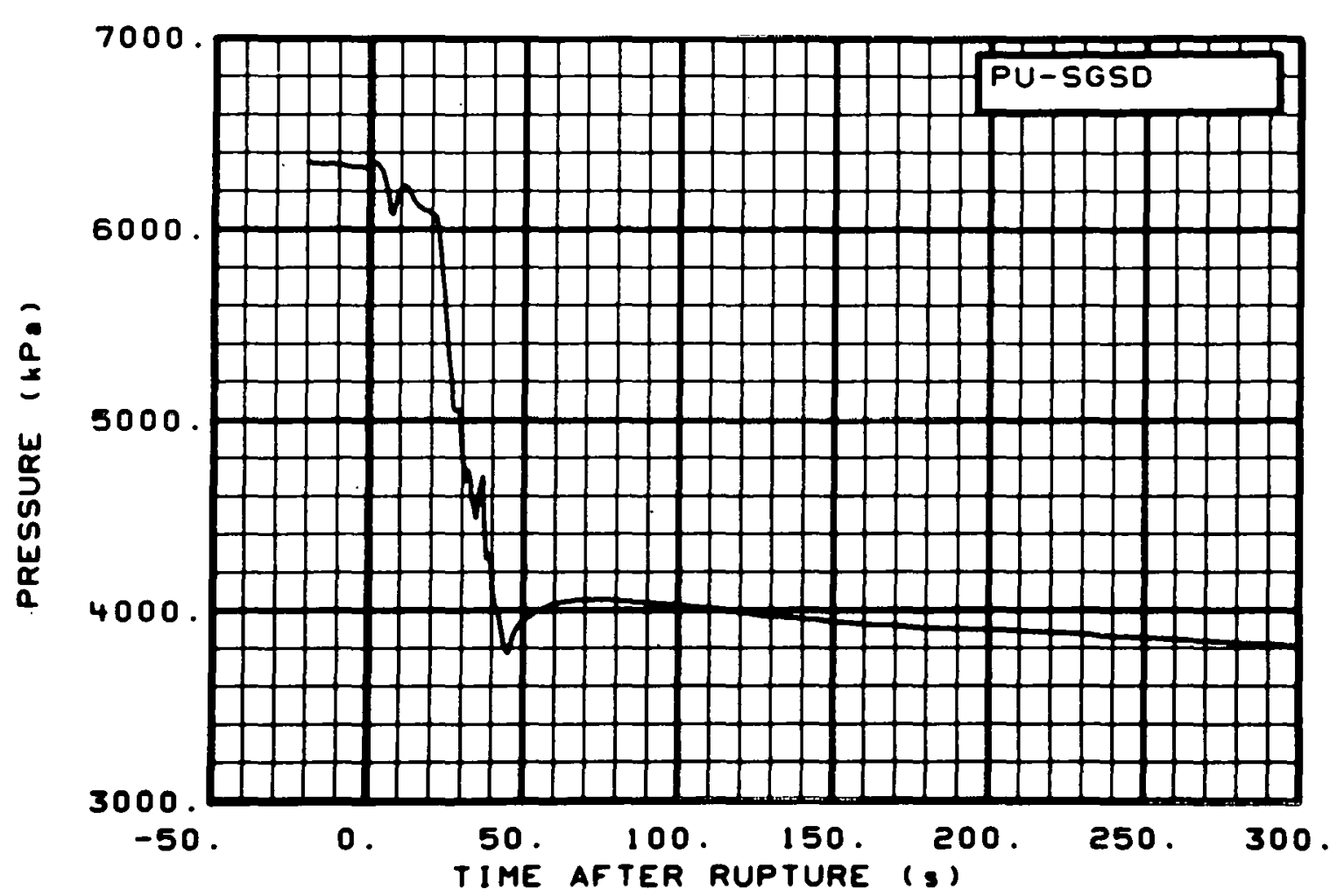

Fig. 135 Pressure in steam generator, secondary side (PU-SGSD), from -20 to $300 \mathrm{~s}$.

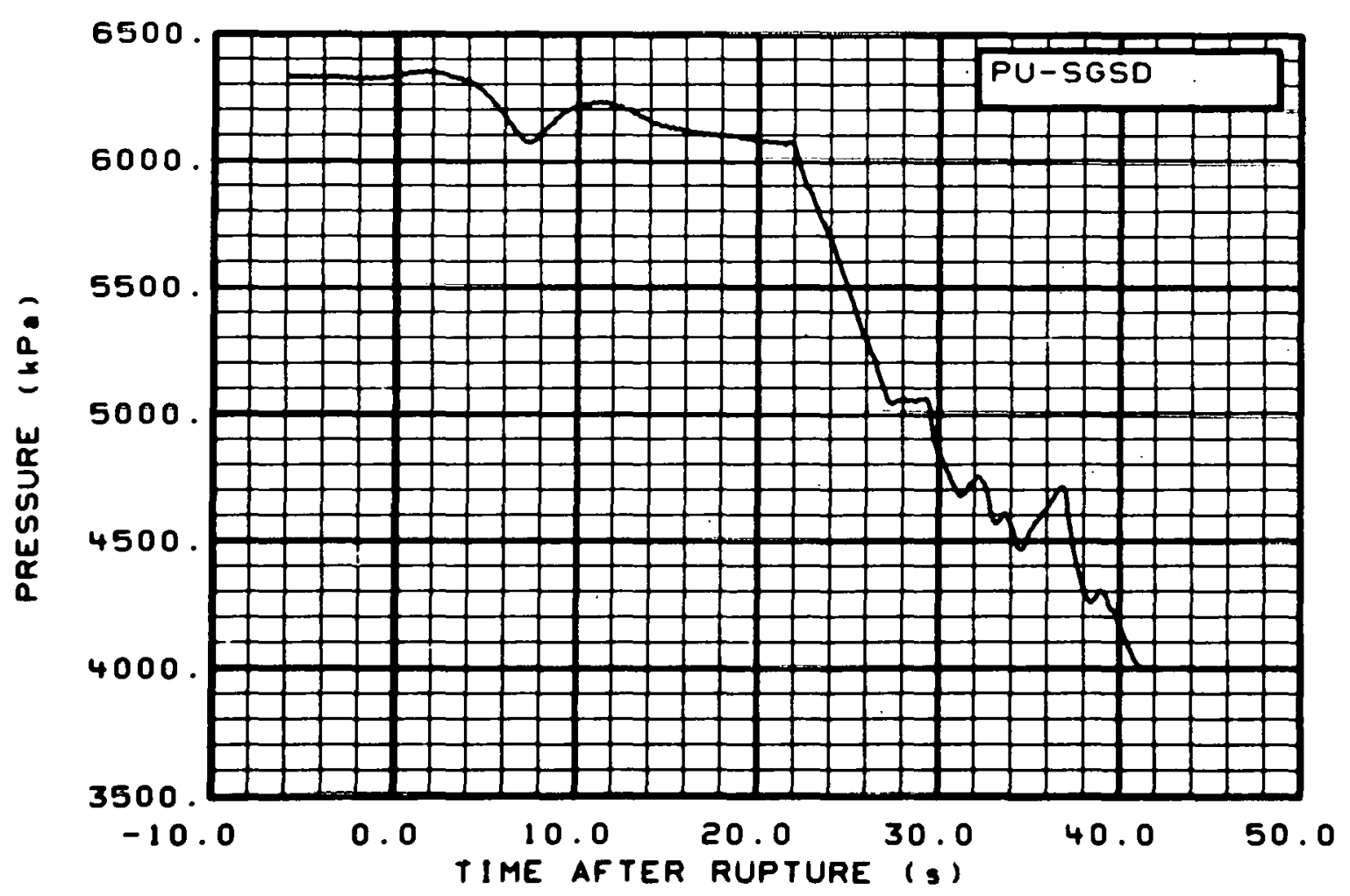

Fig. 136 Pressure in steam generator, secondary side (PU-SGSD), from -6 to 42 s. 


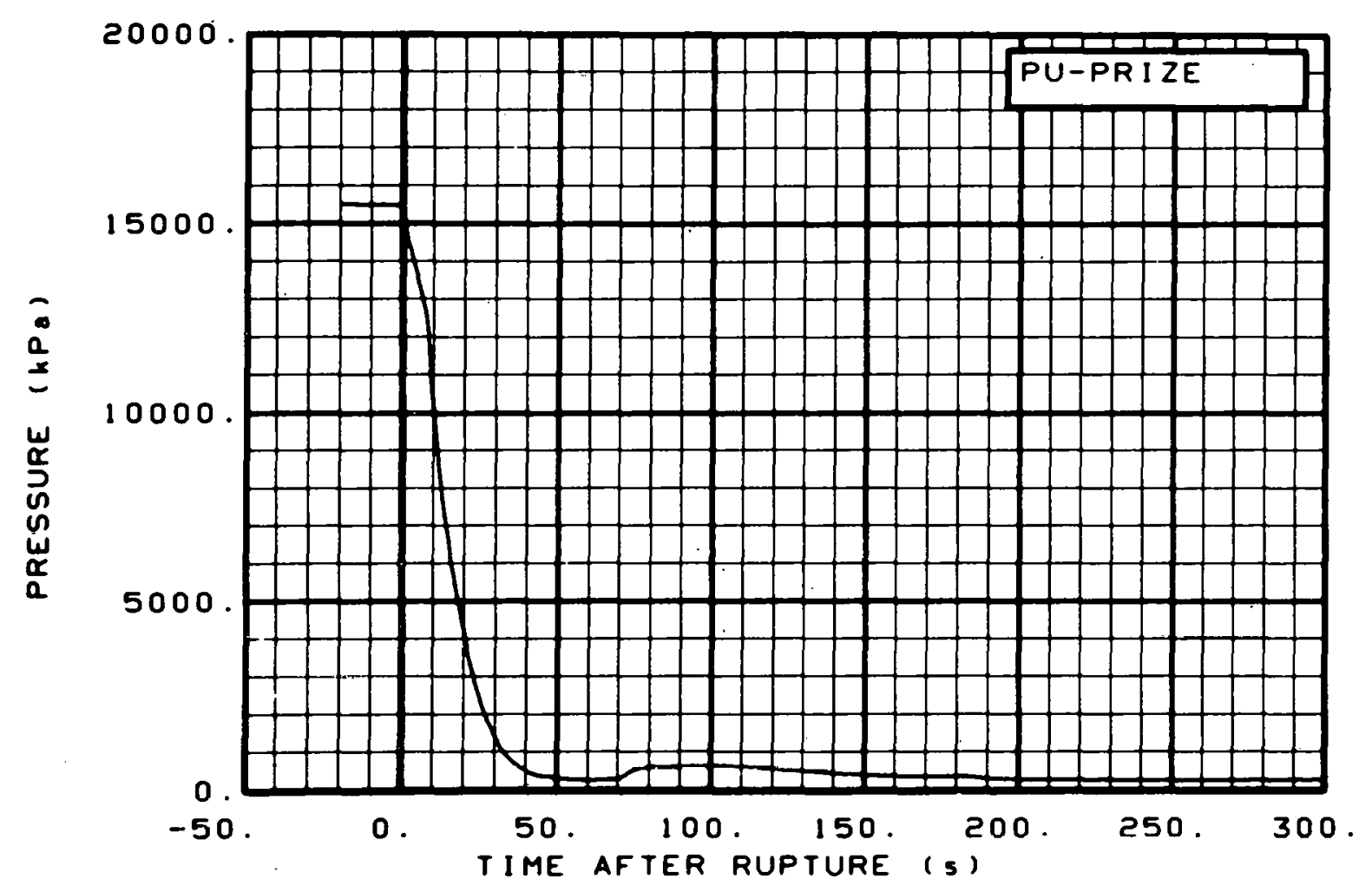

Fig. 137 Pressure in pressurizer (PU-PRIZE), from -20 to $300 \mathrm{~s}$.

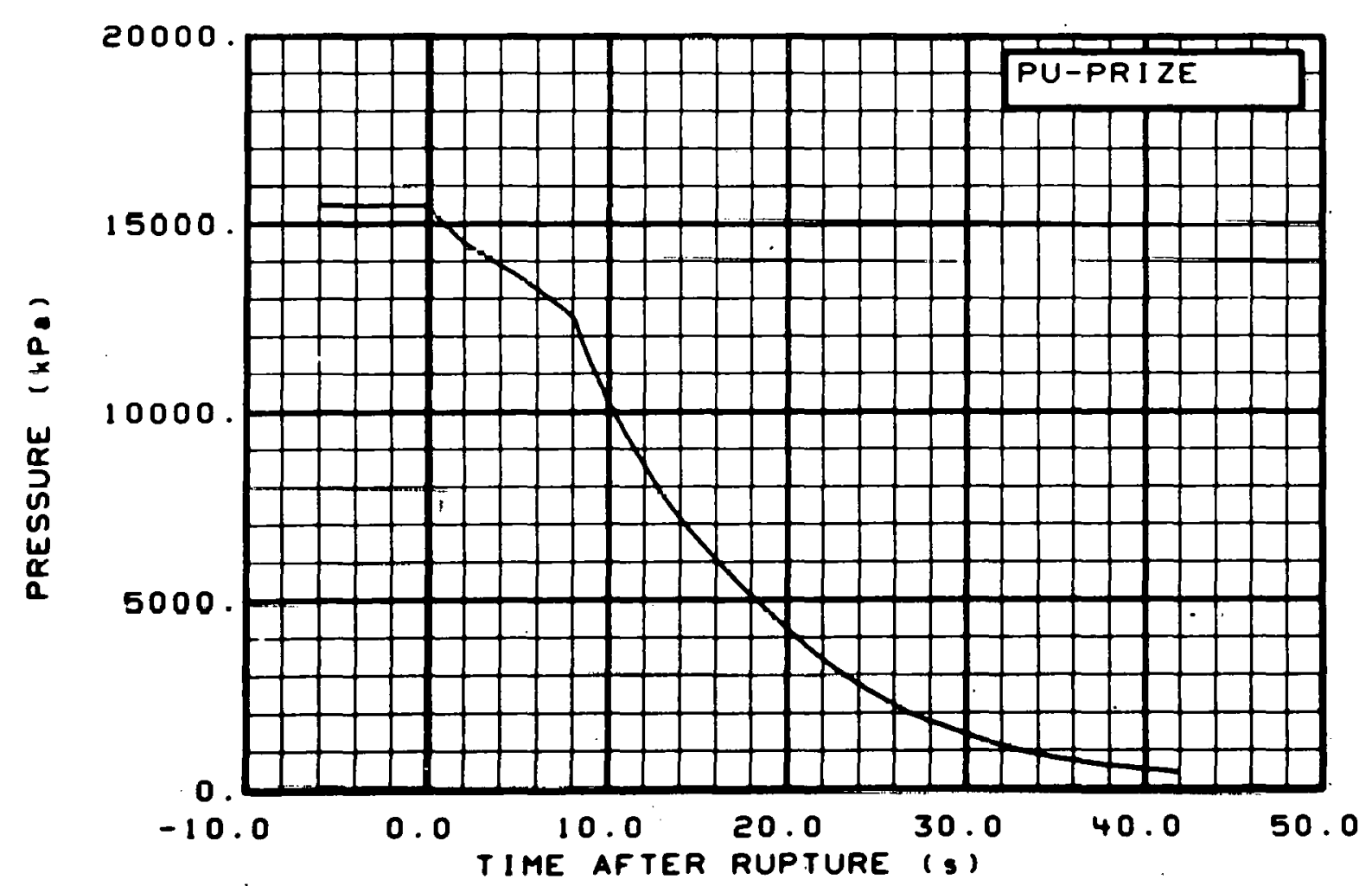

Fig. 138 Pressure in pressurizer (PU-PRIZE), from -6 to $42 \mathrm{~s}$. 


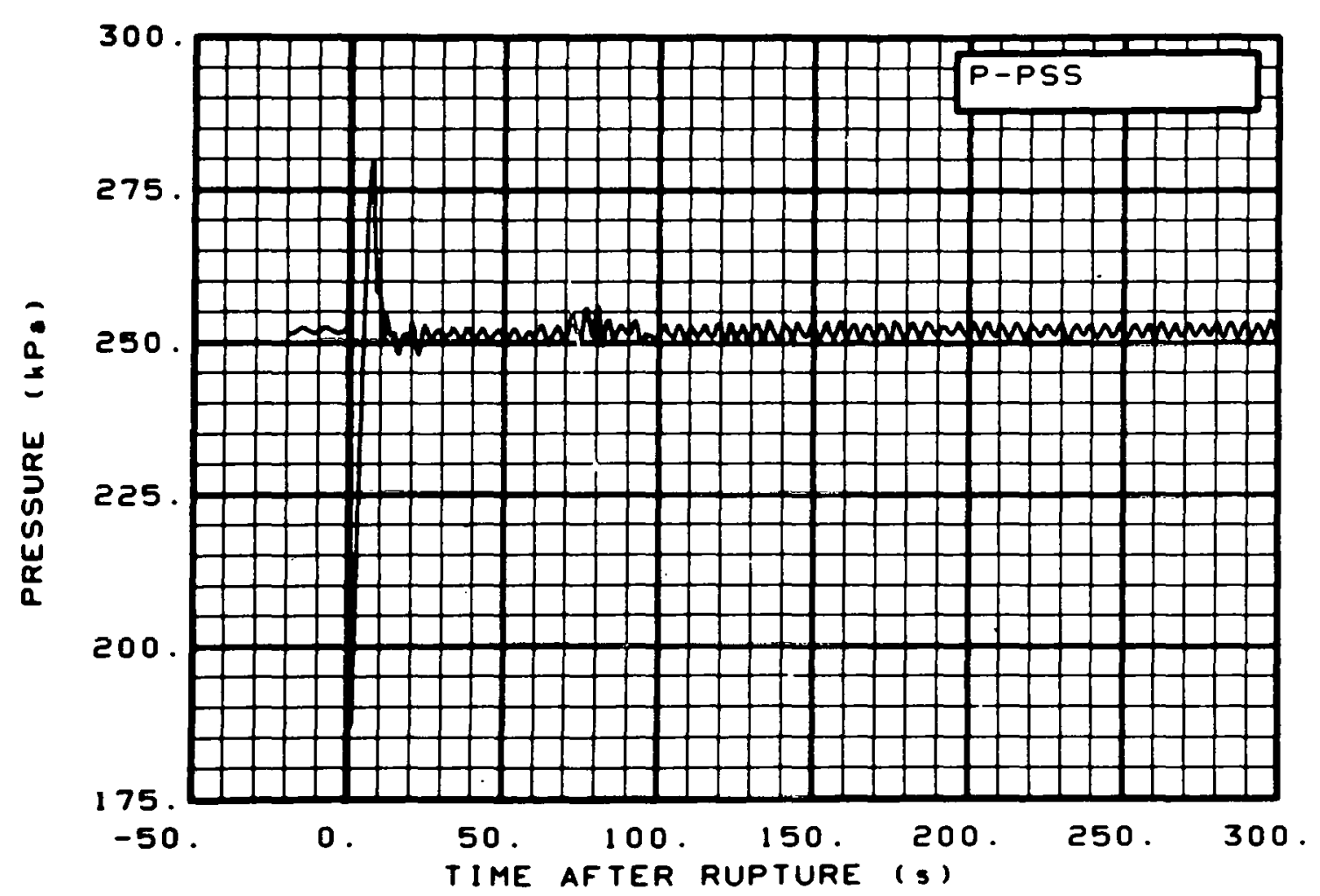

Fig. 139 Pressure in pressure suppression tank (P-PSS), from -20 to $300 \mathrm{~s}$.

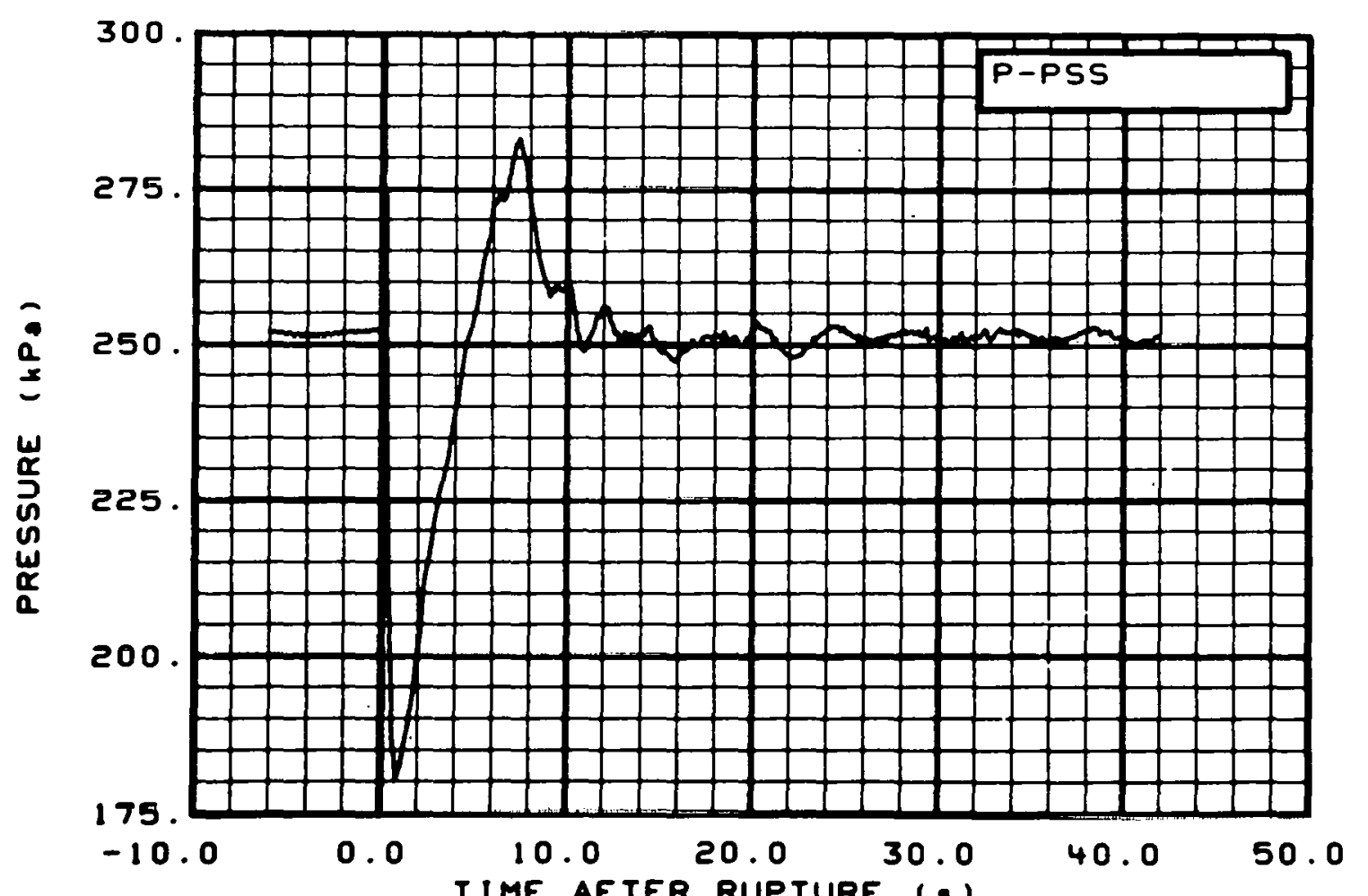

Fig. 140 Pressure in pressure suppression tank (P-PSS), from -6 to $42 \mathrm{~s}$. 


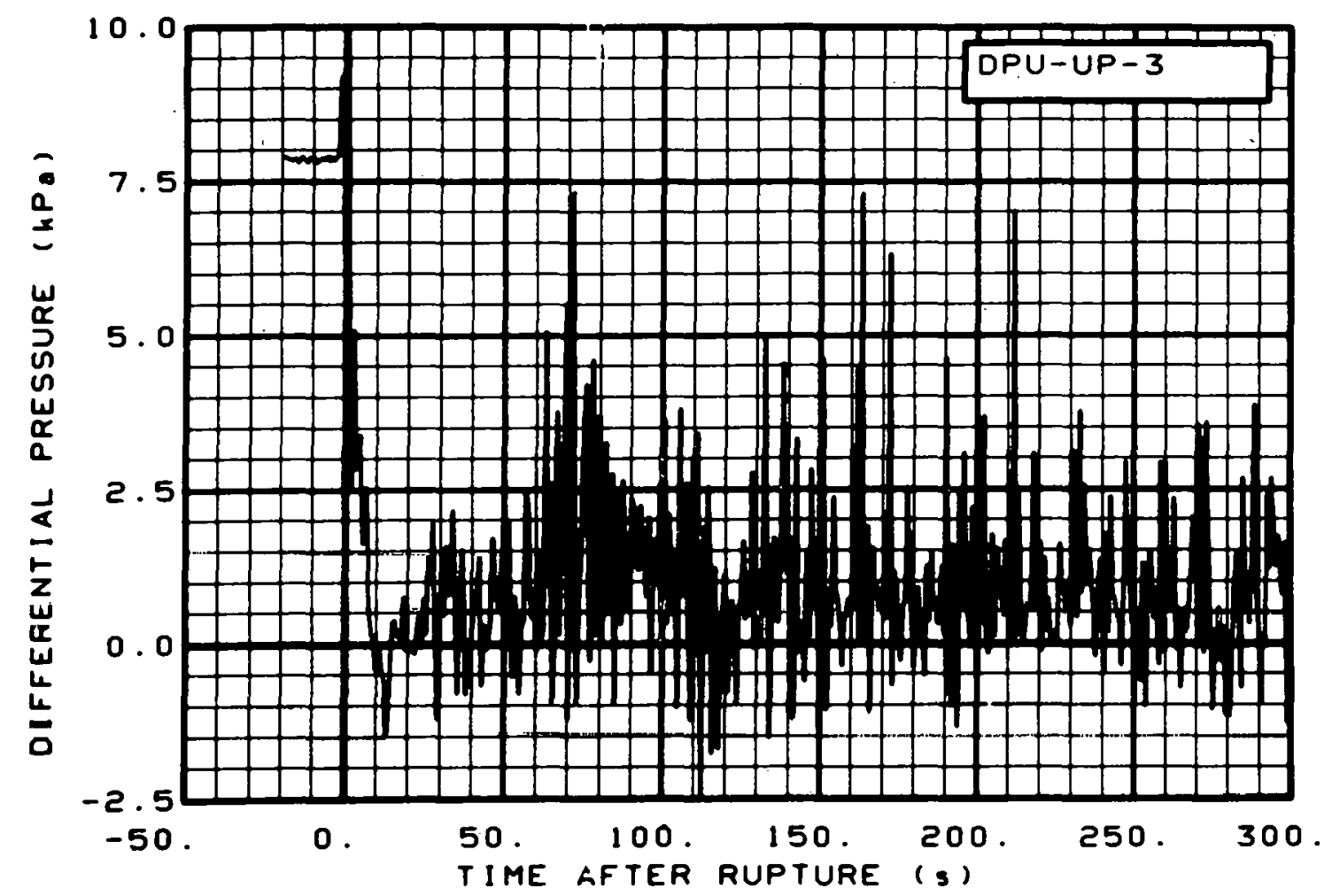

Fig. 141 Differential pressure in intact loop (DPU-UP-3), from -20 to $300 \mathrm{~s}$.

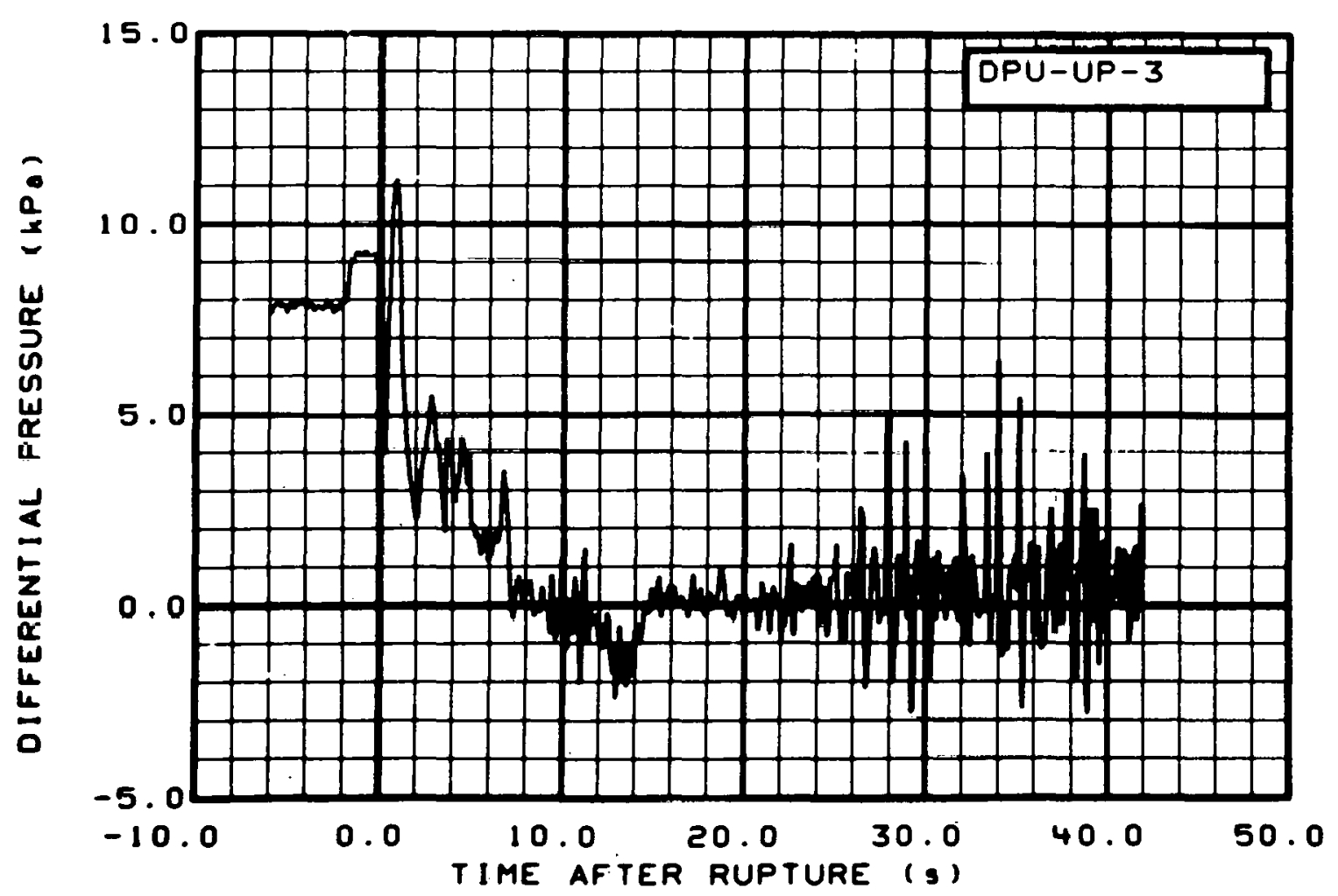

Fig. 142 Differential pressure in intact loop (DPU-UP-3), from -6 to $42 \mathrm{~s}$. 


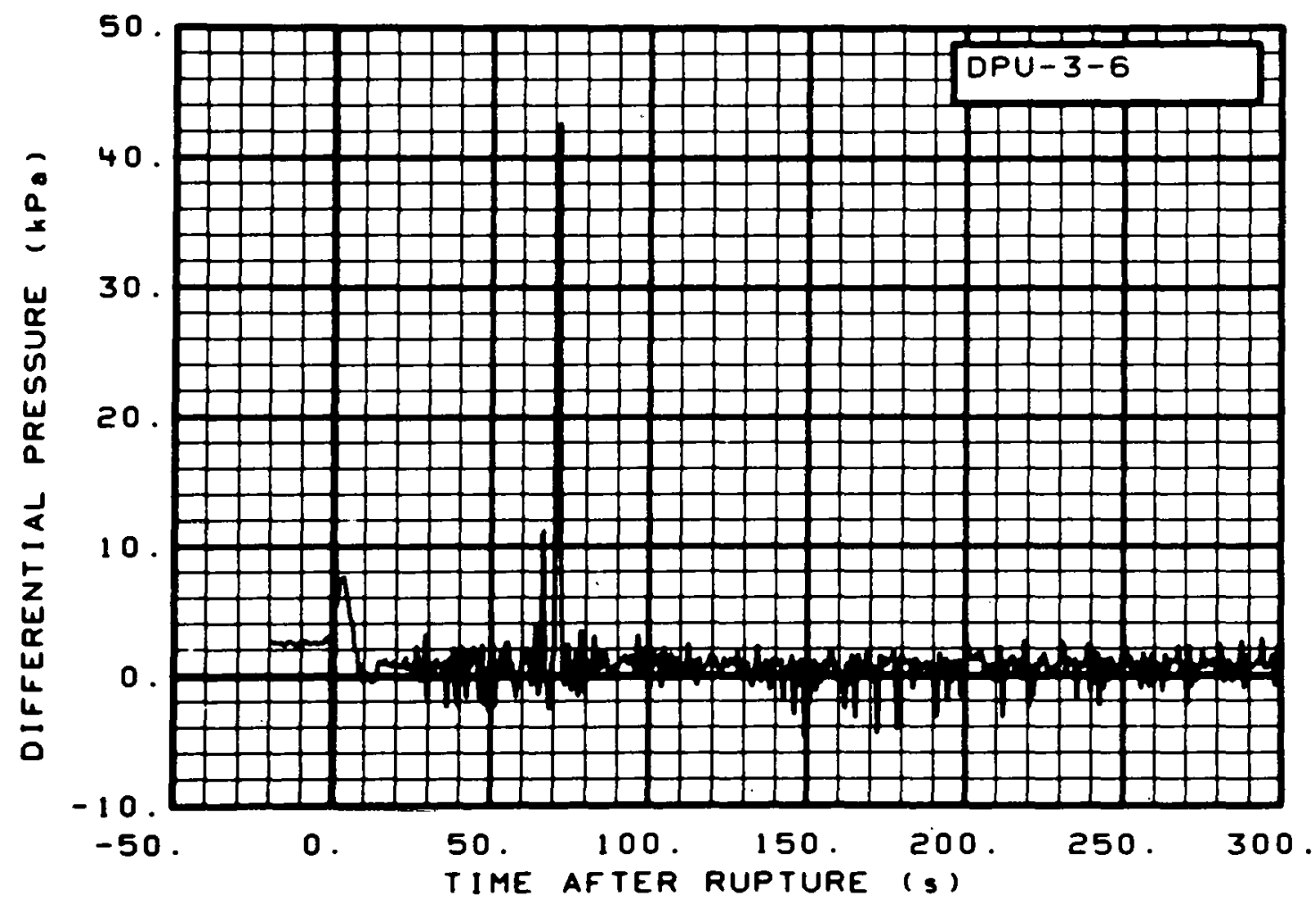

Fig. 143 Differential pressure in intact loop (DPU-3-6), from -20 to $300 \mathrm{~s}$.

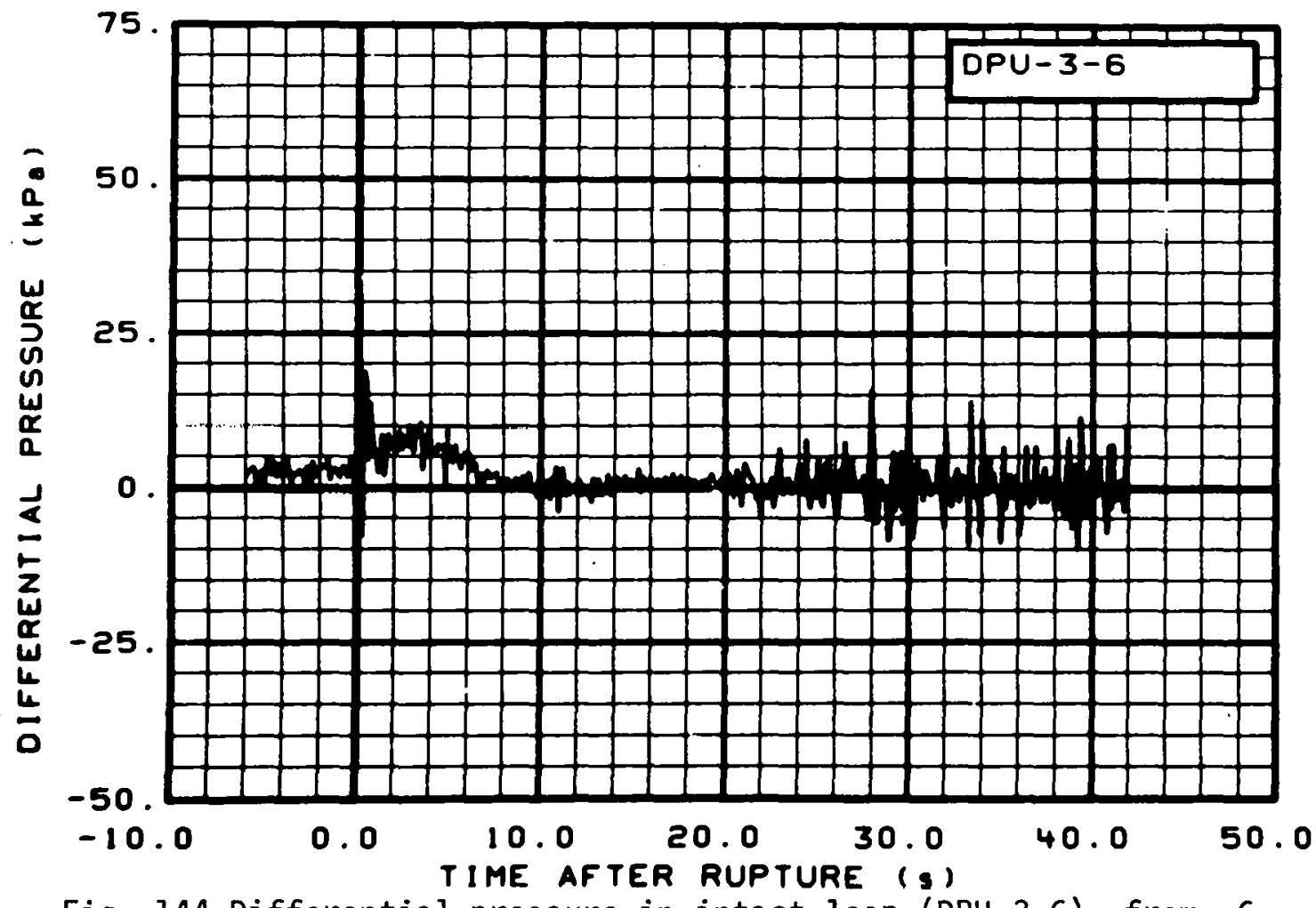

Fig. 144 Differential pressure in intact loop (DPU-3-6), from -6 to $42 \mathrm{~s}$. 


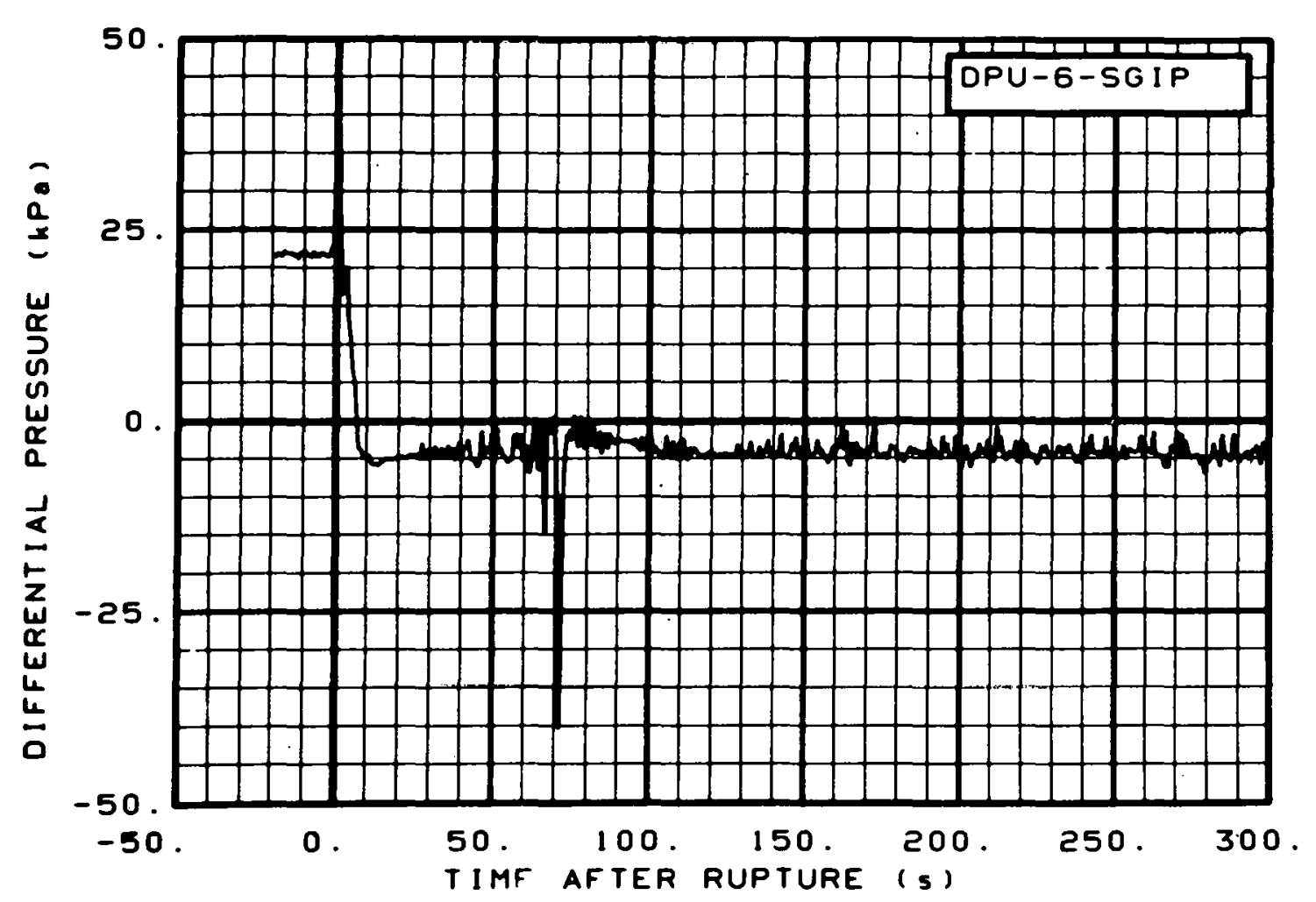

Fig. 145 Differential pressure in intact 10op (DPU-6-SGIP), from -20 to $300 \mathrm{~s}$.

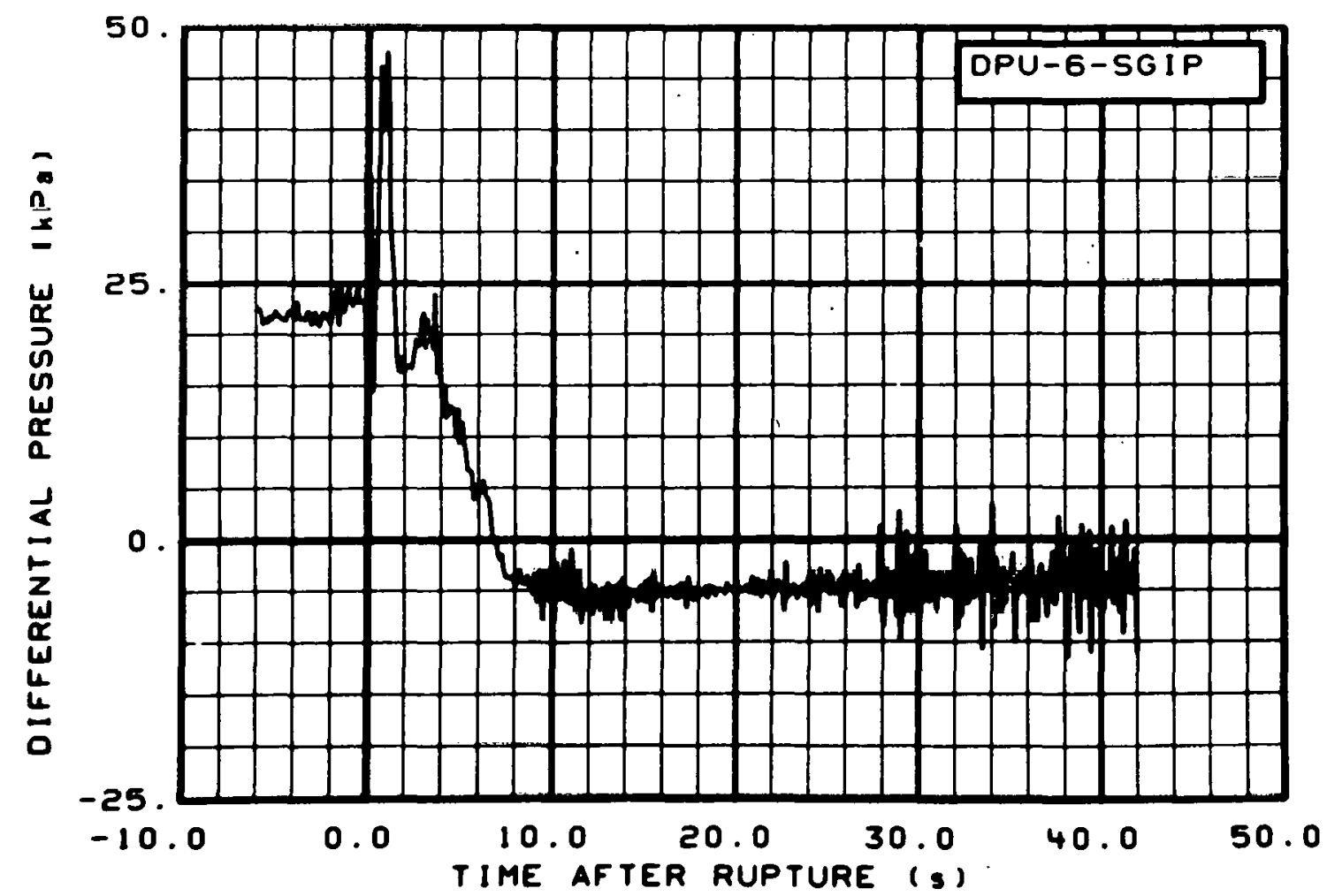

Fig. 146 Differential pressure in intact loop (DPU-6-SGIP), from -6 to $42 \mathrm{~s}$. 


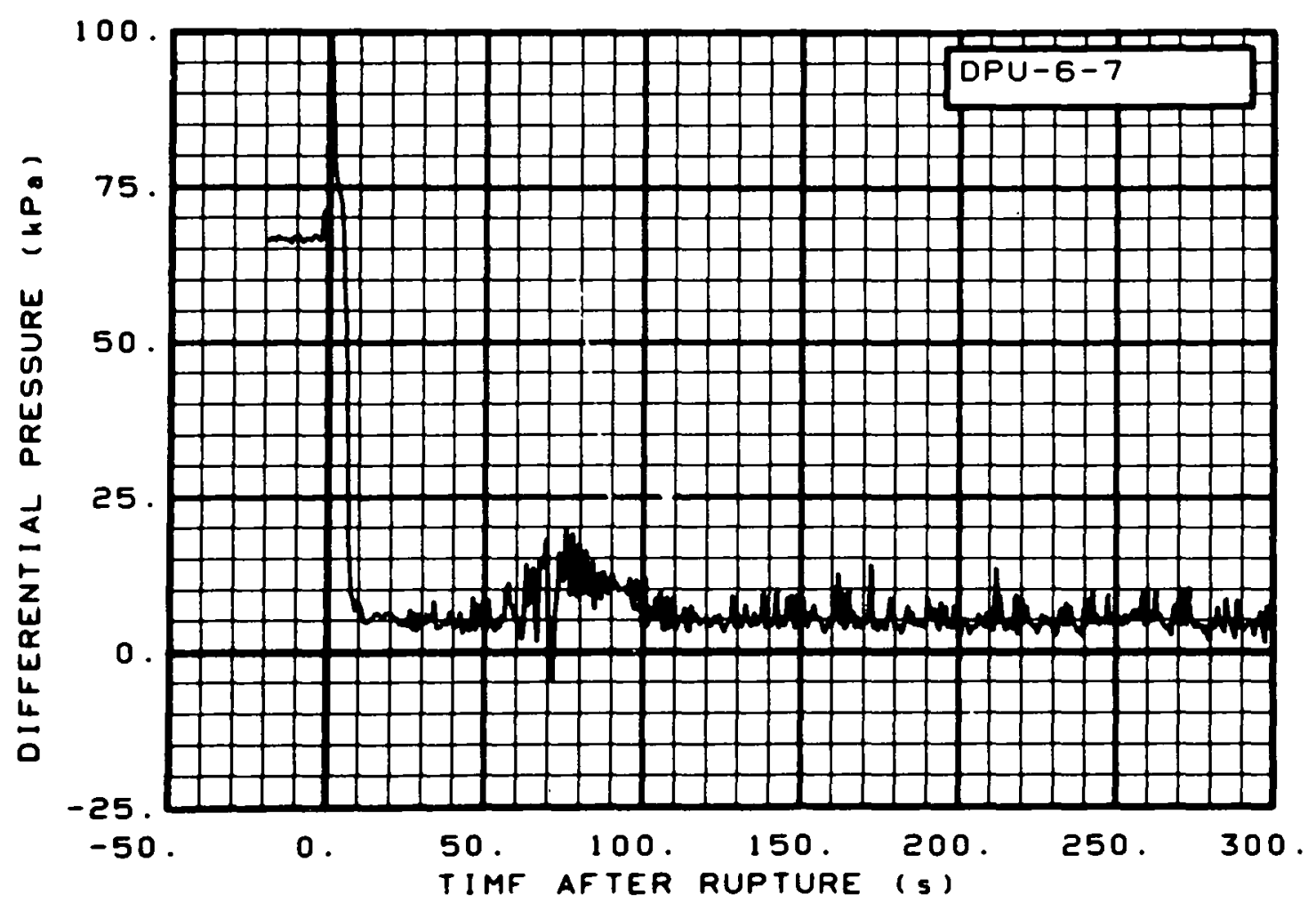

Fig. 147 Differential pressure in intact loop (DPU-6-7), from -20 to $300 \mathrm{~s}$.

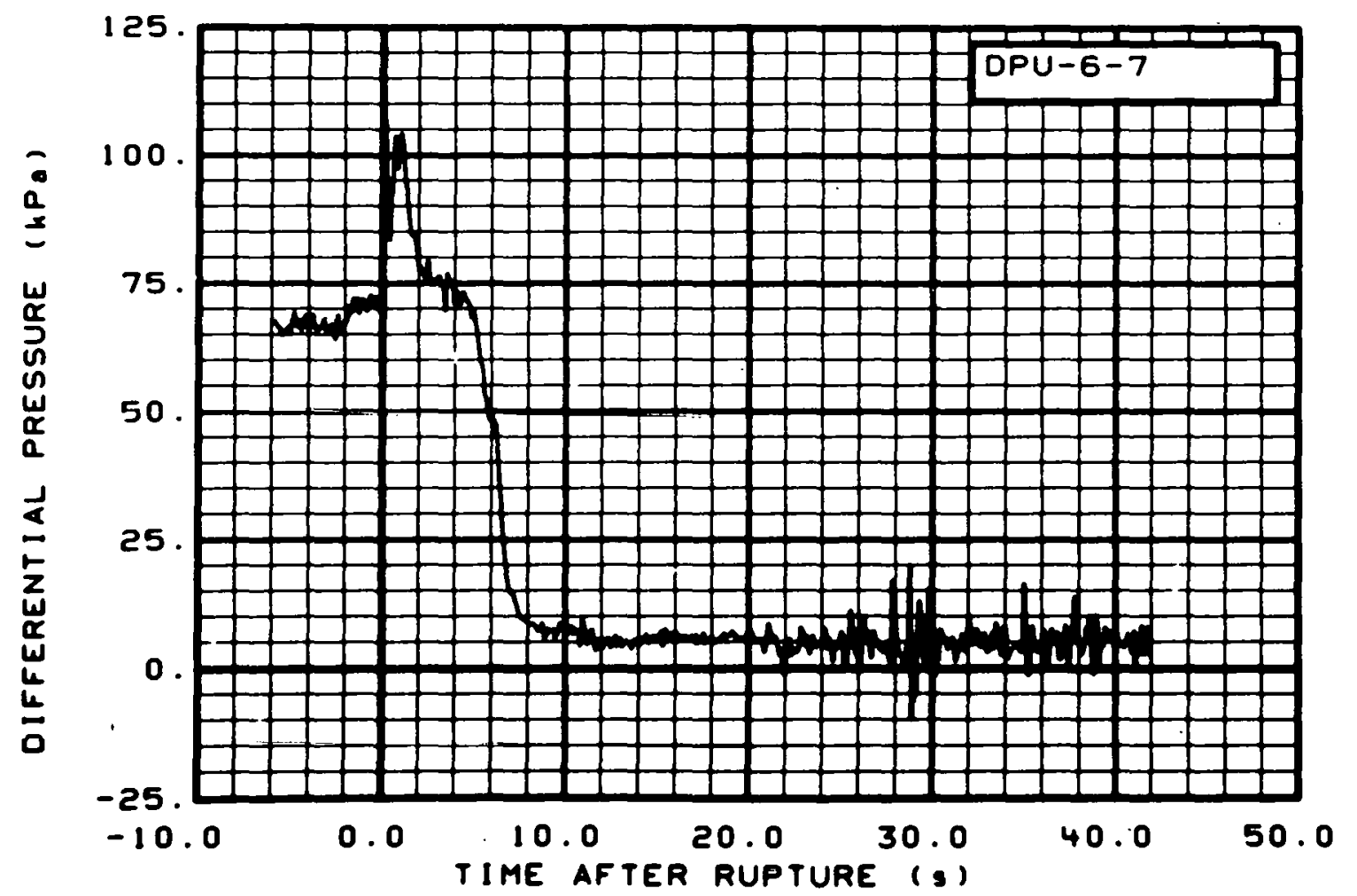

Fig. 148 Differential pressure in intact loop (DPU-6-7), from -6 to $42 \mathrm{~s}$. 


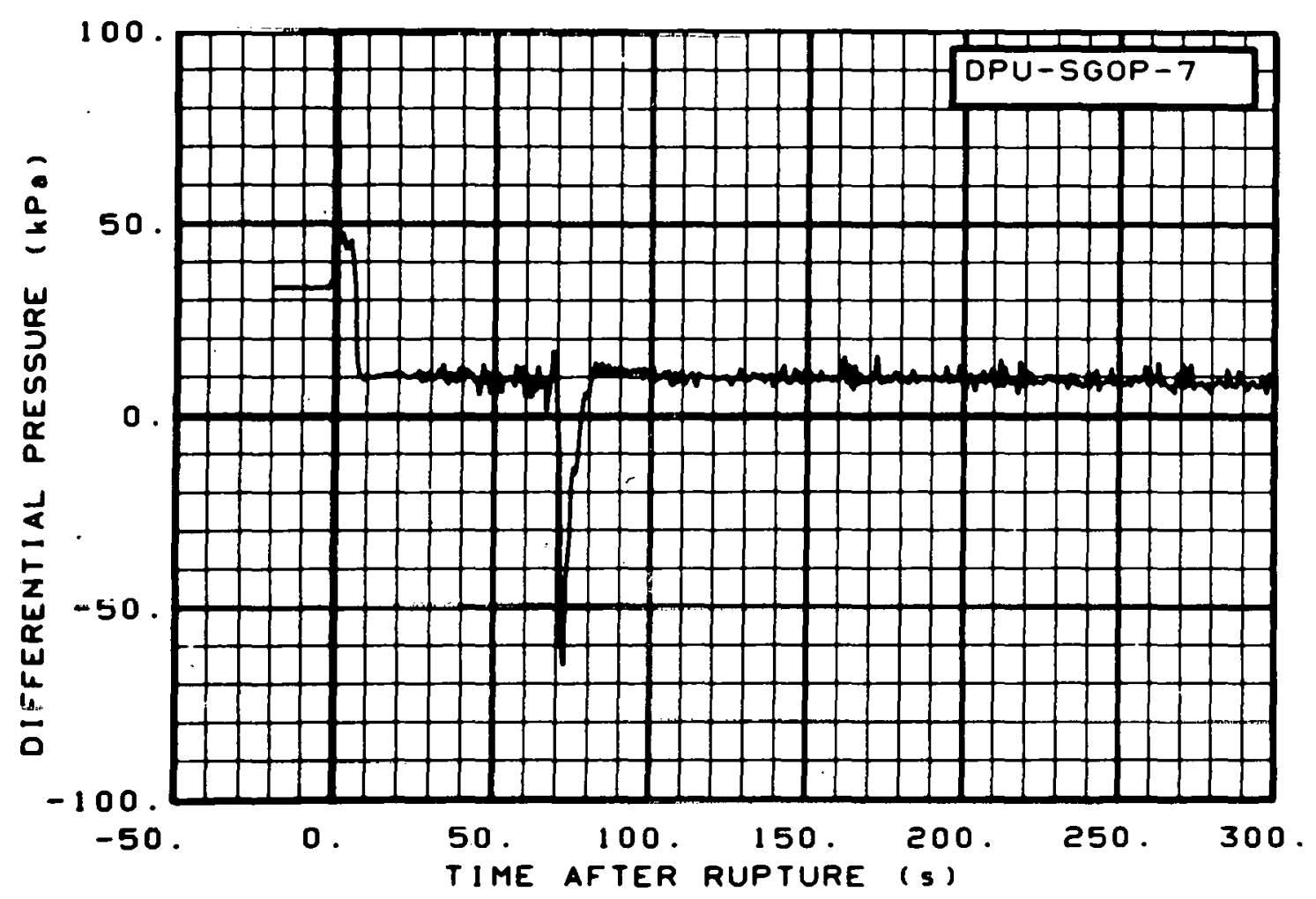

Fig. 149 Differential pressure in intact loop (DPU-SGOP-7), from -20 to $300 \mathrm{~s}$.

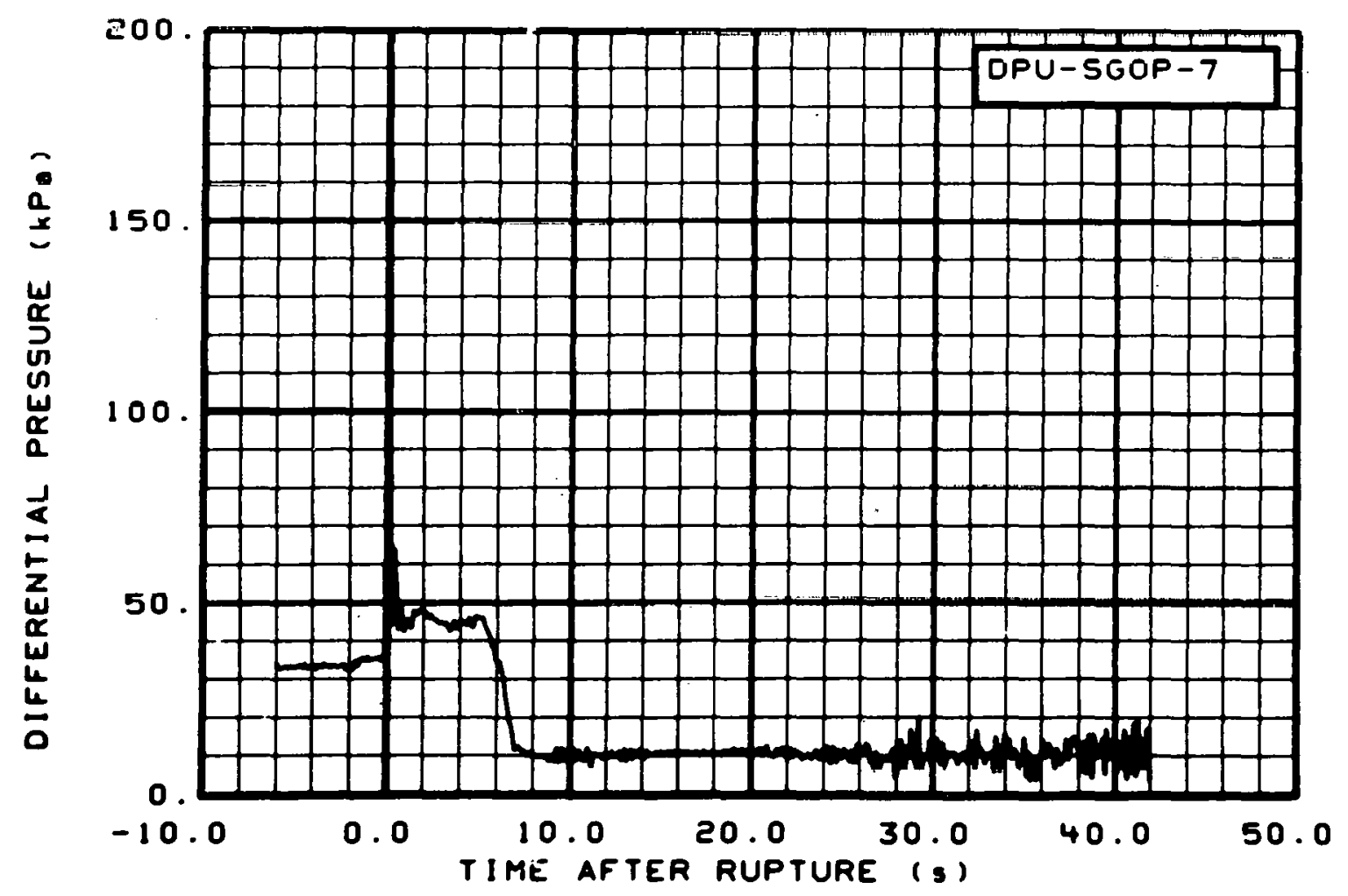

Fig. 150 Differential pressüre in intact loop (DPU-SGOP-7), from -6 to $42 \mathrm{~s}$. 


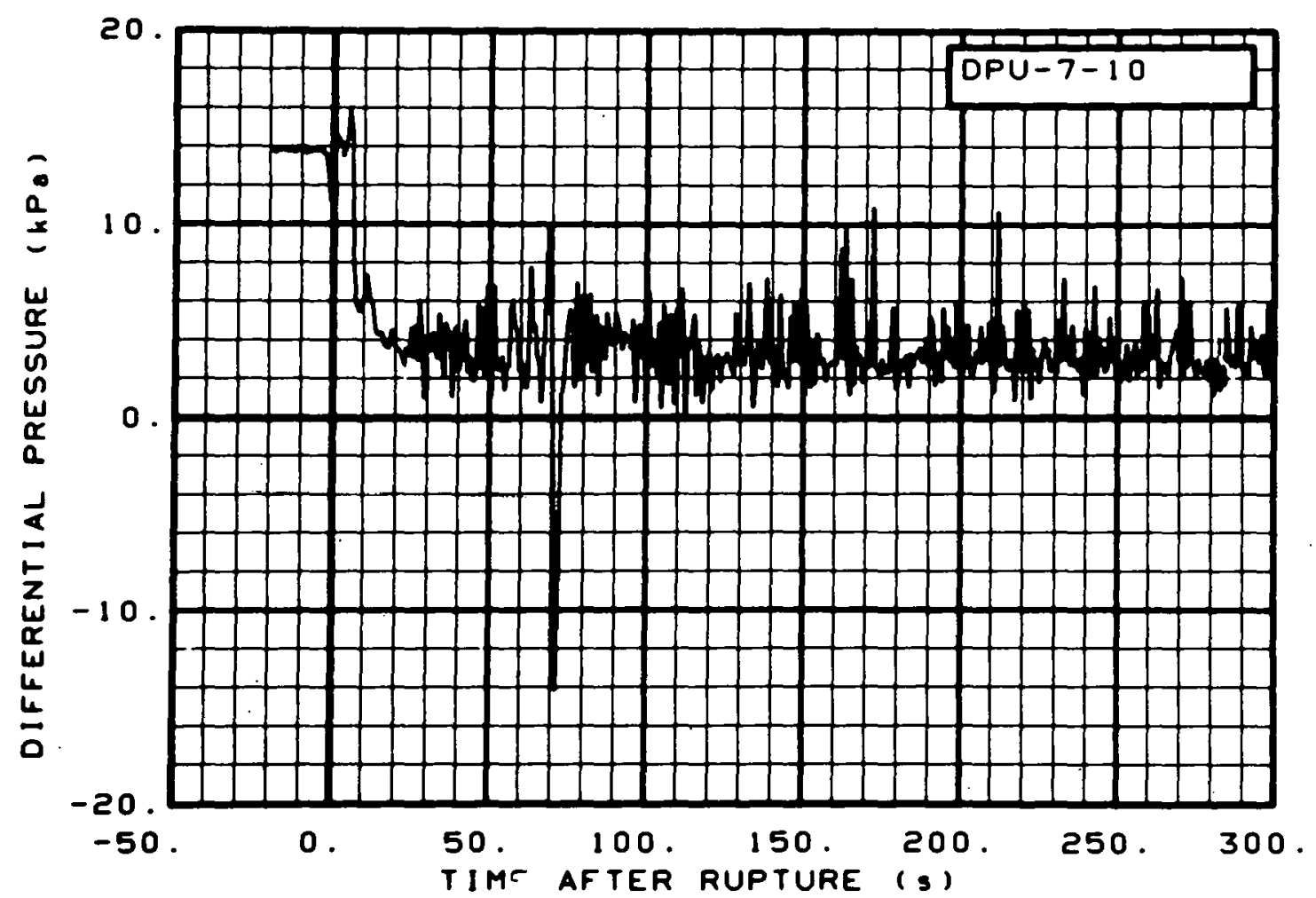

Fig. 151 Differential pressure in intact loop (DPU-7-10), from -20 to $300 \mathrm{~s}$.

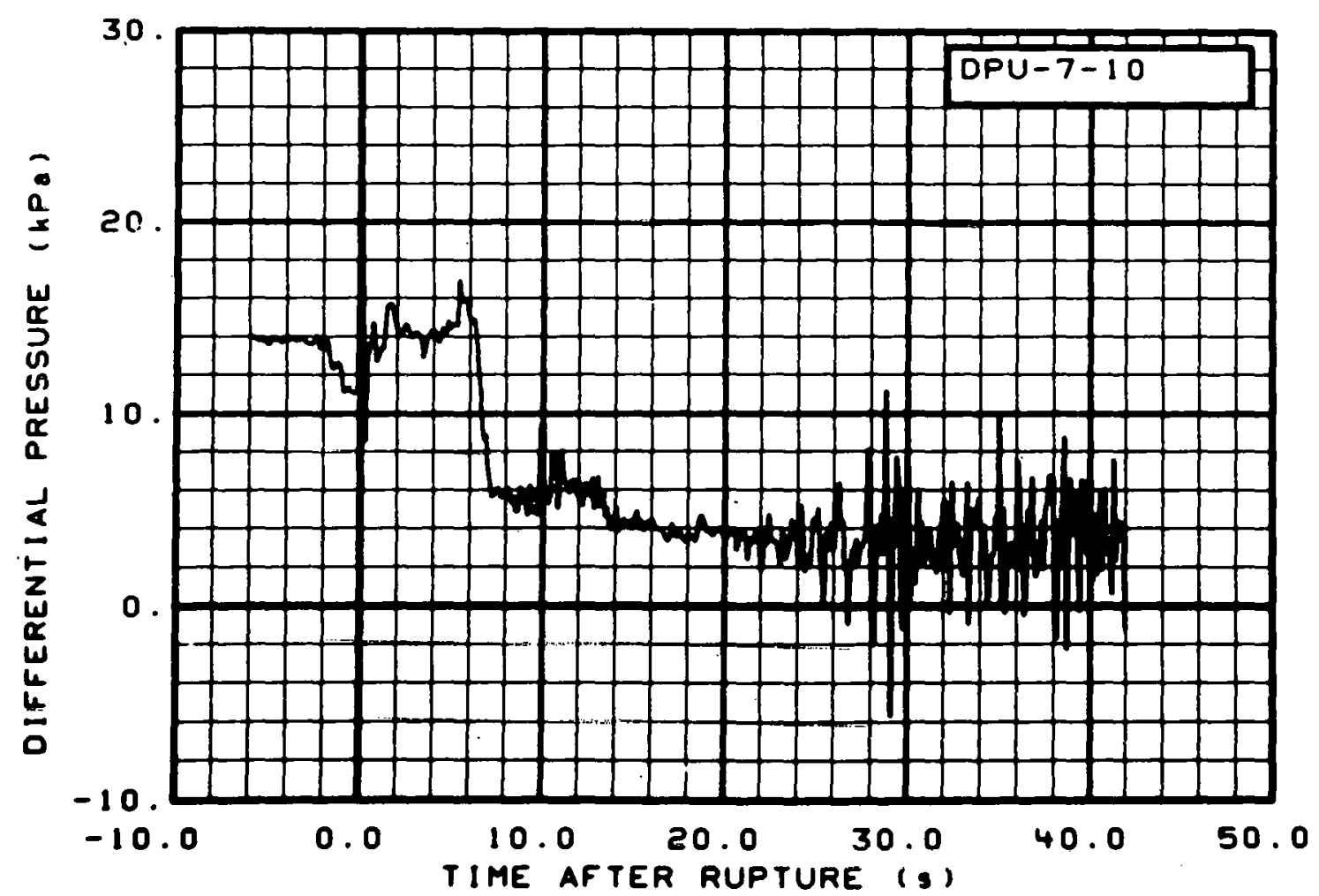

Fig. 152 Differential pressure in intact loop (DPU-7-10), from -6 to $42 \mathrm{~s}$. 


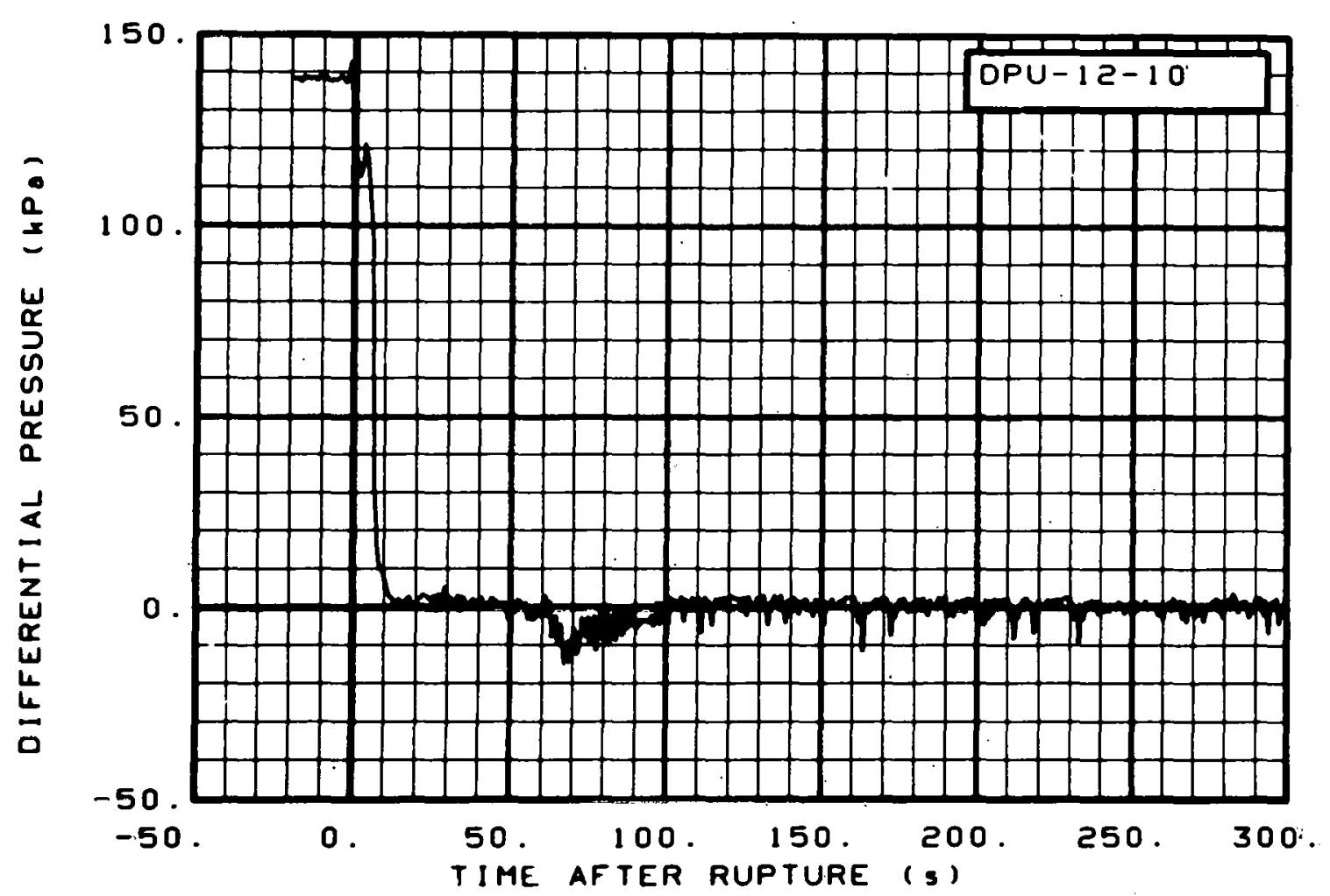

Fig. 153 Differential pressure in intact loop (DPU-12-10), from -20 to $300 \mathrm{~s}$.

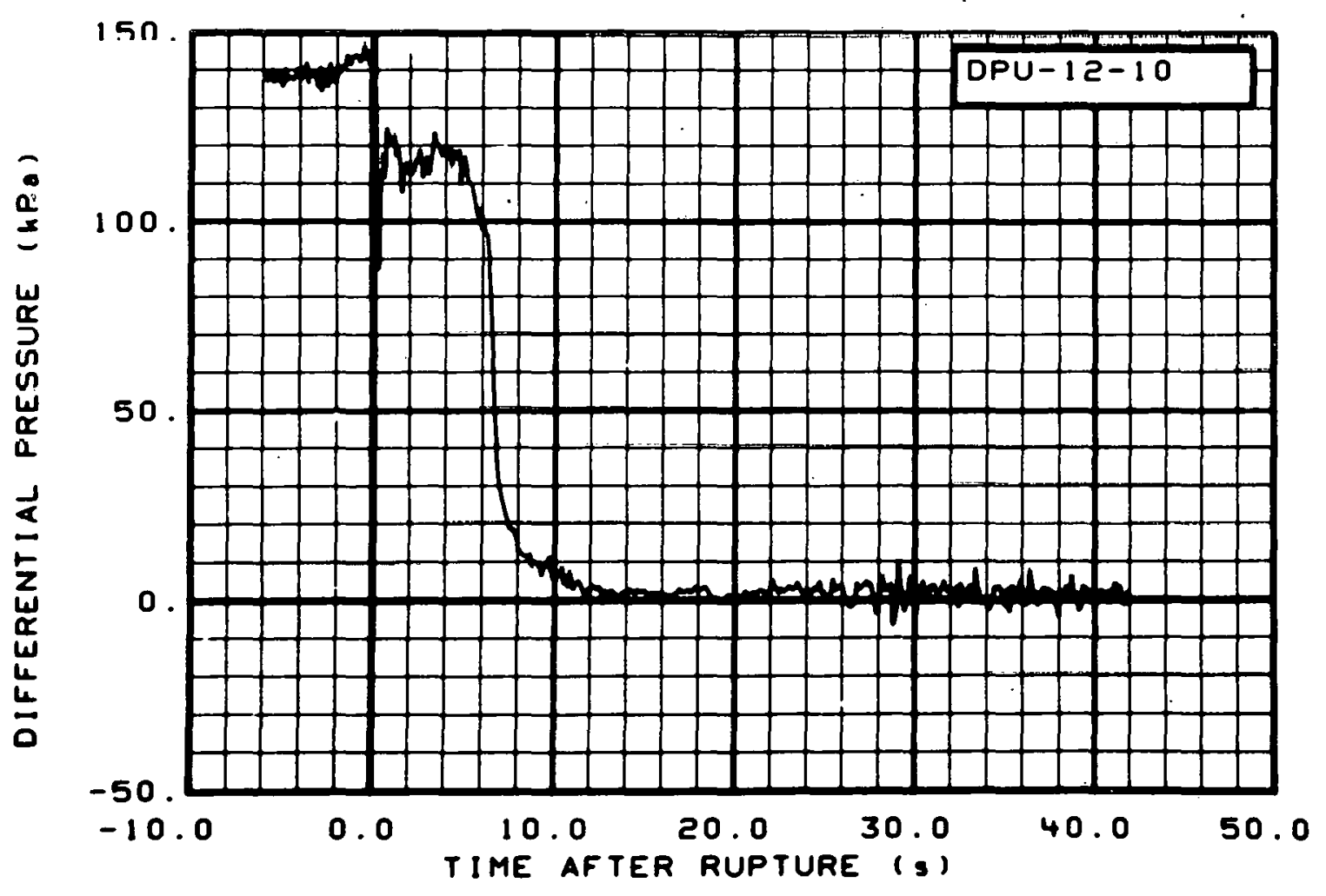

Fig. 154 Differential pressure in intact loop (DPU-12-10), from -6 to $42 \mathrm{~s}$. 


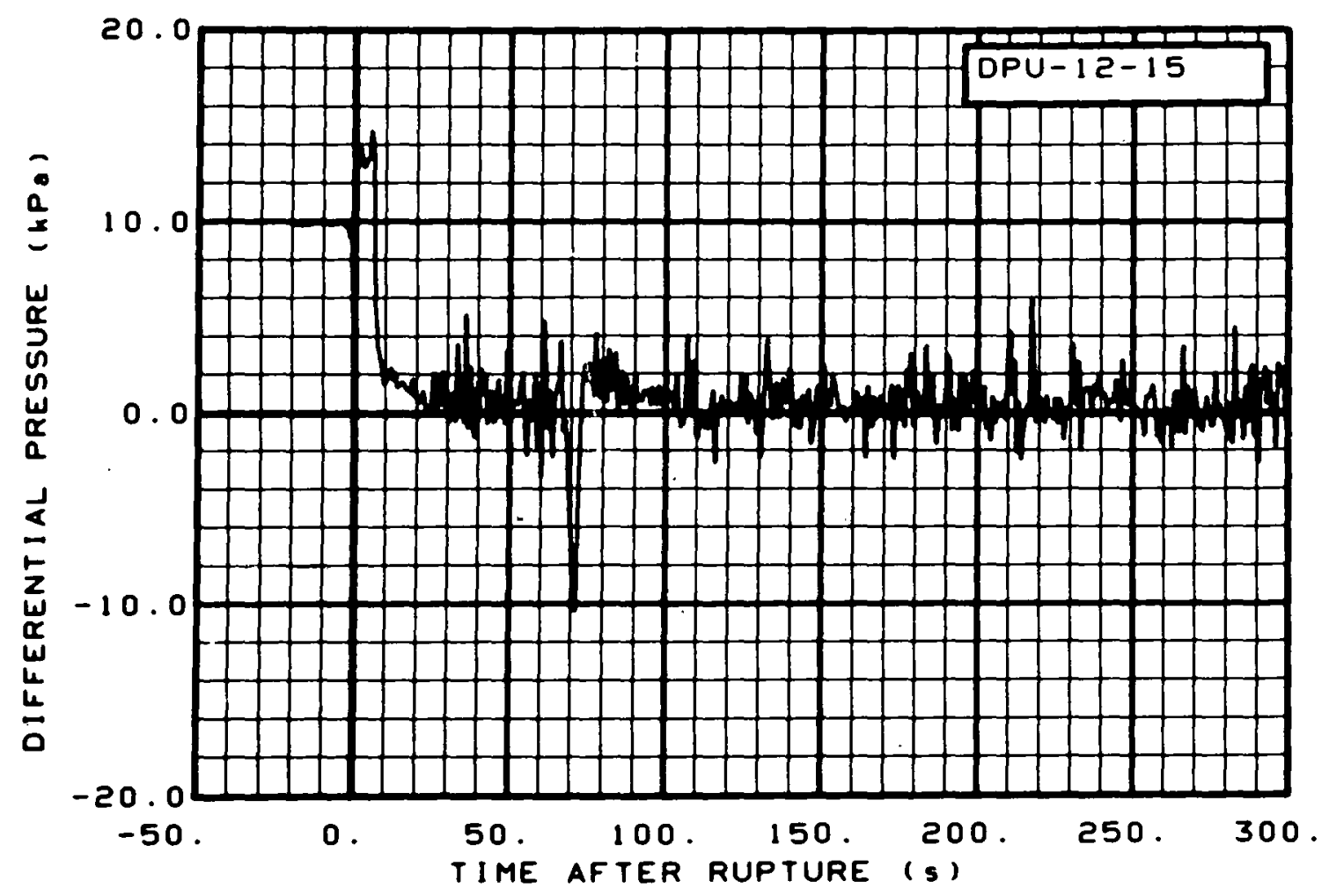

Fig. 155 Differential pressure in intact loop (DPU-12-15), from -20 to $300 \mathrm{~s}$.

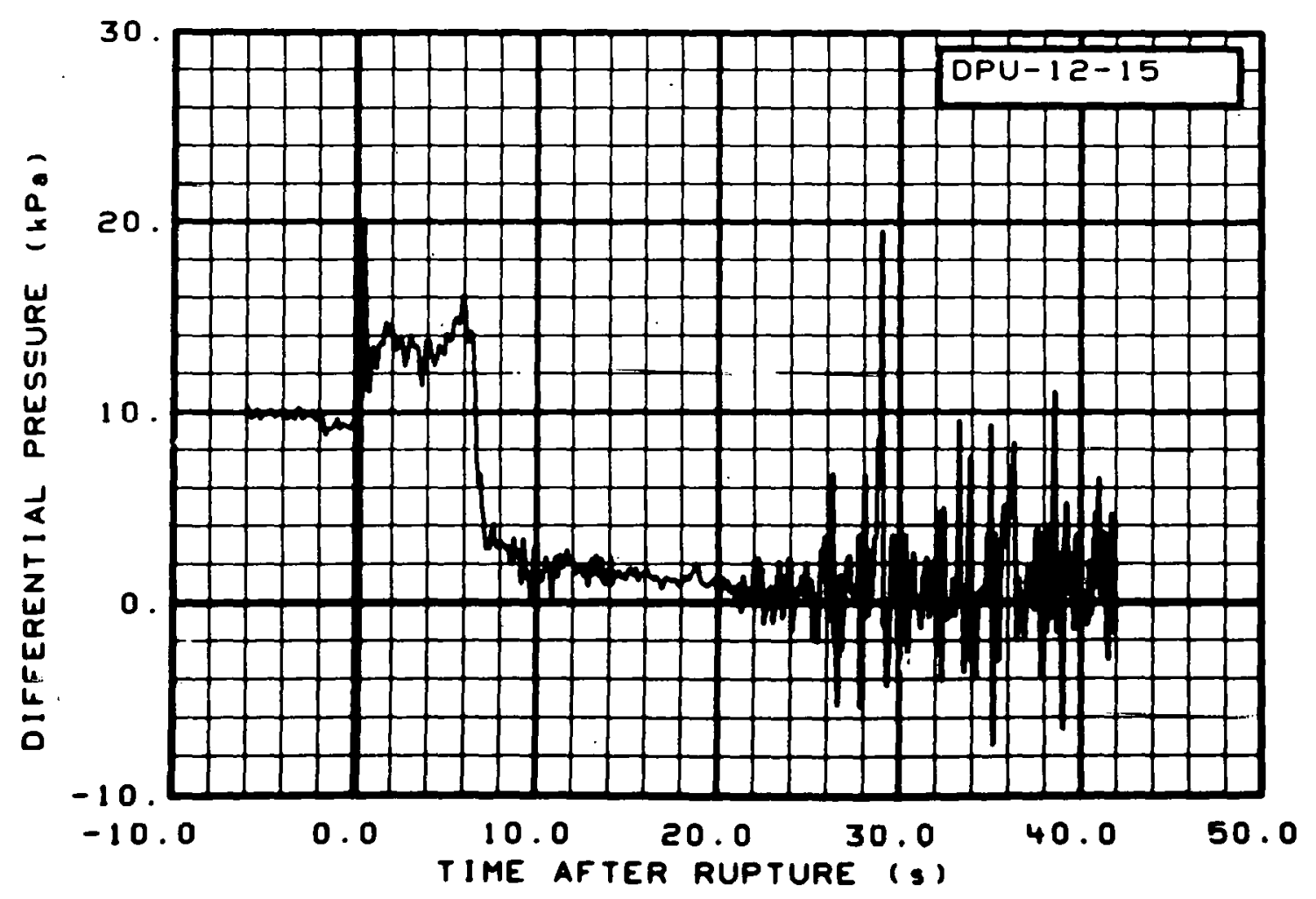

Fig. 156 Differential pressure in intact loop (DPU-12-15), from -6 to $42 \mathrm{~s}$. 


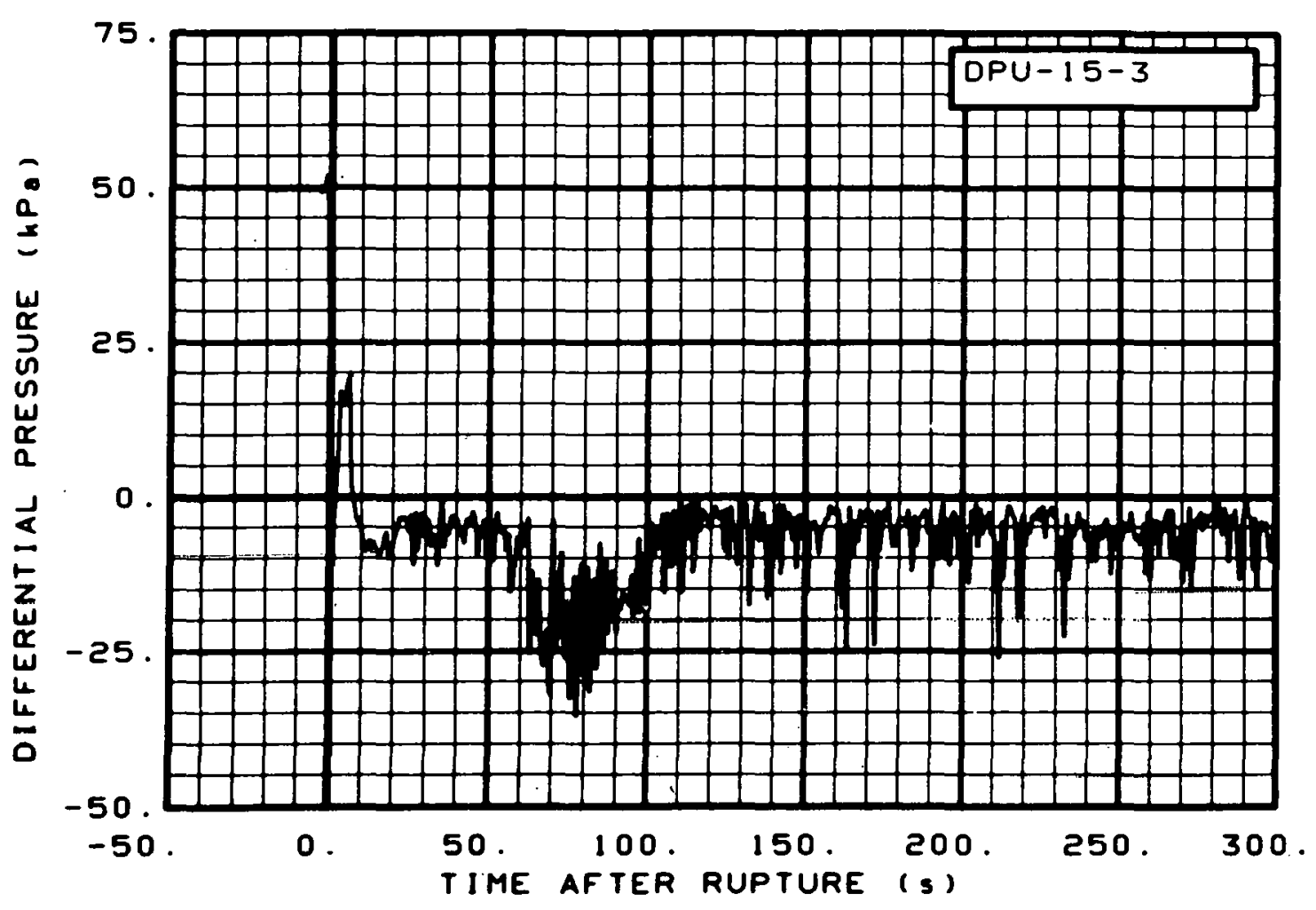

Fig. 157 Differential pressure in intact loop (DPU-15-3), from -20 to $300 \mathrm{~s}$.

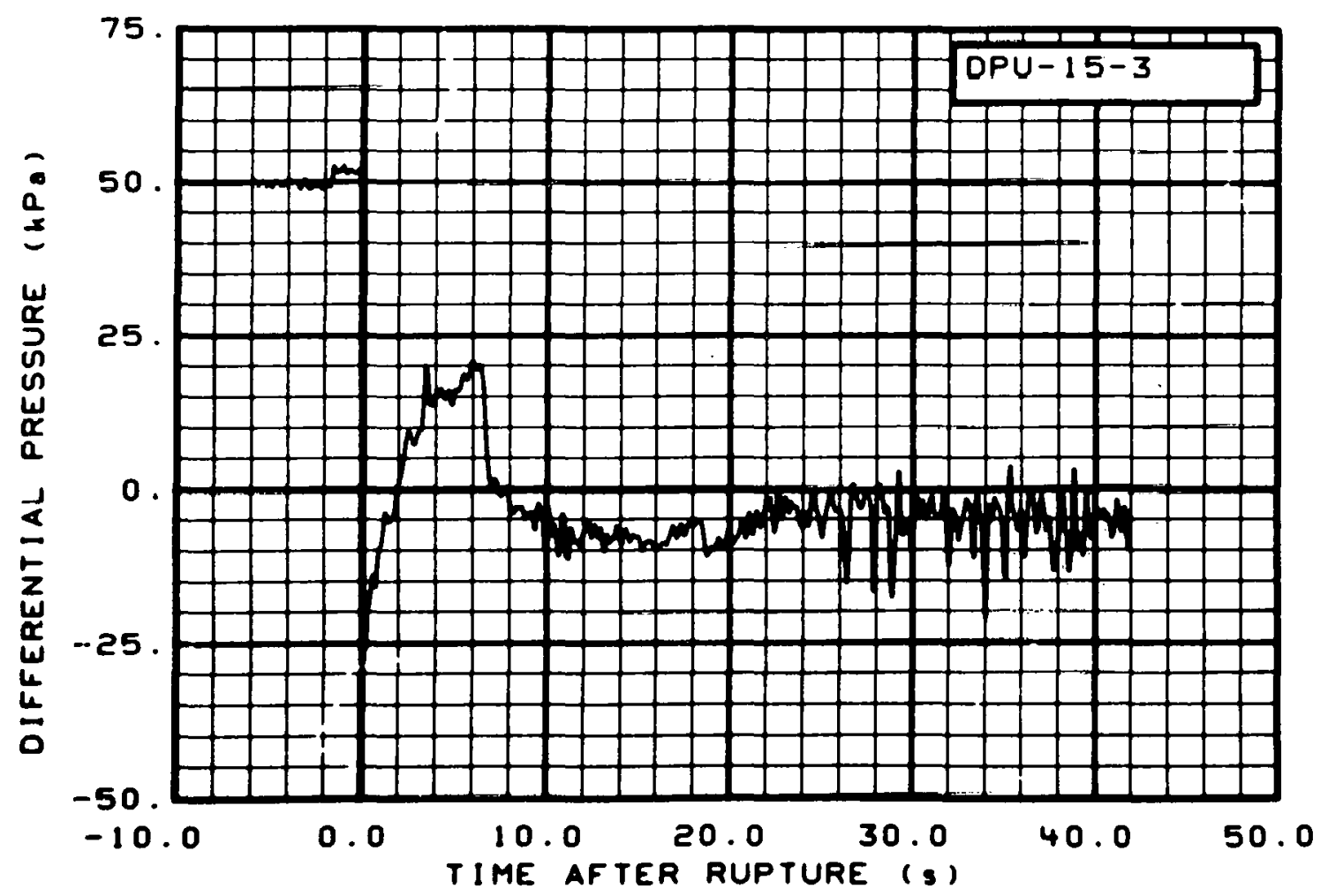

Fig. 158 Differential pressure in intact loop (DPU-15-3), from -6 to $42 \mathrm{~s}$. 


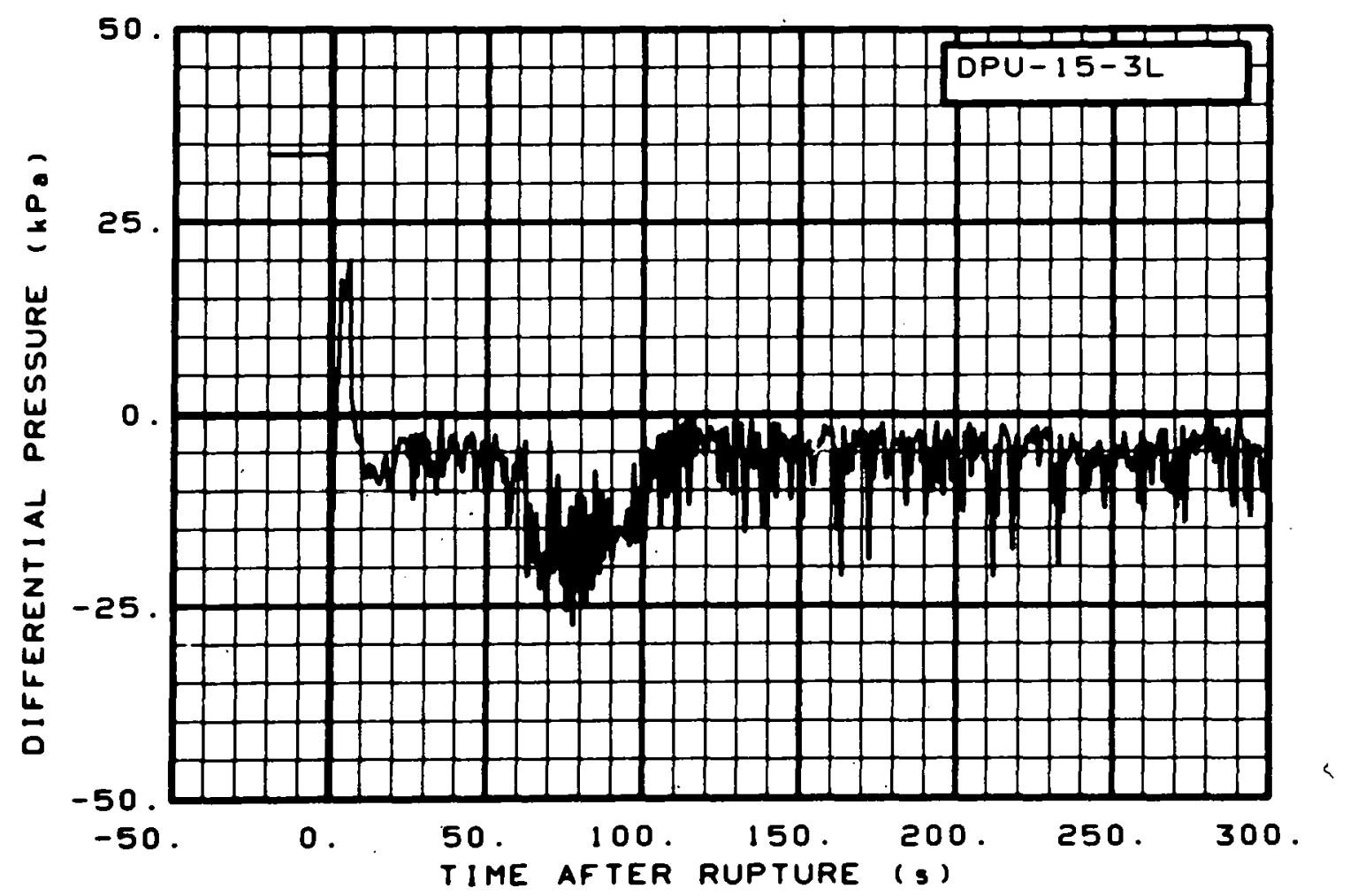

Fig. 159 Differential pressure in intact loop, low range (DPU-15-3L), from -20 to $300 \cdot \mathrm{s}$.

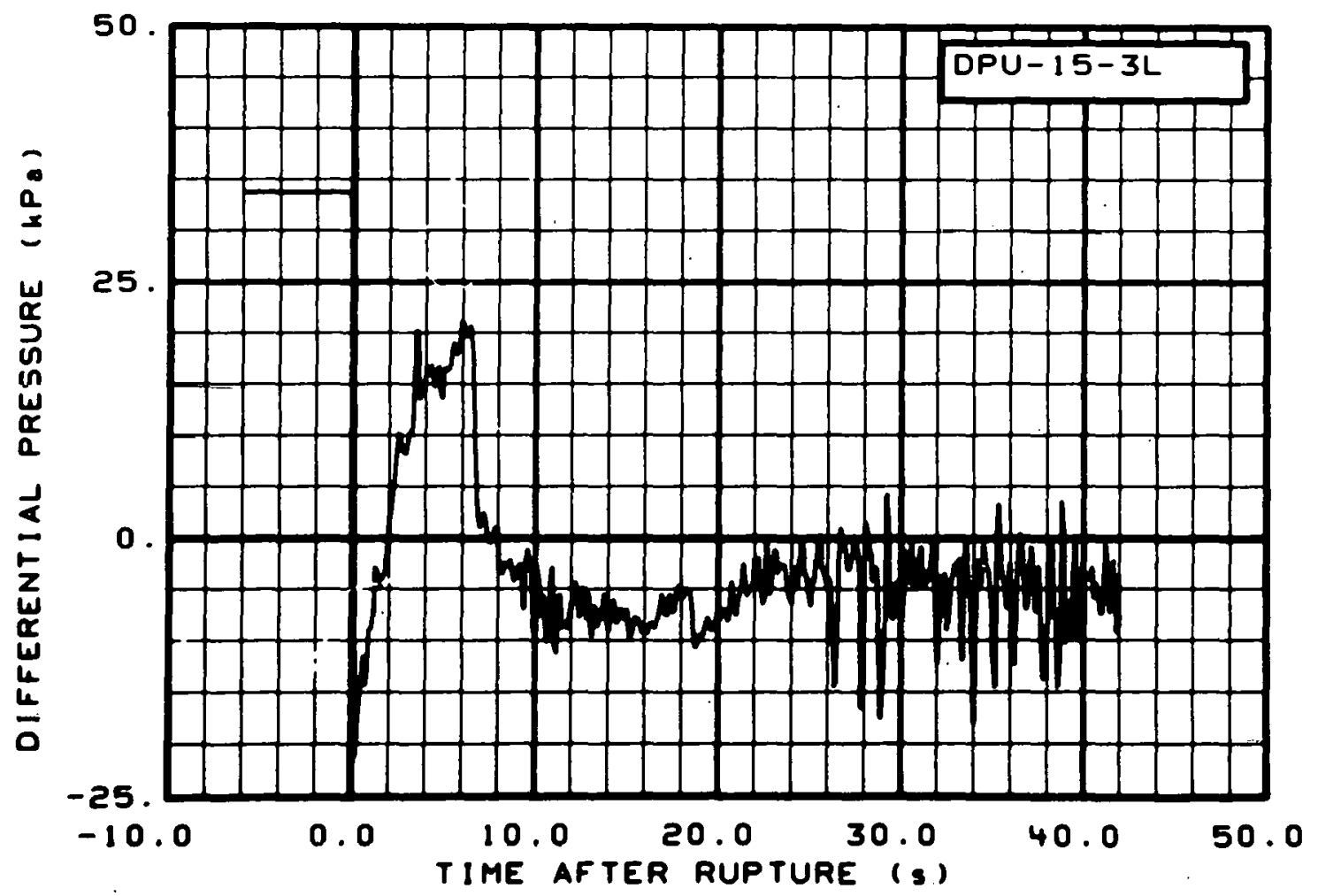

Fig. 160 Differential pressure in intact loop, low range (DPU-15-3L), from -6 to $42 \mathrm{~s}$. 


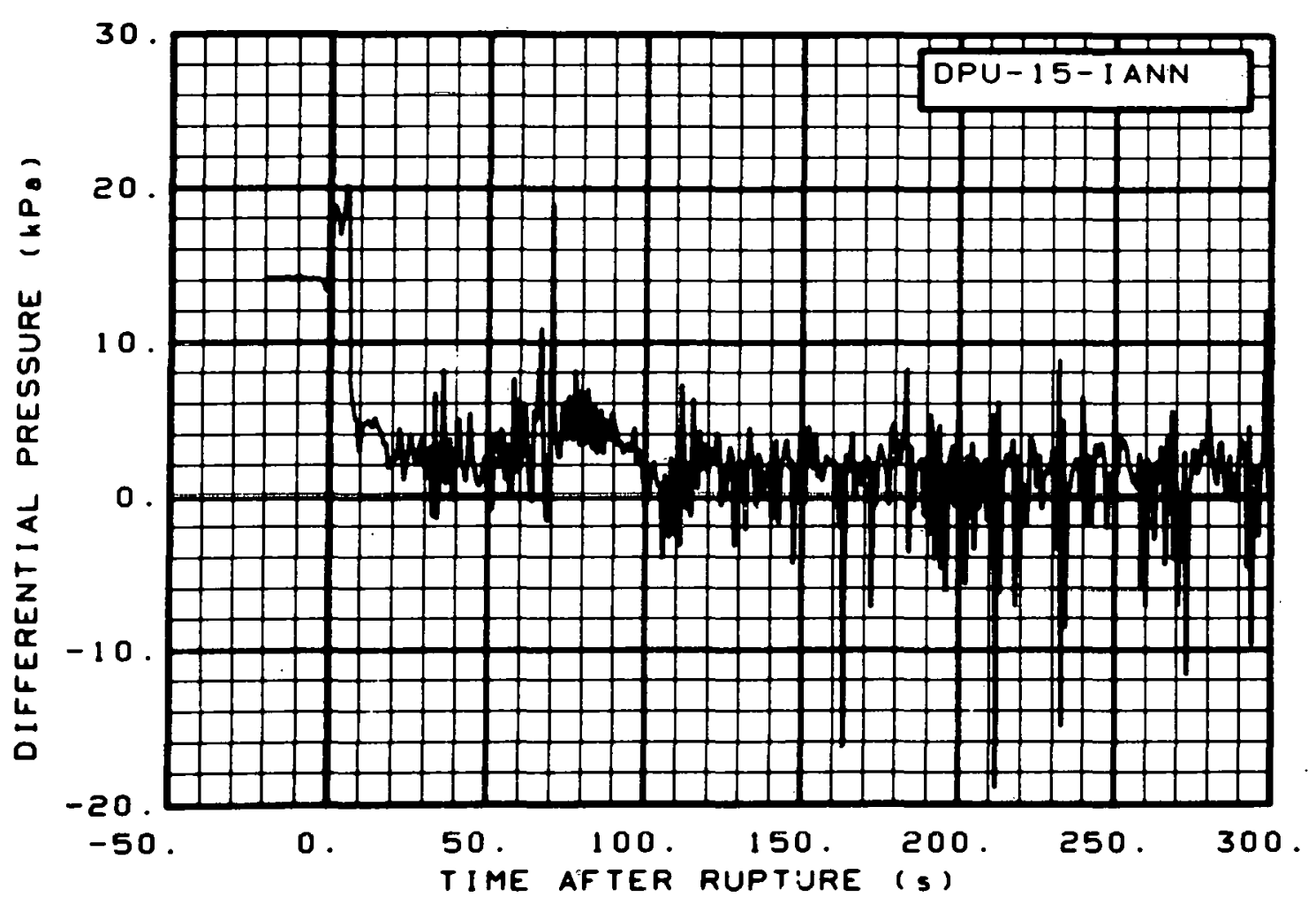

Fig. 161 Differential pressure in intact loop (DPU-15-IANN), from -20 to $300 \mathrm{~s}$.

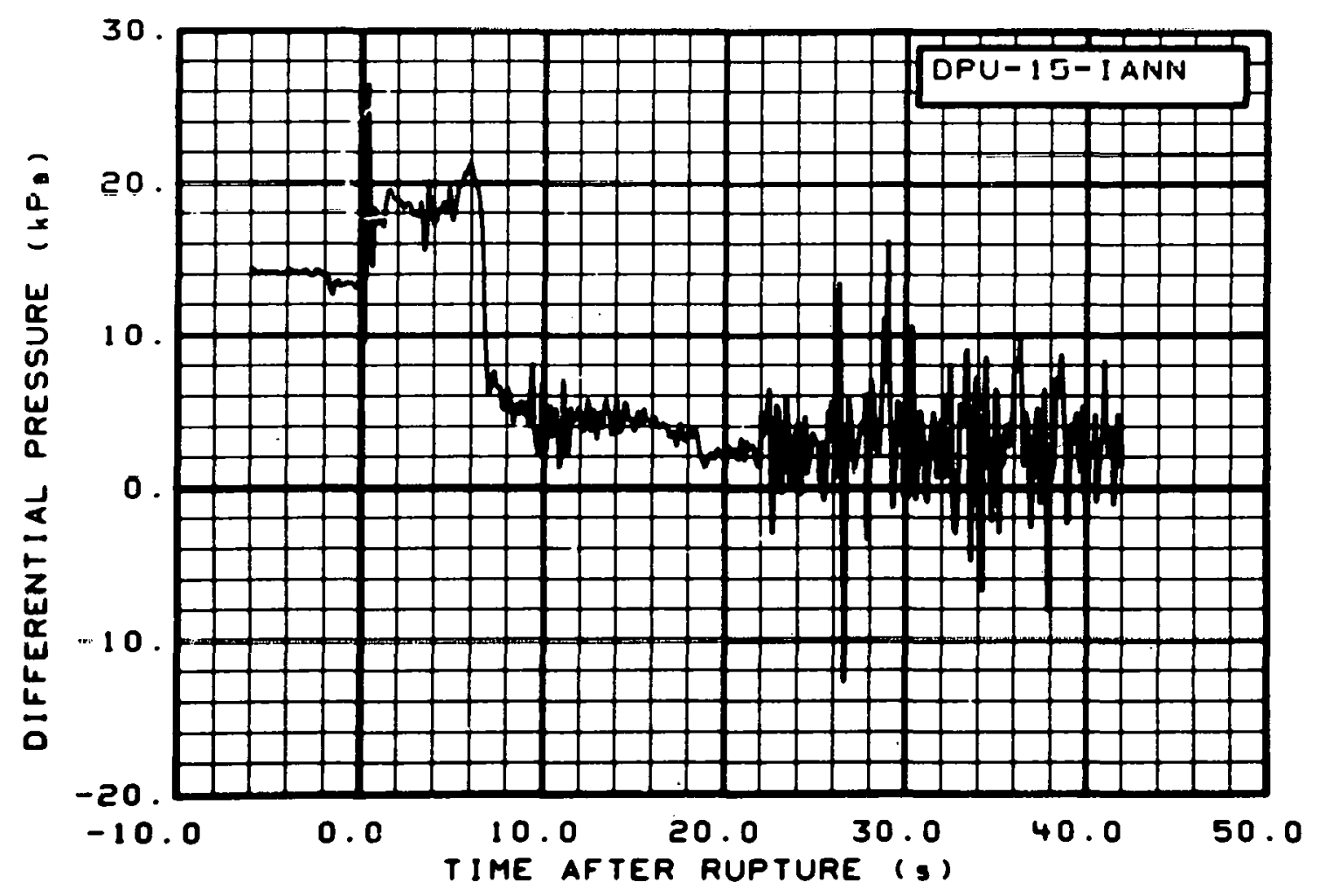

Fig. 162 Differential pressure in intact loop (DPU-15-IANN), from -6 to $42 \mathrm{~s}$. 


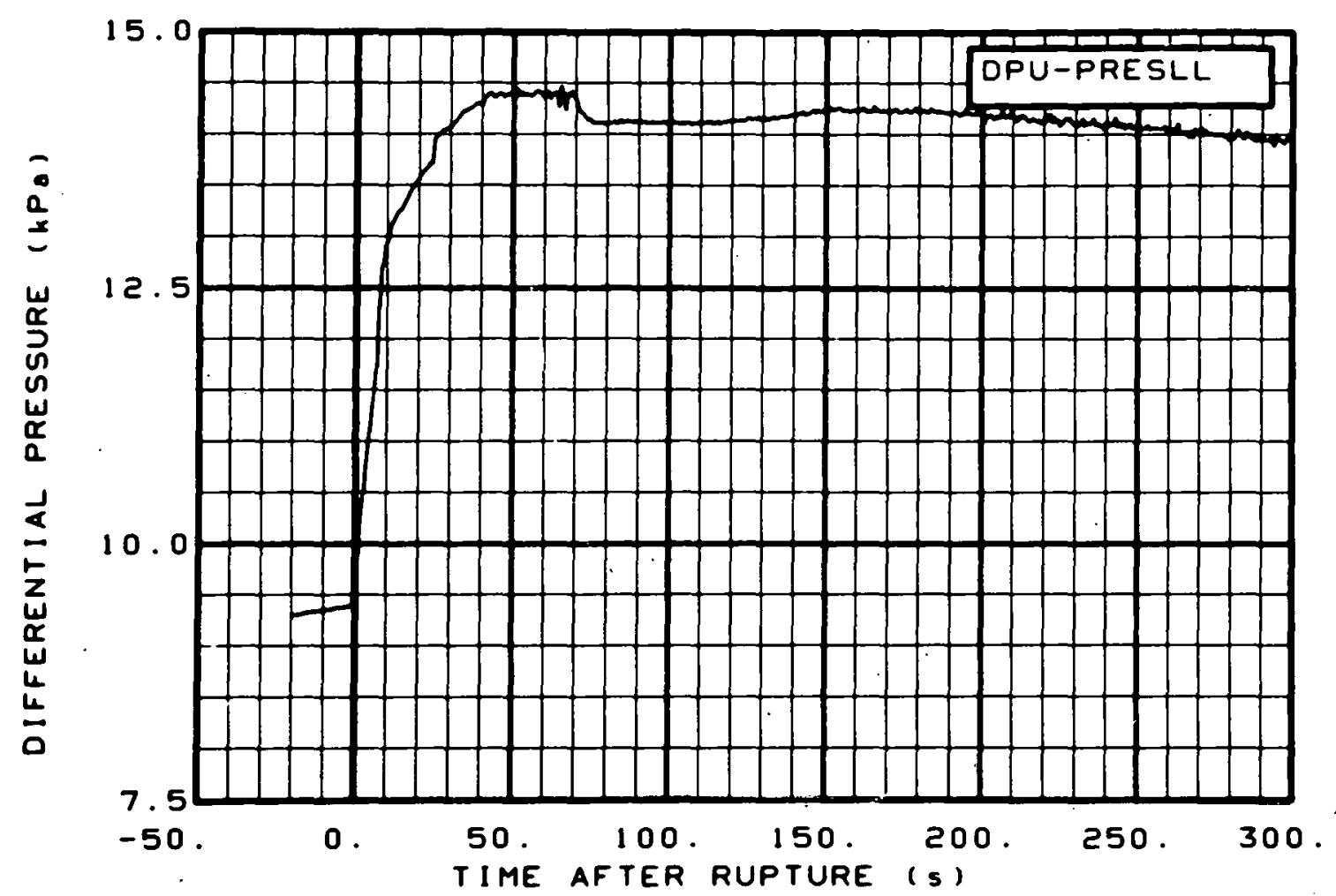

Fig. 163 Differential pressure in intact loop (DPU-PRESLL), from -20 to $300 \mathrm{~s}$.

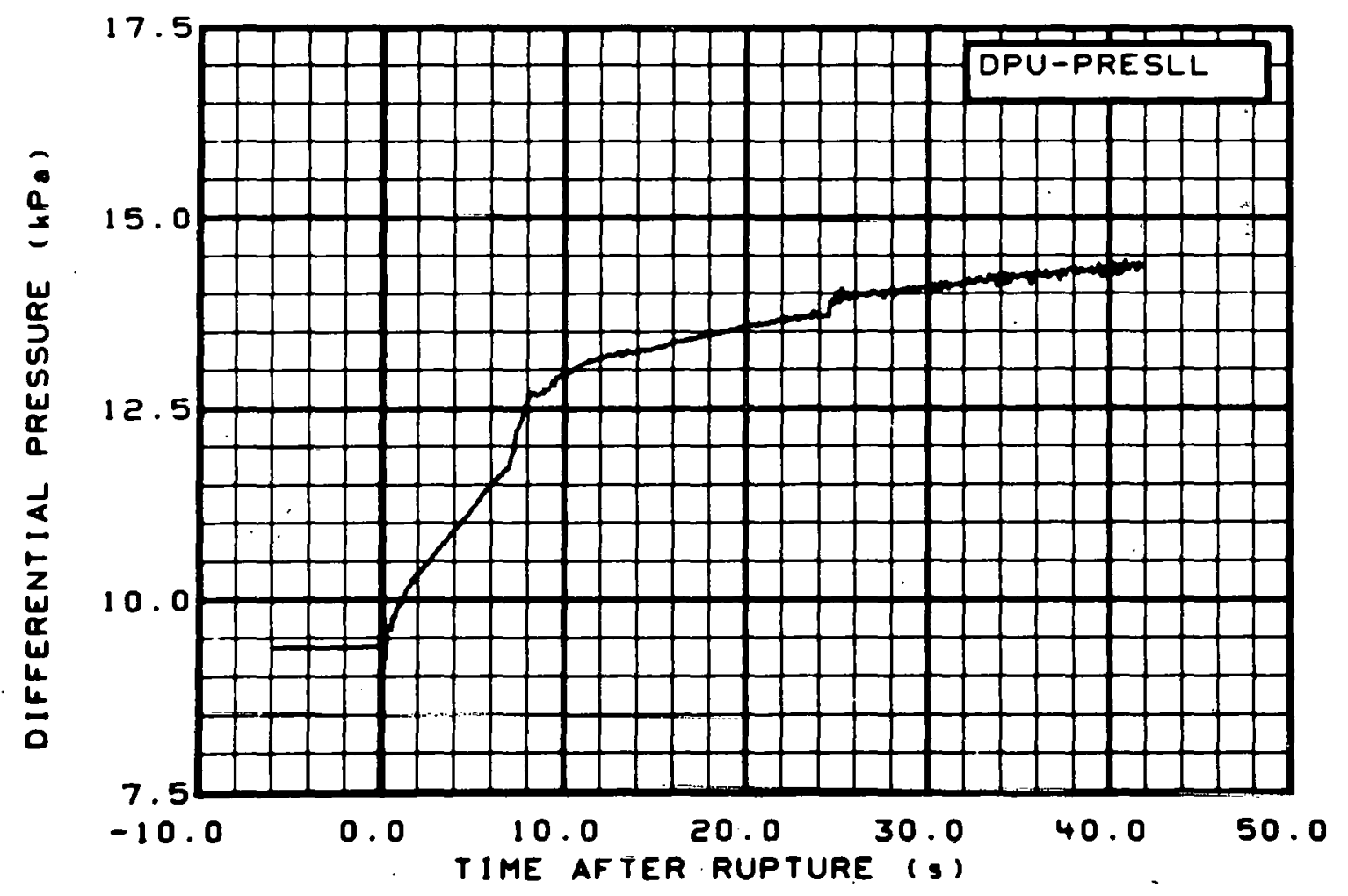

Fig. 164 Differential pressure in intact loop (DPU-PRESLL), from -6 to $42 \mathrm{~s}$. 


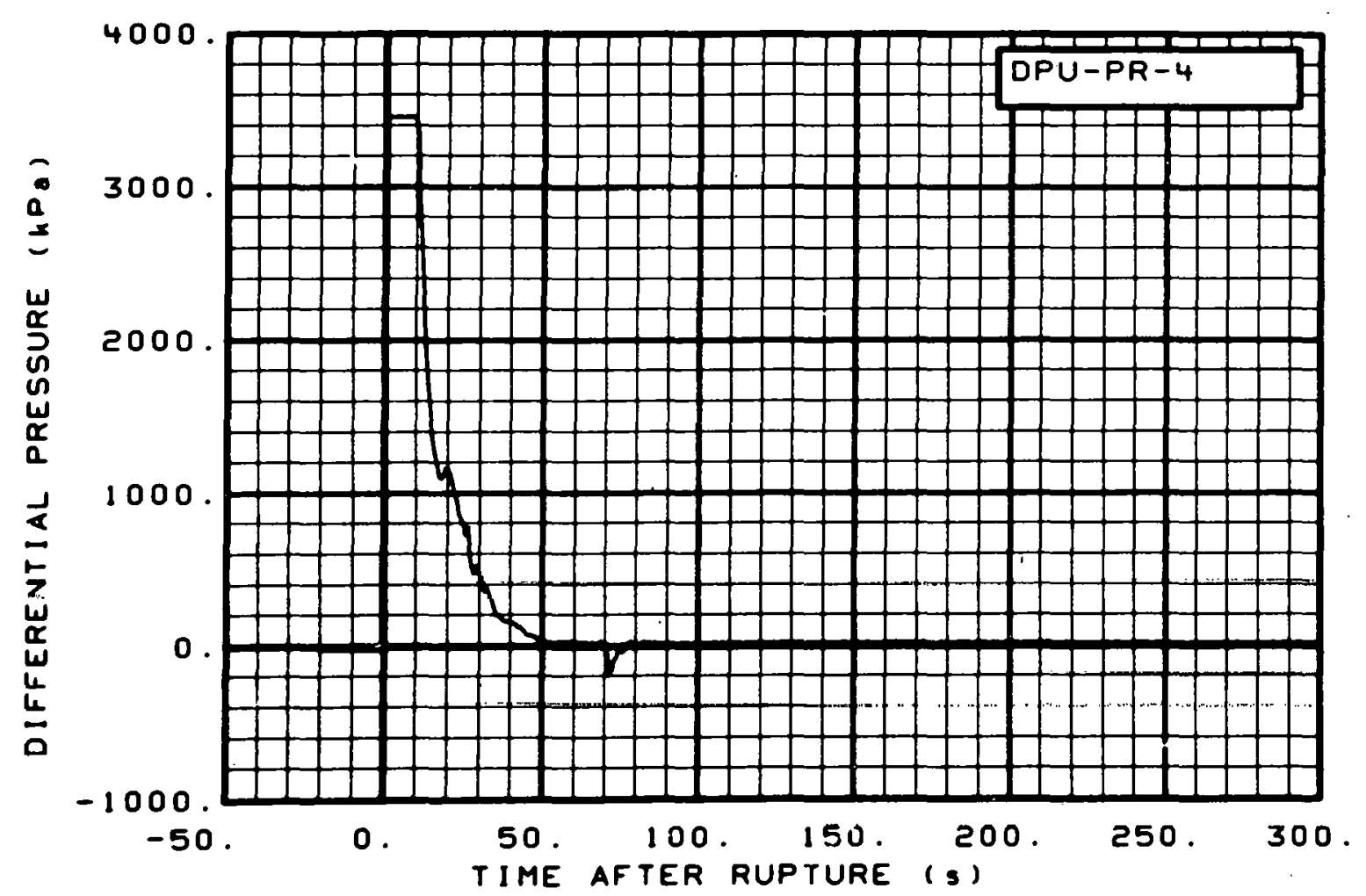

Fig. 165 Differential pressure in intact loop (DPU-PR-4), from -20 to $300 \mathrm{~s}$.

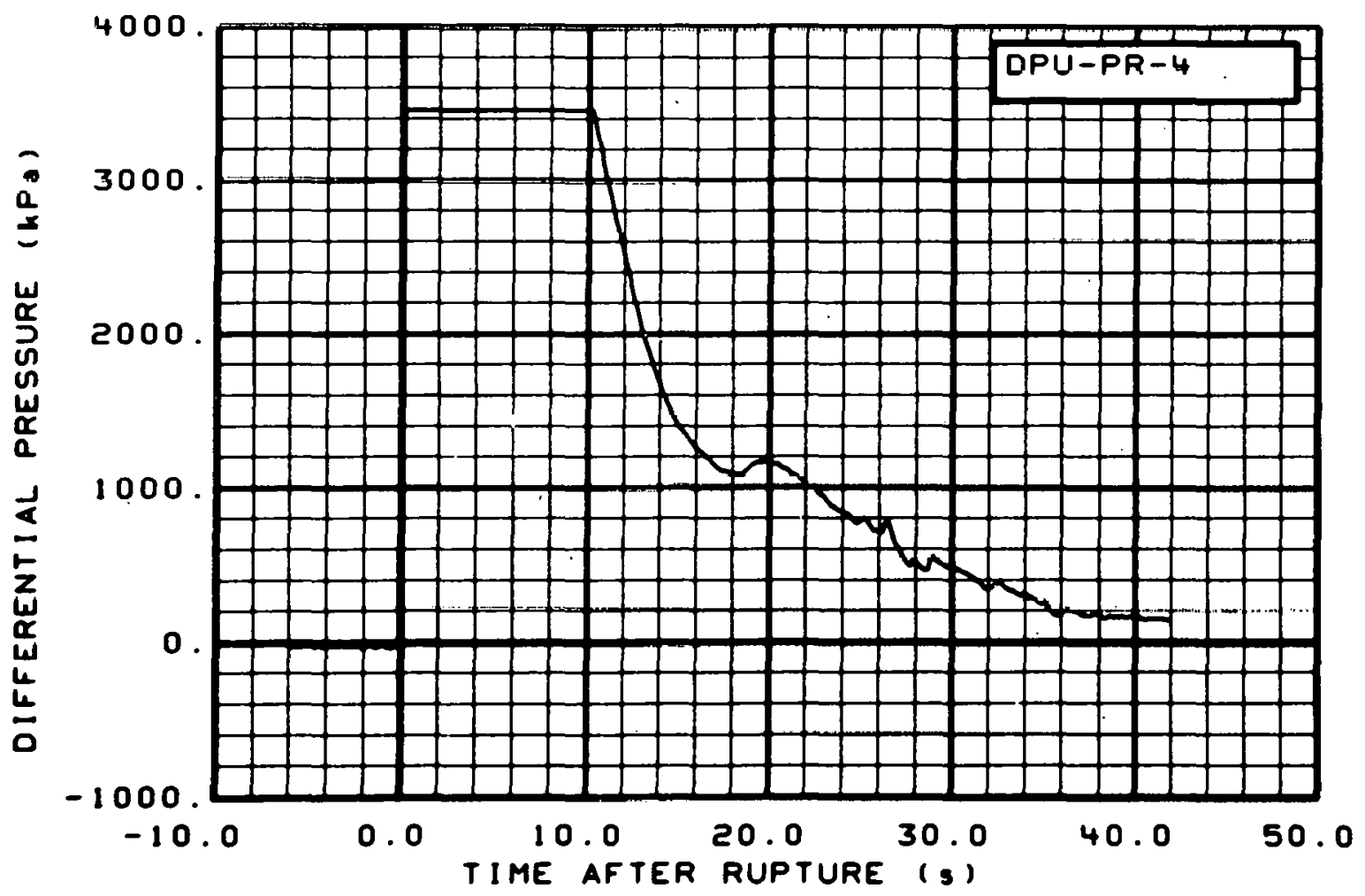

Fig. 166 Differential pressure in intact loop (DPU-PR-4), from -6 to $42 \mathrm{~s}$. 


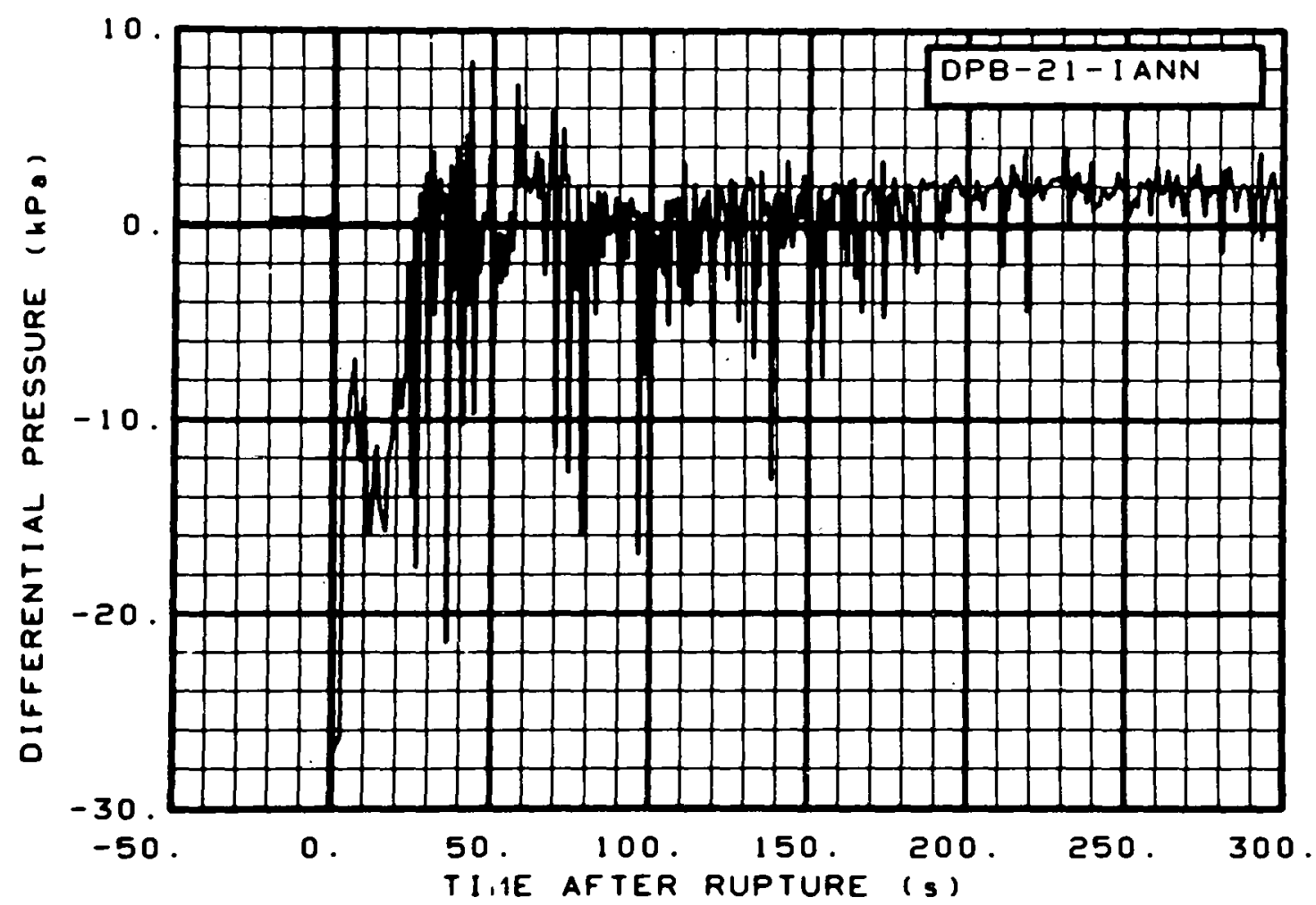

Fig. 167 Differential pressure in broken loop (DPB-21-IANN), from -20 to $300 \mathrm{~s}$.

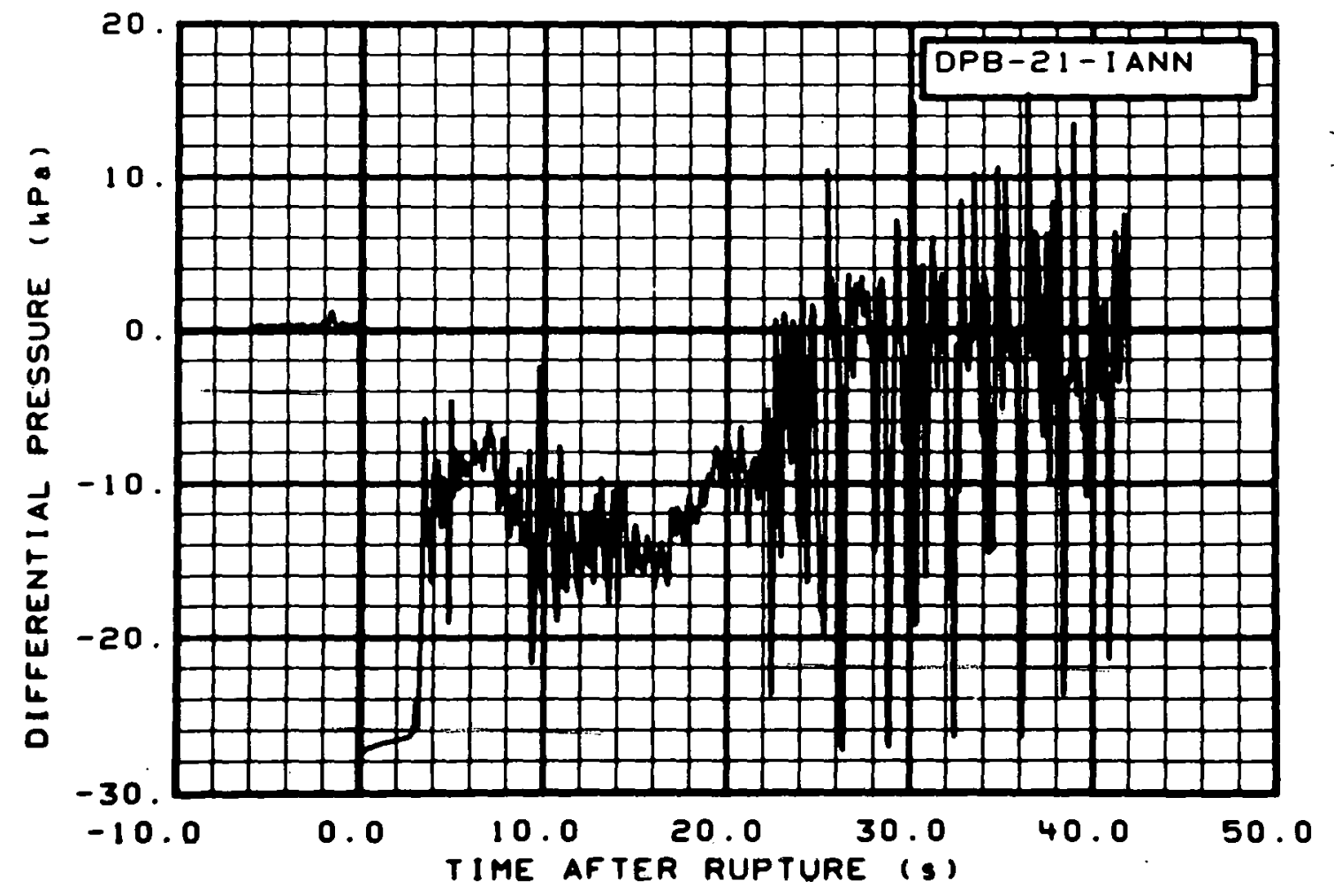

Fig. 168 Differential pressure in broken loop (DPB-27-IANN), from -6 to $42 \mathrm{~s}$. 


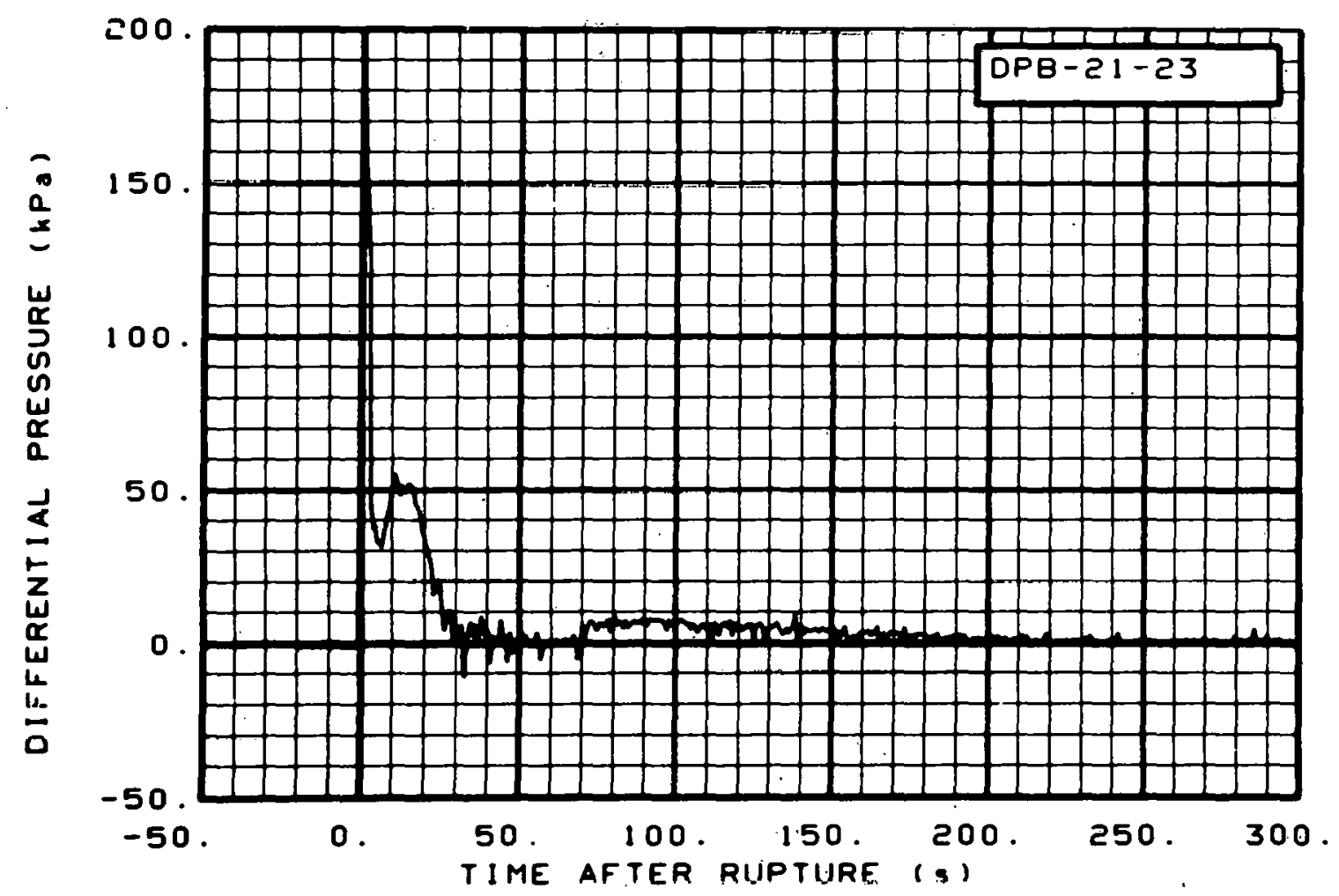

Fig. 169 Differential pressure in broken 1oop (DPB-21-23), from -20 to $300 \mathrm{~s}$.

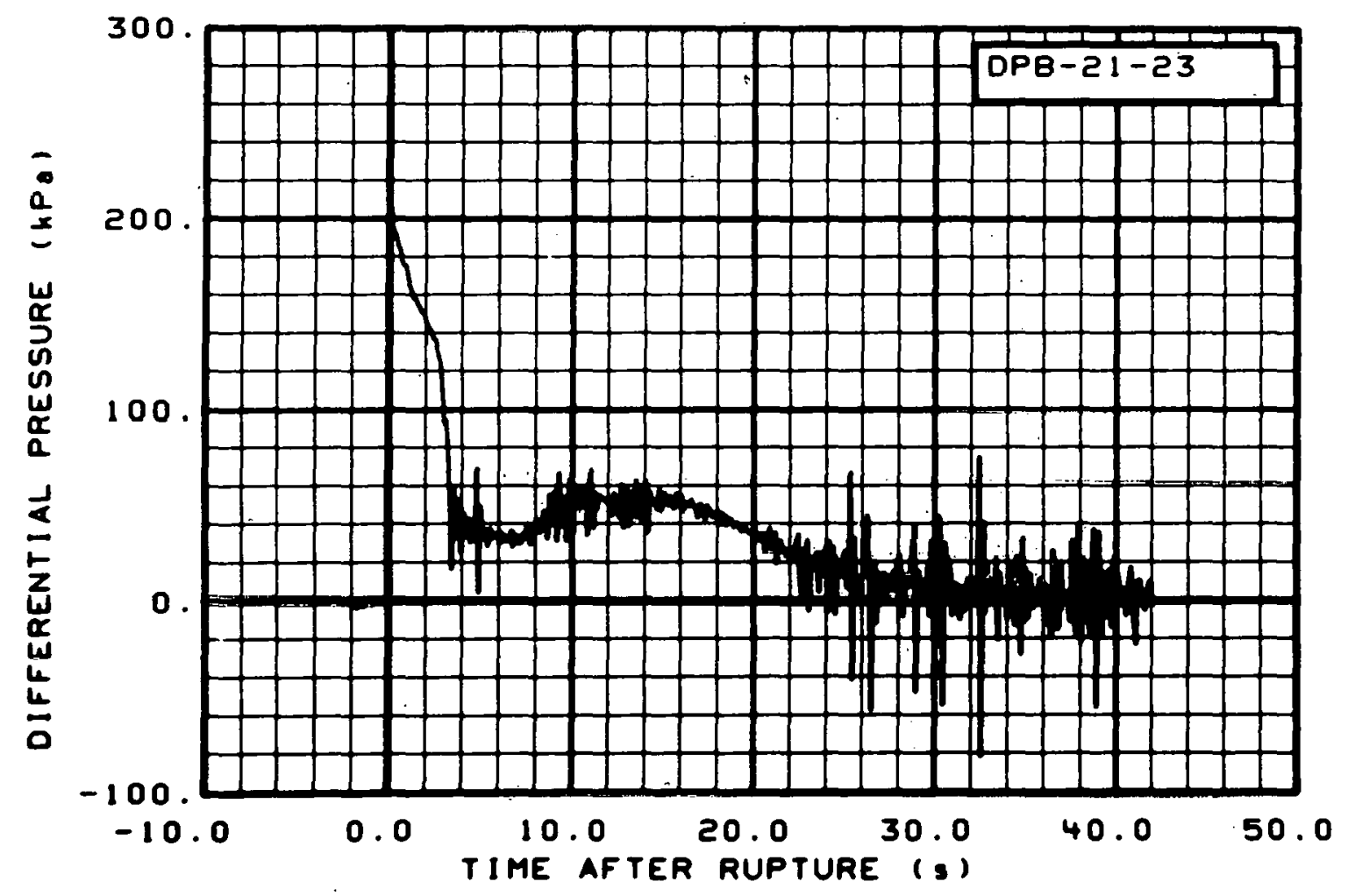

Fig. 170 Differential pressure in broken loop (DPB-21-23), from -6 to $42 \mathrm{~s}$. 


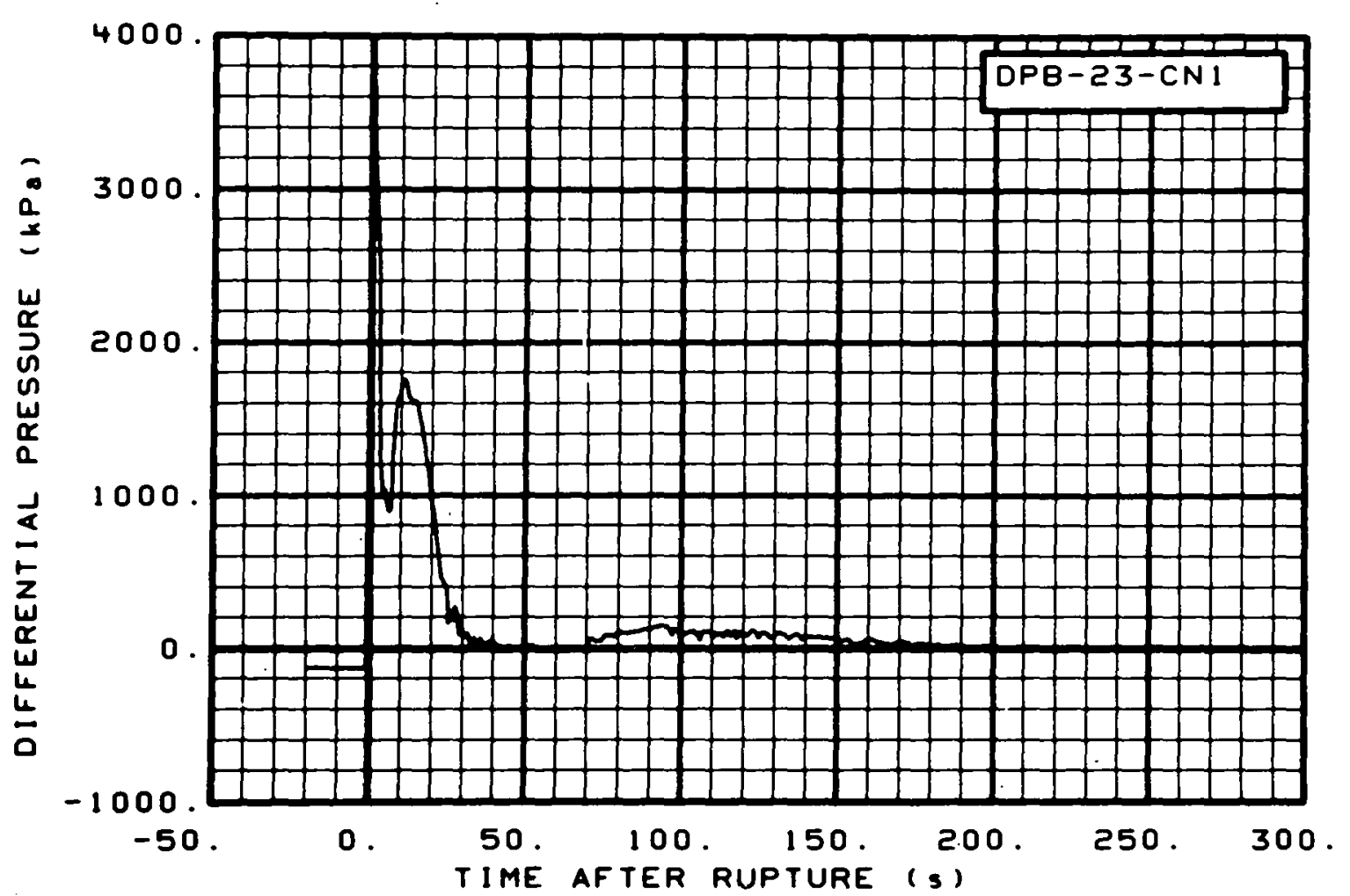

Fig. 171 Differential pressure in broken loop (DPB-23-CN1), from -20 to $300 \mathrm{~s}$.

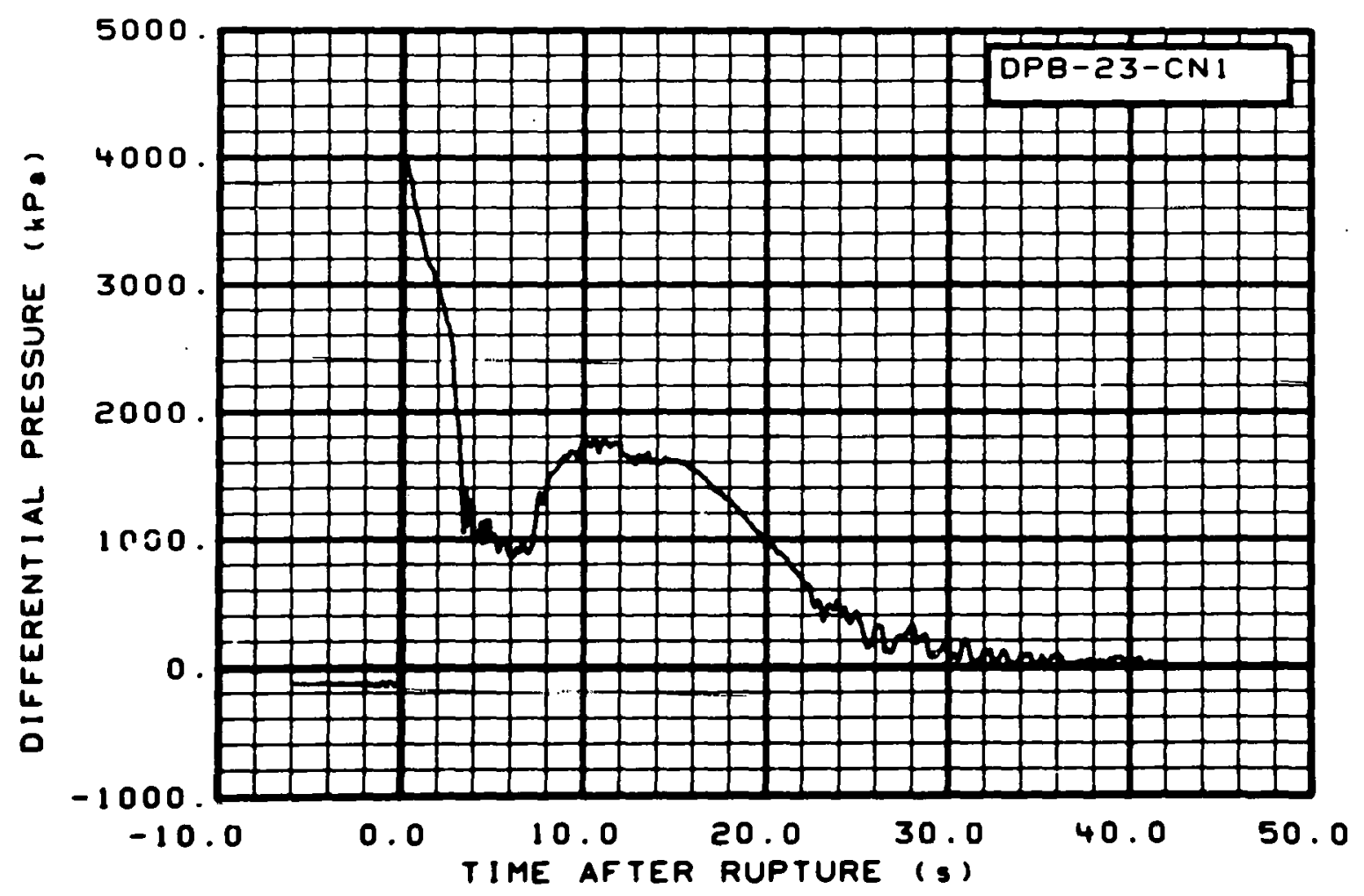

Fig. 172 Differential pressure in broken loop (DPB-23-CN1), from -6 to $42 \mathrm{~s}$. 


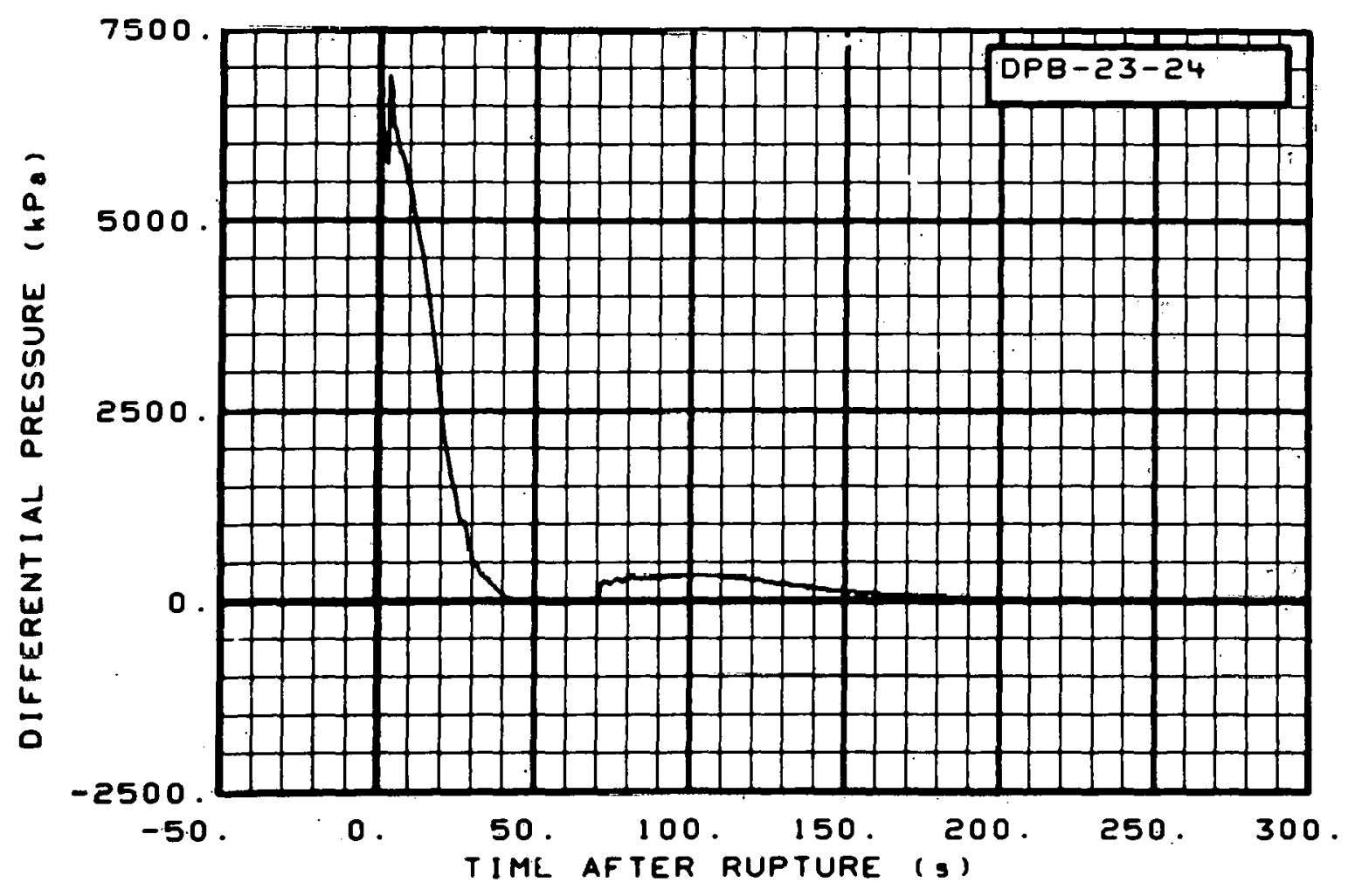

Fig. 173 Differential pressure in broken loop (DPB-23-24.), from -20 to $300 \mathrm{~s}$.

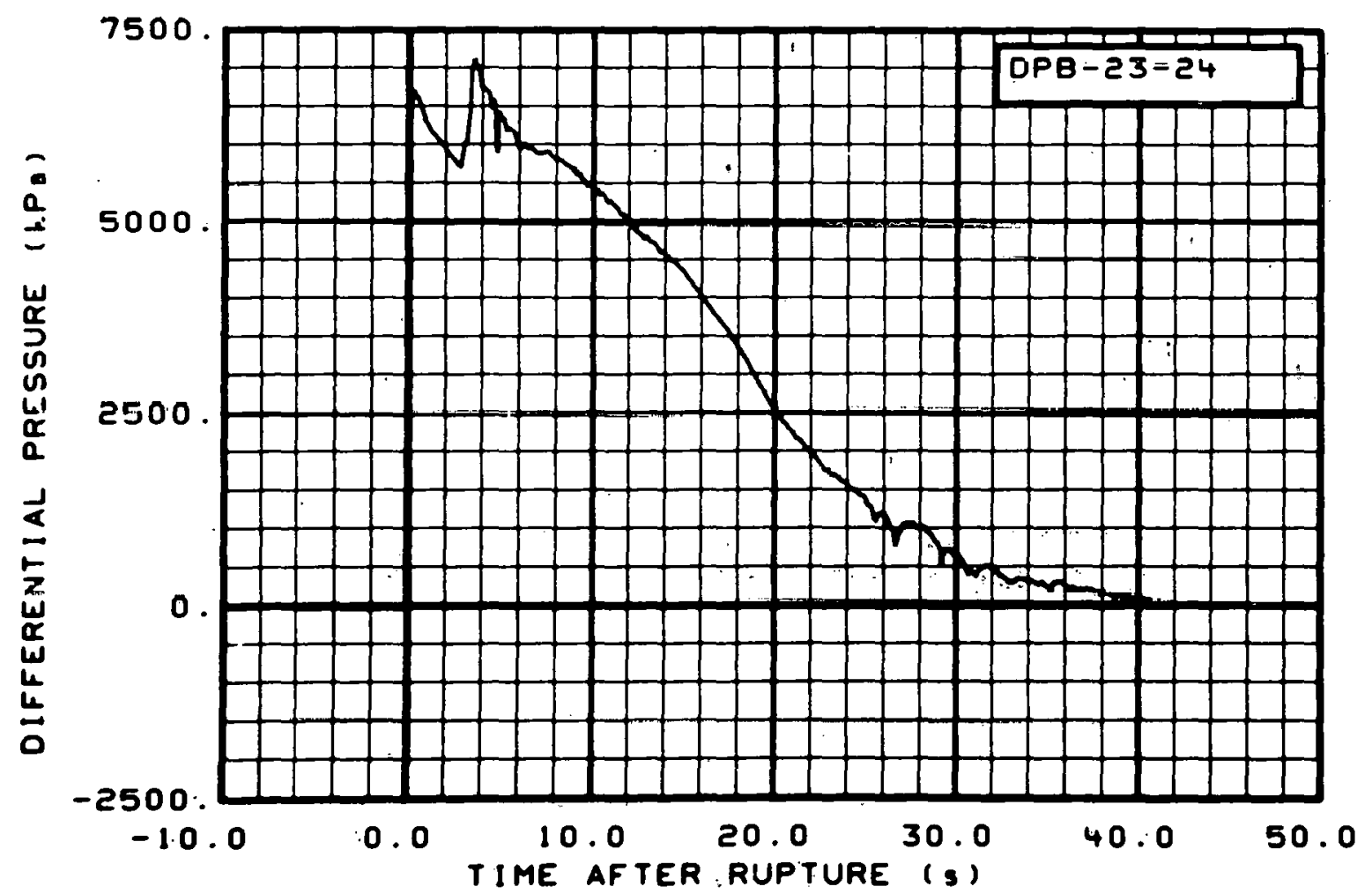

Fig. 174 Differential pressure in broken loop (DPB-23-24), from -6 to " $42 \mathrm{~s}$. 


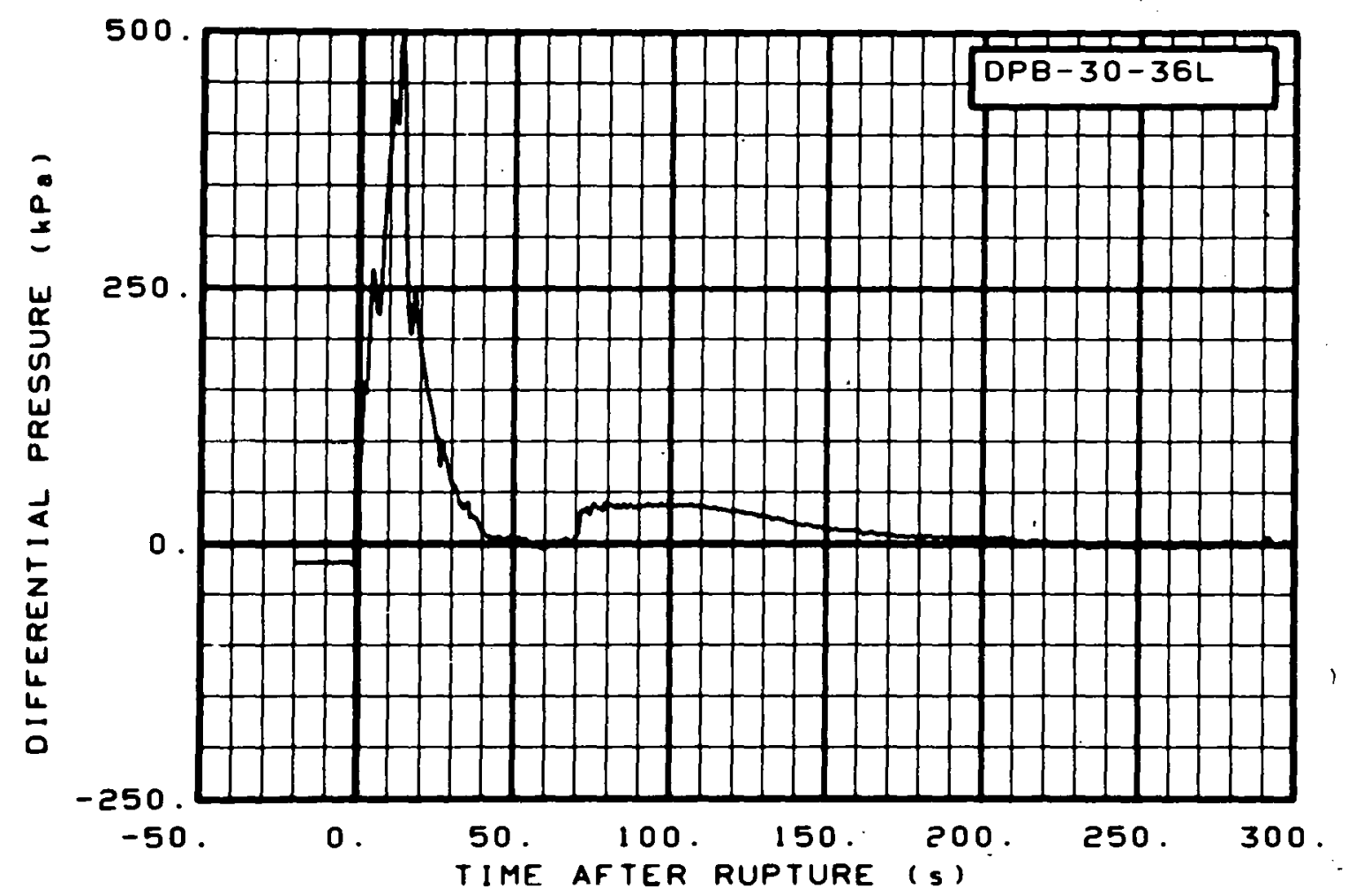

Fig. 175 Differential pressure in broken loop (DPB-30-36L), from -20 to $300 \mathrm{~s}$.

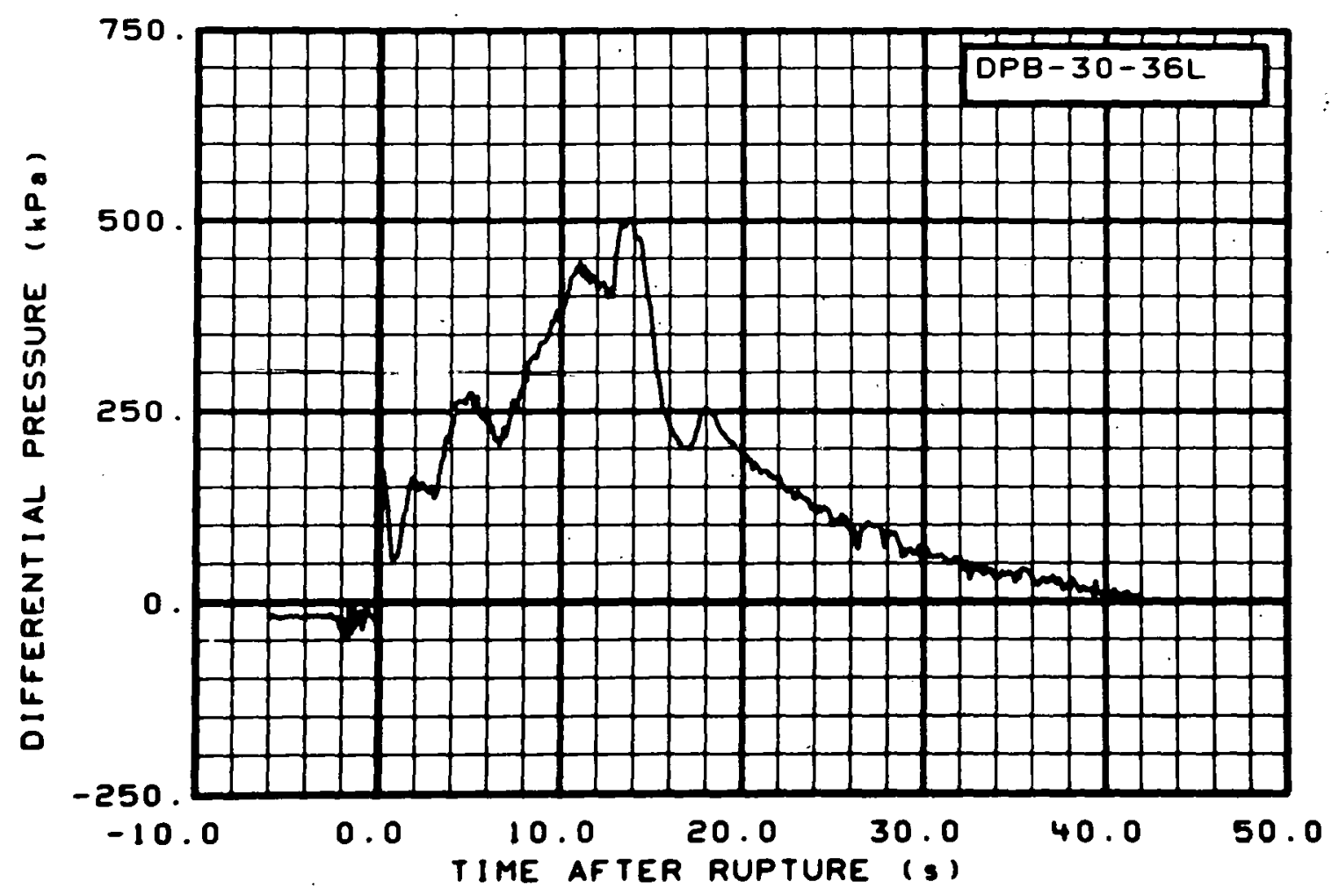

Fig. 176 Differential pressure in broken loop (DPB-30-36L), from -6 to $42 \mathrm{~s}$. 


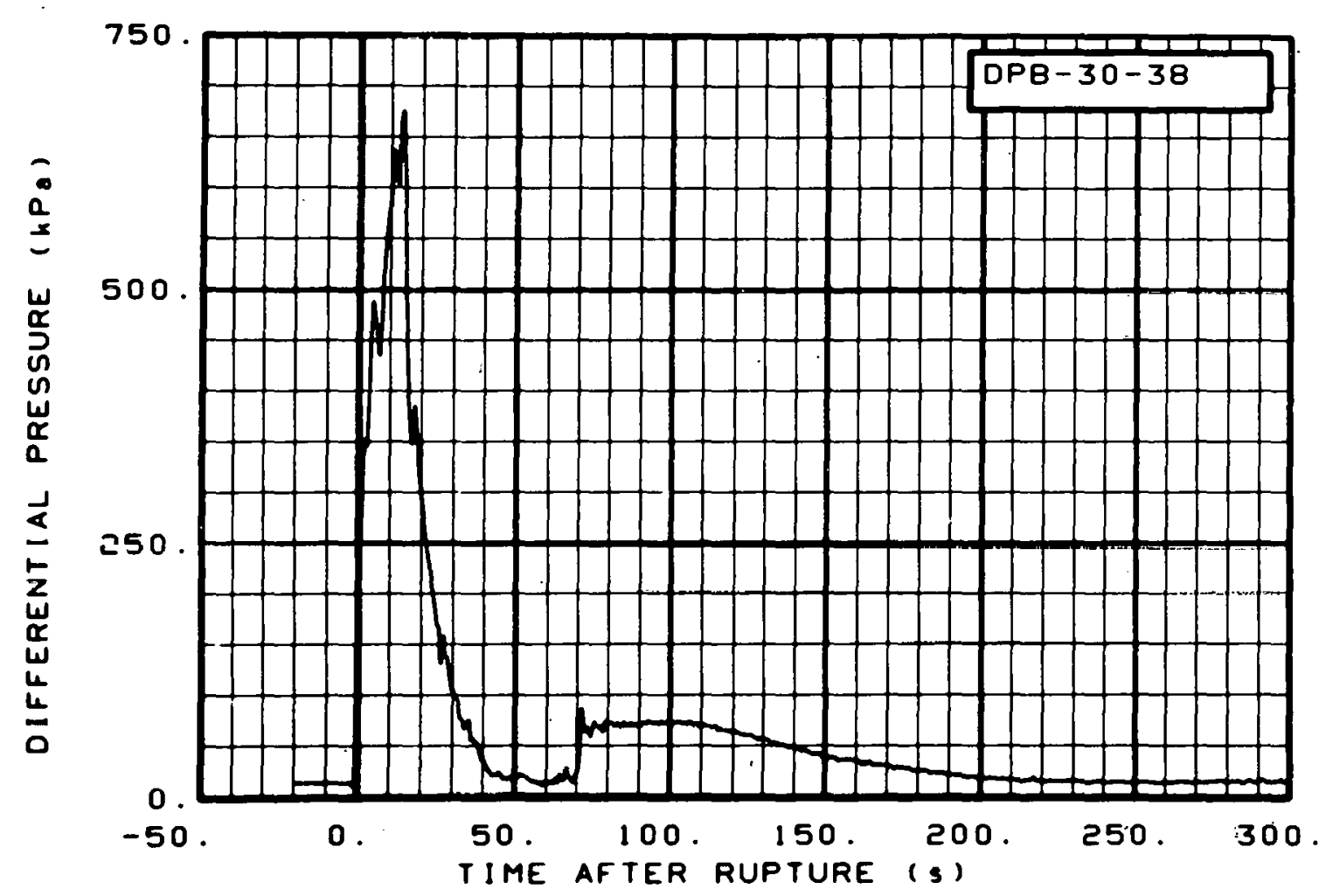

Fig. 177 Differential pressure in broken loop (DPB-30-38), from -20 to $300 \mathrm{~s}$.

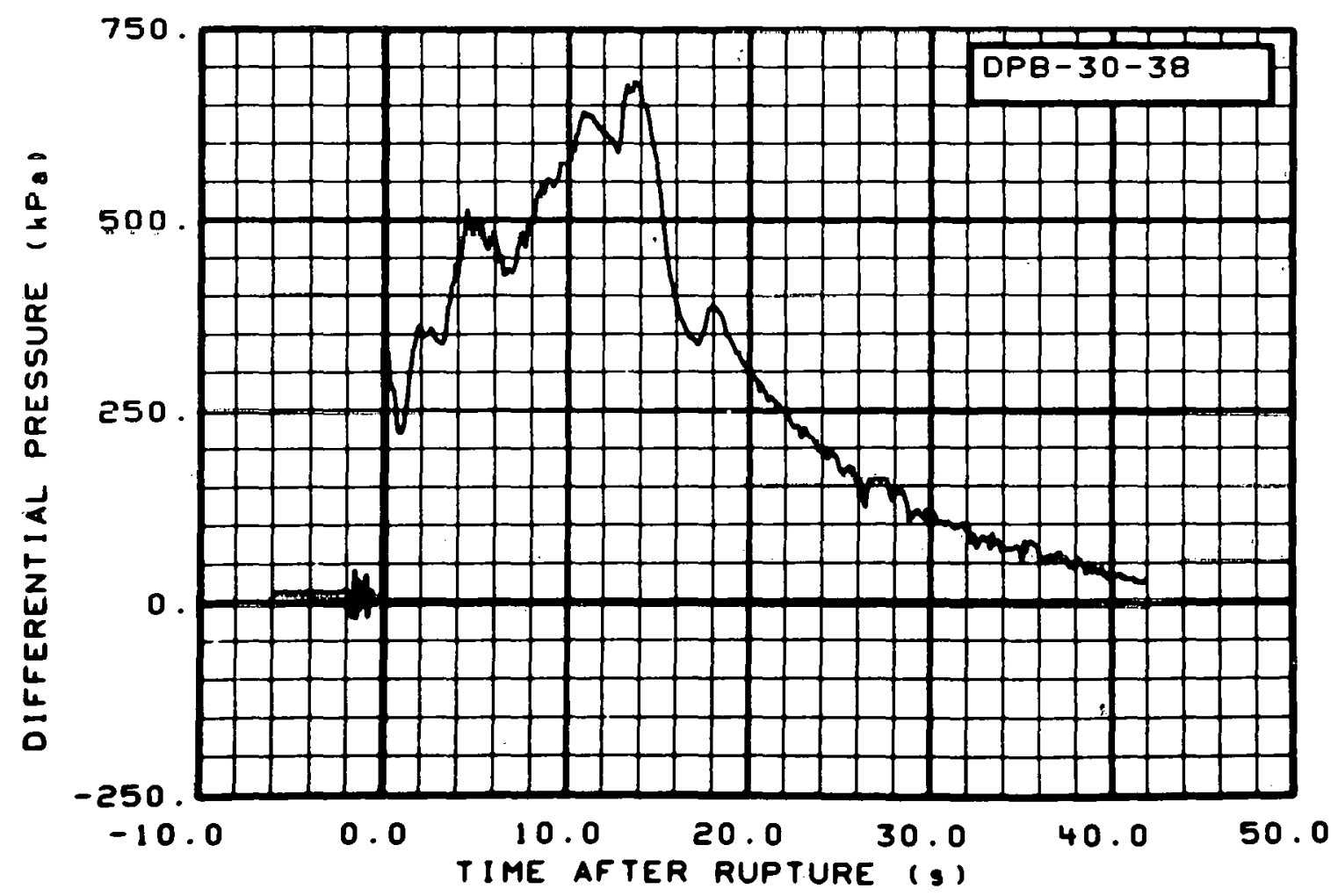

Fig. 178 Differential pressure in broken loop (DPB-30-38), from -6 to $42 \mathrm{~s}$. 


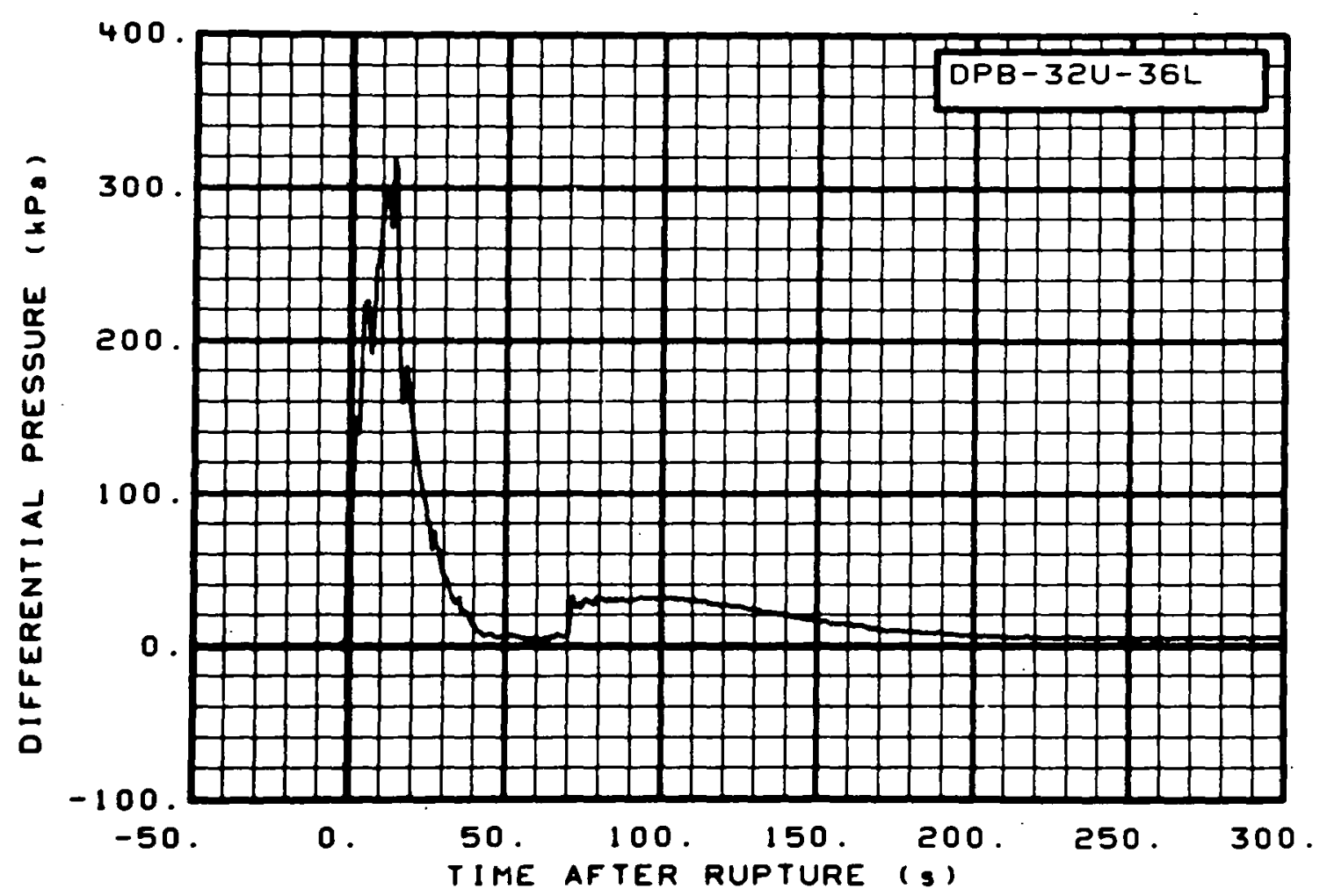

Fig. 179 Differential pressure in broken loop (DPB-32U-36L), from -20 to $300 \mathrm{~s}$.

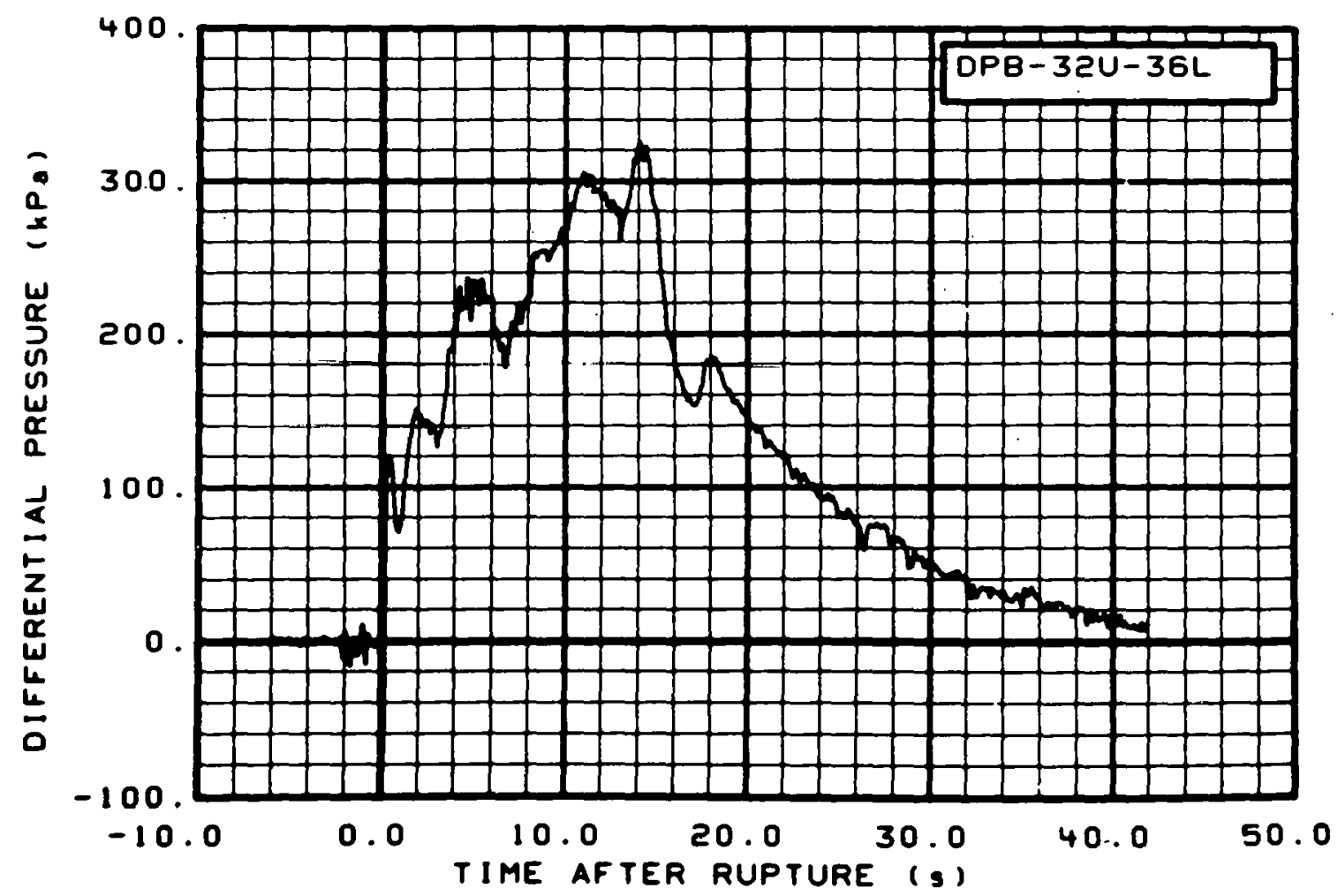

Fig. 180 Differential pressure in broken loop (DPB-32U-36L), from -6 to $42 \mathrm{~s}$. 


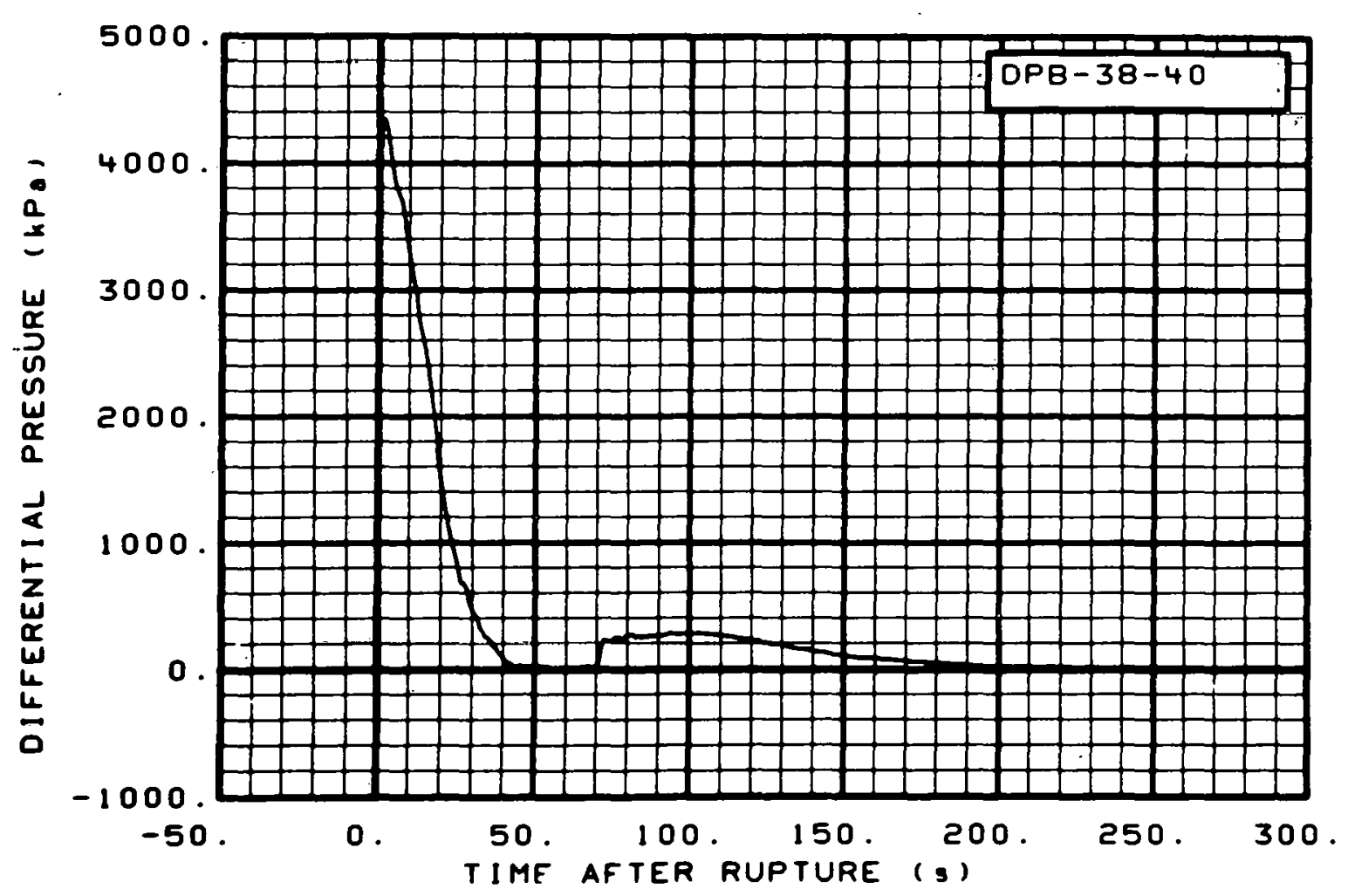

Fig. 181 Differential pressure in broken loop (DPB-38-40), from -20 to $300 \mathrm{~s}$.

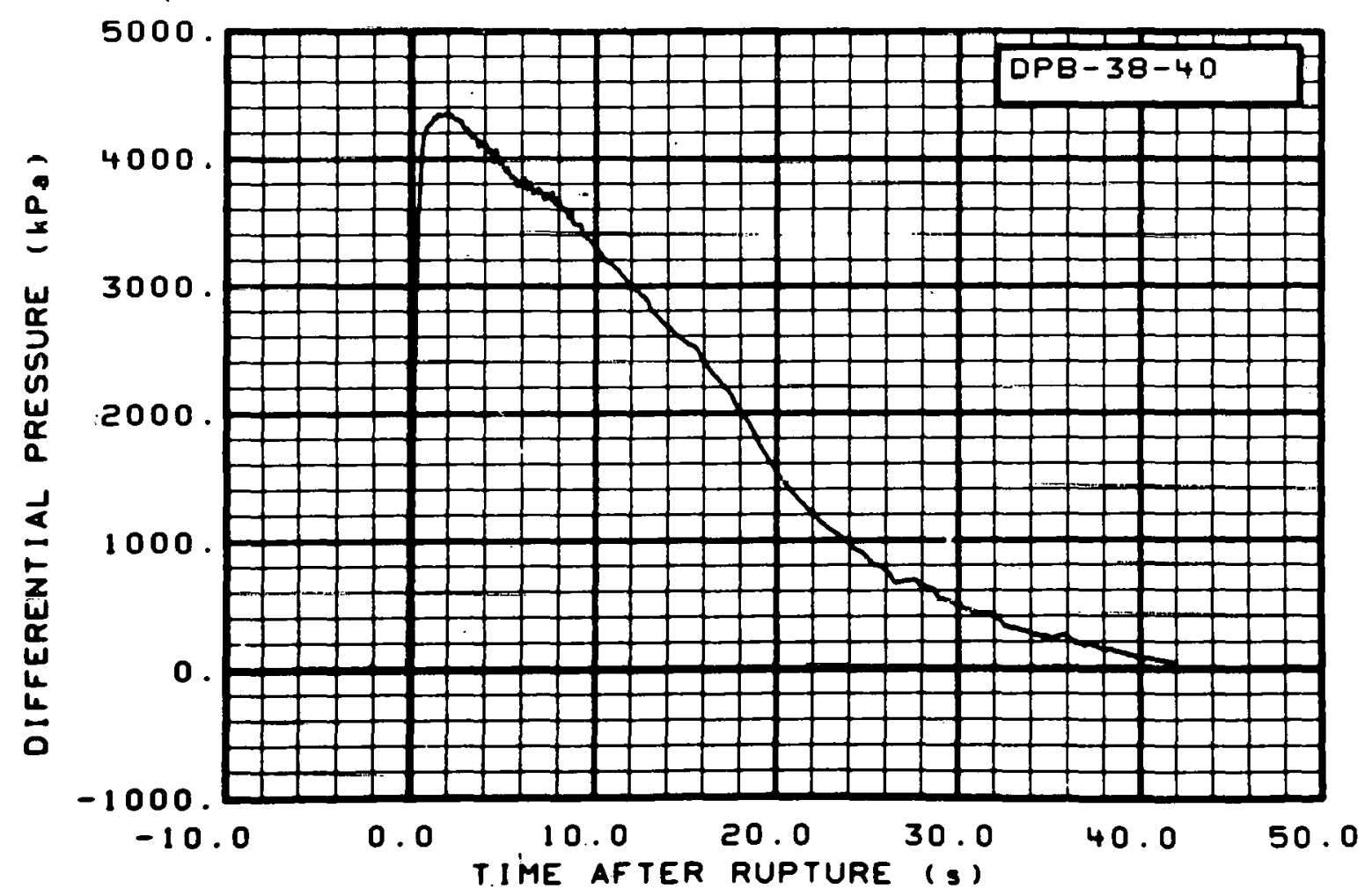

Fig. 182 Differential pressure in broken loop (DPB-38-40), from -6 to $42 \mathrm{~s}$. 


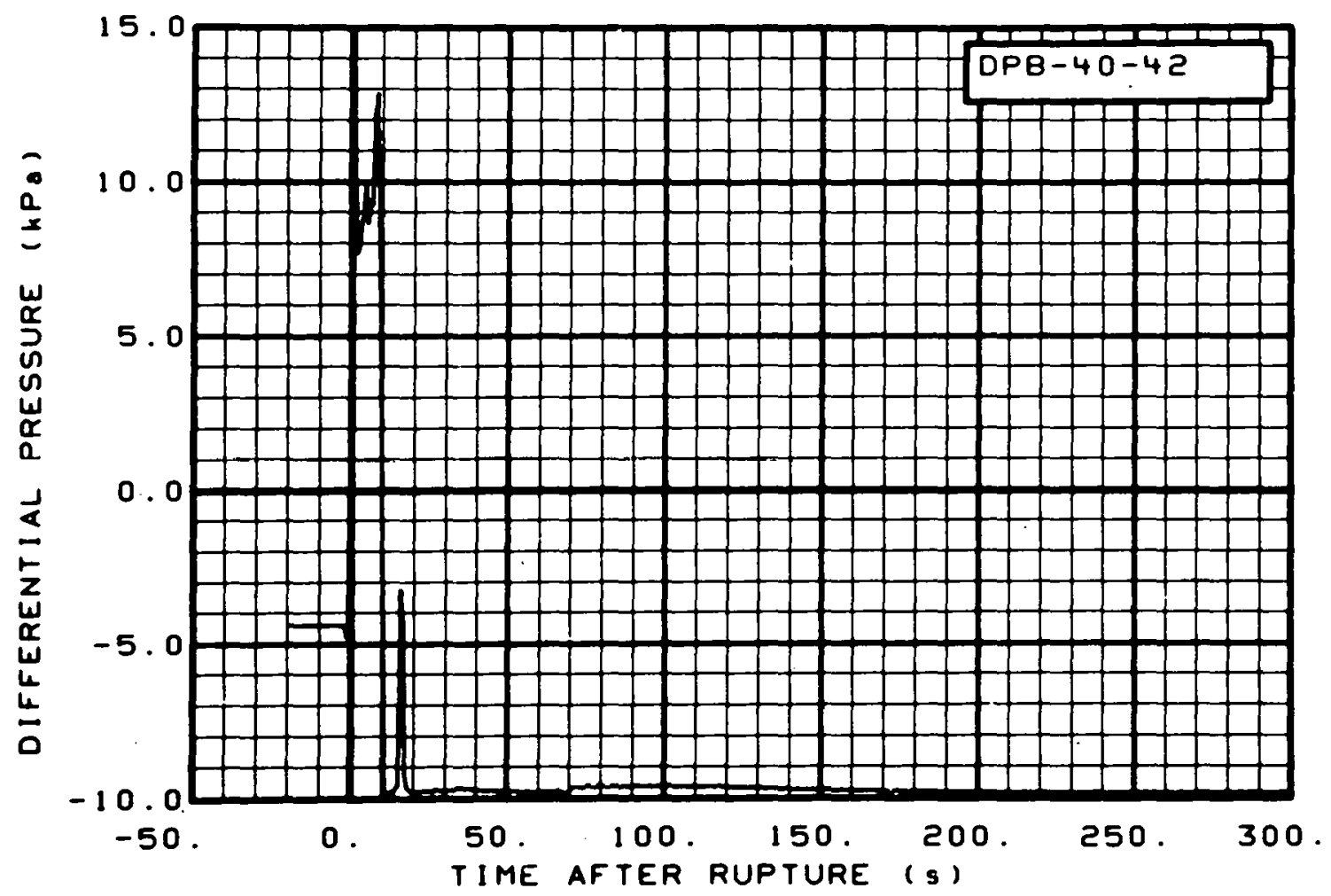

Fig. 183 Differential pressure in broken loop (DPB-40-42), from -20 to $300 \mathrm{~s}$.

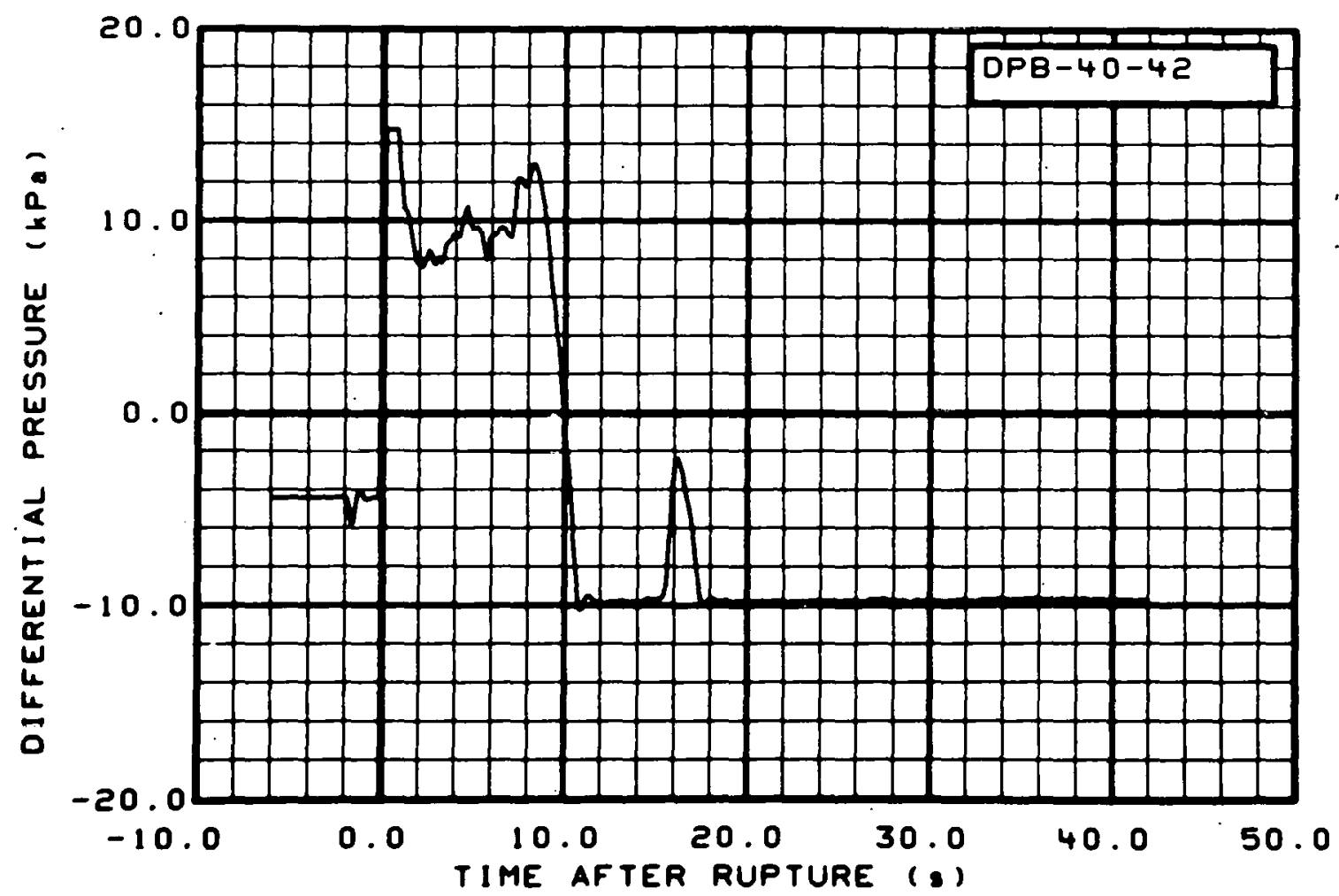

Fig. 184 Differential pressure in broken loop (DPB-40-42), from -6 to $42 \mathrm{~s}$. 


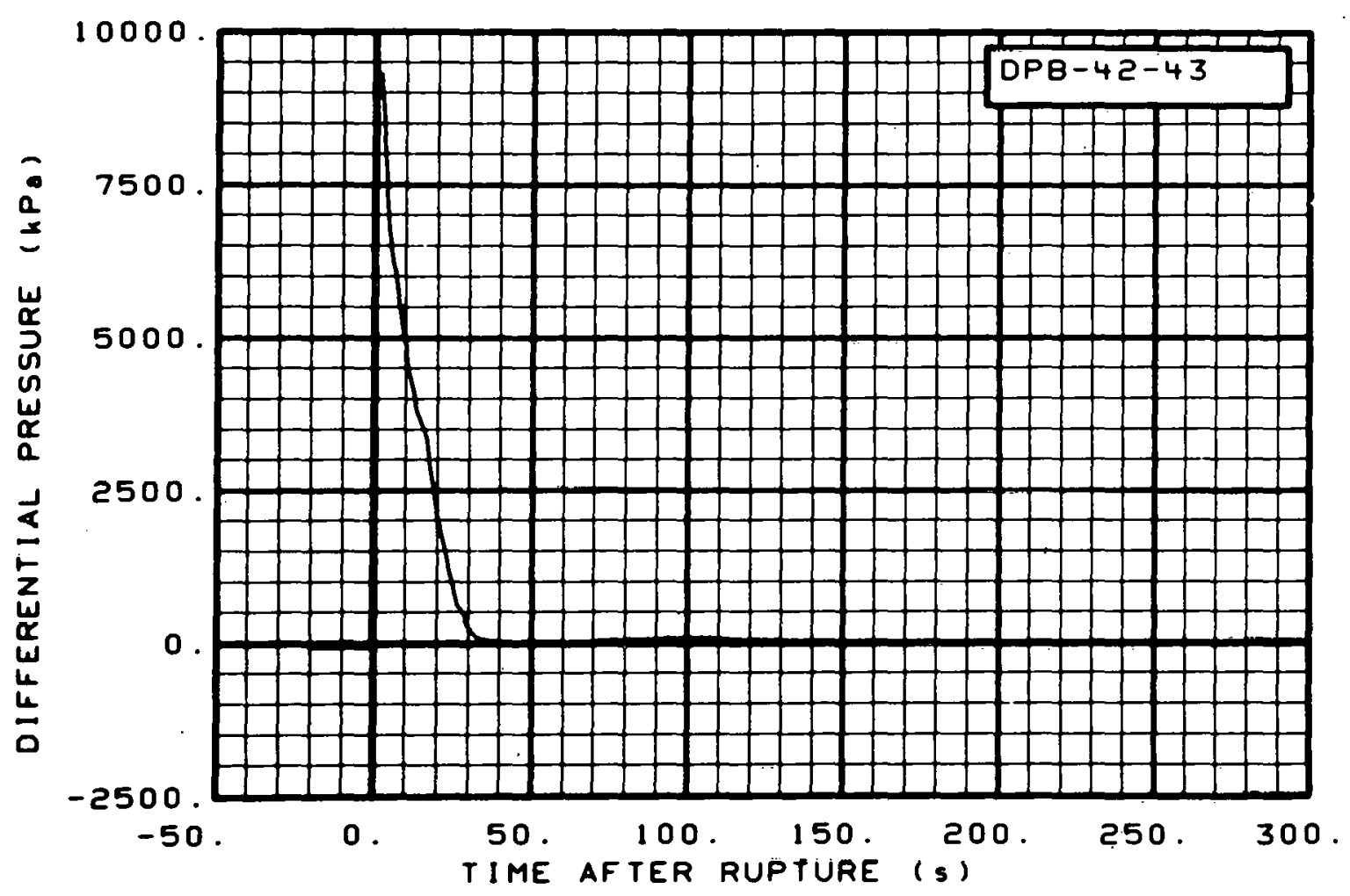

Fig. 185 Differential pressure in broken loop (DPB-42-43), from -20 to $300 \mathrm{~s}$.

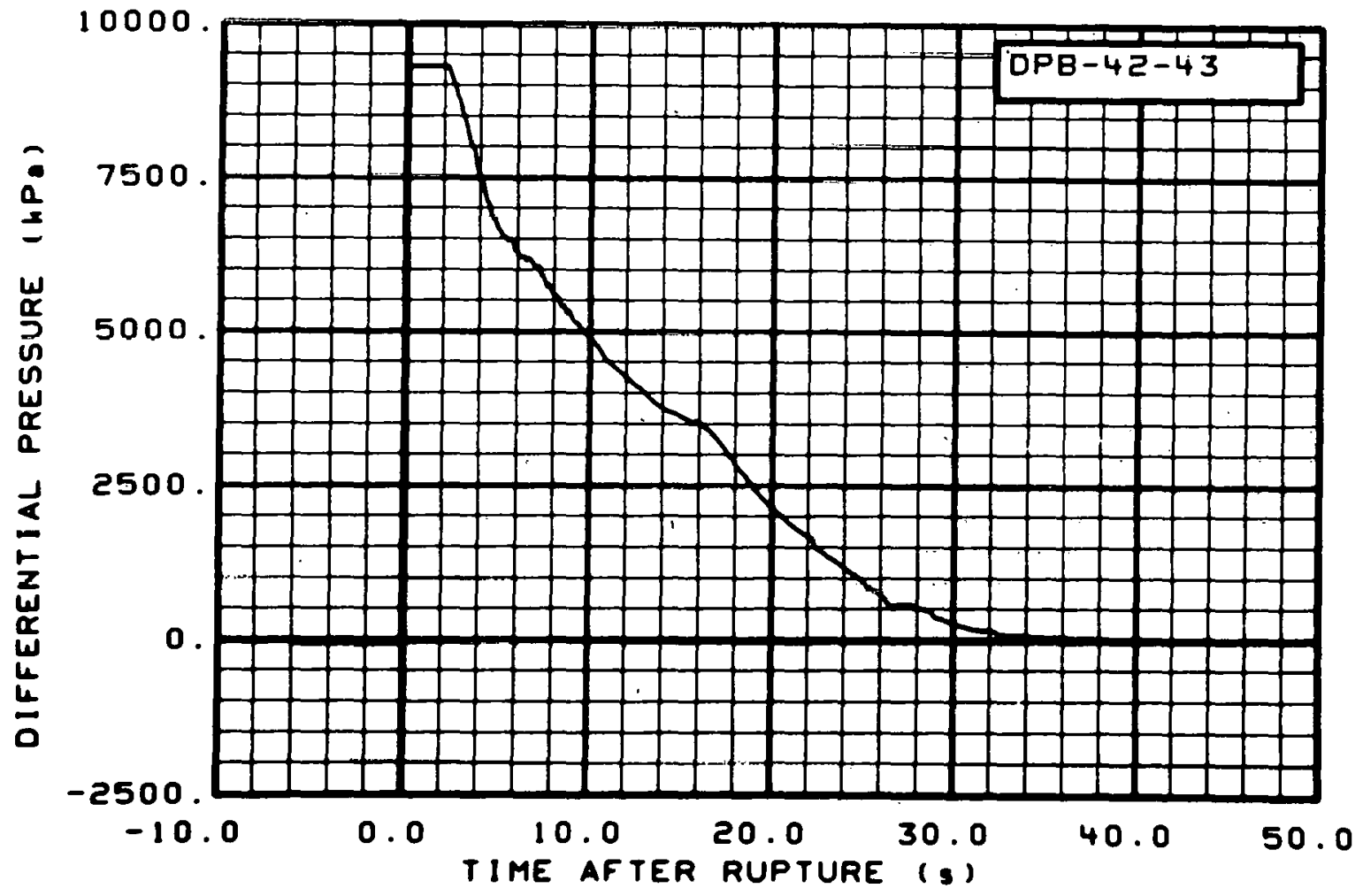

Fig. 186 Differential pressure in broken loop (DPB-42-43), from -6 to $42 \mathrm{~s}$. 


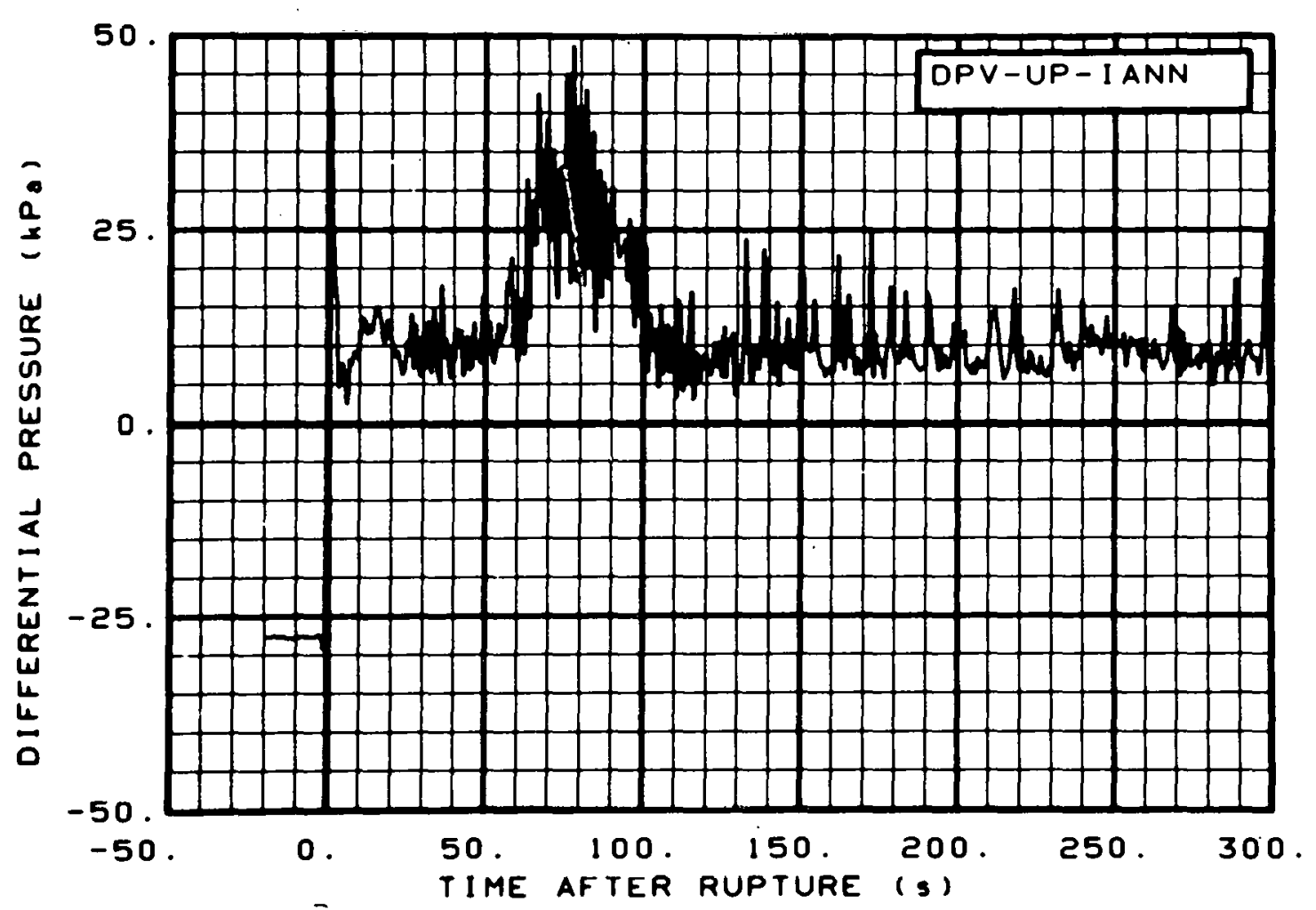

Fig. 187 Differential pressure in vessel (DPV-UP-IANN), from -20 to $300 \mathrm{~s}$.

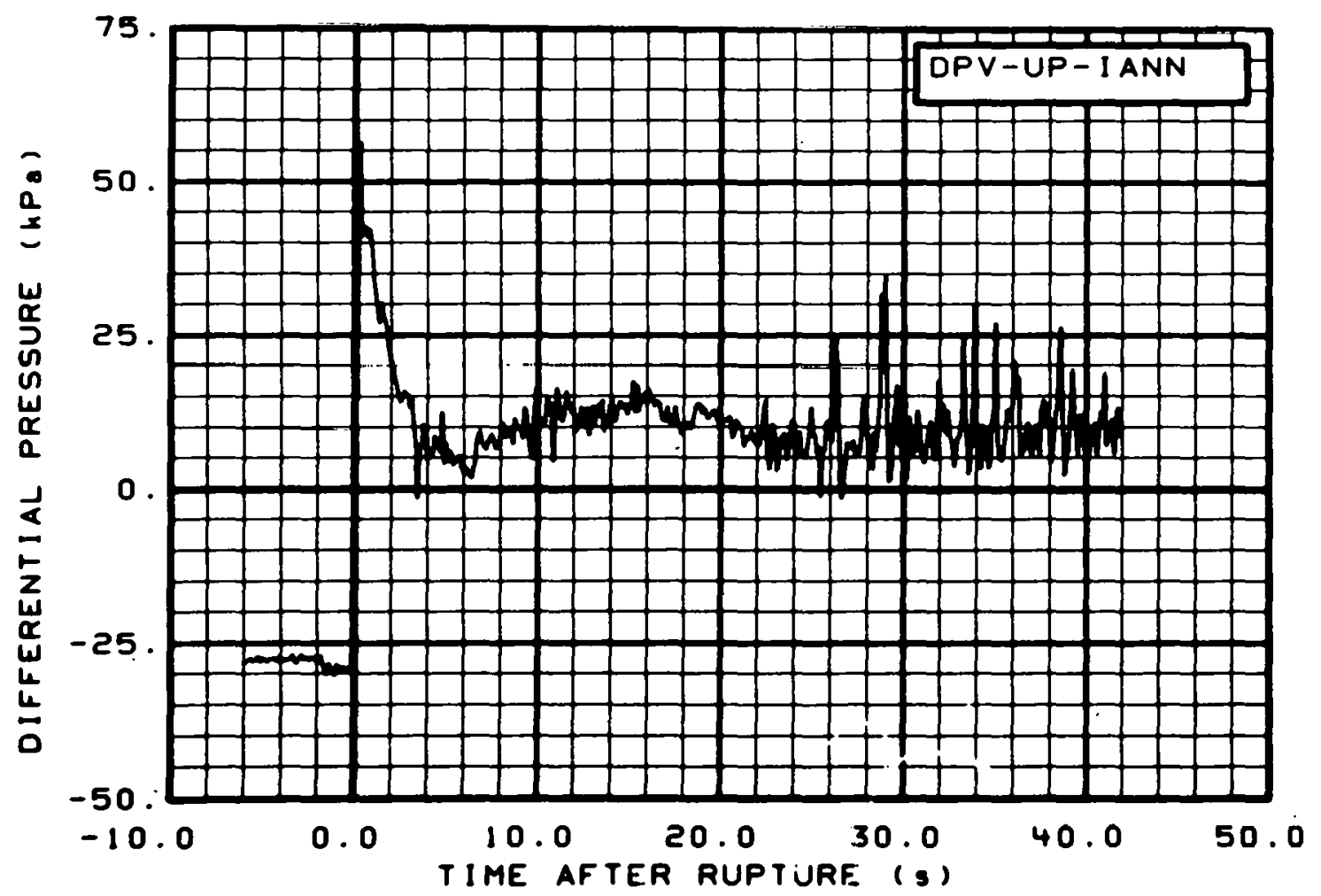

Fig. 188 Differential pressure in vessel (DPV-IIP-IANN), from -6 to $42 \mathrm{~s}$. 


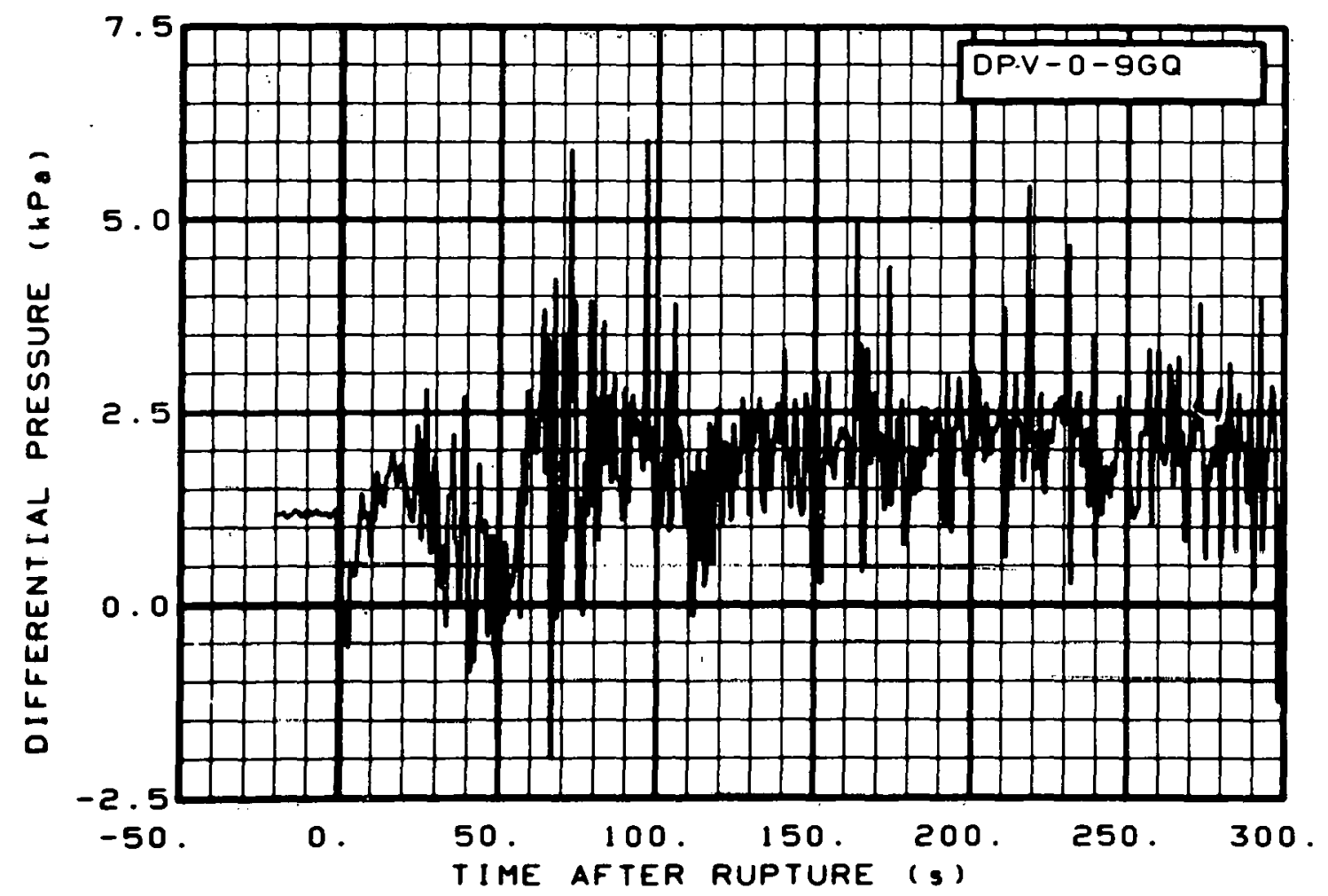

Fig. 189 Differential pressure in vessel (DPV-0-9GQ), from -20 to $300 \mathrm{~s}$.

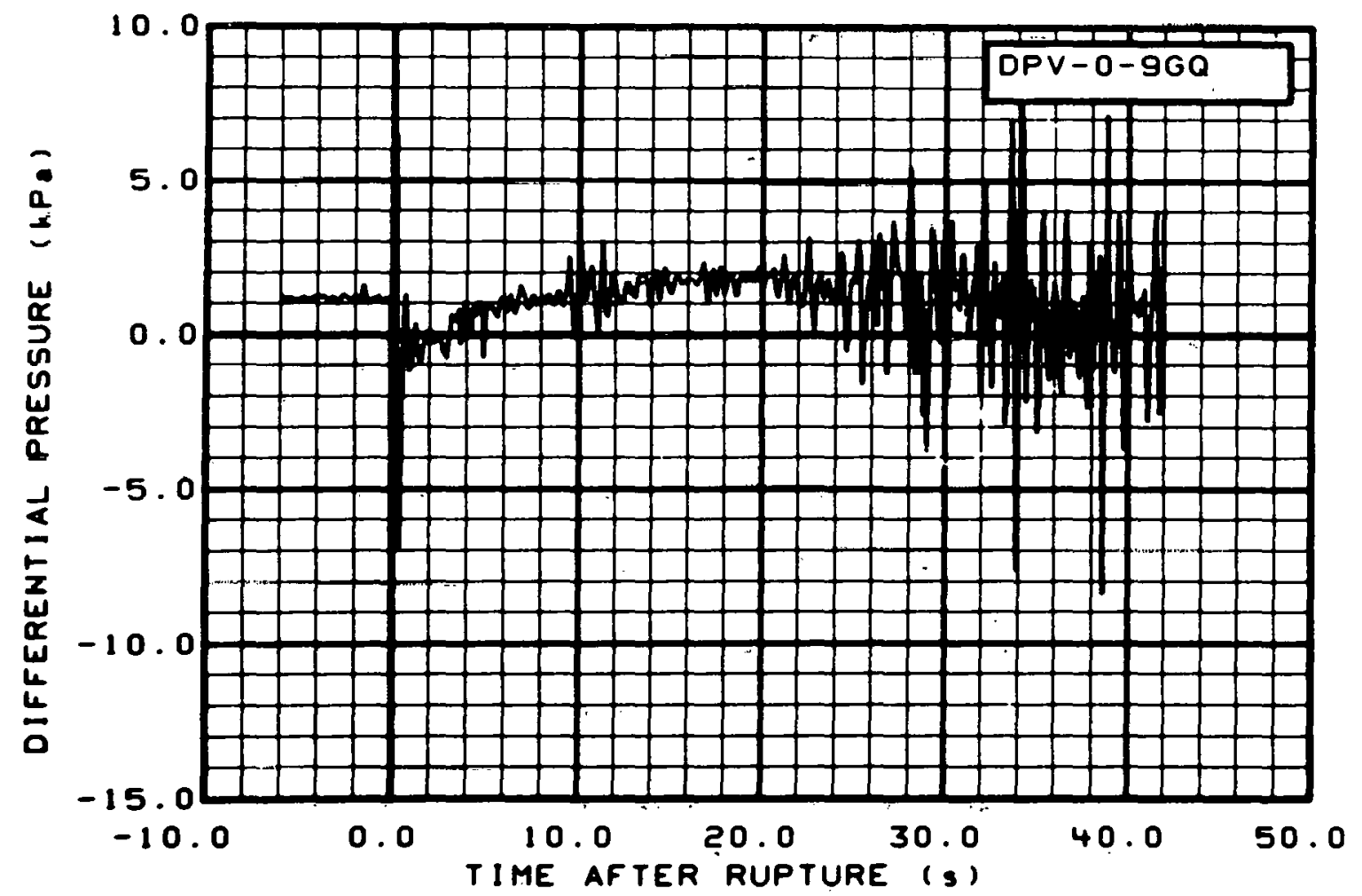

Fig. 190 Differential pressure in vesser (DPV-0-9GQ), from -6 to $42 \mathrm{~s}$. 


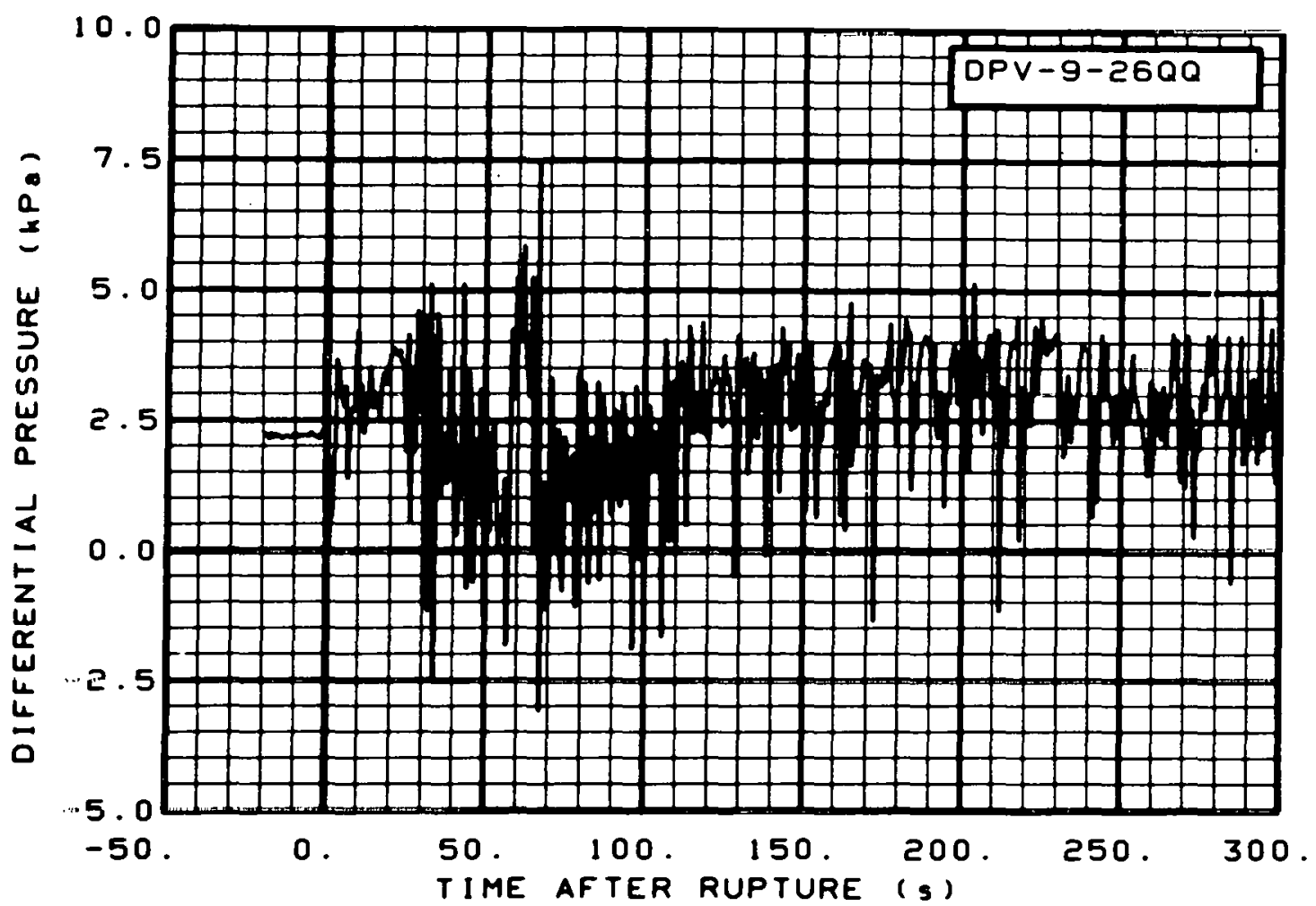

Fig. 191 Differential pressure in vessel (DPV-9-26QQ), from -20 to $300 \mathrm{~s}$.

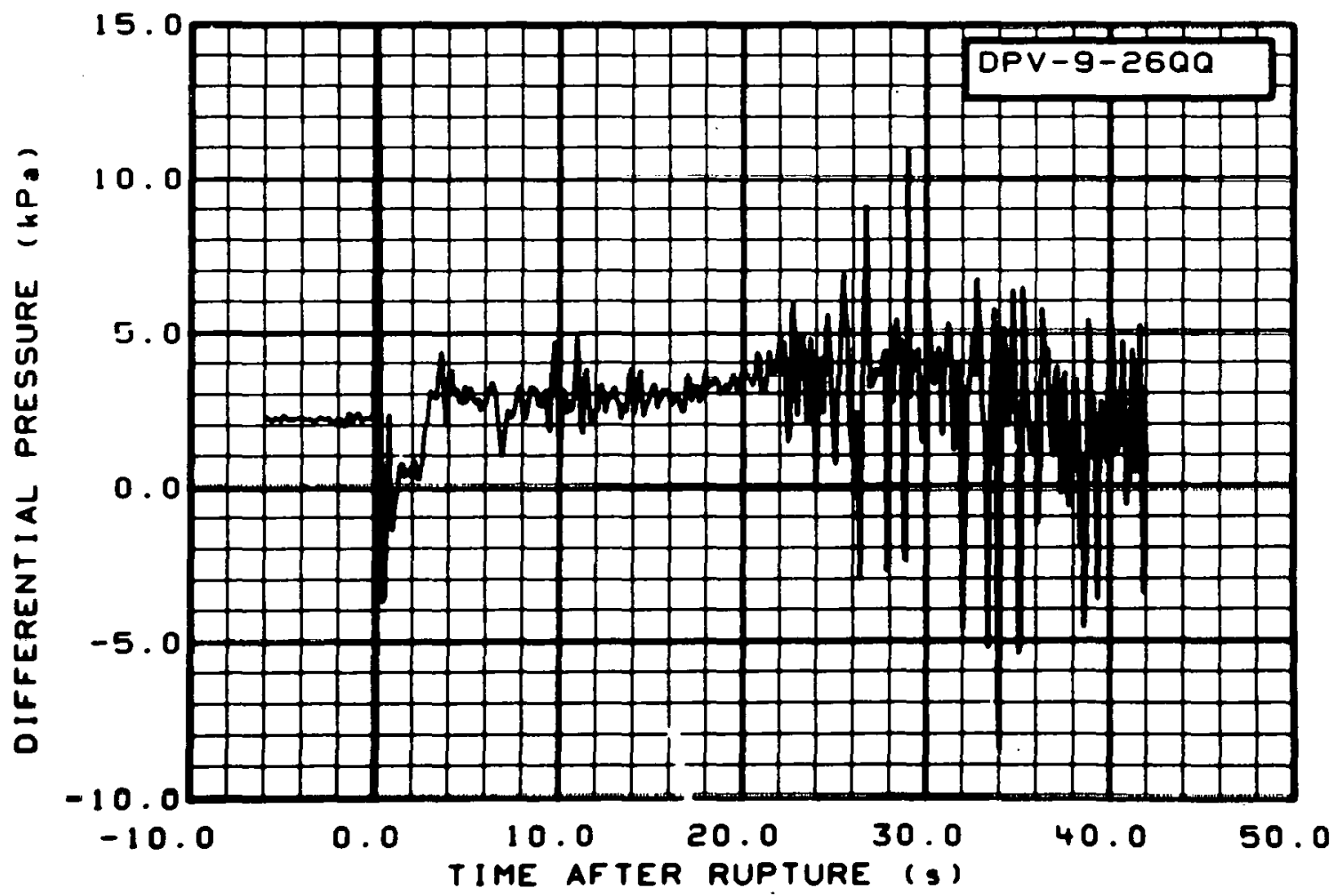

Fig. 19? Differential pressure in vessel (DPV-9-2600), from -6 to $42 \mathrm{~s}$. 


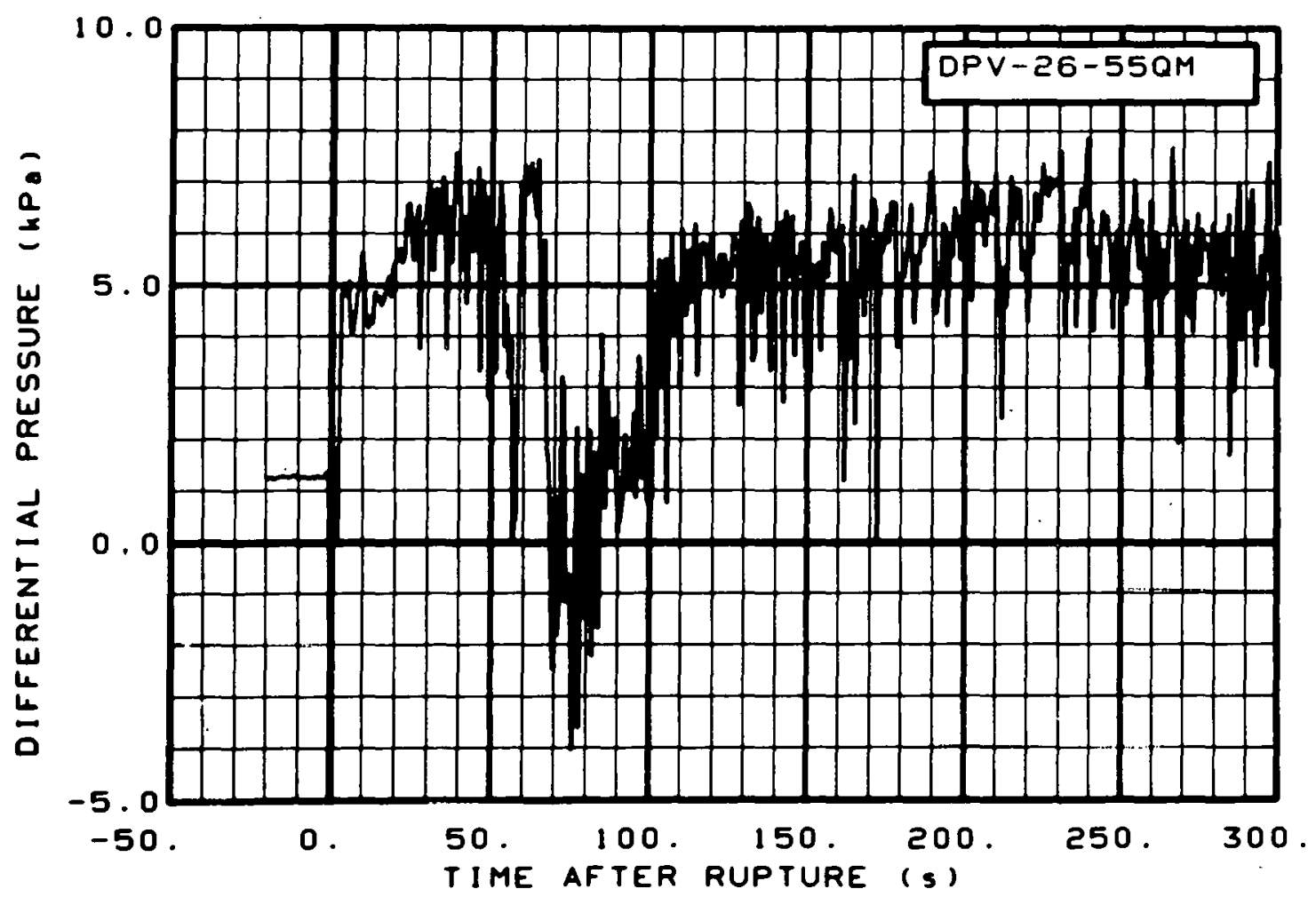

Fig. 193 Differential pressure in vessel (DPV-26-550M), from -20 to $300 \mathrm{~s}$.

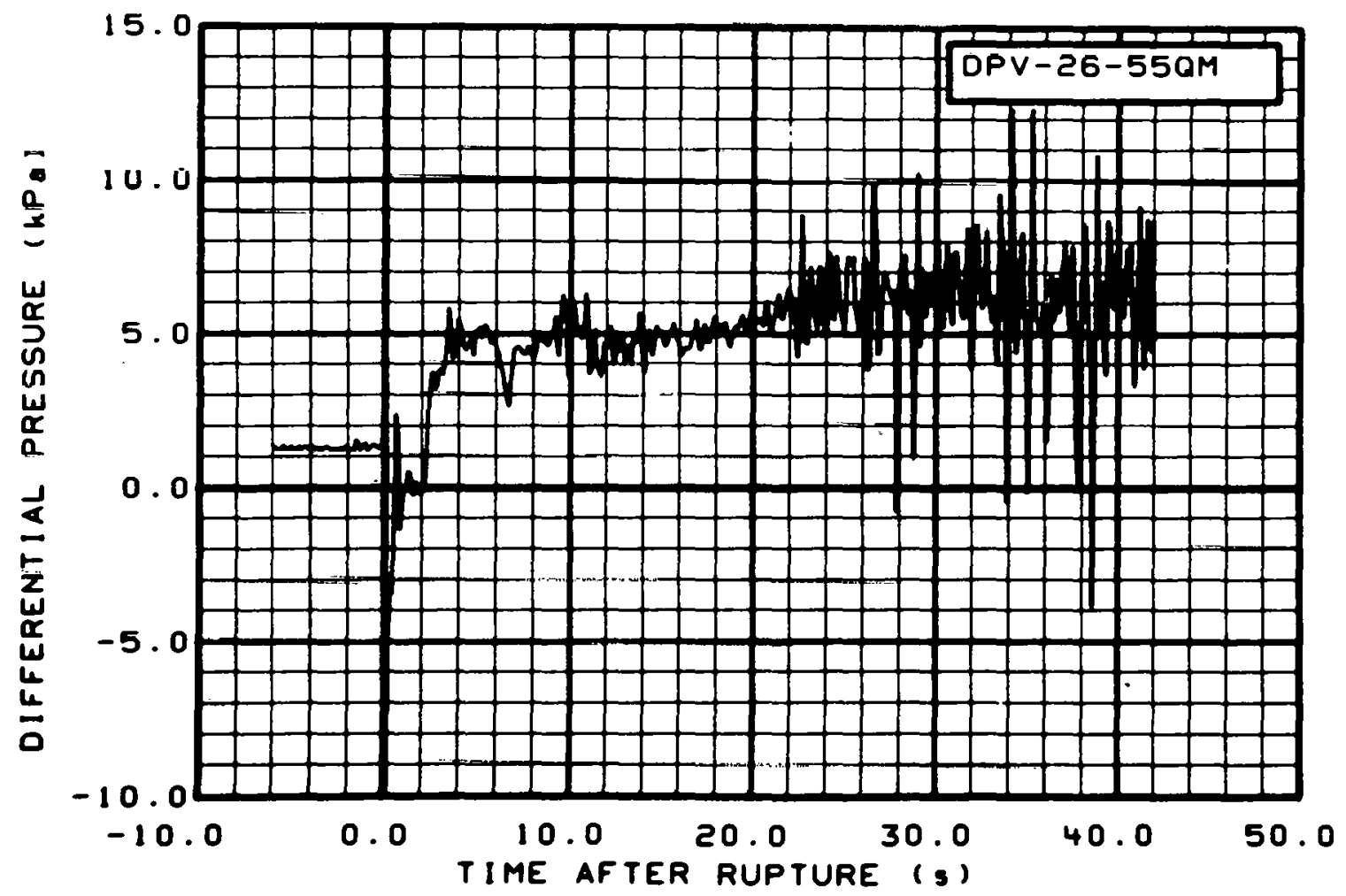

Fig. 194 Differential pressure in vessel (DPV-26-55QM), from -6 to $42 \mathrm{~s}$. 


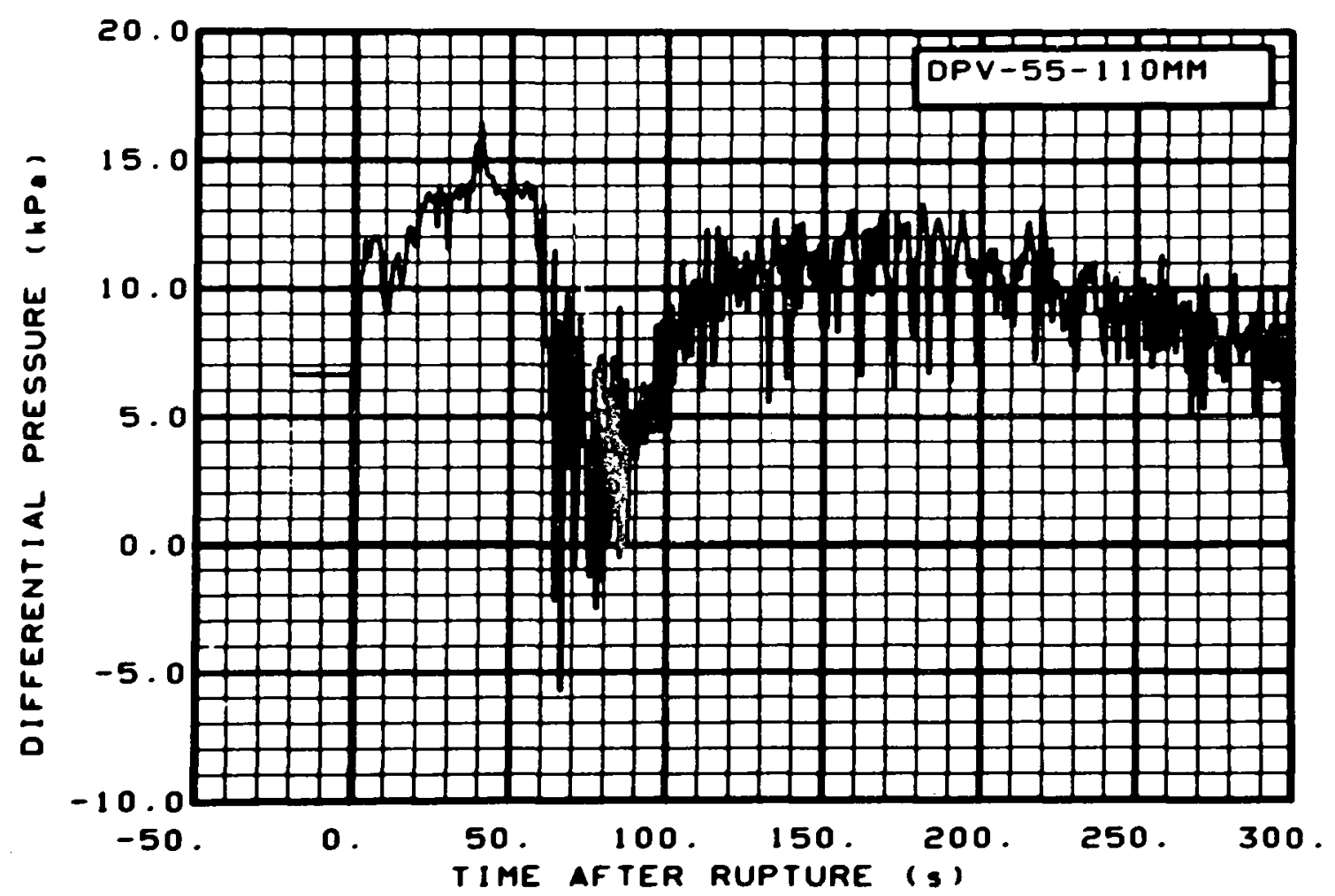

Fig. 195 Differential pressure in vessel (DPV-55-110MM), from -20 to $300 \mathrm{~s}$.

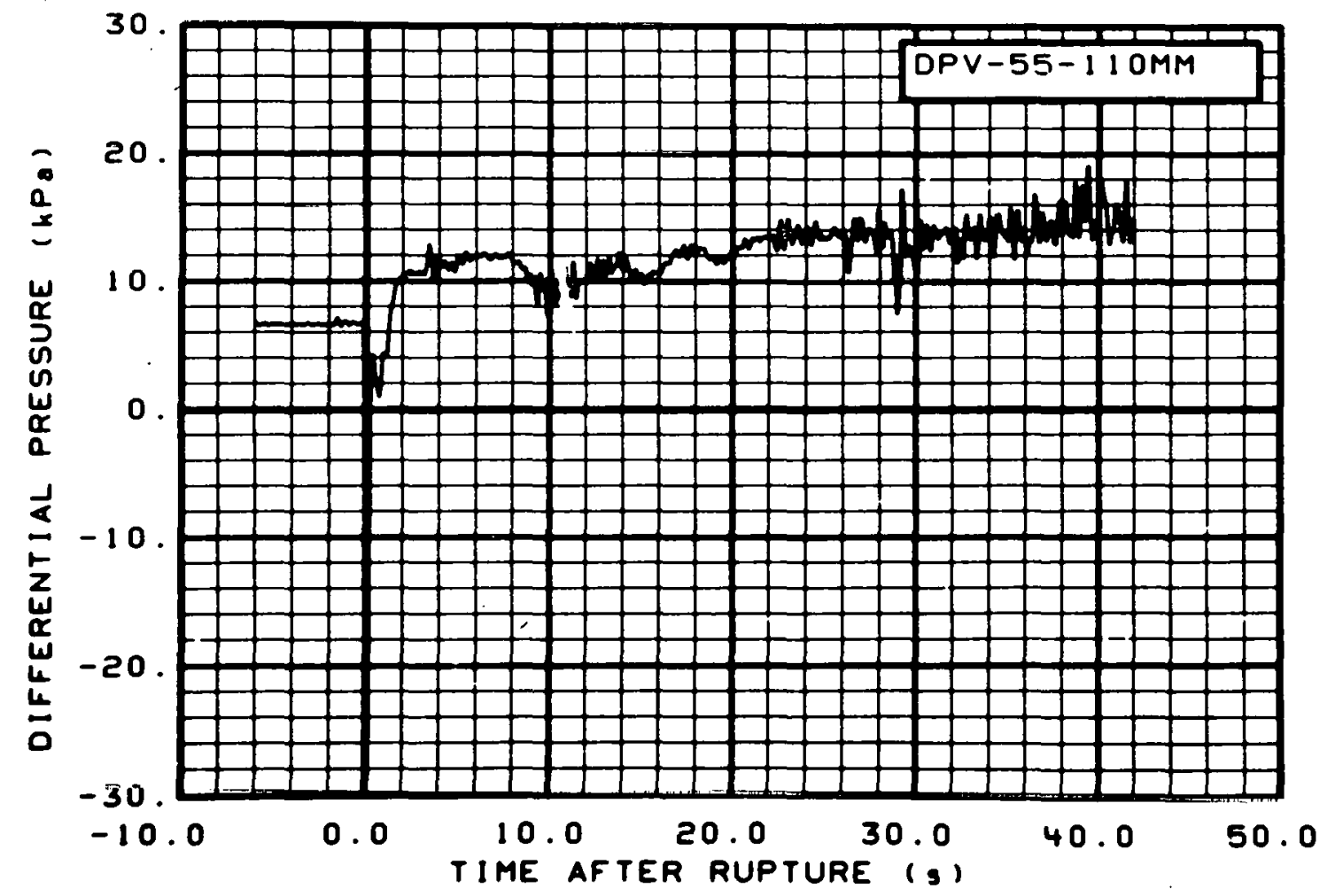

Fig. 196 Differential pressure in vessel (DPV-55-110MM), from -6 to $42 \mathrm{~s}$. 


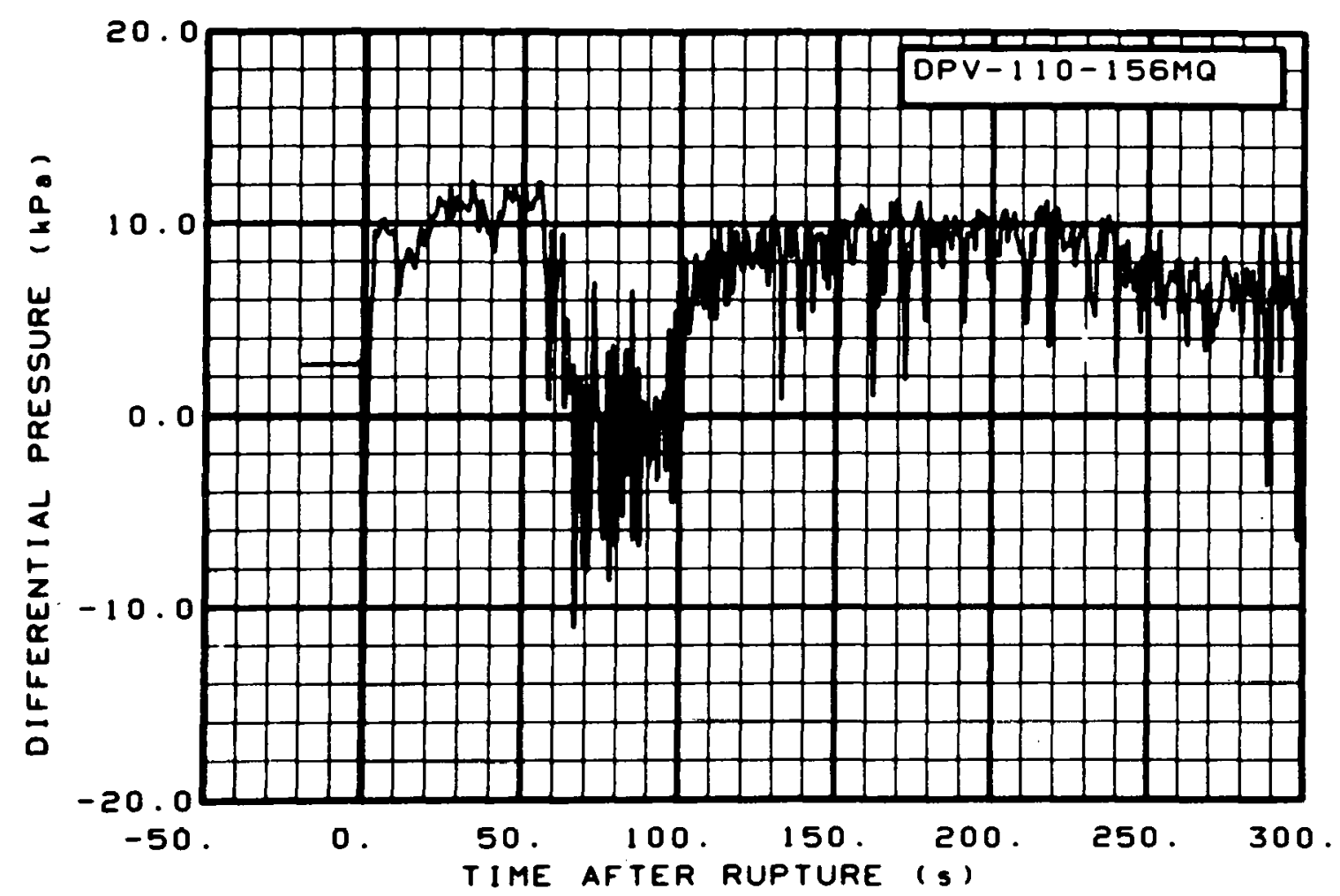

Fig. 197 Differential pressure in vessel (DPV-110-156MQ), from -20 to $300 \mathrm{~s}$.

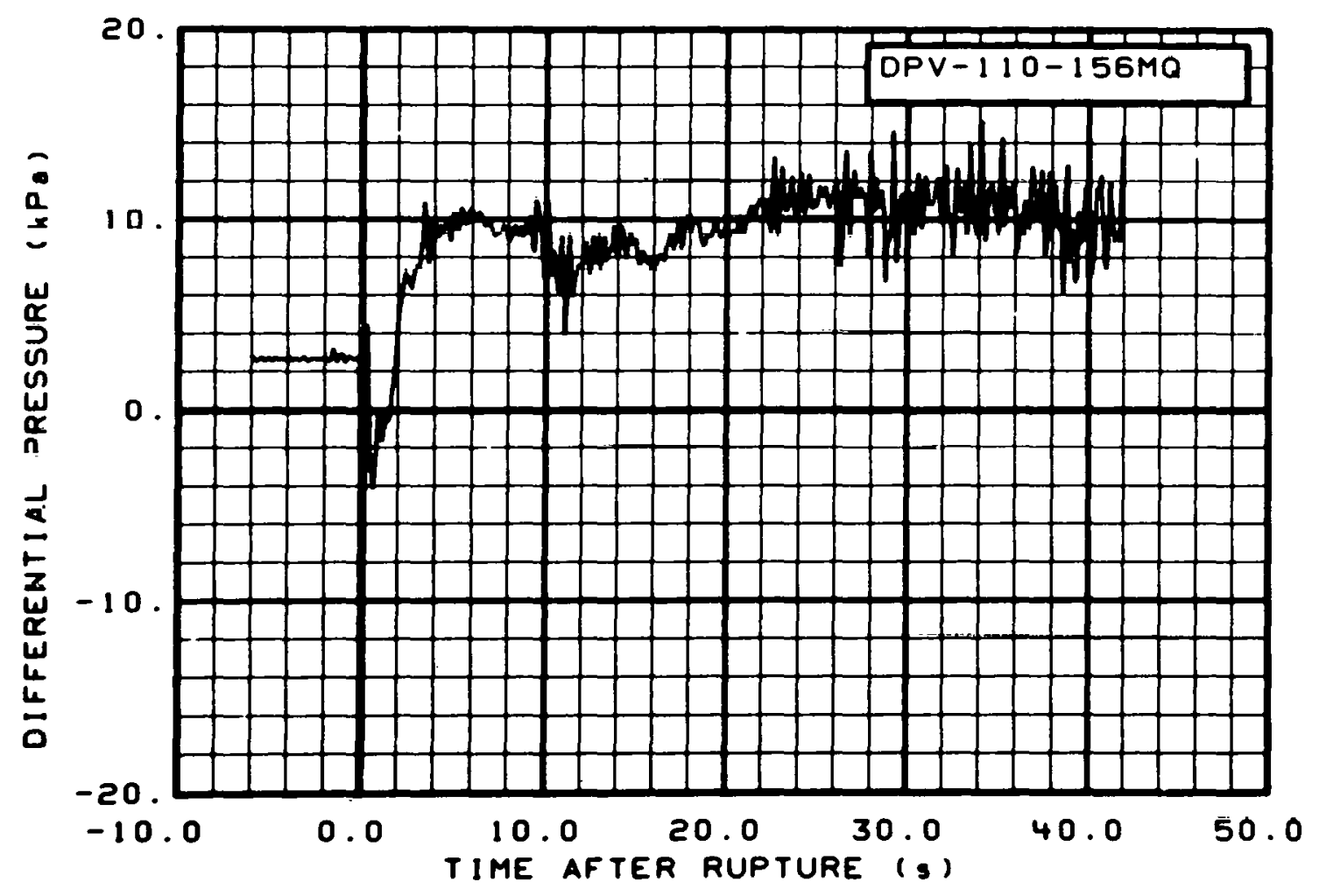

Fig. 198 Differential pressure in vessel (DPV-110-156MQ), from -6 to $42 \mathrm{~s}$ : 


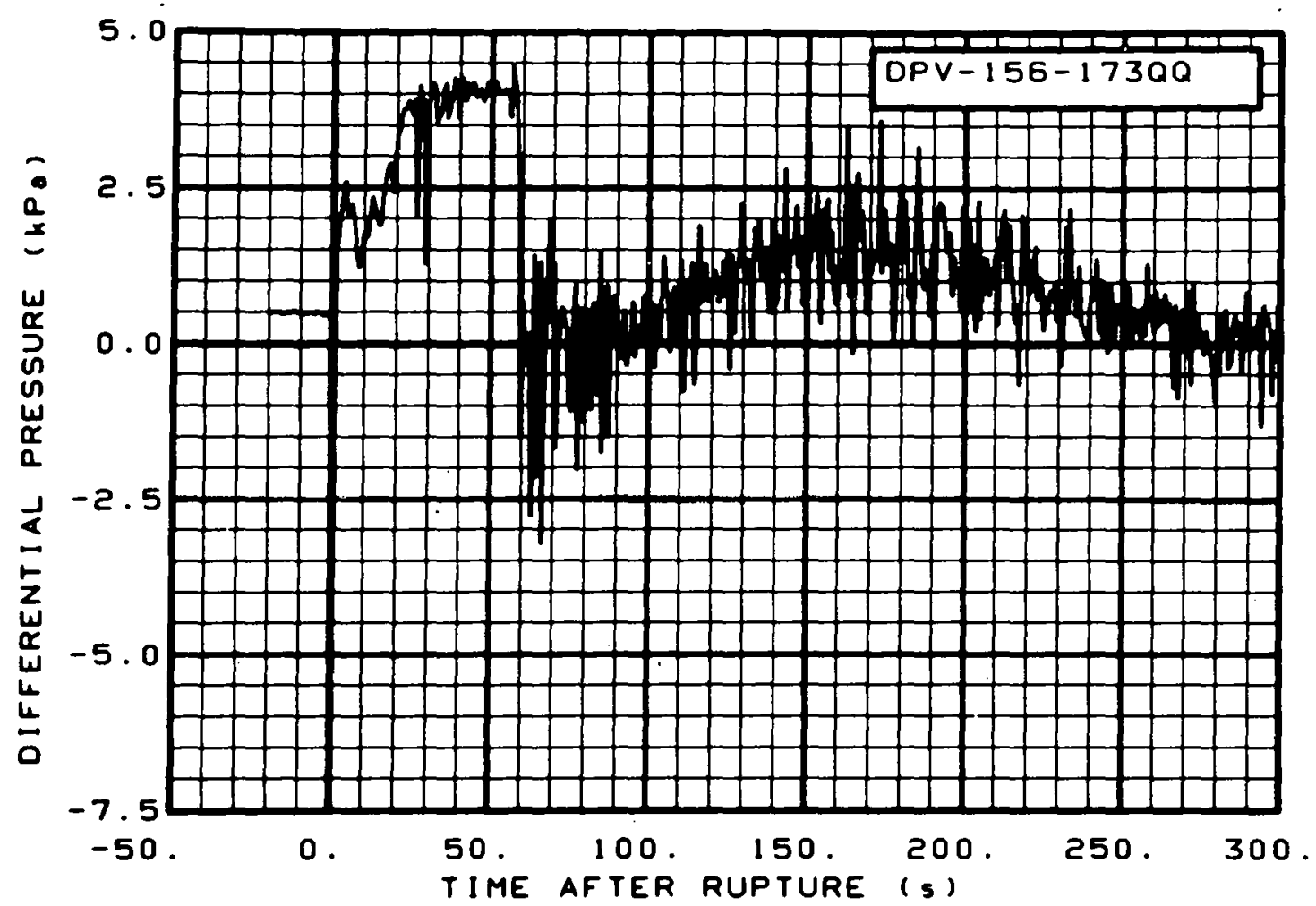

Fig. 199 Differential pressure in vessel (DPV-156-1730Q), from -20 to $300 \mathrm{~s}$.

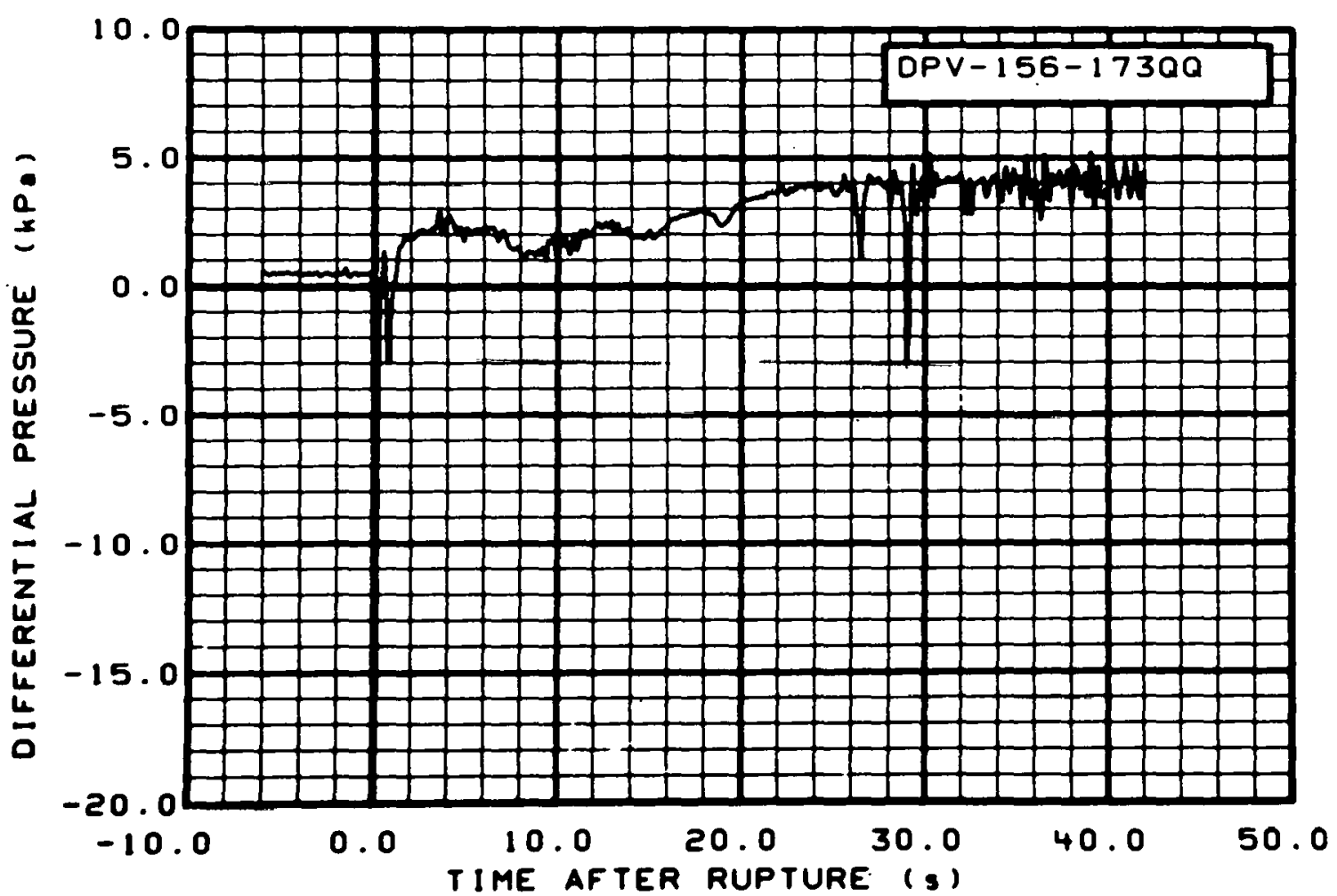

Fig. 200 Differential pressure in vessel (DPV-156-173QQ), from -6 to $42 \mathrm{~s}$. 


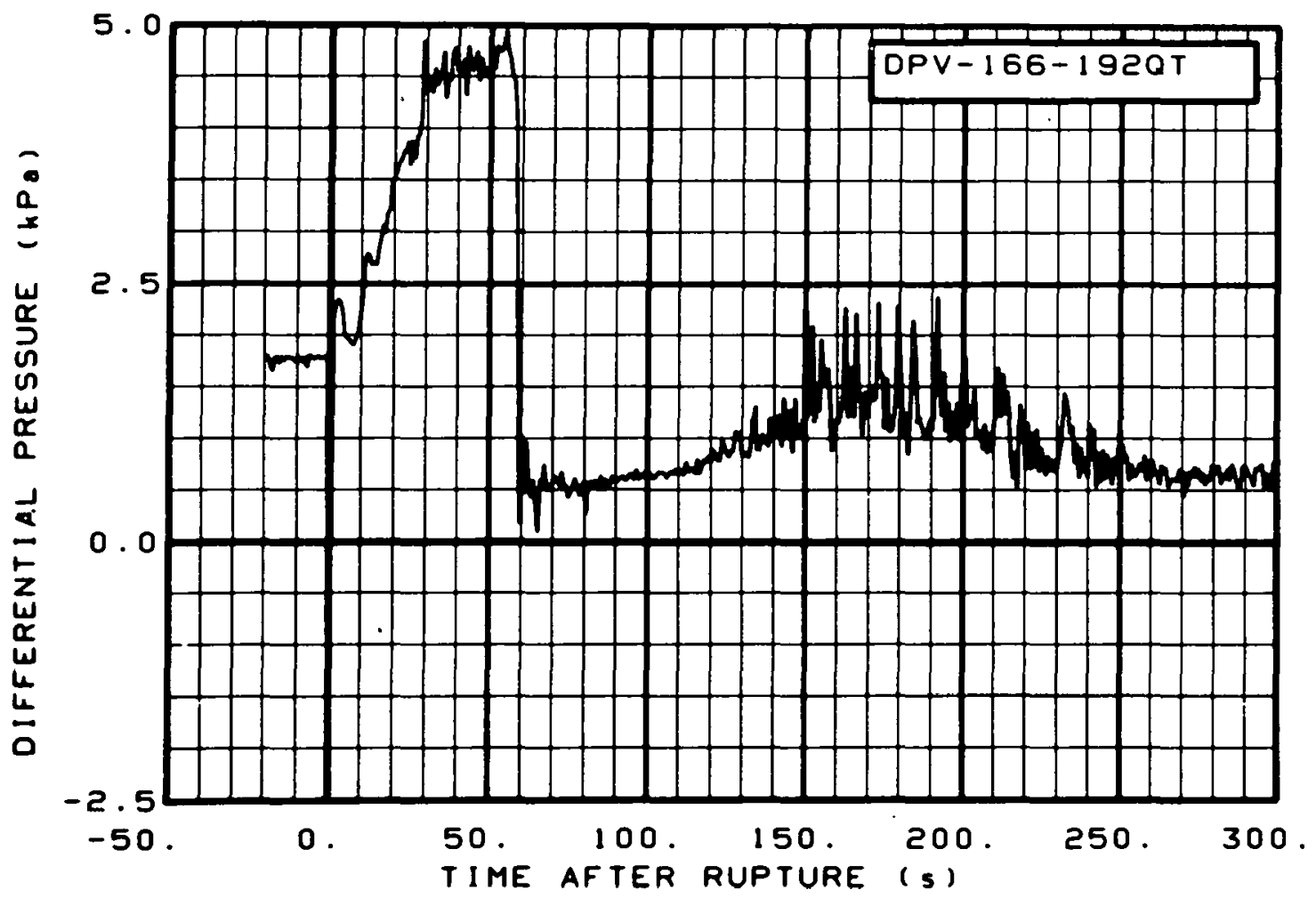

Fig. 201 Differential pressure in vessel (DPV-166-192QT), from -20 to $300 \mathrm{~s}$.

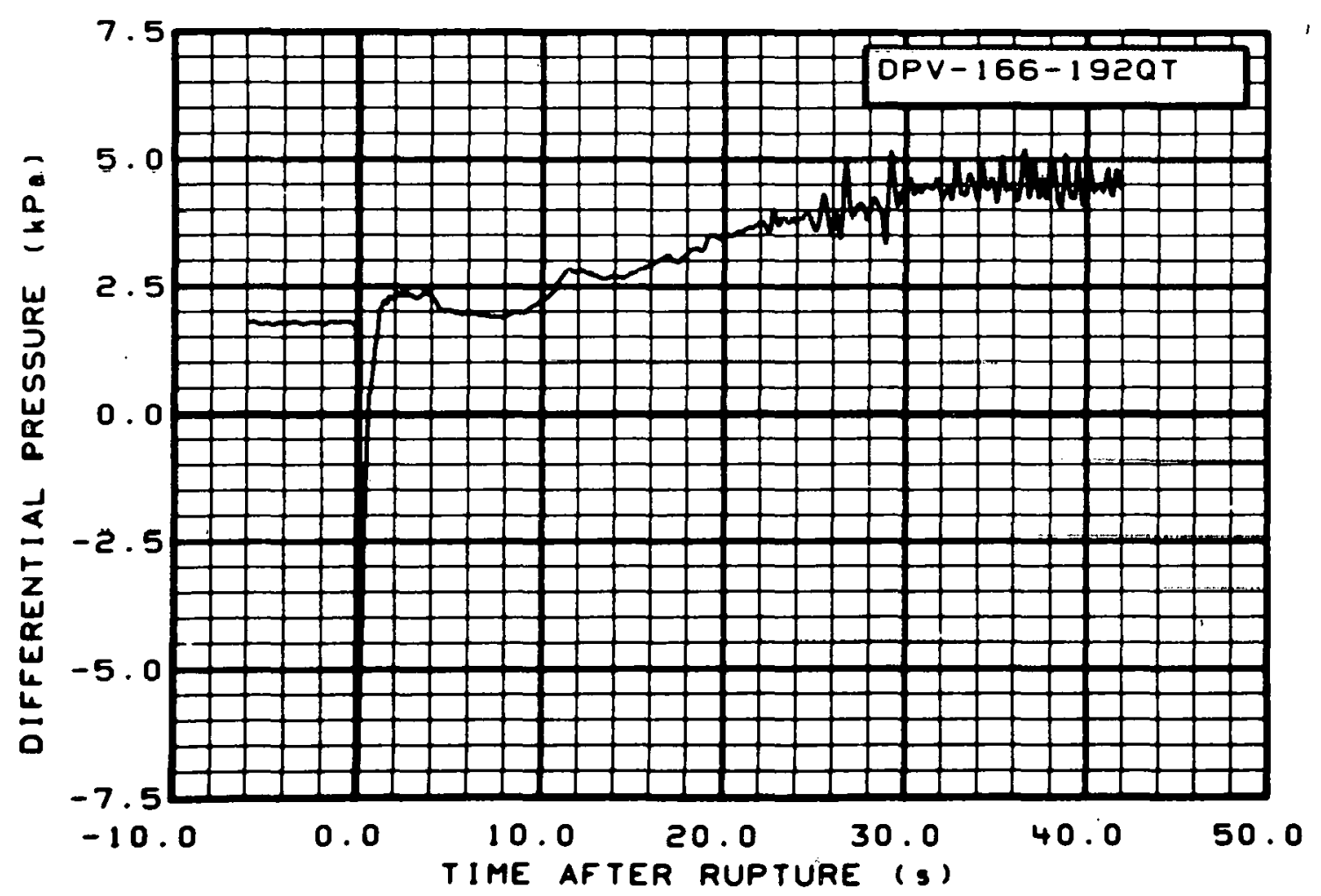

Fig. 202 Differential pressure in vessel (DPV-166-192QT), from -6 to $42 \mathrm{~s}$. 


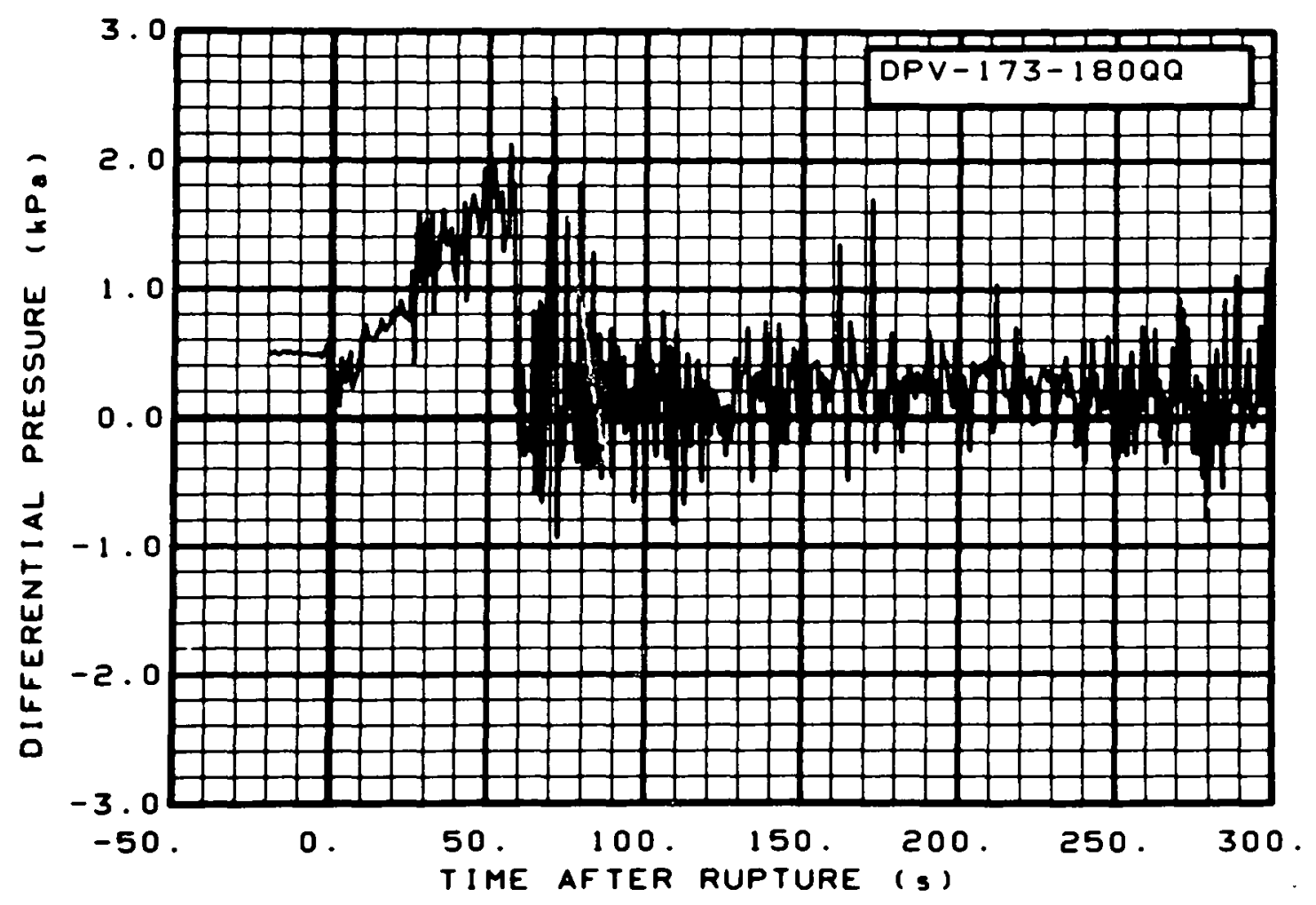

Fig. 203 Differential pressure in vessel (DPV-173-180QQ), from -20 to $300 \mathrm{~s}$.

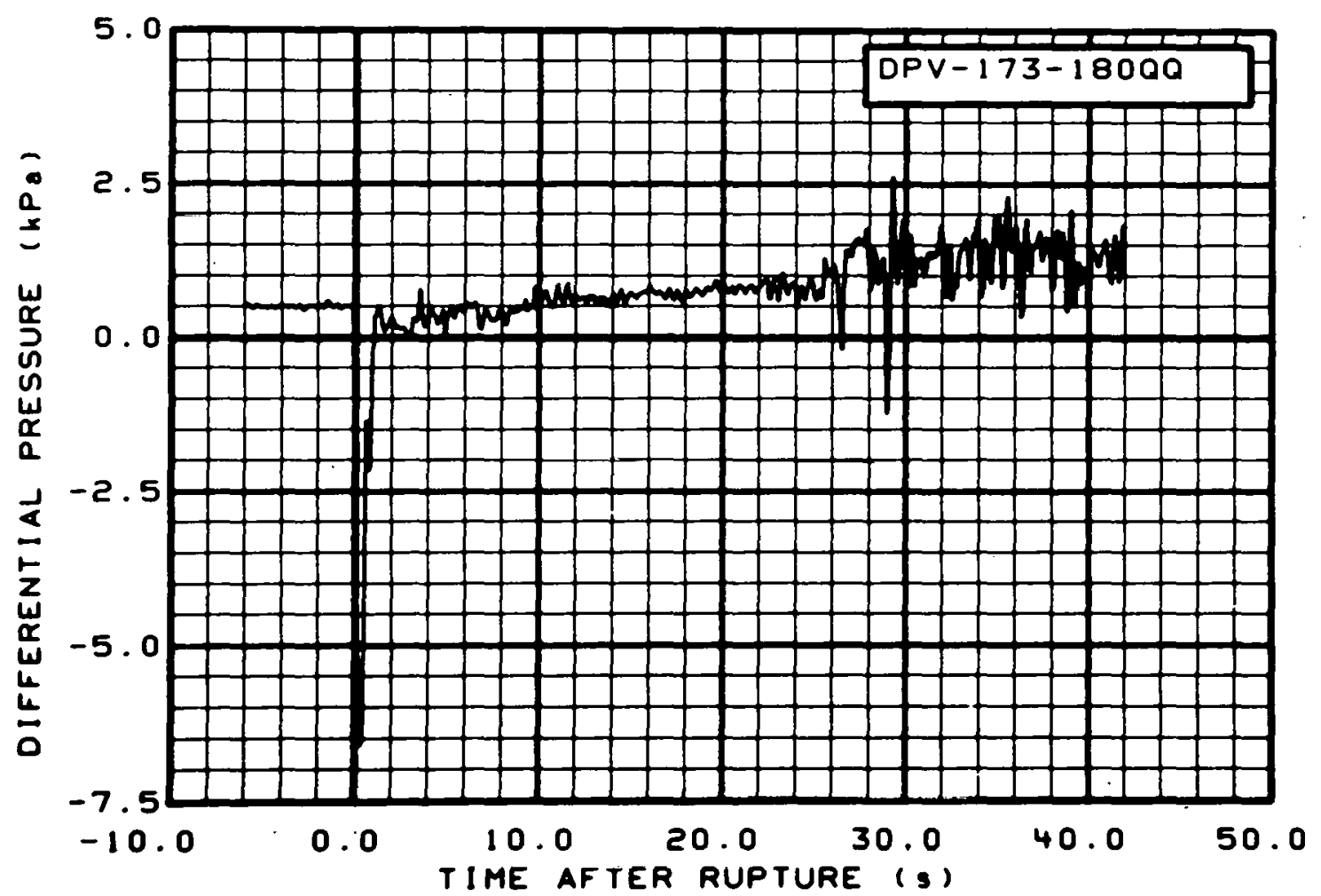

Fig. 204 Differential pressure in vessel (DPV-173-180QQ), from -6 to $42 \mathrm{~s}$. 


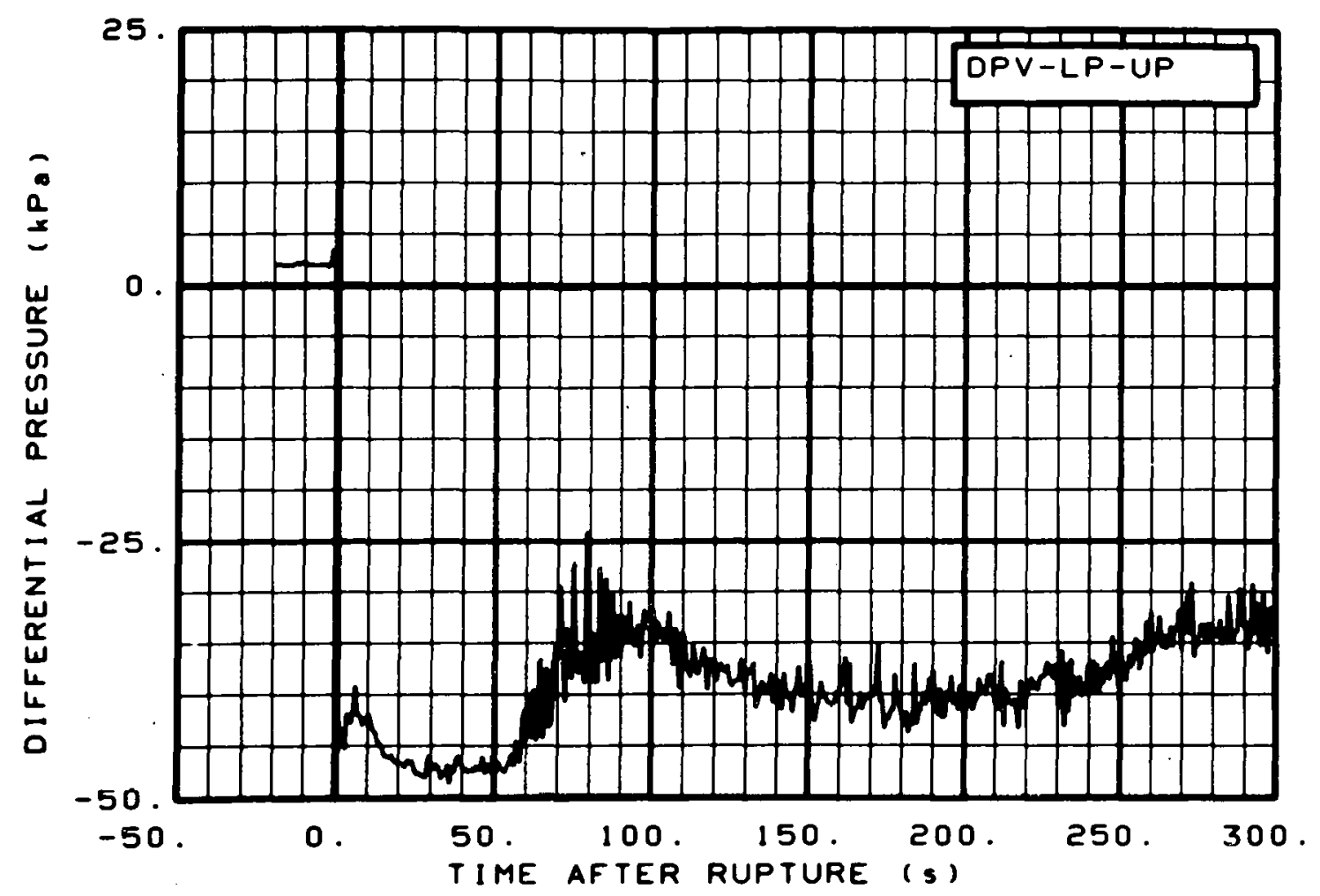

Fig. 205 Differential pressure in vessel (DPV-LP-UP), from -20 to $300 \mathrm{~s}$.

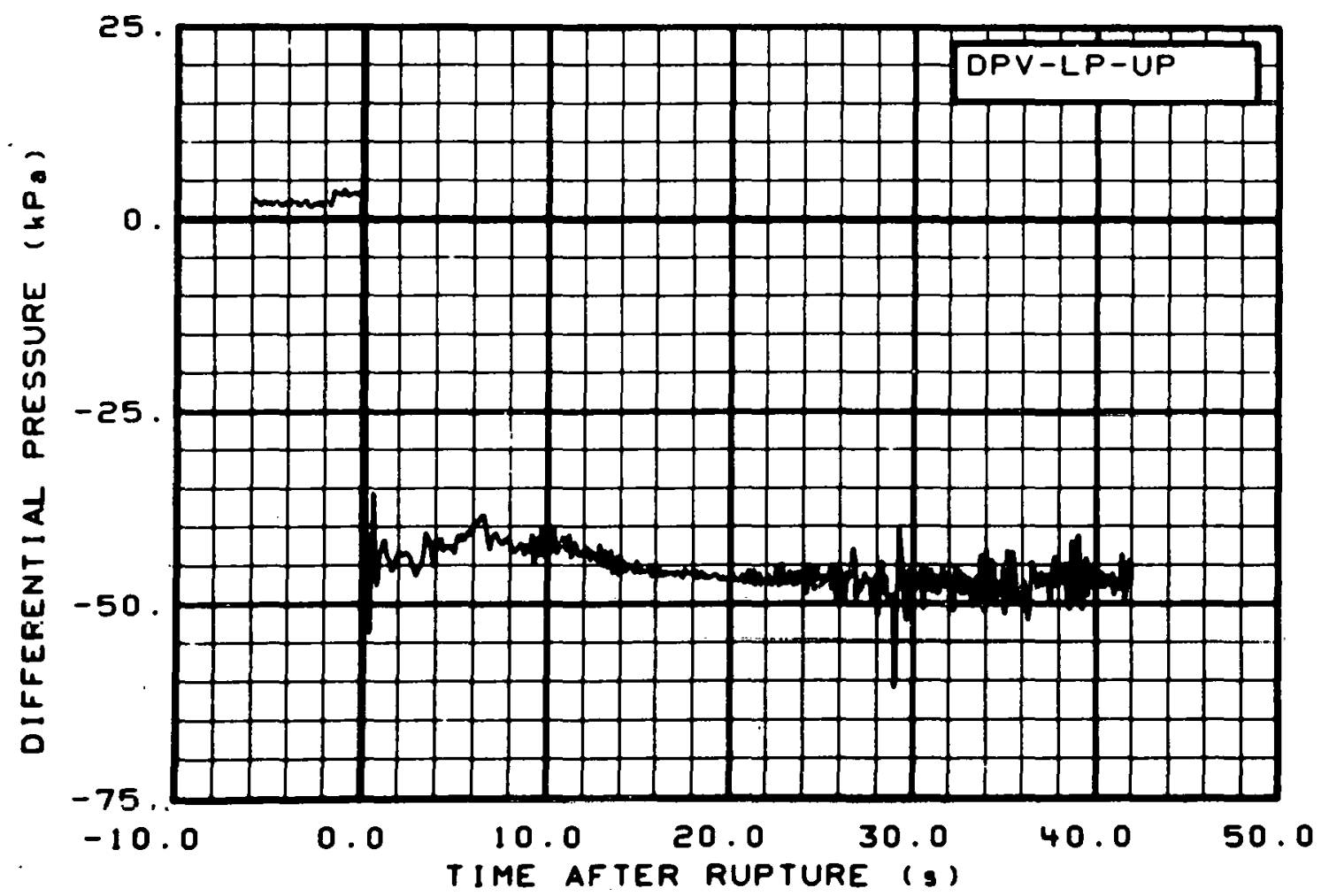

Fig. 206 Differential pressure in vessel (DPV-LP-UP), from -6 to $42 \mathrm{~s}$. 


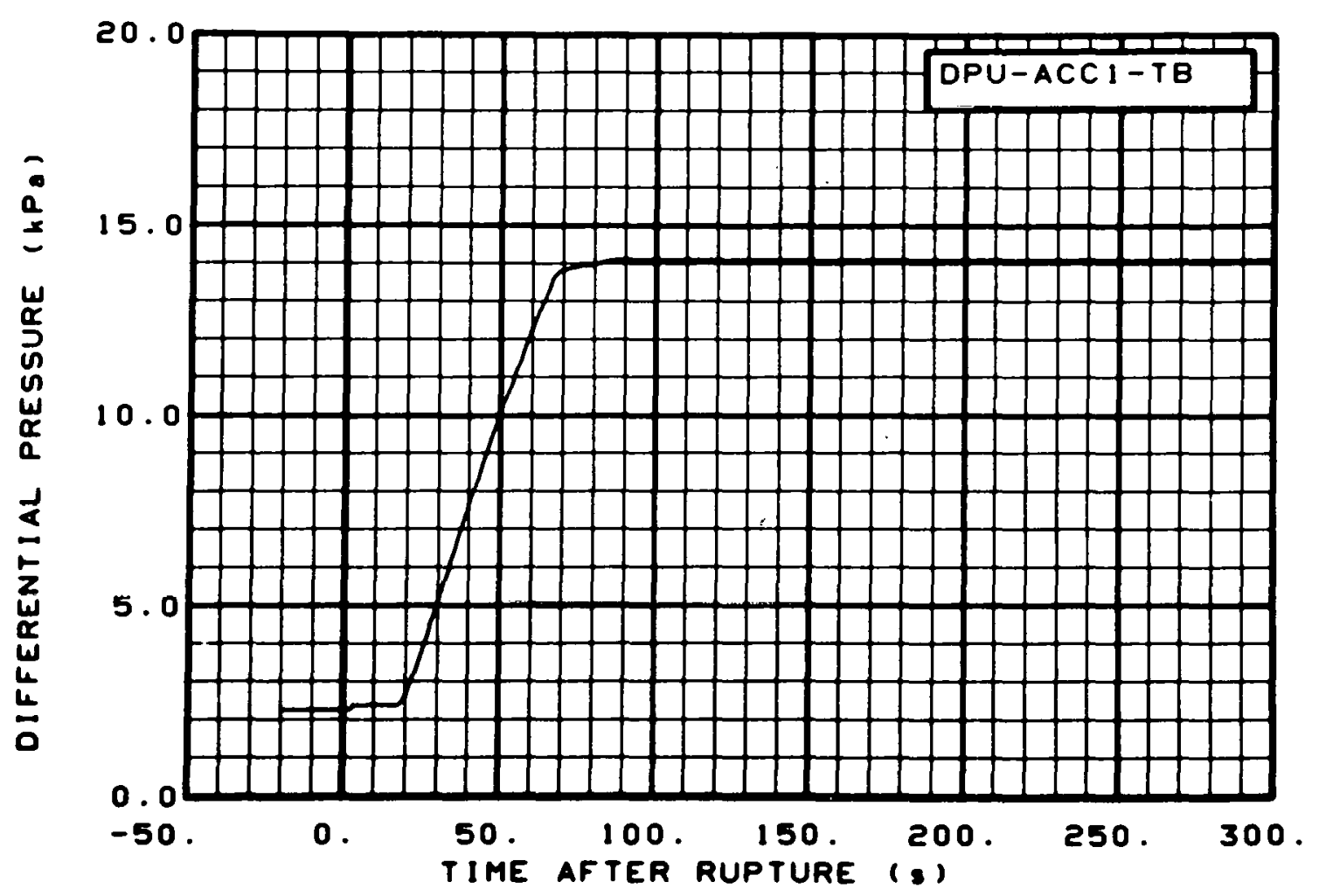

Fig. 207 Differential pressure in intact loop accumulator (DPU-ACCI-TB), from -20 to $300 \mathrm{~s}$.

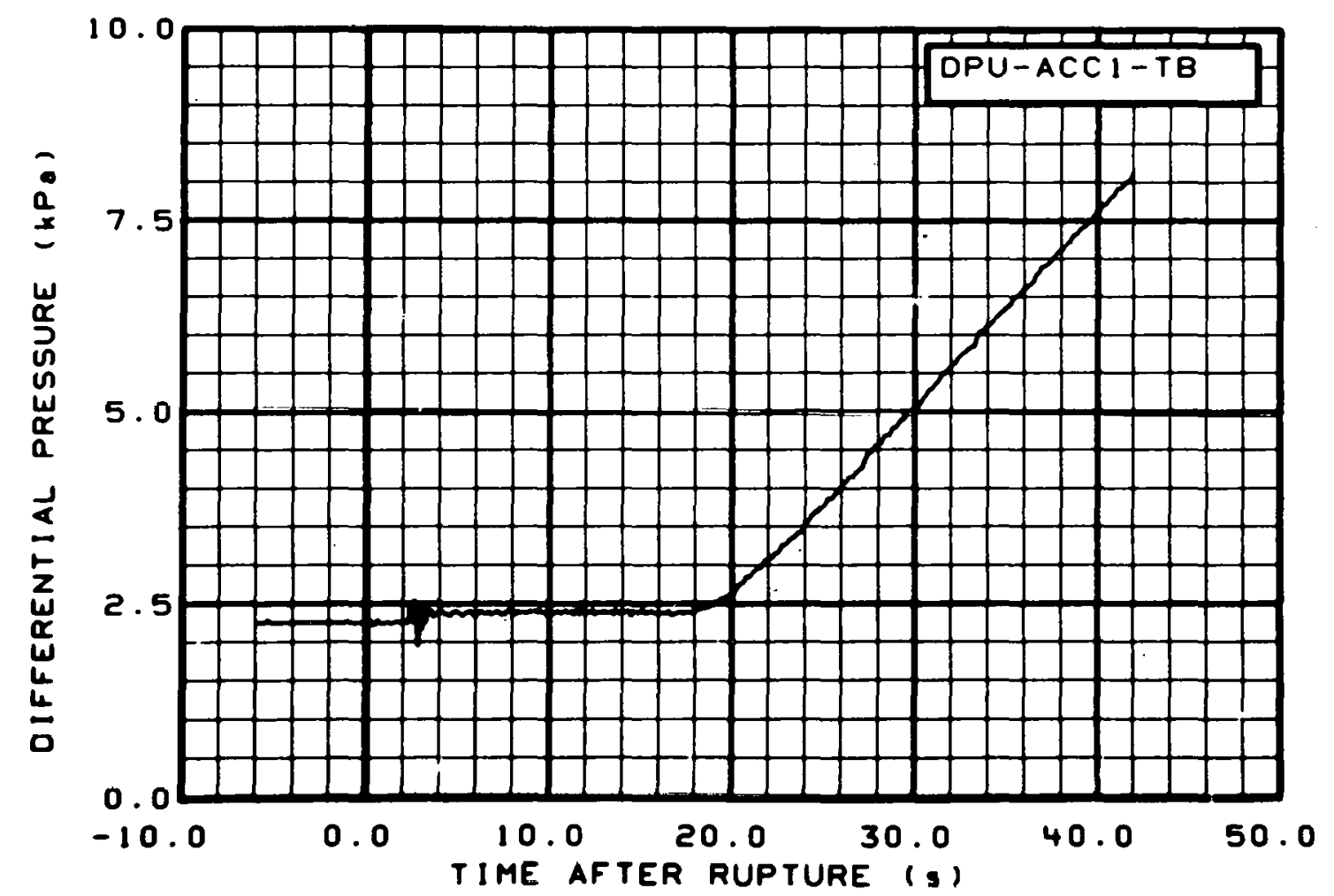

Fig. 208 Differential pressure in intact loop accumulator (DPU-ACC1-TB), from -6 to $42 \mathrm{~s}$. 


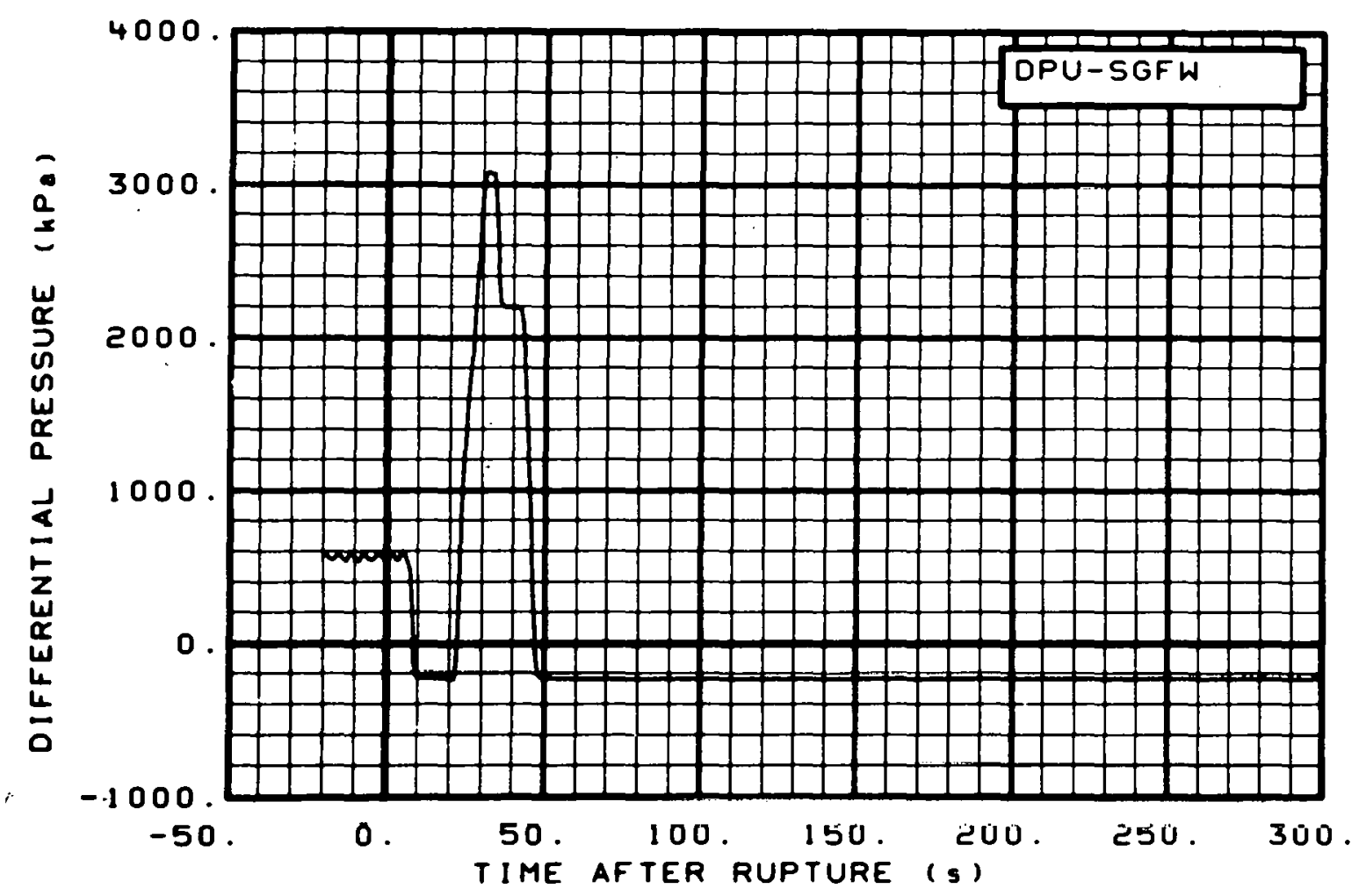

Fig. 209 Differential pressure across steam generator feedwater orifice (DPU-SGFW), from -20 to $300 \mathrm{~s}$.

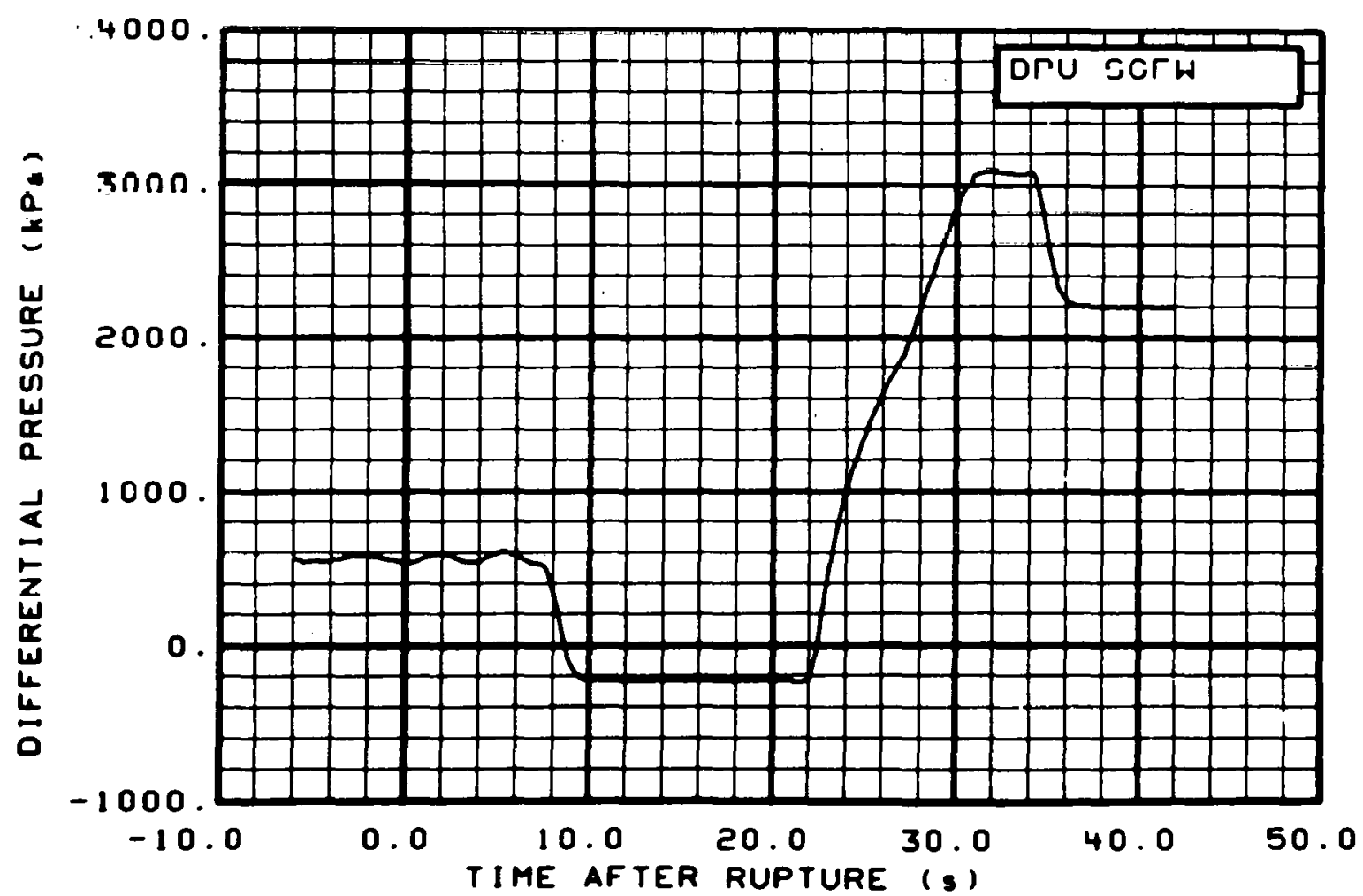

Fig. 210 Differential pressure across steam generator feedwater orifice (DPU-SGFW), from -6 to $42 \mathrm{~s}$. 


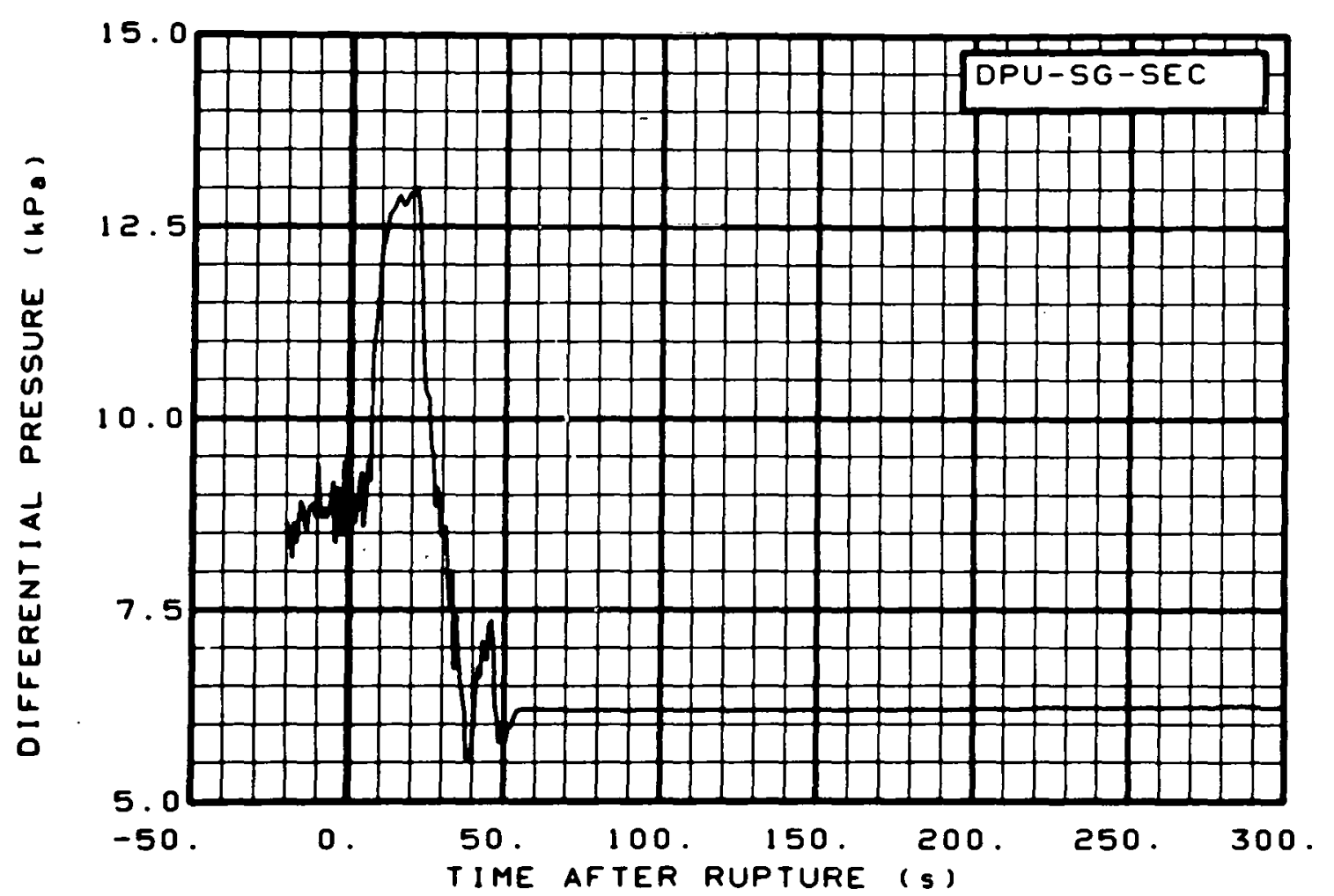

Fig. 211 Differential pressure in steam generator secondary (DPU-SG-SEC), from -20 to $300 \mathrm{~s}$.

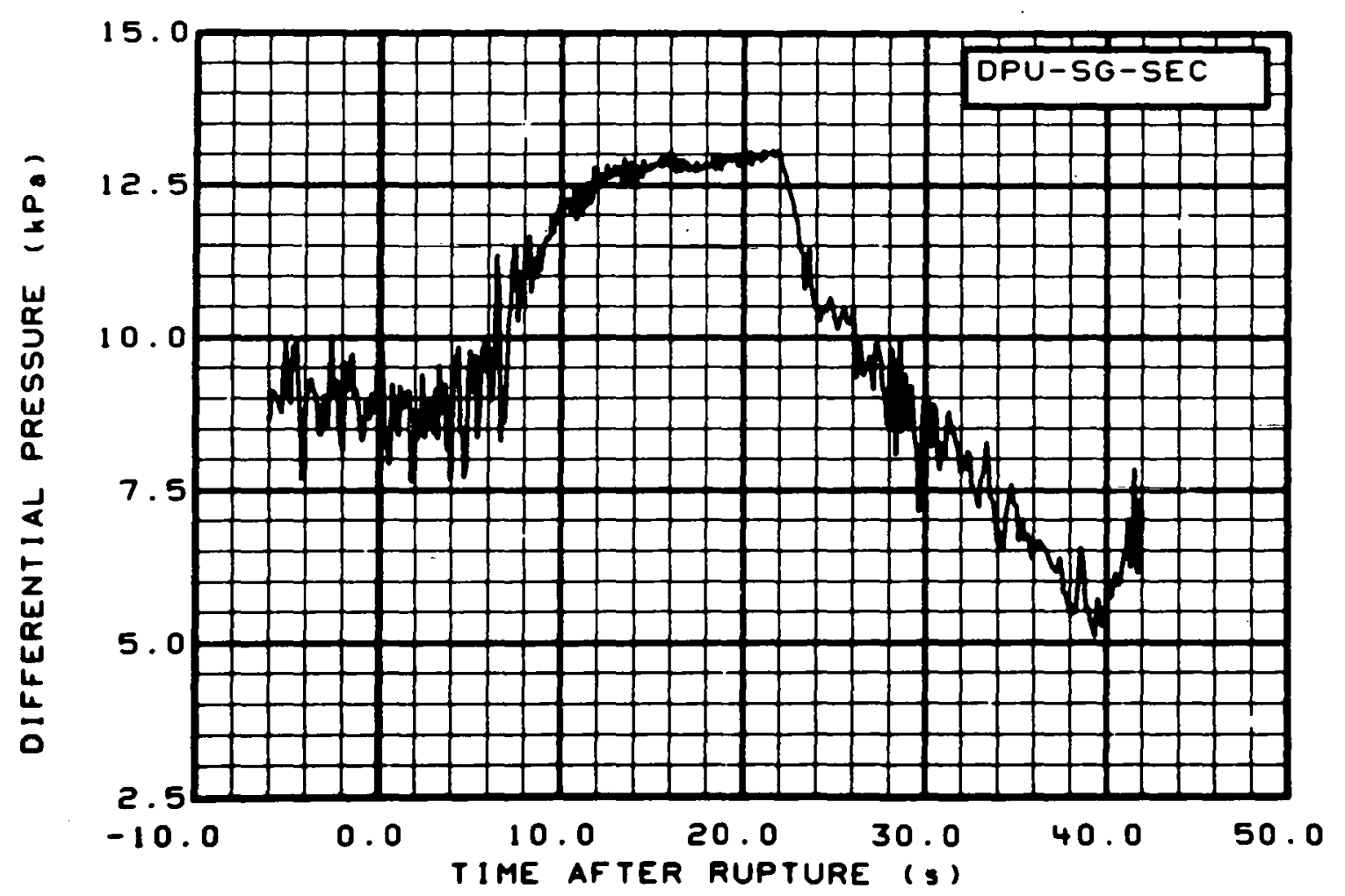

Fig. 212 Differential pressure in steam generator secondary (DPU-SG-SEC), from -6 to $42 \mathrm{~s}$. 


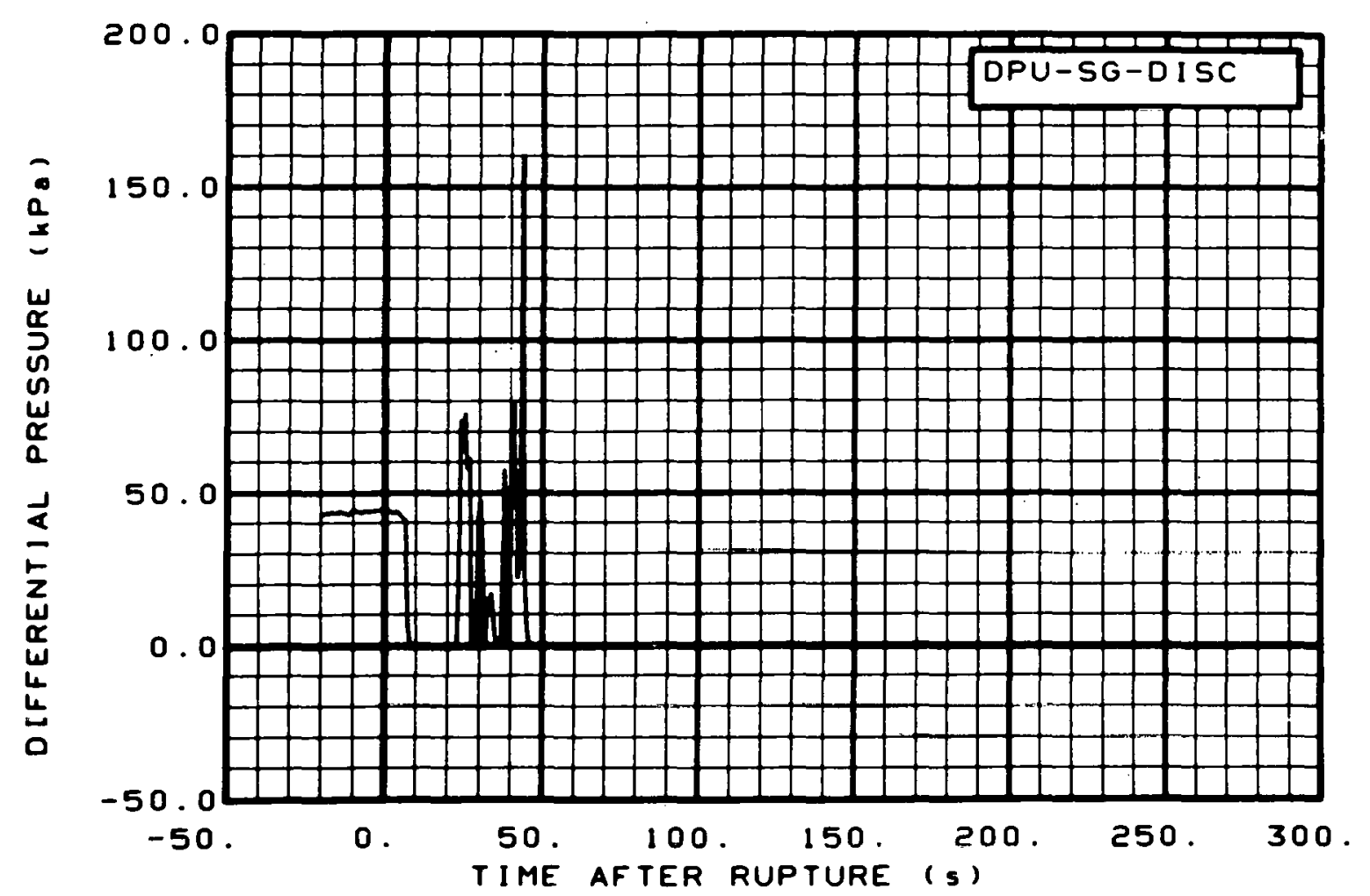

Fig. 213 Differential pressure across steam generator outlet orifice (DPU-SG-DISC), from -20 to $300 \mathrm{~s}$.

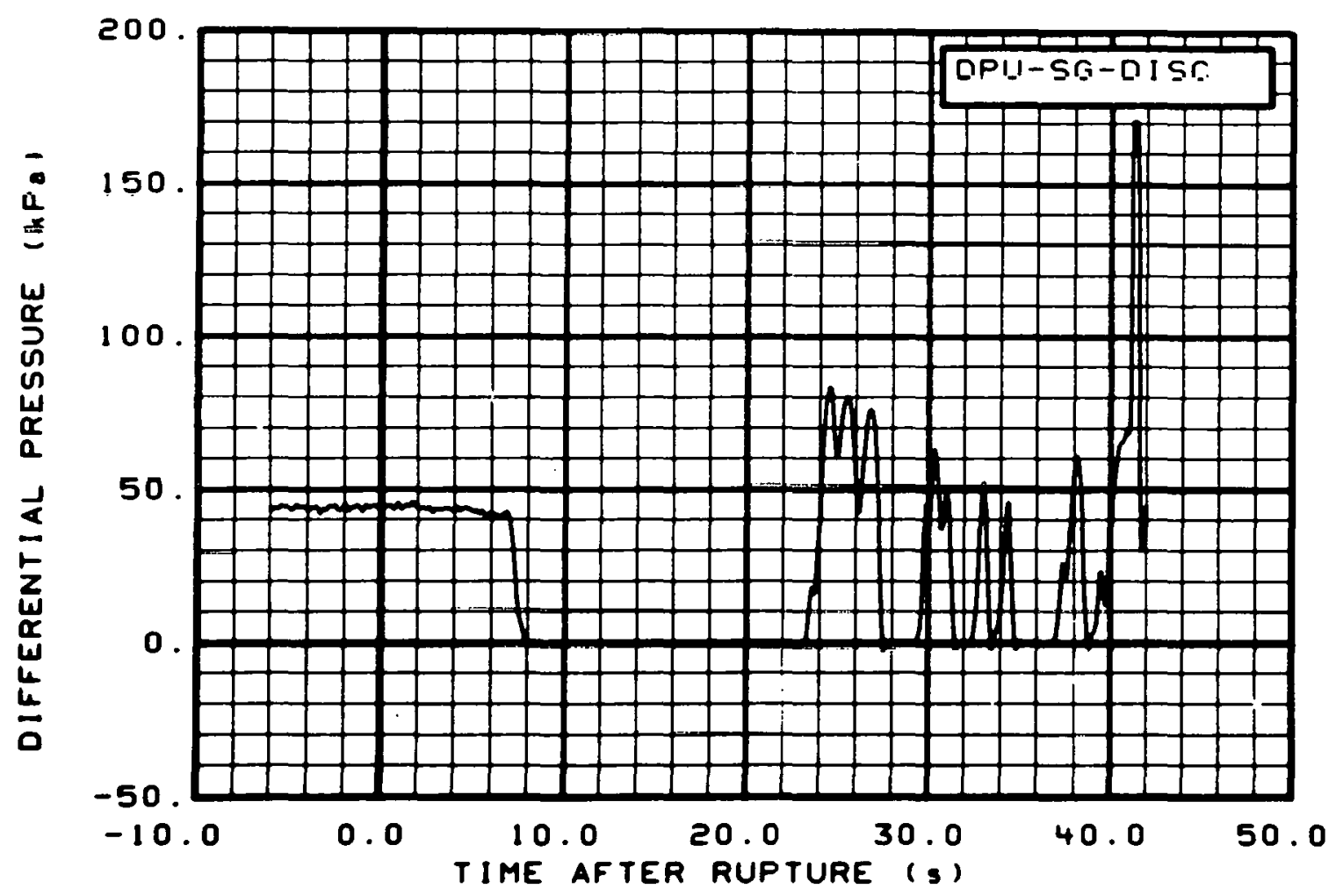

Fig. 214 Differential pressure across steam generator outlet orifice (DPU-SG-DISC), from -6 to $42 \mathrm{~s}$. 


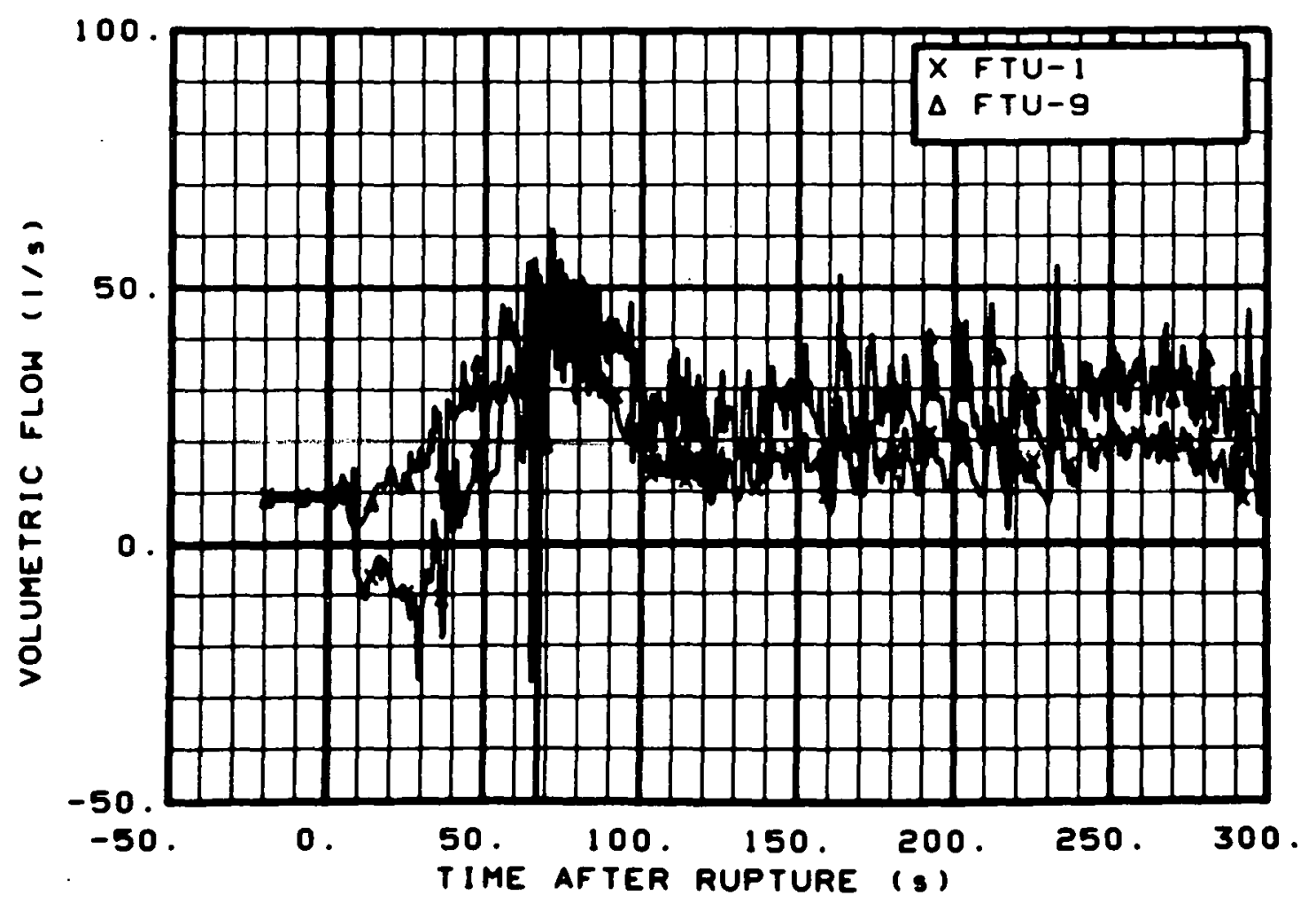

Fig. 215 Volumetric flow in intact loop (FTU-1 and FTU-9), from -20 to $300 \mathrm{~s}$.

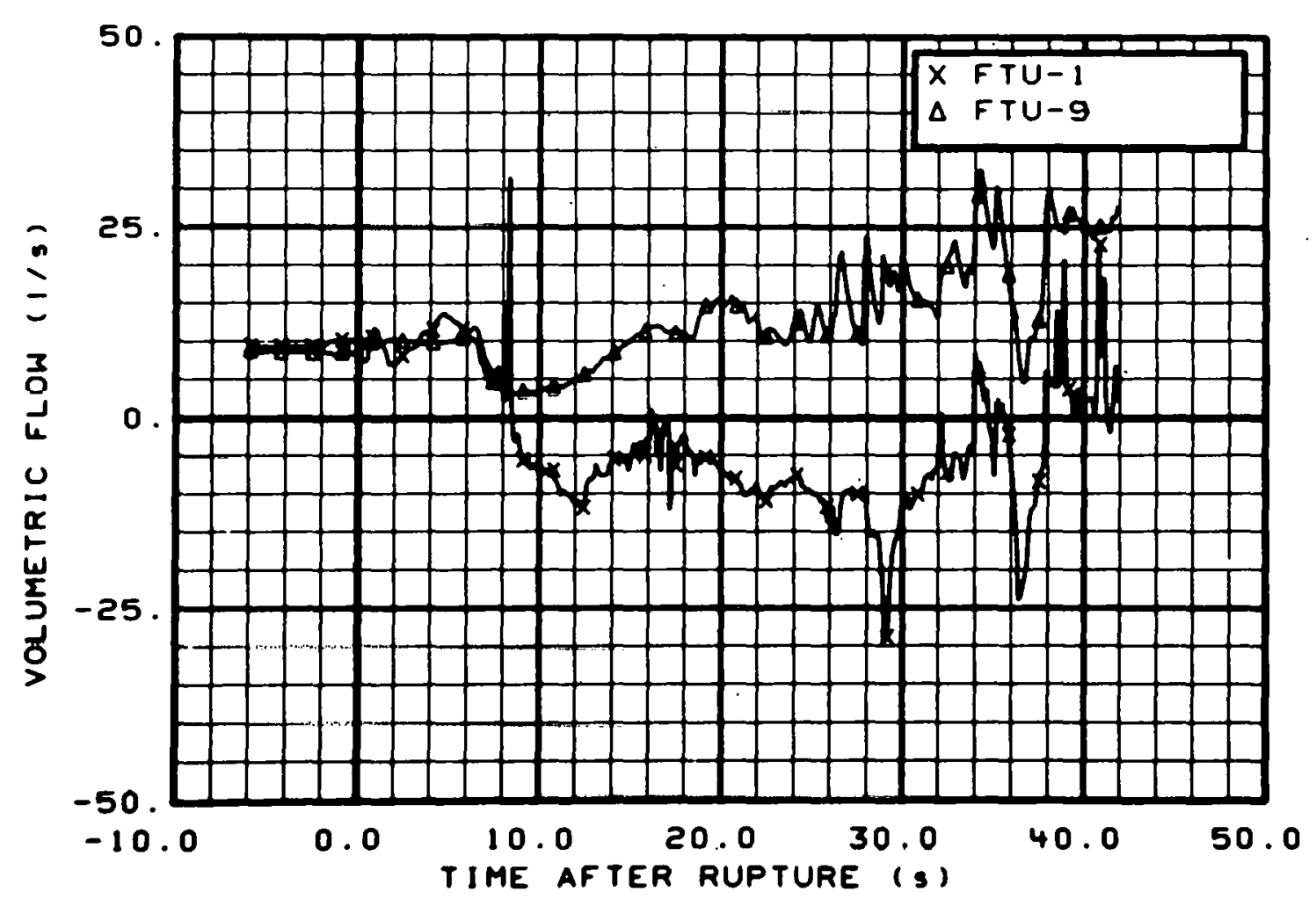

Fig. 216 Volumetric flow in intact loop (FTU-1 and FTU-9), from -6 to $42 \mathrm{~s}$. 


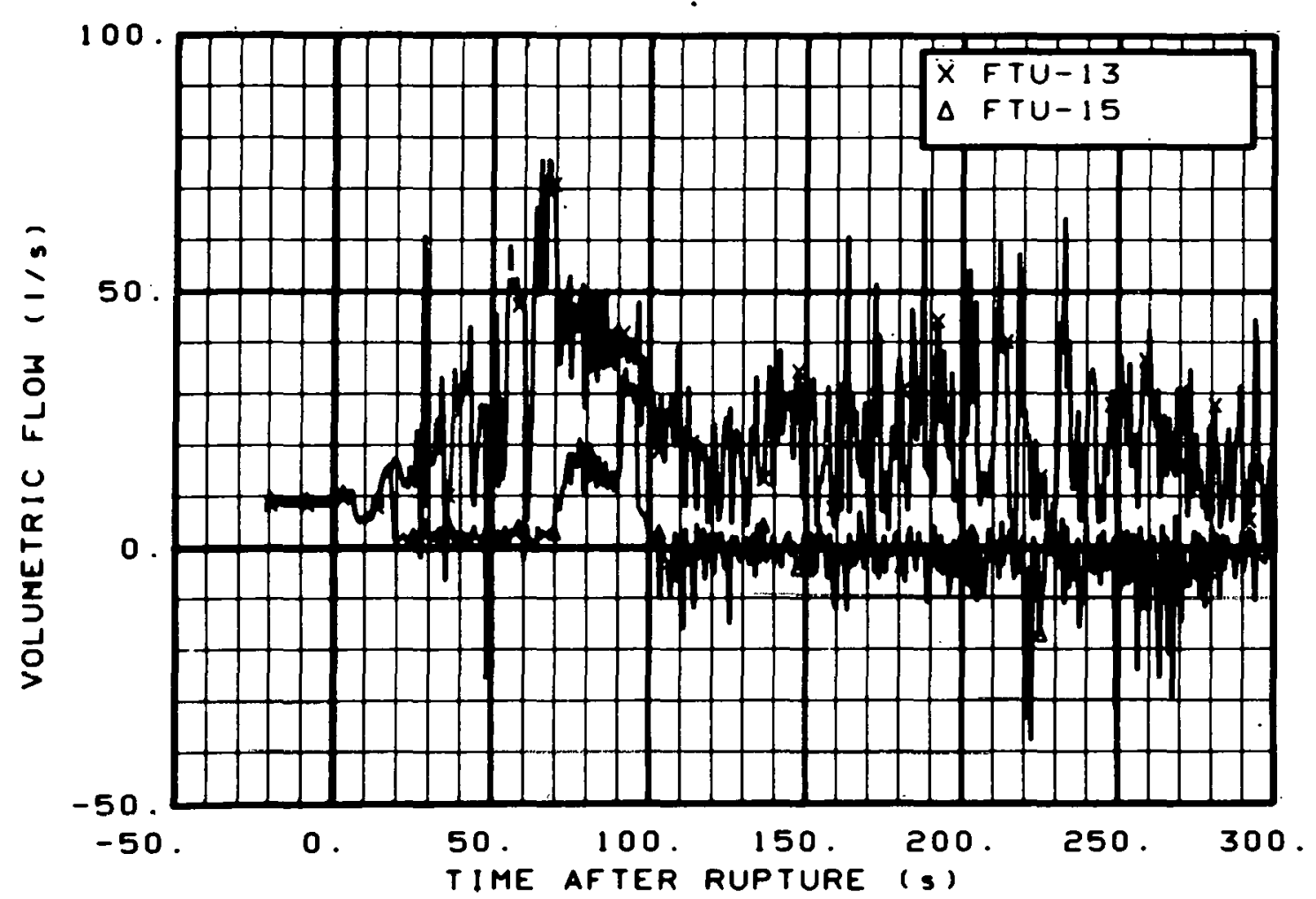

Fig. 217 Volumetric flow in intact loop (FTU-13 and FTU-15), from -20 to $300 \mathrm{~s}$.

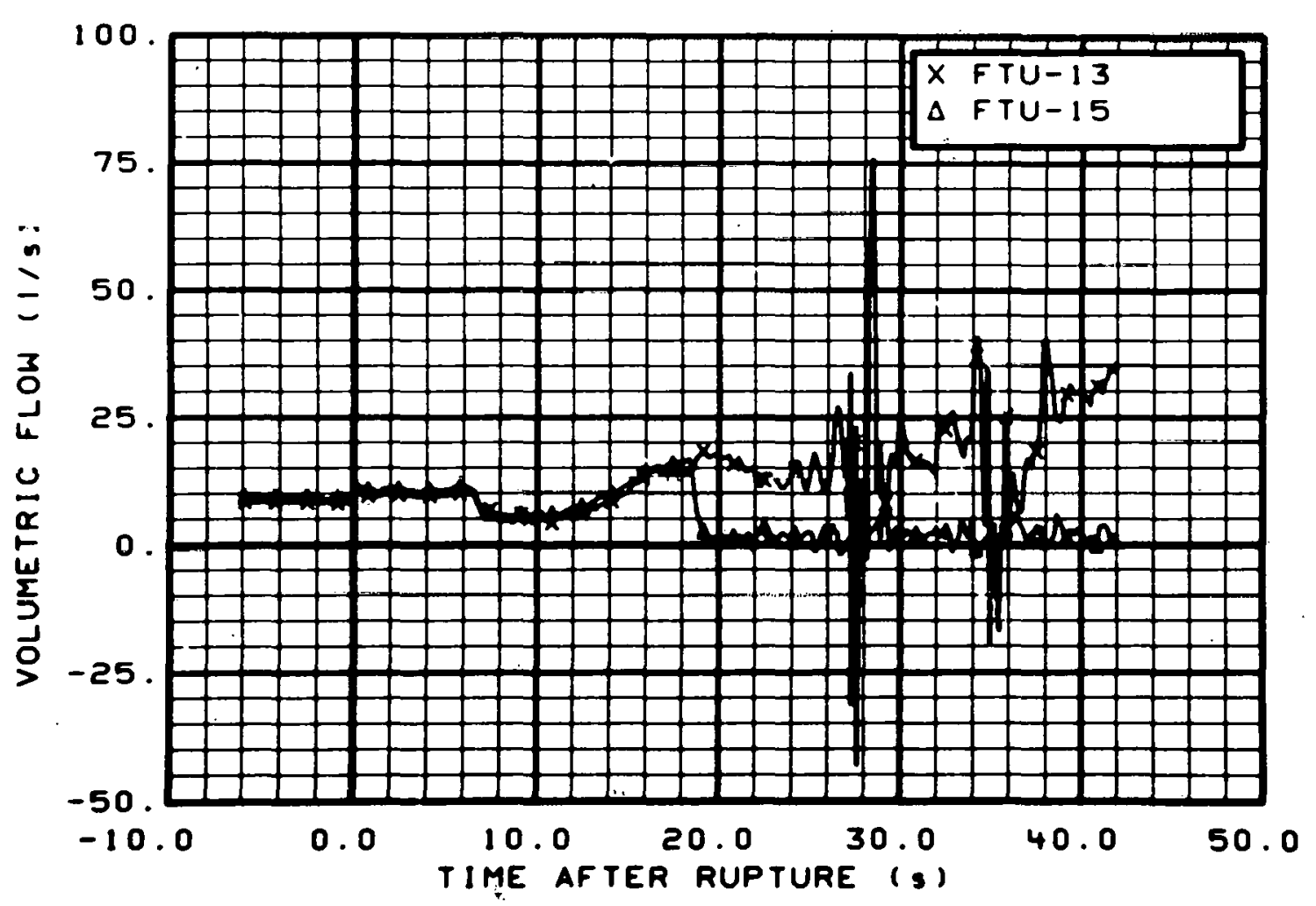

Fig. 218 Volumetric flow in intact loop (FTU-13 and FTU-15), from -6: to $42 \mathrm{~s}$. 


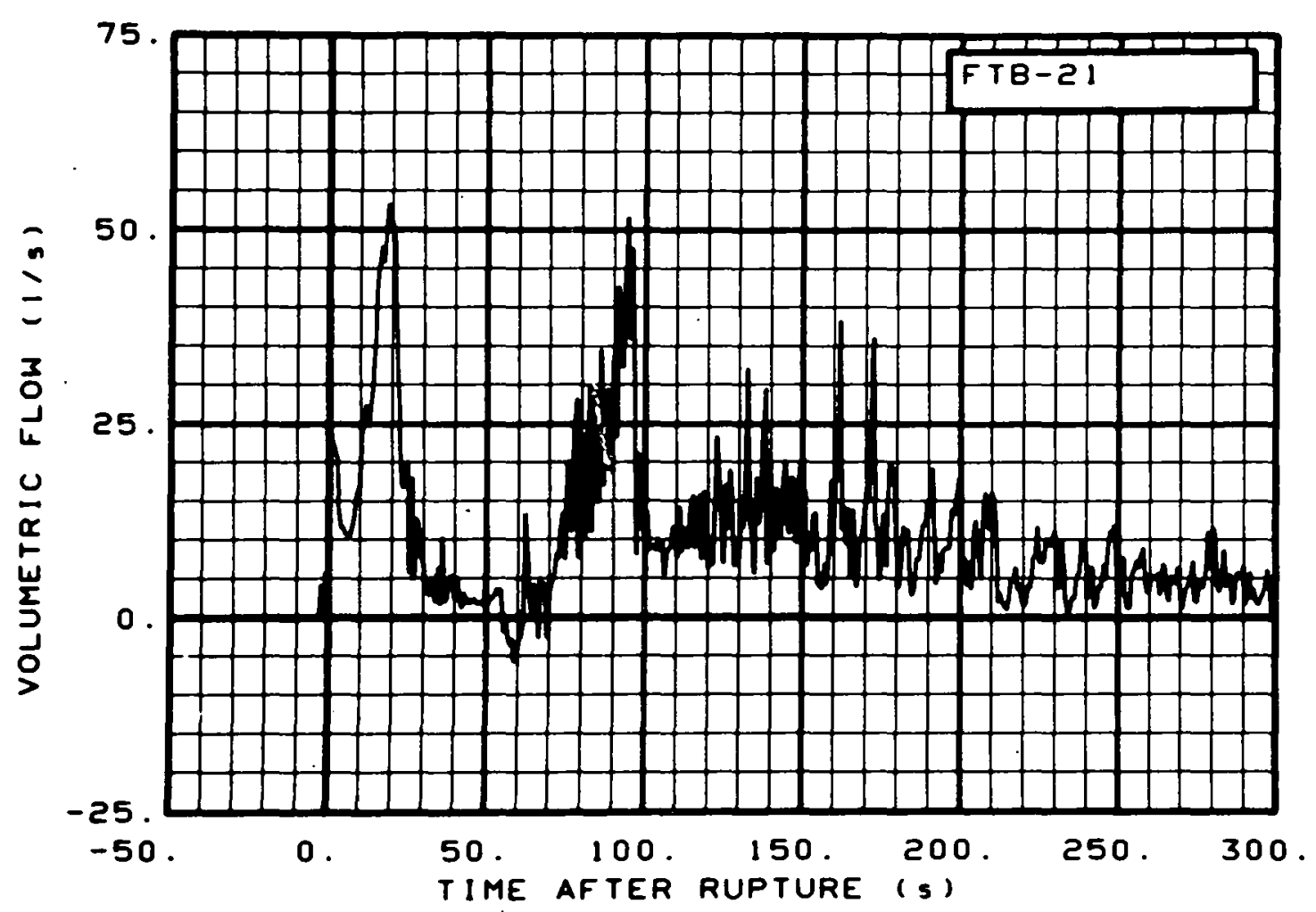

Fig. 219 Volumetric flow in broken loop (FTB-21), from -20 to $300 \mathrm{~s}$.

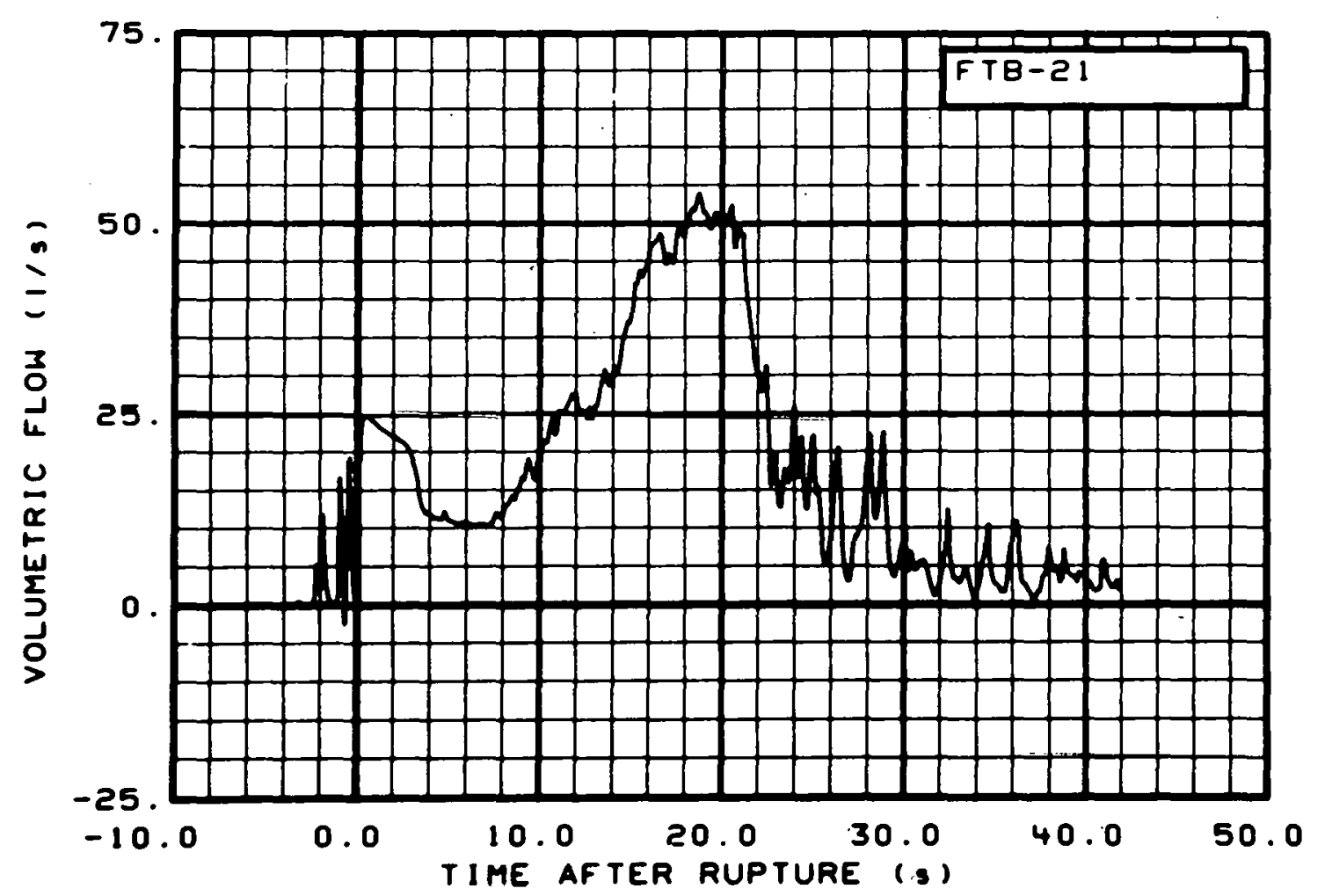

Fig. 220 Volumetric flow in broken loop (FTB-21), from -6 to $42 \mathrm{~s}$. 


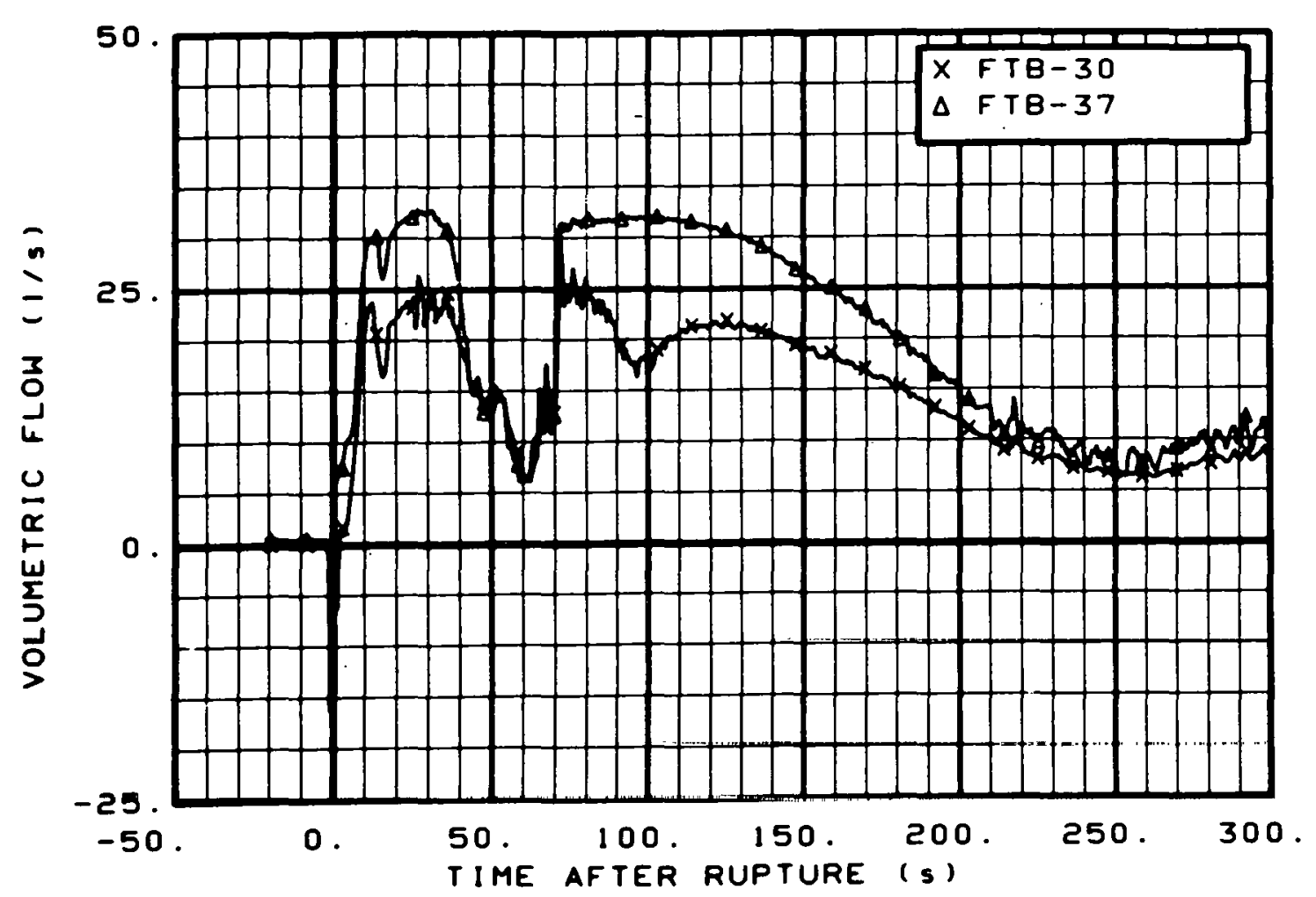

Fig. 221 Volumetric flow in broken loop (FTB-30 and FTB-37), from -20 to $300 \mathrm{~s}$.

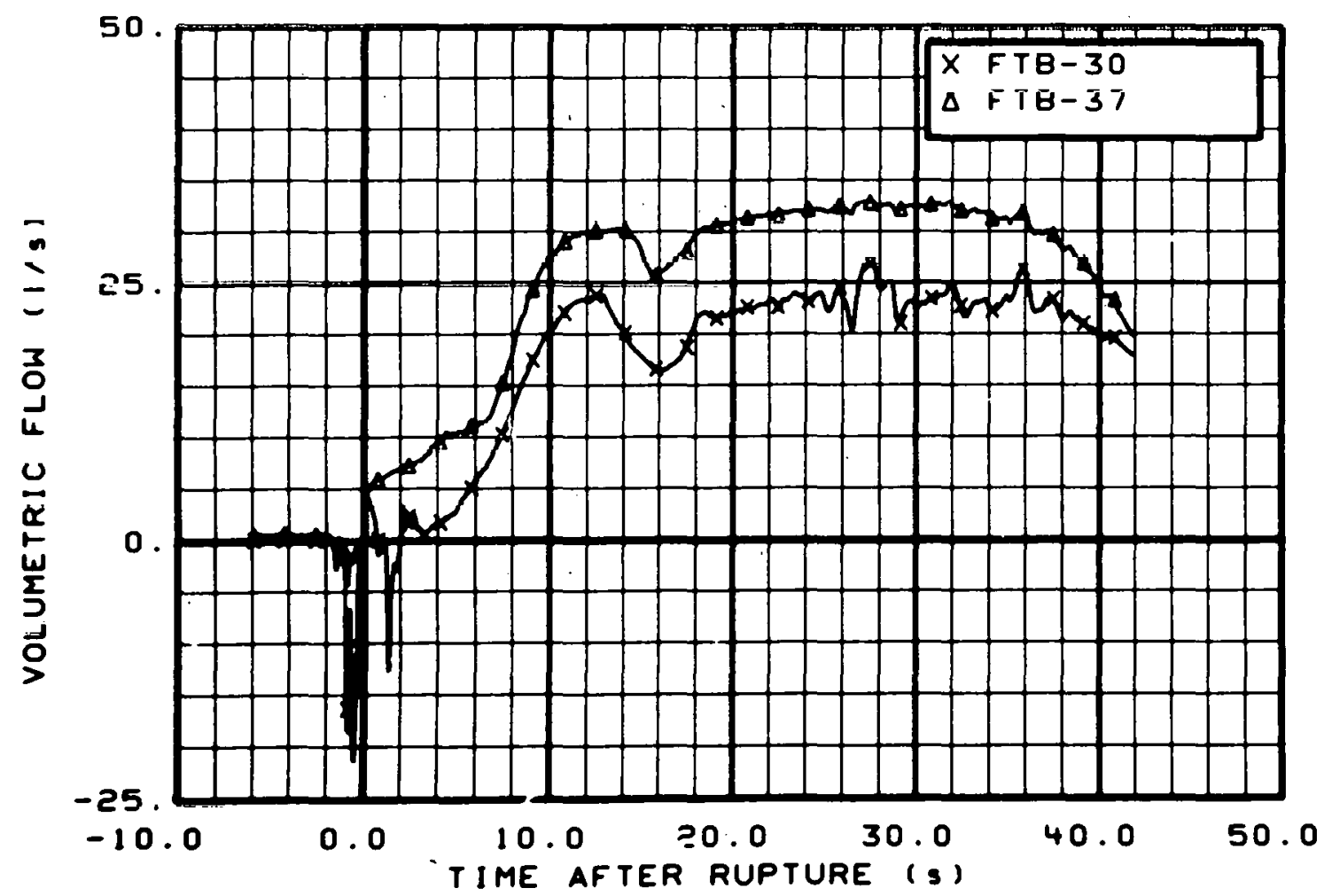

Fig. 222 Volumetric flow in broken loop (FTB-30 and FTB-37), from -6 to $42 \mathrm{~s}$. 


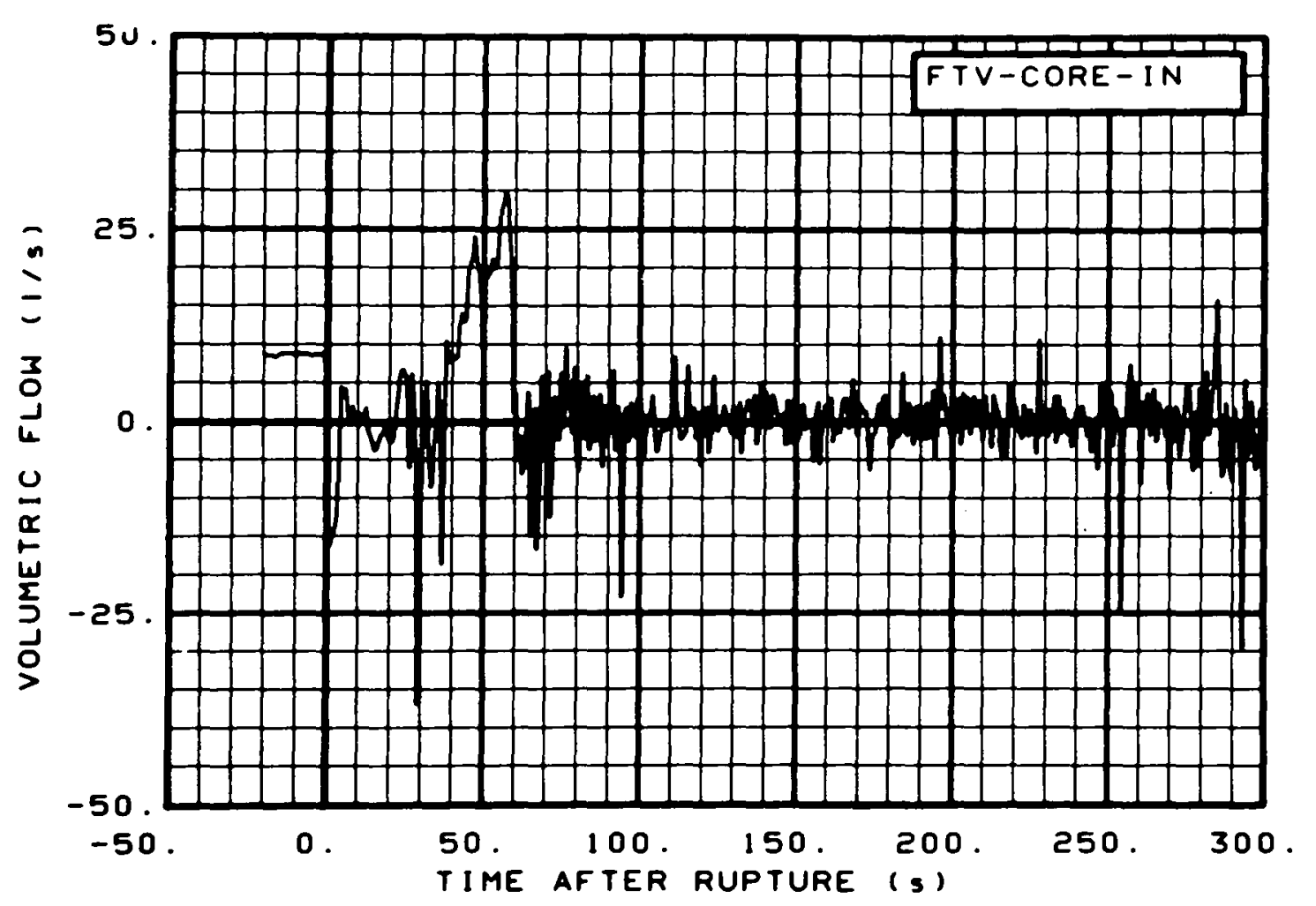

Fig. 223 Volumetric flow in core entrance (FTV-CORE-IN), from -20 to $300 \mathrm{~s}$.

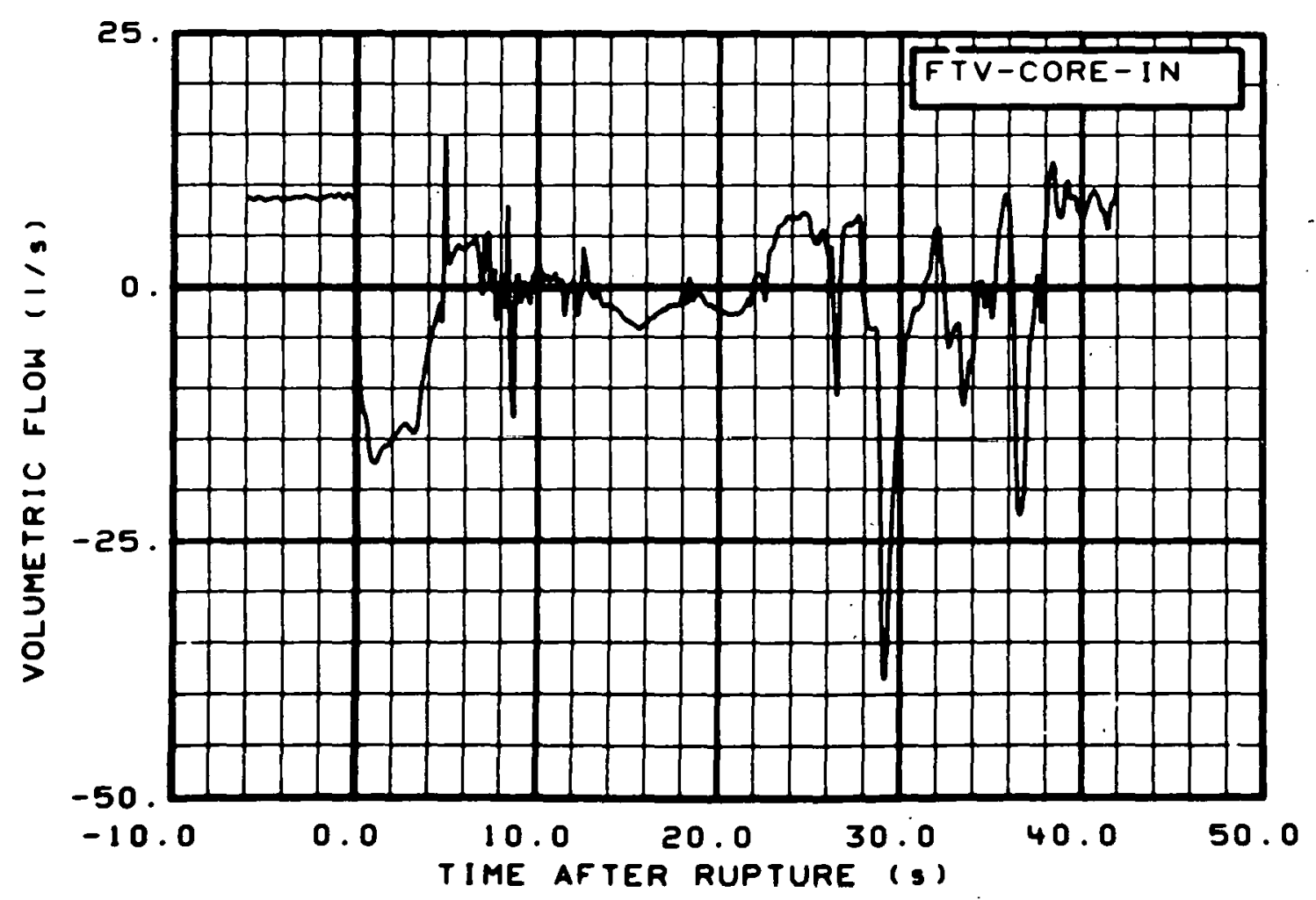

Fig. 224 Volumetric flow in core entrance (FTV-CORE-IN), from -6 to $42 \mathrm{~s}$. 


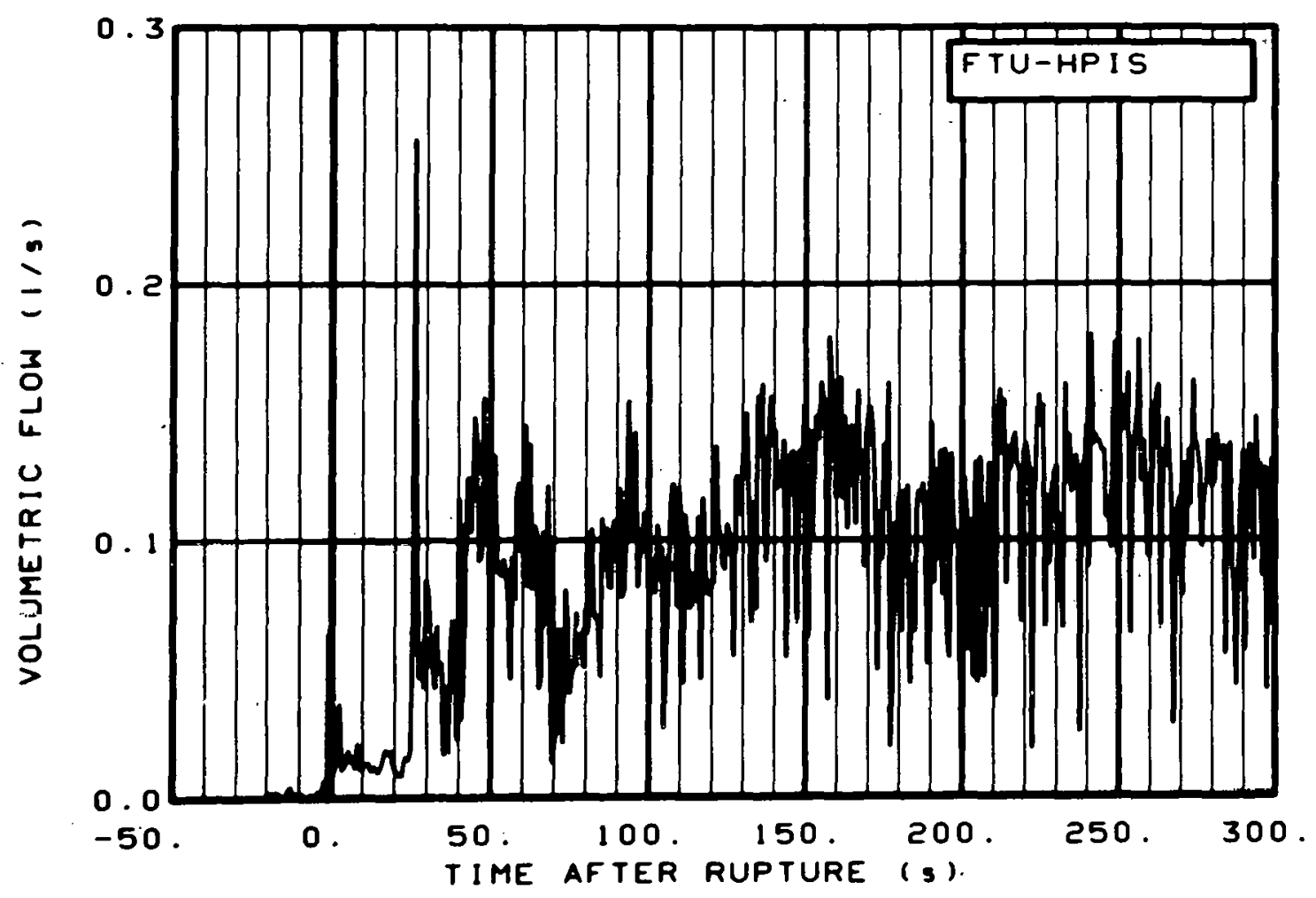

Fig. 225 Volumetric flow in intact loop high pressure injection line (FTU-HPIS), from -20 to $300 \mathrm{~s}$.

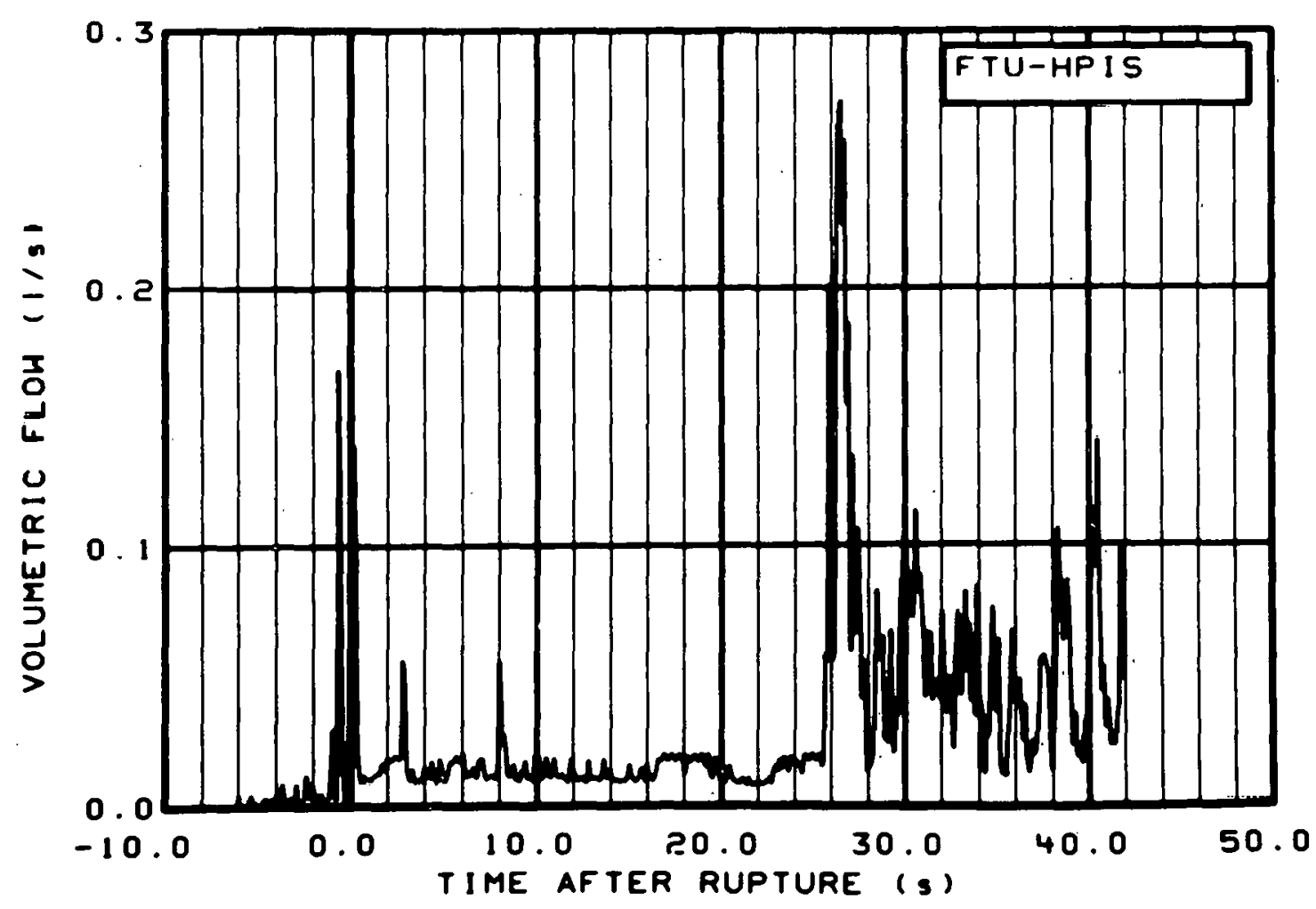

Fig. 226 Volumetric flow in intact loop high pressure injection line (FTU-HPIS), from -6 to $42 \mathrm{~s}$. 


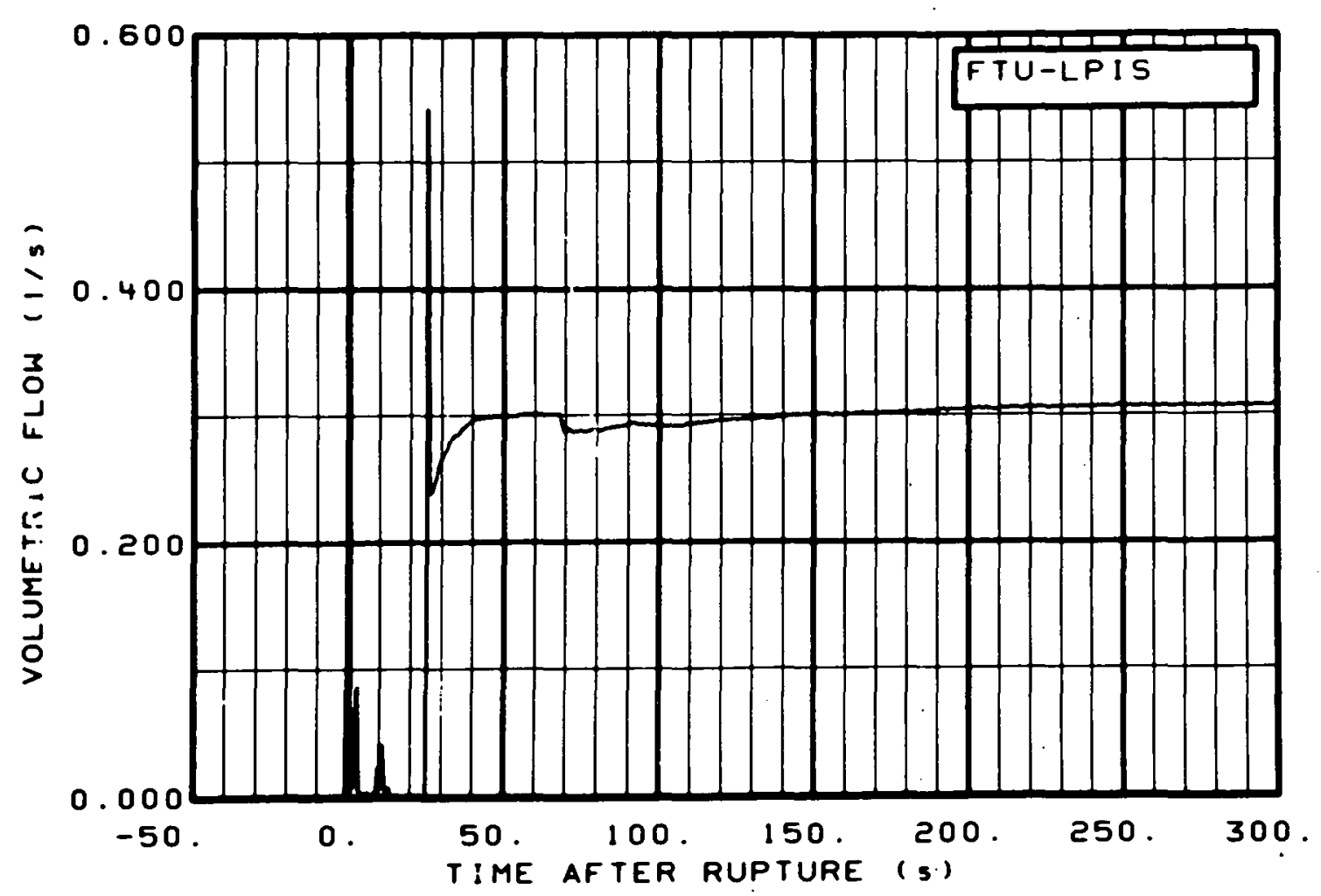

Fig. 227 Volumetric flow in intact loop low pressure injection line (FTU-LPIS), from -20 to $300 \mathrm{~s}$.

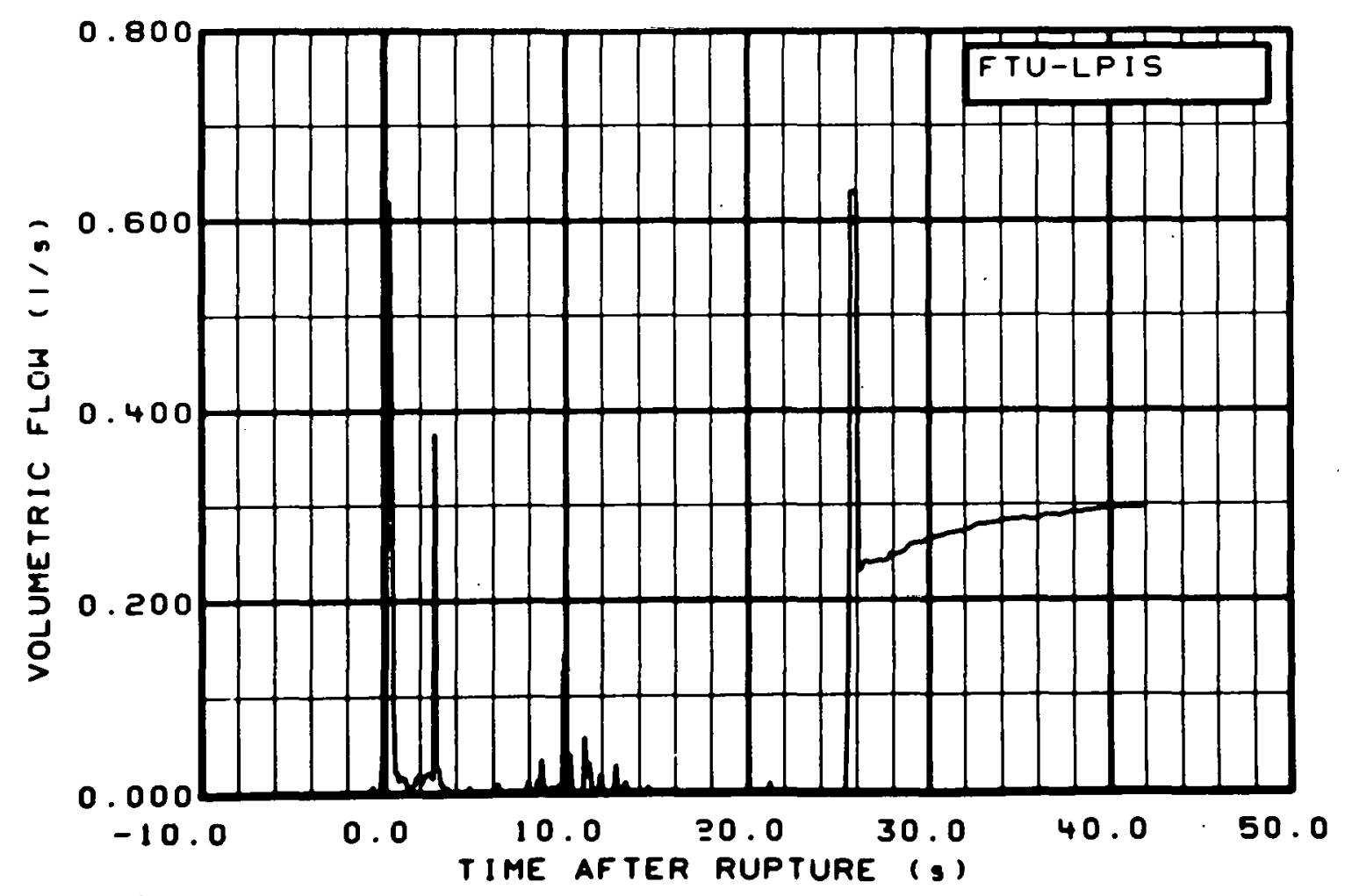

Fig. 228 Volumetric flow in intact loop low pressure injection line (FTU-LPIS), from -6 to $42 \mathrm{~s}$. 


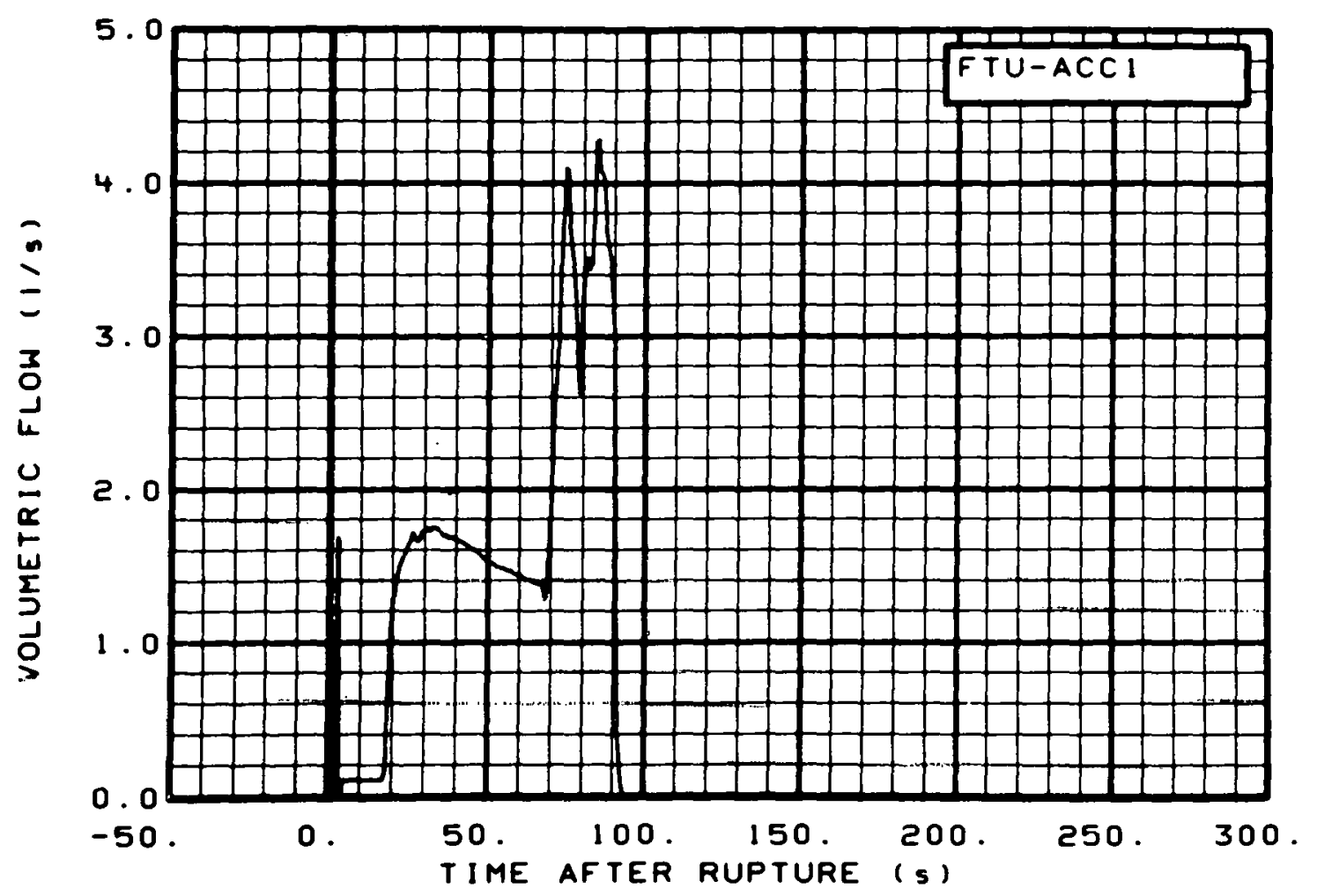

Fig. 229 Volumetric flow in intact loop accumulator discharge line (FTU-ACC 1), from -20 to $300 \mathrm{~s}$.

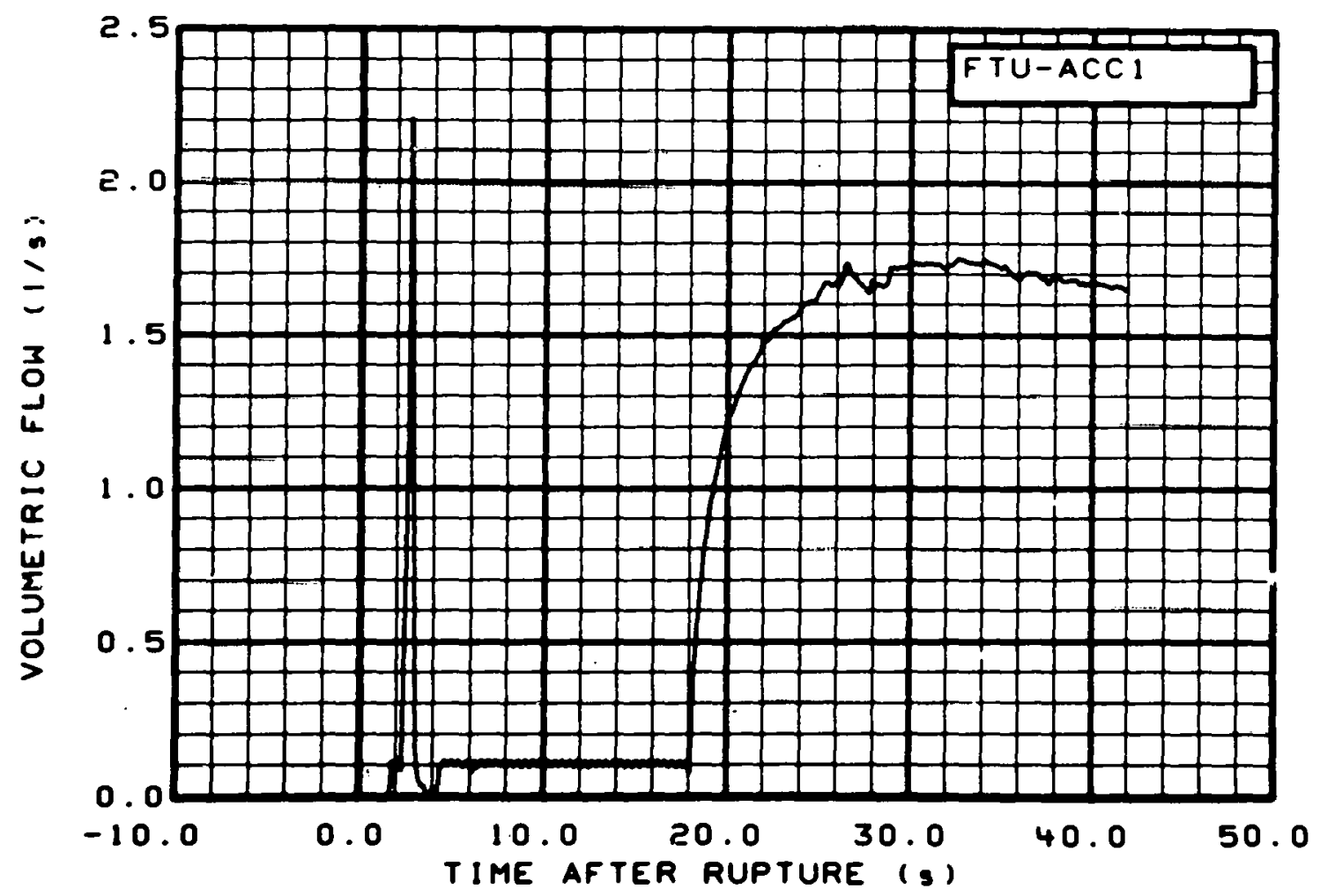

Fig. 230 Volumetric flow in intact loop accumulator discharge line (FTU-ACCl), from -6 to $42 \mathrm{~s}$. 


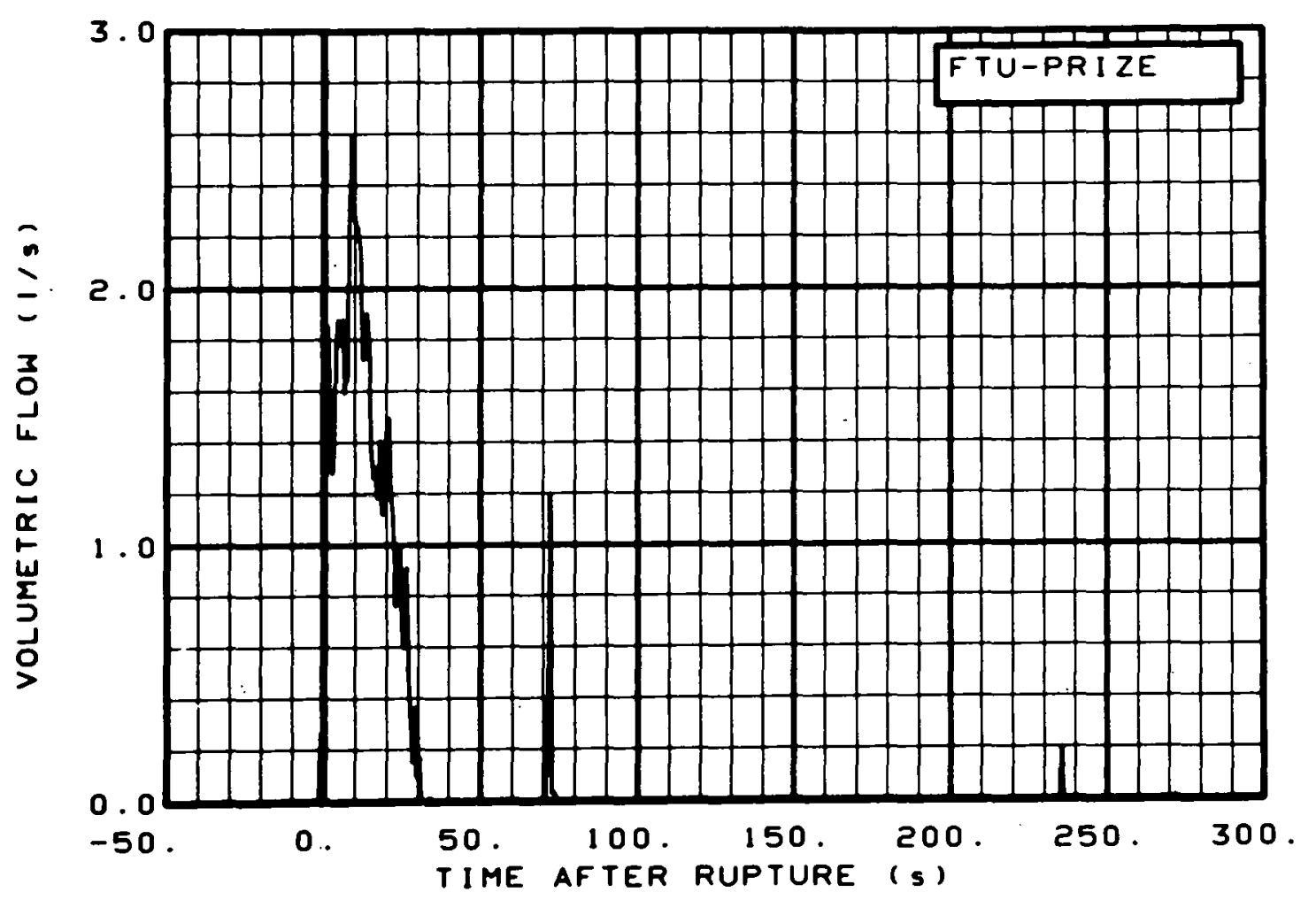

Fig. 231 Volumetric flow from pressurizer (FTU-PRIZE), from -20 to $300 \mathrm{~s}$.

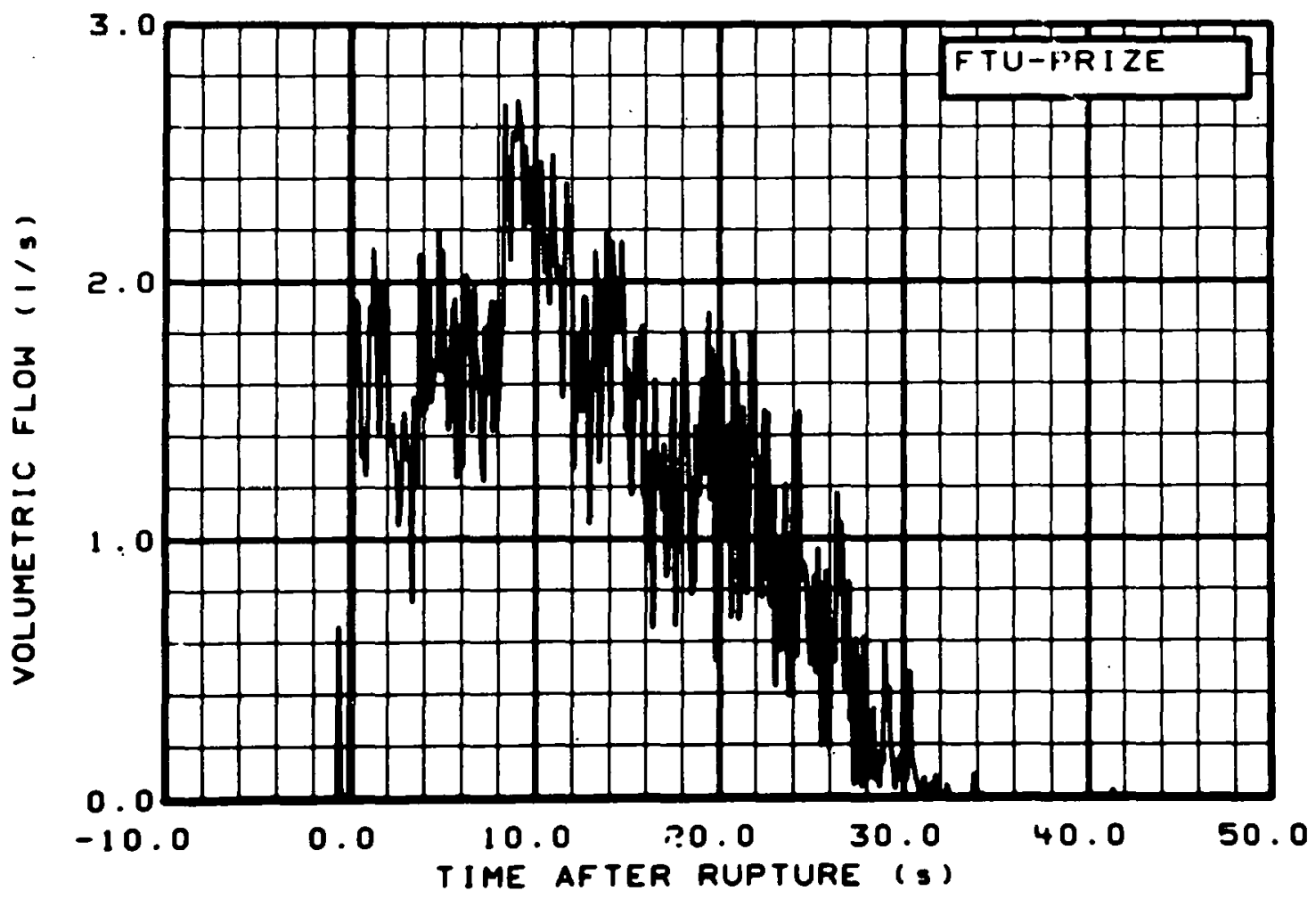

Fig. 232 Volumetric flow from pressurizer (FTU-PRIZE), from -6 to $42 \mathrm{~s}$. 


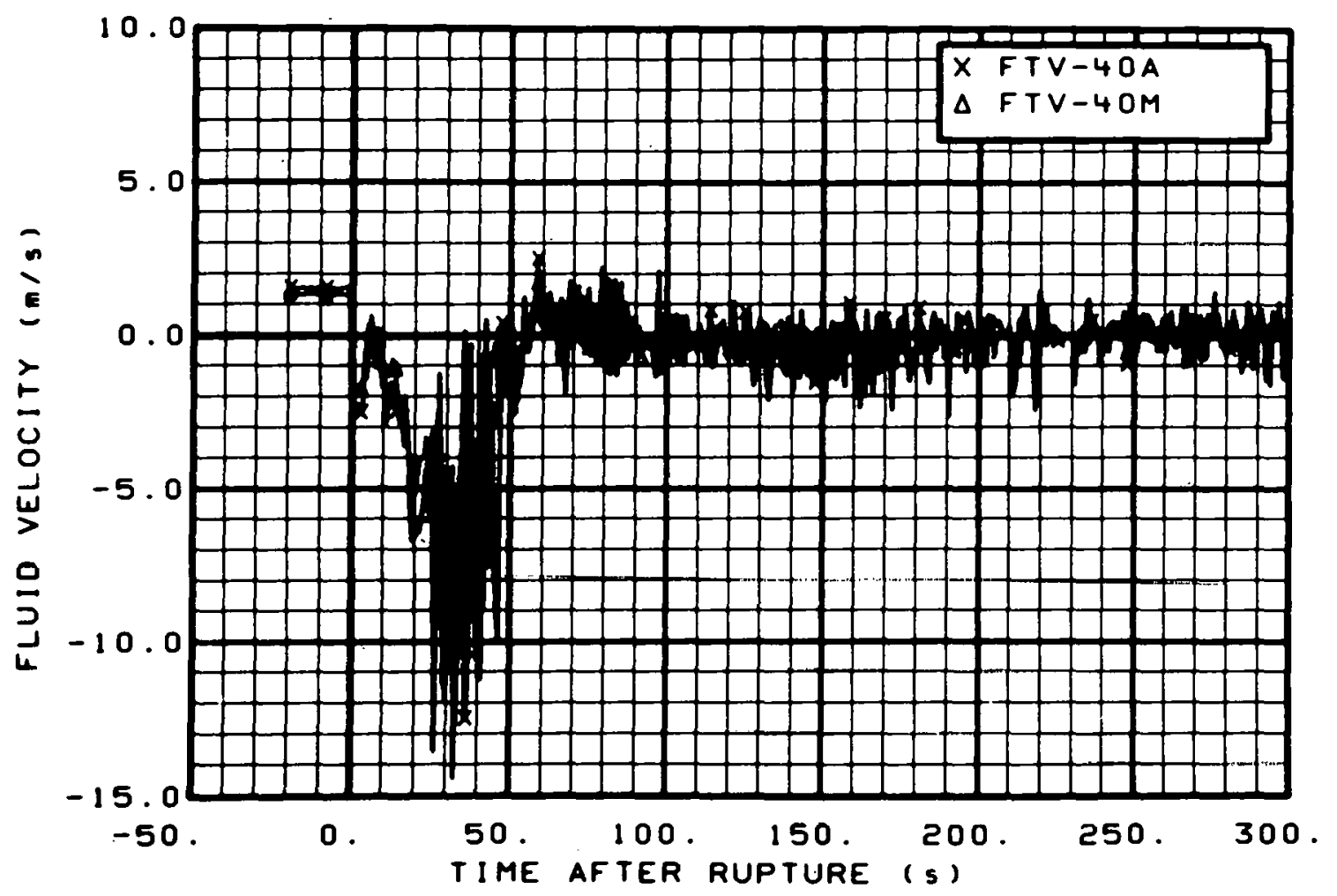

Fig. 233 Fluid velocity in vessel (FTV-40A and FTV-40M), from -20 to $300 \mathrm{~s}$.

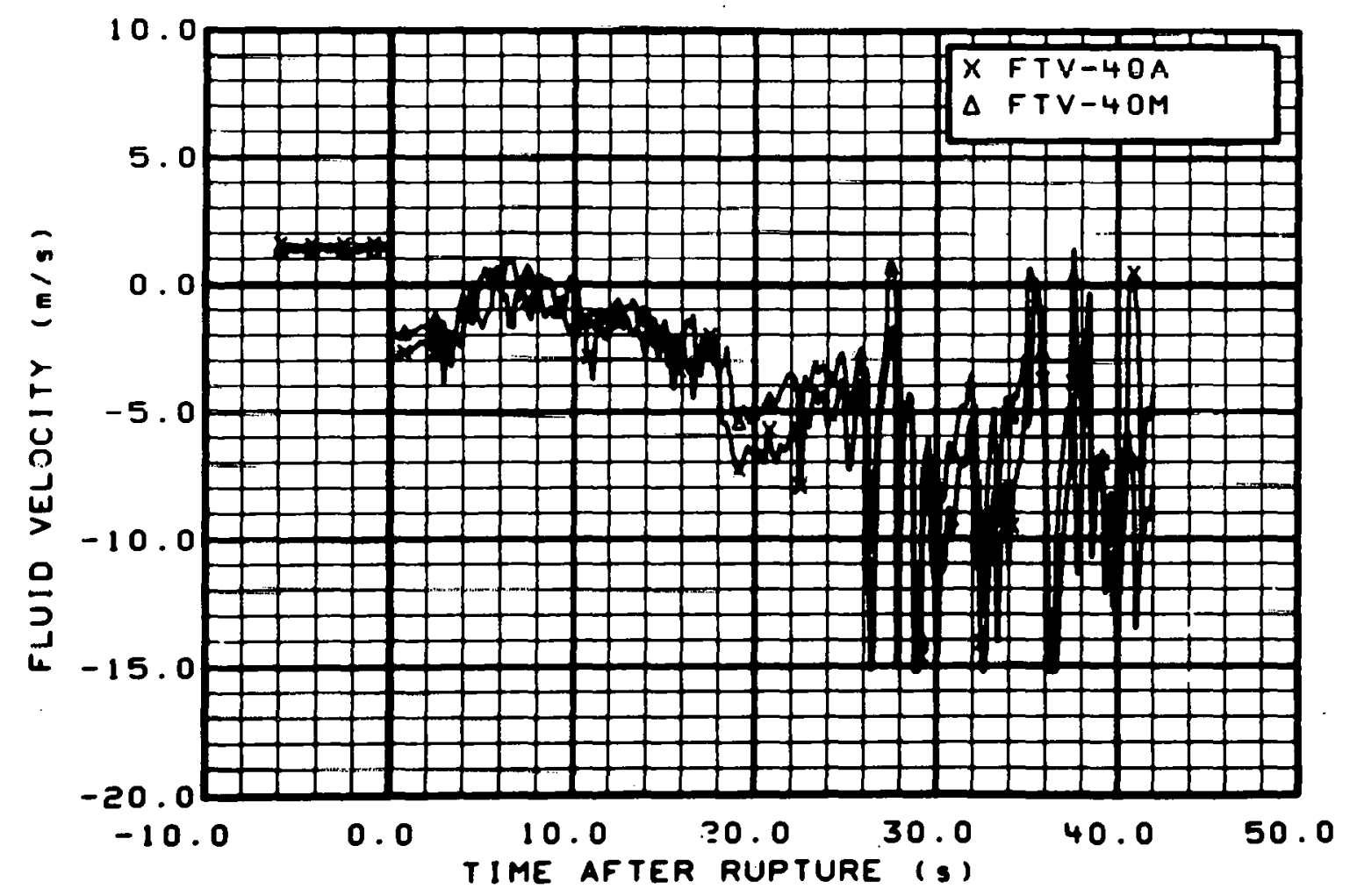

Fig. 234 Fluid velocity in vessel (FTV-40A and FTV-40M), from -6 to $42 \mathrm{~s}$. 


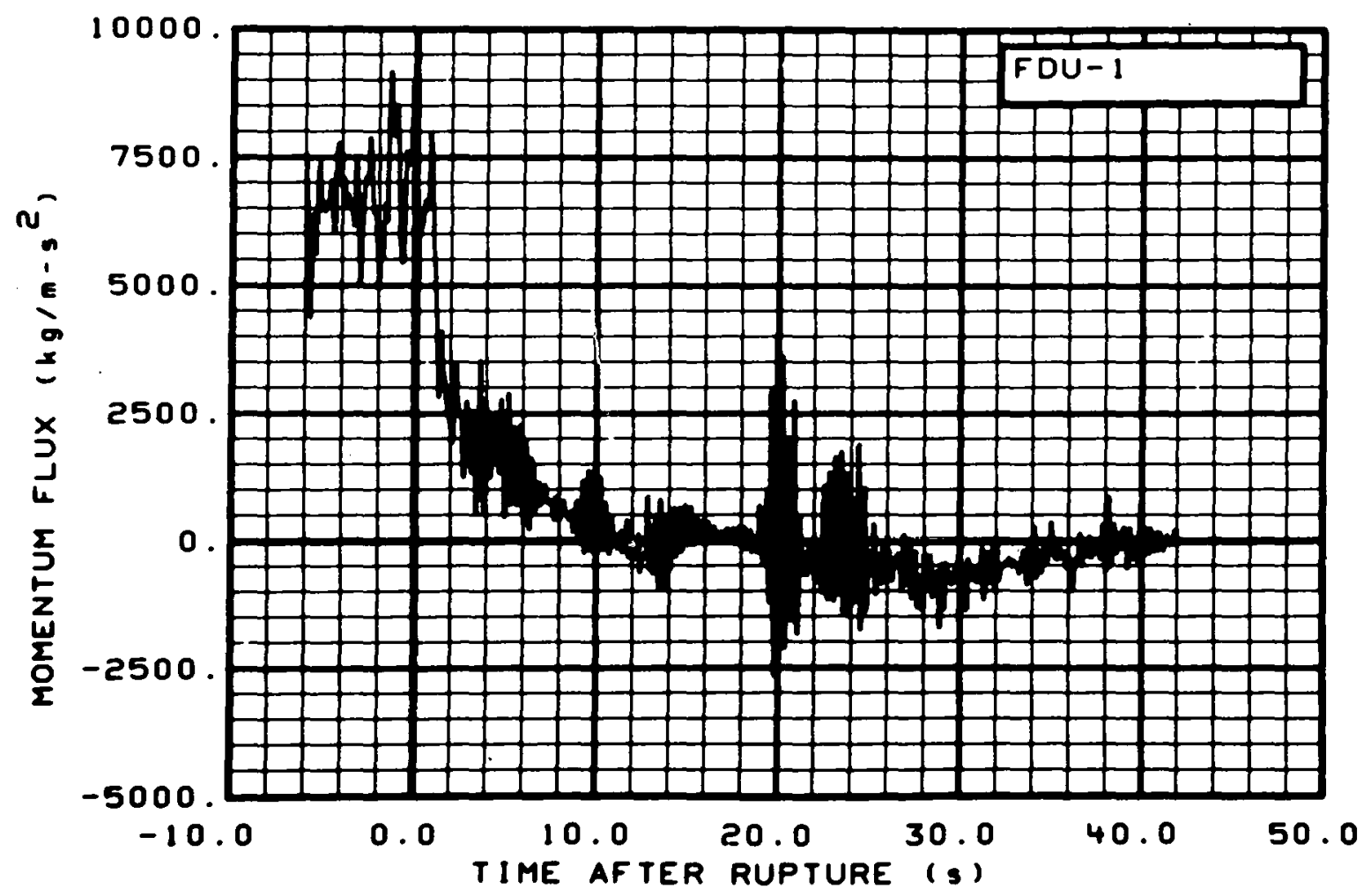

Fig. 235 Momentum flux in intact loop (FDU-1), from -6 to $42 \mathrm{~s}$.

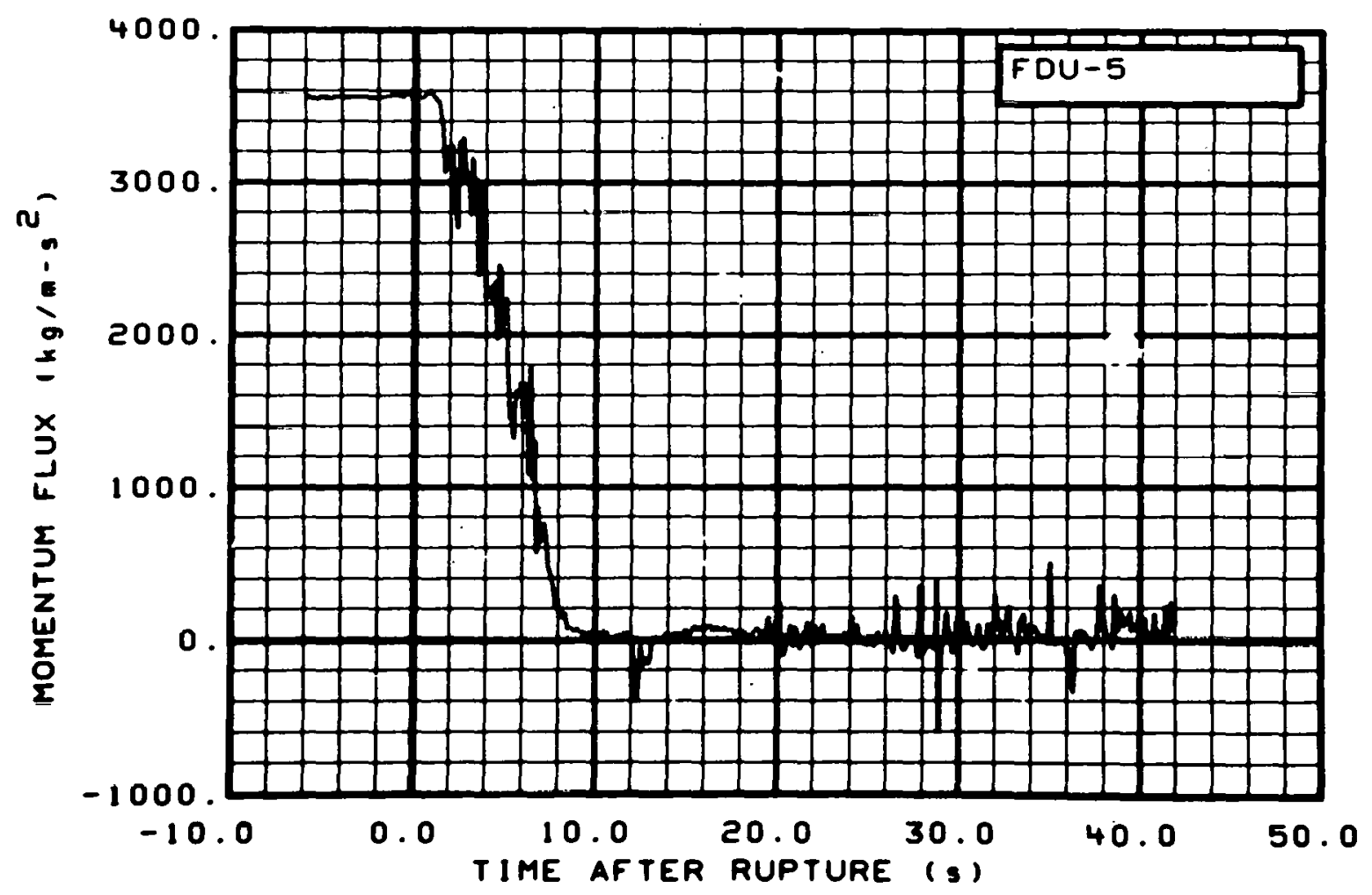

Fig. 236 Momentum flux in intact 1nop (FDU-5), from -6 to $42 \mathrm{~s}$, 


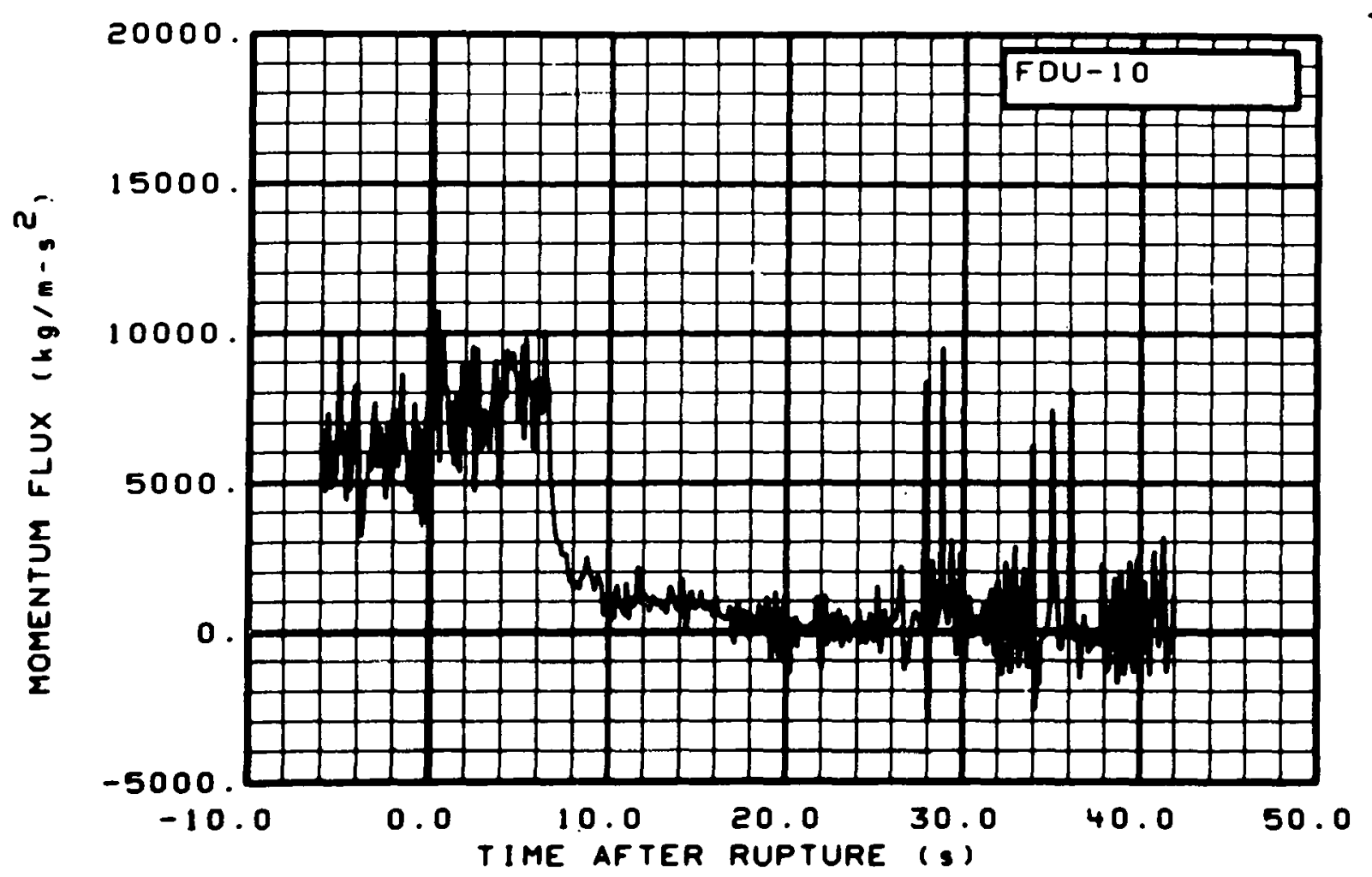

Fig. 237 Momentum flux in intact loop (FDU-10), from -6 to $42 \mathrm{~s}$.

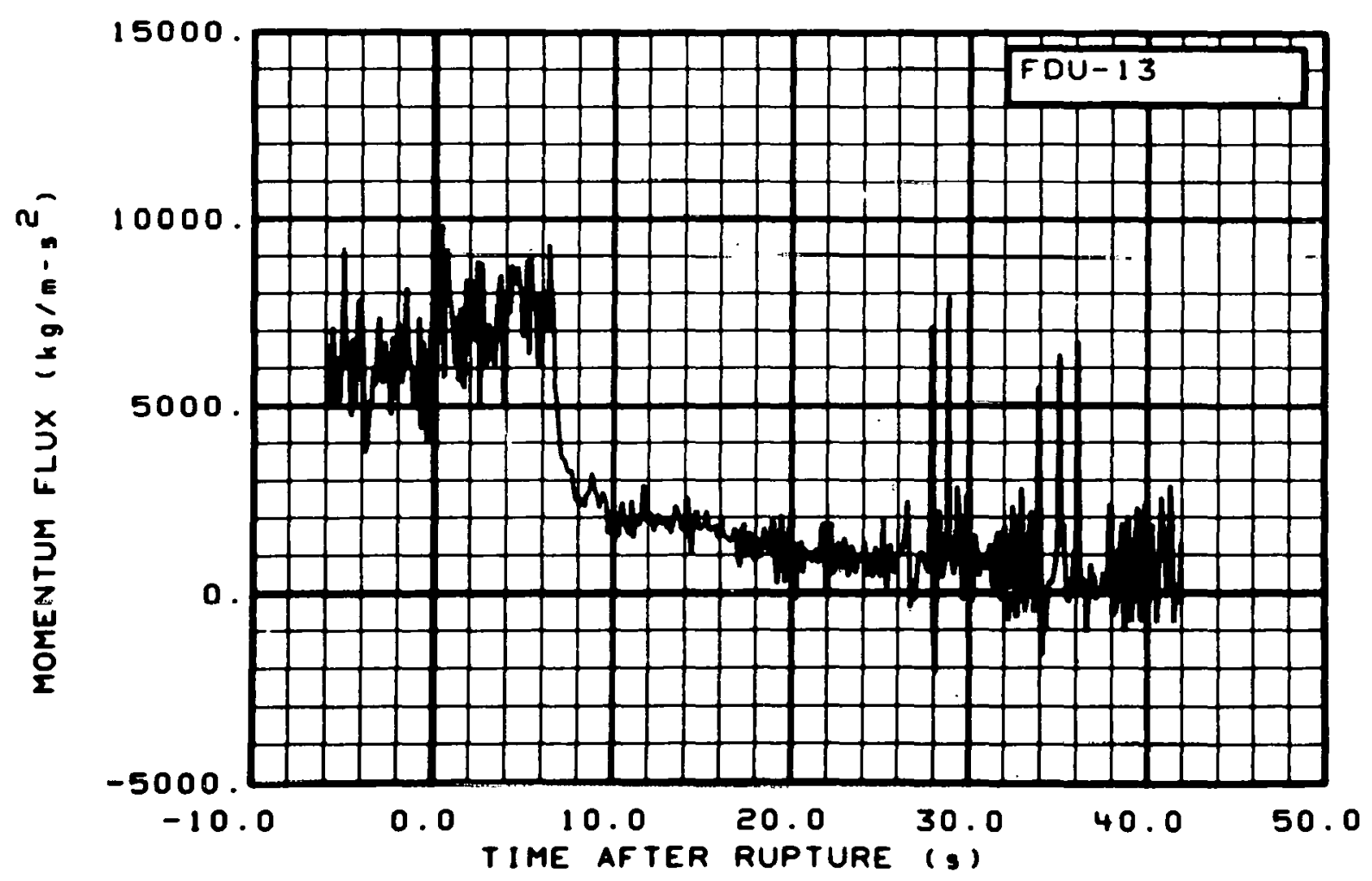

Fig. 238 Momentum flux in intact loop (FDU-13), from -6 to $42 \mathrm{~s}$. 


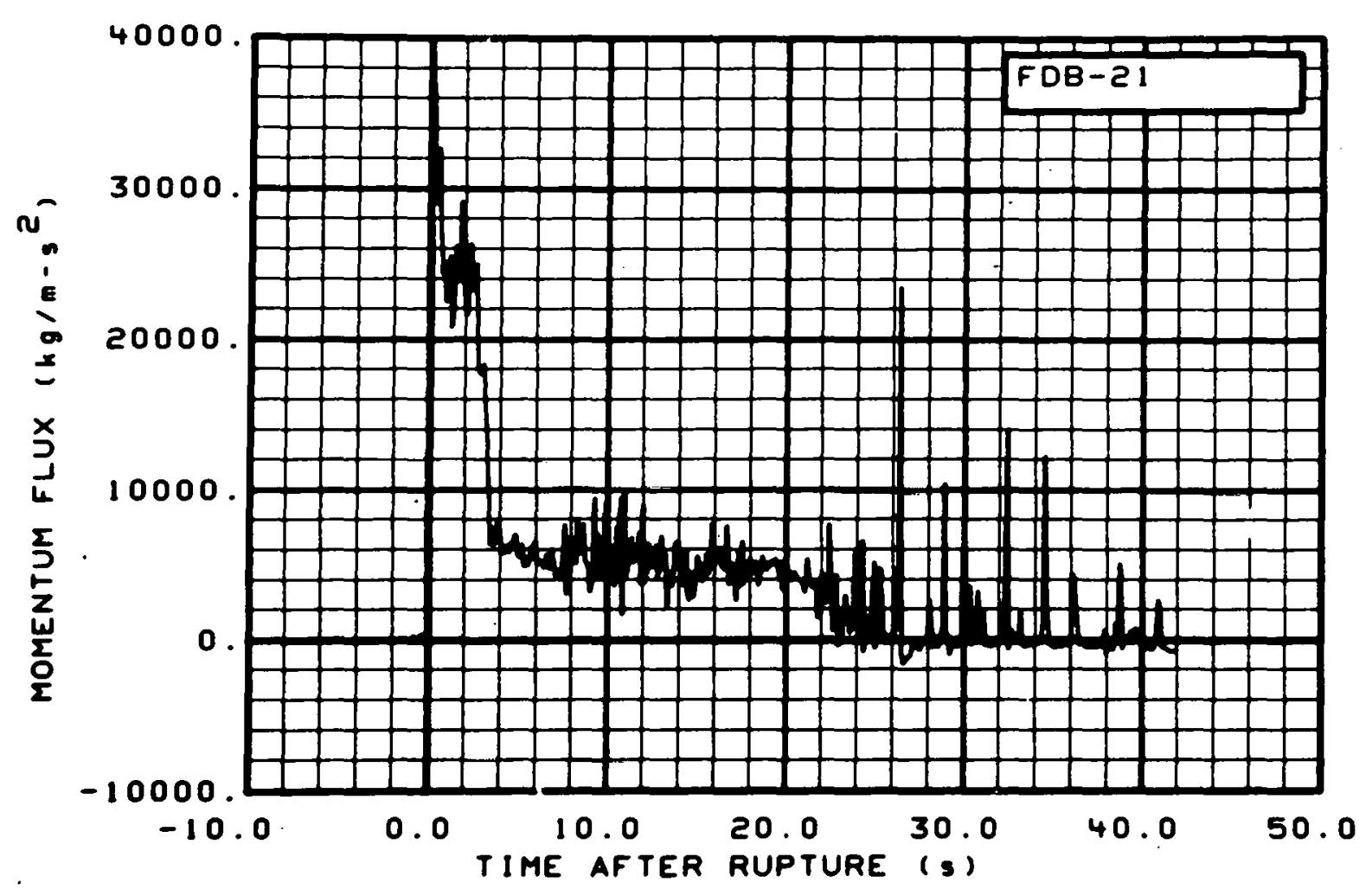

Fig. 239 Momentum flux in broken loop (FDB-21), from -6 to $42 \mathrm{~s}$.

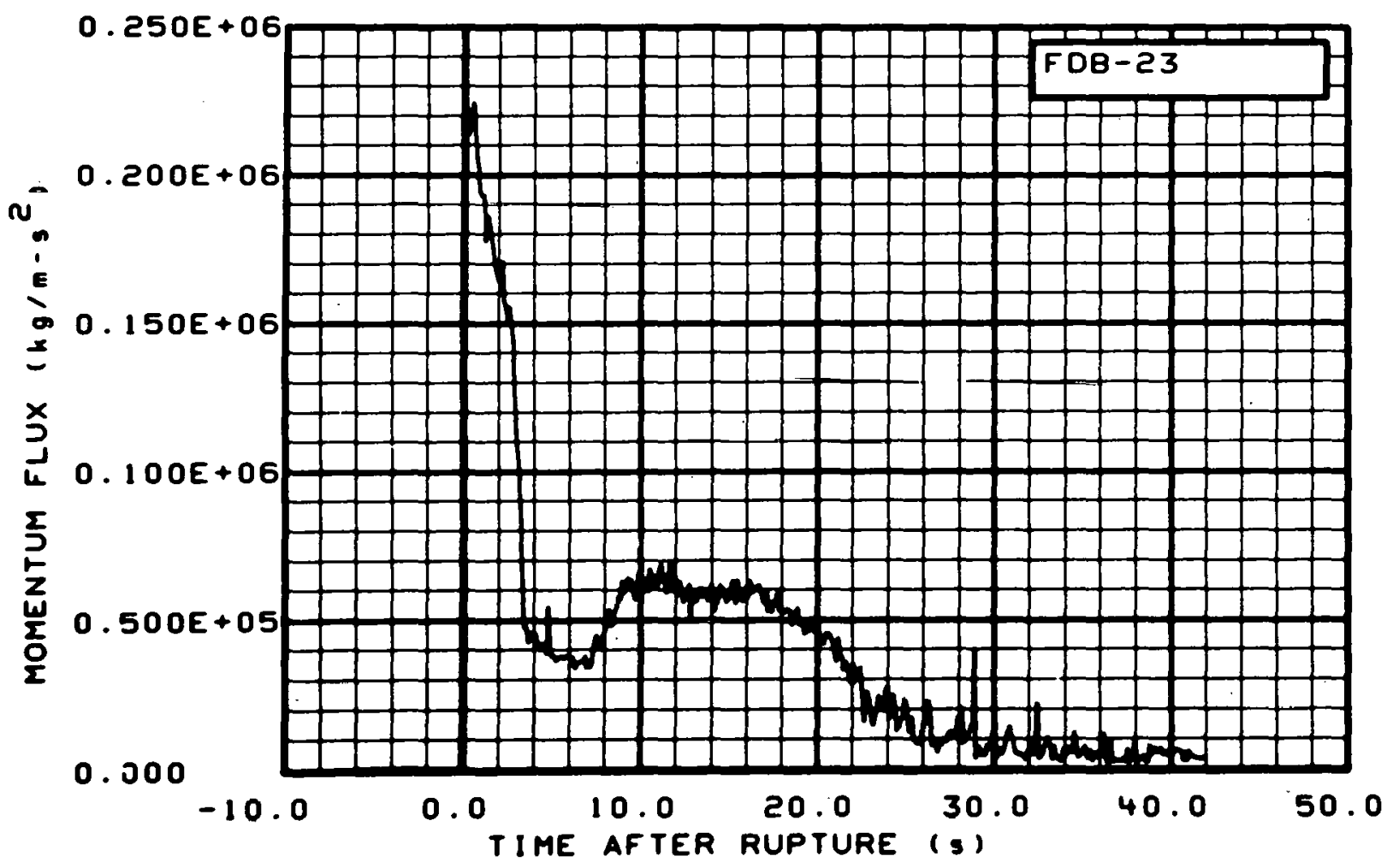

Fig. 240 Momentum flux in broken loop (FDB-23), from -6 to $42 \mathrm{~s}$. 


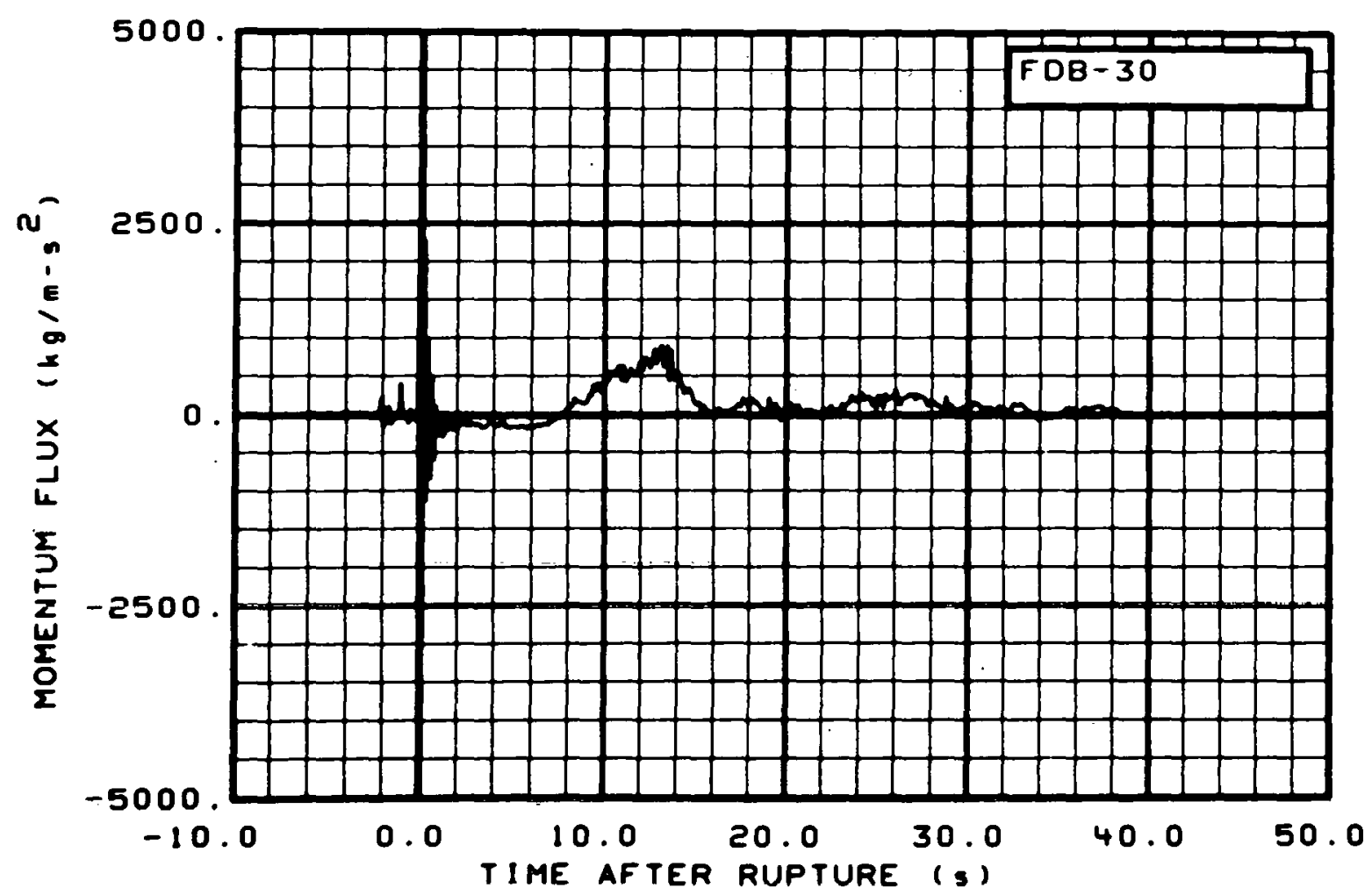

Fig. 241 Momentum flux in broken loop (FDB-30), from -6 to $42 \mathrm{~s}$.

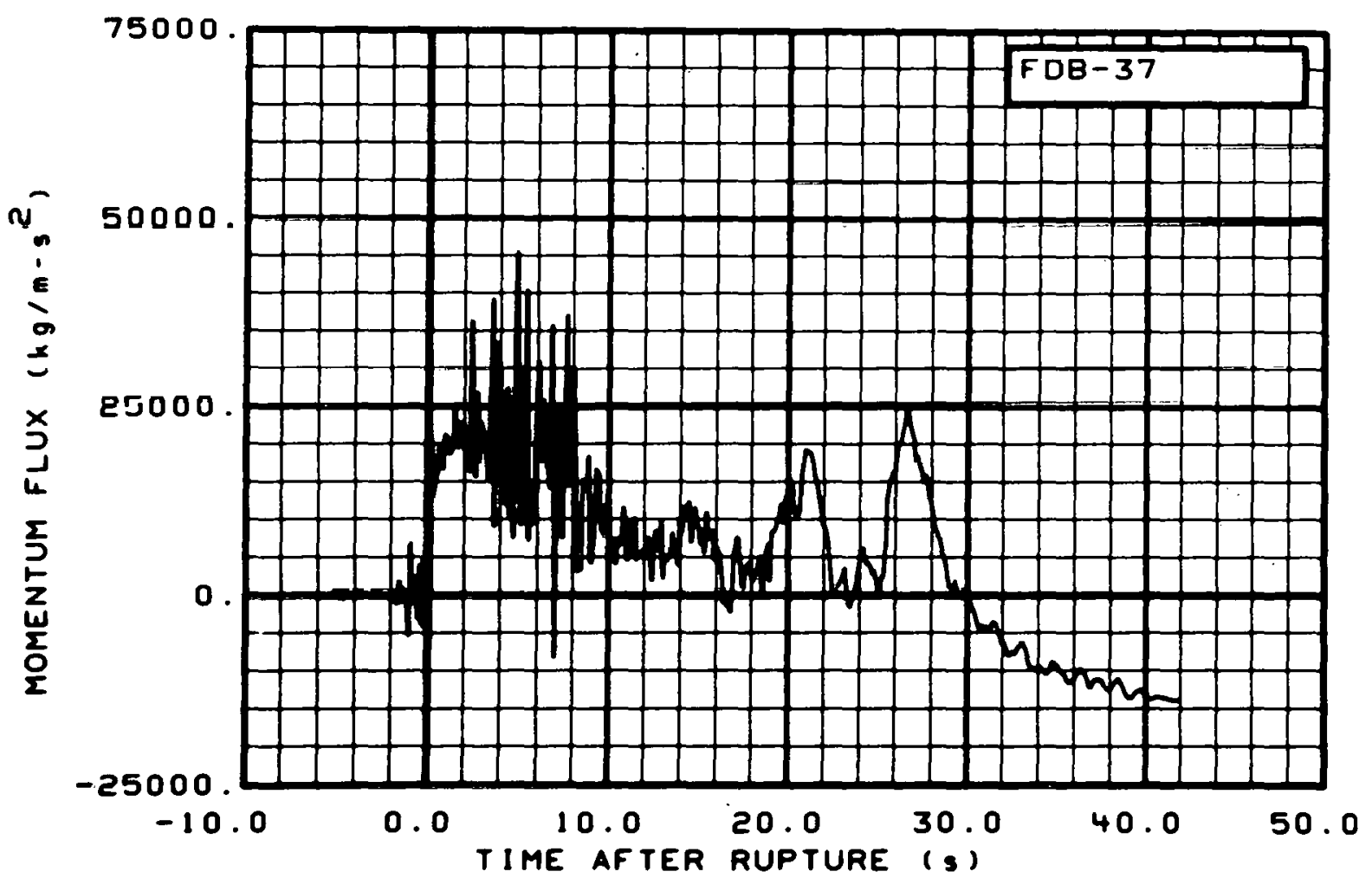

Fig. 242 Momentum flux in broken loop (FDB-37), from -6 to $42 \mathrm{~s}$. 


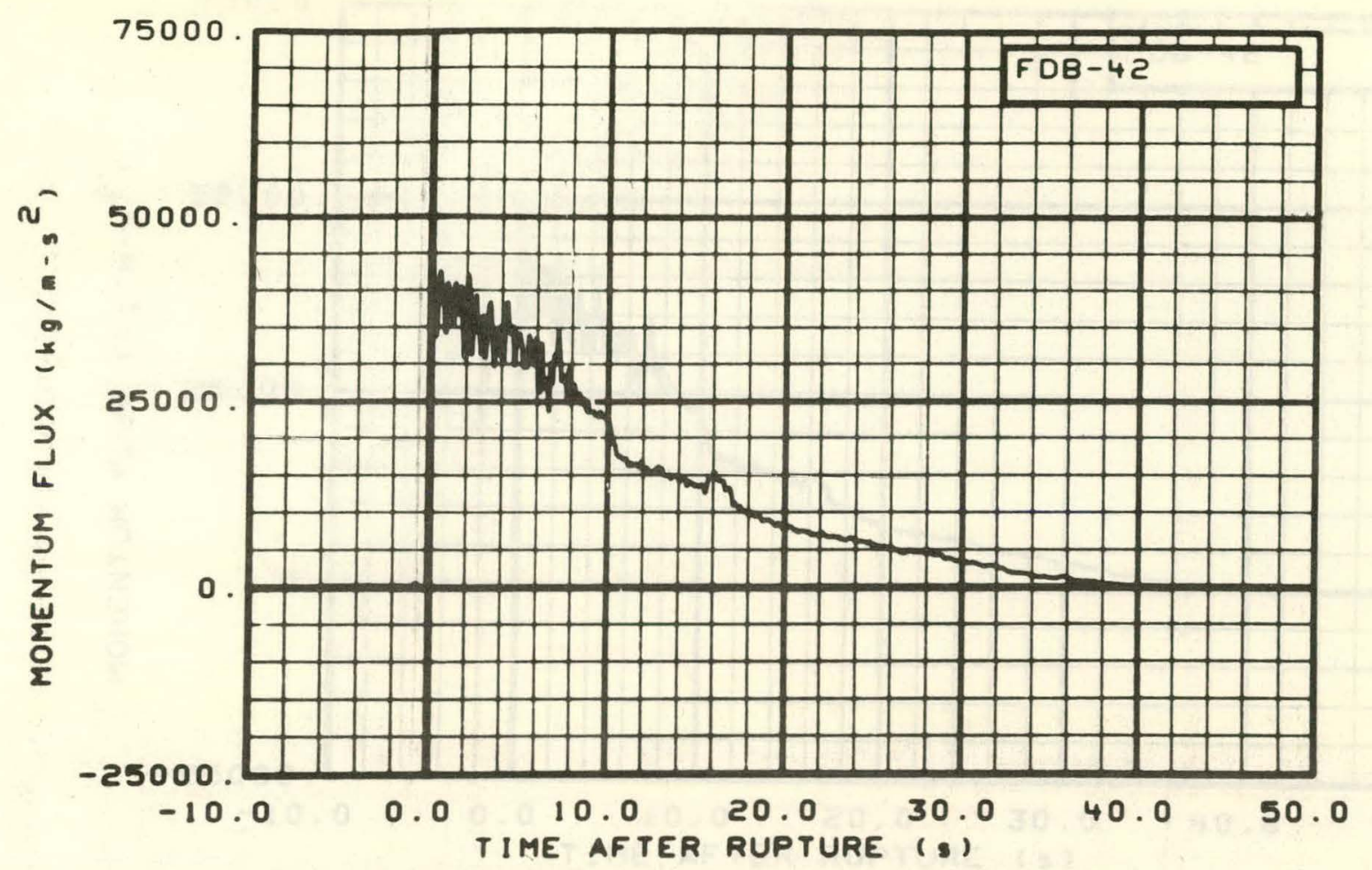

Fig. 243 Momentum flux in broken loop (FDB-42), from -6 to $42 \mathrm{~s}$.

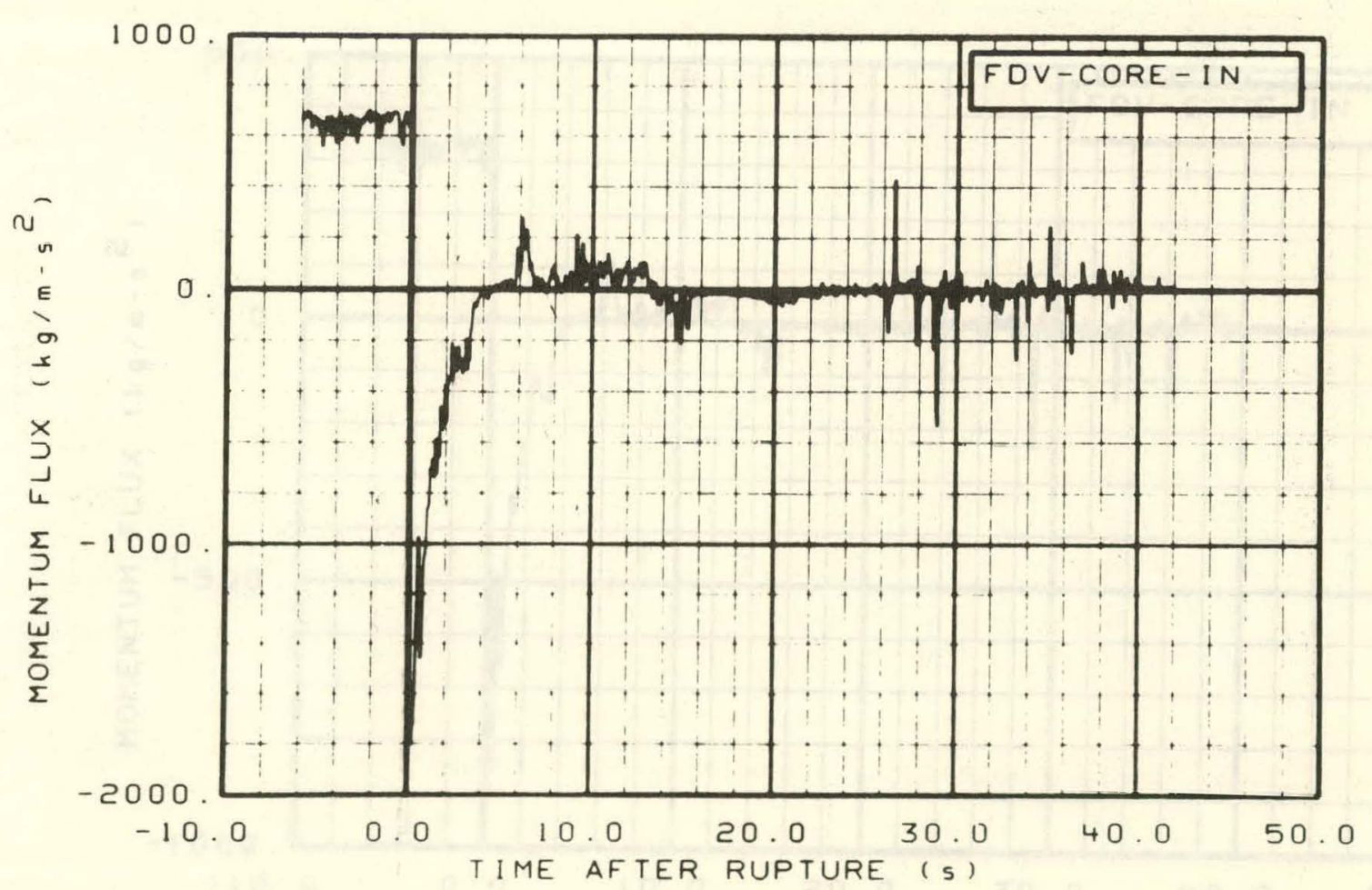

Fig. 244 Momentum flux in core entrance (FDV-CORE-IN), from -6 to $42 \mathrm{~s}$. 


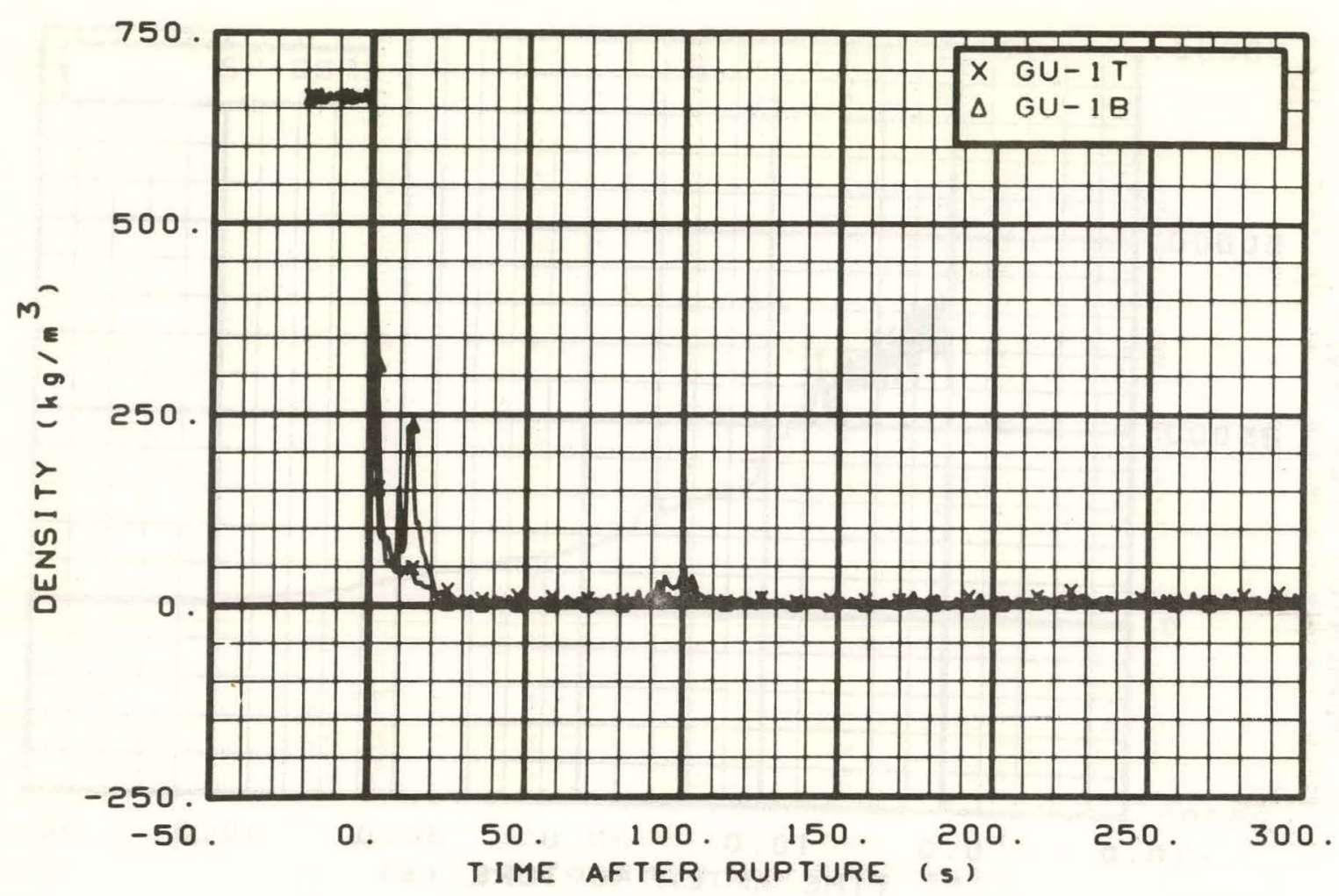

Fig. 245 Density in intact 1oop (GU-1T and GU-1B), from -20 to $300 \mathrm{~s}$.

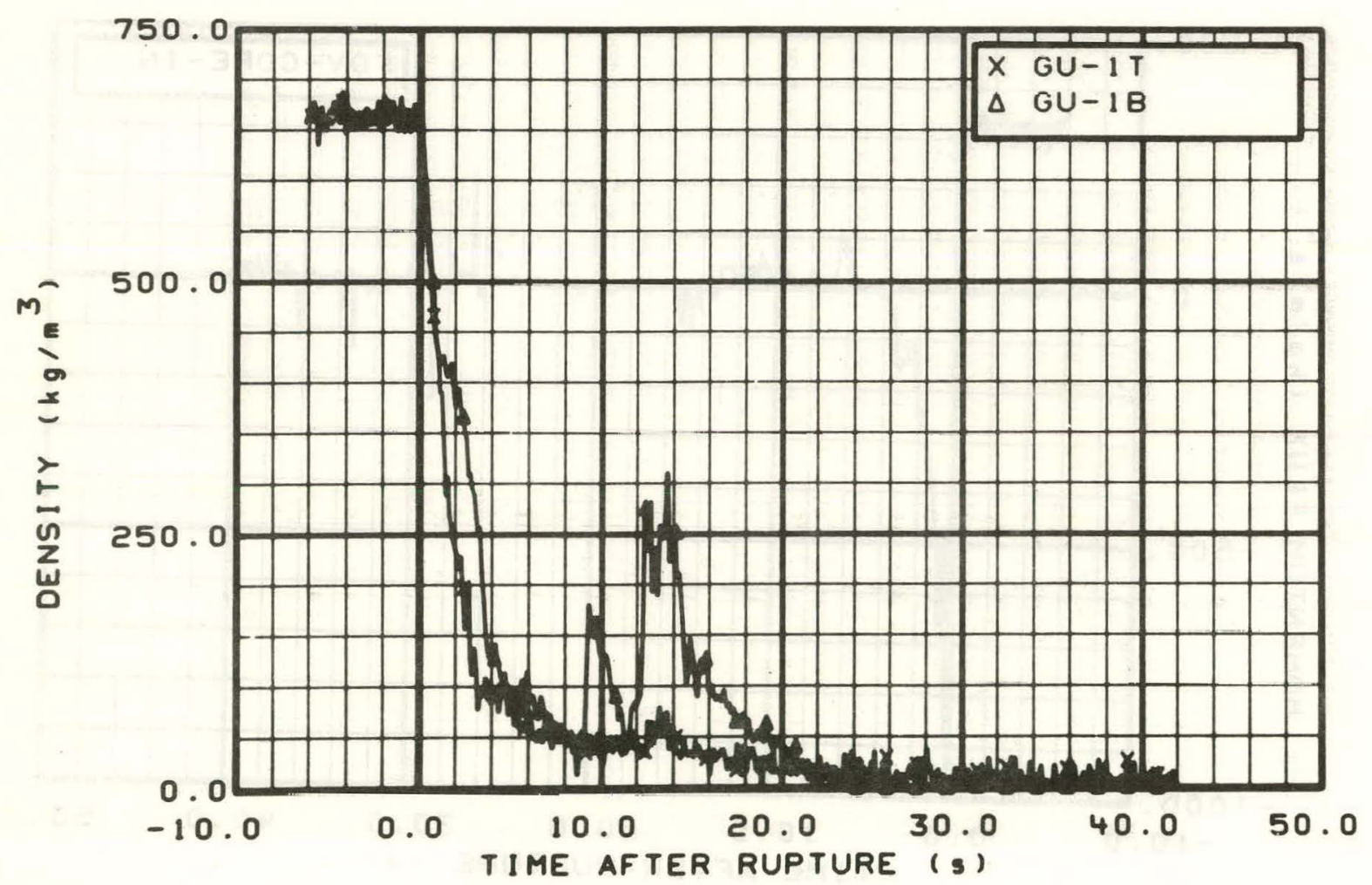

Fig. 246 Density in intact 1oop (GU-1T and GU-1B), from -6 to $42 \mathrm{~s}$. 


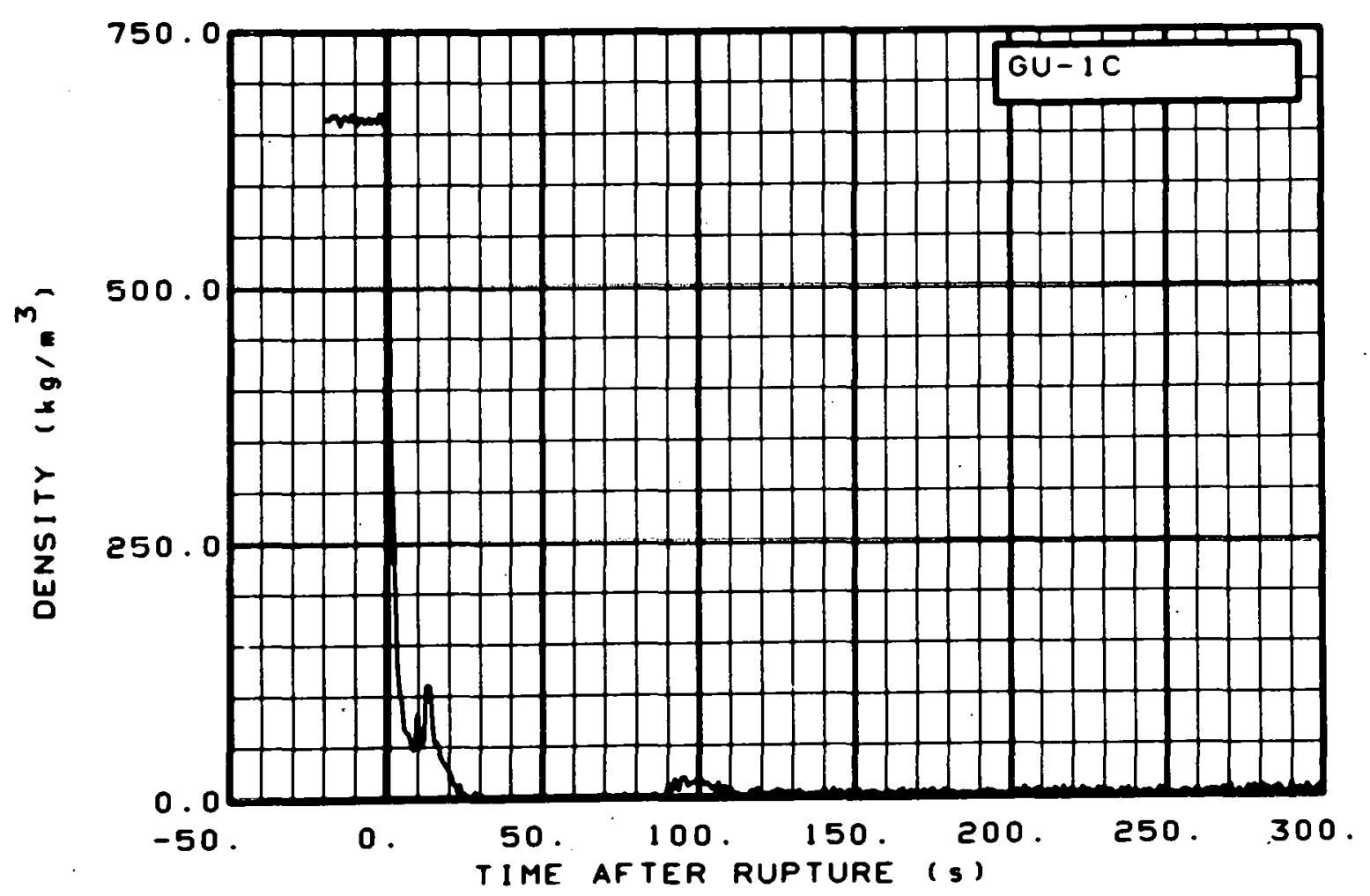

Fig. 247 Density in intact loop (GU-1C), from -20 to $300 \mathrm{~s}$.

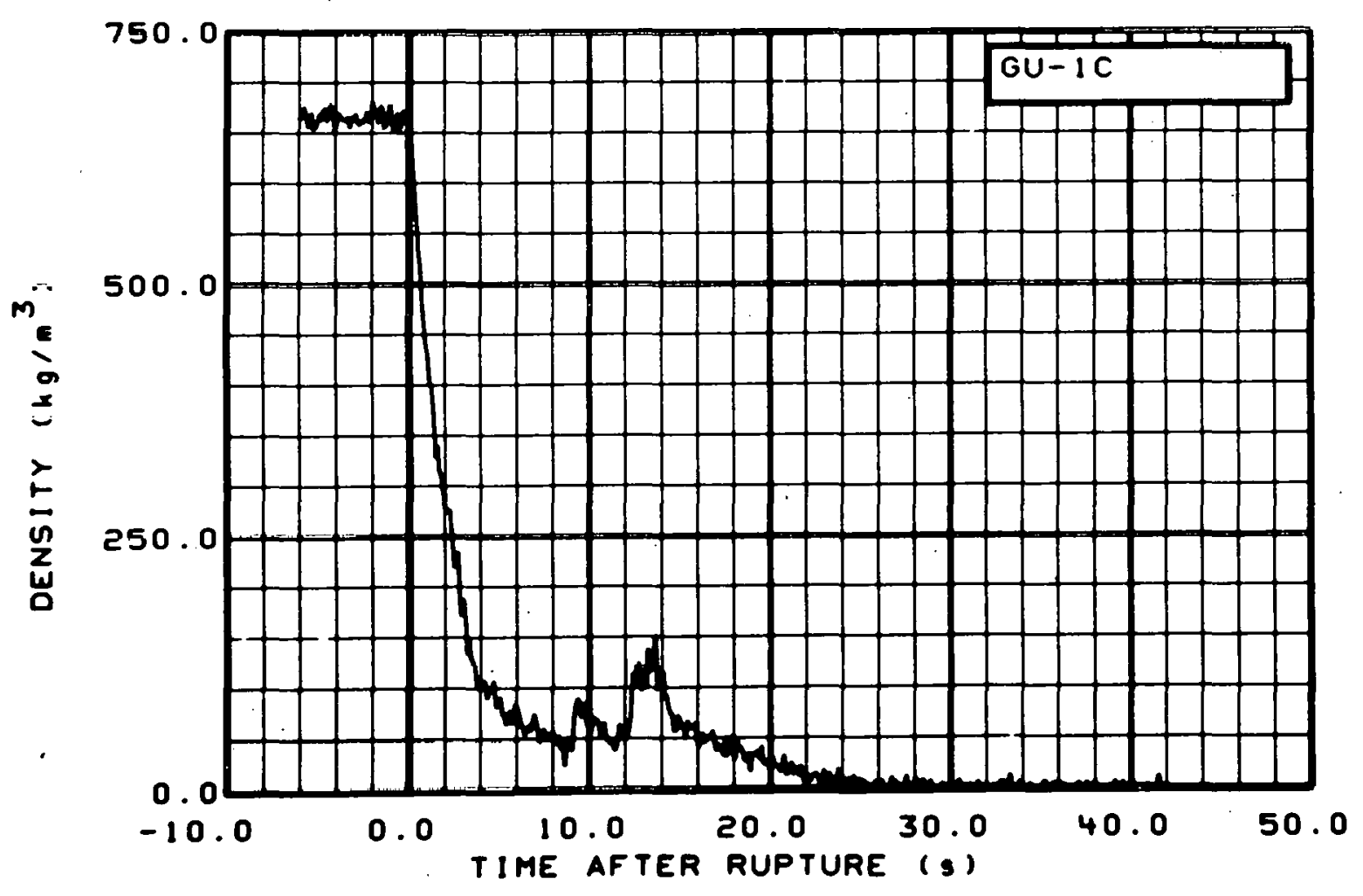

Fig. 248 Density in intact loop (GU-1C), from -6 to $42 \mathrm{~s}$. 


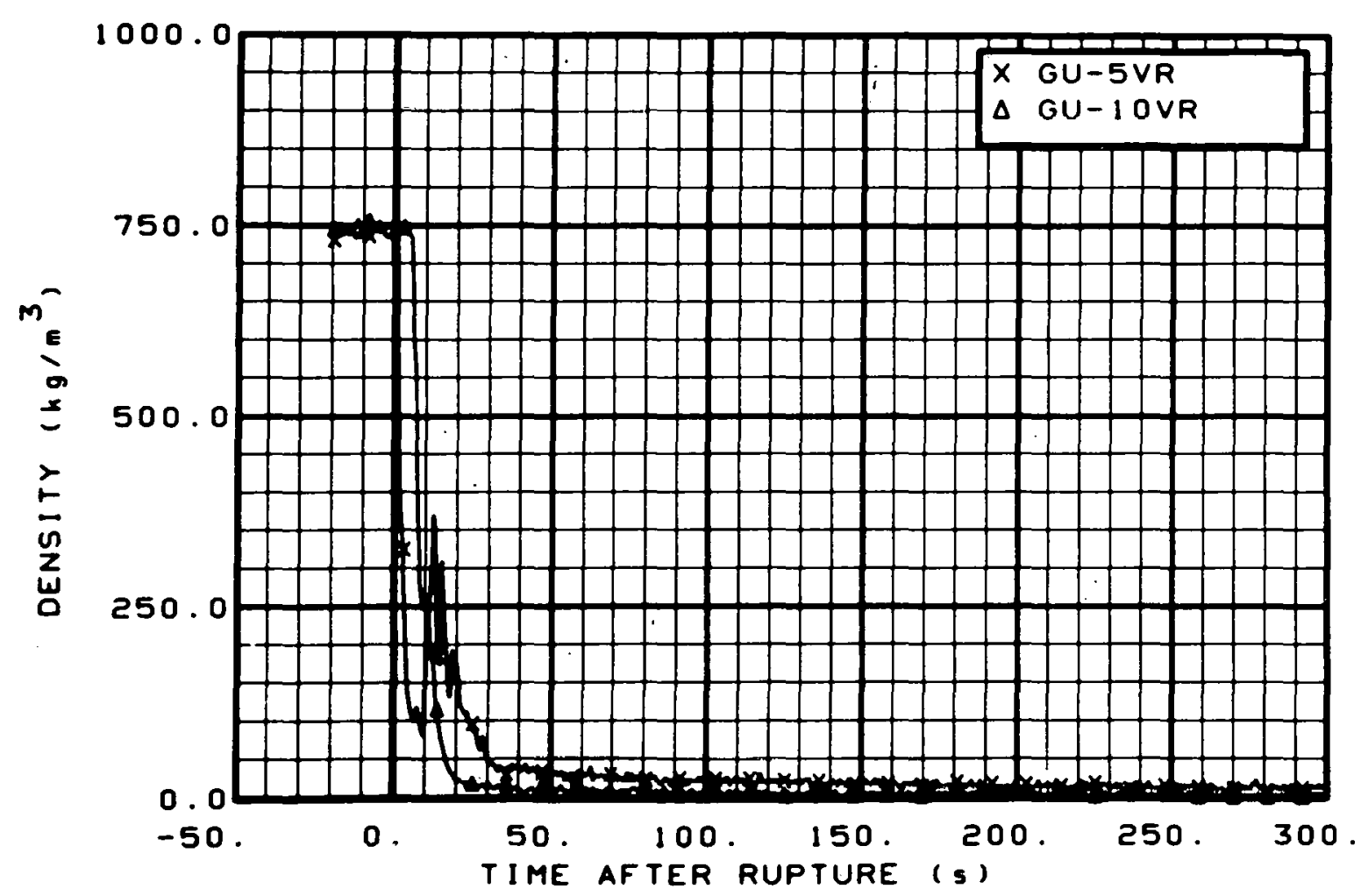

Fig. 249 Density in intact loop (GU-5VR and GU-1UVR), from -20 to $300 \mathrm{~s}$.

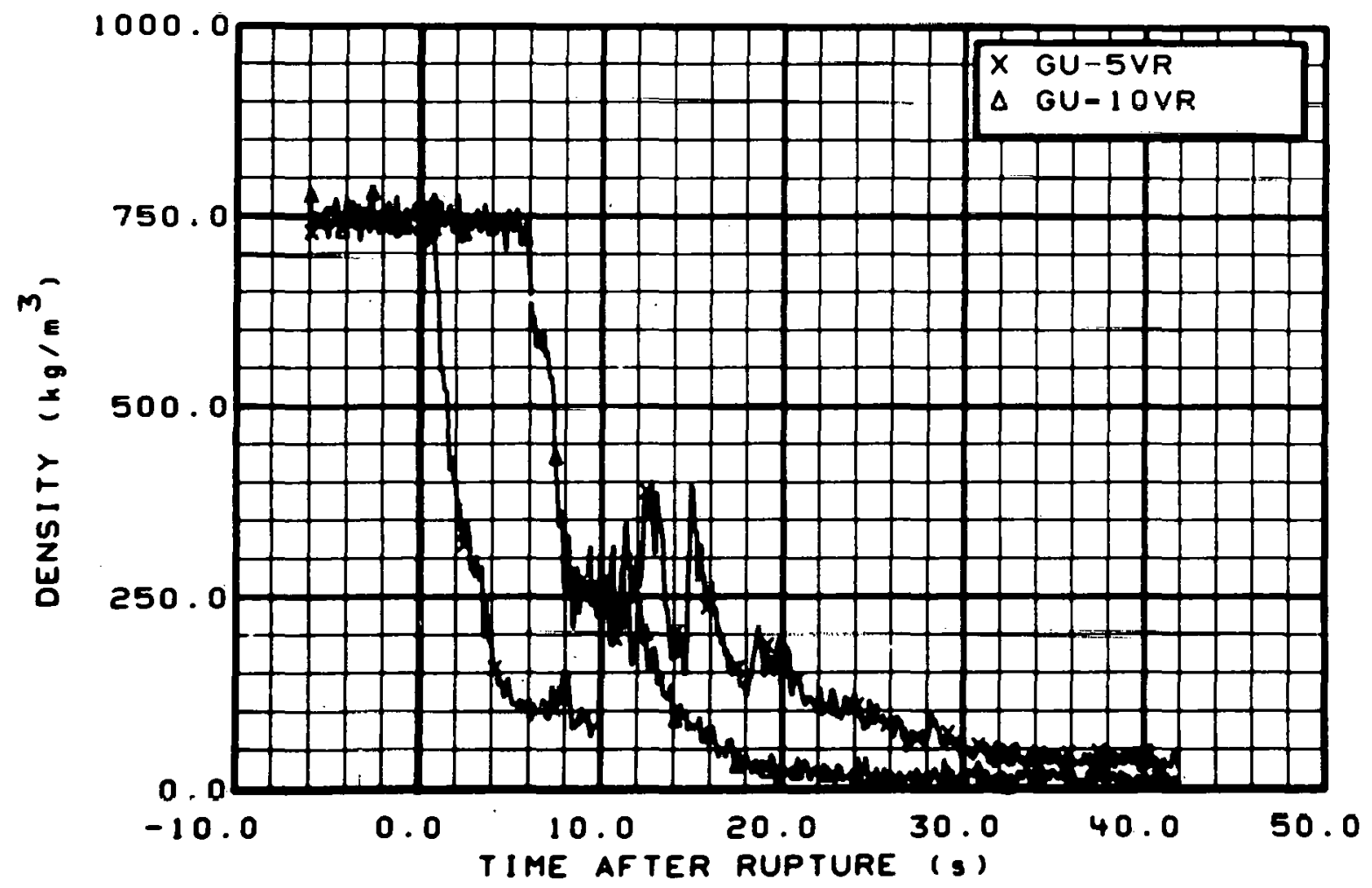

Fig. 250 Density in intact loop (GU-5VR and GU-10VR), from -6 to $42 \mathrm{~s}$. 


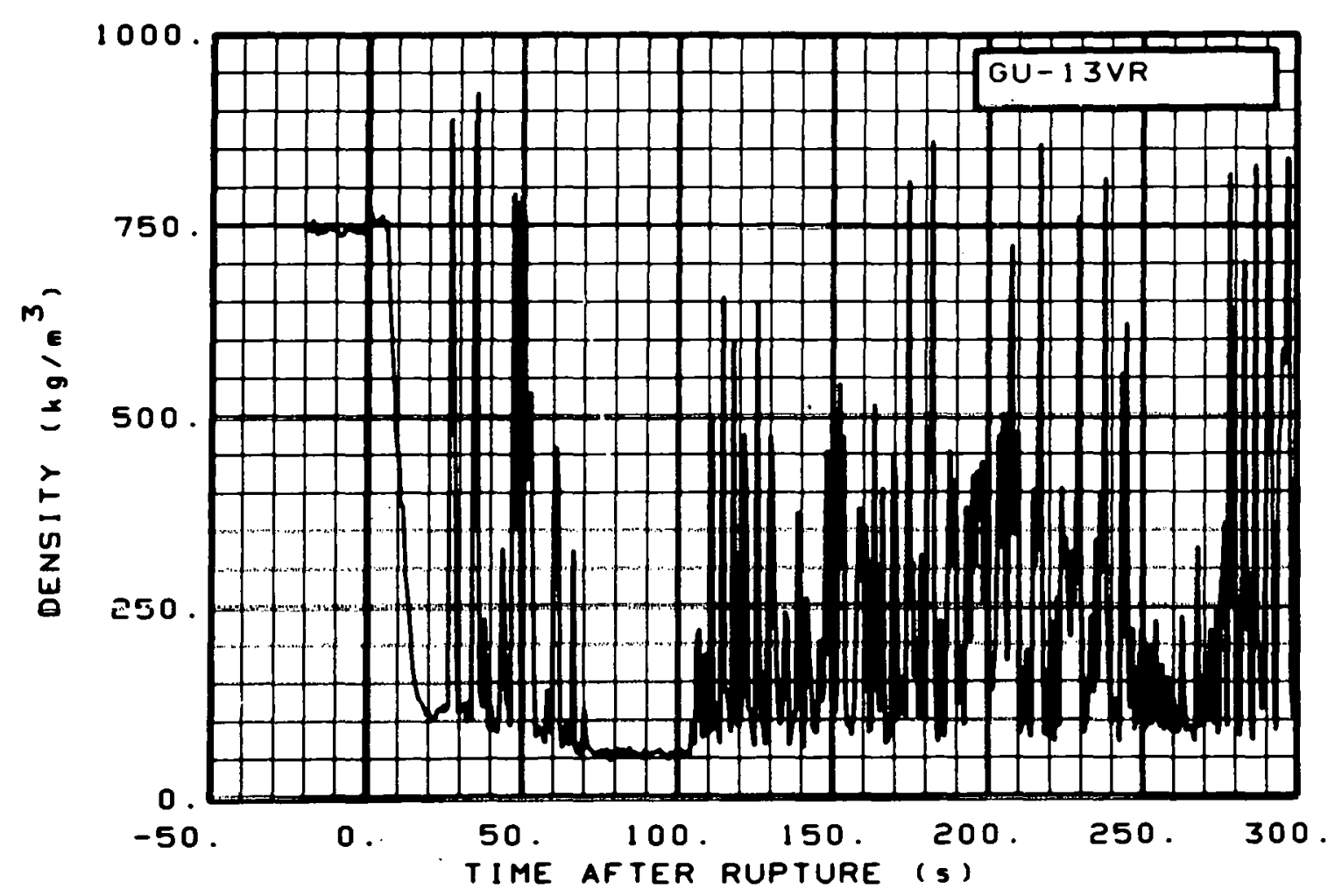

Fig. 251 Density in intact loop (GU-13VR), from -20 to $300 \mathrm{~s}$. .

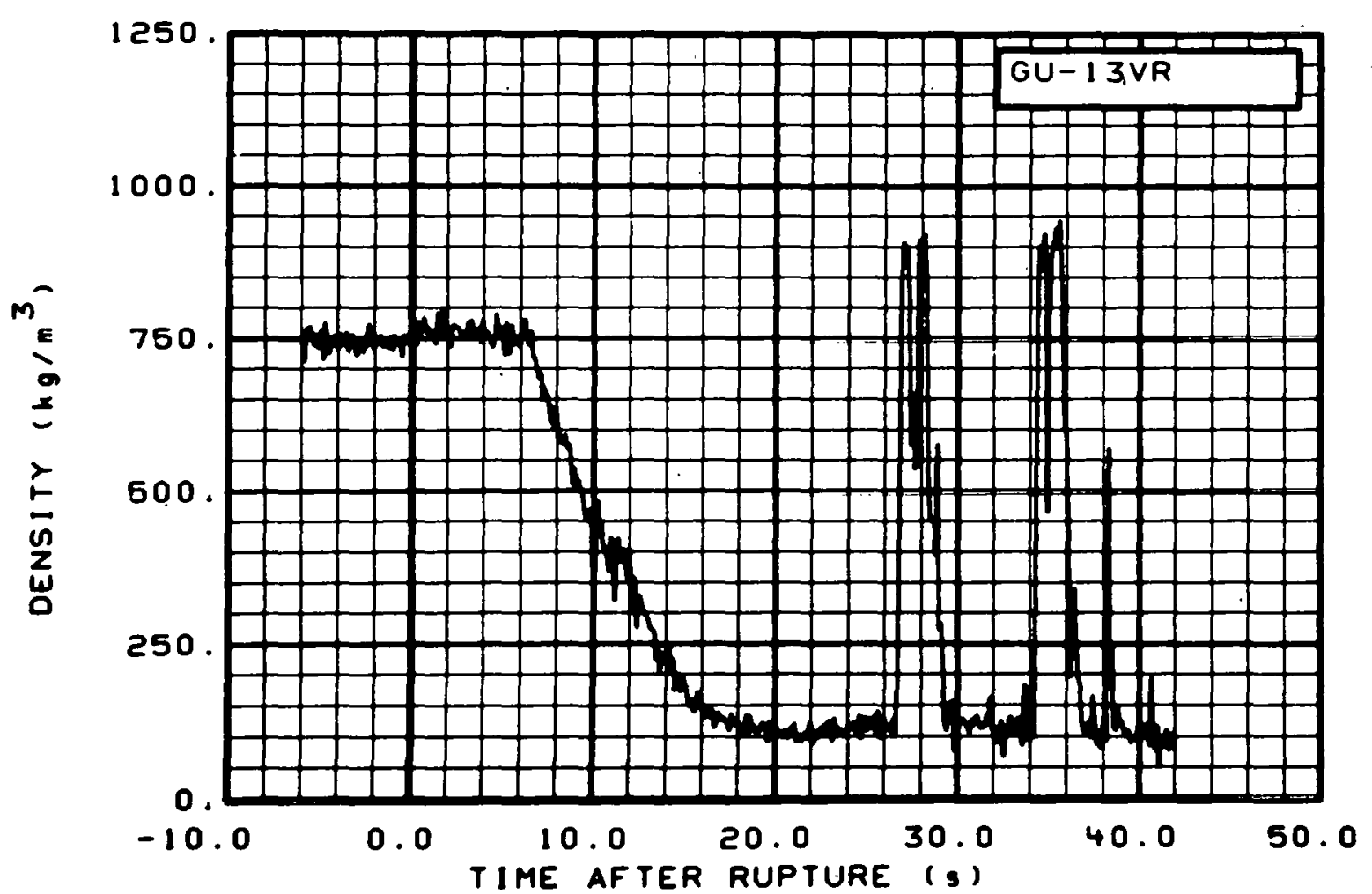

Fig. 252 Density in intact loop (GU-T3VR), from -6 to $42 \mathrm{~s}$. 


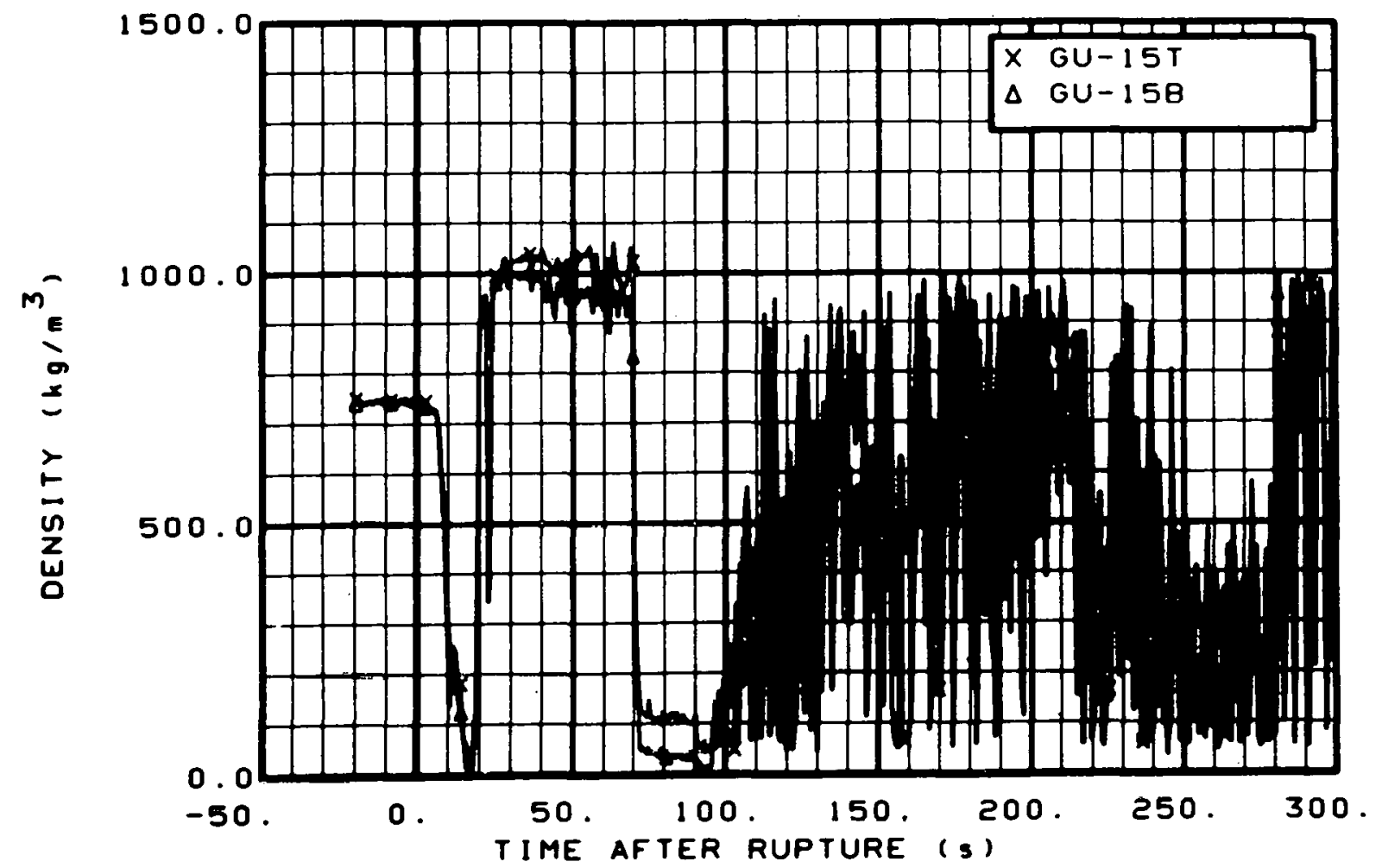

Fig. 253 Density in intact loop (GU-15T and GU-15B), from -20 to $300 \mathrm{~s}$.

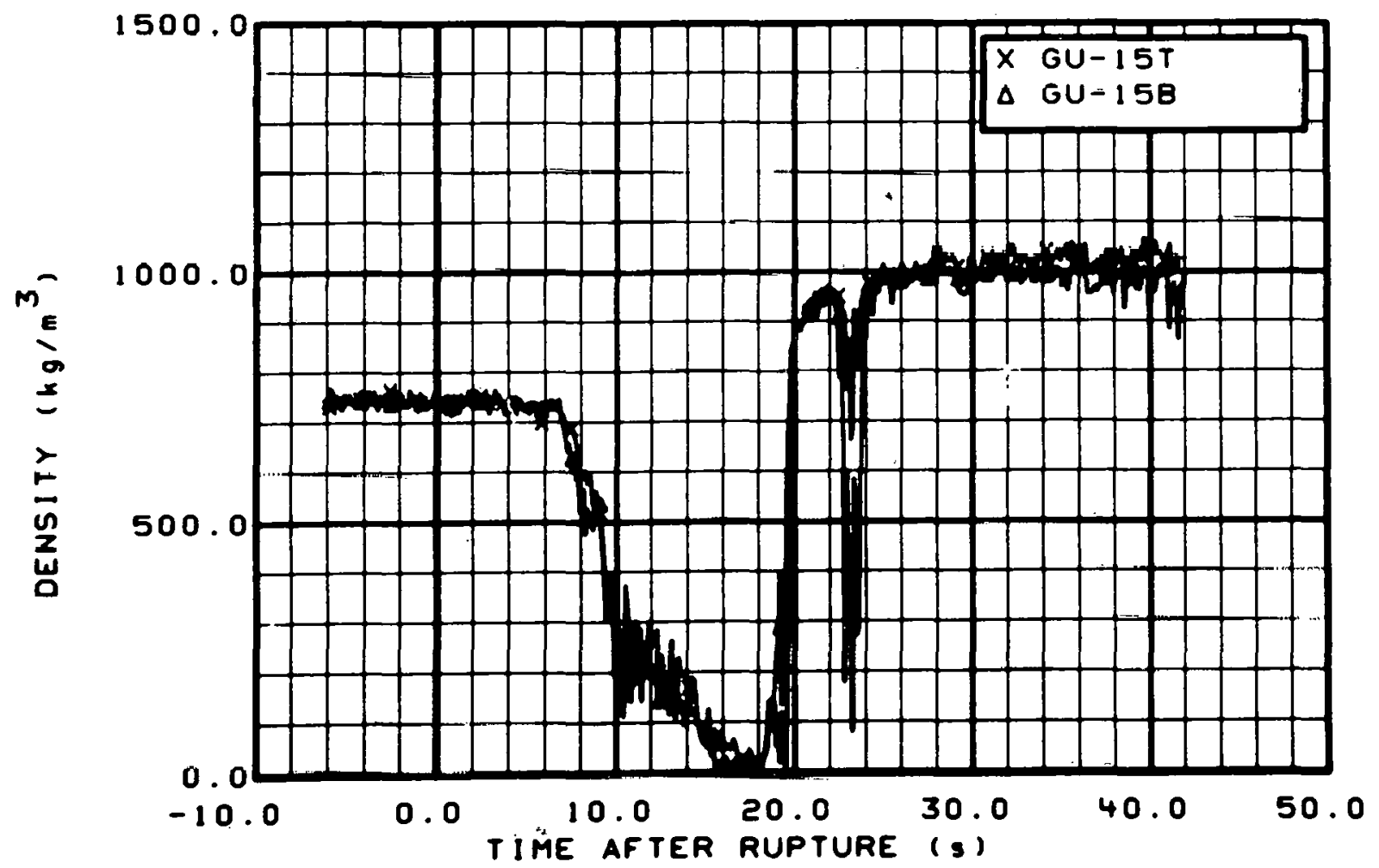

Fig. 254 Density in intact loop (GU-15T and GU-15B), from -6 to $42 \mathrm{~s}$. 


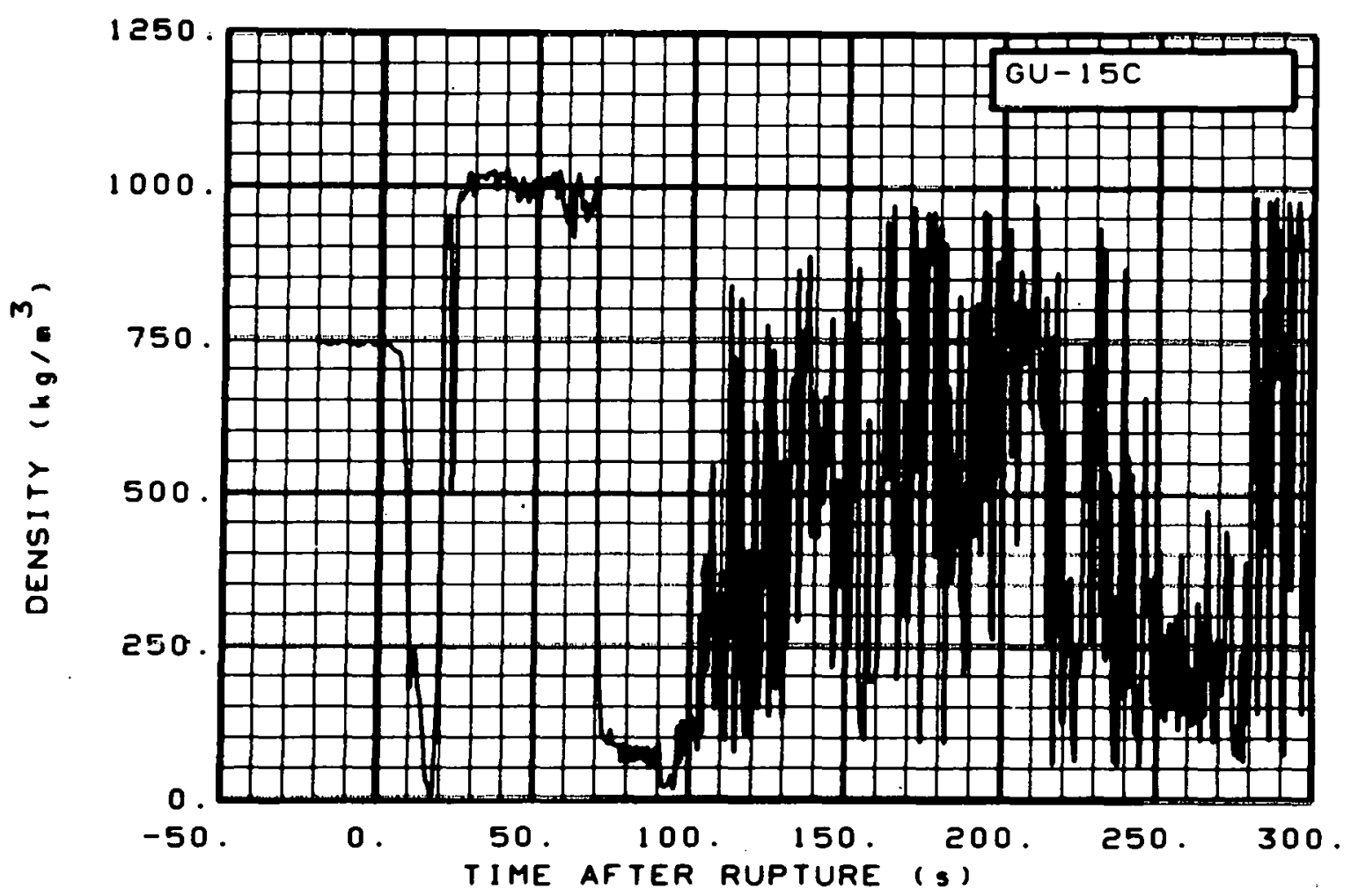

Fig. 255 Density in intact loop (GU-15C), from -20 to $300 \mathrm{~s}$.

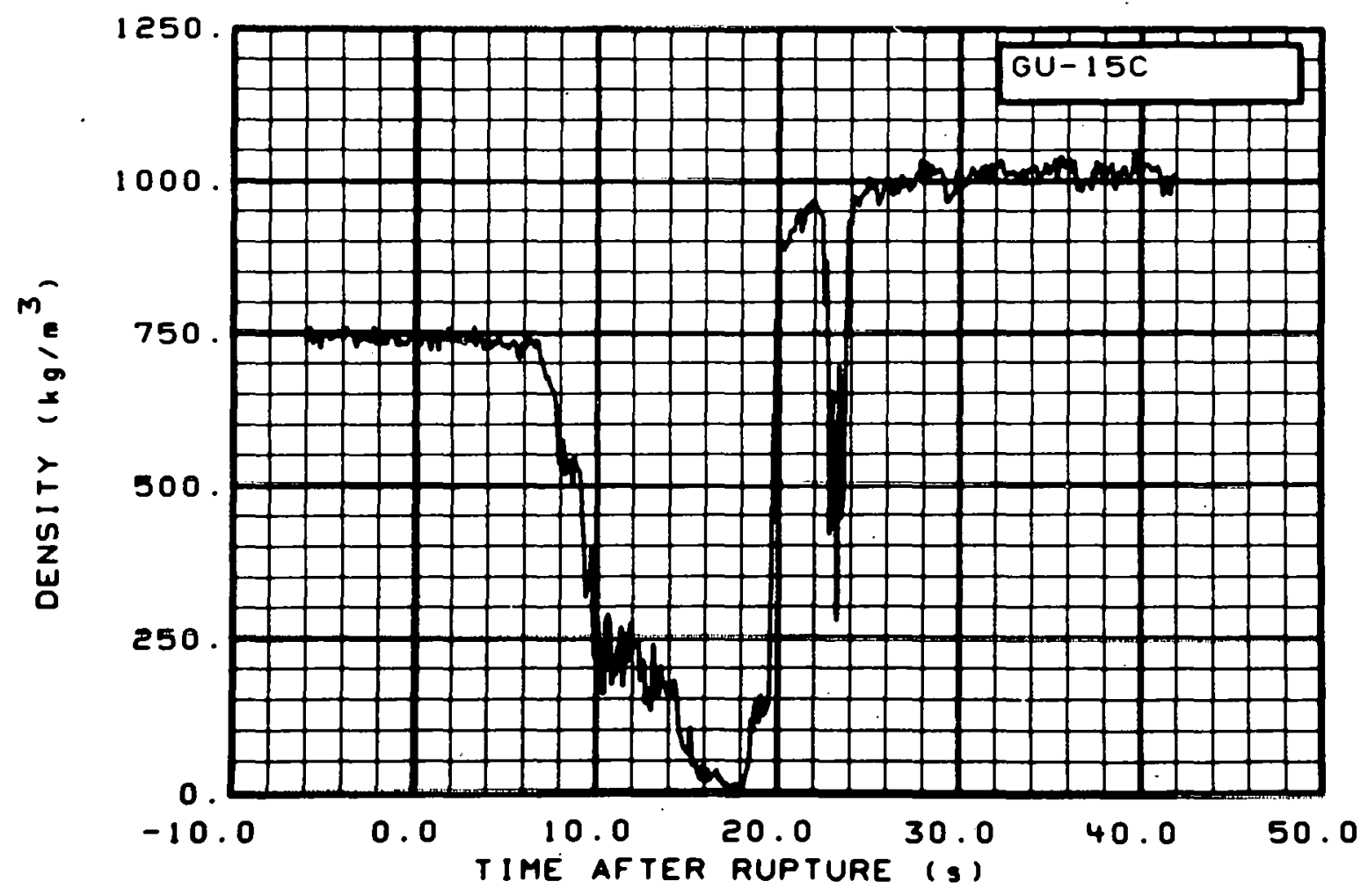

Fig. 256 Density in intact loop (GU-15C), from -6 to $42 \mathrm{~s}$. 


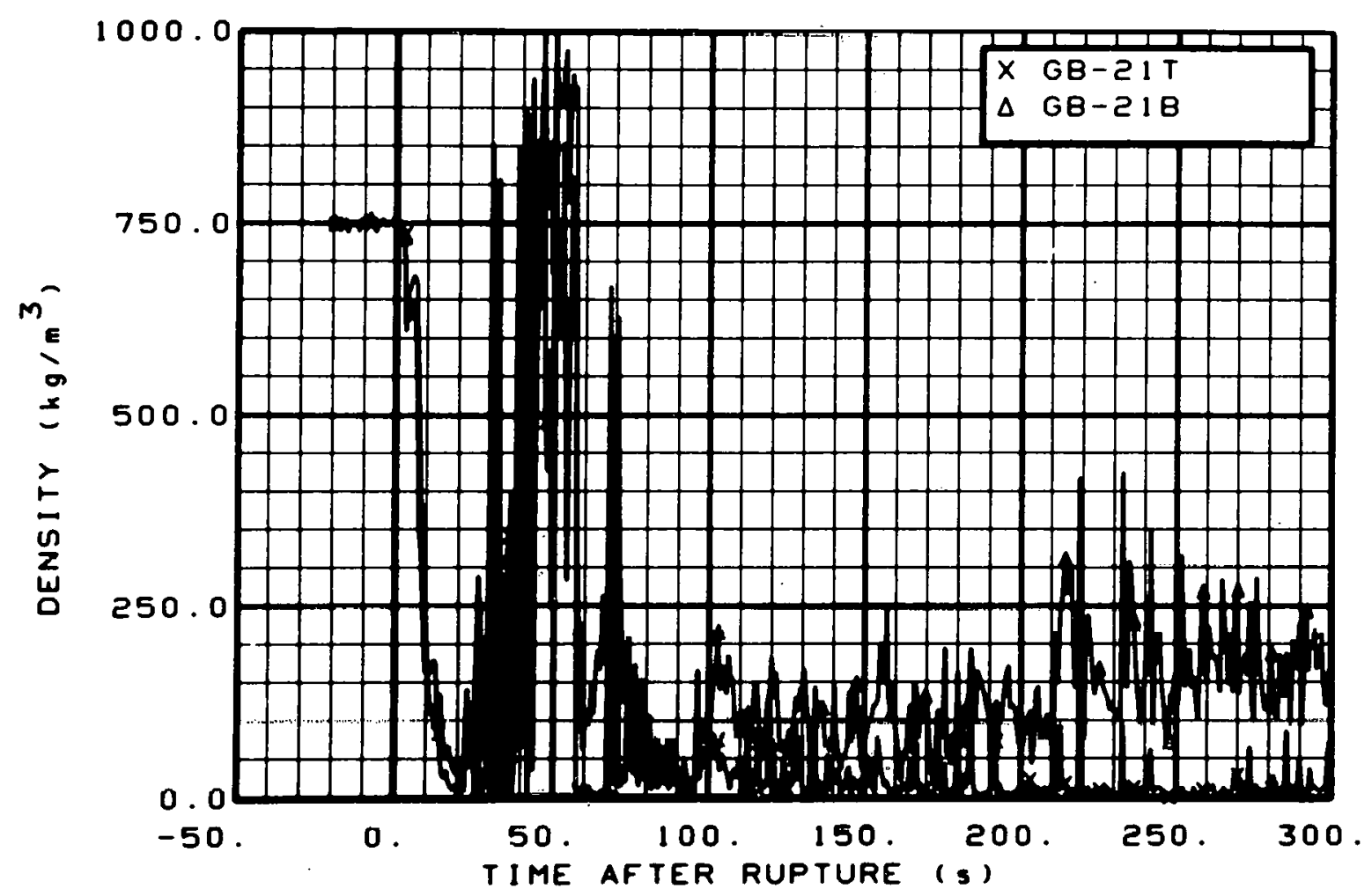

Fig. 257 Density in broken loop (GB-21T and GB-21B), from -20 to $300 \mathrm{~s}$.

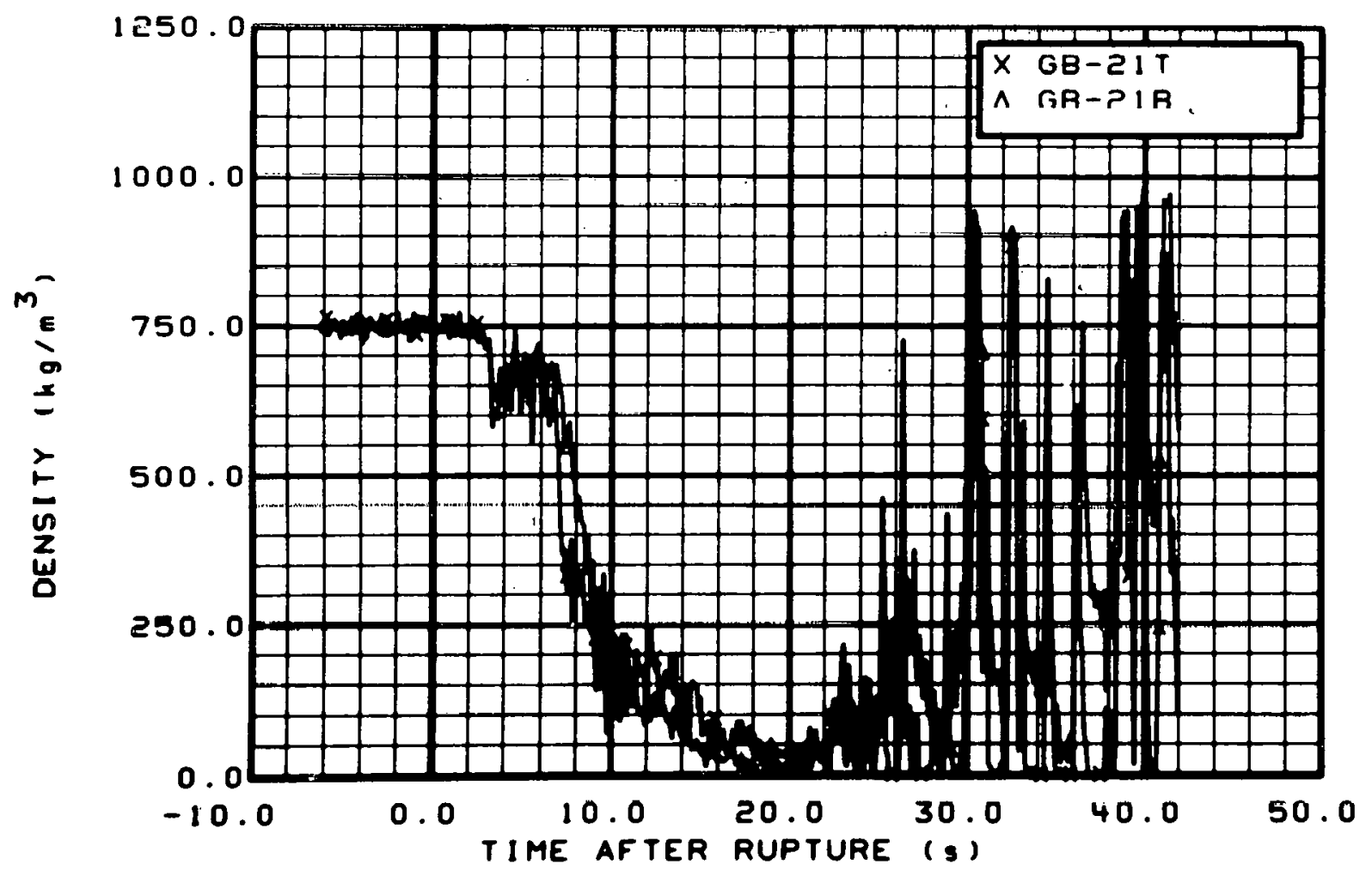

Fig. 258 Density in broken loop (GB-21T and GB-21B), from -6 to $42 \mathrm{~s}$. 


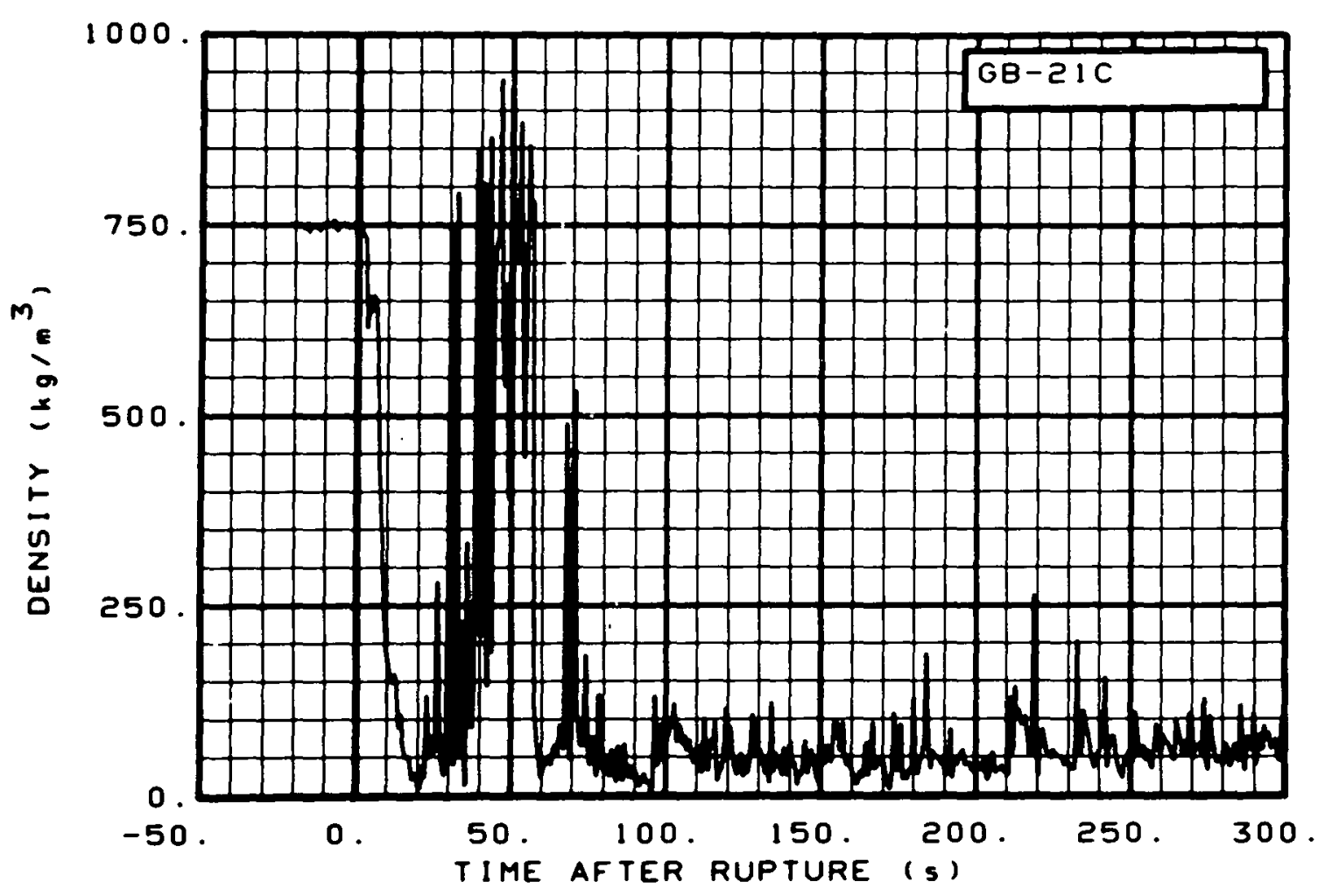

Fig. 259 Density in broken loop (GB-21C), from -20 to $300 \mathrm{~s}$.

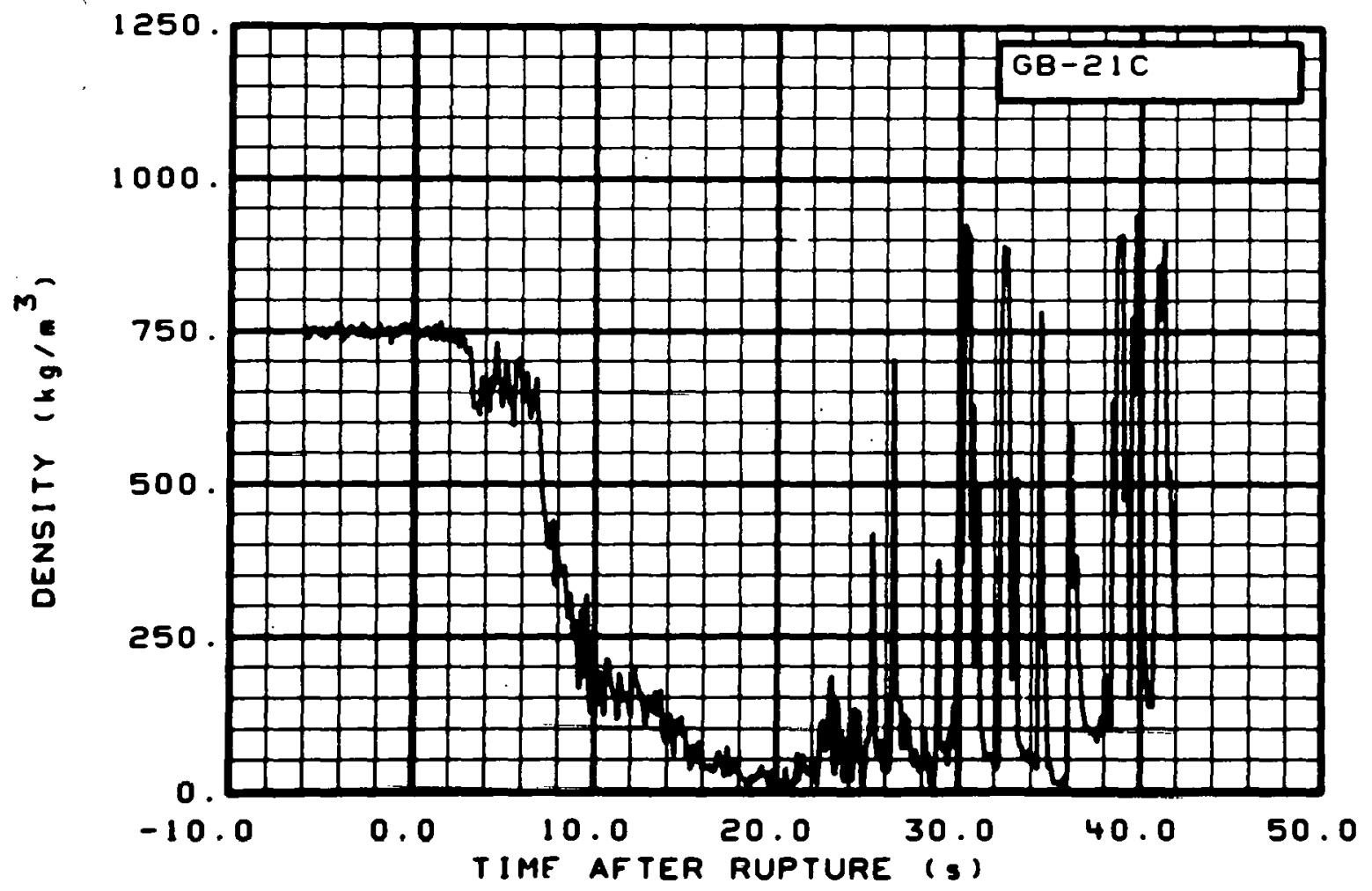

Fig. 260 Density in broken loop (GB-21C), from -6 to $42 \mathrm{~s}$. 


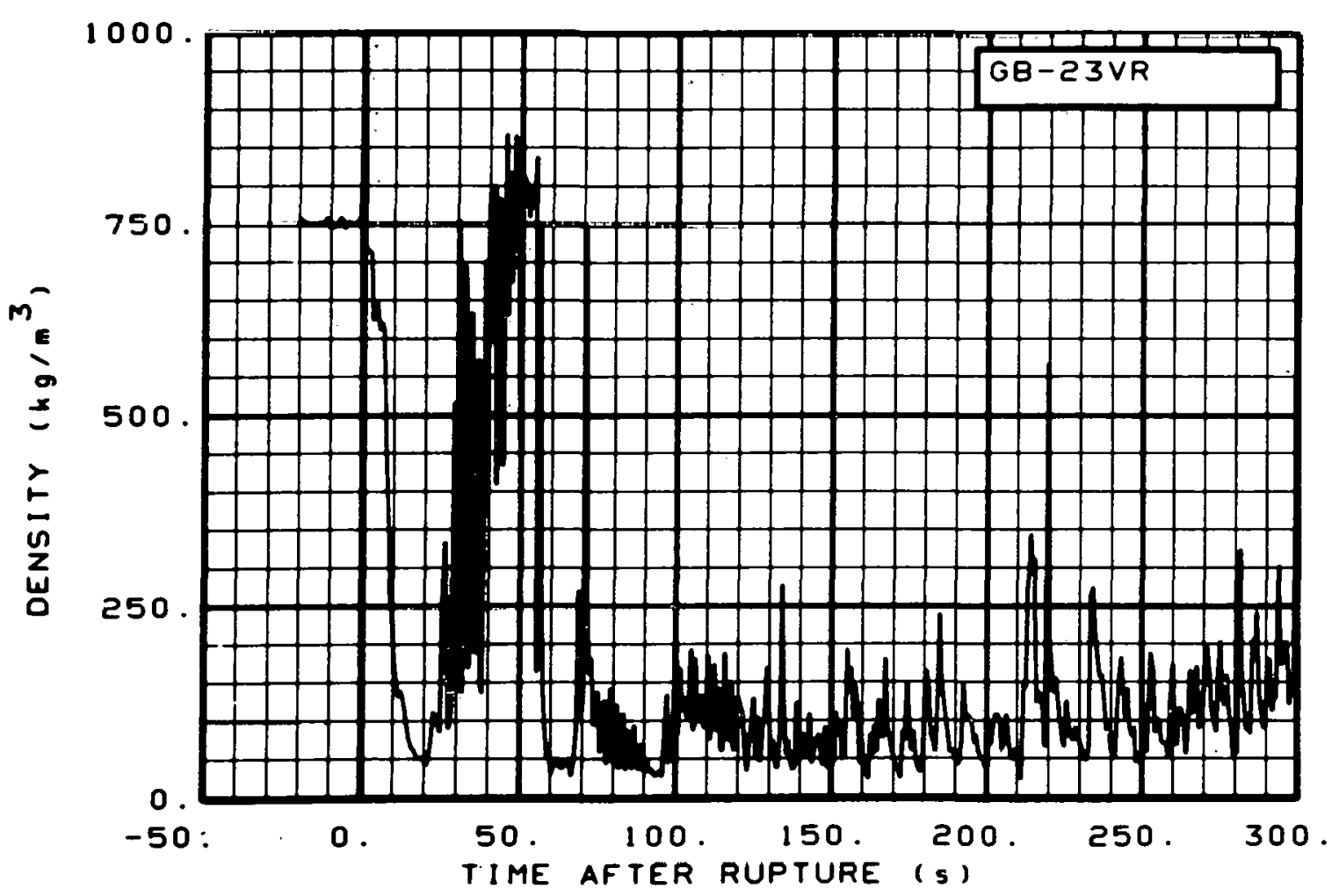

Fig. 261 Density in broken 10op (GB-23VR), from -20 to $300 \mathrm{~s}$.

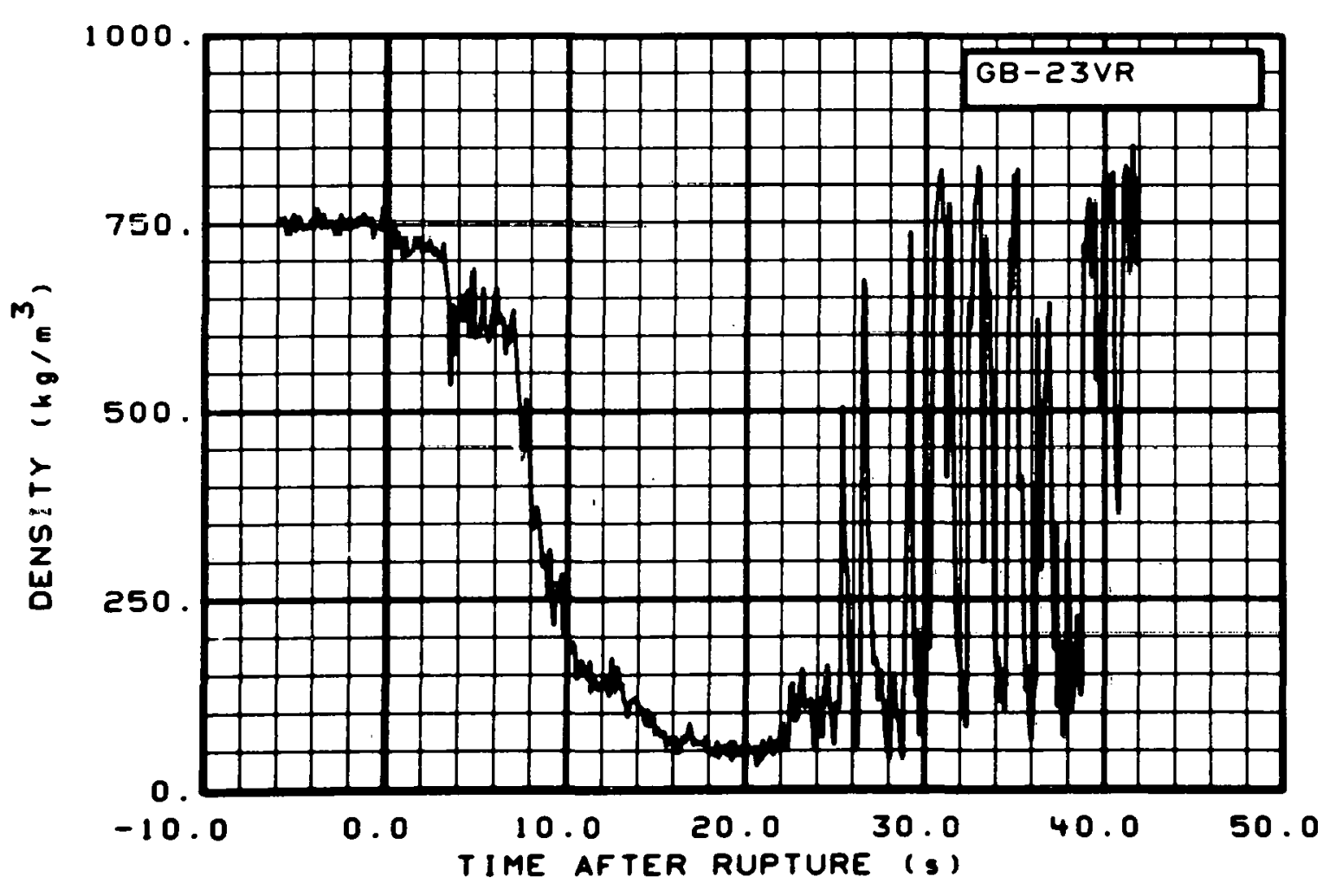

Fig. 262 Density in broken loop (GB-23VR), from -6 to $42 \mathrm{~s}$. 


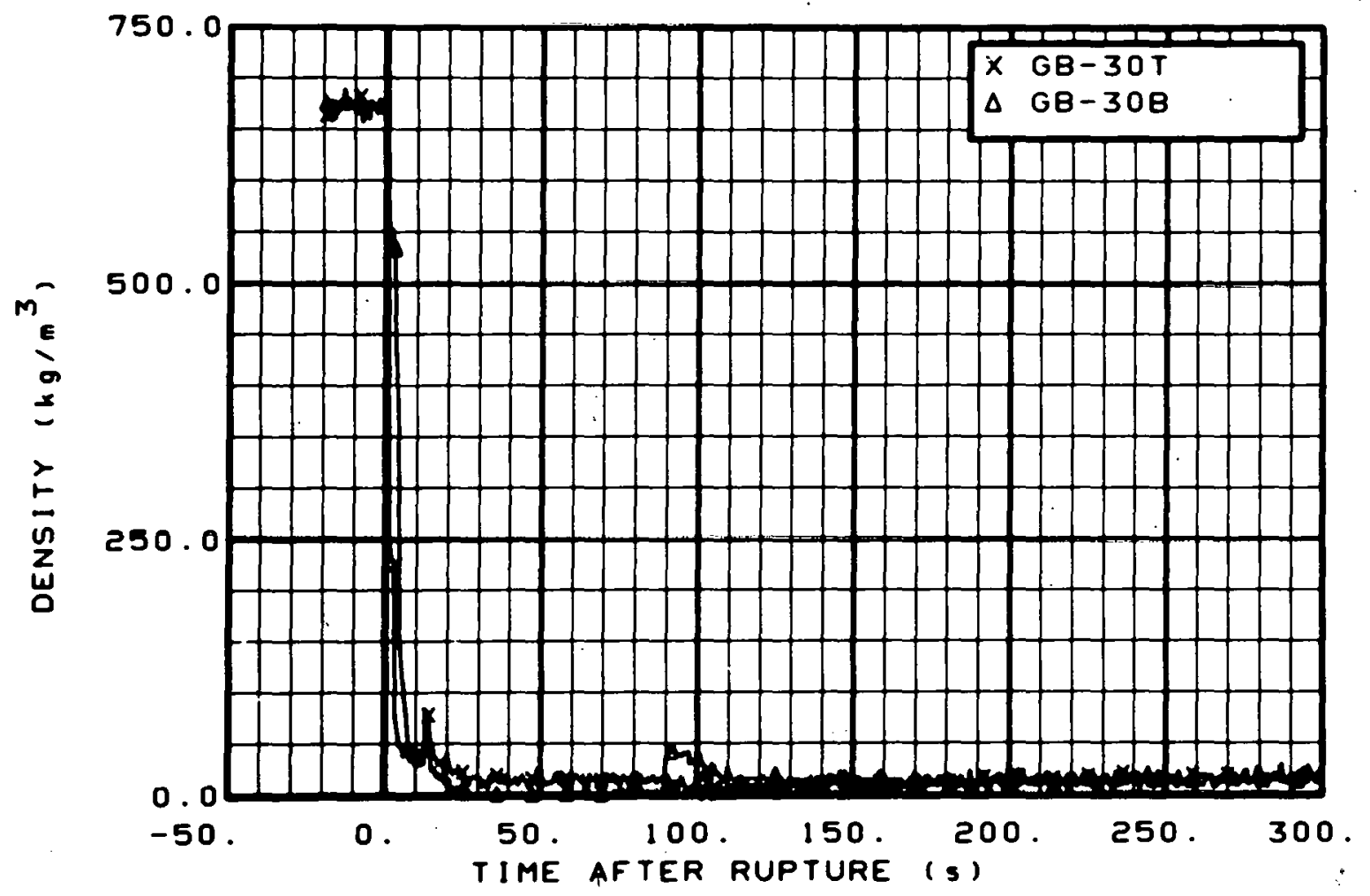

Fig. 263 Density in broken loop (GB-30T and GB-30B), from -20 to $300 \mathrm{~s}:-$

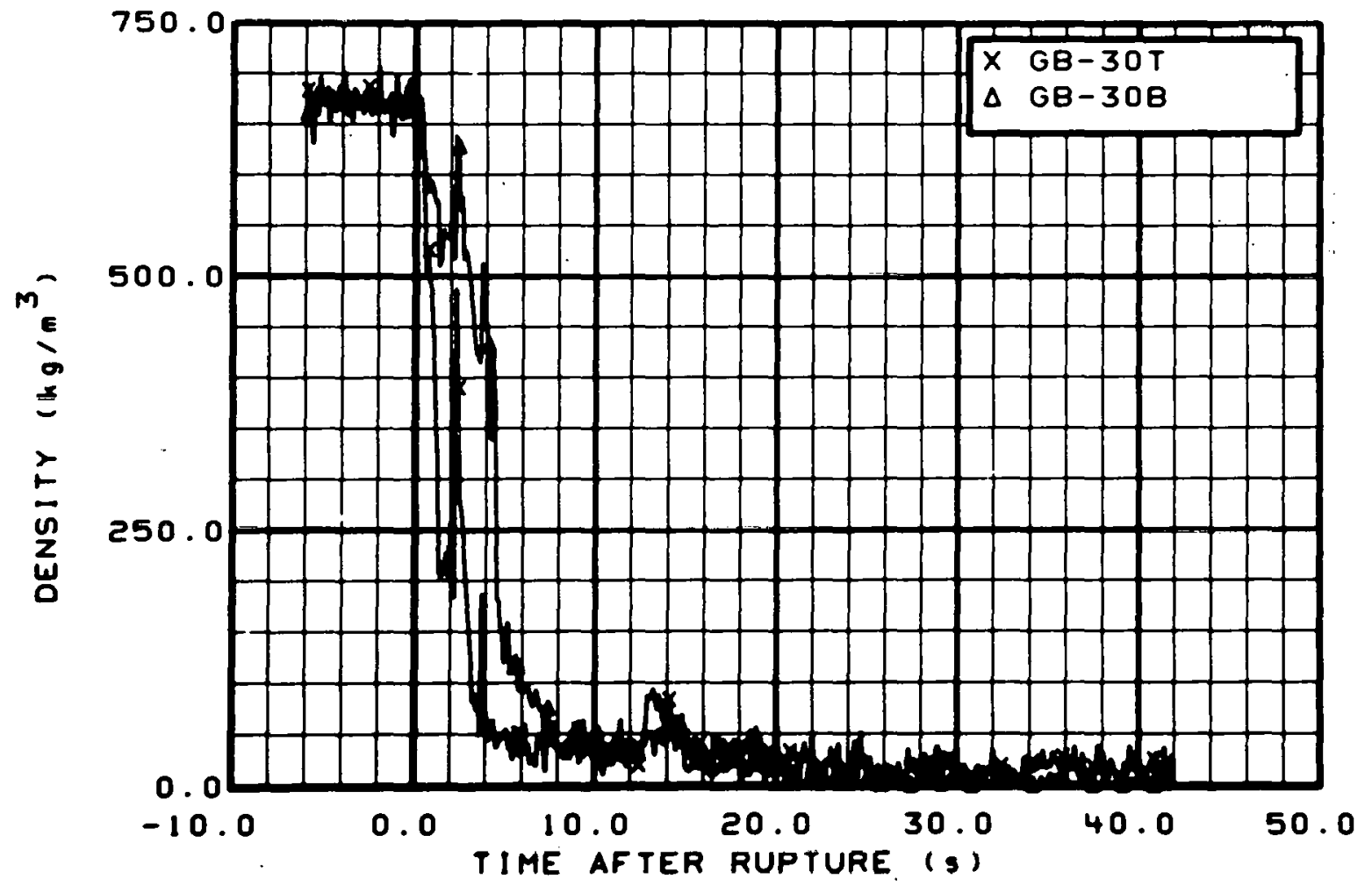

Fig. 264 Density in broken loop (GB-30T and GB-30B), from -6 to $42 \mathrm{~s}$. 


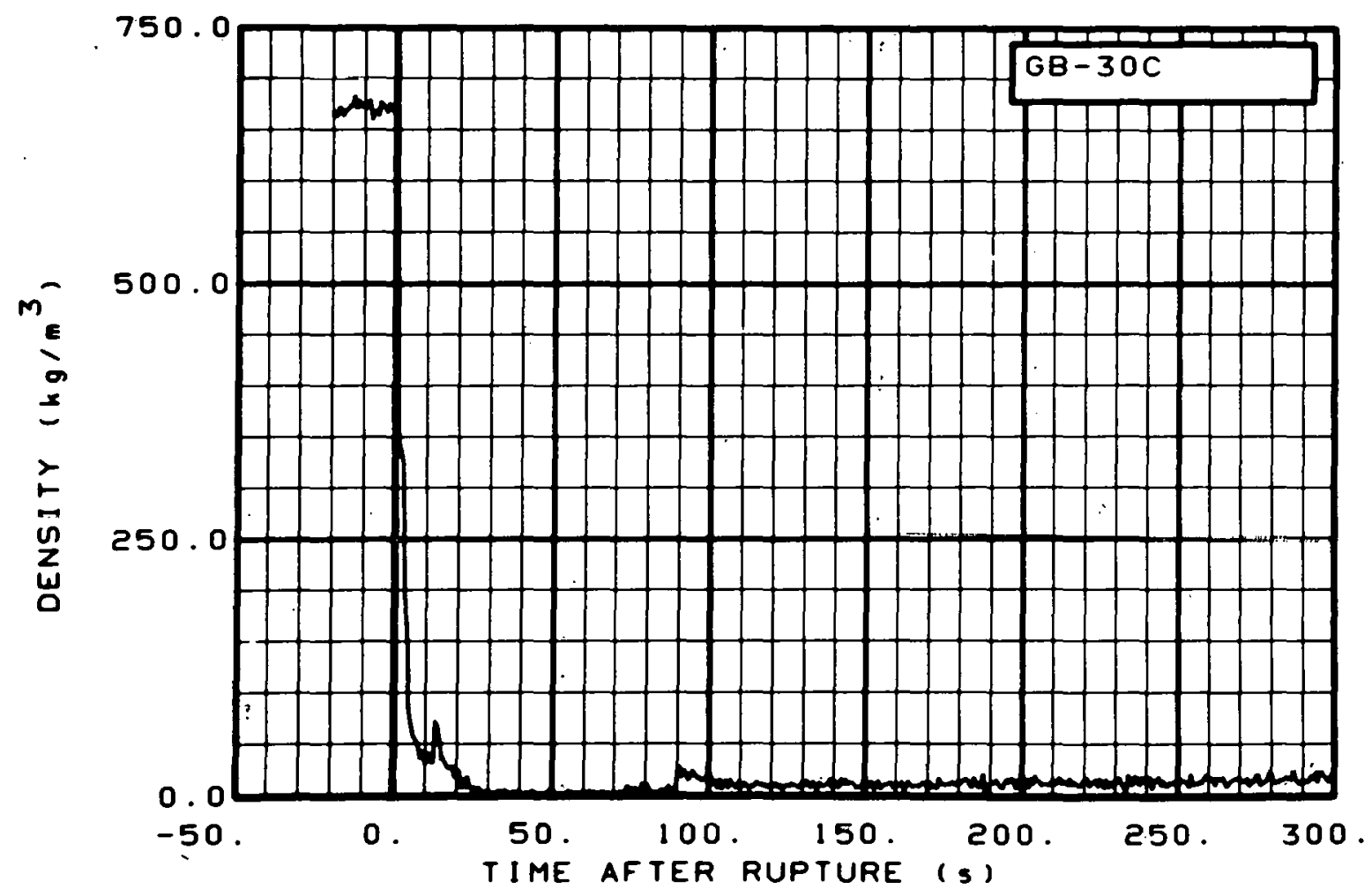

Fig. 265 Density in broken loop $(G B-30 C)$, from -20 to $300 \mathrm{~s}$.

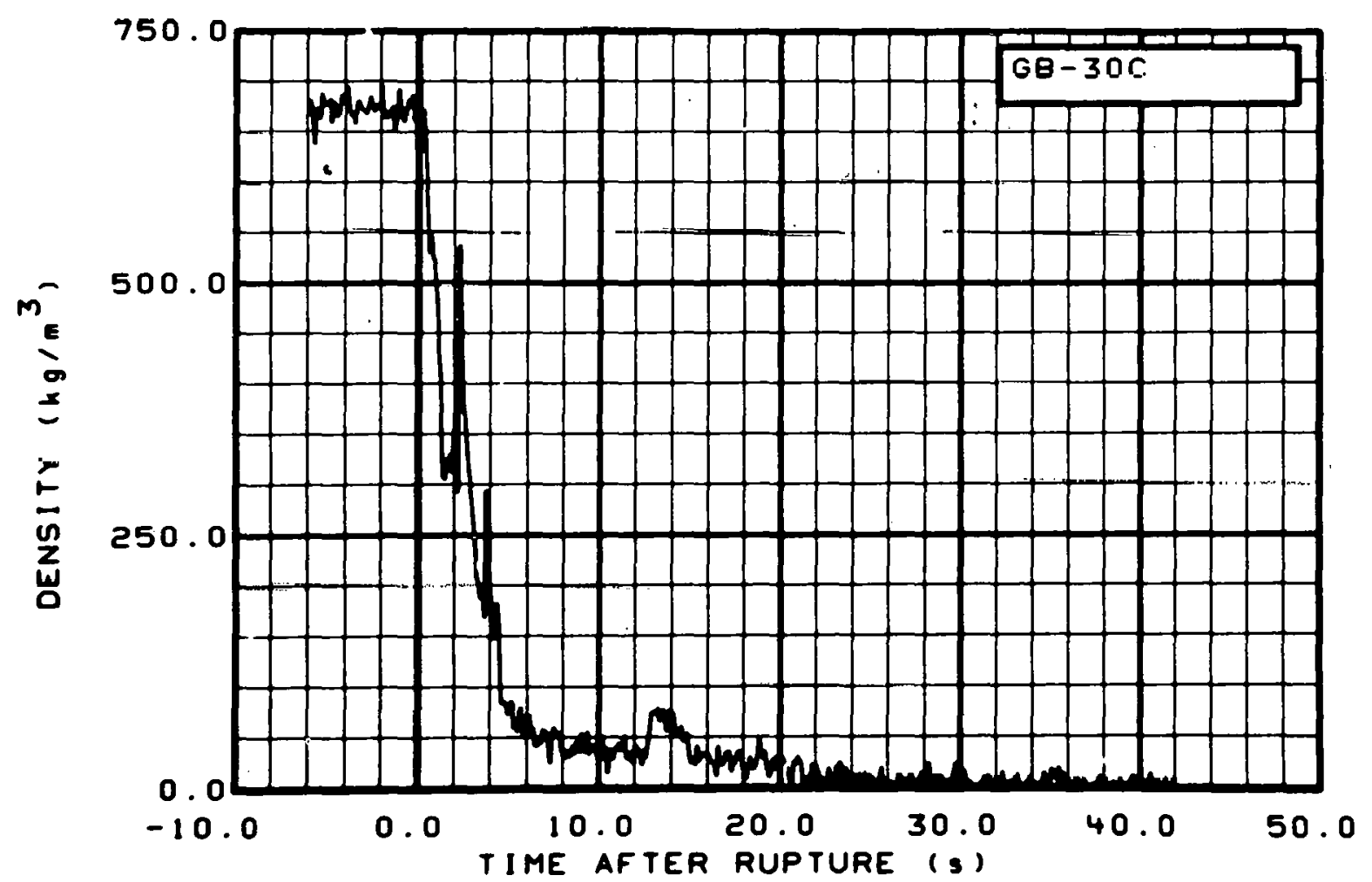

Fig. 266 Density in broken loop $(G B-30 C)$, from -6 to $42 \mathrm{~s}$. 


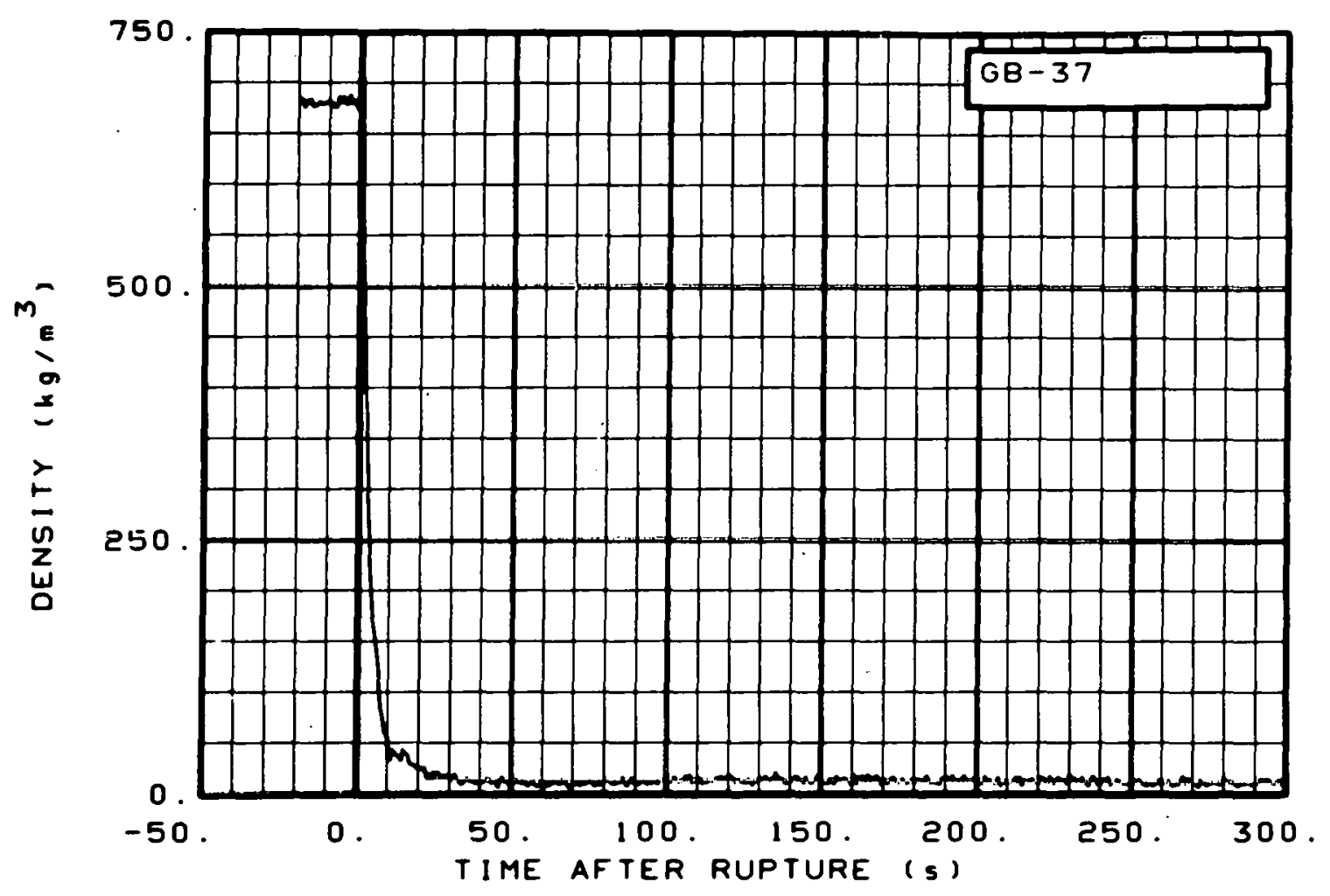

Fig. 267 Density in broken 10op (GB-37), from -20 to $300 \mathrm{~s}$.

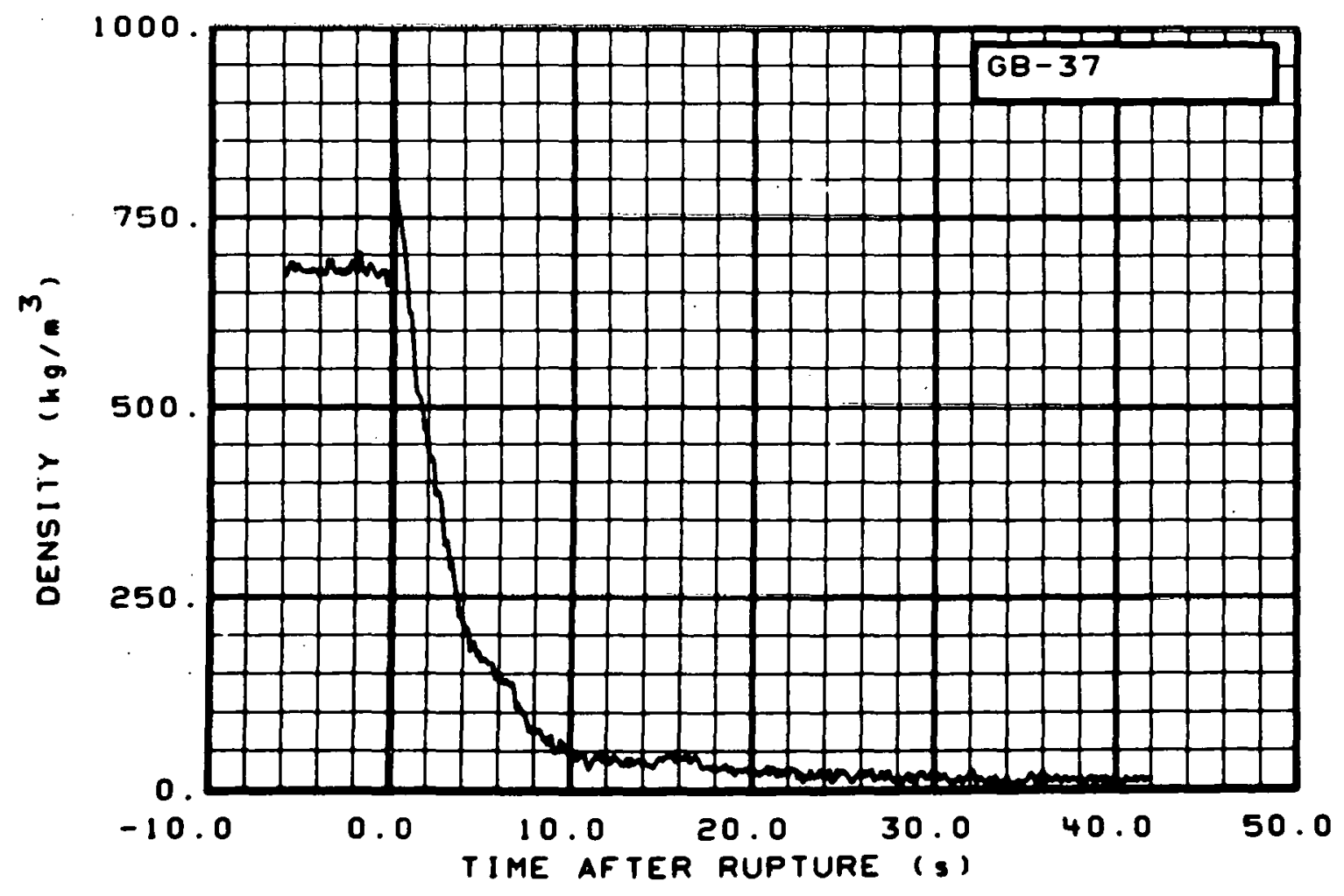

Fig. 268 Density in broken loop (GB-37), from -6 to $42 \mathrm{~s}$. 


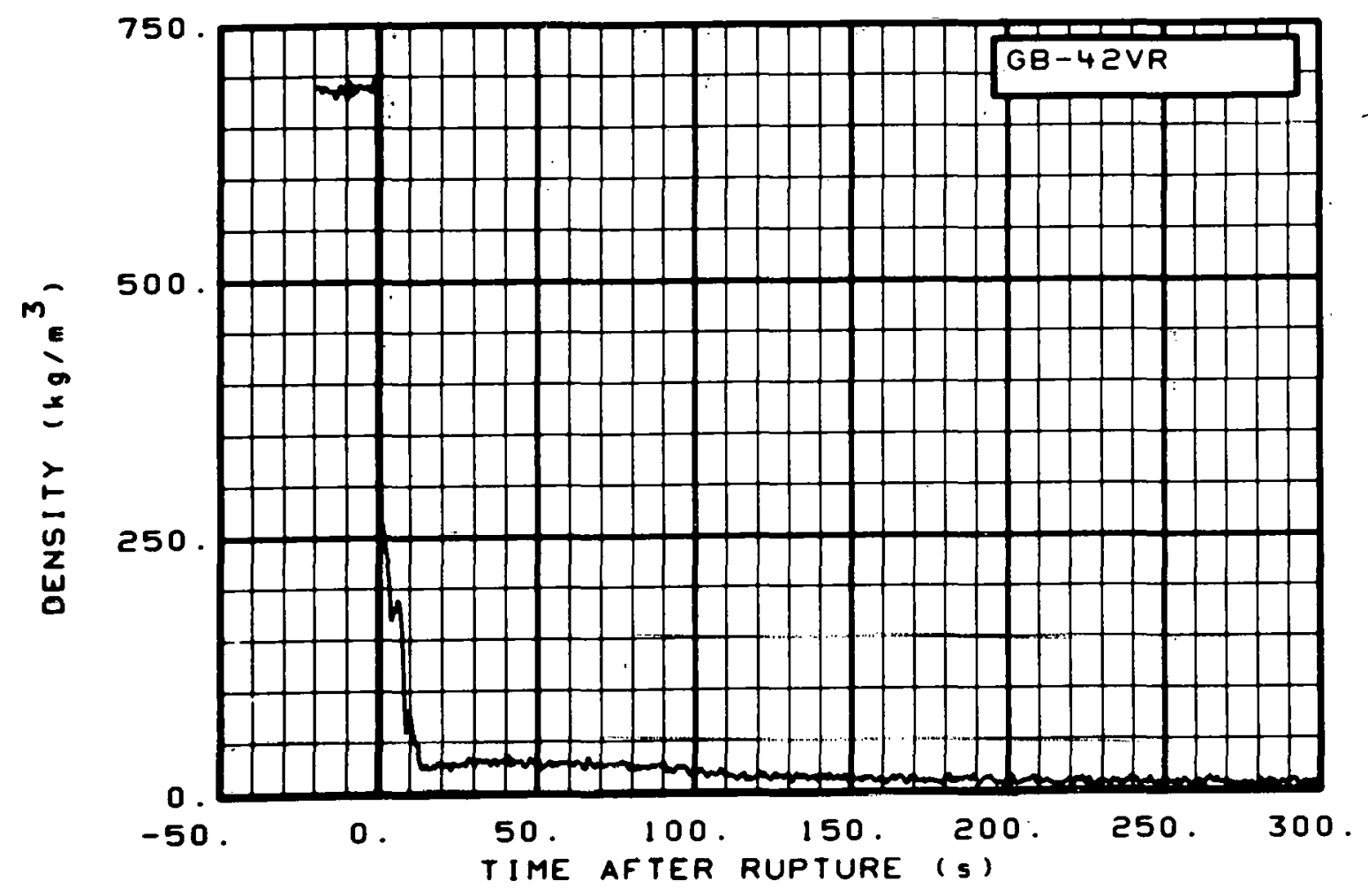

Fig. 269 Density in broken loop (GB-42VR), from -20 to $300 \mathrm{~s}$.

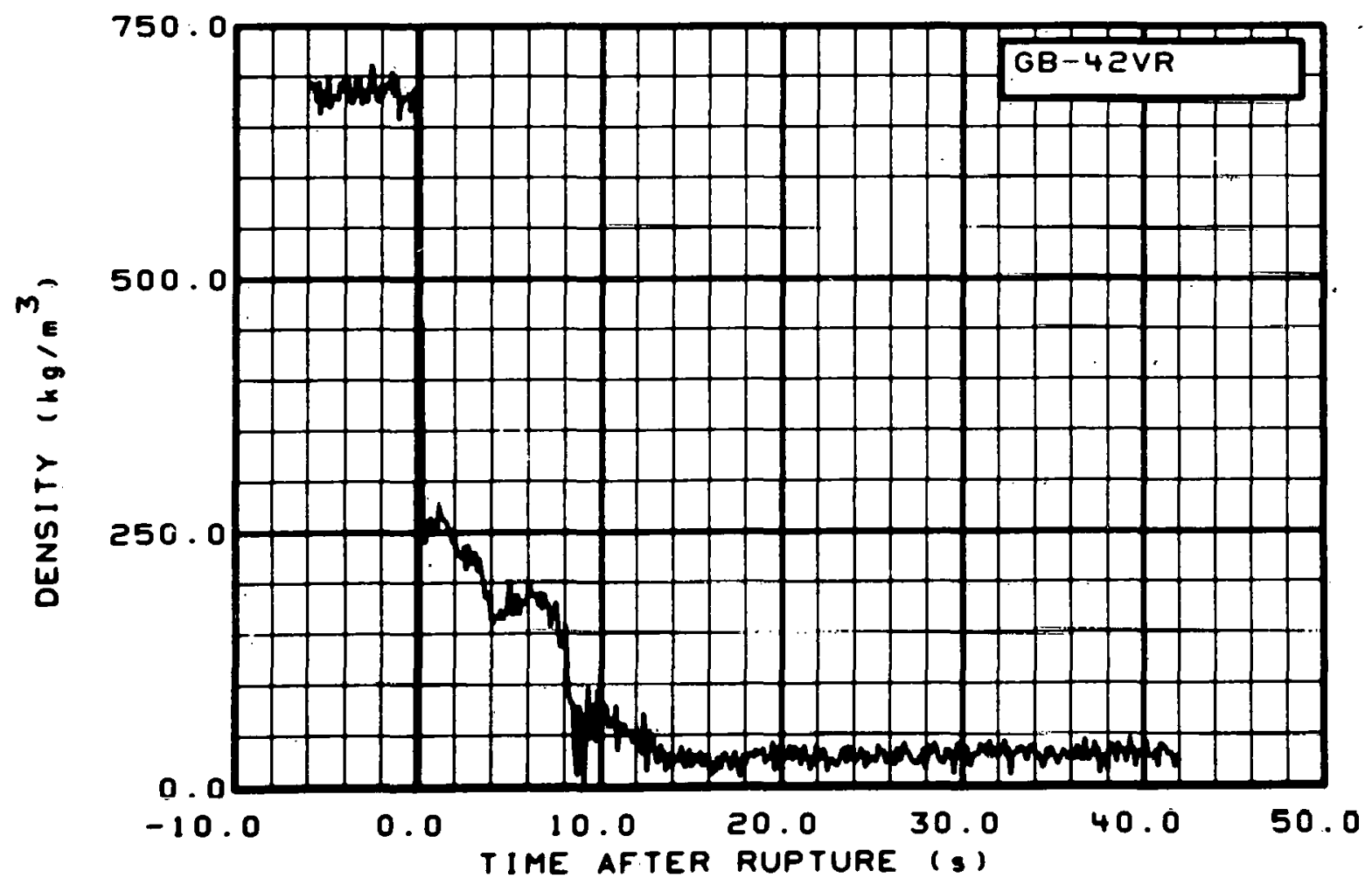

Fig. 270 Density in broken loop (GB-42VR), from -6 to. $42 \mathrm{~s}$. 


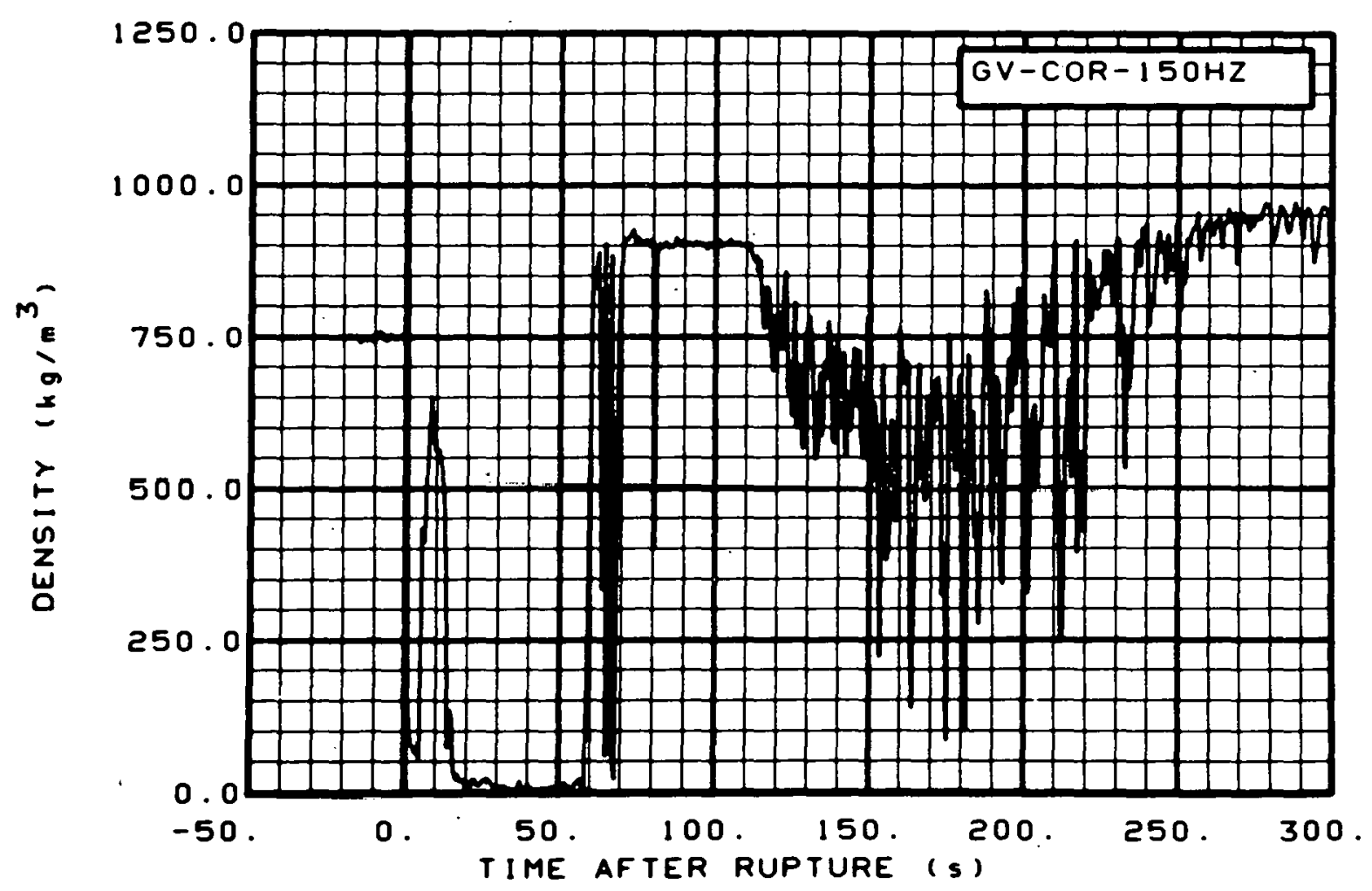

Fig. 271 Density in vessel (GV-COR-150HZ), from -20 to $300 \mathrm{~s}$.

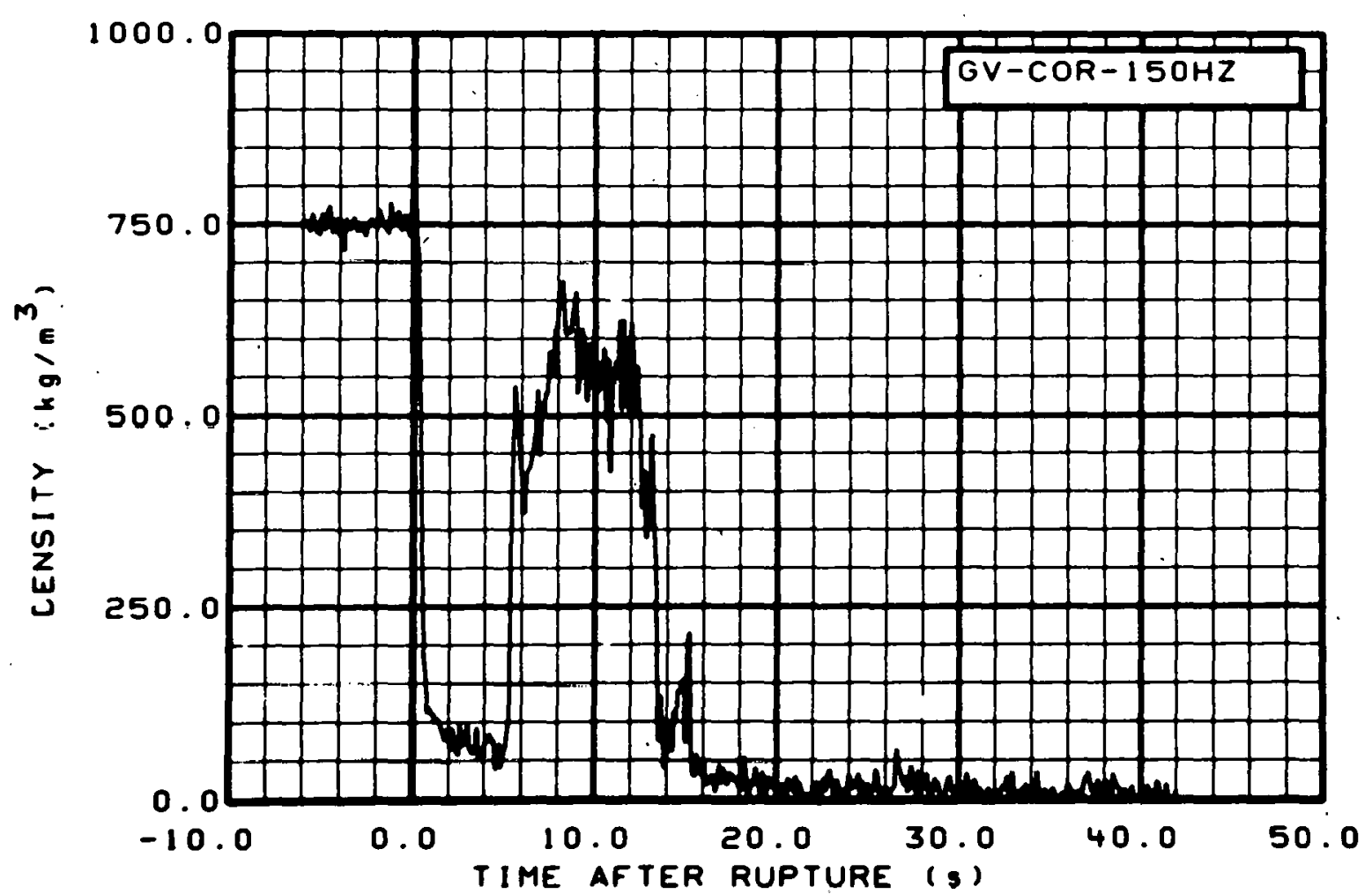

Fig. 272 Density in vessel (GV-COR-150HZ), from -6 to $42 \mathrm{~s}$. 


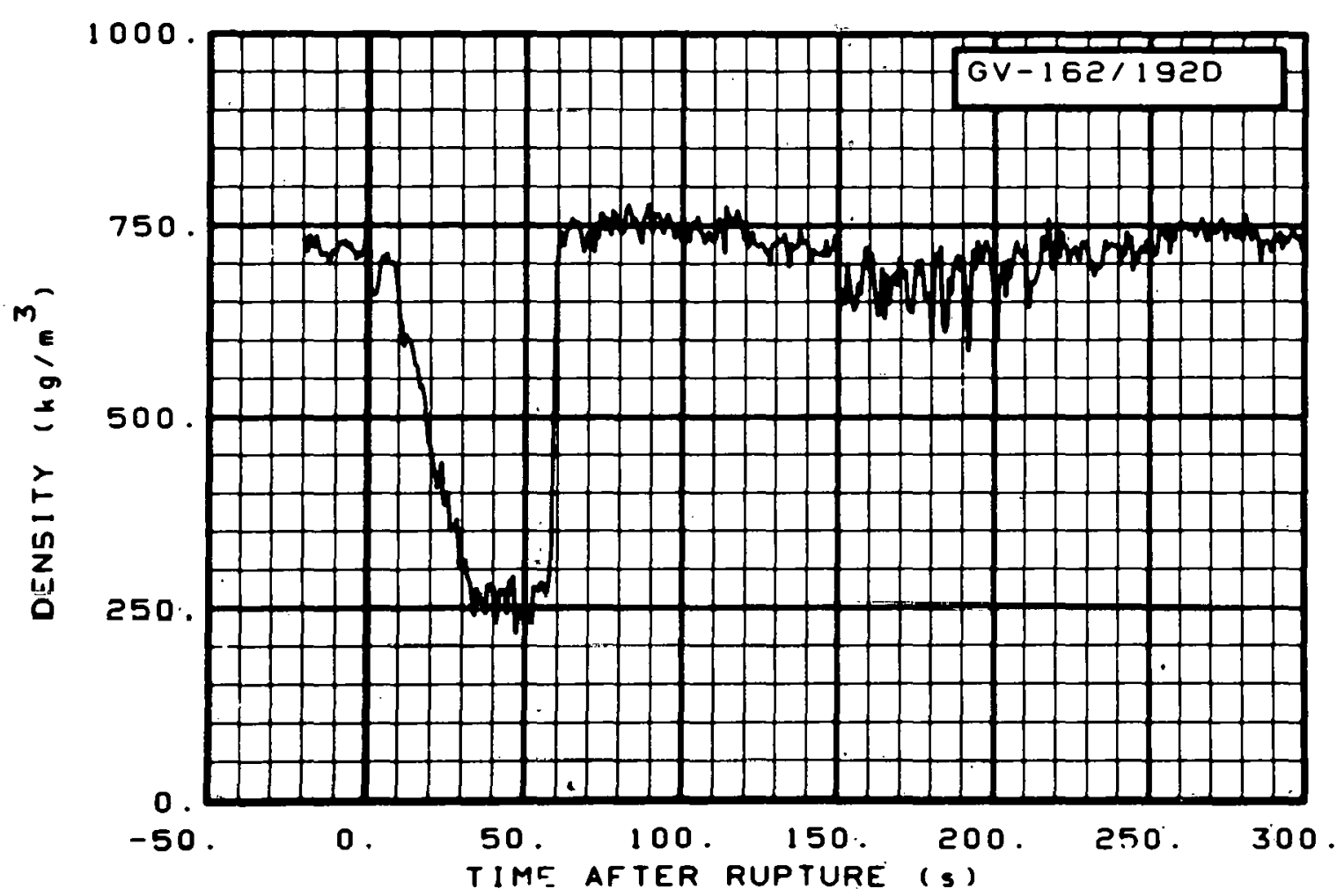

Fig. 273 Density in vessel (GV-162/192D), from -20 to $300 \mathrm{~s}$.

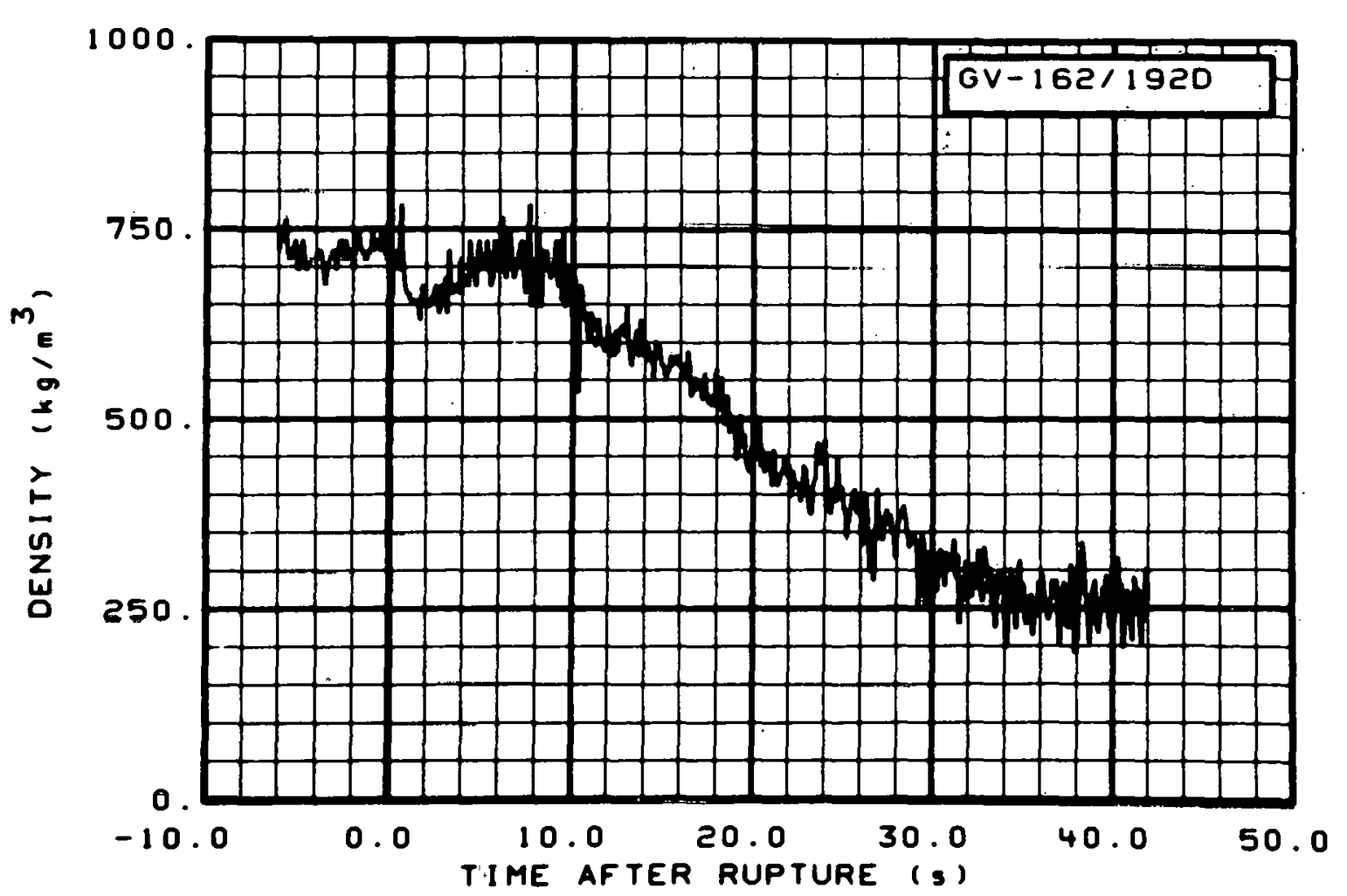

Fig. 274 Density in vessel (GV-162/192D), from -6 to $42 \mathrm{~s}$. 


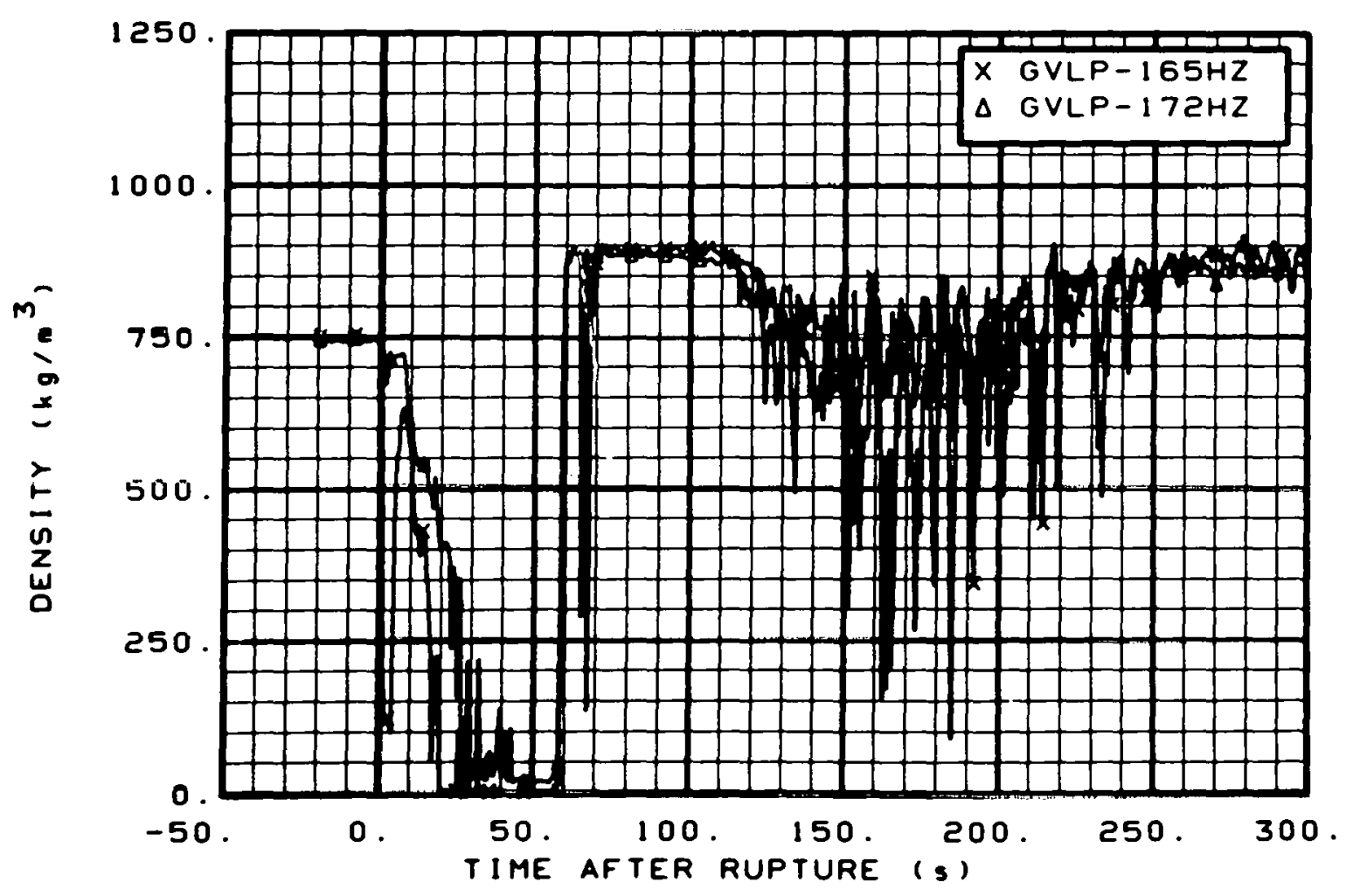

Fig. 275 Density in vessel (GVLP-165HZ and GVLP-172HZ), from -20 to $300 \mathrm{~s}$.

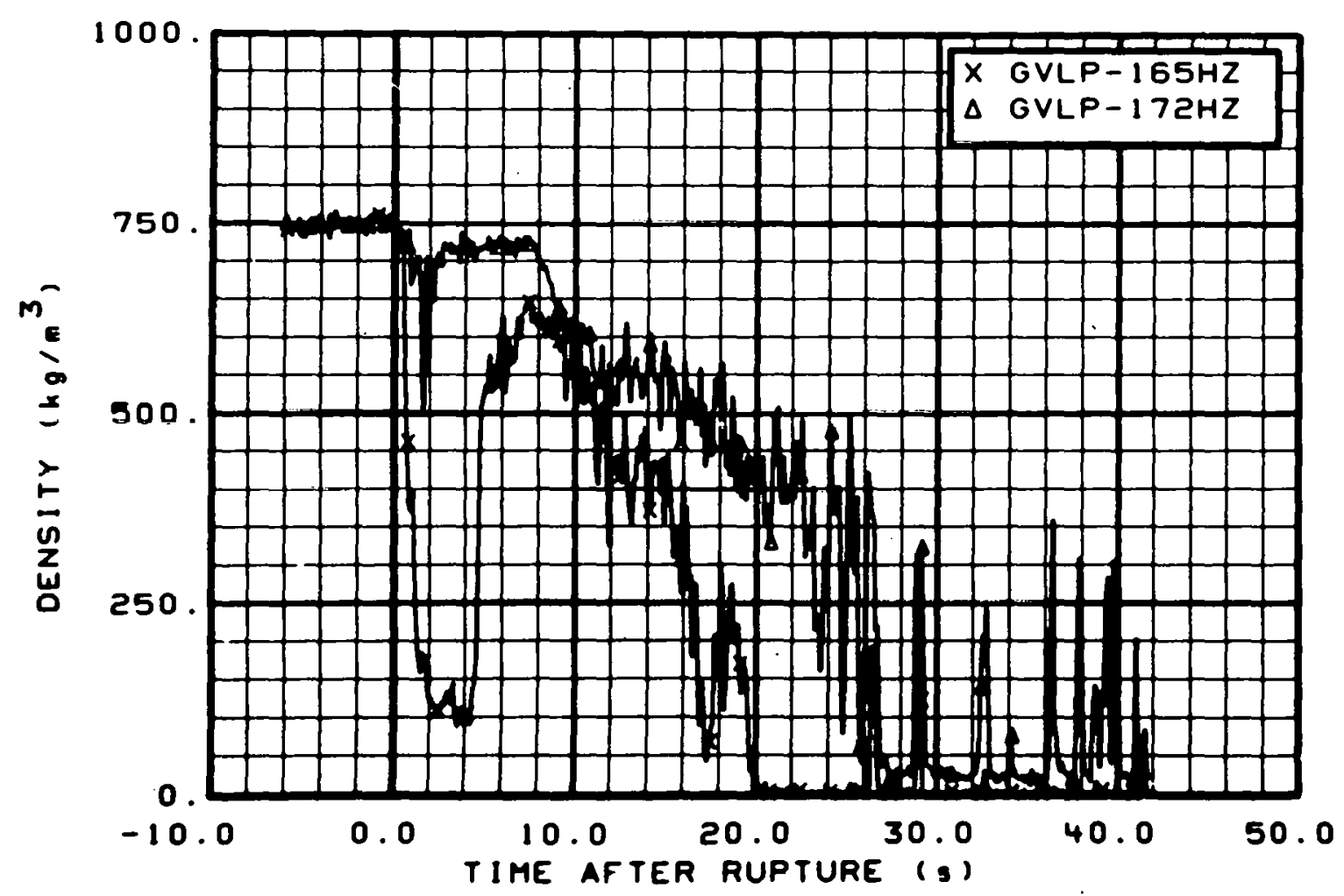

Fig. 276 Density in vessel (GVLP-165HZ and GVLP-172HZ), from -6 to $42 \mathrm{~s}$. 


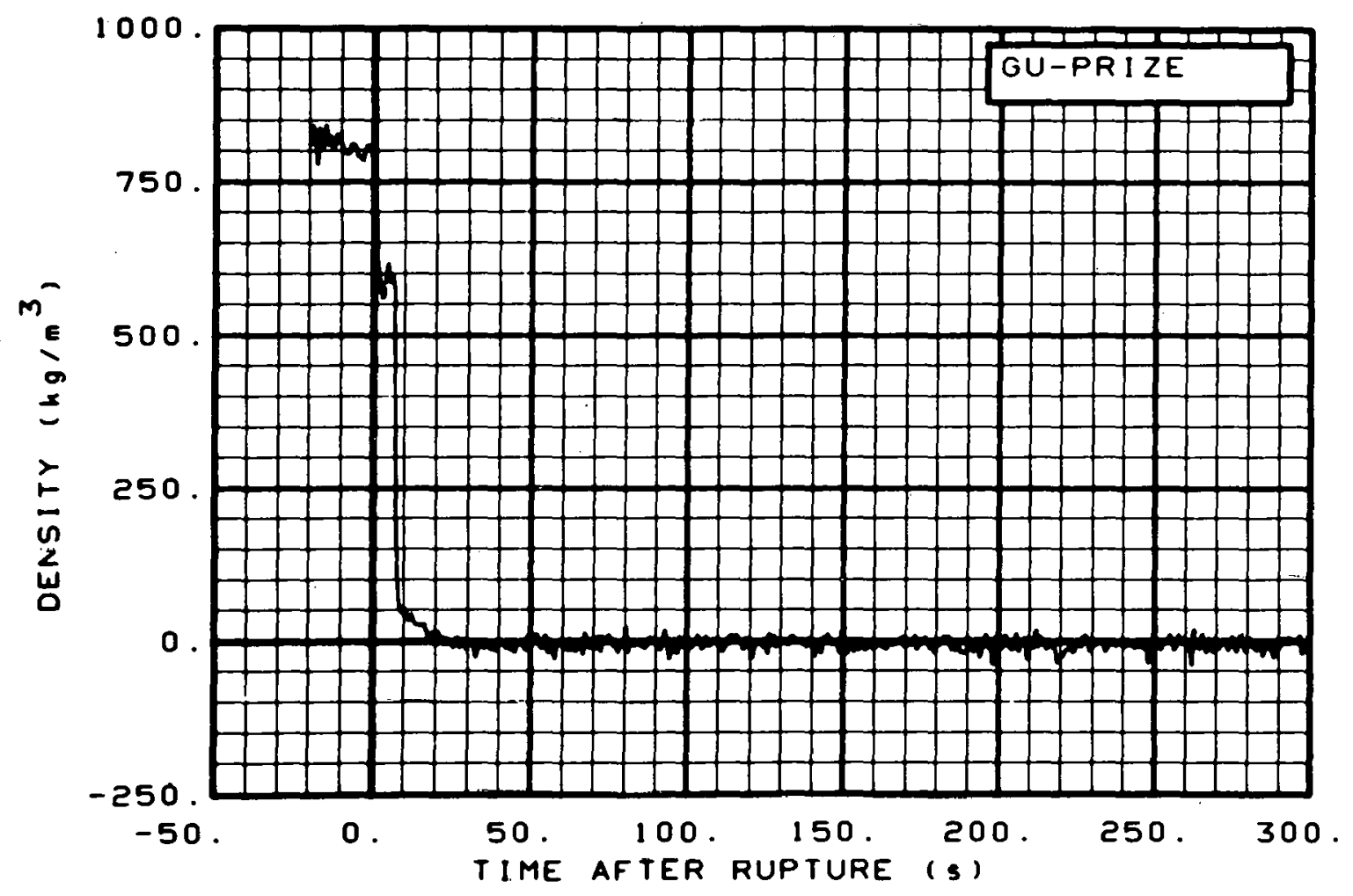

Fig. 277 Density in pressurizer (GU-PRIZE), from -20 to $300 \mathrm{~s}$.

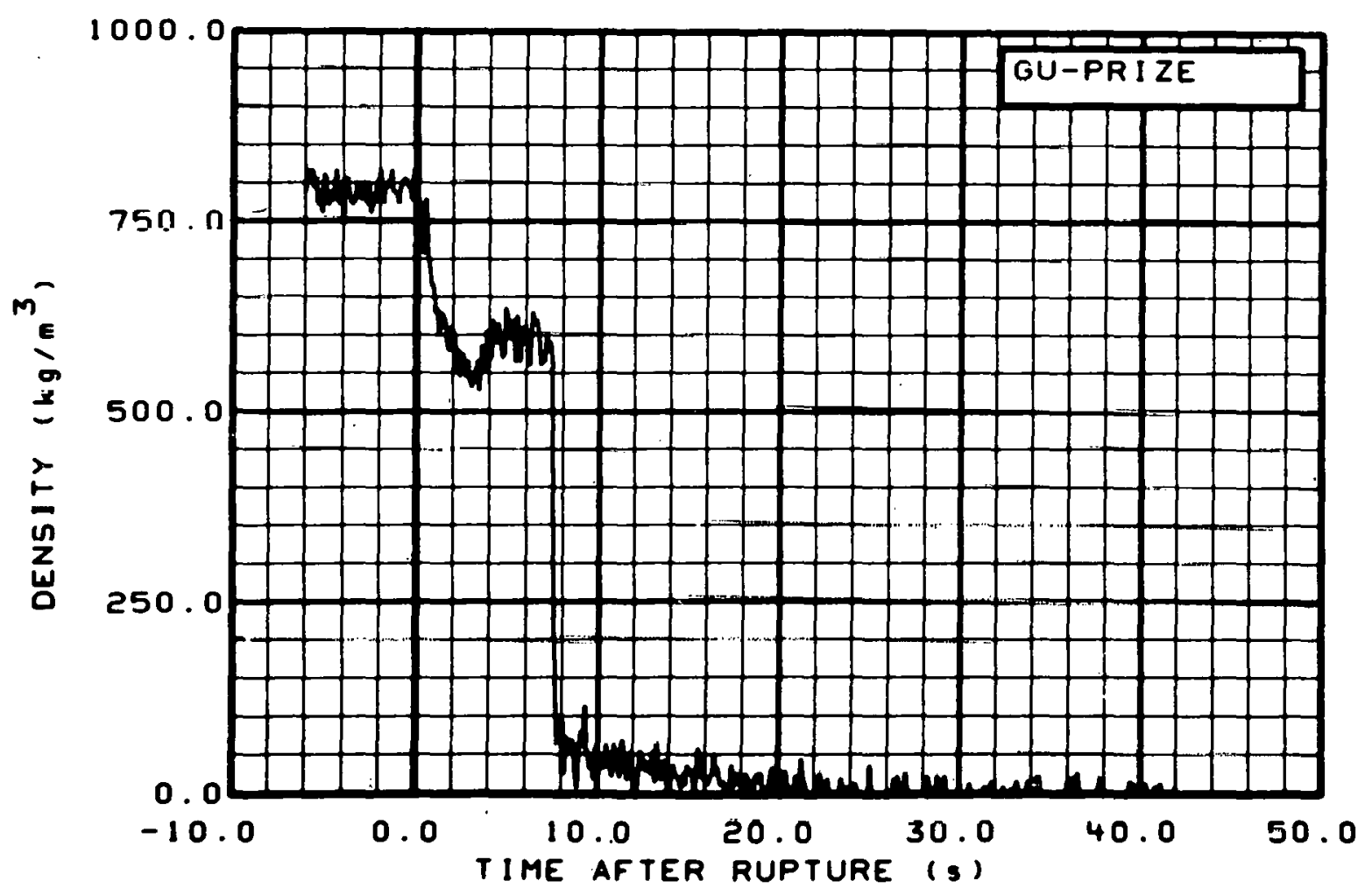

Fig. 278 Density in pressurizer (GU-PRIZE), from -6 to $42 \mathrm{~s}$. 


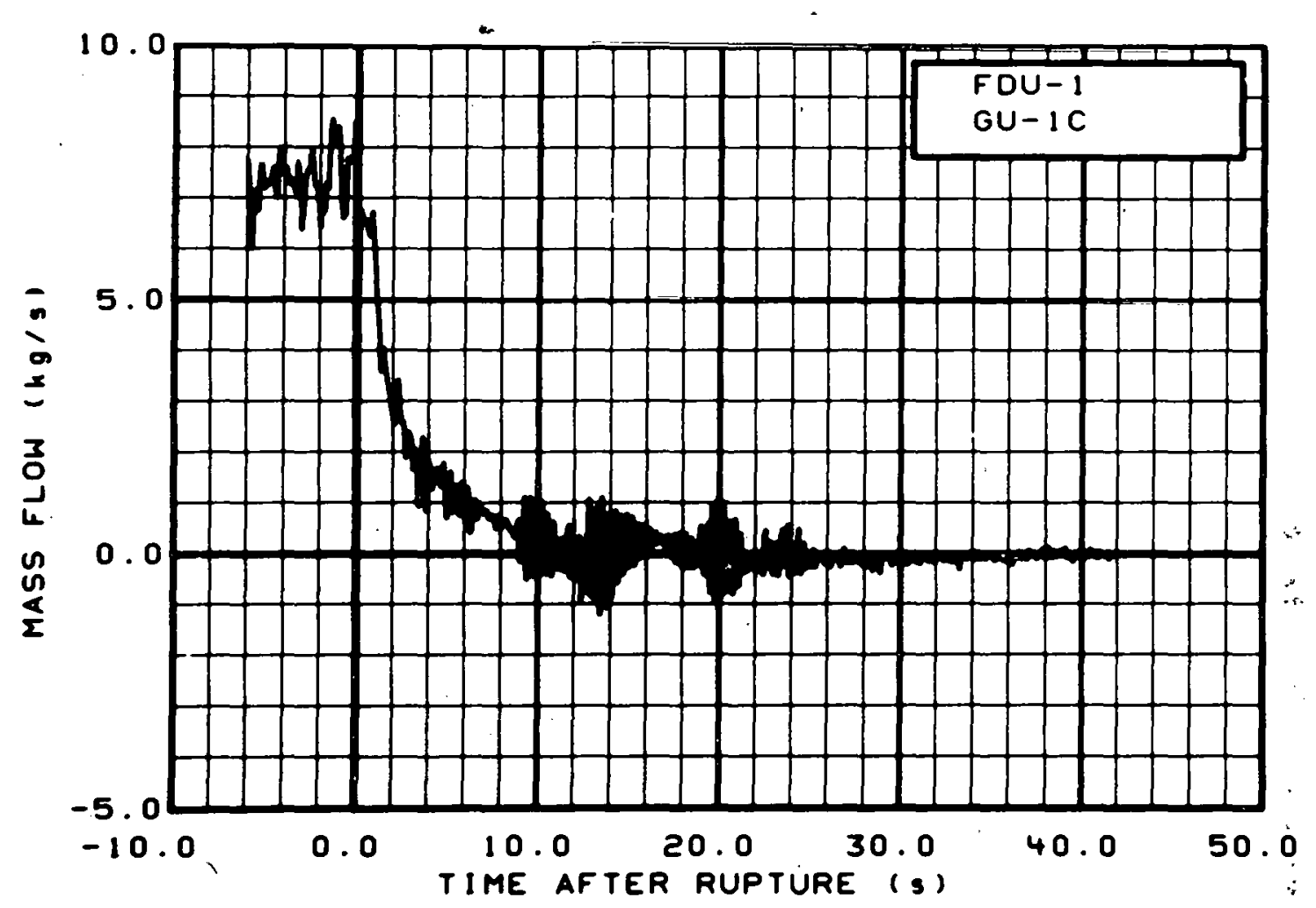

Fig. 279 Mass flow in intact loop (FDU-1, GU-1C), from -6 to $42 \mathrm{~s}$. 


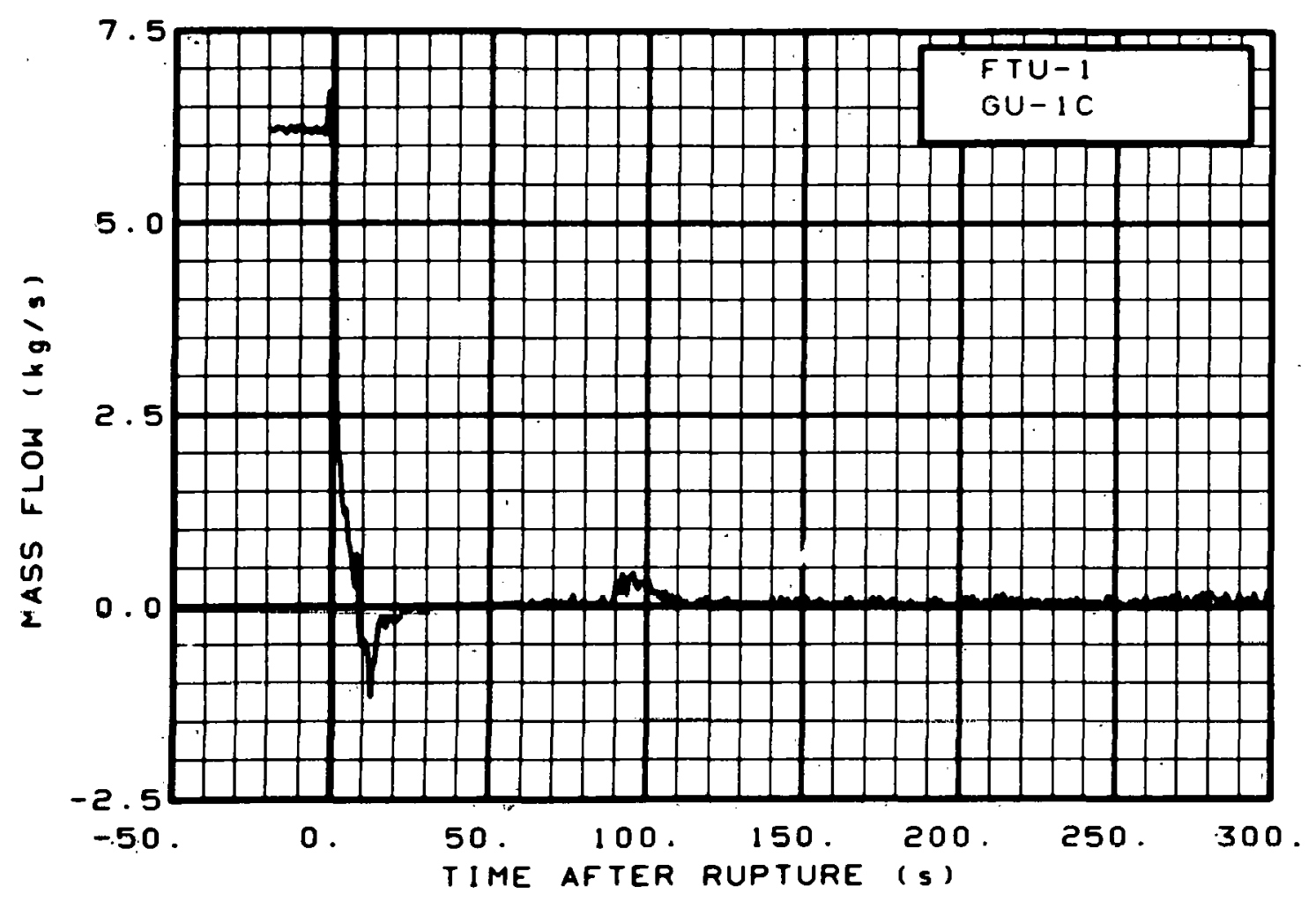

Fig. 280. Mass flow in intact loop (FTU-1, GU-1C), from -20 to $300 \mathrm{~s}$.

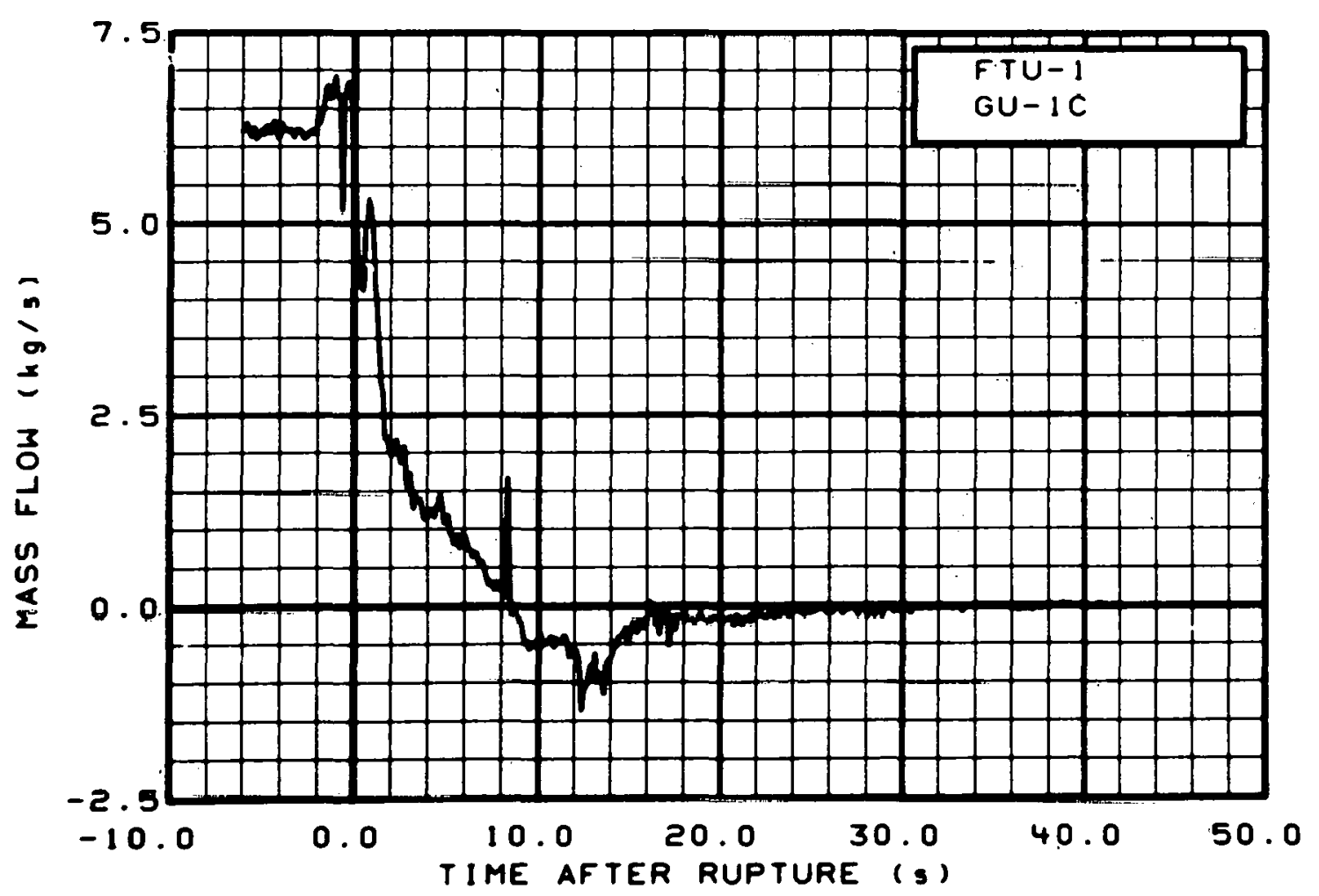

Fig. 281 Mass flow in intact loop (FTU-1, GU-1C), from -6 to $42 \mathrm{~s}$. 


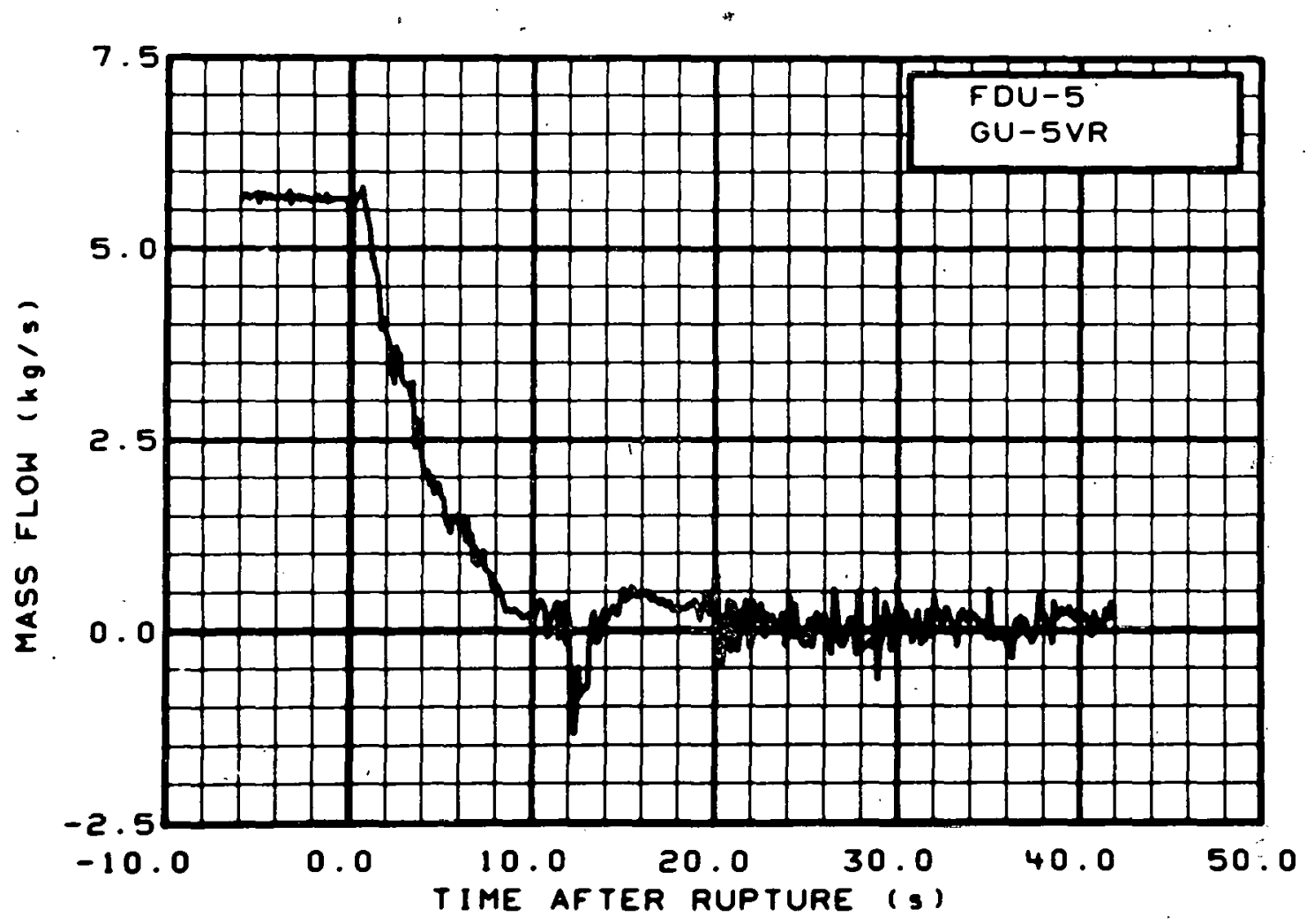

Fig. 282 Mass flow in intact loop (FDU-5, GU-5VR), from -6 to $42 \mathrm{~s}$. 


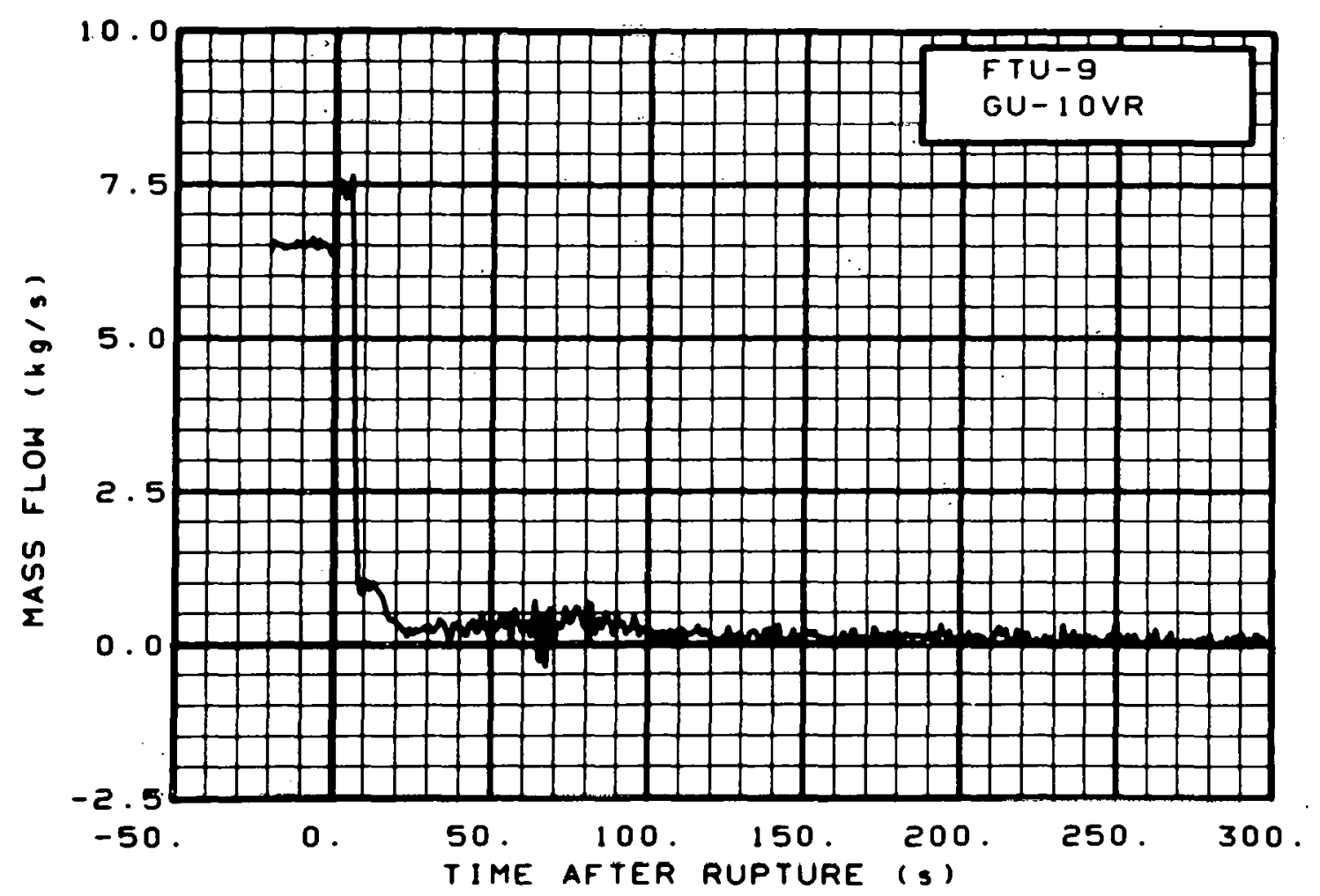

Fig. 283 Mass flow in intact loop (FTU-9, GU-10VR), from -20 to $300 \mathrm{~s}$.

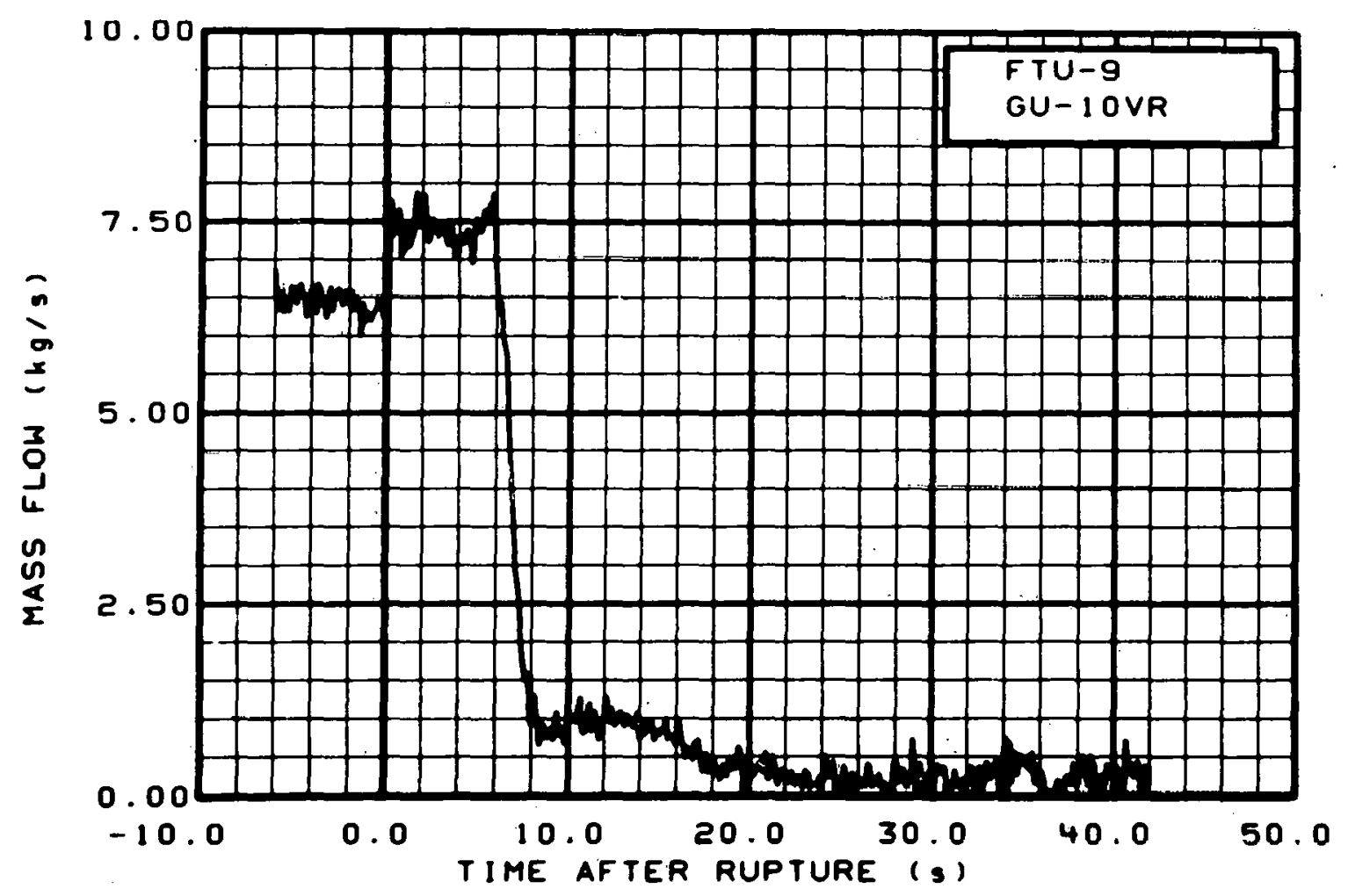

Fig. 284 Mass flow in intact loop (FTU-9, GU-10VR), from -6 to $42 \mathrm{~s}$. 


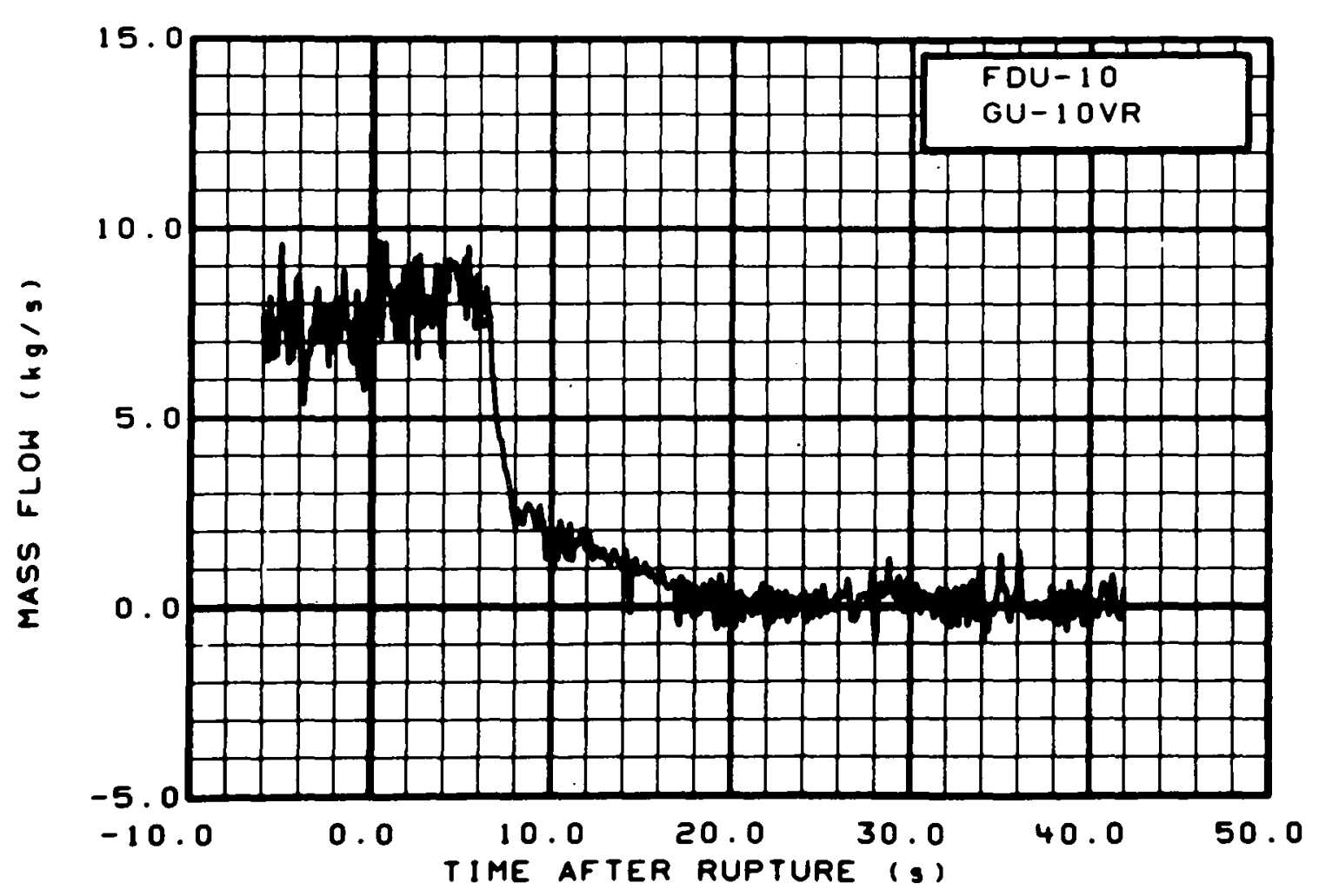

Fig. 285 Mass flow in intact loop (FDU-10, GU-10VR), from -6 to $42 \mathrm{~s}$.

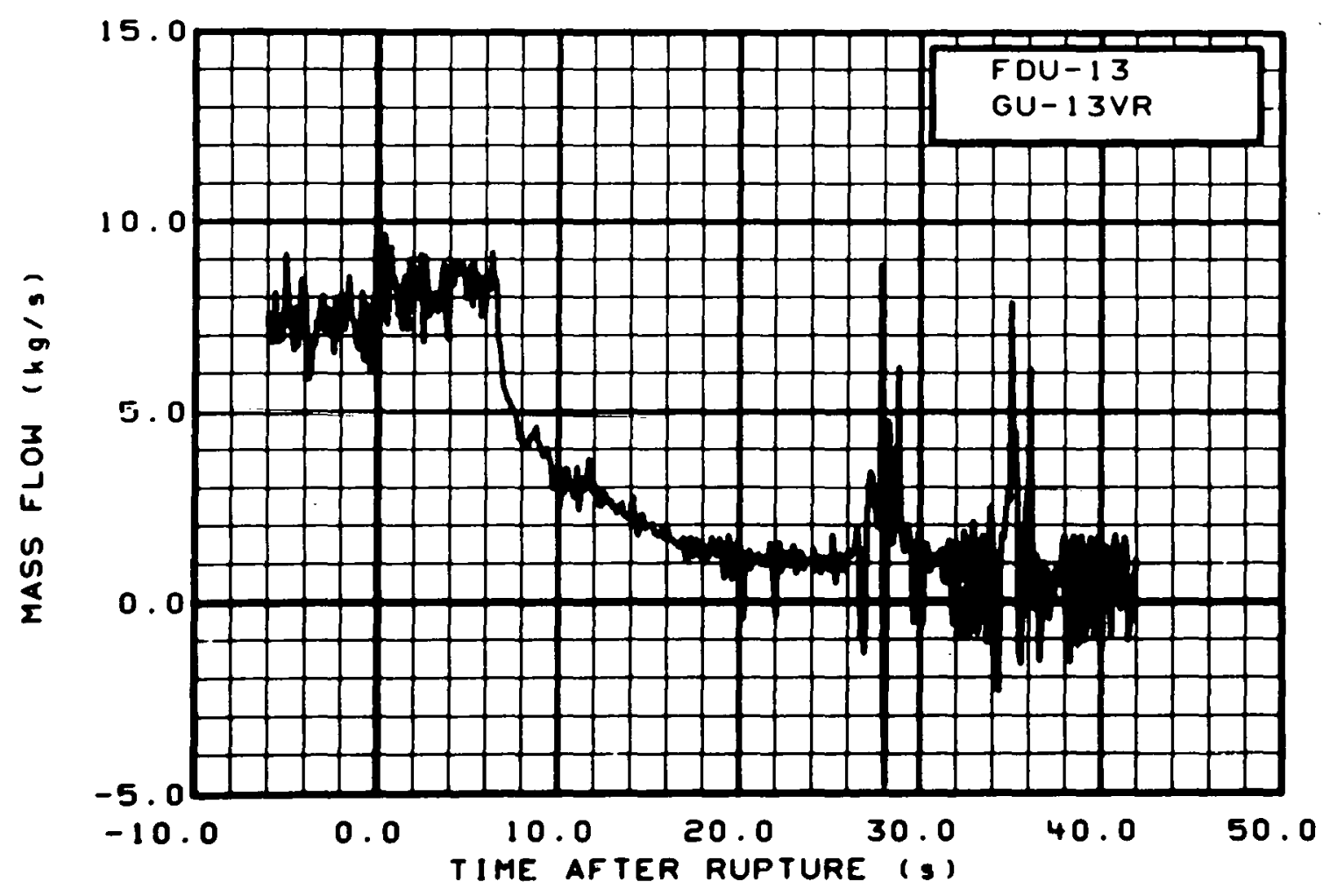

Fig. 286 Mass flow in intact loop (FDU-13, GU-13VR), from -6 to $42 \mathrm{~s}$. 


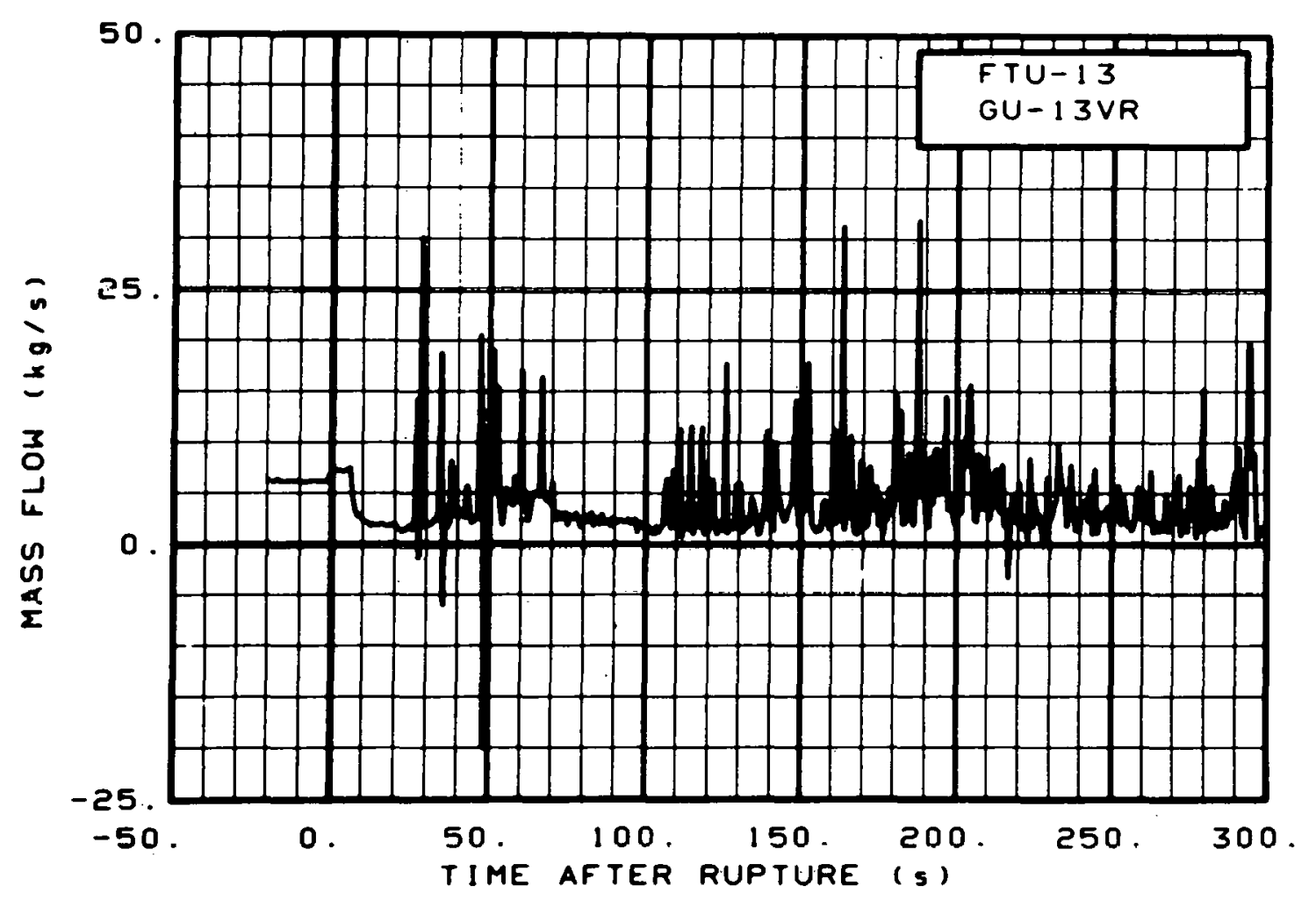

Fig. 287 Mass flow in intact loop (FTU-13, GU-13VR), from -20 to $300 \mathrm{~s}$.

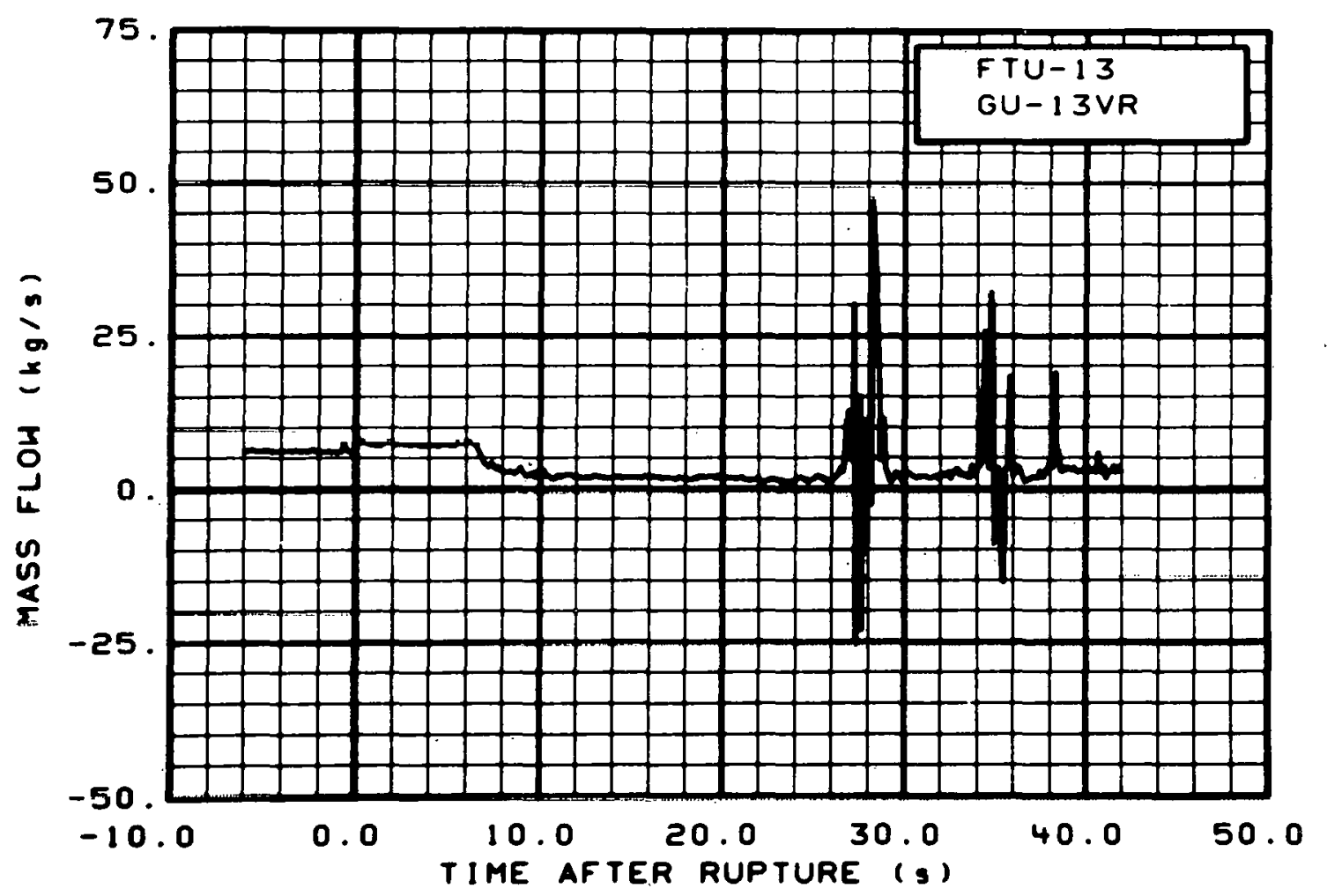

Fig. 288 Mass flow in intact loop (FTU-13, GU-13VR), from -6 to $42 \mathrm{~s}$. 


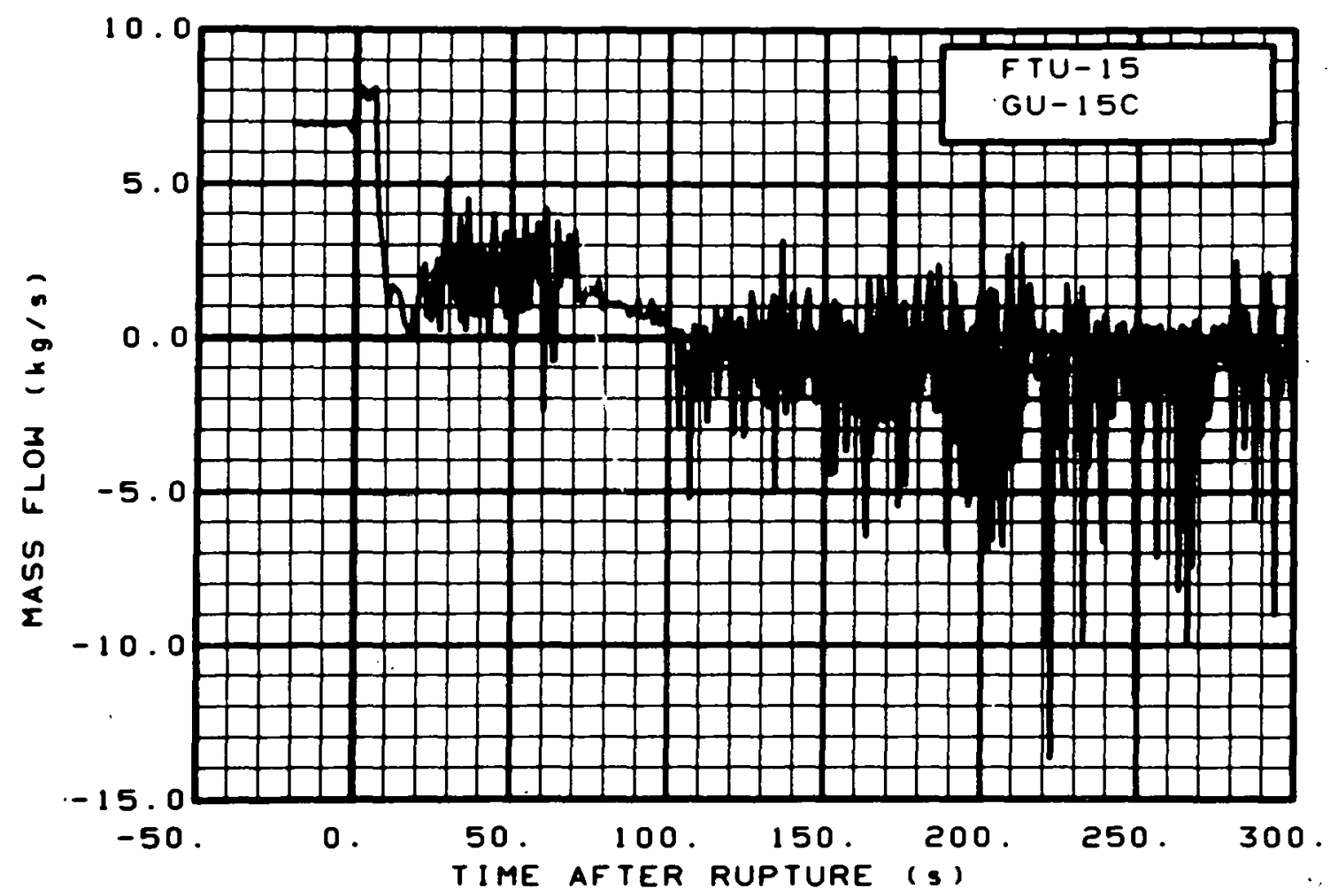

Fig. 289 Mass flow in intact 1oop (FTU-15, GU-15C), from -20 to $300 \mathrm{~s}$.

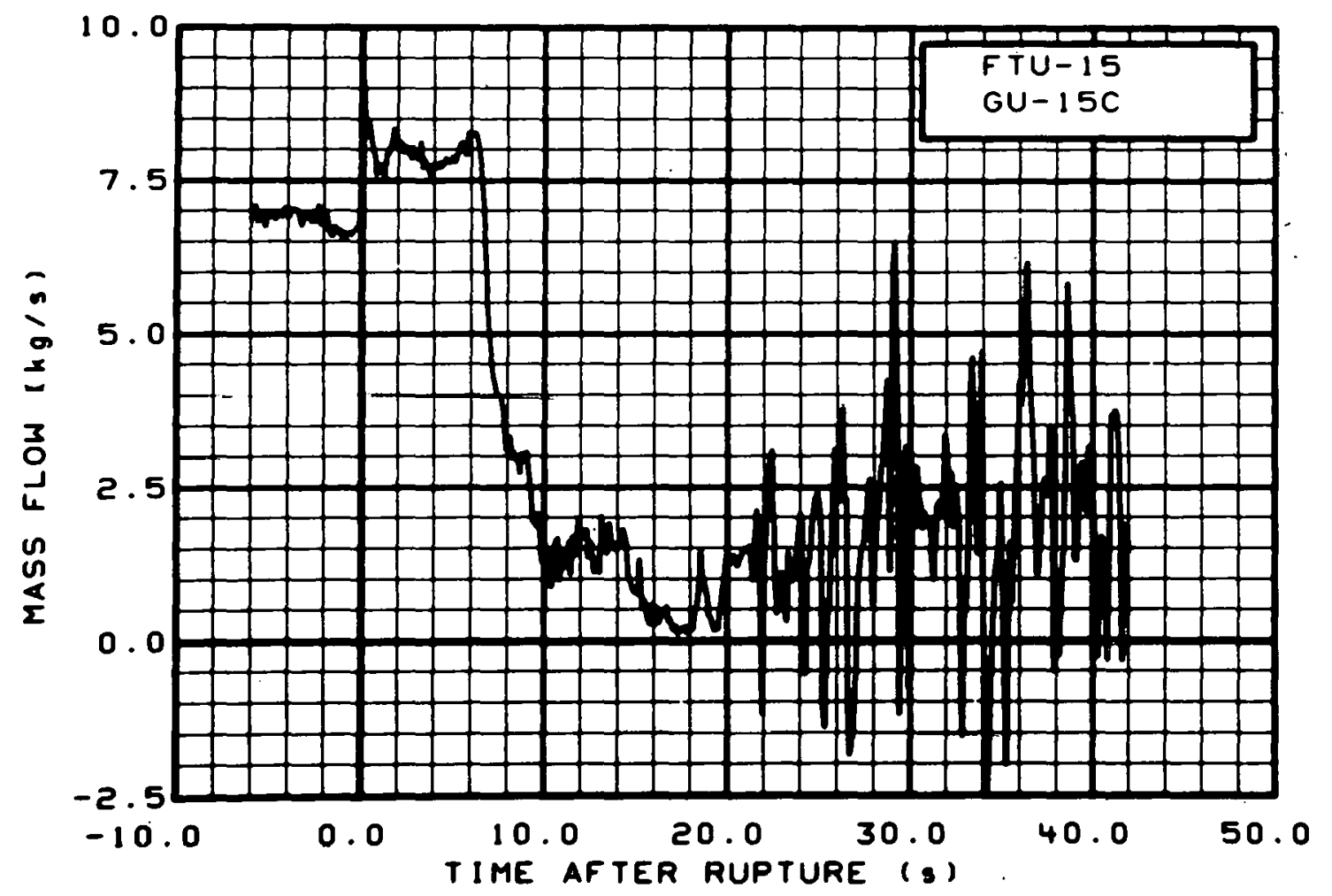

Fig. 290 Mass flow in intact loop (FTU-15, GU-15C), from -6 to $42 \mathrm{~s}$. 


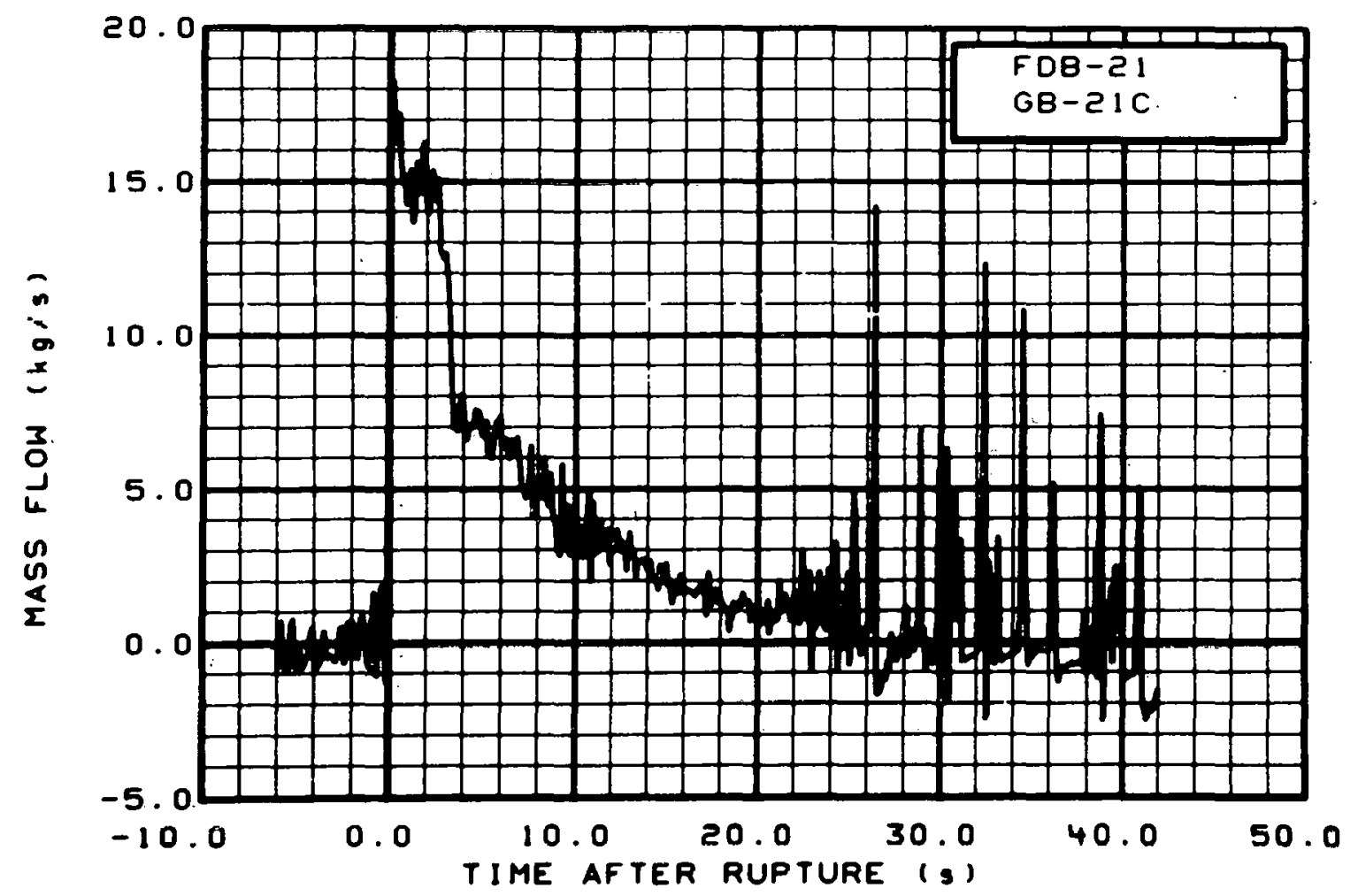

Fig. 291 Mass flow in intact loop (FDB-21, GB-21C), from -6 to $42 \mathrm{~s}$.

b. 


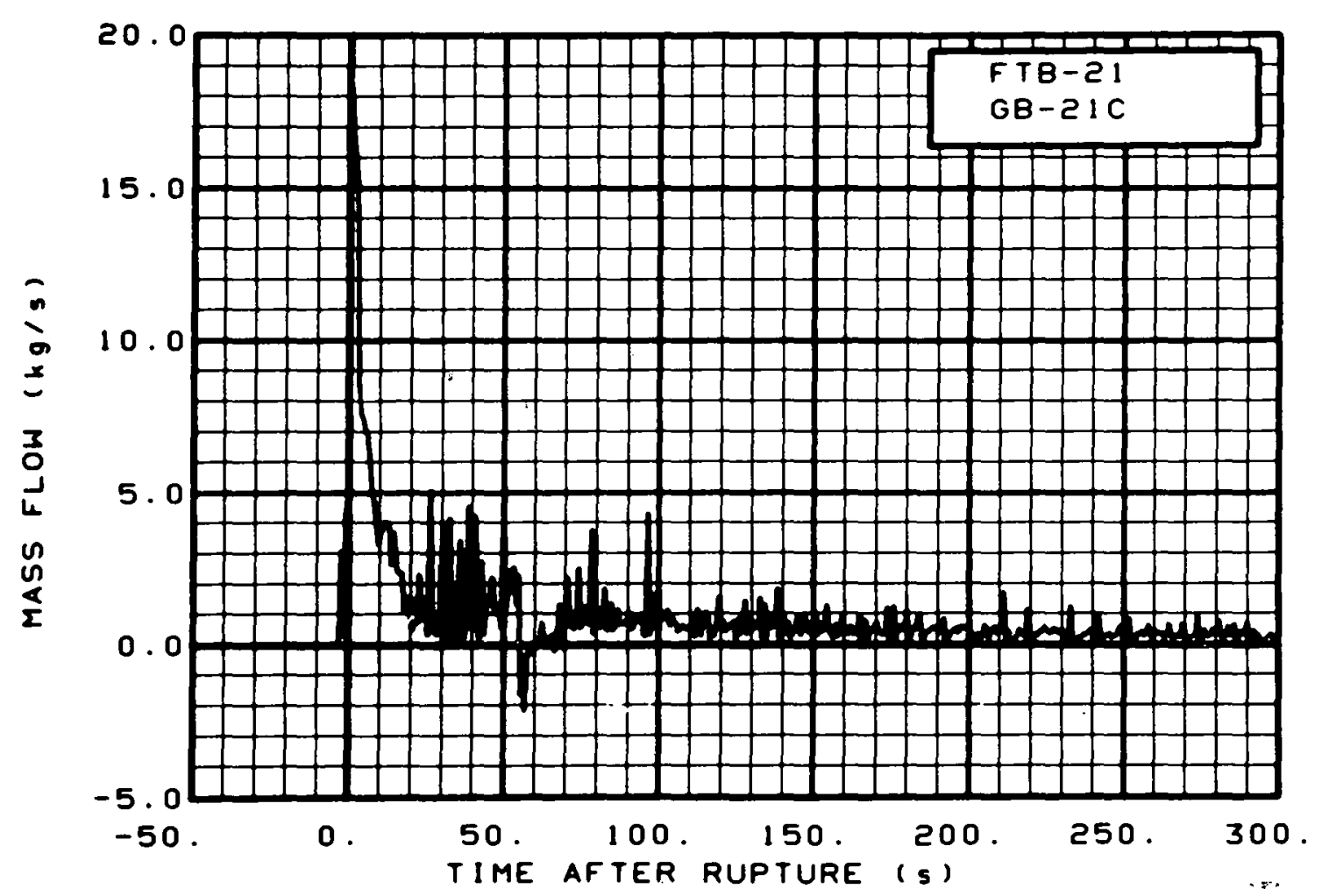

Fig. 292 Mass flow in broken loop (FTB-21, GB-21C), from -20 to $300 \mathrm{~s}$.

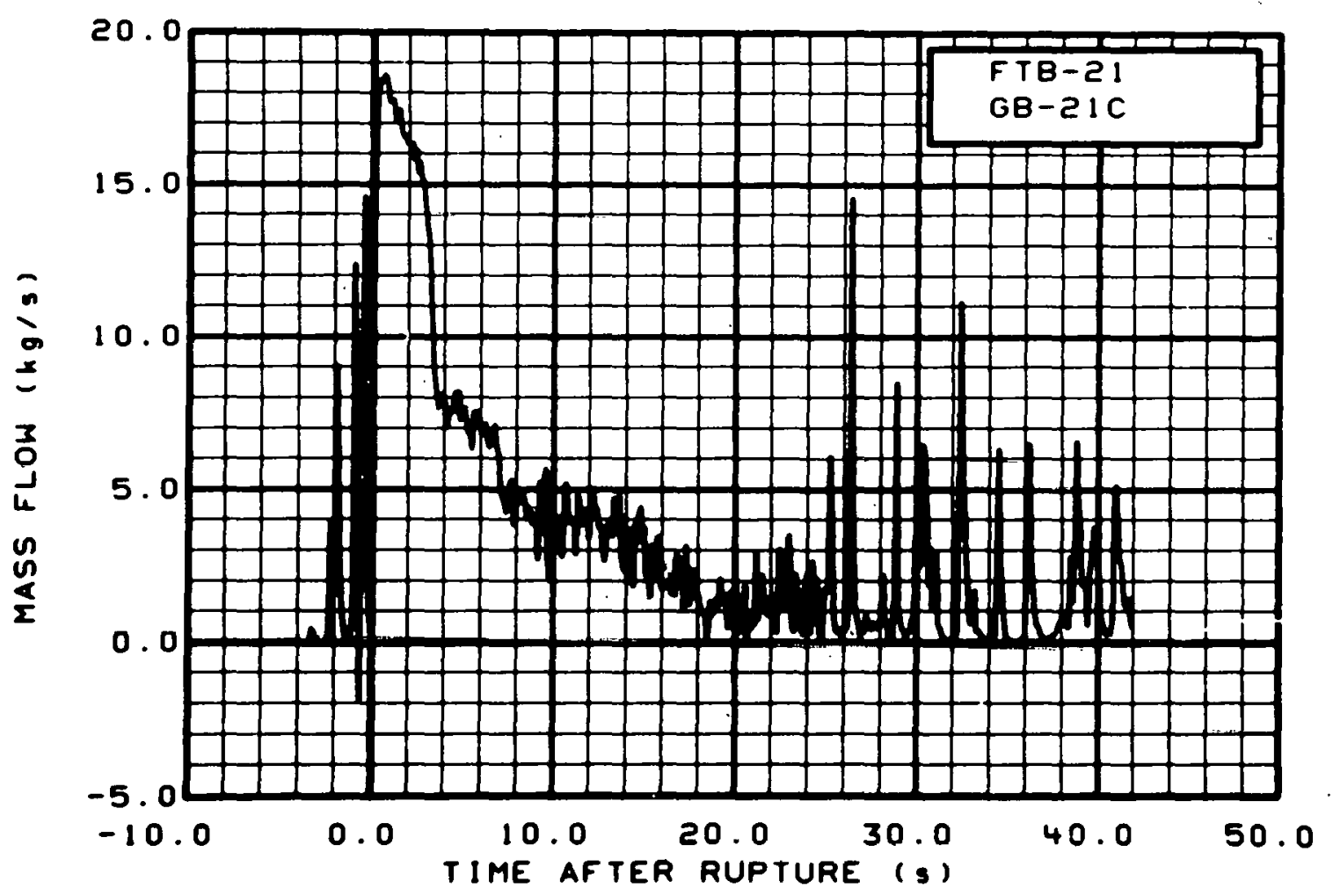

Fig. 293 Mass flow in broken loop (FTB-21, GB-21C), from -6 to $42 \mathrm{~s}$. 


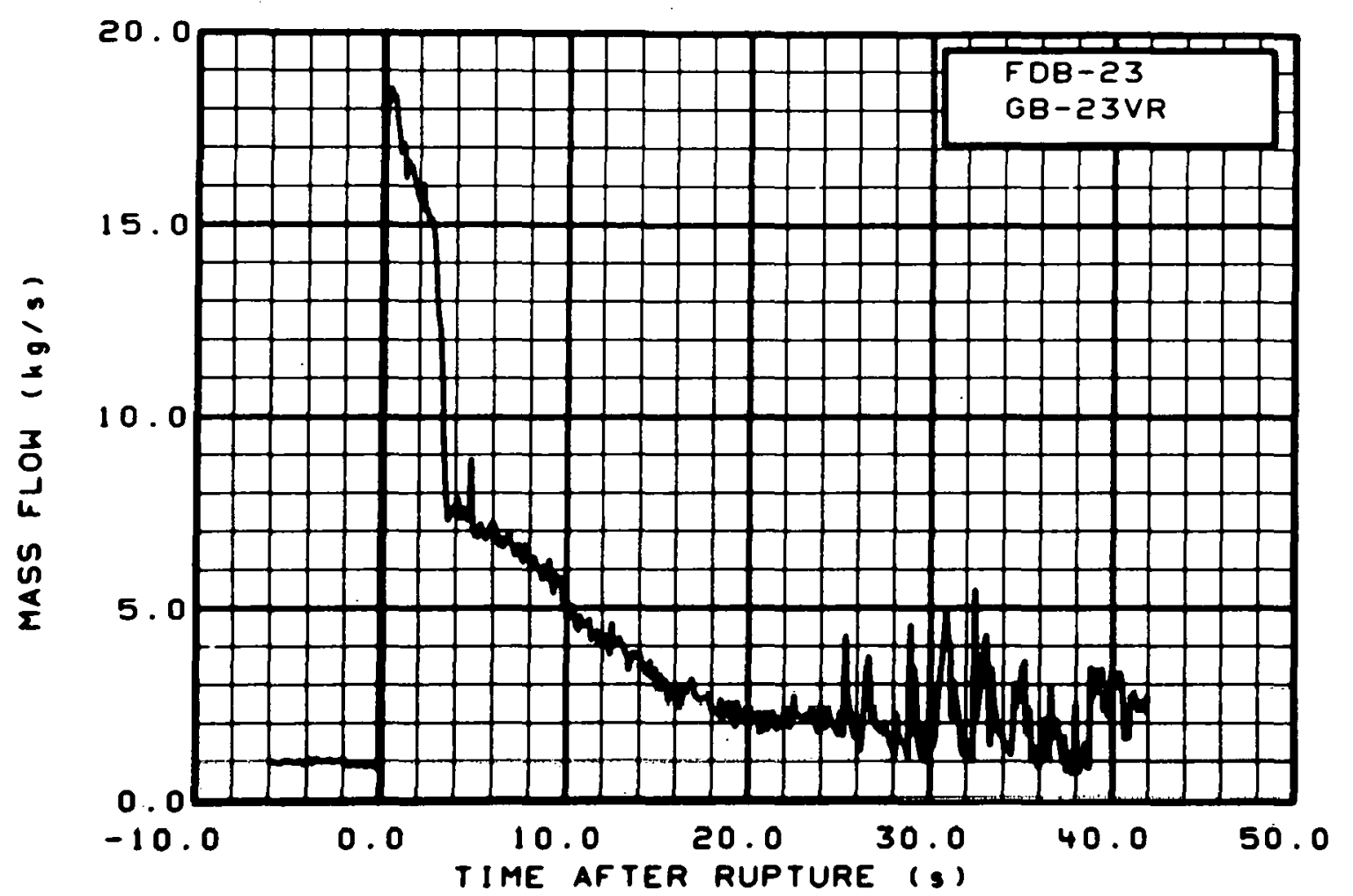

Fig. 294 Mass flow in broken loop (FDB-23, GB-23VR), from -6 to $42 \mathrm{~s}$.

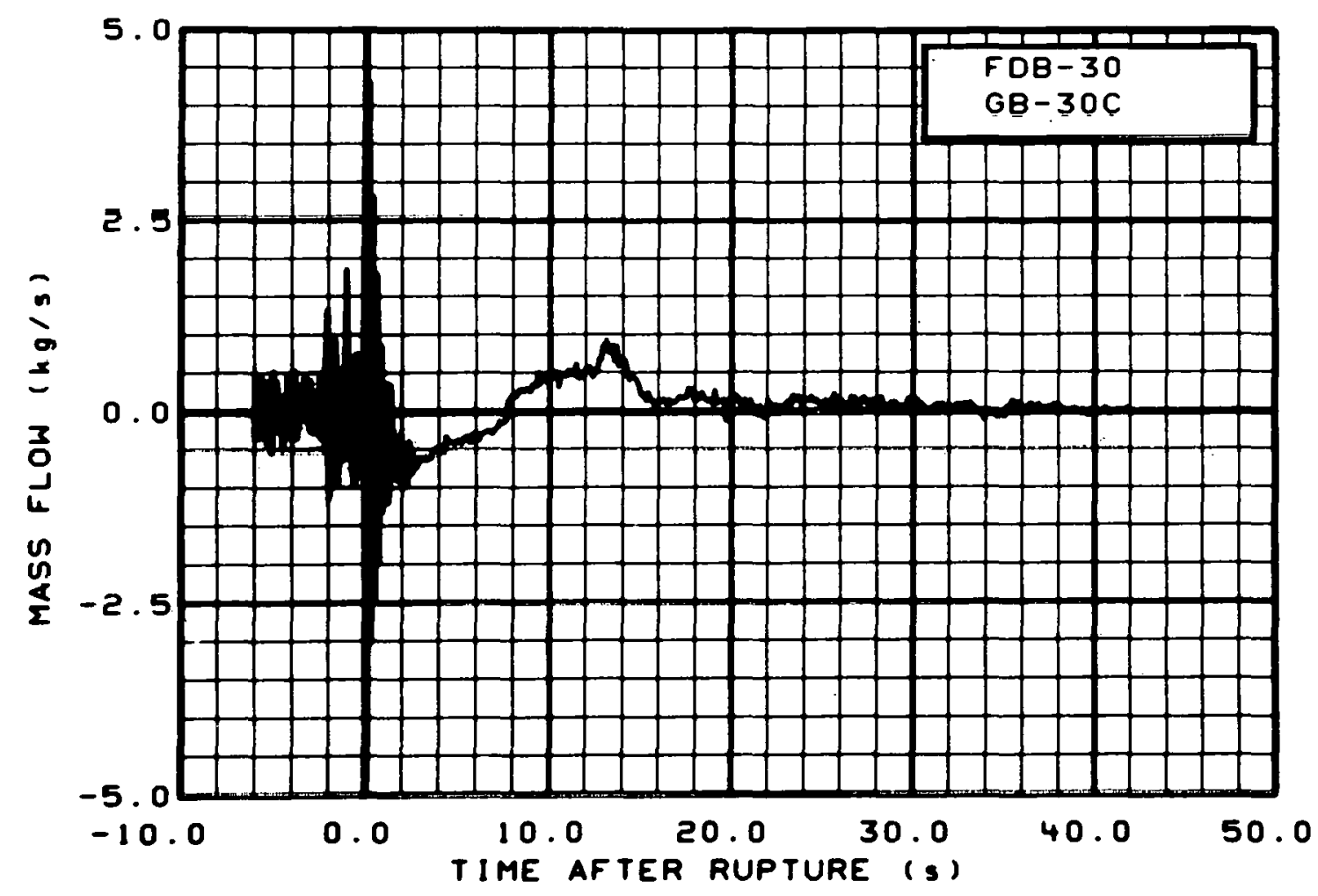

Fig. 295 Mass flow in broken loop (FDB-30, GB-30C), from -6 to $42 \mathrm{~s}$. 


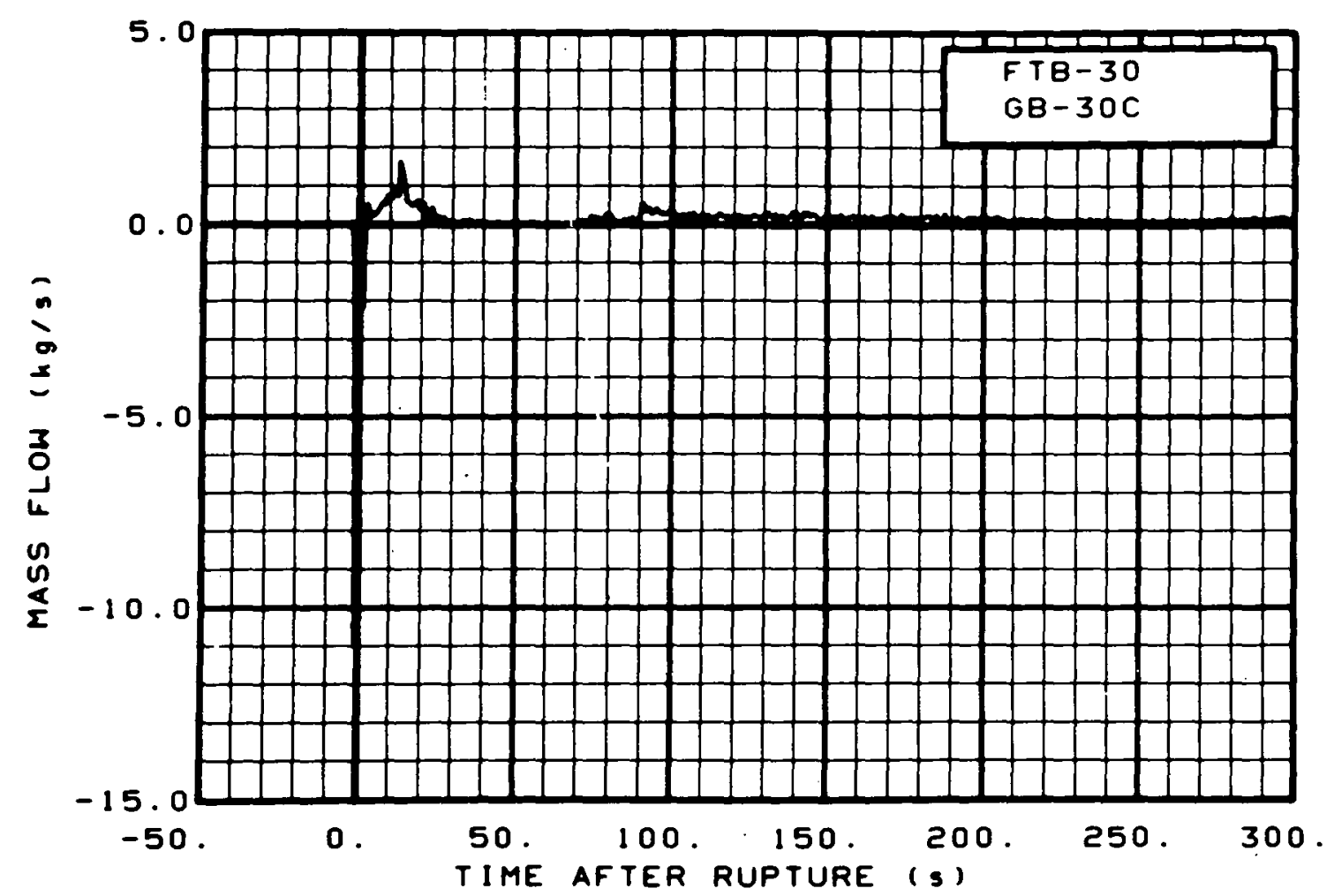

Fig. 296 Mass flow in broken loop (FTB-30, GB-30C), from -20 to $300 \mathrm{~s}$.

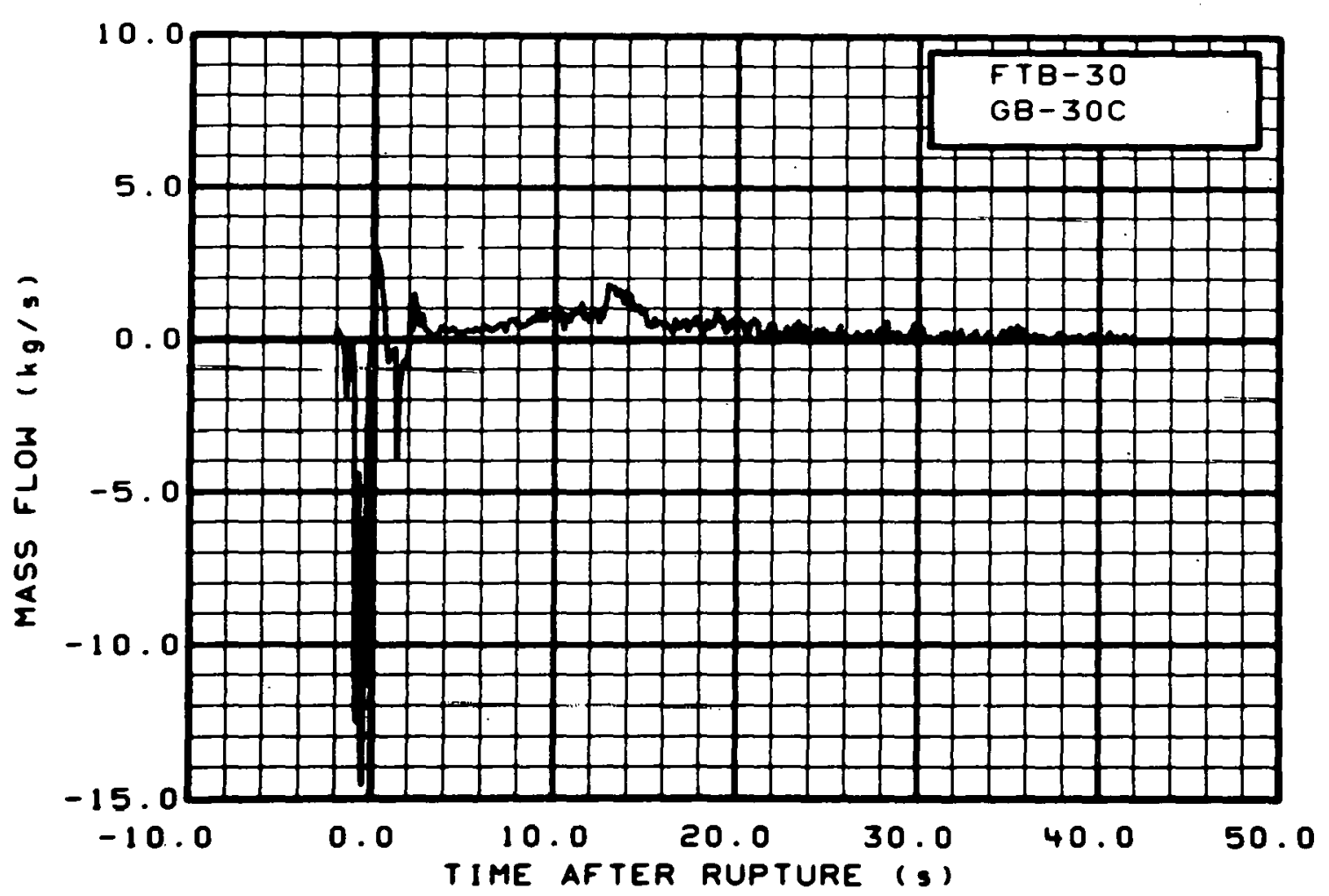

Fig. 297 Mass flow in broken loop (FTB-30, GB-30C), from -6 to $42 \mathrm{~s}$. 


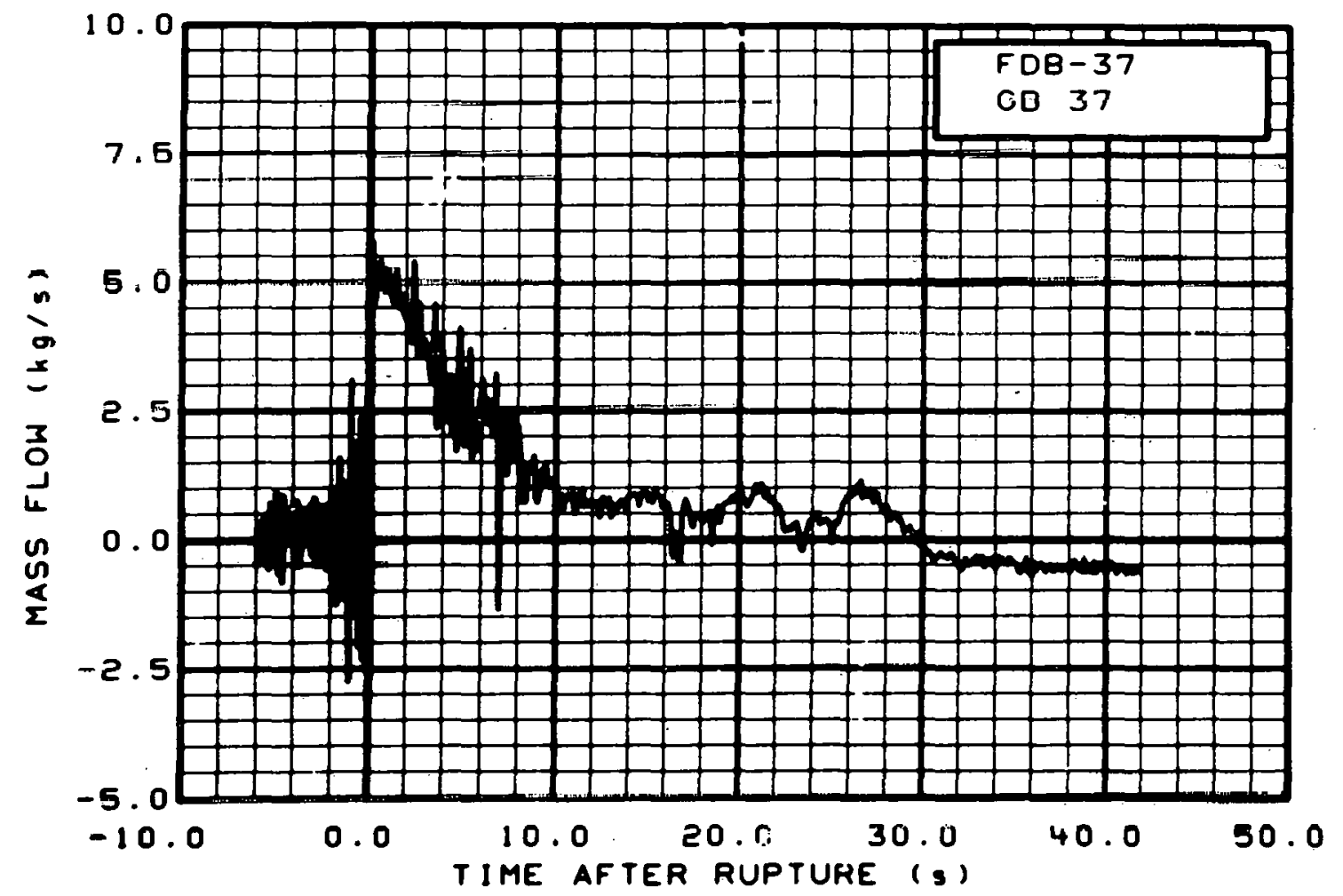

Fig. 298 Mass flow in broken loop (FDB-37, GB-37), from -6 to $42 \mathrm{~s}$. 


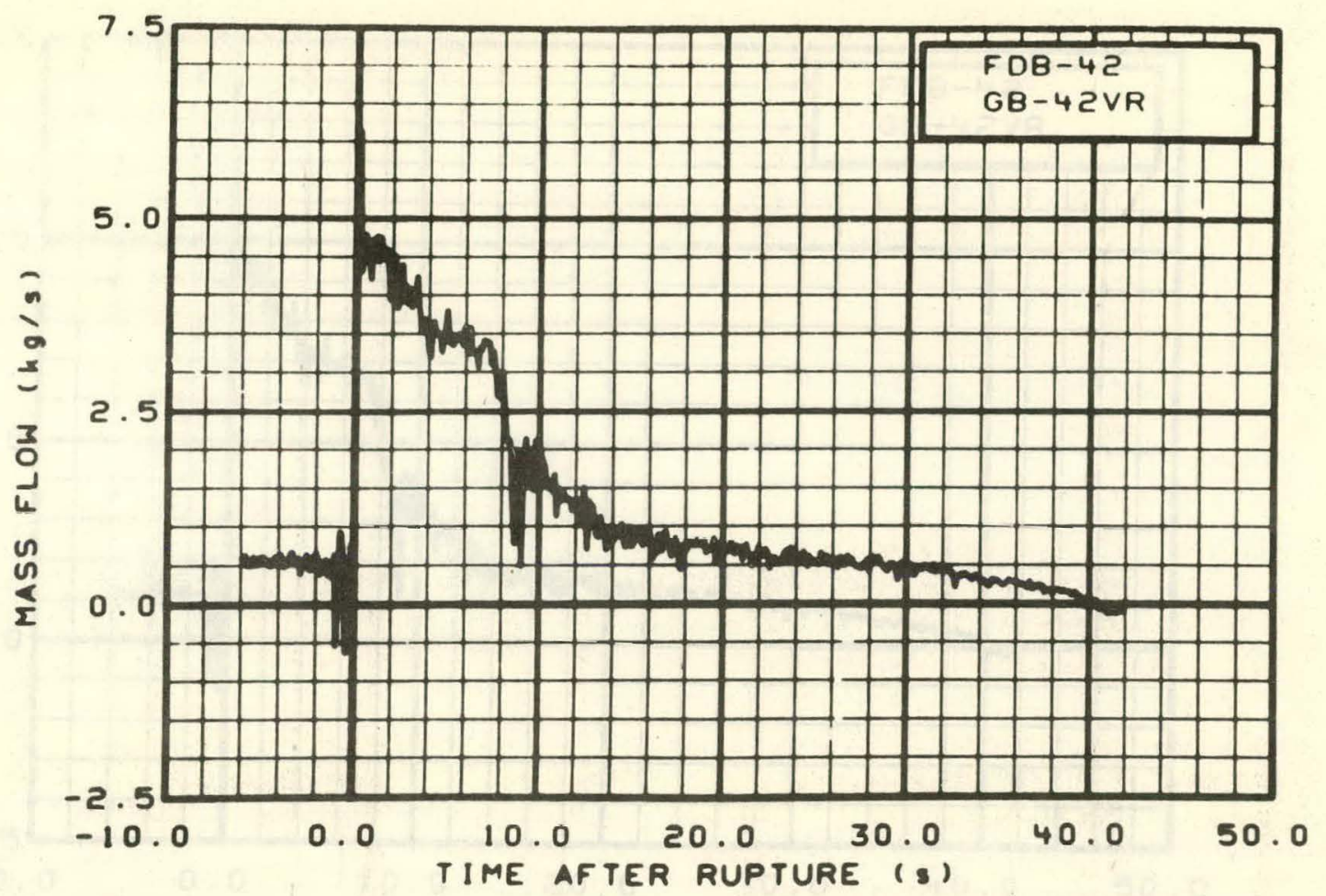

Fig. 301 Mass flow in broken loop (FDB-42, GB-42VR), from -6 to $42 \mathrm{~s}$.

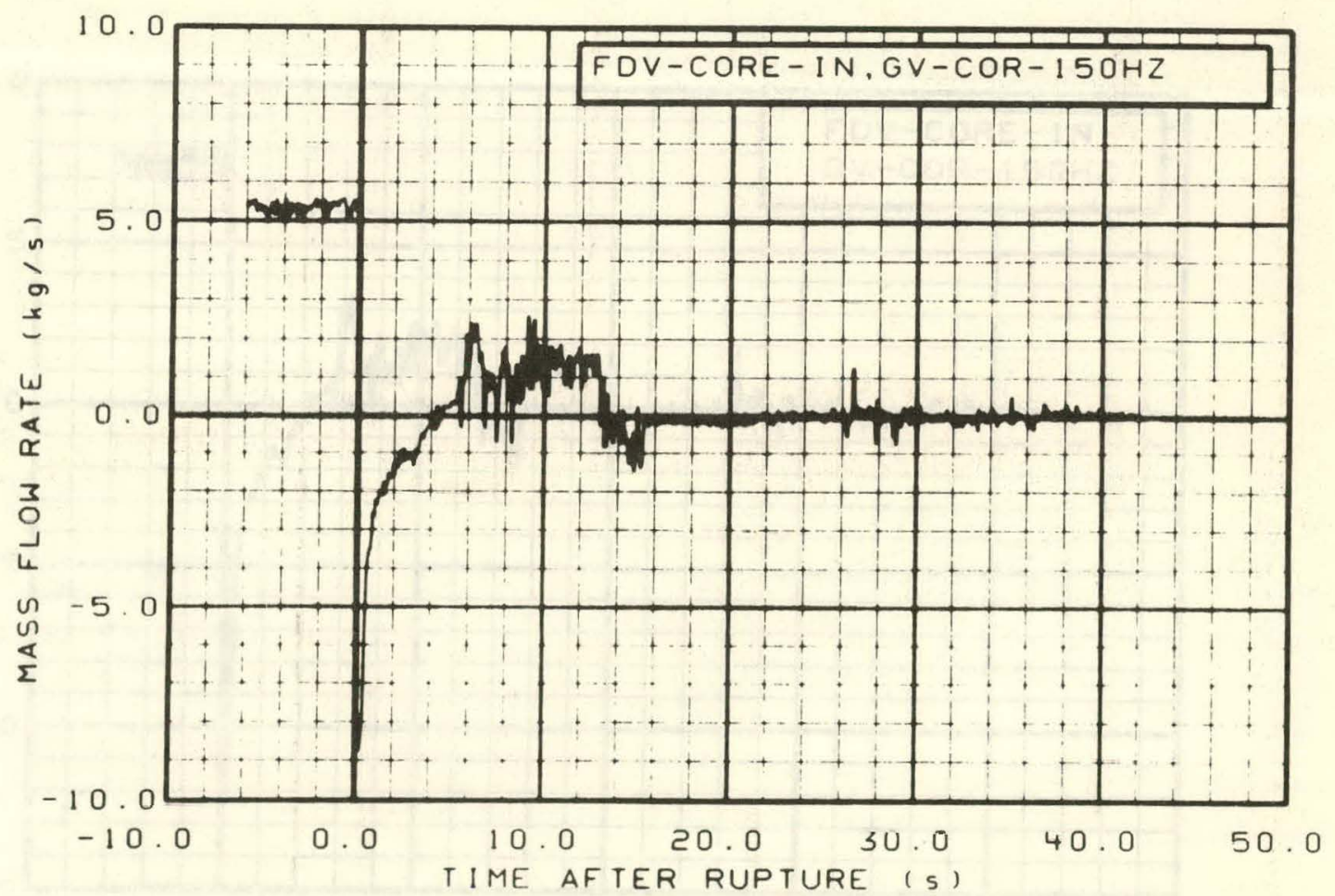

Fig. 302 Mass flow in vessel (FDV-CORE-IN, GV-COR-150HZ), from -6 to $42 \mathrm{~s}$. 


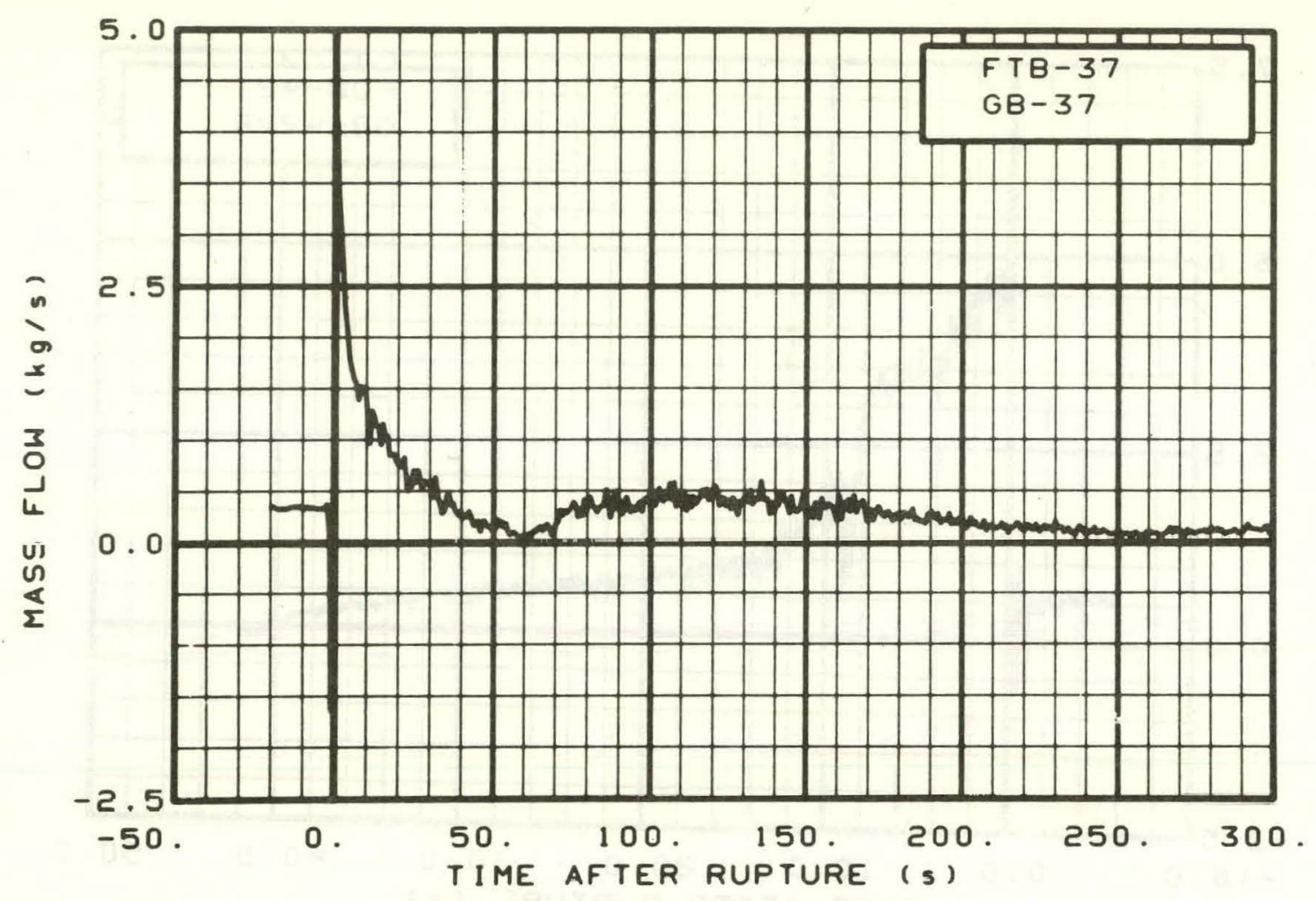

Fig. 299 Mass flow in broken loop (FTB-37, GB-37), from -20 to $300 \mathrm{~s}$.

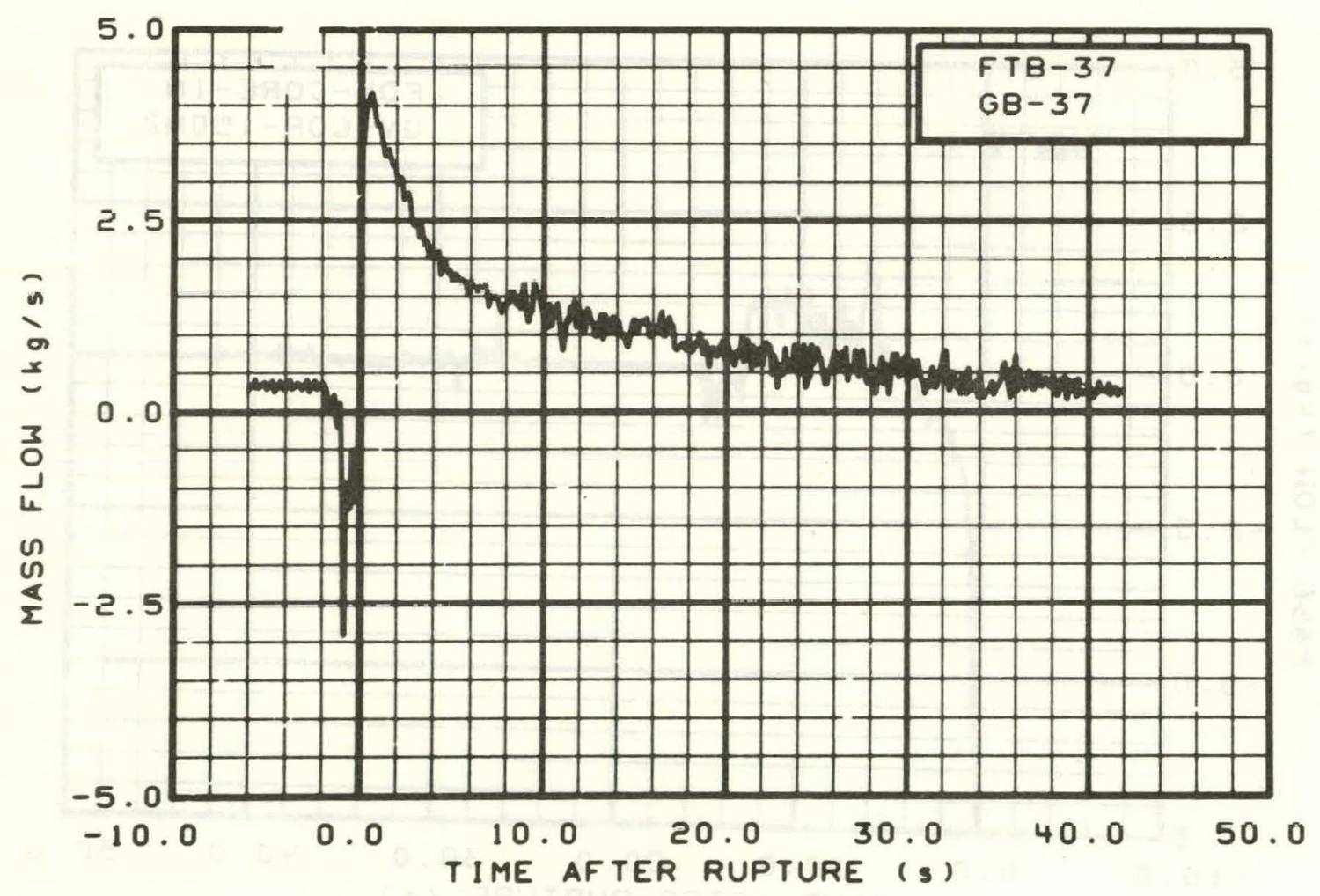

Fig. 300 Mass flow in broken loop (FTB-37, GB-37), from -6 to $42 \mathrm{~s}$. 


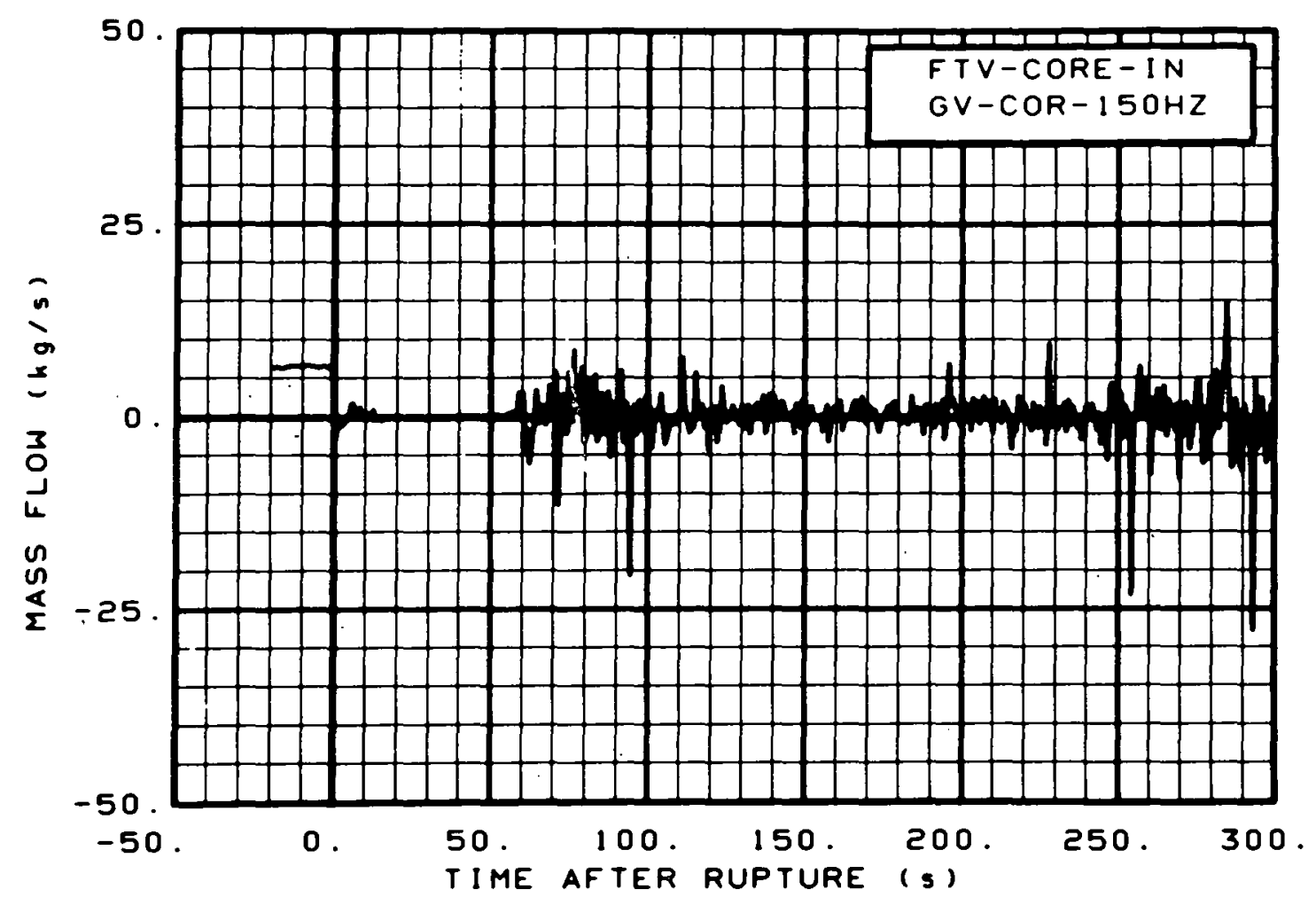

Fig. 303 Mass flow in vessel (FTV-CORE-IN, GV-COR-150HZ), from -20 to $300 \mathrm{~s}$.

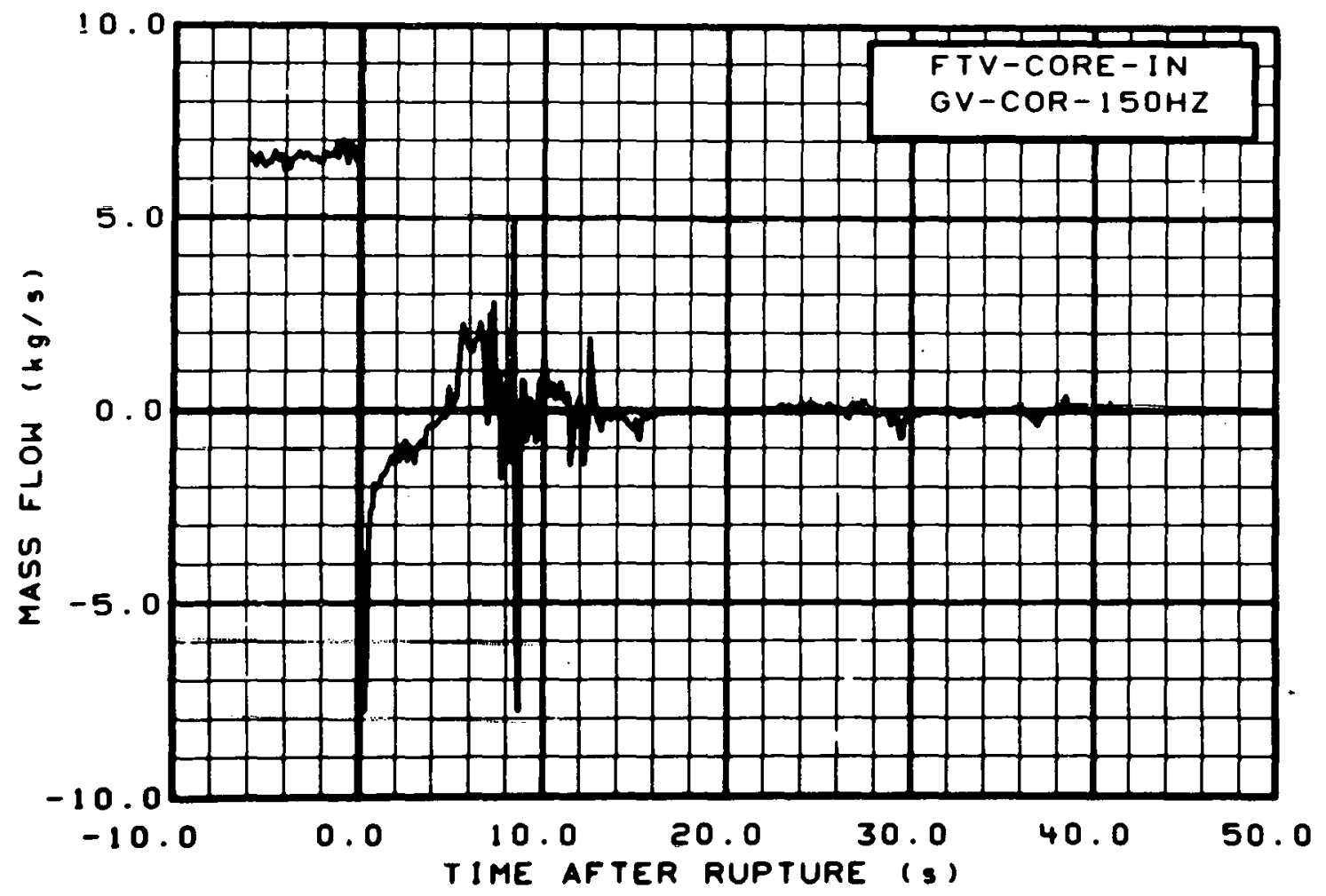

Fig. 304 Mass flow in vessel (FTV-CORE-IN, GV-COR-150HZ), from -6 to $42 \mathrm{~s}$. 


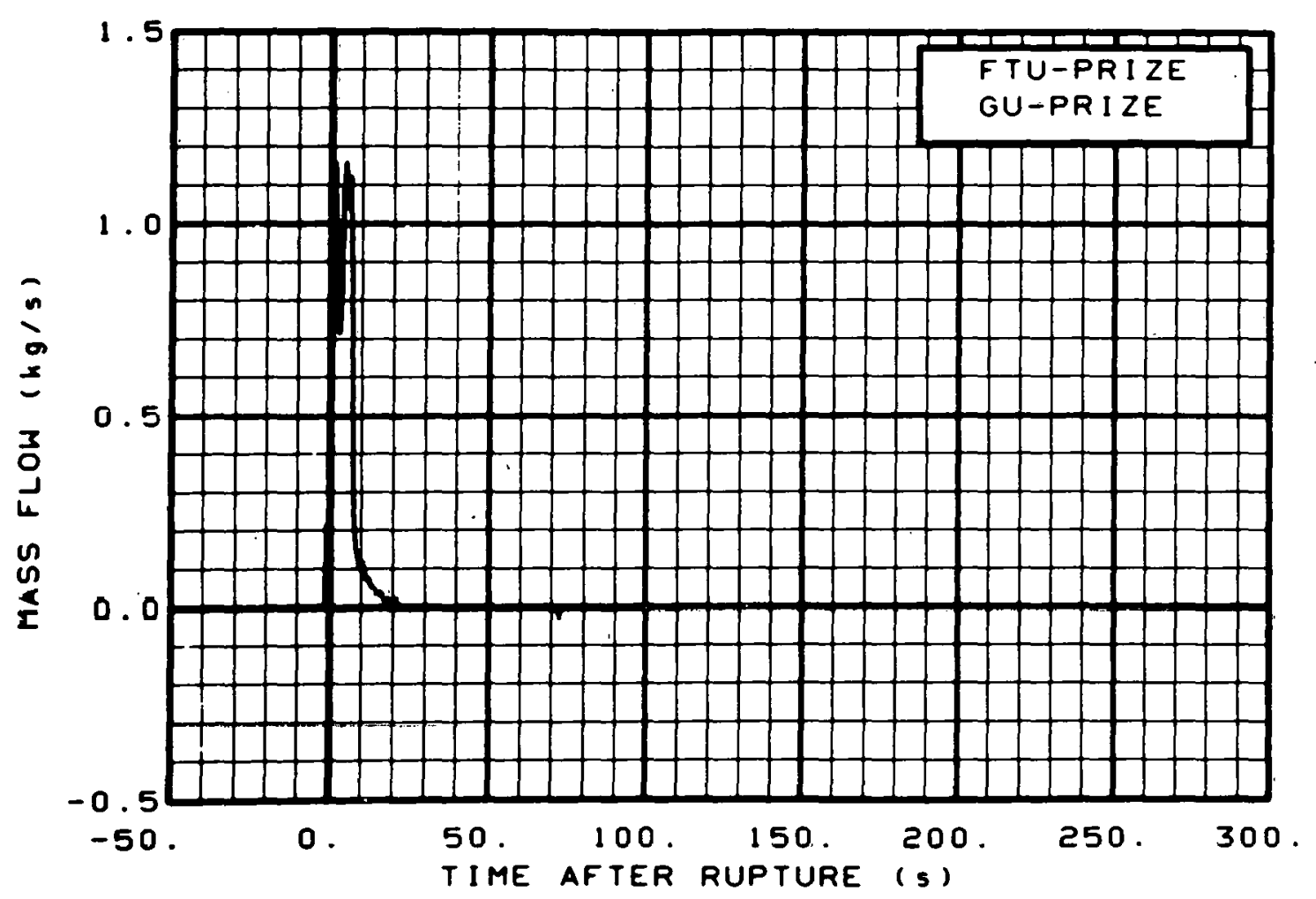

Fig. 305 Mass flow in pressurizer (FTU-PRIZE, GU-PRIZE), from -20 to $300 \mathrm{~s}$.

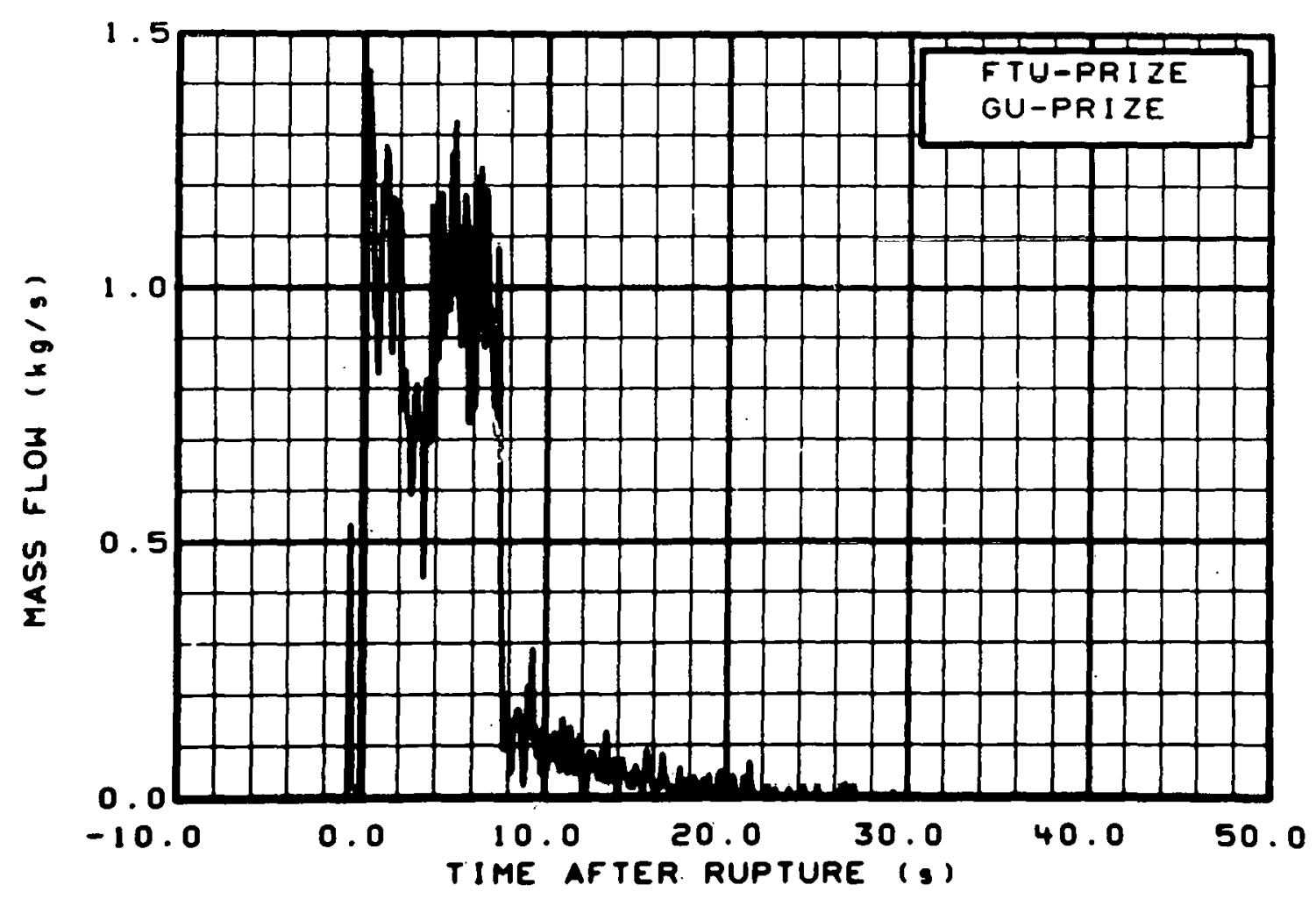

Fig. 306 Mass flow in pressurizer (FTU-PRIZE, GU-PRIZE), from -6 to $42 \mathrm{~s}$. 


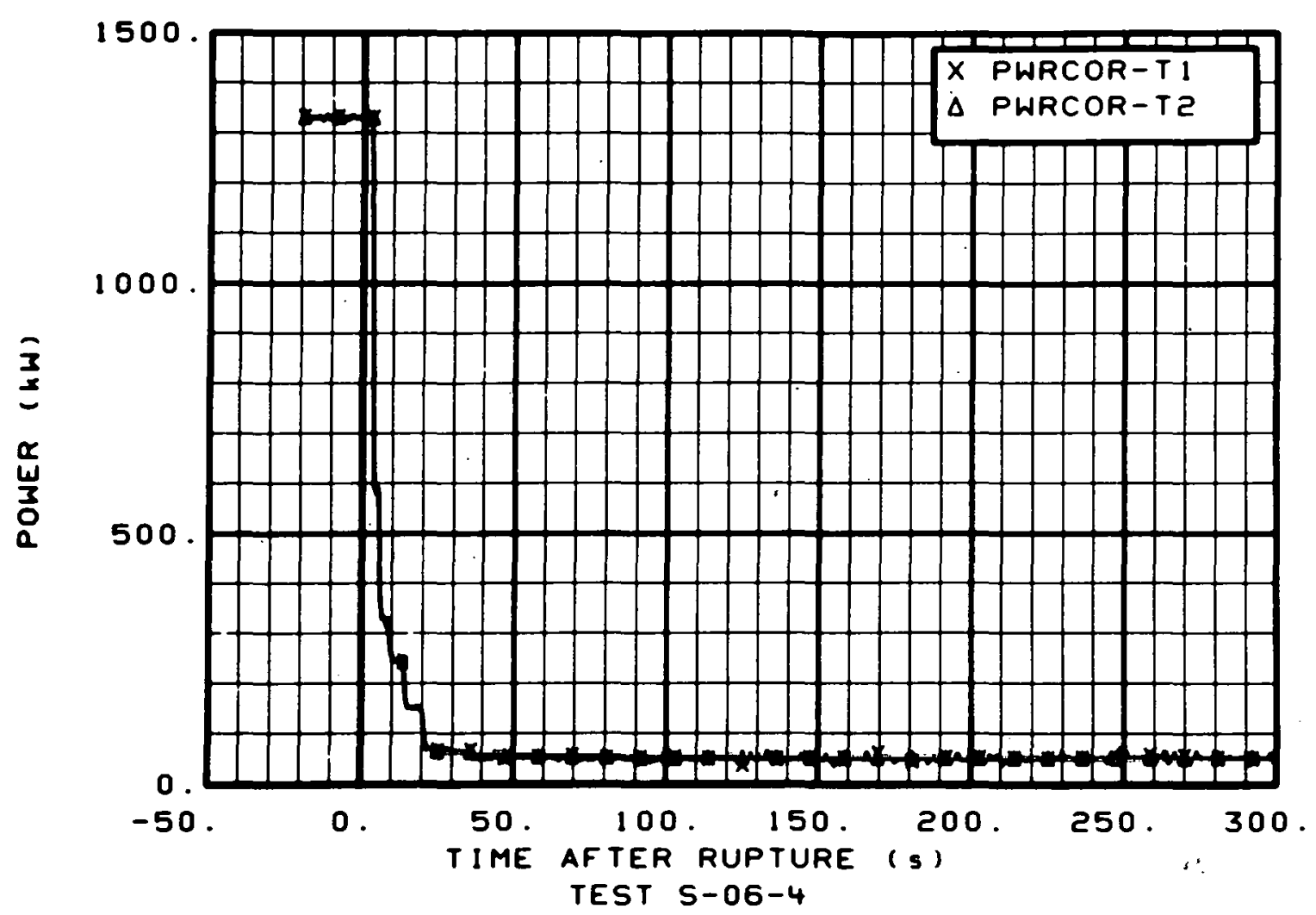

Fig. 307 Core heater rod total power (PWRCOR T-1 and PWRCOR T-2), from -20 to $300 \mathrm{~s}$.

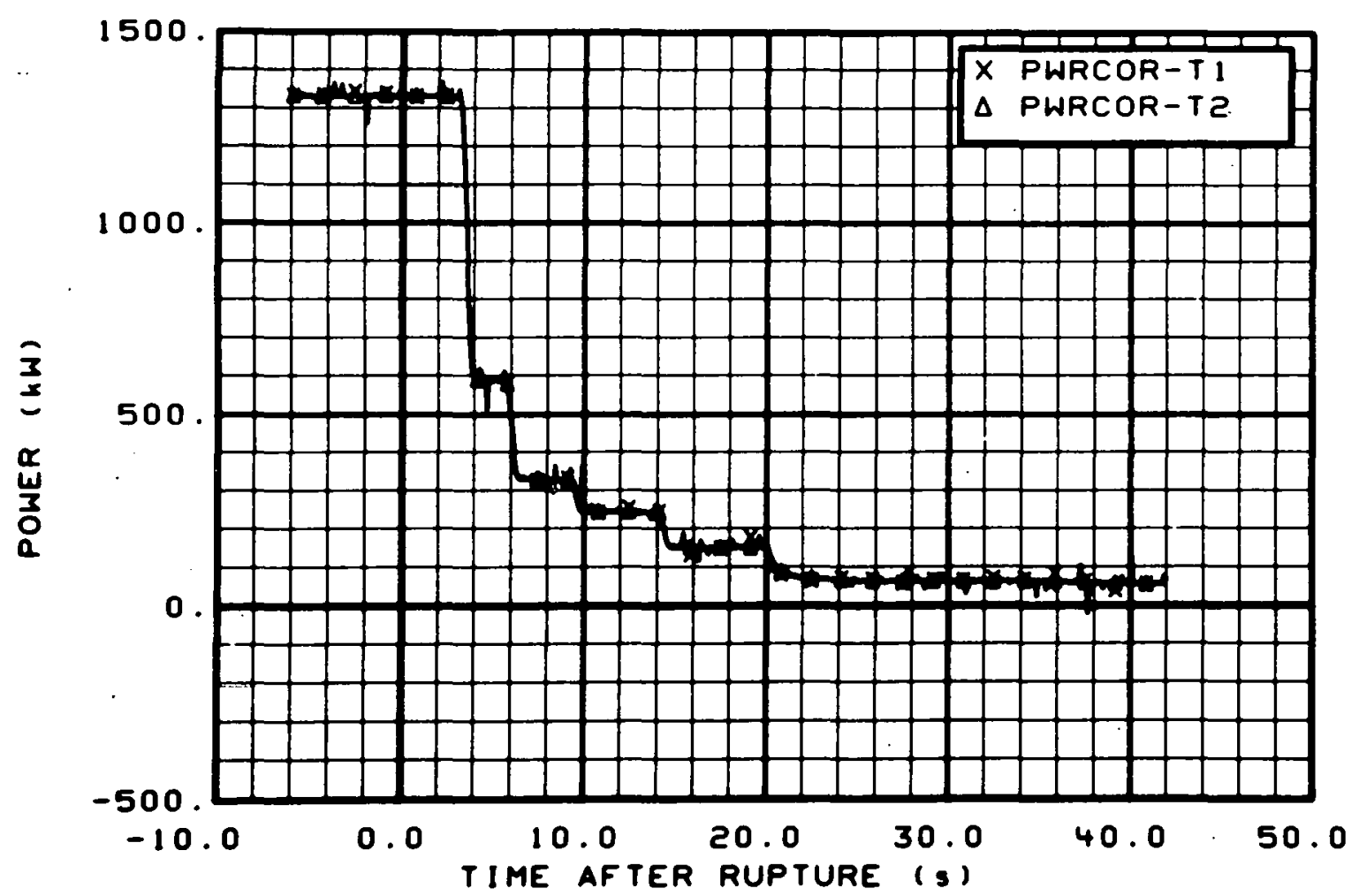

Fig. 308 Core heater rod total power (PWRCOR T-1 and PWRCOR T-2), from -6 to $42 \mathrm{~s}$. 


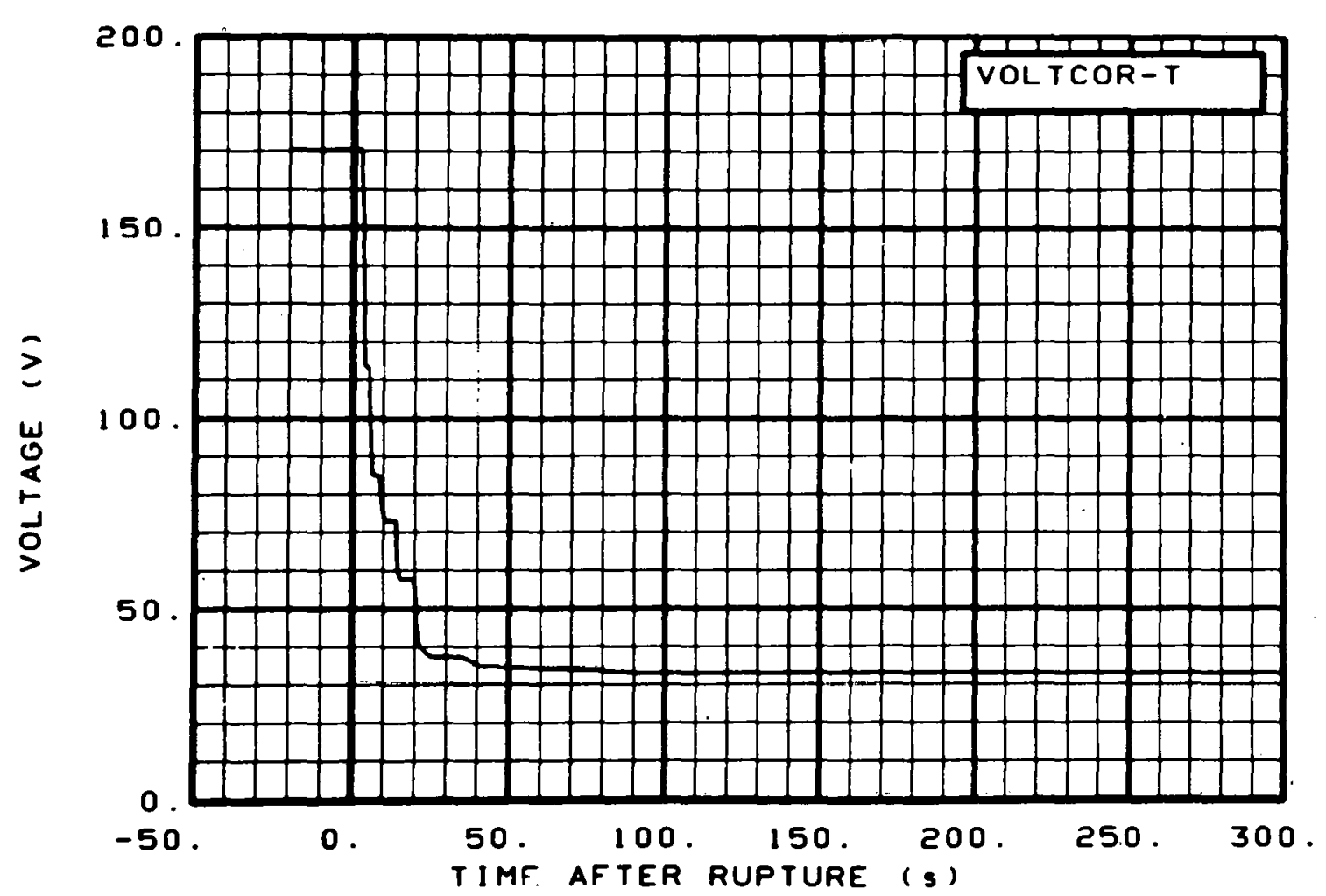

Fig. 309 Core heater voltage (VOLTCOR-T), from -20 to $300 \mathrm{~s}$.

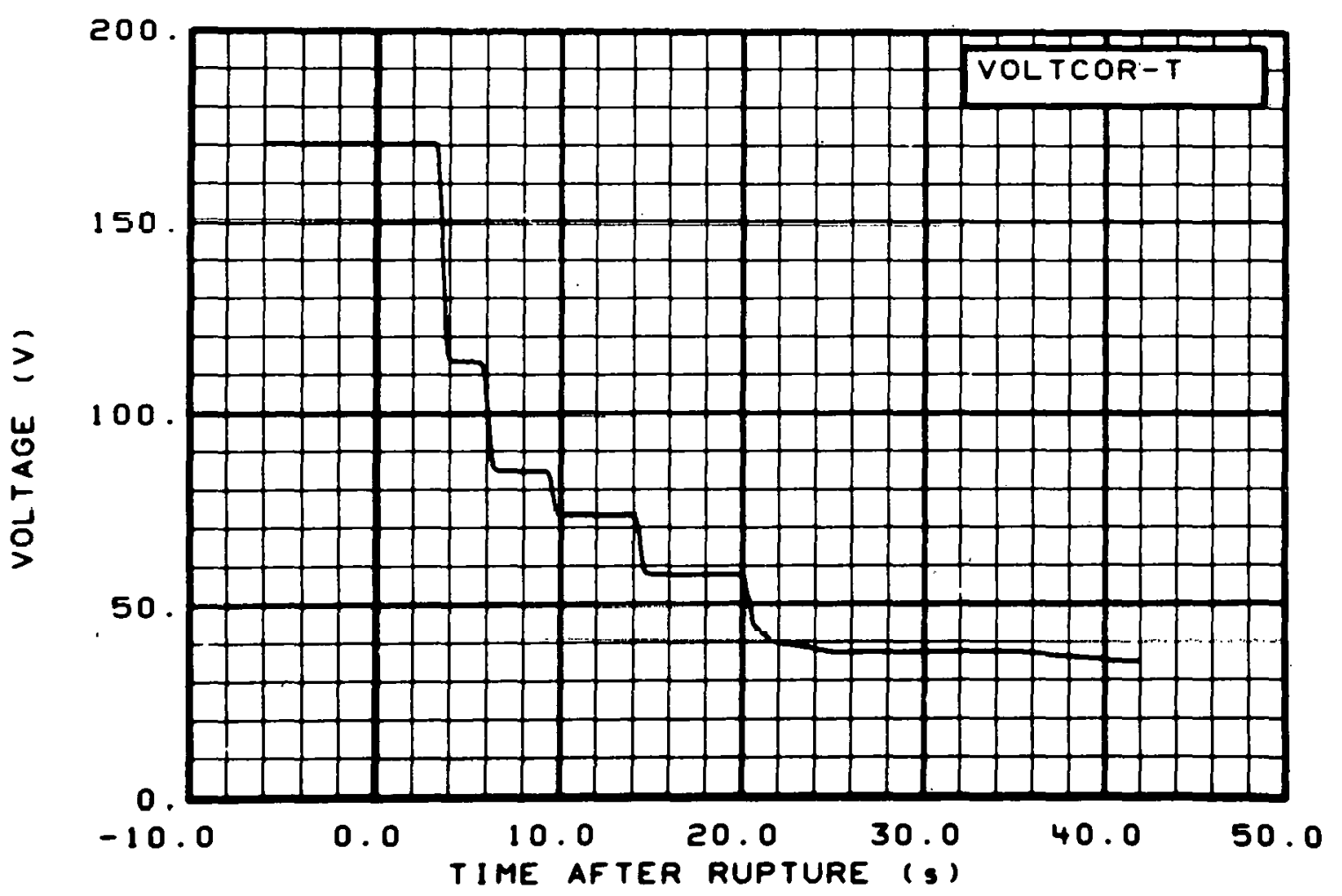

Fig. 310 Core heater voltage (VOLTCOR-T), from -6 to $42 \mathrm{~s}$. 


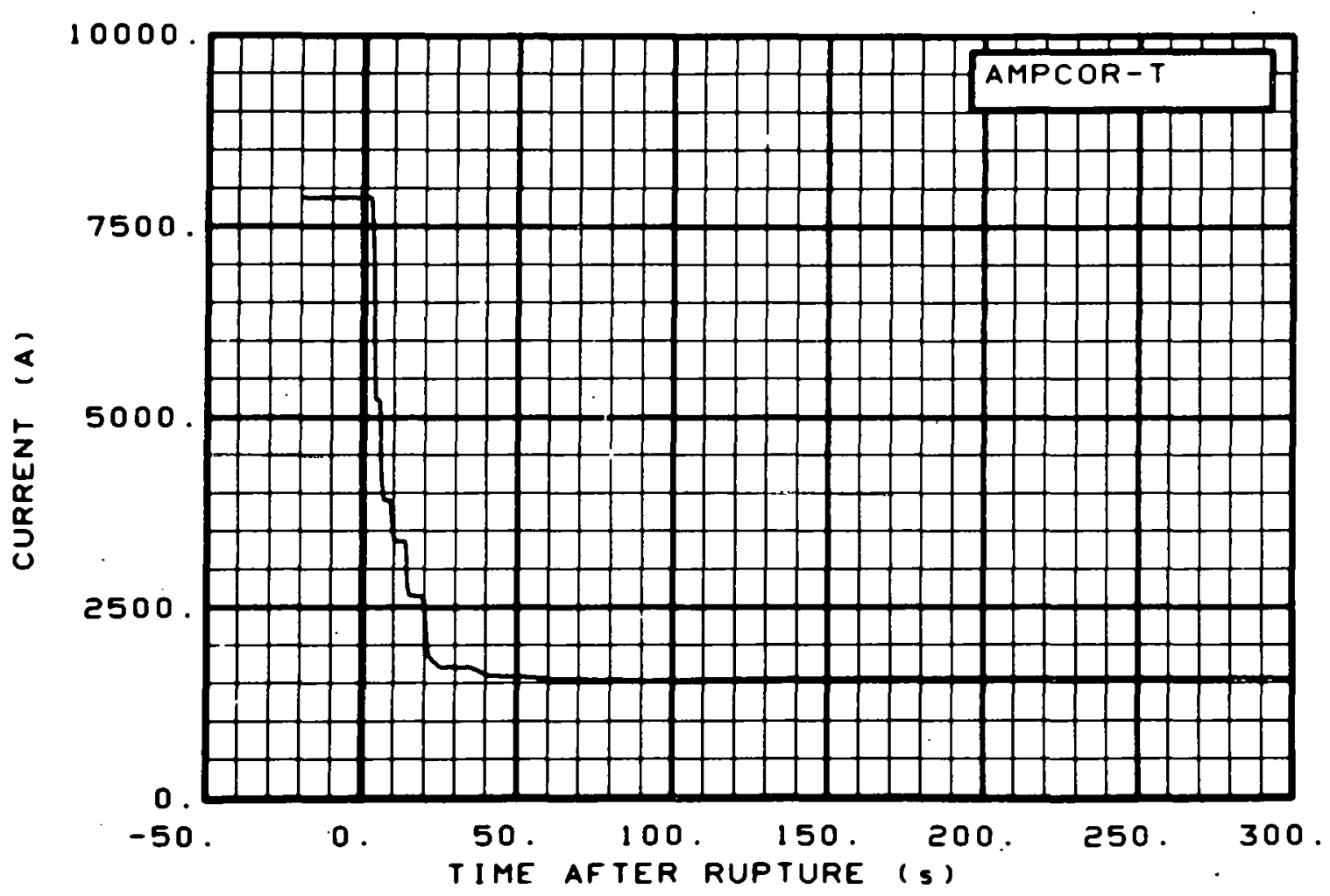

Fig. 311 Core heater total current (AMPCOR-T), from -20 to $300 \mathrm{~s}$.

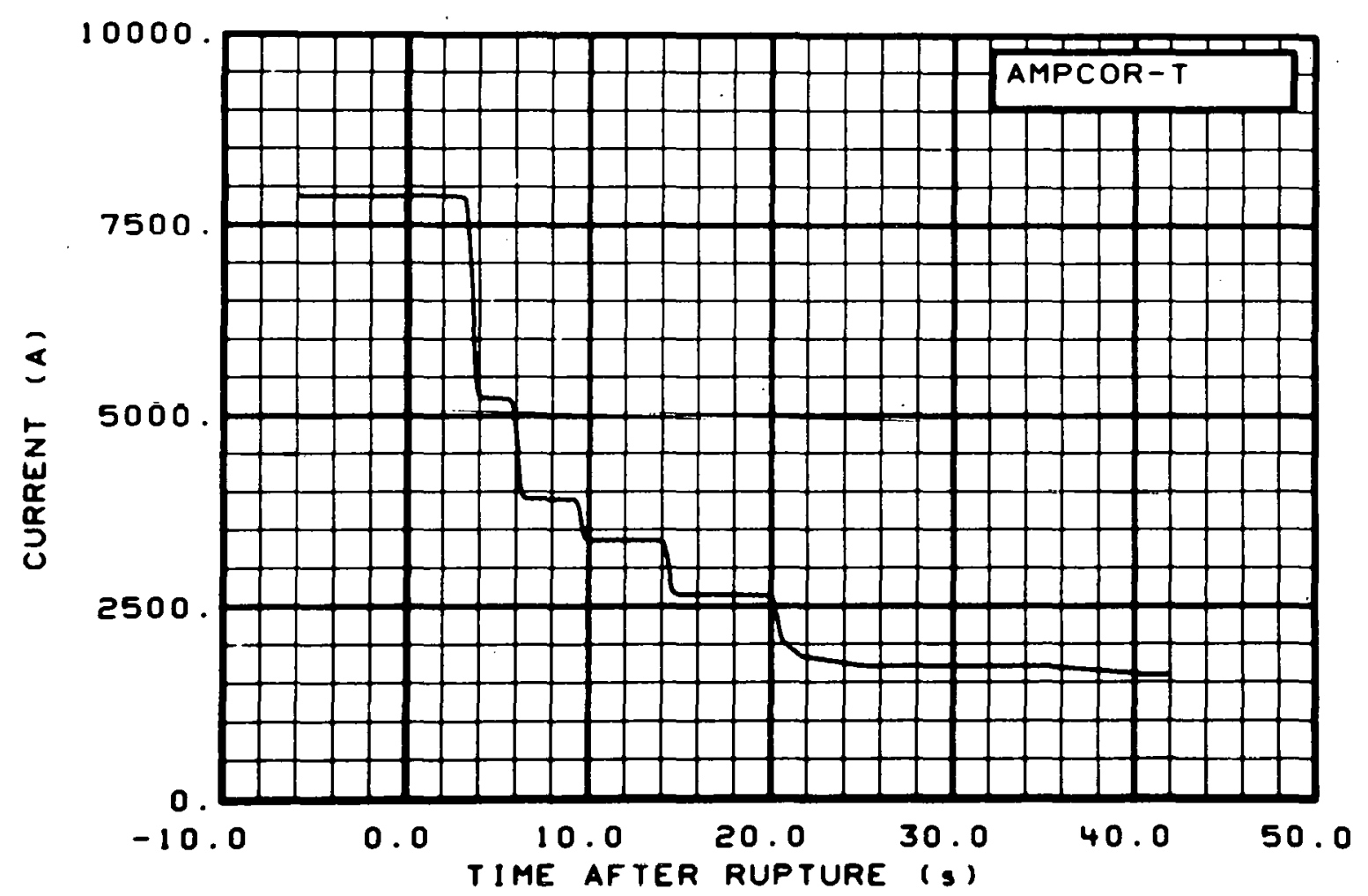

Fig. 312 Core heater total current (AMPCOR-T), from -6 to $42 \mathrm{~s}$. 


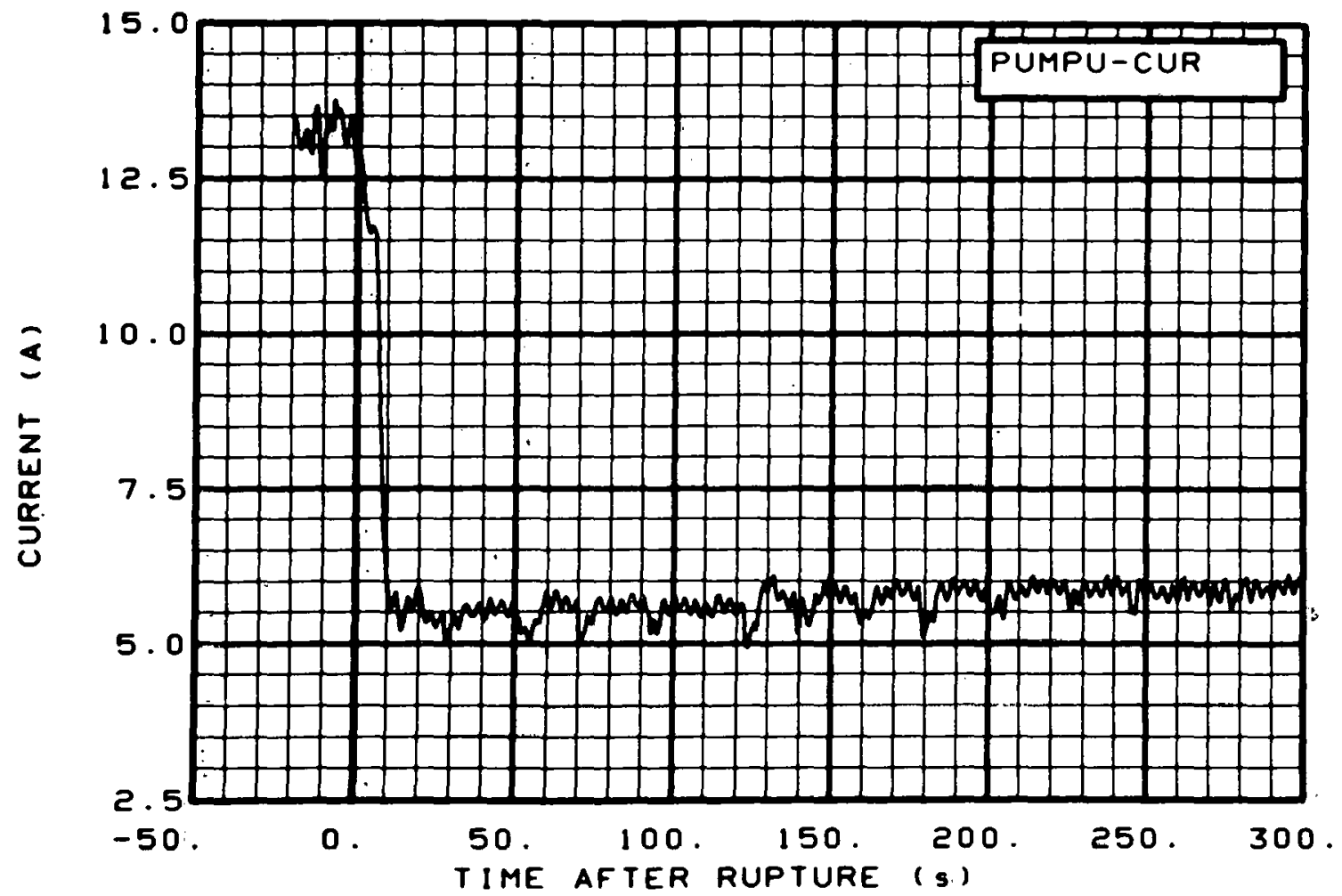

Fig. 313 Primary pump current (PUMPU-CUR), from -20 to $300 \mathrm{~s}$.

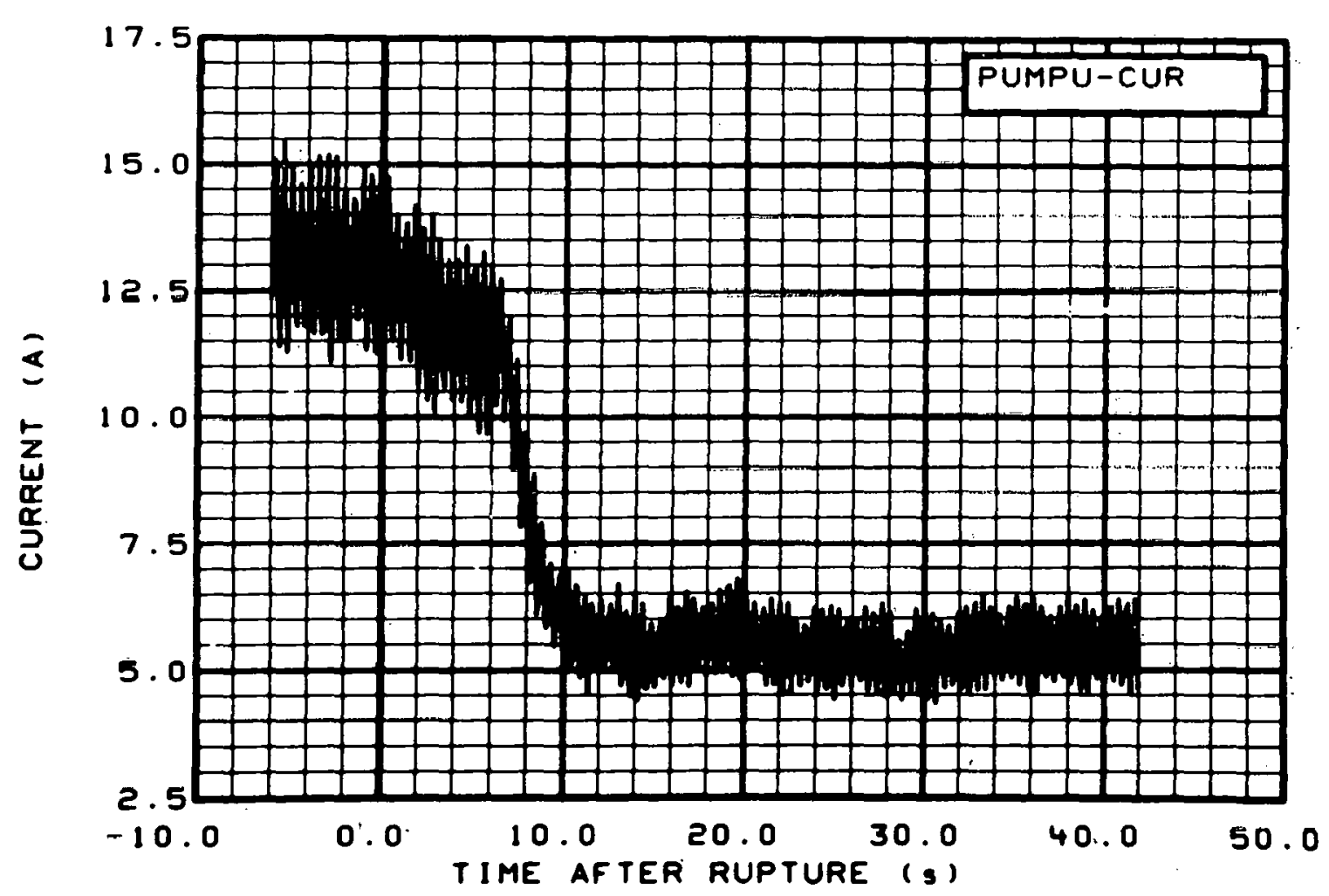

Fig. 314 Primary pump current (PUMPU-CUR), from -6 to $42 \mathrm{~s}$. 


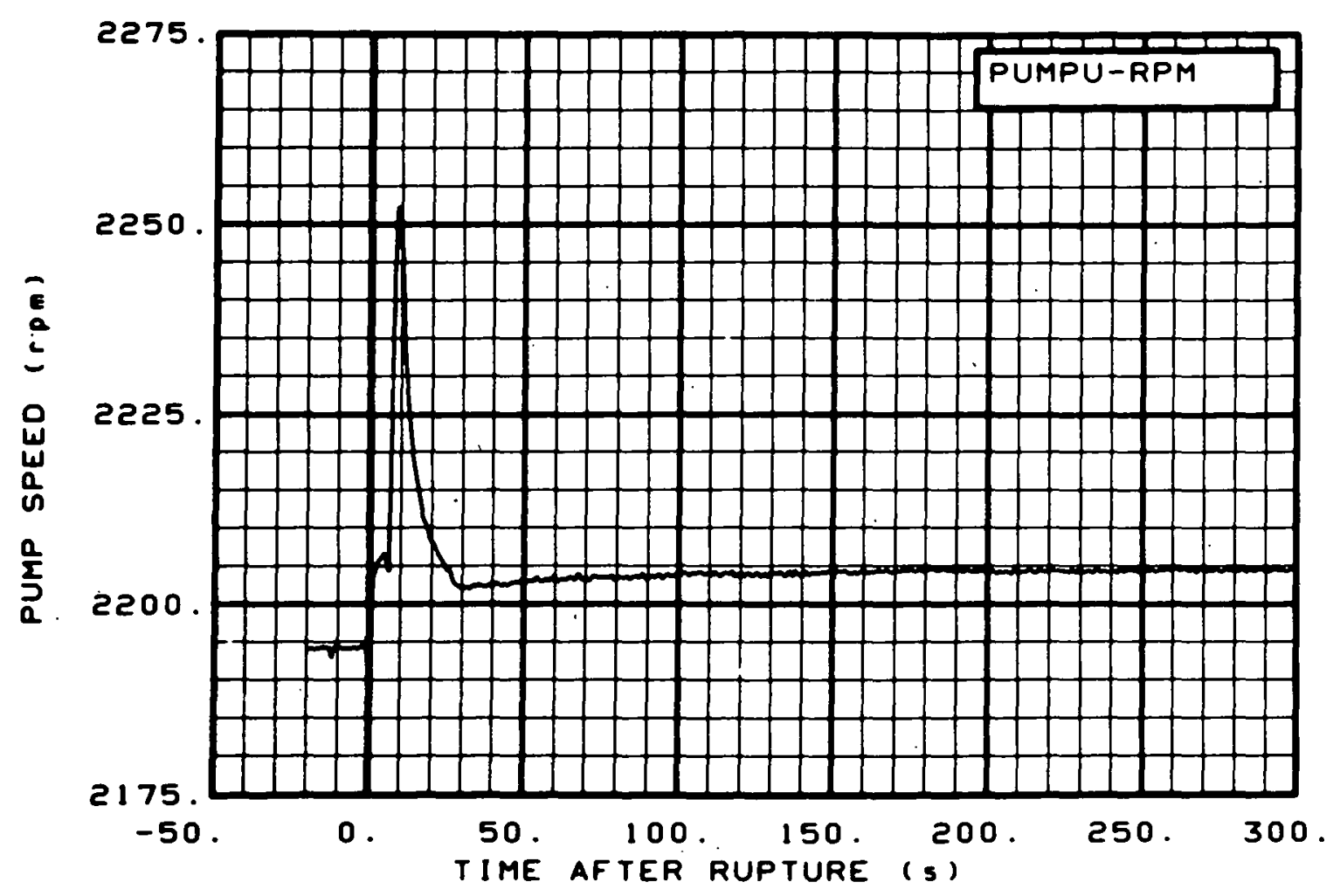

Fig. 315 Primary pump speed (PUMPU-RPM), from -20 to $300 \mathrm{~s}$.

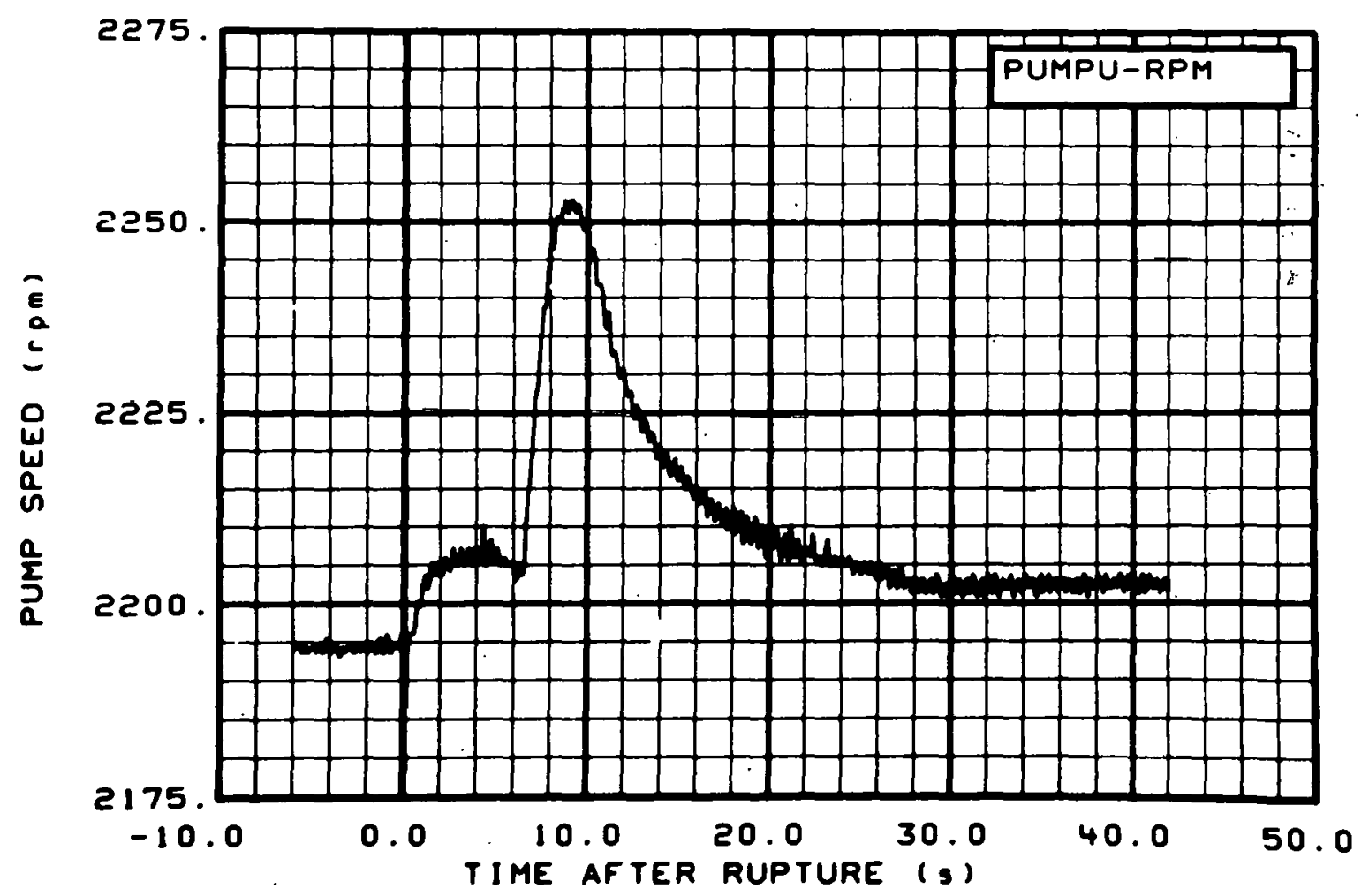

Fig. 316 Primary pump speed (PUMPU-RPM), from -6 to $42 \mathrm{~s}$. 


\section{REFERENCES}

1. E. M. Feldman and D. J. Olson, Semiscale Mod-1 Program and System Description for the Blowdown.Heat Transfer Tests (Test Series 2), ANCR-1230 (August 1975).

2. V. Esparza and K. E. Sackett, Experiment Data Report for Semiscale Mod-1 Test S-06-5 (LOFT Counterpart Test), TREE-NUREG-1125 (June 1977). 


\section{APPENDIX A}

\section{DATA ACQUISITION SYSTEM CAPABILITIES}




\section{THIS PAGE}

\section{WAS INTENTIONALLY \\ LEFT BLANK}




\section{APPENDIX A \\ DATA ACQUISITION SYSTEM CAPABILITIES}

The Semiscale Mod-1 system provides for the acquisition, processing, and presentation of test data. Detectors, signal conditioners, signal processors, and recording and display equipment comprise the test data system. The data obtained are principally recorded on an on-line digital system. Selected data channels are also recorded on an analog system.

The on-line digital system is called the digital data acquisition and processing system (DDAPS). The DDAPS has dual and single speed capabilities with identical storage and data output limitations. The dual speed mode is used to extend the recording time when obtaining high frequency data.

From each of up to 240 data channels, the test data system stores 20 blocks of data. Each block of data contains 920 words (each word is the abscissa and ordinate of a data point) of digital information. These 920 words represent a fixed storage display.

The maximum measured throughput rate for the system is 24000 words per second. This throughput rate can be reduced in increments of 100 words per second. The throughput rate, the number of data channels recorded, and the fixed display of 920 words per block determine the time base for displaying the data.

After the data have been stored, data reduction can be made for presentation and analysis purposes. Because of hardware limitations and aesthetic considerations of data presentation, only certain time bases are used when the data are reduced. For data displayed from -20 to $300 \mathrm{~s}$, the recorded data are made to occupy a $320 \mathrm{~s}$ span yielding a time base of $16 \mathrm{~s}$, which is the $320 \mathrm{~s}$ span divided by the 20 blocks of recorded data.

Generally, 920 words from a given data channel are displayed in the nominal time base of $16 \mathrm{~s}$. Integral ( 1 to 20 ) multiples of $16 \mathrm{~s}$ may be used as variations on the nominal time base. Because the output is fixed at 920 words, data compression is done by averaging adjacent data points to give the desired compression. 
THIS PAGE

WAS INTENTIONALLY

LEFT BLANK 
APPENDIX B

POSTTEST ADJUSTMENTS TO DATA FROM SEMISCALE MOD-1

TEST S-06-4 
THIS PAGE

\section{WAS INTENTIONALLY LEFT BLANK}




\title{
APPENDIX B \\ POSTTEST ADJUSTMENTS TO DATA FROM SEMISCALE MOD-1
}

\author{
TEST S-06-4
}

Many of the transducers used in the Semiscale Mod-1 system exhibit significant sensitivity to one or more spurious inputs. Strain gage bridge circuits used in pressure transducers, differential pressure transducers, and drag discs are sensitive to changes in ambient temperature. Differential pressure cells are also sensitive to changes in system pressure. Photomultiplier tubes used as gamma ray detectors in the density transducers are sensitive to temperature changes, as well as to random variations in the locations of the radiation sources. Core power measurements depend on a calibrated resistor, whose resistance changes in value as a function of time and power level as it heats up.

Although the errors introduced into the data by spurious secondary inputs generally do not exceed the specified error ranges of the transducers, significant improvement in measurement accuracy can be achieved if the secondary sensitivity can be identified and removed. In the case of the drag discs, corrections are absolutely necessary because the signal due to temperature fluctuations can exceed that due to flow by several hundred percent. Since the exact values of the spurious inputs to which different transducers might be sensitive cannot often be easily predicted and are sometimes inconvenient to measure, secondary effects have been accounted for by correcting the data after the test rather than by using elaborate real time programs in the data acquisition system computer. The methods and results of the posttest data correction analysis for Test S-06-4 are presented in the following paragraphs and tables.

\section{PRESSURE MEASUREMENTS}

Corrections to pressure transducer measurements in the main system loop are based on data taken from the standard reference (Heise) gauge at Spool 4, taken $15 \mathrm{~s}$ before initiation of blowdown and at $300 \mathrm{~s}$ after initiation of blowdown. The pressure readings are adjusted to account for pressure variations around the main loop, using the readings of nearby differential pressure cells. A linear correction is then applied to the pressure data to match the data to the calculated reference data at the two specified time points.

Correction of the pressurizer pressure (PU-PRIZE) is done in the same manner as for the main loop pressures using a Heise gauge installed expressly for this purpose.

The data from the pressure transducer for the pressure suppression system (P-PSS) are corrected to match the process instrumentation at preblowdown conditions. 
Pressure measurement corrections are performed using the data acquisition system (DAS) computer using the following equation:

$$
F^{\prime}(t)=c_{0}+C_{1}[F(t)]
$$

where

$$
\begin{aligned}
& F^{\prime}(t)=\text { corrected data } \\
& F(t)=\text { raw data } \\
& C_{0}=\text { offset, } \mathrm{kPa} \\
& \mathrm{C}_{1}=\text { scaling factor. }
\end{aligned}
$$

The values of the offset and scaling factor are given in Table B-I.

\section{TABLE B-I.}

CONSTANTS FOR PRESSURE MEASUREMENT'

CORRECTIONS: (TEST S-06-4)

\begin{tabular}{lcc}
$\begin{array}{c}\text { Detector } \\
\text { Identification }\end{array}$ & $\frac{\mathrm{C}_{0}}{\mathrm{C}_{1}}$ \\
PU-PRIZE & 0 & 0.9896 \\
PB-24 & 0 & $0.98 ? ?$ \\
\hline
\end{tabular}

\section{DIFFERENTIAL PRESSURE MEASUREMENTS}

Pressure sensitivity in the differentlal pressure cells in the niaili. system loop is determined from the pretest system pressure check. Digital data are recorded for all measurements at ambient temperature, with no system flow, at pressures of ambient, 2010 , $5100,6880,8580,12040$ and $15060 \mathrm{kPa}$. 'l'he output of the diffcrential pressure cells is plotted against system pressure, with the resulting plots used to describe the pressure response of the transducers.

Pressure cell ambient offsets are evaluated using a posttest, system-drained digital data scan. The measured transducer outputs are compared with values calculated from the differences in standing leg heights for the sense lines to each pressure cell. The difference between the measured value and the calculated value is the ambient offset. 
For some differential pressure measurements, other references are used. The liquid level measurement in the intact loop accumulator (DPU-ACC1-TB) is referenced to a calculated value based on geometrical considerations at the time when the water is depleted from the accumulator and gas flow begins. The reading from the steam generator discharge venturi (DPU-SG-DISC) is shifted to read zero after flow is stopped.

In correcting differential pressure data for pressure sensitivity, the data are initially corrected for errors in amplification and ambient offsets. The data are then corrected for pressure sensitivity to arrive at the final values. Corrections were made using the following equations:

$$
F^{\prime}(t)=K[F(t)]+C_{0}
$$

where

$$
\begin{aligned}
& F^{\prime}(t)=\text { data corrected for amplification and ambient offsets, } k P a \\
& K=\text { amplification factor } \\
& F(t)=\text { raw data, } \mathrm{kPa} \\
& C_{0}=\text { ambient offset, } \mathrm{kPa} \\
& F^{\prime \prime}(t)=F^{\prime}(t)+c_{1} \text { for } t<t_{1} \\
& F^{\prime \prime}(t)=F^{\prime}(t)+C_{i}+\frac{t-t_{i}}{t_{i+1}-t_{i}}\left(C_{i+1}-c_{i}\right) \text { for } t_{i} \leq t \leq t_{i+1}
\end{aligned}
$$

where $\mathrm{i}$ takes on values 1 to $\mathrm{n}-1$

$$
F^{\prime \prime}(t)=F^{\prime}(t)+C_{n} \text { for } t>t_{n}
$$

where

$$
\begin{aligned}
& \mathrm{t}=\text { time } \\
& \mathrm{F}^{\prime \prime}(\mathrm{t})=\text { final corrected data, } \mathrm{kPa} \\
& \mathrm{C}_{\mathrm{i}}=\text { data corrcction points, } \mathrm{kPa} \\
& \mathrm{t}_{\mathrm{i}}=\text { times corresponding to data correction points, } \mathrm{s} .
\end{aligned}
$$

The values of the constants are given in Table B-II. 
TABLE B-II

CONSTANTS FOR DIFFERENTIAL PRESSURE

MEASUREMENT CORRECTIONS (TEST S-06-4)

\begin{tabular}{|c|c|c|c|c|c|c|c|c|}
\hline $\begin{array}{c}\text { Detector } \\
\text { Identification } \\
\end{array}$ & $K$ & $c_{0}$ & $c_{1}$ & $t_{1}$ & $\mathrm{C}_{2}$ & $t_{2}$ & $\mathrm{C}_{3}$ & $\underline{t_{3}}$ \\
\hline DPU-12-10 & 1 & & -6.3 & 0 & -4.7 & 0.01 & -0.3 & 32 \\
\hline DPU-ACCl-TB & 1 & -10.3 & & & & & & \\
\hline DPB-23-24 & 1 & -5.5 & & & & & & \\
\hline DPB-30-38 & 1 & & -29.6 & 0 & -24.1 & 0.01 & -1.4 & 32 \\
\hline DPB-32U-36L & 1 & & 63.4 & 0 & 46.9 & 0.01 & 2.8 & 32 \\
\hline DPV-UP_-IANN & 1 & 2.6 & & & & & & \\
\hline DPV-9-26QQ & 1 & & 0.23 & 0 & 0.21 & 0.01 & 0.06 & 32 \\
\hline DPV-26-550M & 1 & & -1.7 & 0 & -1.4 & 0.01 & -0.3 & 32 \\
\hline DPV-173-180QQ & 1 & -0.15 & & & & & & \\
\hline DPV-LP-UP & 1 & -95.0 & & & & & & \\
\hline
\end{tabular}

\section{MUMEN'IUM FLUX MEASUREIMENTS}

The temperature sensitivity of drag discs is determined from pretest warmup data taken at 366 and $533 \mathrm{~K}$ with no system flow. The temperature and pressure sensitivities are removed before the data are converted to momentum flux. The temperature and pressure of each transducer are :taken from the signal ul a nearby fluid or mctal tomporatury thermocouple. Slight corrections for errors in setting the transducer output to zero at ambient conditions are also made at this time. Corrections are made using the following equation:

$$
\Gamma^{\prime}(t)=F(t)+C_{0}-T_{1} \cdot T(t)=P_{1} P(t)
$$

where

$$
\begin{aligned}
& F^{\prime}(t)=\text { corrected data, DAS volts } \\
& F(t)=\text { raw data, DAS volts } \\
& T_{1}=\text { temperature sensitivity, DAS volts } / K
\end{aligned}
$$




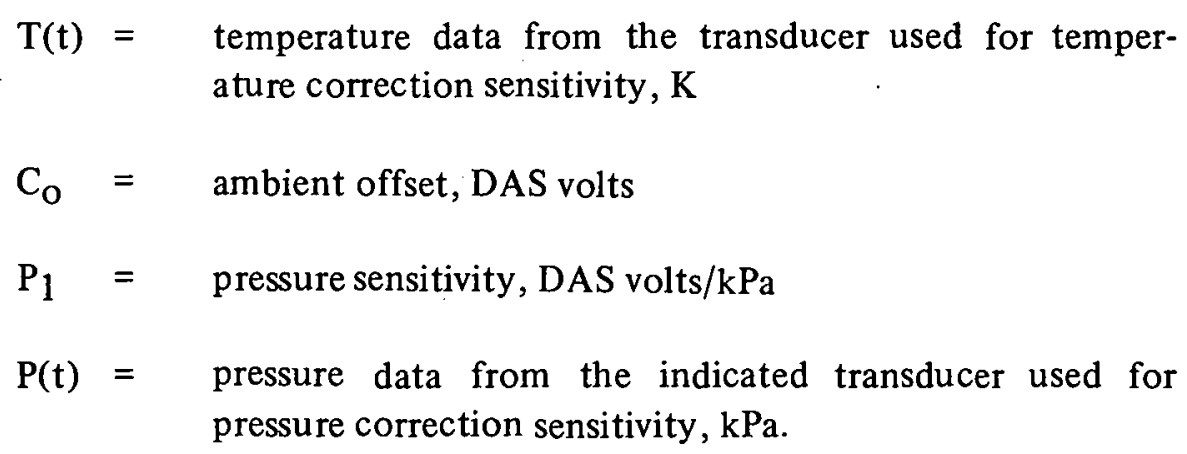

Values of the constants are given in Table B-III. 
$\underline{T A B L E} \cdot B-I I I$

CONSTANTS FOR MOMENTUM FLUX

MEASUREMENT CORRECTIONS (TEST S-06-4)

\begin{tabular}{|c|c|c|c|c|c|c|}
\hline ID & $\mathrm{C}_{\mathrm{O}}$ & $T_{1}$ & $T(t)^{[a]}$ & $P_{1}$ & & $P(t)^{[b]}$ \\
\hline FDU -5 & 0 & 0.000943 & TFU-7 & -0.000001 & 6 & $P V=U P+10$ \\
\hline FDV-CORE-IN & 0 & -0.000 .038 & TFV-CORE-IN & & & \\
\hline FDU-10 & 0 & 0.000198 & TFU -10 & 0.000000 & 7 & $P V-U P+10$ \\
\hline FDU -13 & 0 & 0.001327 & RBU-14A & & & \\
\hline $\mathrm{FDB}-30^{[\mathrm{c}]}$ & -0.092 & -0.001436 & TFB-30 & 0.000000 & 7 & $P V-U P+10$ \\
\hline FDB -21 & 0 & -0.001046 & TFB-20 & $-0.000 \quad 003$ & 9 & $\mathrm{~PB}-21$ \\
\hline FDB -2.3 & 0 & -0.001885 & TFB -23 & -0.000003 & 8 & $\mathrm{~PB}-2 \cdot 3$ \\
\hline$F D B-42$ & -0.037 & 0.001413 & TFB -42 & -0.000003 & 2 & $P B-42$ \\
\hline $\mathrm{FDB}-37^{[\mathrm{c}]}$ & -0.125 & 0.000839 & TFB -37 & -0.000000 & 5 & $P B-42$ \\
\hline FDU-1 & 0 & -0.002225 & RBU-2 & -0.000 .004 & 3 & $P V-U P+10$ \\
\hline
\end{tabular}

[a] $T(t)$ is the temperature data used for temperature sensitivity correction. The symbols listed identify the thermnonuples from which the data are obtained.

[b] $P(t)$ is the pressure data from the indicated transducer used for pressure sensitivity correction. The symbols listed identify the pressure transducers from which the data are obtained.

[c] Temperature sensitivity corrections were applied as usual; however, because FDB-30 and FDB-37 are mounted horizontally, during blowdown they were partially filled with subcooled water which affected the temperature sensitivity. Therefore, the temperature sensitivity correction is more uncertain that that applied to other detectors: 


\section{DENSITY MEASUREMENTS}

Density calculations are based on the voltage output of the photomultiplier tubes in the gamma-attenuation densitometer assemblies. The equation used for converting voltage to density is as follows:

$$
\rho=(1 / C) \ln \{D /[A F(t)+B]\}
$$

where

$$
\begin{aligned}
& \rho=\text { the density in } \mathrm{kg} / \mathrm{m}^{3} \\
& \mathrm{C}=\begin{array}{l}
\text { a constant based on the length of the gamma beam path, } \\
\text { DAS volts } / \mathrm{kg} / \mathrm{m}^{3}
\end{array} \\
& \mathrm{D}=\text { a theoretical voltage for zero attenuation inside the vessel, DAS volts } \\
& \mathrm{A}=\text { an amplification factor } \\
& \mathrm{B}=\text { a biasing factor, DAS volts } \\
& \mathrm{F}(\mathrm{t})=\text { the transducer voltage output, DAS volts. }
\end{aligned}
$$

Constants $\mathrm{A}$ and $\mathrm{B}$ are adjusted to match the final data to density values calculated from measured pressure and temperature values at the preblowdown and postdrain conditions, effectively giving the data an in-place calibration. The values of the constants for various transducers are given in Table B-IV.

The density measurements GVLP-172HZ and GV-COR-150HZ use amplifiers which precalculate the logarithm function, and hence have a simpler conversion formula:

$$
\begin{array}{ll}
\text { GVLP-172HZ } & \rho=-20.936 F(t)-546.7 \\
\text { GV-COR-150HZ } & \rho=-60.068 F(t)-2877.9 .
\end{array}
$$

Some density measurements are obtained using a two-beam gamma densitometer which operates on the same basic principle of gamma attenuation as does the single-beam gamma densitometer. Each beam originates from the same gamma source and is allowed to pass through separate portions of the piping cross-sectional flow area to obtain an average density measurement in that particular region. The geometrical relationship of the gamma beam path through the piping and geometrically related variables used for processing of data from a two-beam gamma densitometer are shown in Figure B-1.

The average density measured by each individual gamma beam is obtained using the same equation as is used for the single-beam gamma densitometers. Values for the constants for the single-beam density measurements obtained with the two-beam gamma densitometers are presented in Table B-IV along with the constants for single-beam gamma densitometers. 
TABLE B-IV

CONSTANTS FOR : DENSITY MEASUREMENT

CONVERSIONS TO ENGINEERING UNITS (TEST S-06-4)

\begin{tabular}{|c|c|c|c|c|}
\hline $\begin{array}{c}\text { Detector } \\
\text { Identification }\end{array}$ & A & B & C & D \\
\hline GU-TT & 1.031 & -0.201 & 0.00036 & $6: 48$ \\
\hline GU-1B & 0.987 & 0.150 & 0.00050 & 7.18 \\
\hline GU-5VR & 1.100 & -0.450 & 0.00059 & 4.51 \\
\hline GIII-THVR & 1.076 & -0.468 & 0.00059 & 6.72 \\
\hline GU-13VR. & 0.839 & 0.315 & 0.00059 & 1.94 \\
\hline GU-75T & 1.151 & $-0: 952$ & 0.00036 & 6.34 \\
\hline GU-1.5B & 0.992 & 0.055 & 0.00050 & 6.76 \\
\hline GB-21T & 1.057 & -0.348 & 0.00036 & 6.50 \\
\hline$G B-21 . B$ & 1.112 & -0.814 & 0.00050 & 7.40 \\
\hline$G B-23 V R$ & 0.977 & 0.187 & 0.00037 & 7.40 \\
\hline GB-30T & 1.119 & -0.711 & 0.00036 & 5.98 \\
\hline$G B-30 B$ & 1.014 & -0.085 & 0.00050 & 7.73 \\
\hline$G B-37$ & 1.918 & -3.621 & 0.00059 & 3.95 \\
\hline$G B-42 V R$ & -0.621 & 7.624 & 0.00037 & 4.71 \\
\hline$G V-162 / 192 D$ & 1.389 & -0.578 & 0.00540 & 1.60 \\
\hline GVLP $165 \mathrm{HZ}$ & 1,0 1. & -0.138 & 0.00150 & 10.50 \\
\hline GU-PRIZE & 1.207 & -0.193 & $0.000 \quad 59$ & 0.955 \\
\hline
\end{tabular}




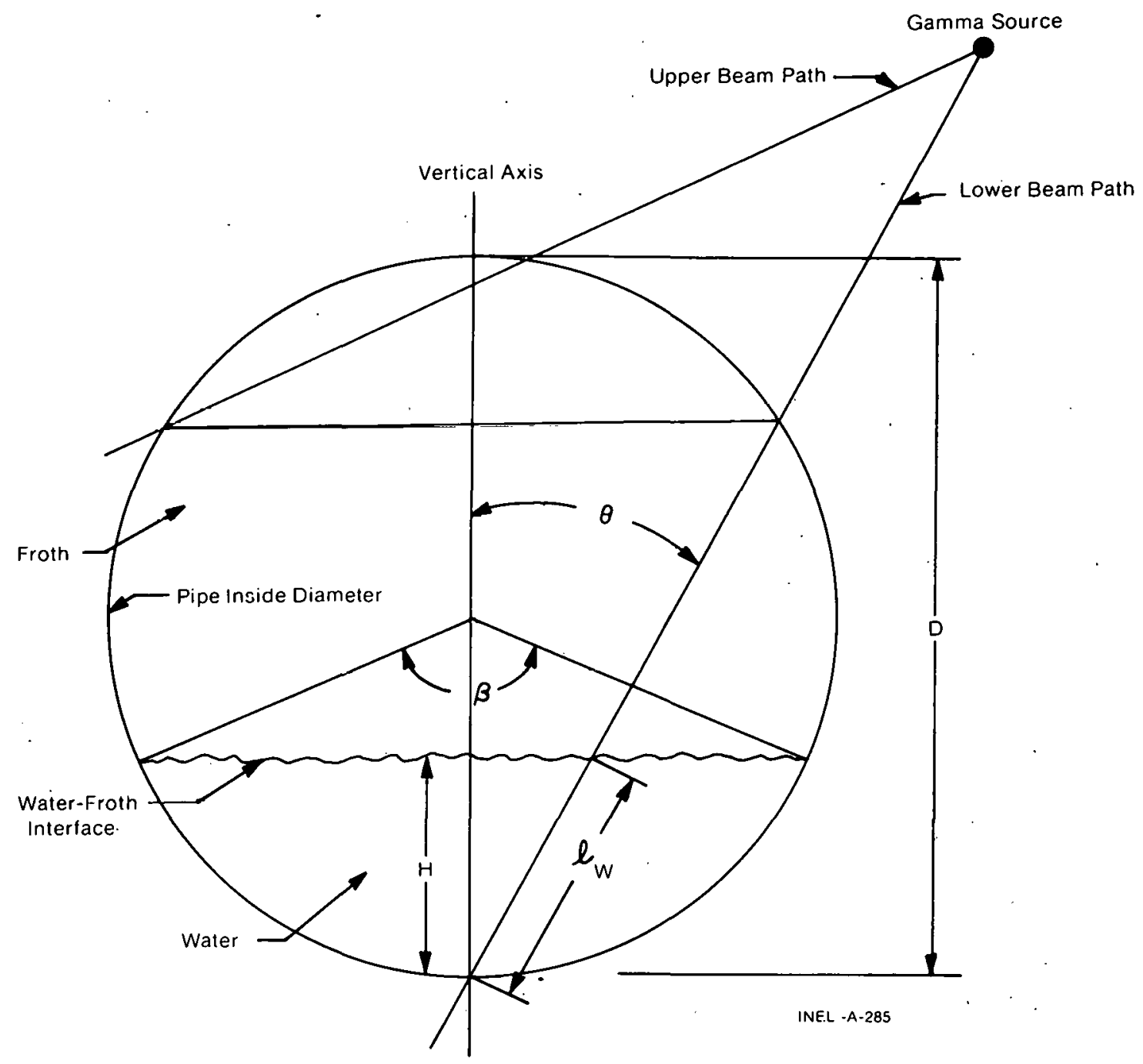

Fig. B-1 Geometry used for processing of density data obtained from two-beam garma densitometers.

In the Semiscale Mod-1 system, two-beam gamma densitometers provide added infonnation which allows the calculation of a better average density than that obtained from a single beam. A mathematical model is used for processing the two-beam data to obtain the improved average density information. The processing method used is based on a froth-water model coupled with information from the two individual gamma beams and related beam path and piping cross-sectional geometry. The resulting information is recorded and reported under the density measurement identification ending with a " $C$ ", for example, GB-21C.

The use of the froth-water model for obtaining average density from a two-beam gamma densitometer is based on observations indicating that flow regimes in the Semiscale Mod-1 system can be modeled by a layer of water on the bottom of the pipe with a degree of froth on the surface. For homogeneous flow conditions such as all froth or all liquid the 
model remains valid. At any point in time, slug flow is also modeled. The froth-water model does not model annular or inverted annular flows very well. However, these flows are not expected to exist for significant portions of a Semiscale Mod-1 system blowdown in horizontal piping. Density gradients from the top to the bottom of the pipe may exist showing no distinct location change from water to froth. This flow is neither totally homogeneous nor stratified, but the froth-water model does provide an adequate approximation of the average density characteristic of this flow pattern.

The average density obtained by using the gamma beam geometry shown in Figure B-1 and by applying the froth-water model is given by

$$
\bar{\rho}=\alpha_{f} \rho_{1}+\left(1-\alpha_{f}\right) \rho_{w} \cdot \mathrm{kg} / \mathrm{m}^{3}
$$

where

$$
\begin{aligned}
& \bar{\rho}=\text { average cross-sectional density } \\
& \rho_{1}=\begin{array}{l}
\text { average density measured by the upper gamma beam } \\
\text { (measures the froth density) }
\end{array} \\
& \rho_{\mathrm{W}}=\text { density of liquid water (at local system conditions) } \\
& \alpha_{\mathrm{f}}=: 1+(1 / 2 \pi)(\sin \beta-\beta)=\text { froth fraction. }
\end{aligned}
$$

The angle which $\beta$ represents is shown in Figure $B=1$. Values for $\beta$ are obtained as follows:

$$
\beta=2 \cos ^{-1}(1-2 h)
$$

where

$$
\mathrm{h}=\frac{\mathrm{H}}{\mathrm{D}}=\cos ^{2} \theta \frac{\rho_{2}-\rho_{1}}{\rho_{\mathrm{W}}-\rho_{1}}
$$

where

$$
\begin{aligned}
& \mathrm{H} \quad-\quad \ell_{\mathrm{W}} \cos \theta\left(\ell_{\mathrm{W}} \text { and } \theta \text { are defined in Figure } \mathrm{B}-1\right) \\
& \mathrm{D}=\text { piping inside diameter } \\
& \rho_{2}=\text { the average density measured by the lower gamma beam. }
\end{aligned}
$$

Average density is not calculated using the two-beam froth-water model when the angle $\theta$ is not favorable due to system hardware restrictions in positioning the source. The froth-water model requires separate density sampling in both the upper and lower portions of the piping cross section. 
APPENDIX C

SELECTED DATA WITH ESTIMATED TOTAL ERROR

BANDS FROM SEMISCALE MOD-1 TEST S-06-4 
THIS PAGE

\section{WAS INTENTIONALLY \\ LEFT BLANK}




\section{APPENDIX C \\ SELECTED DATA WITH ESTIMATED TOTAL ERROR \\ BANDS FROM SEMISCALE MOD-1 TEST S-06-4}

Analysis has been performed on selected data from Test S-06-4 to provide a guide to the uncertainty associated with data measurements in the Semiscale Mod-1 system. The end result of the analysis is presented as error bands about the measured data which represent a $95 \%$ confidence level.

The error bands are obtained by combining uncertainties obtained from analysis of the data itself (random error) and engineering analysis of the measurement system (engineering error). The procedure by which error bands were established for the data presented in this appendix is described in the following paragraphs.

The data trace under analysis was empirically fitted with a linear difference equation, which was subject to a white noise input at each sampling time point. The objective of the empirical fitting procedure was to characterize the white noise, which was taken to represent the random error. The procedures for fitting the difference equation are discussed in depth in Reference C-1. A data trace was often segmented and different equations were fitted to each segment with statistical correlations between successive observations accounted for by the fitting procedure. The white noise input was assumed to arise from a normally distributed population. The standard deviation of the white noise, as found during the fitting procedures, was taken as an estimate of the random error standard deviation and is shown in Table C-I. The data traces of the error band analysis are shown in Figures C-1 through $\mathrm{C}-43$.

\section{TABLE C-I}

\section{RANDOM ERROR VARIANCE (TEST S-06-4)}

\begin{tabular}{|c|c|c|c|c|}
\hline Measurement & $\begin{array}{c}\text { Random Error } \\
\text { Variance } \\
\sigma_{\mathrm{R}} \\
\end{array}$ & $\begin{array}{l}\text { Period of } \\
\text { Application } \\
\text { (s) }\end{array}$ & Figure & Comments \\
\hline TFB-2.3 & $\begin{array}{r}9.888 \\
3.545\end{array}$ & 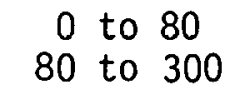 & $C=1$ & \\
\hline TFV-ANN-35A & $\begin{array}{l}7.795 \\
1.289\end{array}$ & $\begin{aligned} 0 & \text { to } \\
80 & \text { to } 300\end{aligned}$ & $C-2$ & \\
\hline TFV-LP-8 & 1.194 & 0 to 300 & $C-3$ & \\
\hline
\end{tabular}


TABLE C-I (continued)

\begin{tabular}{|c|c|c|c|c|}
\hline Measurement & $\begin{array}{c}\text { Random Error } \\
\text { Variance } \\
\sigma_{R} \\
\end{array}$ & $\begin{array}{c}\text { Period of } \\
\text { Application } \\
\text { (s) }\end{array}$ & Figure & Comments \\
\hline TFV-LP- 15 & 1.557 & 0 to 300 & $C-4$ & \\
\hline TFV-LP-22 & 1.855 & 0 to 300 & $C-5$ & \\
\hline TFG-5CD-45 & $\begin{array}{r}16.213 \\
31.205 \\
4.333\end{array}$ & 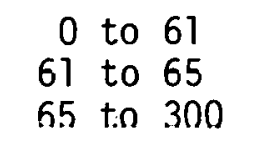 & $c-6$ & \\
\hline TFU-SG3 & 0.175 & 0 to 300 & $C-7$ & \\
\hline TFU-PRIZE & 1.403 & 0 to 300 & $C-8$ & \\
\hline TH-E4-09 & $\begin{array}{r}11.622 \\
27.372 \\
0.387\end{array}$ & $\begin{array}{l}0 \text { to } 86 \\
86 \text { to } 93 \\
93 \text { to } 300\end{array}$ & $C-9$ & \\
\hline$T H-E 4-27$ & 7.846 & 0 to 300 & $C=10$ & \\
\hline TH-E4-55 & $\begin{array}{r}1.420 \\
23.746 \\
0.136\end{array}$ & $\begin{array}{r}0 \text { to } 268 \\
268 \text { to } 275 \\
275 \text { to } 300\end{array}$ & $C-11$ & \\
\hline PU-13 & 2.493 & 0 to 300 & $c-12$ & \\
\hline$P B-23$ & 3.595. & 0 to 300 & $c-13$ & \\
\hline DPU-6-SGIP & $\begin{array}{l}0.406 \\
0.770 \\
0.146\end{array}$ & $\begin{array}{r}0 \text { to } 66 \\
66 \text { to } 80 \\
80 \text { to } 300\end{array}$ & C. -14 & \\
\hline DPU-SGOP-7 & $\begin{array}{l}0.425 \\
0.852 \\
0.223\end{array}$ & $\begin{aligned} 0 & \text { to } \\
66 & \text { to } 80 \\
80 & \text { to } 300\end{aligned}$ & $c-15$ & \\
\hline DPU-7-10 & $\begin{array}{l}0.128 \\
0.267\end{array}$ & $\begin{array}{c}0 \text { to } 14 \\
14 \text { to } 300\end{array}$ & $c-16$ & - \\
\hline DPU-12-10 & $\begin{array}{l}0.850 \\
0.348\end{array}$ & $\begin{aligned} 0 & \text { to } 14 \\
14 & \text { to } 300\end{aligned}$ & $c-17$ & \\
\hline DPU-12-15 & $\begin{array}{l}0.157 \\
0.221\end{array}$ & $\begin{aligned} 0 & \text { to } 25 \\
25 & \text { to } 300\end{aligned}$ & $c-18$ & \\
\hline FTU-1 & $\begin{array}{l}59.772 \\
54.819\end{array}$ & 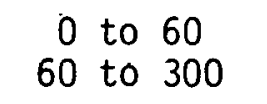 & $C-19$ & \\
\hline
\end{tabular}


TABLE C-I (continued)

\begin{tabular}{|c|c|c|c|c|}
\hline Measurement & $\begin{array}{c}\text { Random Error } \\
\text { Variance } \\
\sigma_{\mathrm{R}} \\
\end{array}$ & $\begin{array}{c}\text { Period of } \\
\text { Application } \\
\text { (s) } \\
\end{array}$ & Figure & Comments \\
\hline FTU-15 & $\begin{array}{r}7.457 \\
31.998 \\
22.977 \\
88.407\end{array}$ & $\begin{array}{l}0 \text { to } 15 \\
15 \text { to } 19 \\
19 \text { to } 66 \\
66 \text { to } 300\end{array}$ & $C-20$ & . \\
\hline FTU-HPIS & $\begin{array}{l}0.069 \\
0.730 \\
0.473\end{array}$ & $\begin{aligned} 0 & \text { to } 22 \\
22 & \text { to } 27 \\
27 & \text { to } 300\end{aligned}$ & $C-21$ & \\
\hline FTU-LPIS & $\begin{array}{l}0.192 \\
1.879 \\
0.011\end{array}$ & 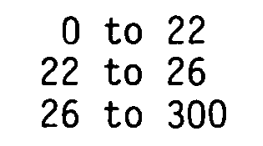 & $C-22$ & \\
\hline FTU-ACC 1 & $\begin{array}{l}3.516 \\
1.393 \\
0.191 \\
1.033 \\
2.585 \\
4.193\end{array}$ & $\begin{array}{l}0 \text { to } 15 \\
15 \text { to } 19 \\
19 \text { to } 65 \\
65 \text { to } 68 \\
68 \text { to } 87 \\
87 \text { to } 91\end{array}$ & $C-23$ & $\begin{array}{l}\text { Error bands are not } \\
\text { presepted from } t=91 \text { to } \\
300 \mathrm{~s}[a] \text {. }\end{array}$ \\
\hline FTV-40A & $\begin{array}{l}7.284 \\
2.263\end{array}$ & $\begin{aligned} 0 & \text { to } \\
57 & \text { to } 300\end{aligned}$ & $C-24$ & - \\
\hline FTV-40M & $\begin{array}{l}5.881 \\
1.692\end{array}$ & $\begin{aligned} 0 & \text { to } \\
57 & \text { to } 300\end{aligned}$ & $C-25$ & \\
\hline GU-1T & $\begin{array}{l}0.666 \\
0.250\end{array}$ & $\begin{array}{c}0 \text { to } 14 \\
14 \text { to } 300\end{array}$ & $C-26$ & \\
\hline$G U-1 B$ & $\begin{array}{l}1 . .859 \\
0.042 \\
0.206\end{array}$ & $\begin{aligned} 0 & \text { to } 22 \\
22 & \text { to } 87 \\
87 & \text { to } 300\end{aligned}$ & $C-27$ & \\
\hline GU-1C & $\begin{array}{l}0.858 \\
0.179\end{array}$ & $\begin{aligned} 0 & \text { to } 14 \\
14 & \text { to } 300\end{aligned}$ & $C-28$ & \\
\hline GU-10VR & $\begin{array}{l}1.662 \\
0.195\end{array}$ & $\begin{aligned} 0 & \text { to } 14 \\
14 & \text { to } 300\end{aligned}$ & $C-29$ & \\
\hline GU-15T & $\begin{array}{r}2.538 \\
9.604 \\
3.684 \\
7.919 \\
0.858 \\
15.823\end{array}$ & $\begin{array}{r}0 \text { to } 15 \\
15 \text { to } 24 \\
24 \text { to } 67 \\
67 \text { to } 72 \\
72 \text { to } 98 \\
98 \text { to } 300\end{array}$ & $C-30$ & \\
\hline
\end{tabular}


TABLE C-I (continued)

\begin{tabular}{|c|c|c|c|c|}
\hline Measurement & $\begin{array}{c}\text { Random Error } \\
\text { Variance } \\
\sigma_{\mathrm{R}} \\
\end{array}$ & $\begin{array}{l}\text { Period of } \\
\text { Application } \\
\text { (s) } \\
\end{array}$ & Figure & Comments \\
\hline GU-15B & $\begin{array}{r}1.388 \\
4.589 \\
4.301 \\
10.280 \\
1.530 \\
12.972\end{array}$ & $\begin{array}{l}0 \text { to } 15 \\
15 \text { to } 23 \\
23 \text { to } 67 \\
67 \text { to } 72 \\
72 \text { to } 98 \\
98 \text { to } 300\end{array}$ & $C-31$ & \\
\hline GU $-15 C$ & $\begin{array}{r}2.024 \\
7.237 \\
3.648 \\
8.555 \\
2.614 \\
14.535\end{array}$ & $\begin{aligned} 0 & \text { to } 15 \\
15 & \text { to } 24 \\
24 & \text { to } 67 \\
67 & \text { to } 72 \\
72 & \text { to } 98 \\
98 & \text { to } 300\end{aligned}$ & $C-32$ & \\
\hline$G B-23 V R$ & $\begin{array}{r}1.202 \\
10.397 \\
3.71 .7\end{array}$ & $\begin{array}{l}0 \text { to } 19 \\
19 \text { to } 57 \\
57 \text { to } 300\end{array}$ & $C-33$ & \\
\hline GV-COR-150HZ & $\begin{array}{r}4.355 \\
1.086 \\
10.863 \\
13.912 \\
0.720 \\
5.866\end{array}$ & $\begin{array}{r}0 \text { to } 14 \\
14 \text { to } 55 \\
55 \text { to } 59 \\
59 \text { to } 81 \\
81 \text { to } 112 \\
112 \text { to } 300\end{array}$ & $C-34$ & \\
\hline GVLP-165HZ & $\begin{array}{l}3.699 \\
0.735 \\
6.128 \\
9.212 \\
0.743 \\
6.073 \\
1.391 .\end{array}$ & $\begin{array}{r}0 \text { to } 20 \\
20 \text { to } 55 \\
55 \text { to } 59 \\
59 \text { to } 7 \cdot 3 \\
7.3 \text { to } 117 \\
117 \text { to } 239 \\
239 \text { to } 300\end{array}$ & $C-35$ & . \\
\hline GVLP-172HZ & $\begin{array}{l}3.136 \\
8.004 \\
0.284 \\
2.095\end{array}$ & $\begin{aligned} 0 & \text { to } 55 \\
55 & \text { to } 59 \\
59 & \text { to } 121 \\
121 & \text { to } 300\end{aligned}$ & $c-36$ & . \\
\hline FTU-1, GU-1C & $\begin{array}{l}0.689 \\
0.105\end{array}$ & $\begin{aligned} 0 & \text { to } 15 \\
15 & \text { to } 300\end{aligned}$ & $\mathrm{C}-37$ & \\
\hline $\begin{array}{l}\text { FDU-5, } \\
\text { GU-5VR }\end{array}$ & $\begin{array}{l}0.431 \\
0.174\end{array}$ & $\begin{array}{r}0 \text { to } 14 \\
14 \text { to } 42\end{array}$ & $C-38$ & $\begin{array}{l}\text { Only short-term ( } 0 \text { to } \\
42 \text { s.) error bands } \\
\text { reported. }\end{array}$ \\
\hline
\end{tabular}


TABLE.C-I (continued)

\begin{tabular}{|c|c|c|c|c|}
\hline Measurement & $\begin{array}{c}\text { Random Error } \\
\text { Variance } \\
\sigma_{\mathrm{R}} \\
\end{array}$ & $\begin{array}{c}\text { Period of } \\
\text { Application } \\
\text { (s) } \\
\end{array}$ & Figure & Comments \\
\hline $\begin{array}{l}\text { FTU-13, } \\
\text { GU-13VR }\end{array}$ & $\begin{array}{r}0.830 \\
10.854 \\
0.771 \\
9.082\end{array}$ & $\begin{aligned} 0 & \text { to } 23 \\
23 & \text { to } 71 \\
71 & \text { to } 101 \\
101 & \text { to } 300\end{aligned}$ & $C-39$ & $\therefore$ \\
\hline $\begin{array}{l}\text { FTU-15, } \\
\text { GU-15C }\end{array}$ & $\begin{array}{l}0.817 \\
3.098 \\
1.066 \\
5.261\end{array}$ & $\begin{array}{r}0 \text { to } 18 \\
18 \text { to } 70 \\
70 \text { to } 100 \\
100 \text { to } 300\end{array}$ & $C-40$ & ; \\
\hline $\begin{array}{l}\text { FDB-21, } \\
G B-21 C\end{array}$ & $\begin{array}{l}1.349 \\
3.618\end{array}$ & $\begin{array}{r}0 \text { to } 19 \\
19 \text { to } 42\end{array}$ & $C-41$ & $\begin{array}{l}\text { Only short-term ( } 0 \text { to } \\
42 \text { s) error bands } \\
\text { reported. }\end{array}$ \\
\hline $\begin{array}{l}\text { FTB-21, } \\
\text { GB-21C }\end{array}$ & $\begin{array}{l}1.790 \\
2.318 \\
0.926 \\
0.510\end{array}$ & $\begin{array}{c}0 \text { to } 14 \\
14 \text { to } 79 \\
79 \text { to } 177 \\
177 \text { to } 300\end{array}$ & $C-42$ & \\
\hline $\begin{array}{l}\text { FTV-CORE-IN, } \\
\text { GV-COR-150HZ }\end{array}$ & $\begin{array}{l}1.255 \\
7.915\end{array}$ & $\begin{aligned} 0 & \text { to } \\
57 & \text { to } 300\end{aligned}$ & $C-43$ & 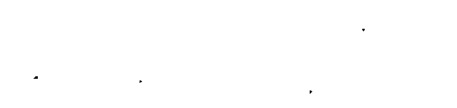 \\
\hline \multicolumn{5}{|c|}{$\begin{array}{l}\text { [a] Error bands are not represented in this region. The sensor gave } \\
\text { a constant reading due to one of the following: (a) sensor } \\
\text { saturation, (b) sensor dropout, or (c) sensor deadband. Random } \\
\text { error in measurement cannot be modeled under these conditions. }\end{array}$} \\
\hline
\end{tabular}




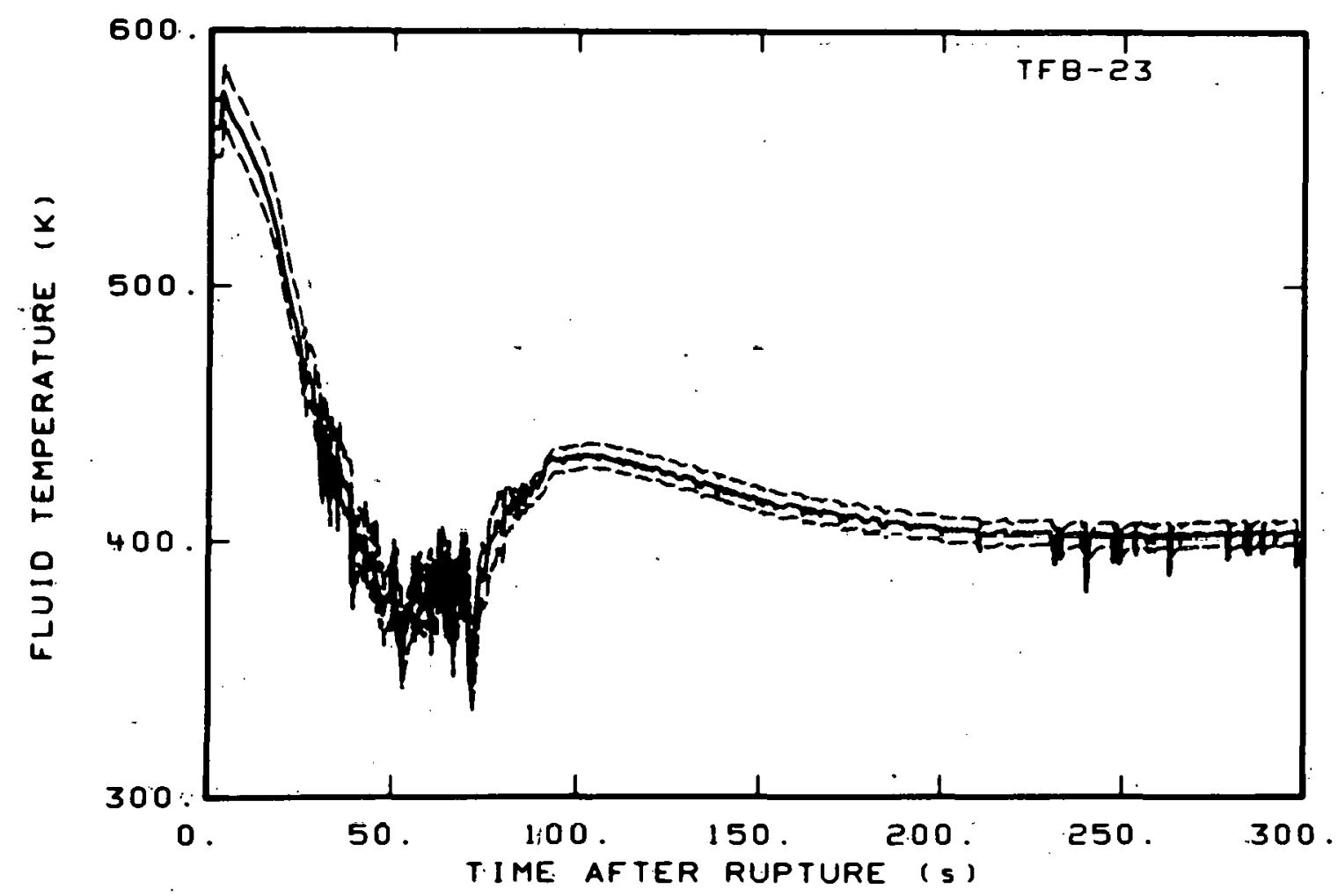

Fig. C-1 Fluid temperature in broken loop (TFB-23).

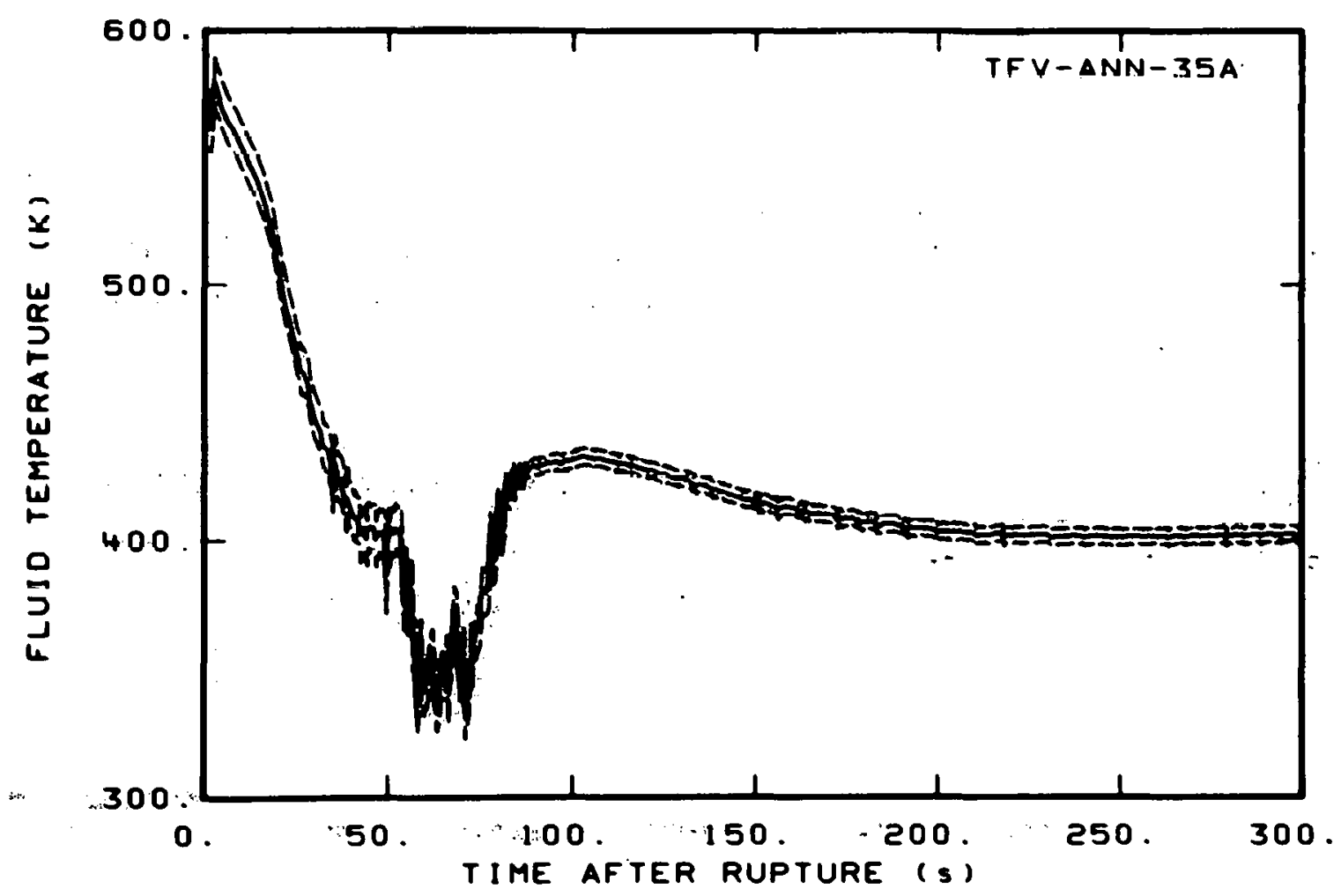

Fig. C-2 Fluid temperature in downcomer annulus (TFV-ANN-35A). 


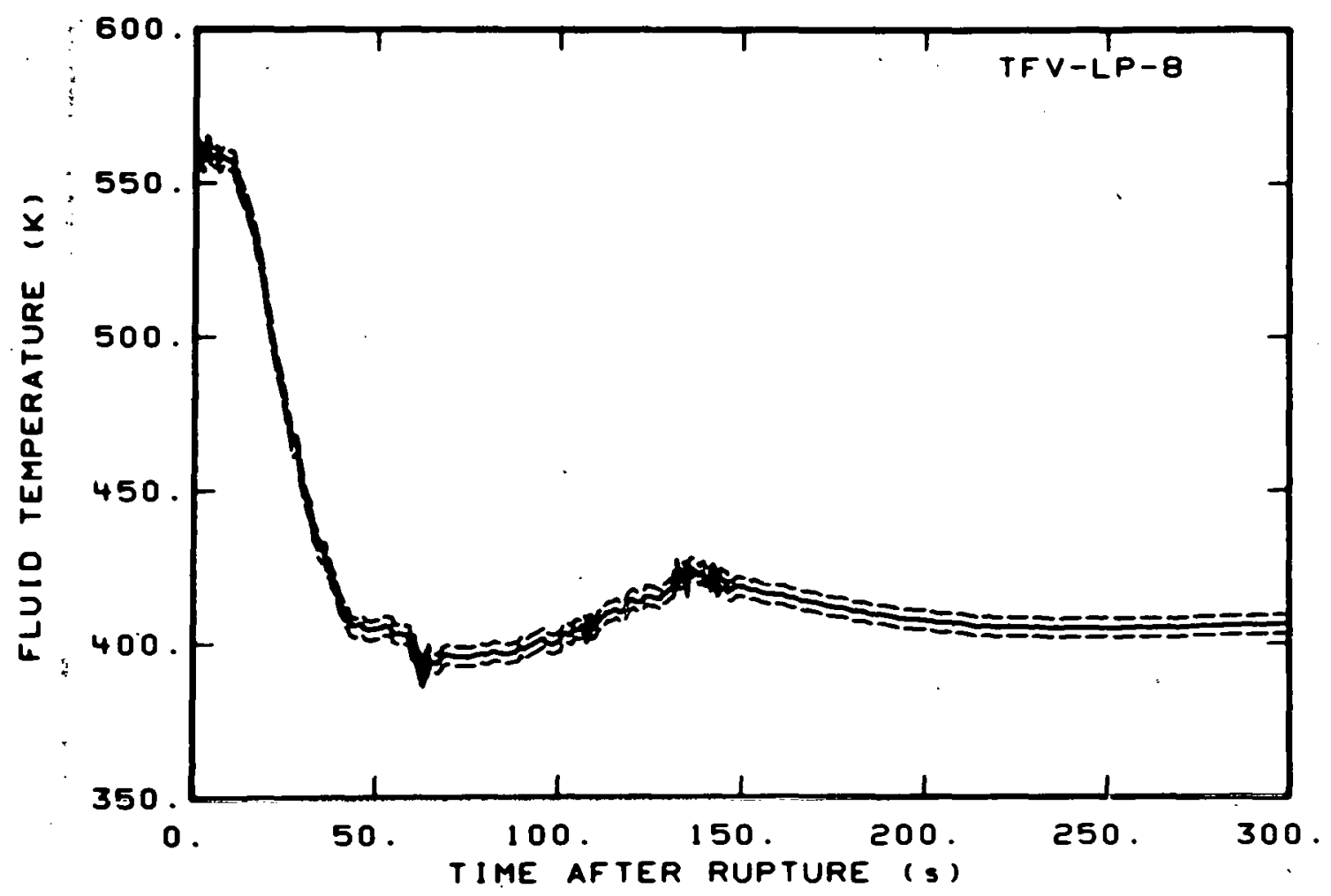

Fig. C-3 Fluid temperature in lower plenum (TFV-LP-8).

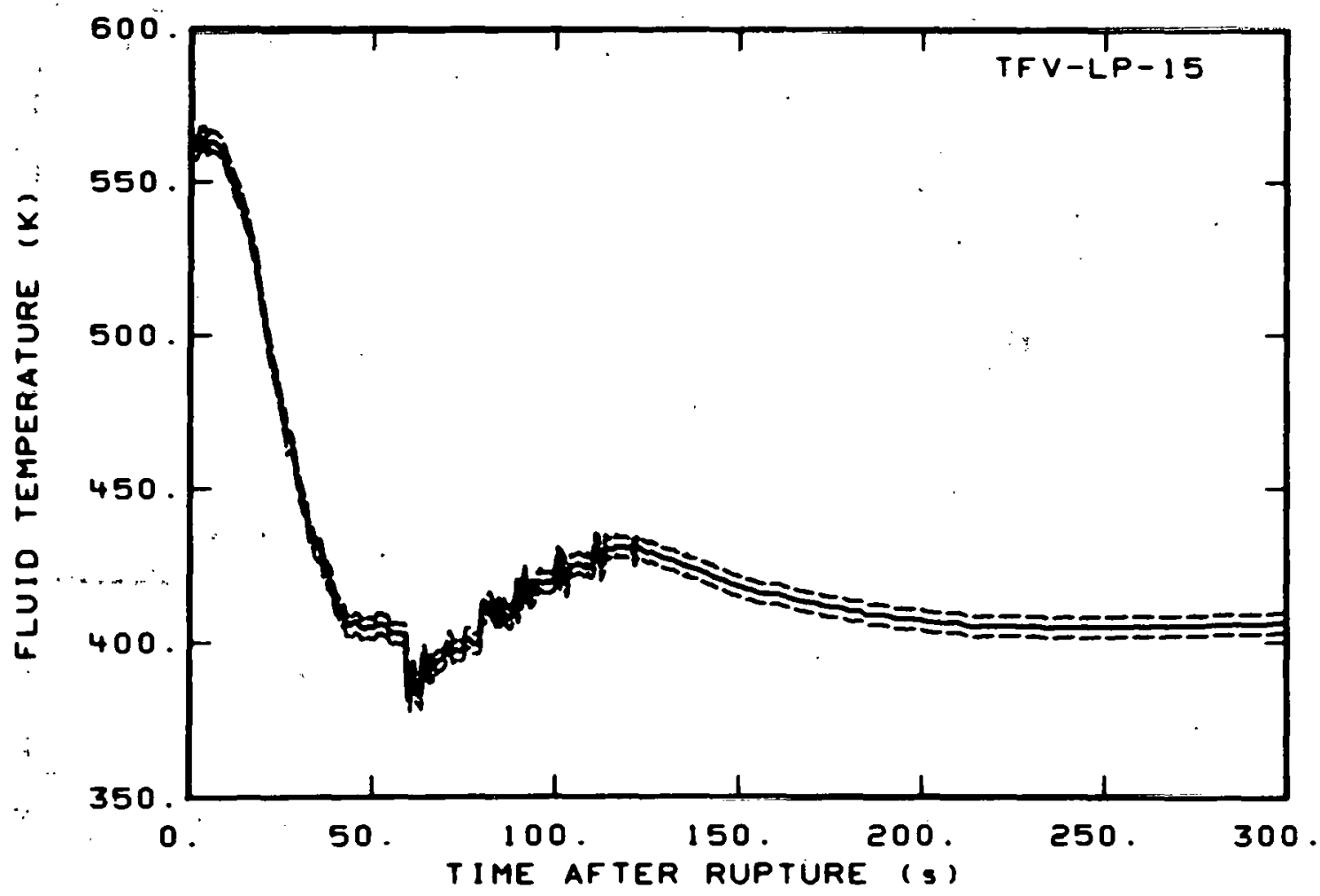

Fig. C-4 Fluid temperature in lower plenum (TFV-LP-15). 


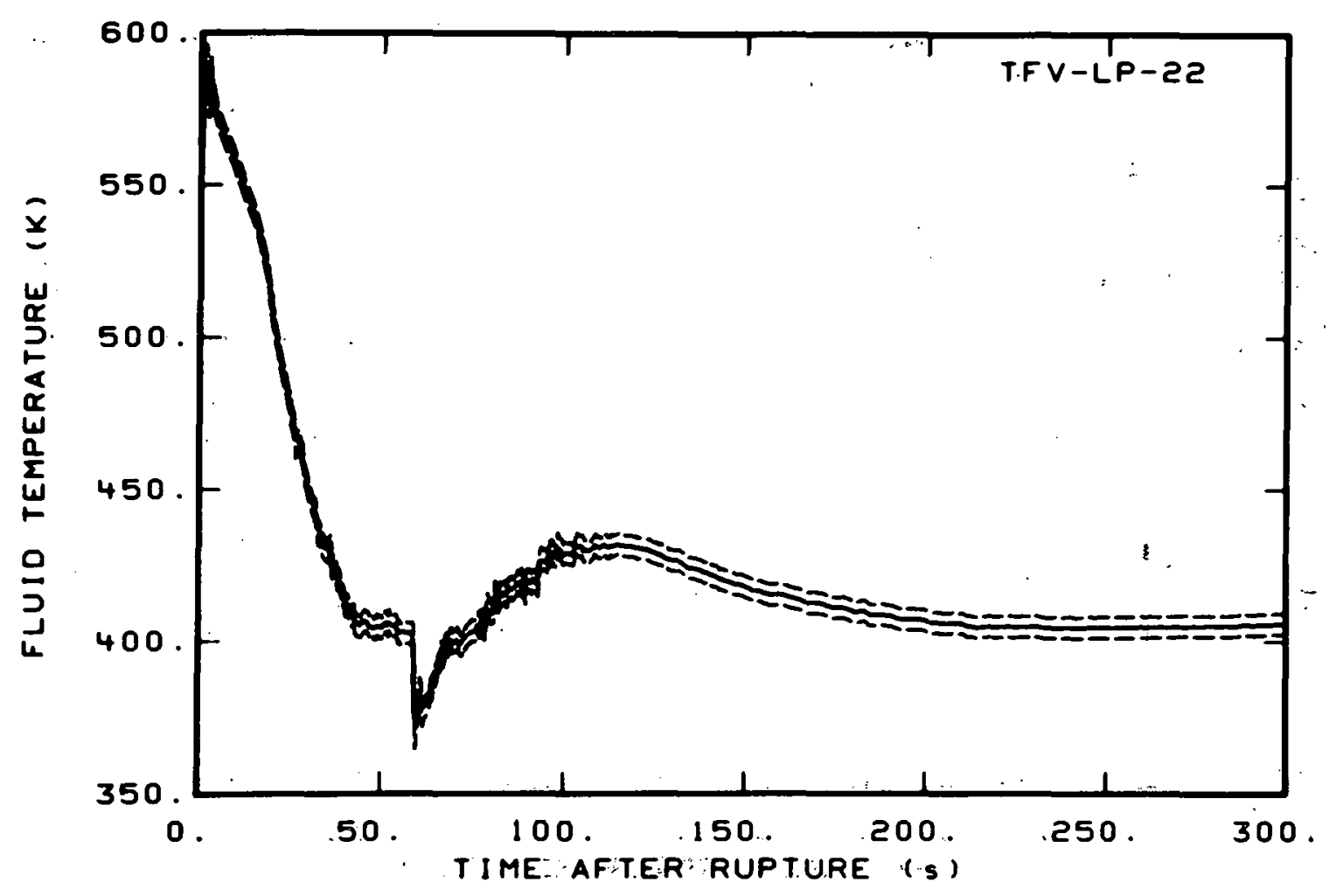

Fig. C-5 Fluid temperature in lower plenum (TFV-LP-22).

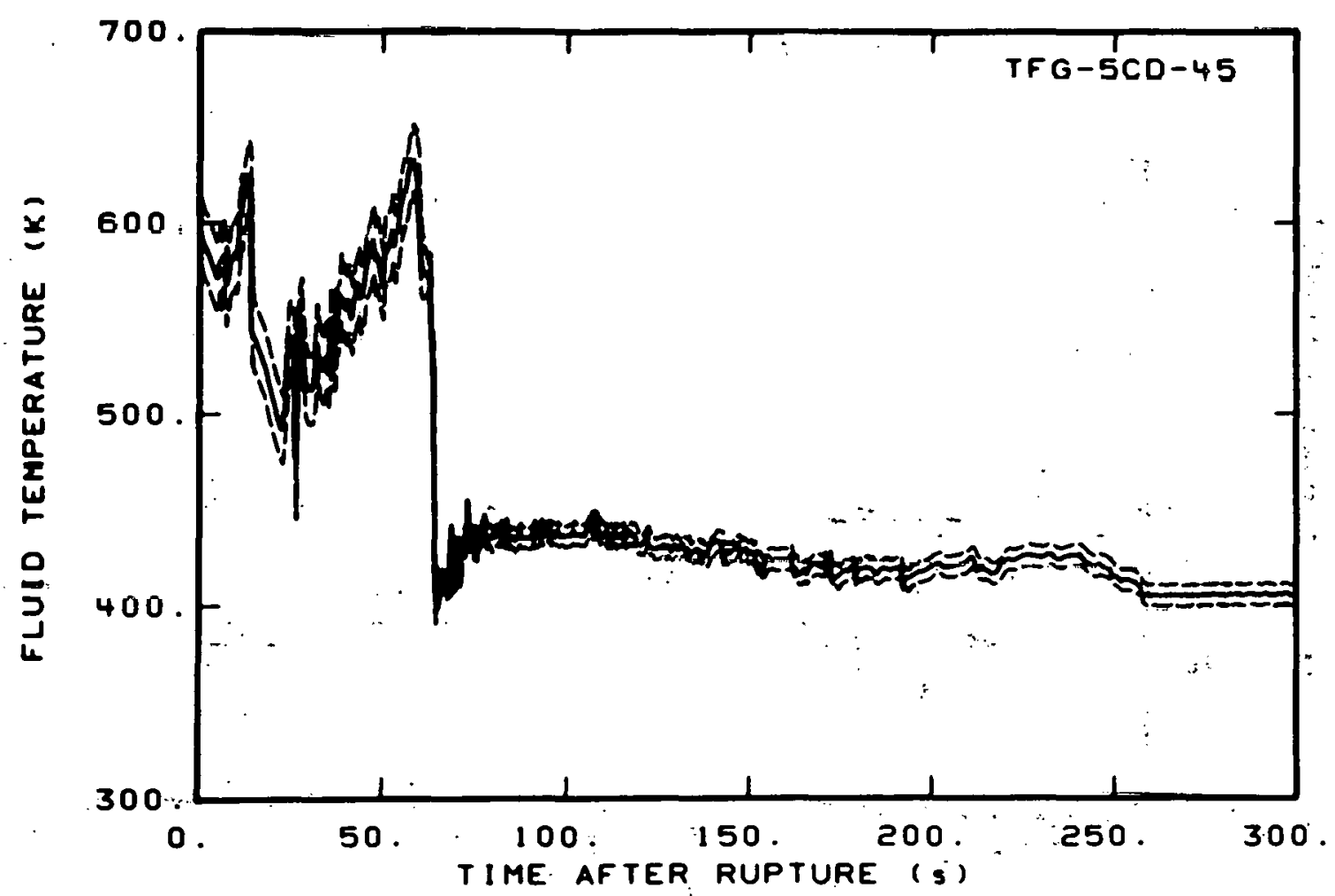

Fig. C-6 Fluid temperature in core, Grid spacer: 5 . (TFG-5CD-45). 


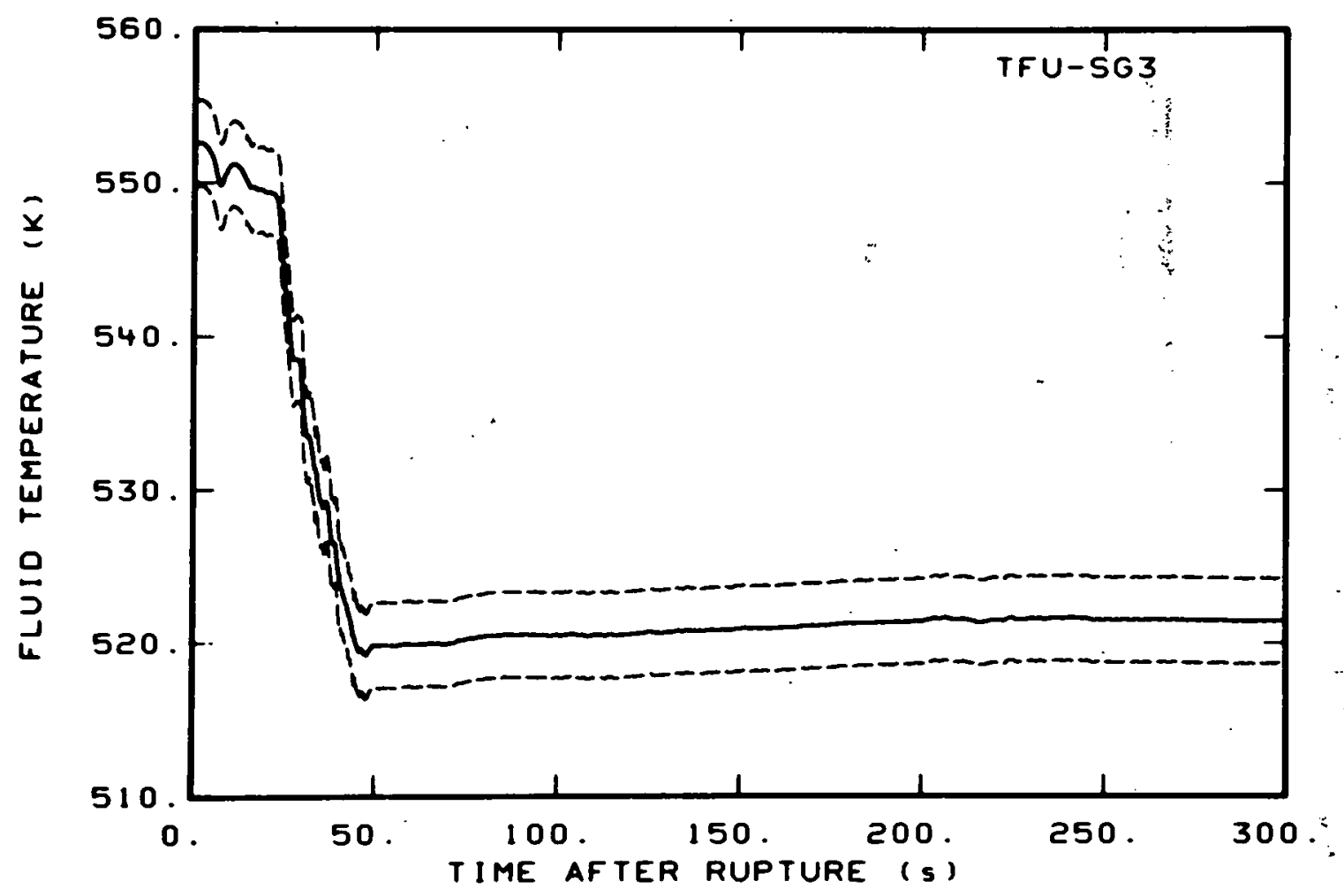

Fig. C-7 Fluid temperature in steam generator (TFU-SG3).

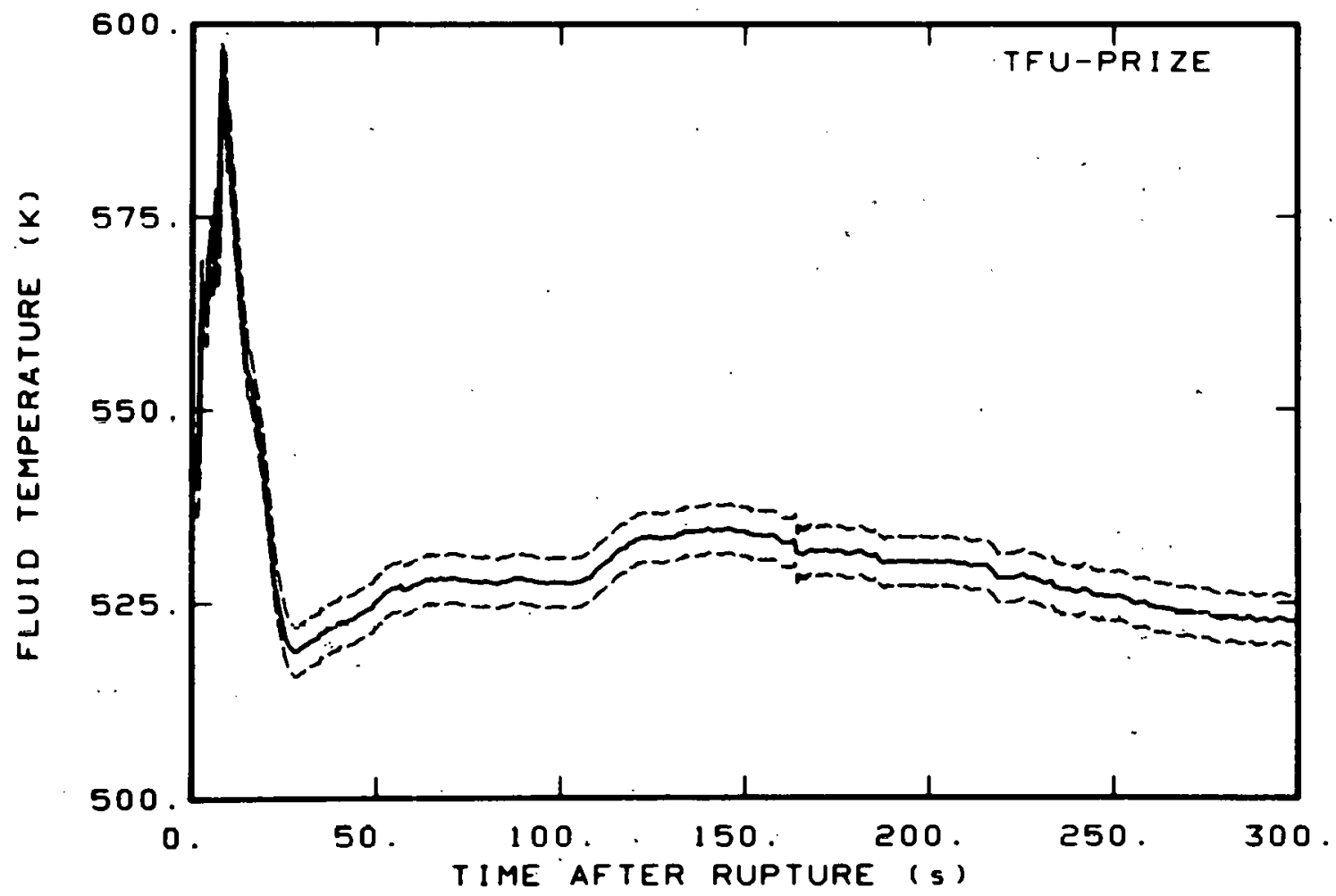

Fig. C-8 Fluid temperáture in pressuriżer surge line (TFU-PRIZE). 


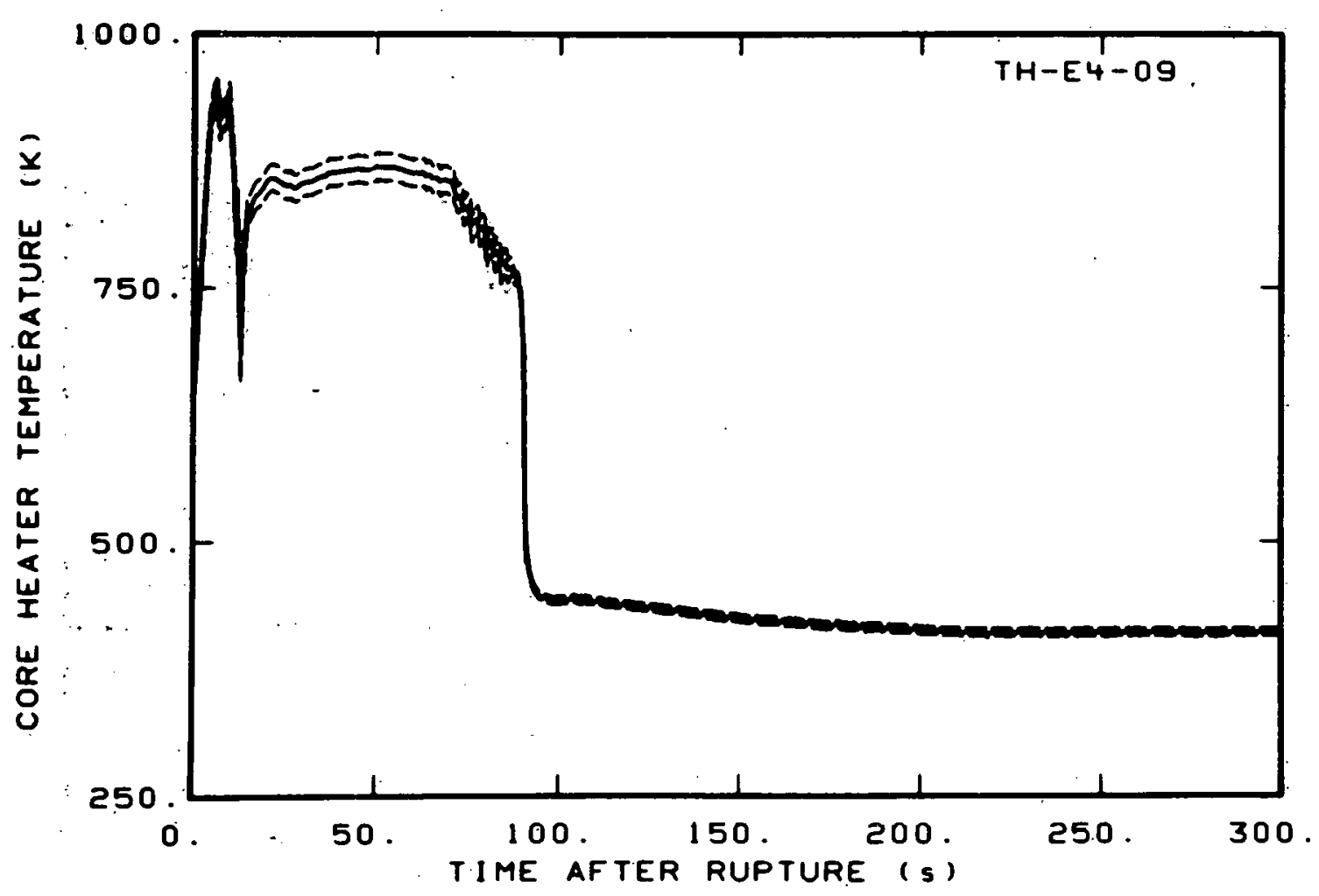

Fig. C-9 Core heater temperature, Rod E-4 (TH-E-4-09).

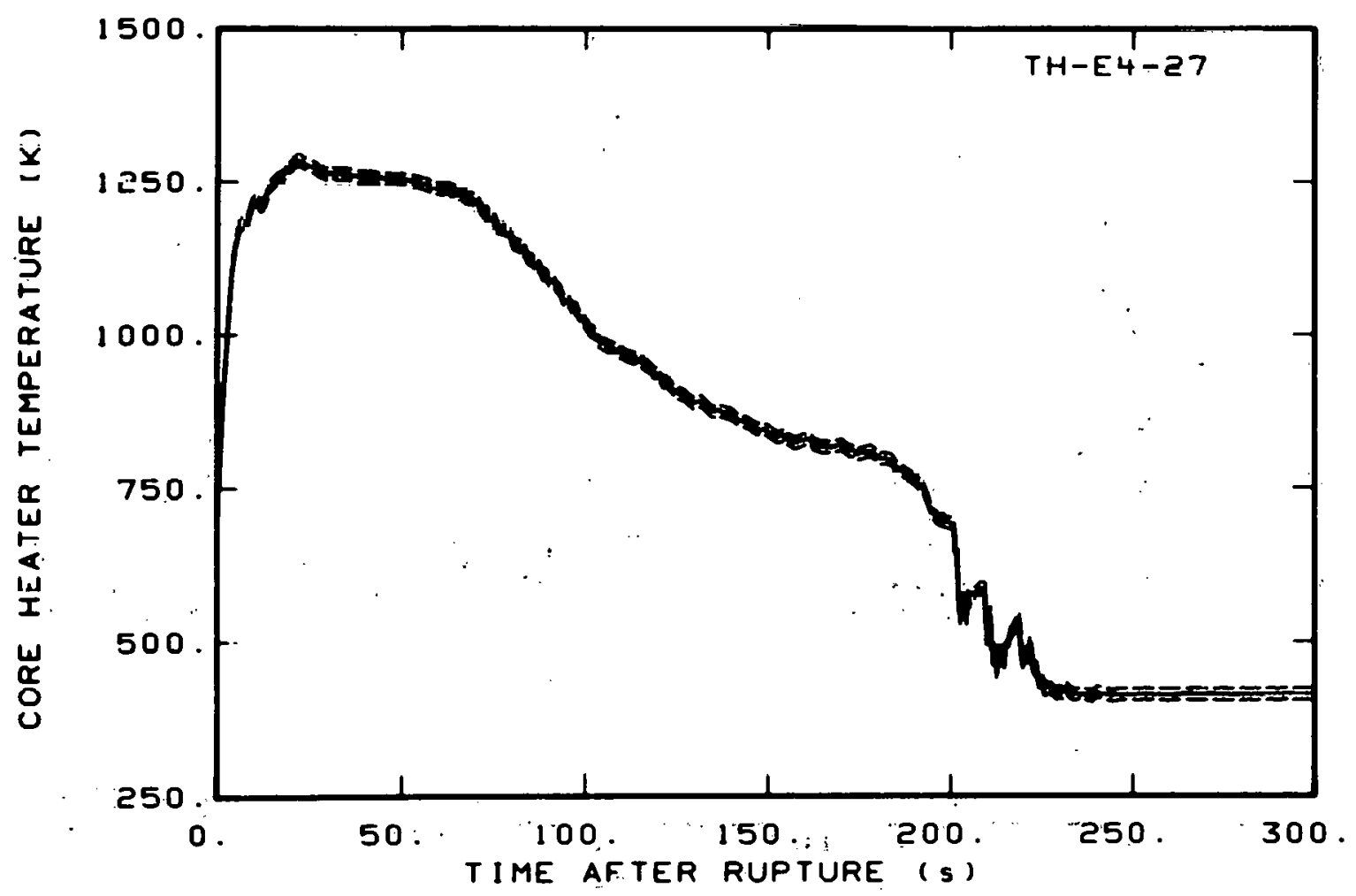

Fig. C-10 Core heater temperature, Rod E-4 (TH-E4-27). 


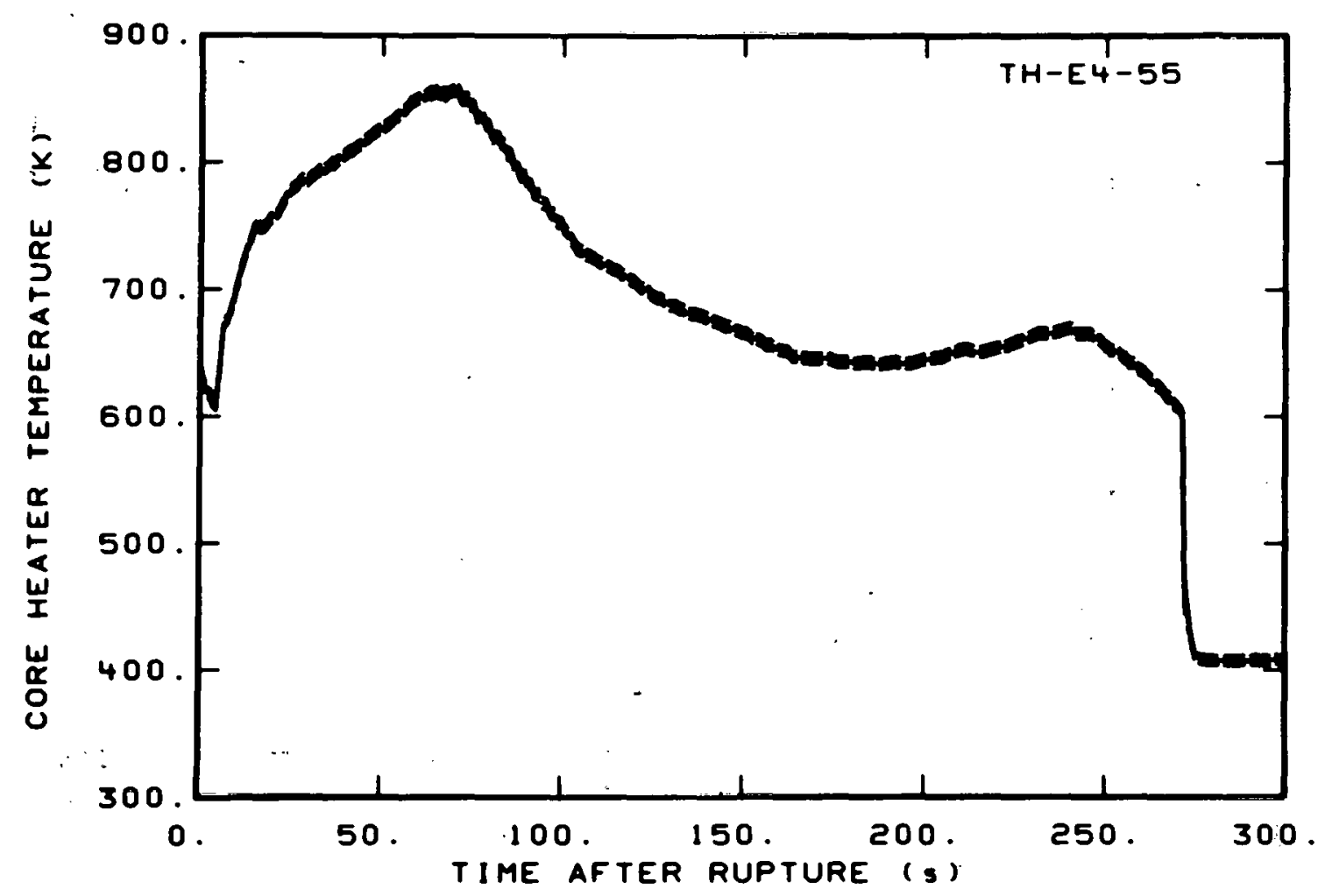

Fig. C-11 Core heater temperature, Rod E-4 (TH-E4-55).

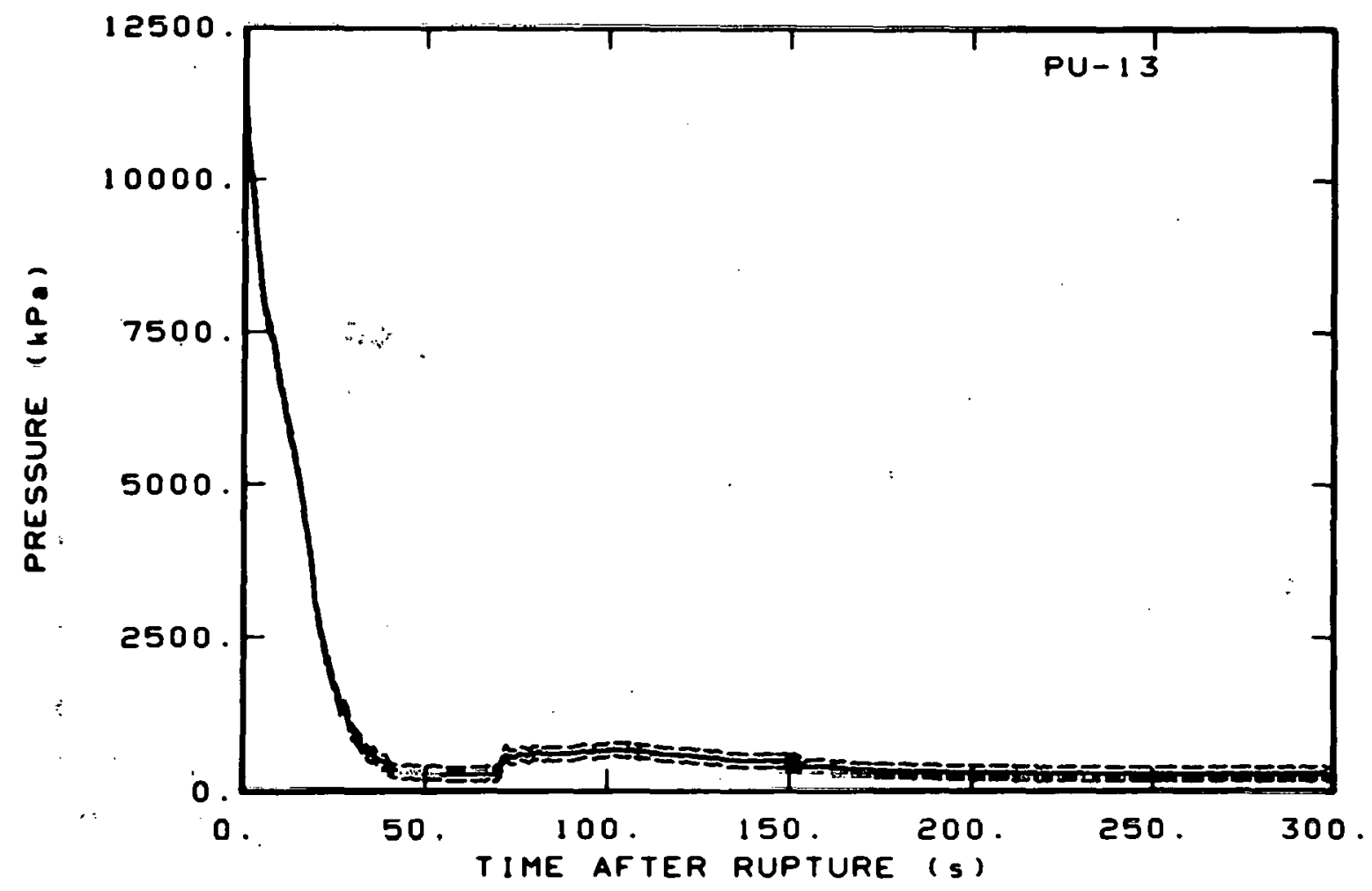

Fig. C-12 Pressure in intact 10op, Spool 13 (PU-13). 


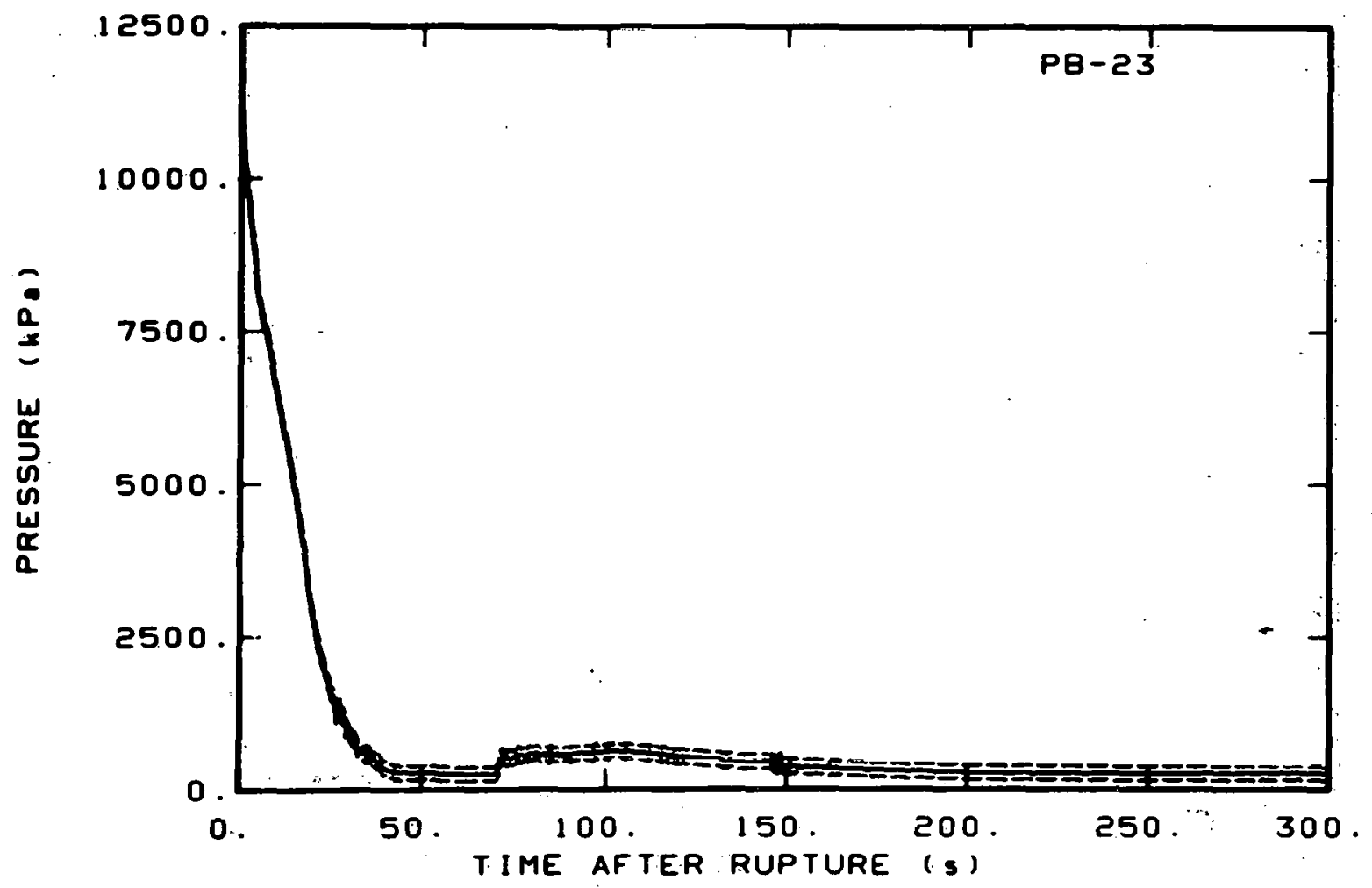

Fig. C-13 Pressure in broken loop, Spool 23 (PB-23).

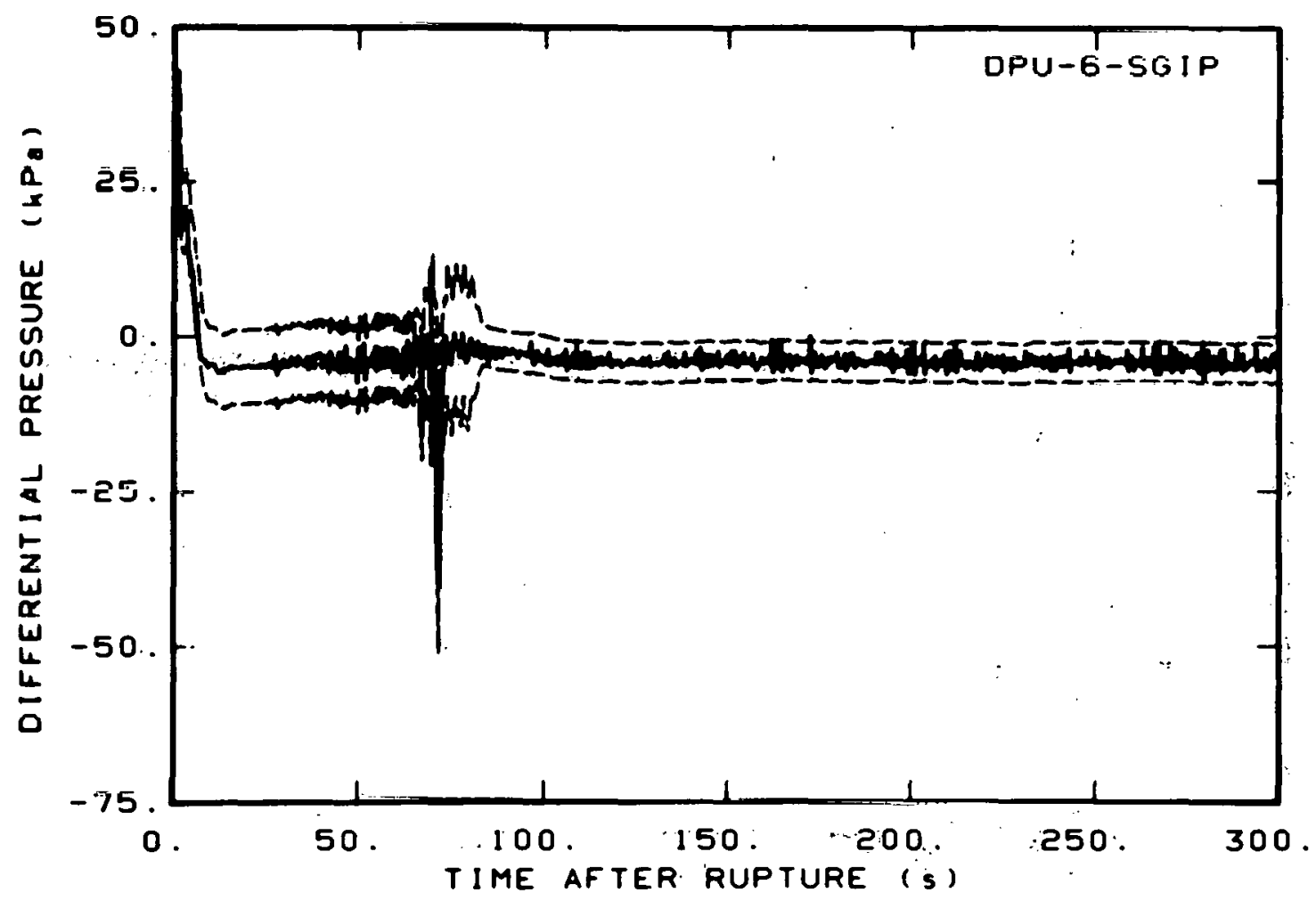

Fig. C-14 Differential pressure in intact loop (DPU-6-SGIP). 


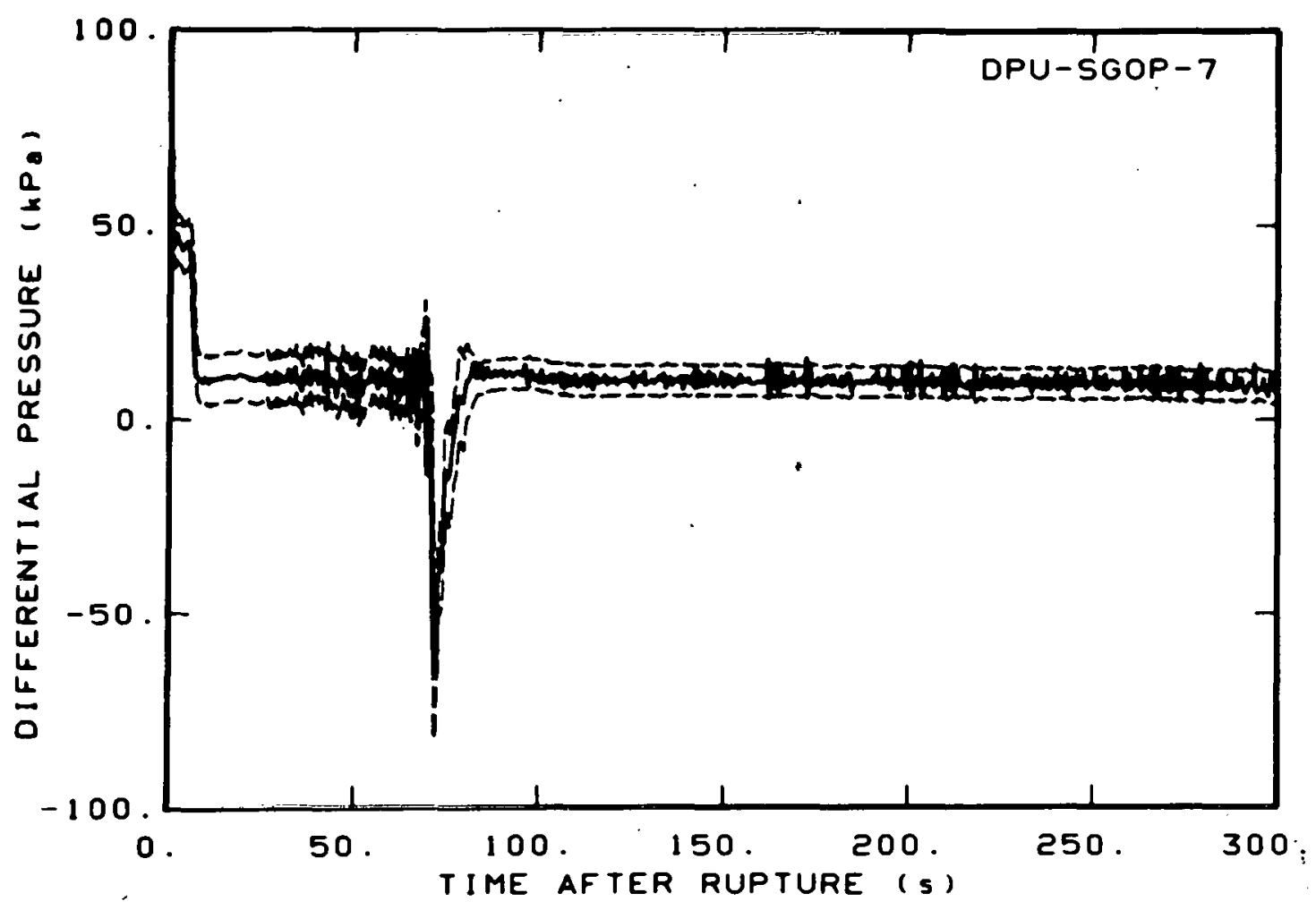

Fig. C-15 Differential pressure in intact loop (DPU-SGOP-7).

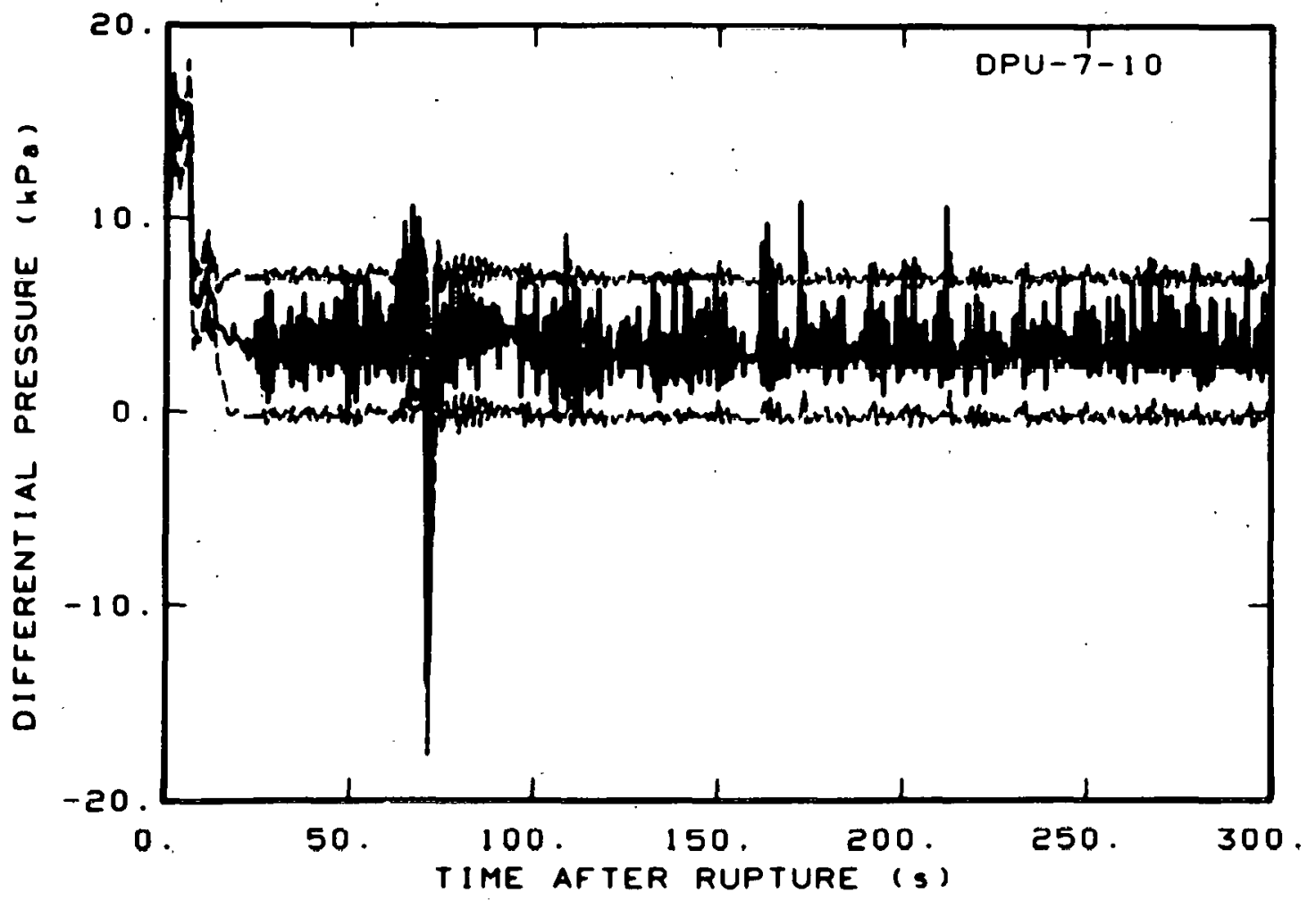

Fig. C-16 Differential pressure in intact loop (DPU-7-10). 


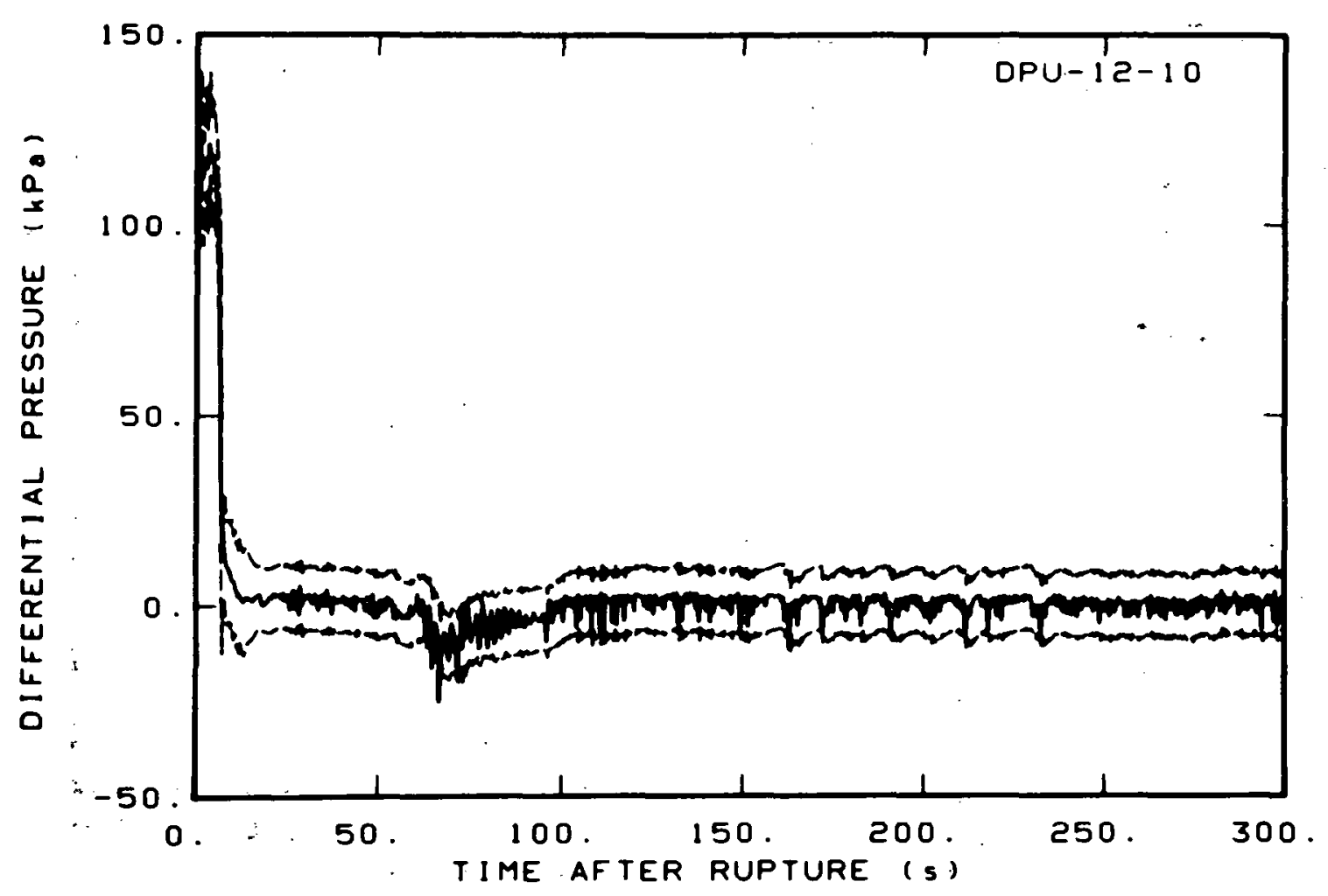

Fig. C-17 Differential pressure in intact loop (DPU-12-10).

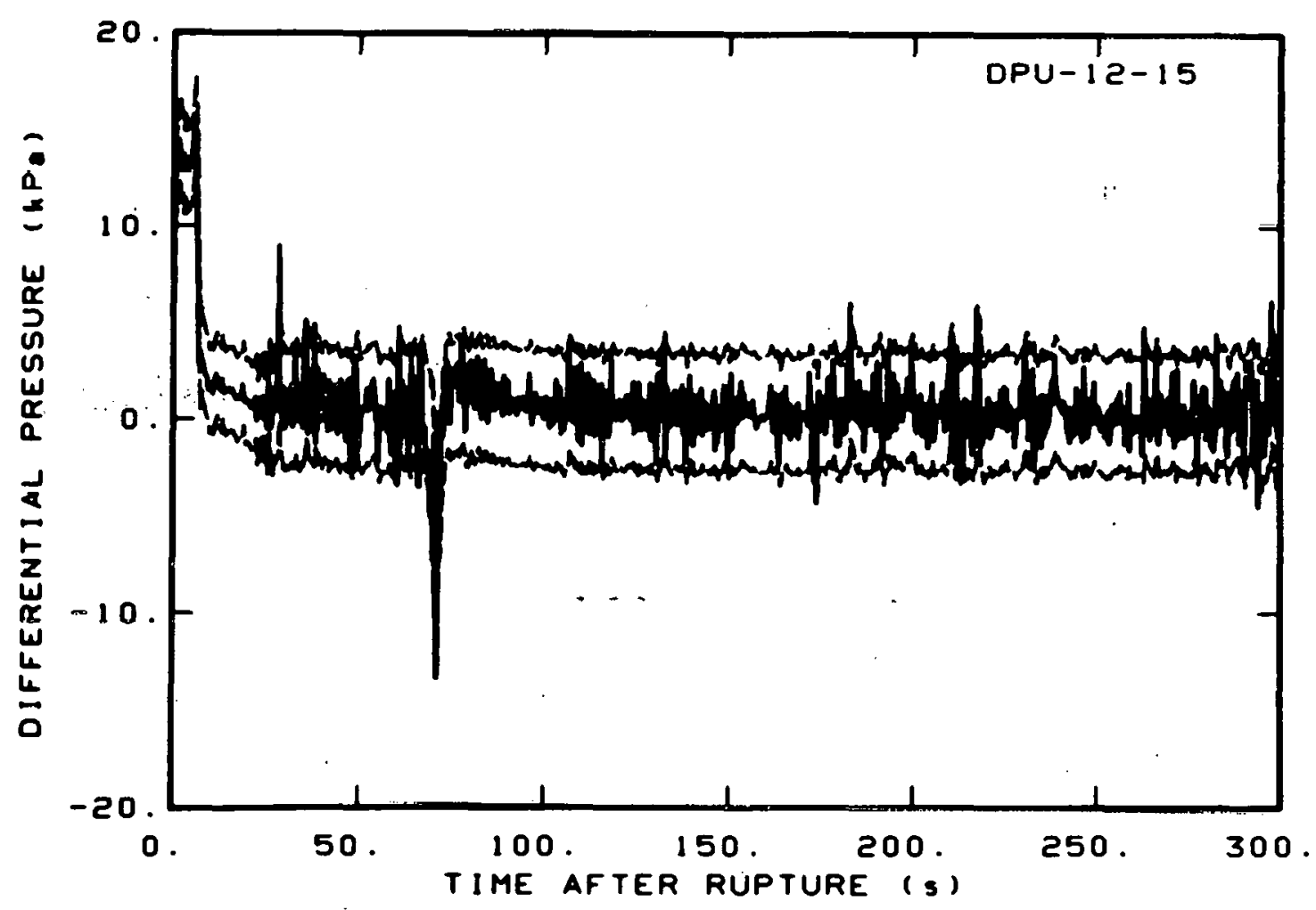

Fig. C-18 Differential pressure in intact loop (DPU-12-15). 


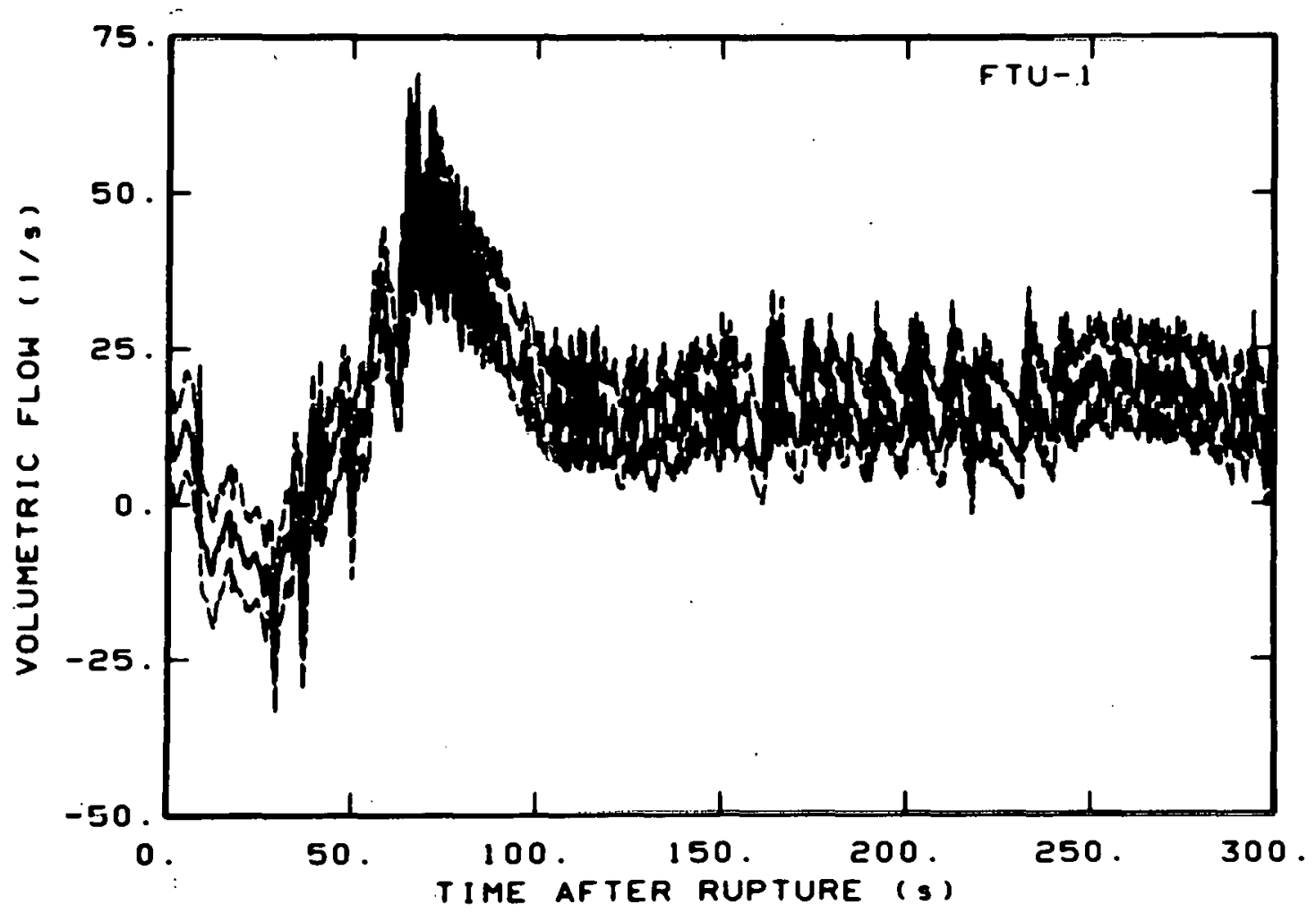

Fig. C-19 Volumetric flow in intact loop (FTU-1).

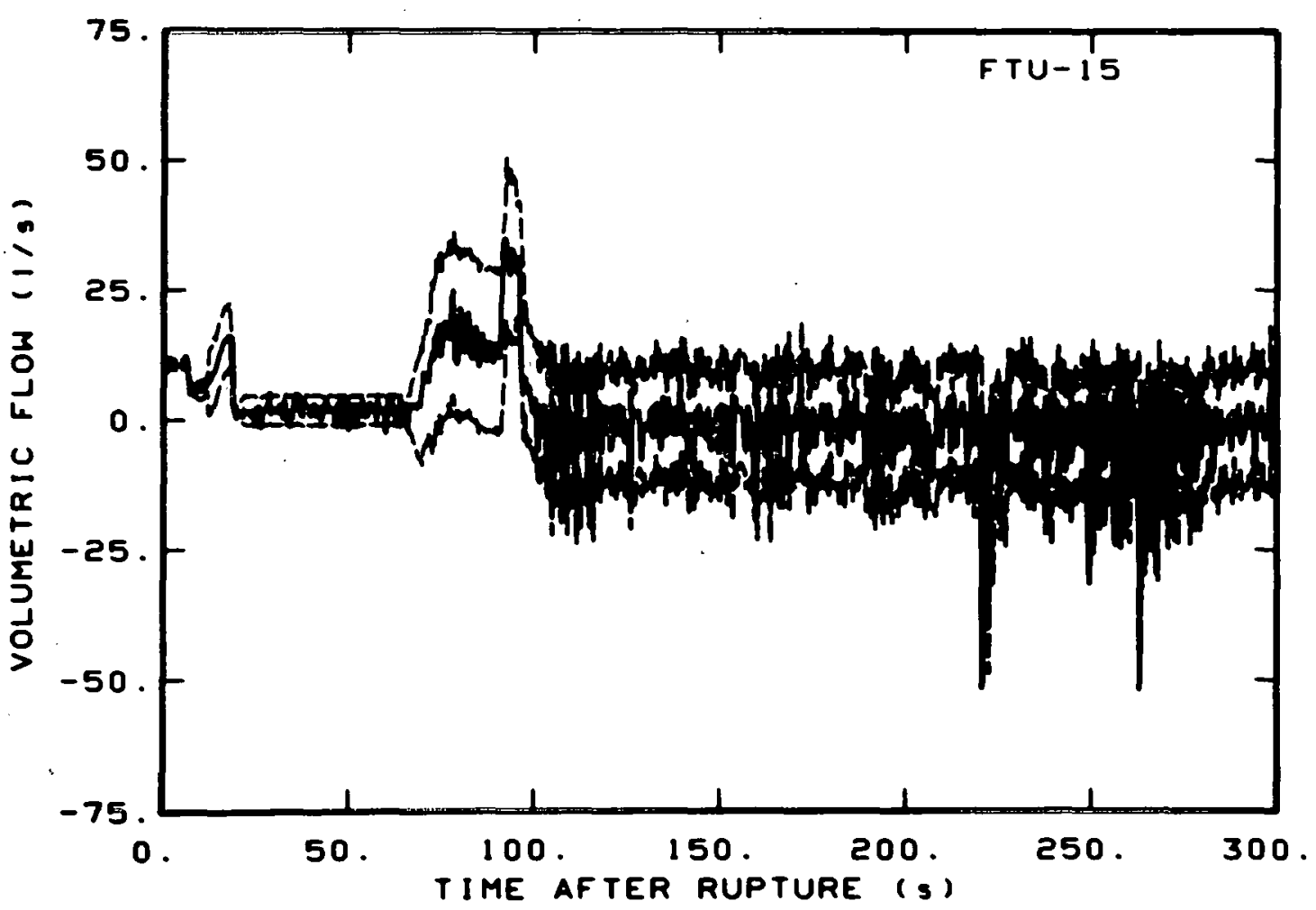

Fig. C-20 Volumetric flow in intact loop (FTU-15). 


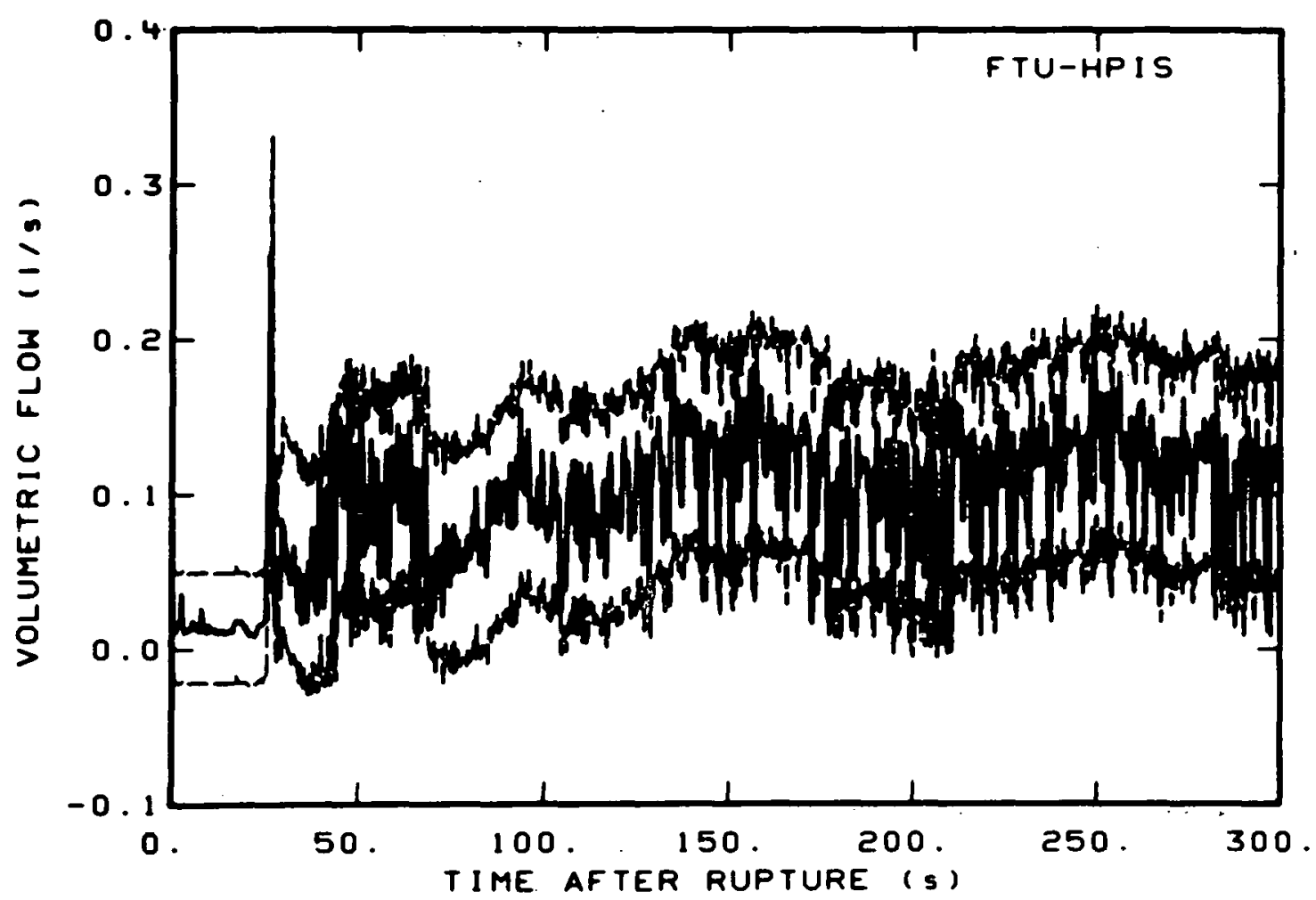

Fig. C-21 Volumetric flow in intact loop high pressure injection line 1 (FTU-HPIS).

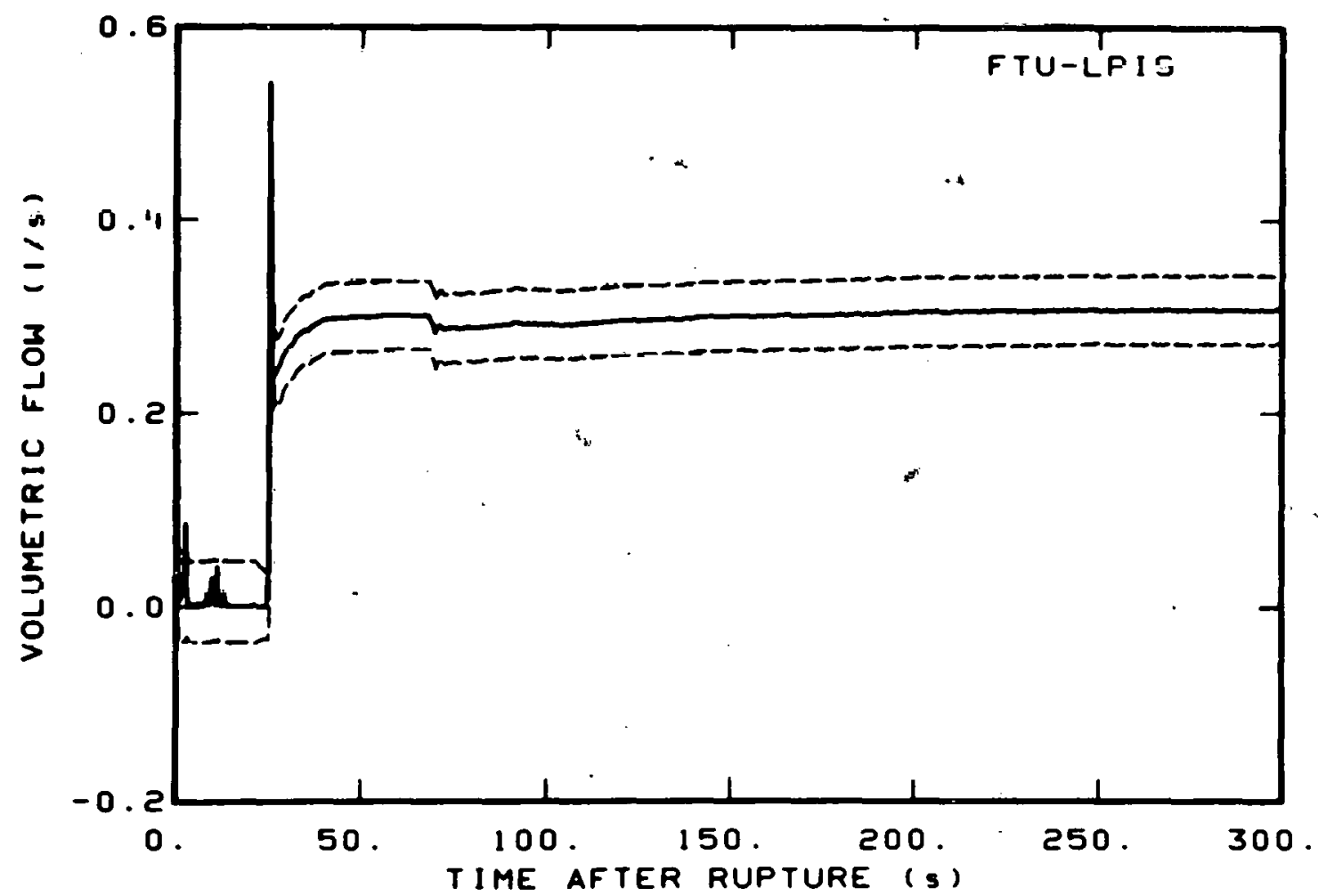

Fig. C-22 Volumetric flow in intact loop low pressure injection line (FTU-LPIS). 


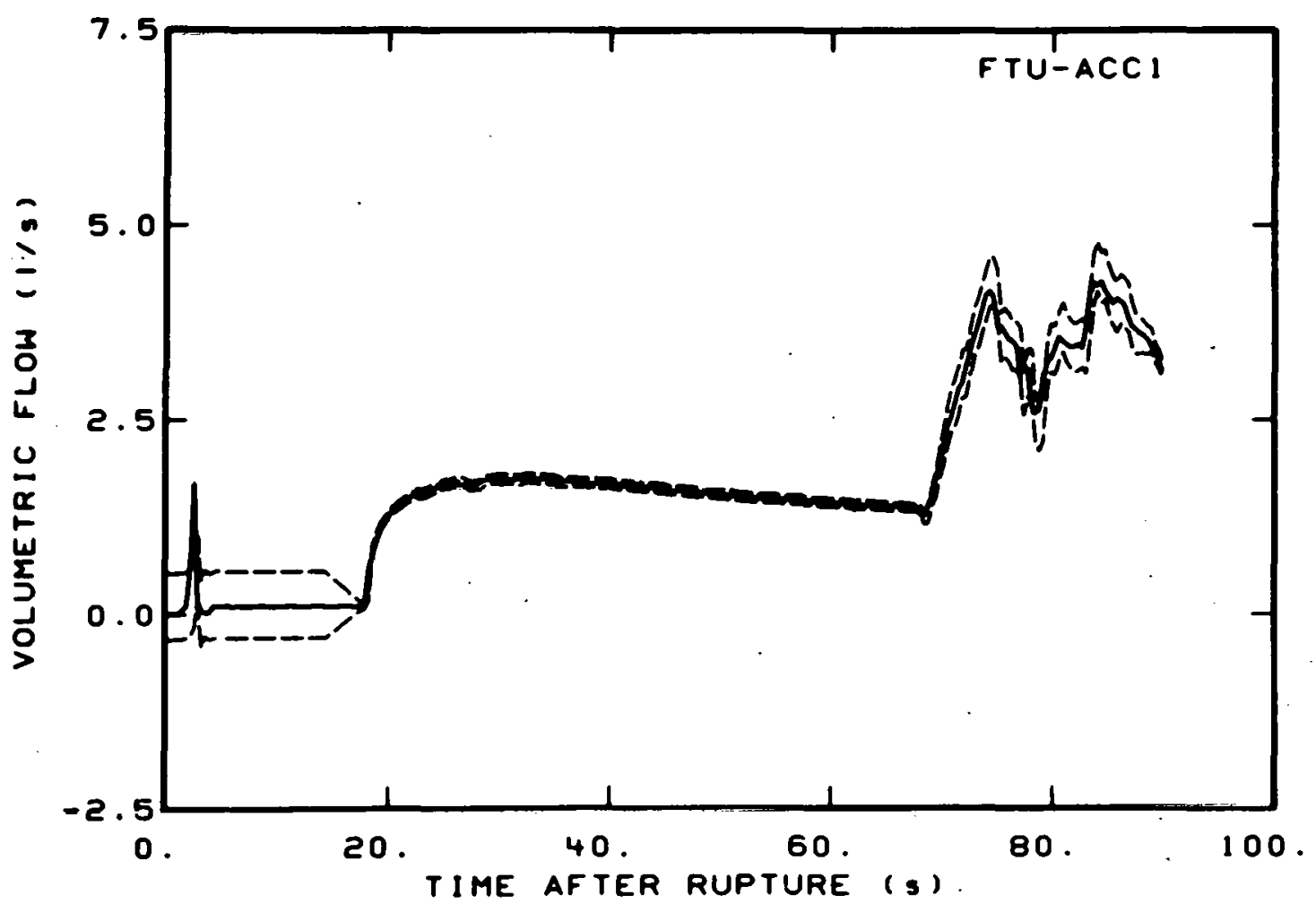

Fig. C-23 Volumetric flow in intact loop accumulator discharge line: (FTU-ACCI).

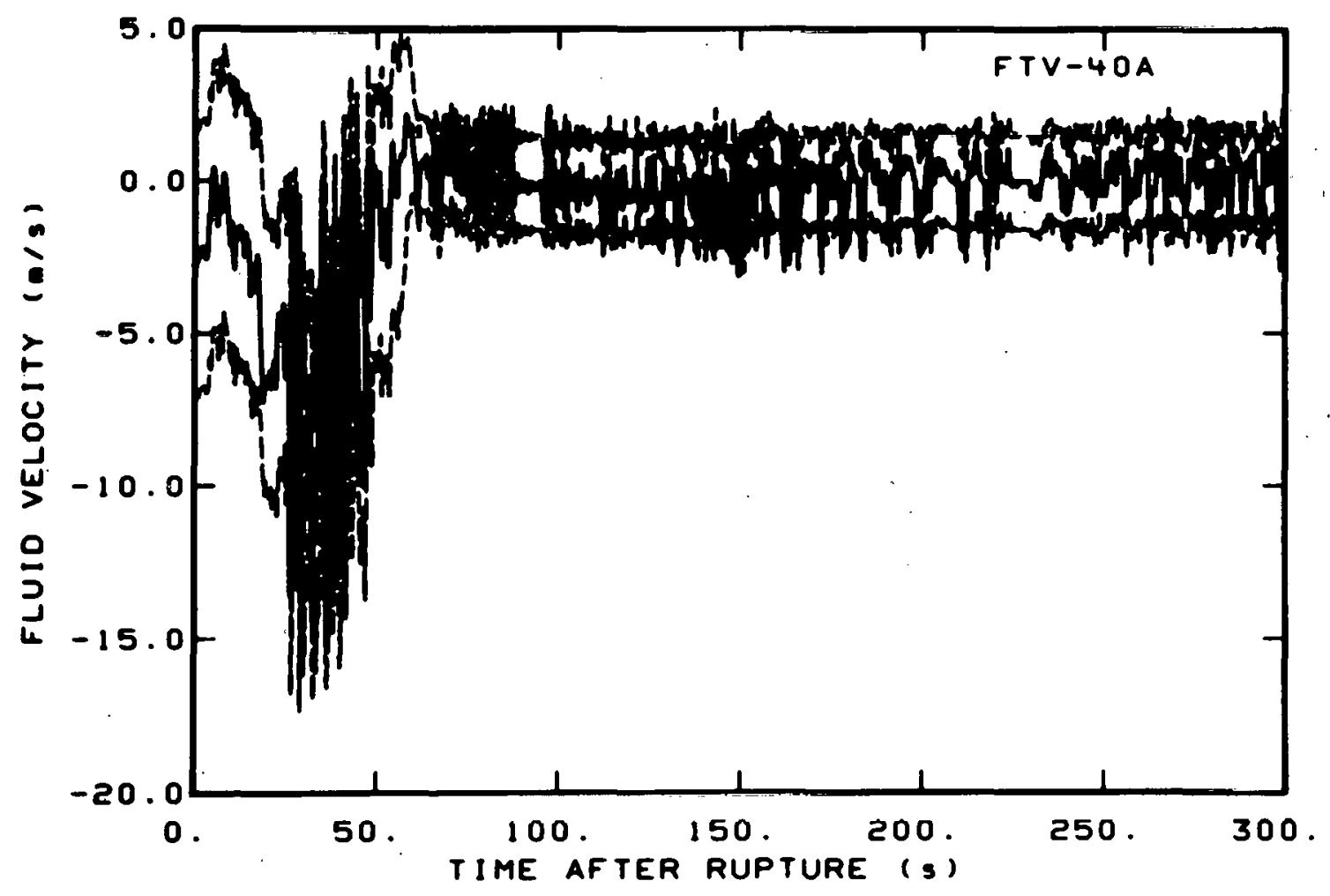

Fig. C-24 Fluid velocity in vesset (FTV-40A). 


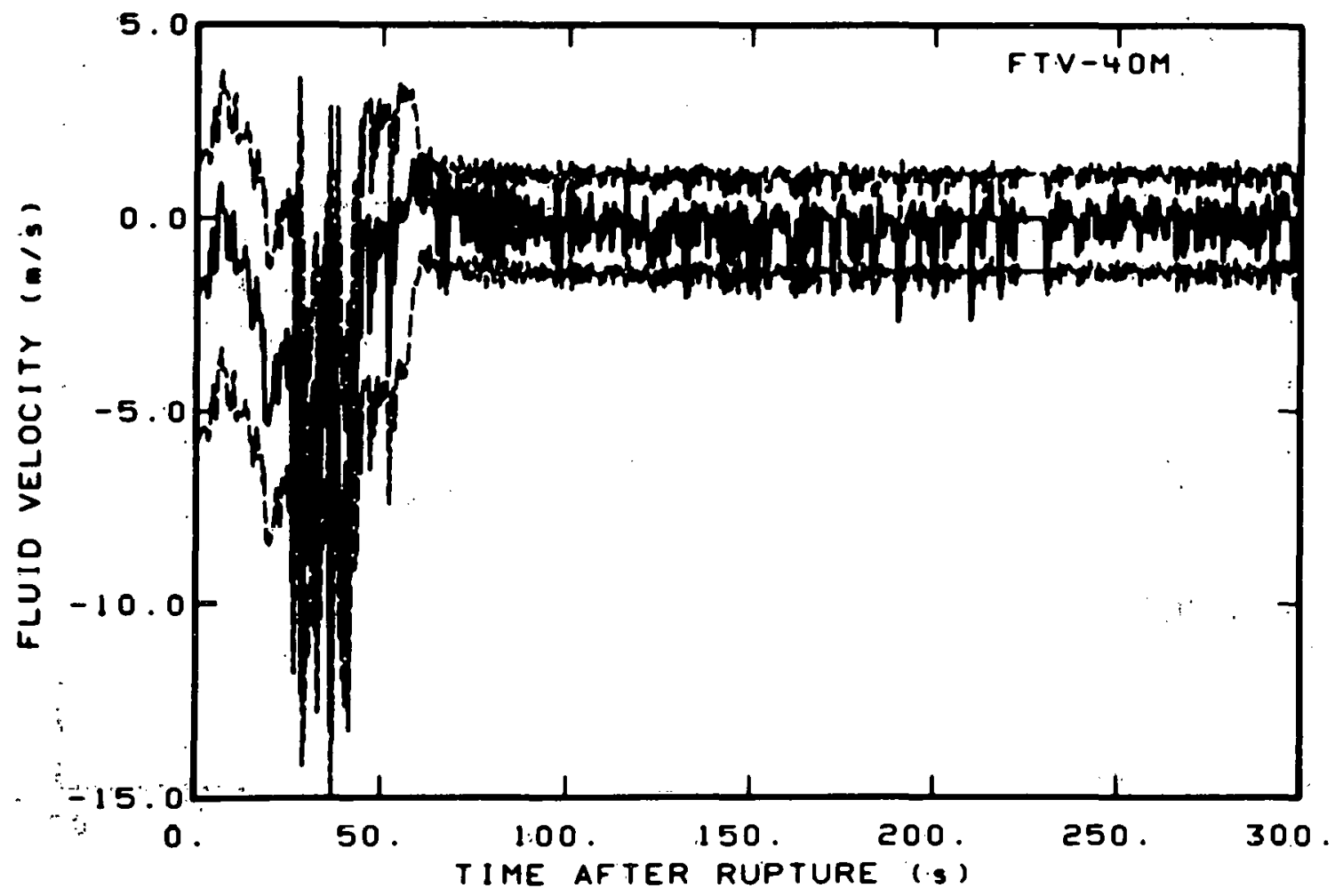

Fig. C-.25 Fluid velocity in vessel (FTV-40M).

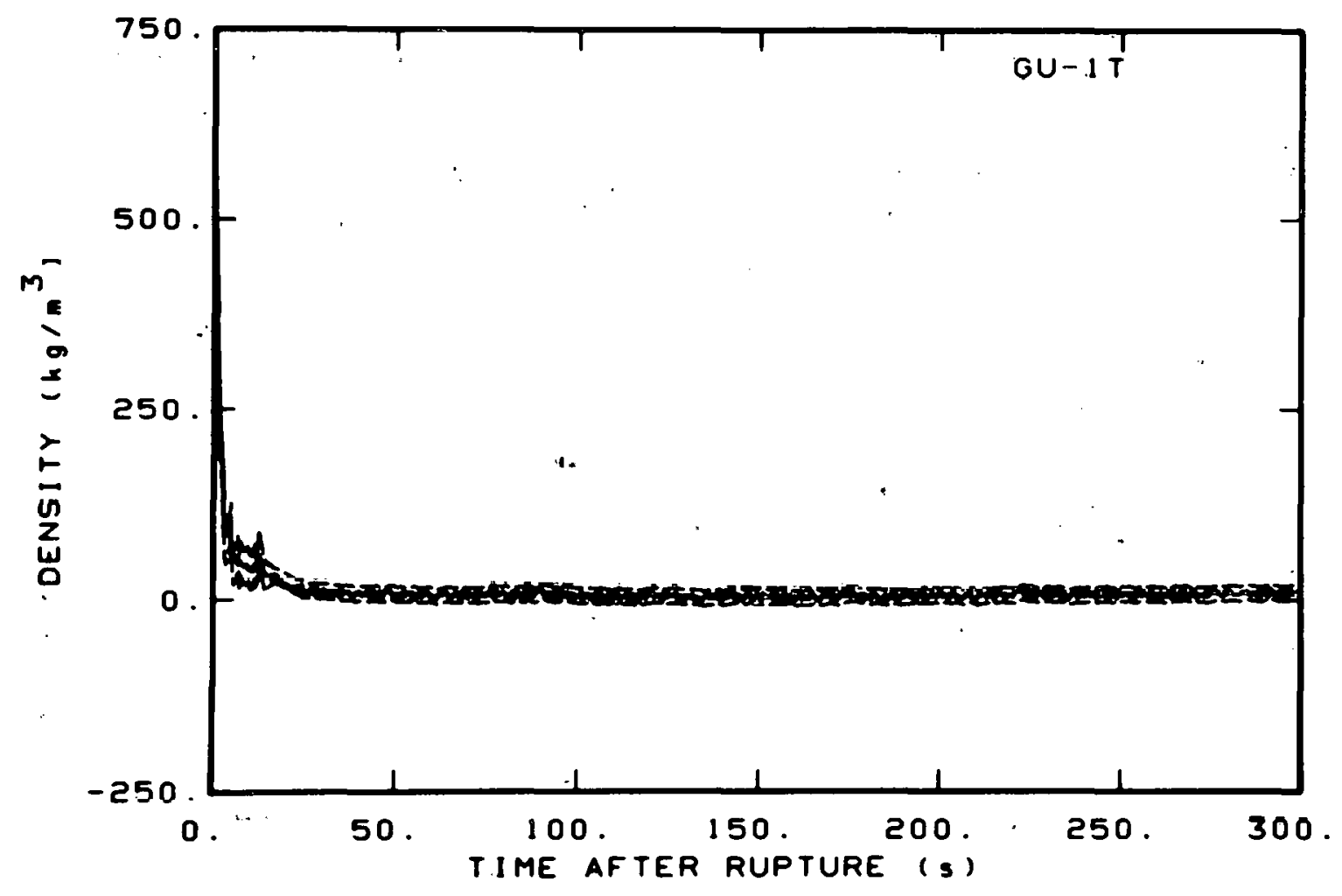

Fig. C-26 Density in intact 1oop (GU-1T). 


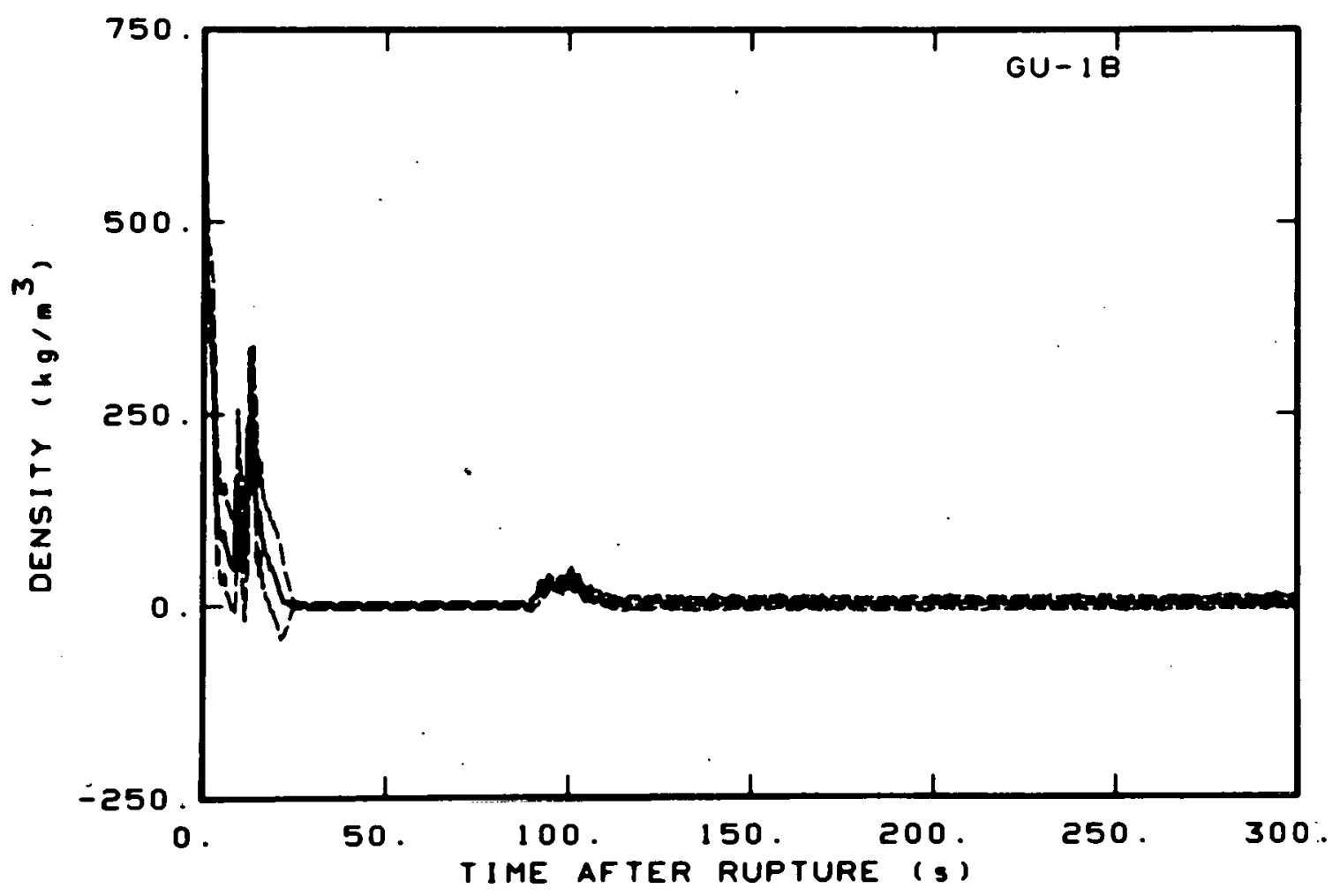

Fig. C-27 Density in intact loop (GU-IB).

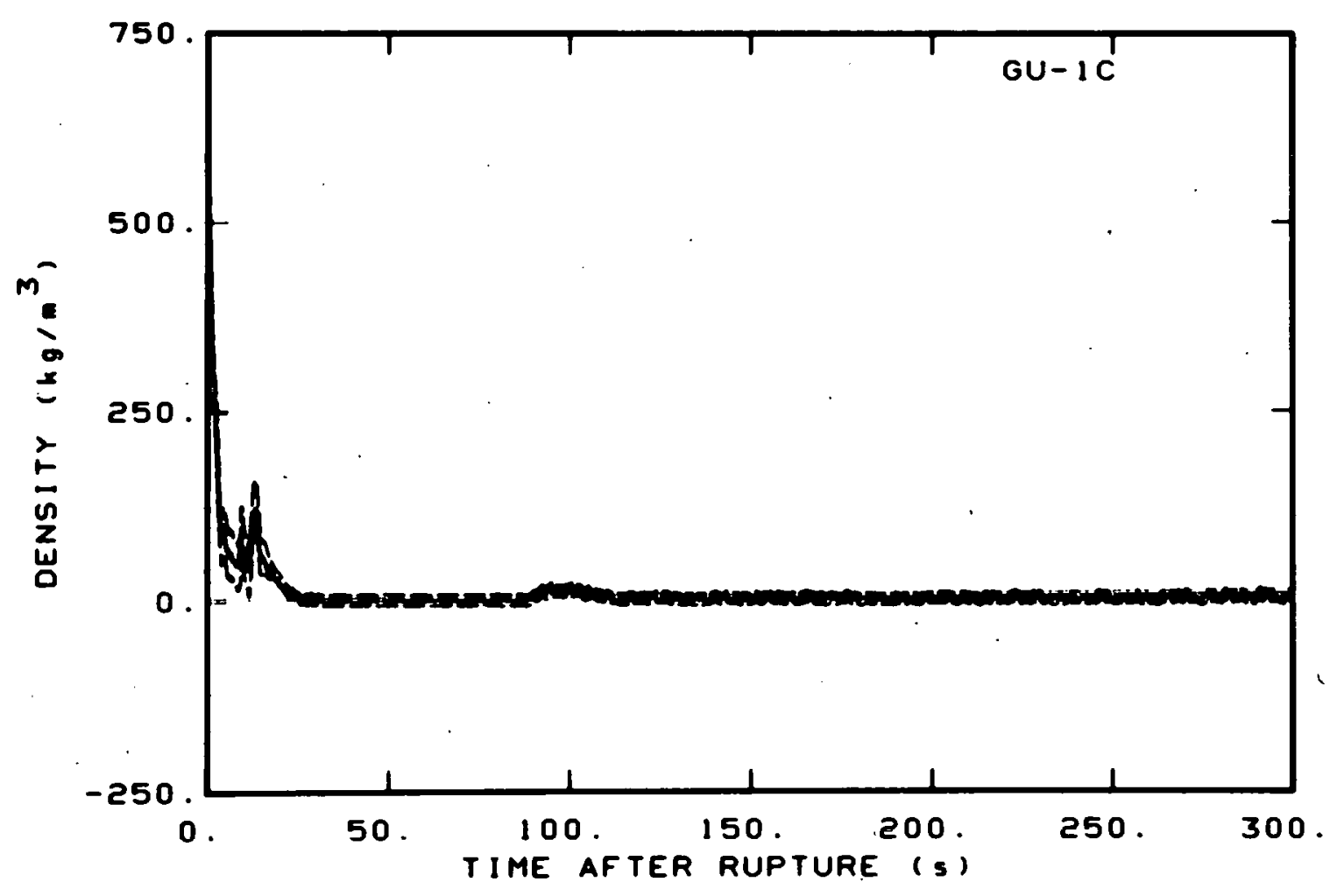

Fig. C-28 Denstiy in intact 10op (GII-1C). 


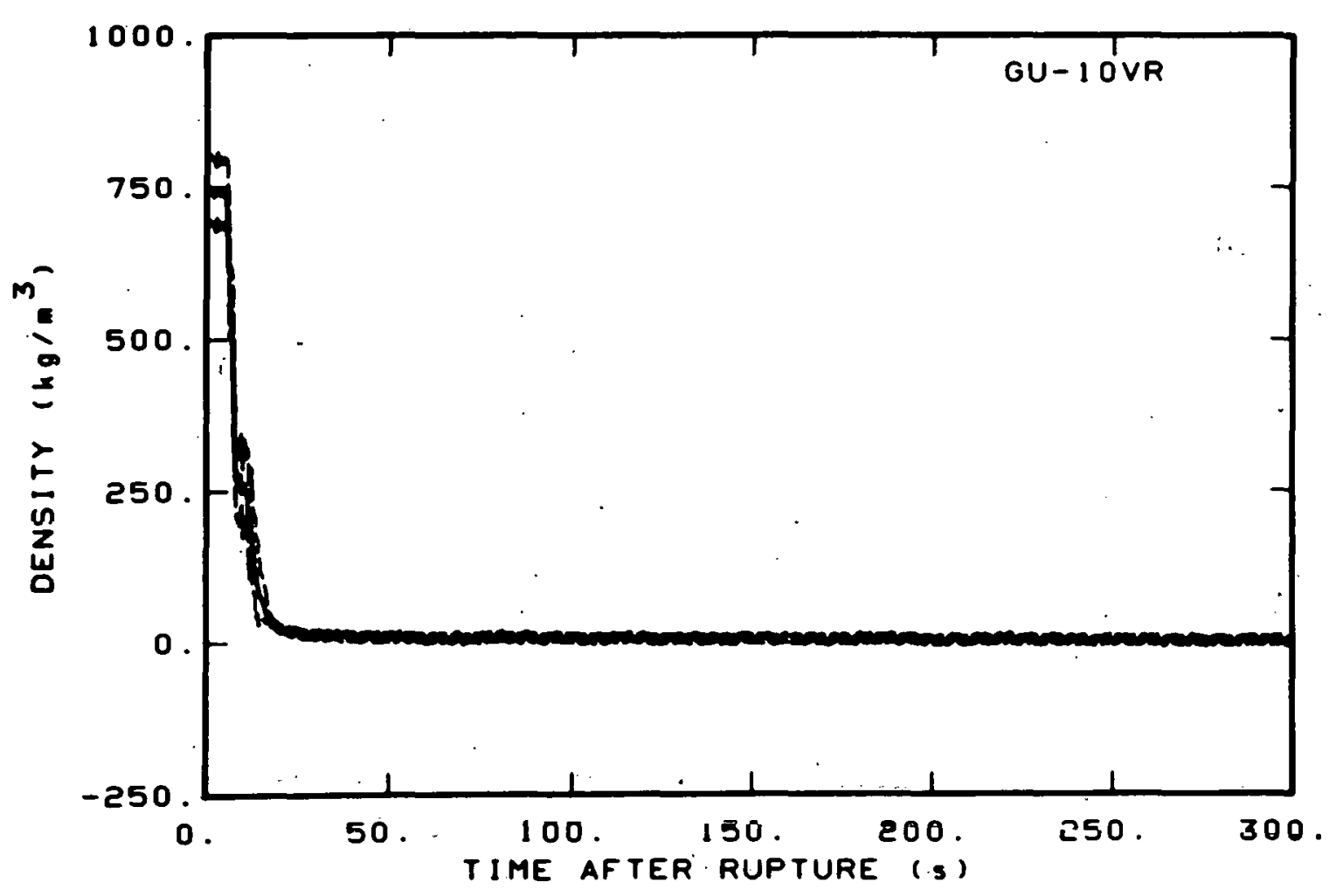

Fig. C-29 Density in intact loop (GU-10VR).

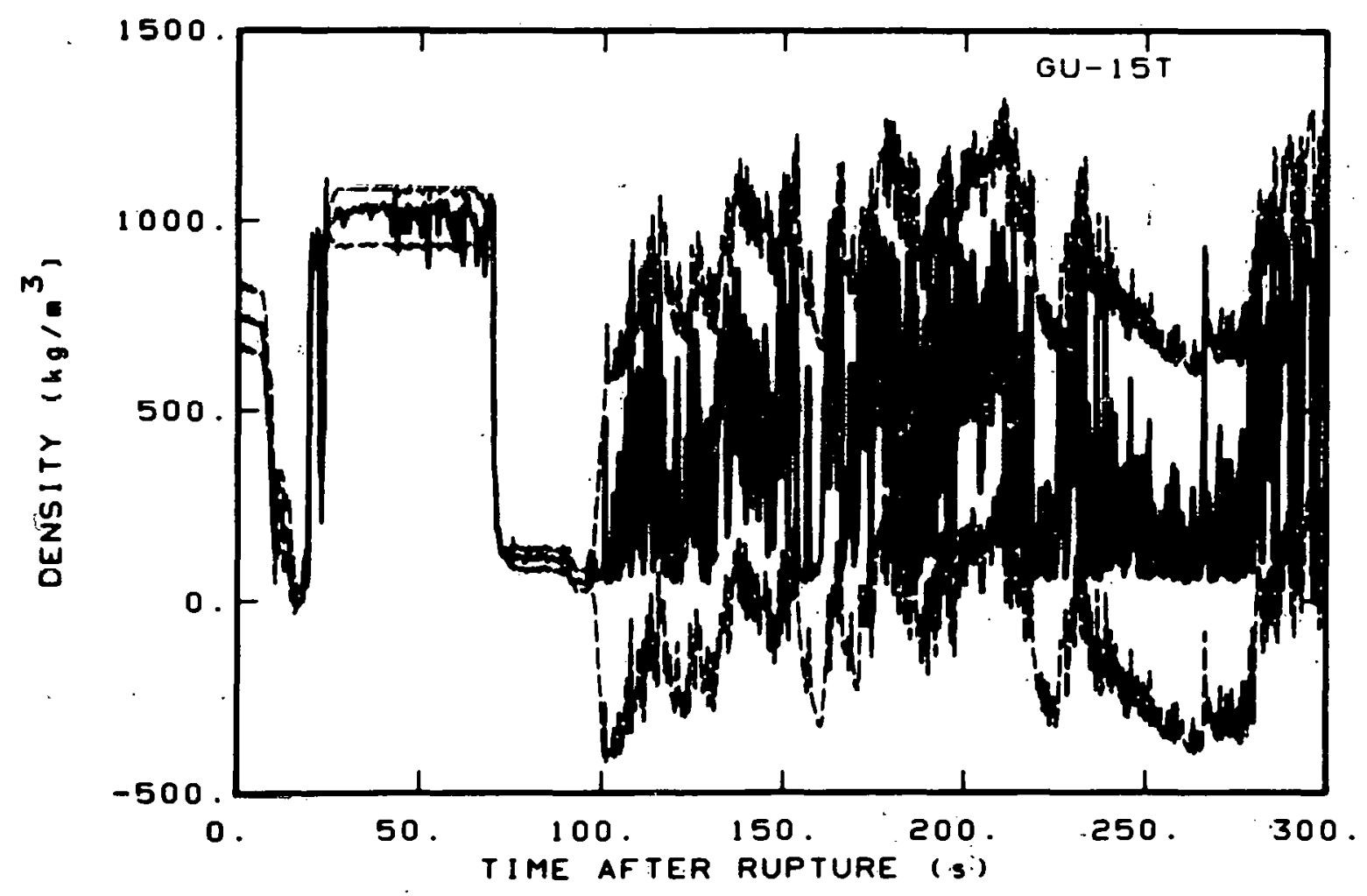

Fig. C-30 Density in intact loop (GU-15T). 


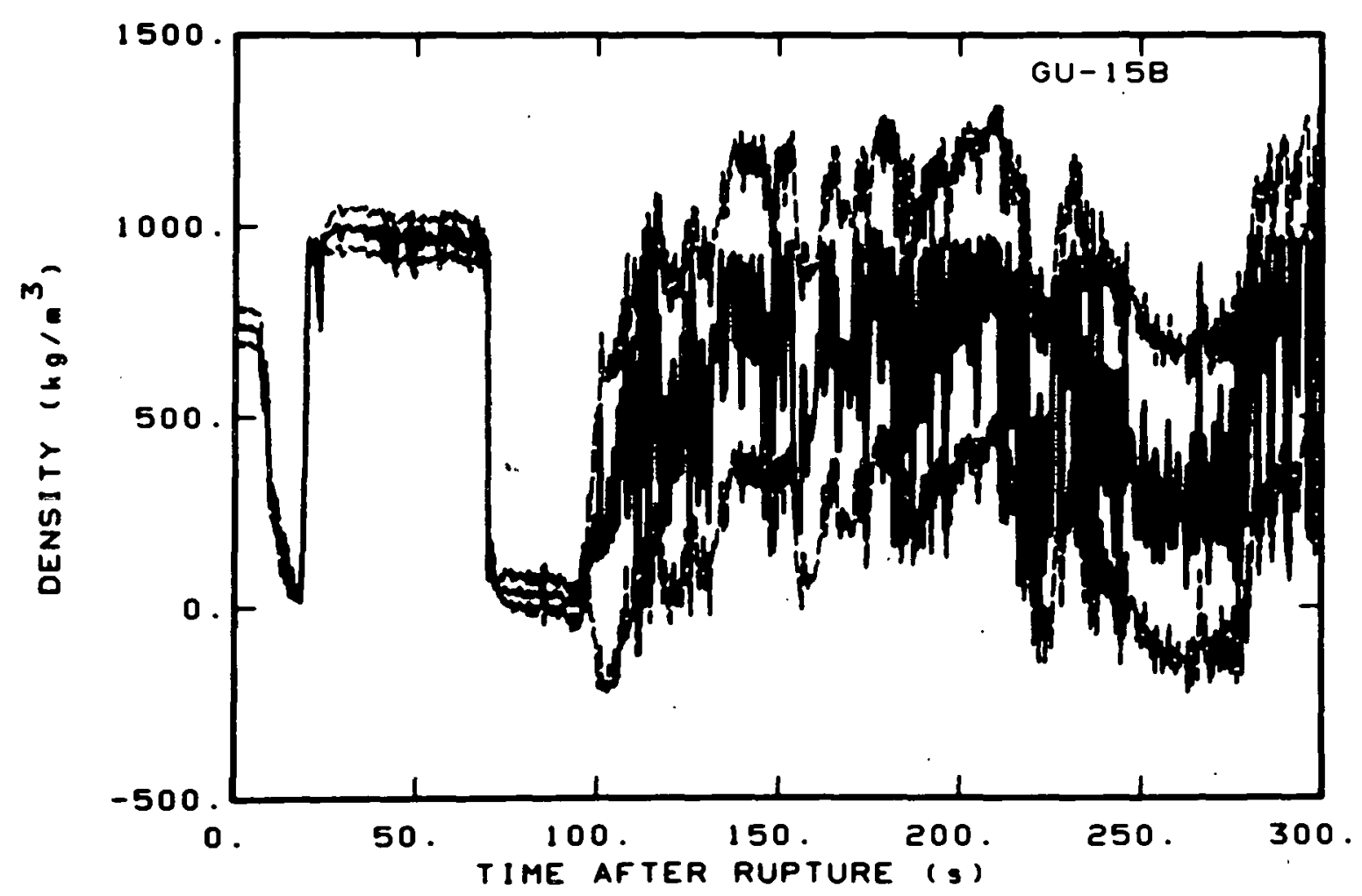

Fig. C-31 Density in intact loop (GU-15B).

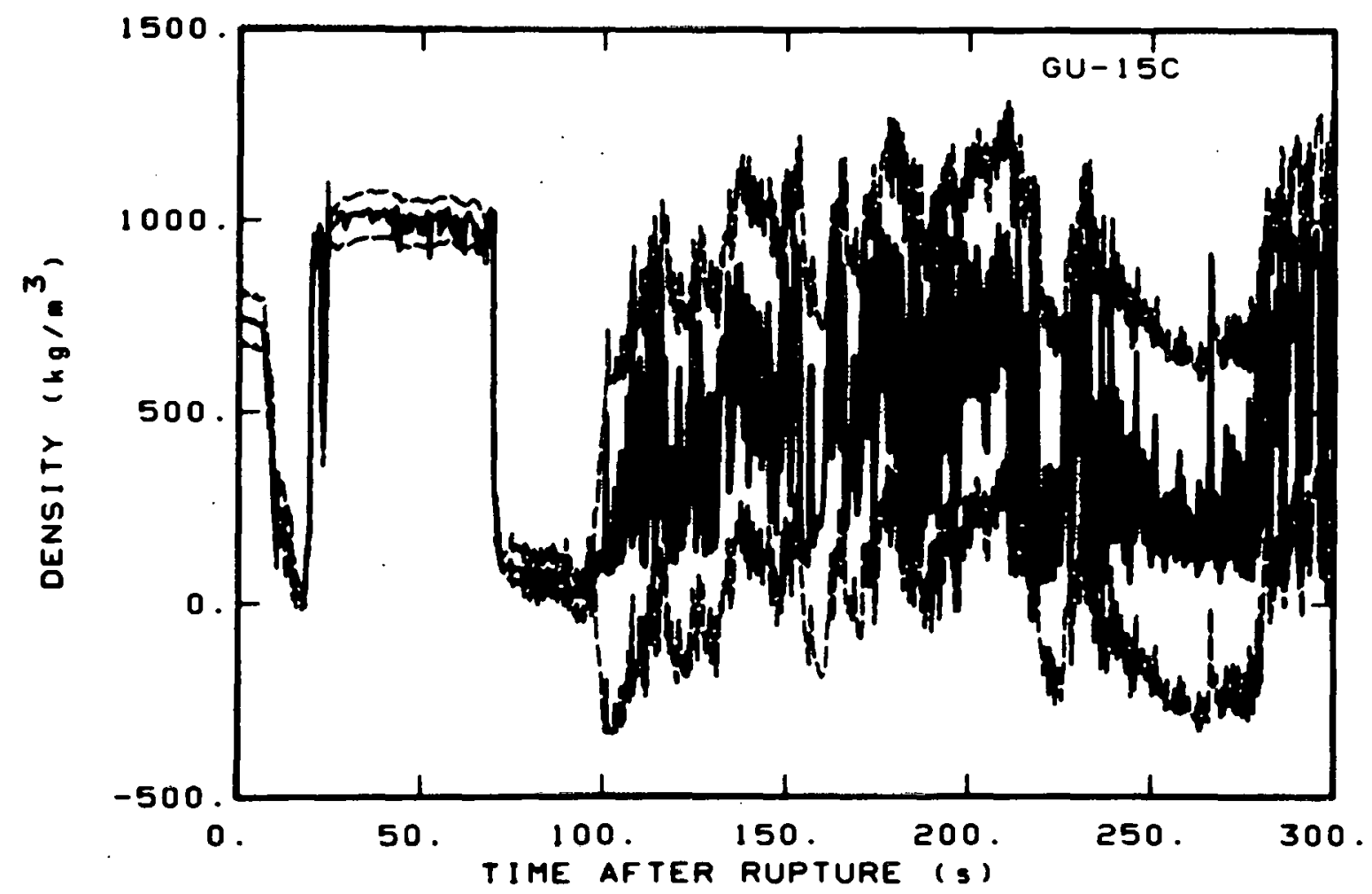

Fig. C-32 Density in intact loop (GU-15C). 


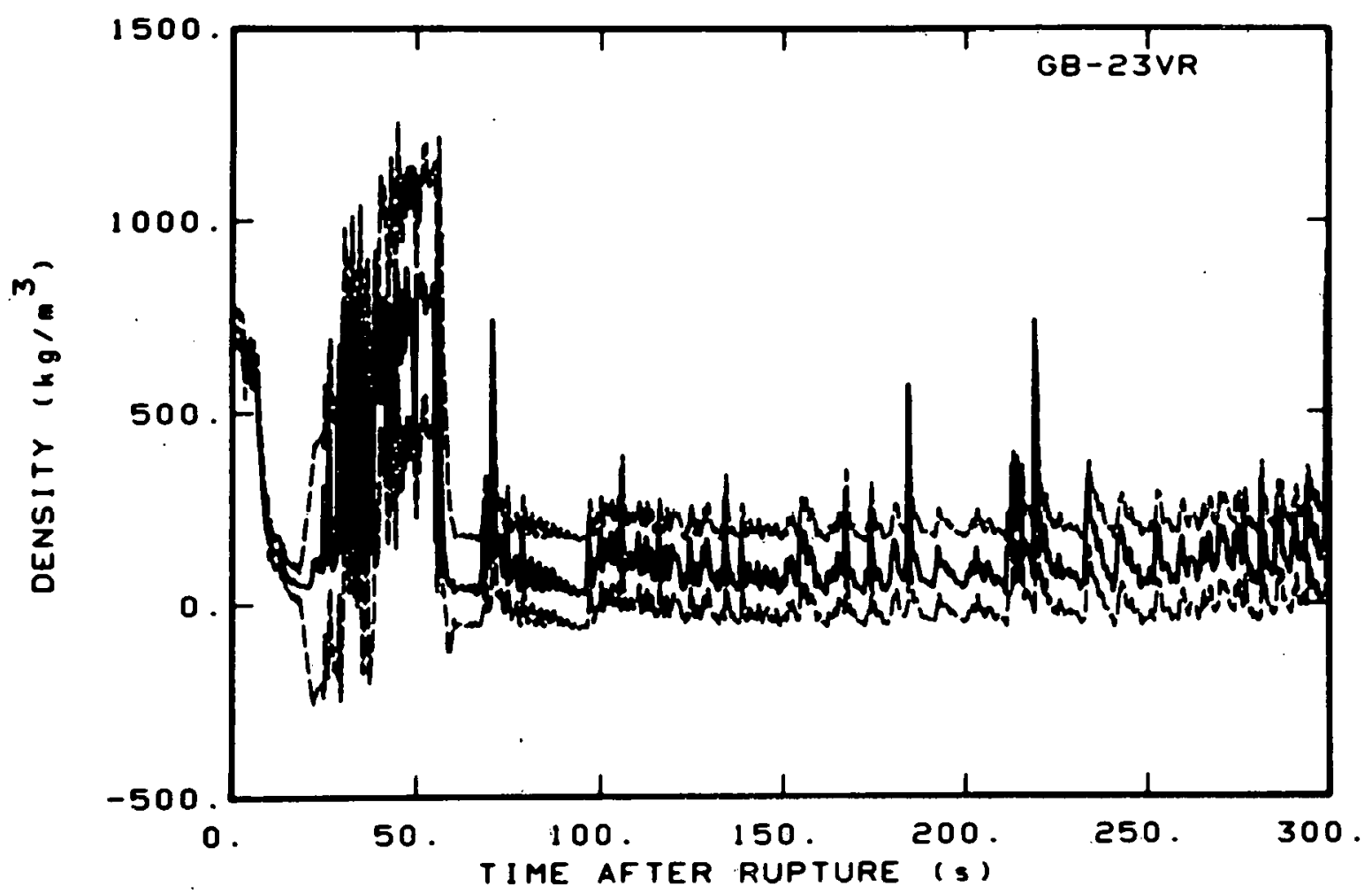

Fig. C-33 Density in broken loop (GB-23VR).

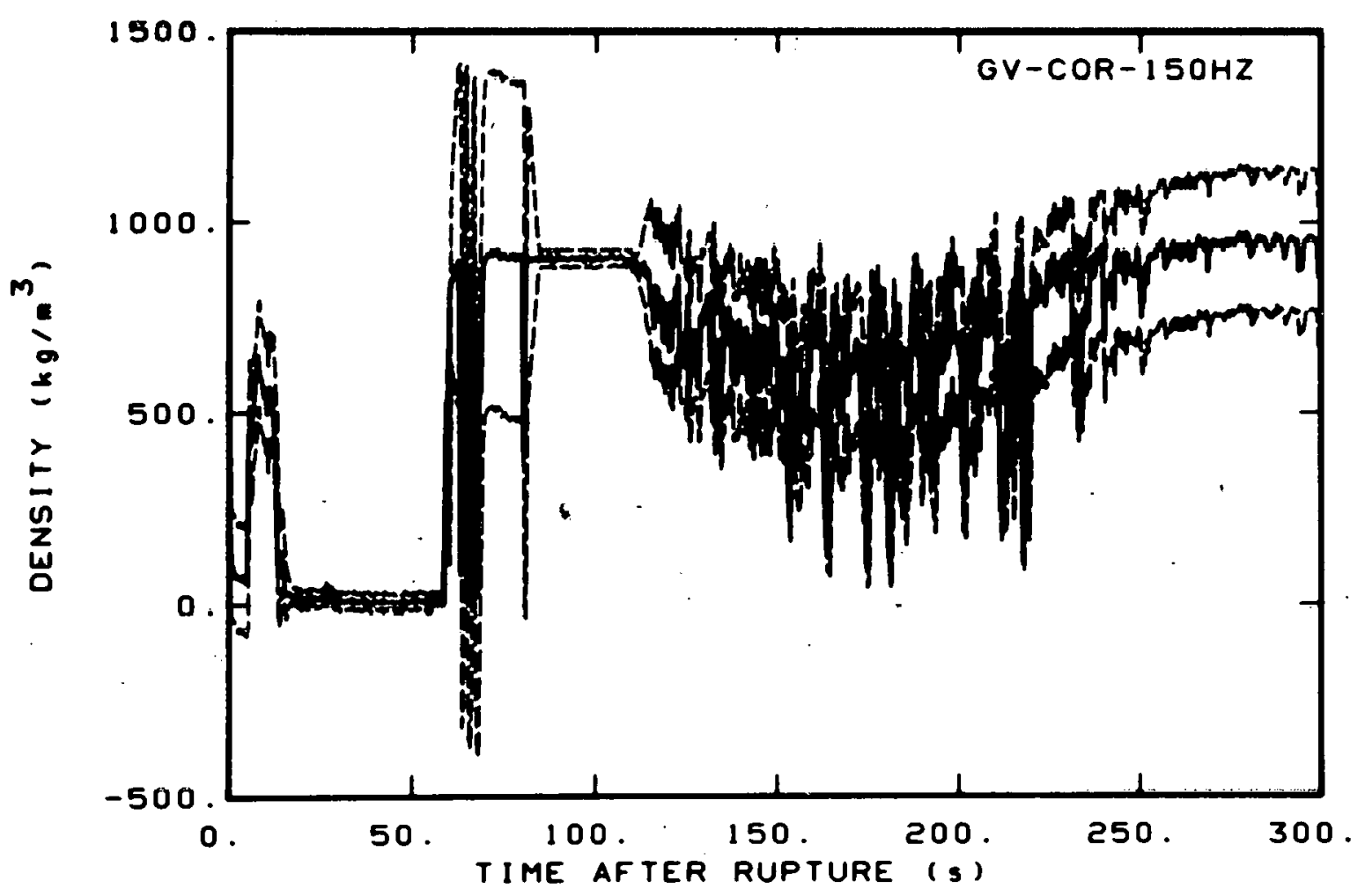

Fig. C-34 Density in vessel (GV-COR-150HZ).

224 


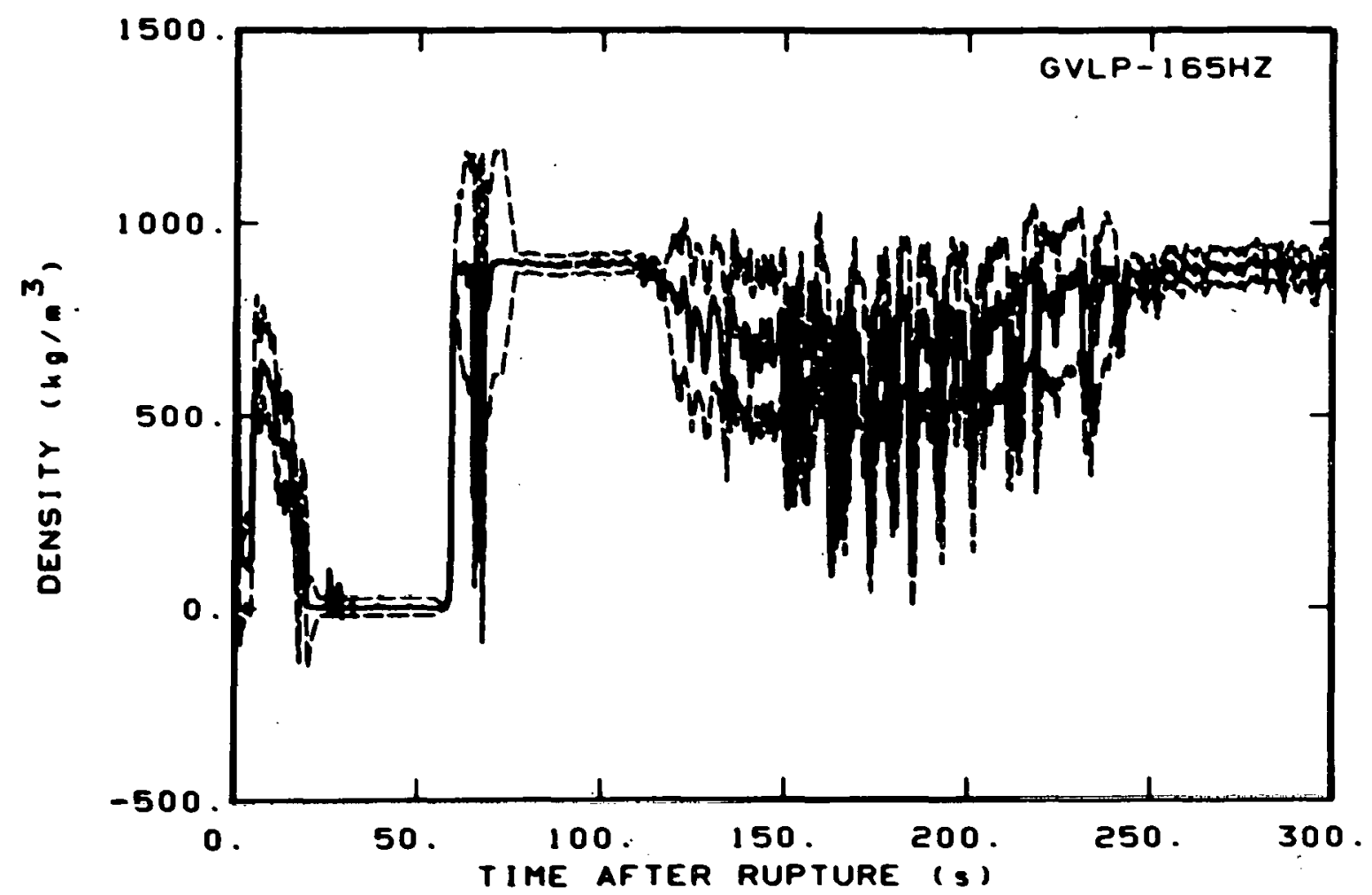

Fig. C-35 Density in vessel (GVLP-155HZ).

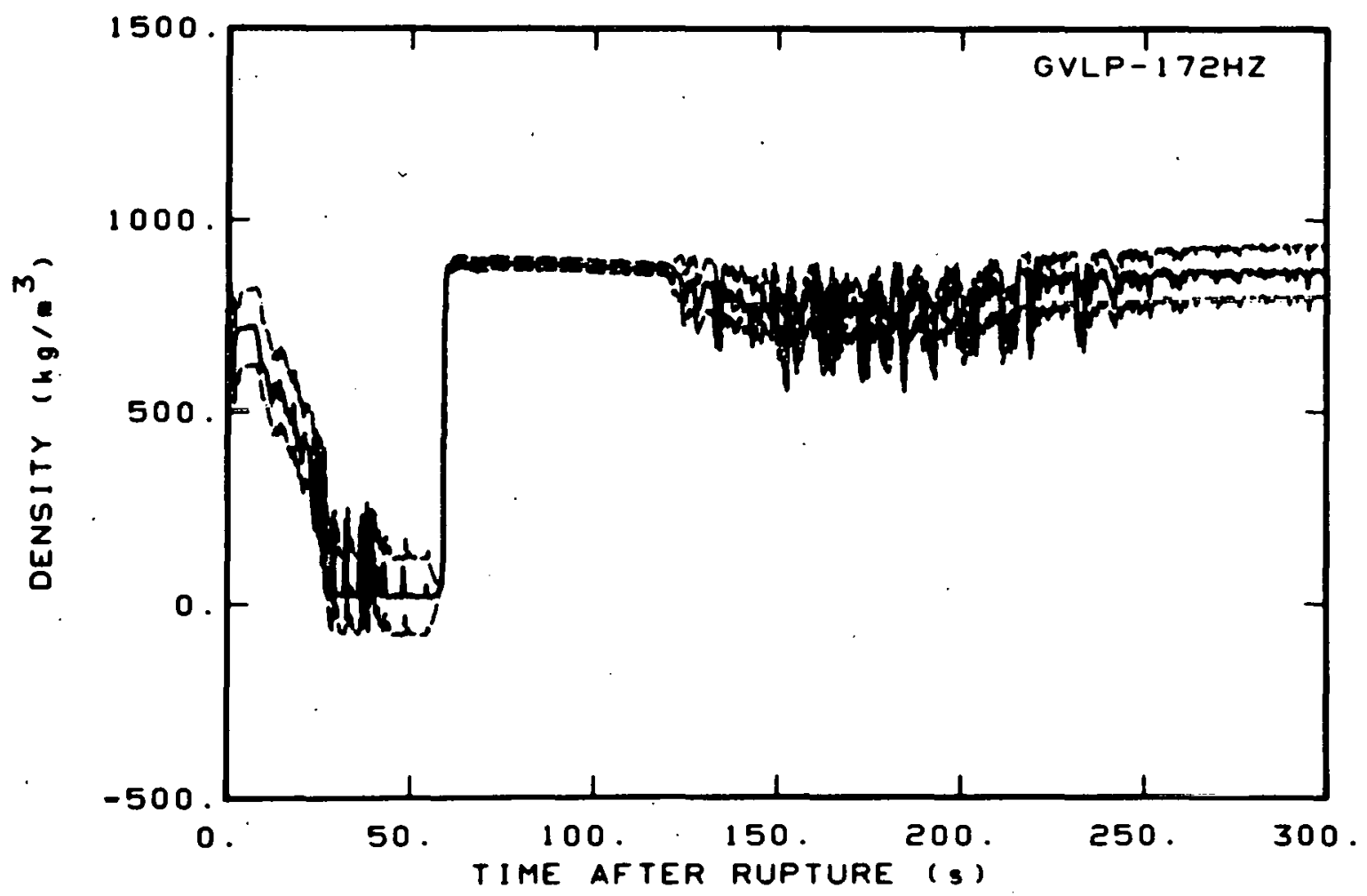

Fig. C-36 Density in vessel (GVLP-172HZ). 


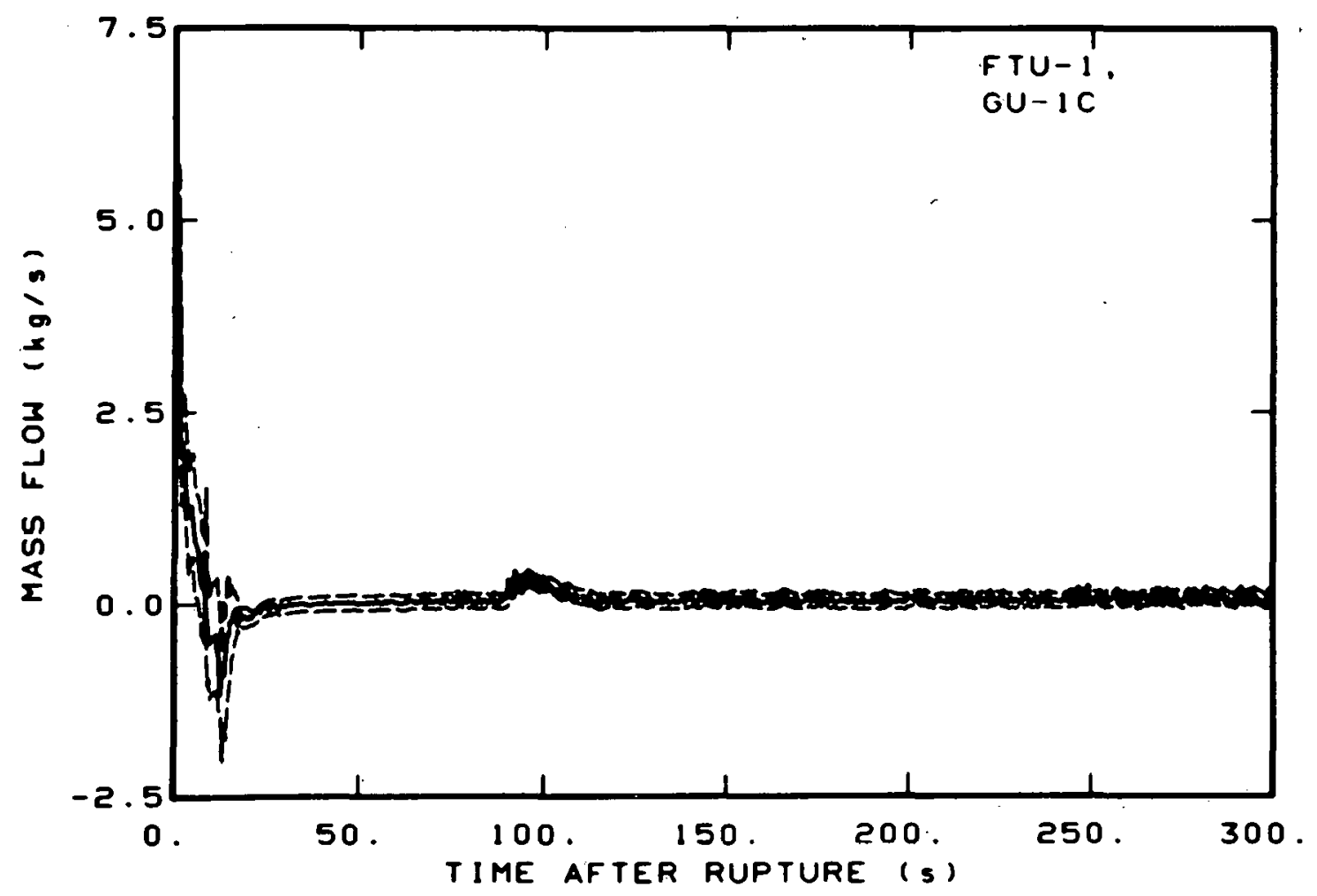

Fig. C-37 Mass flow in intact loop (FTU-1, GU-1C).

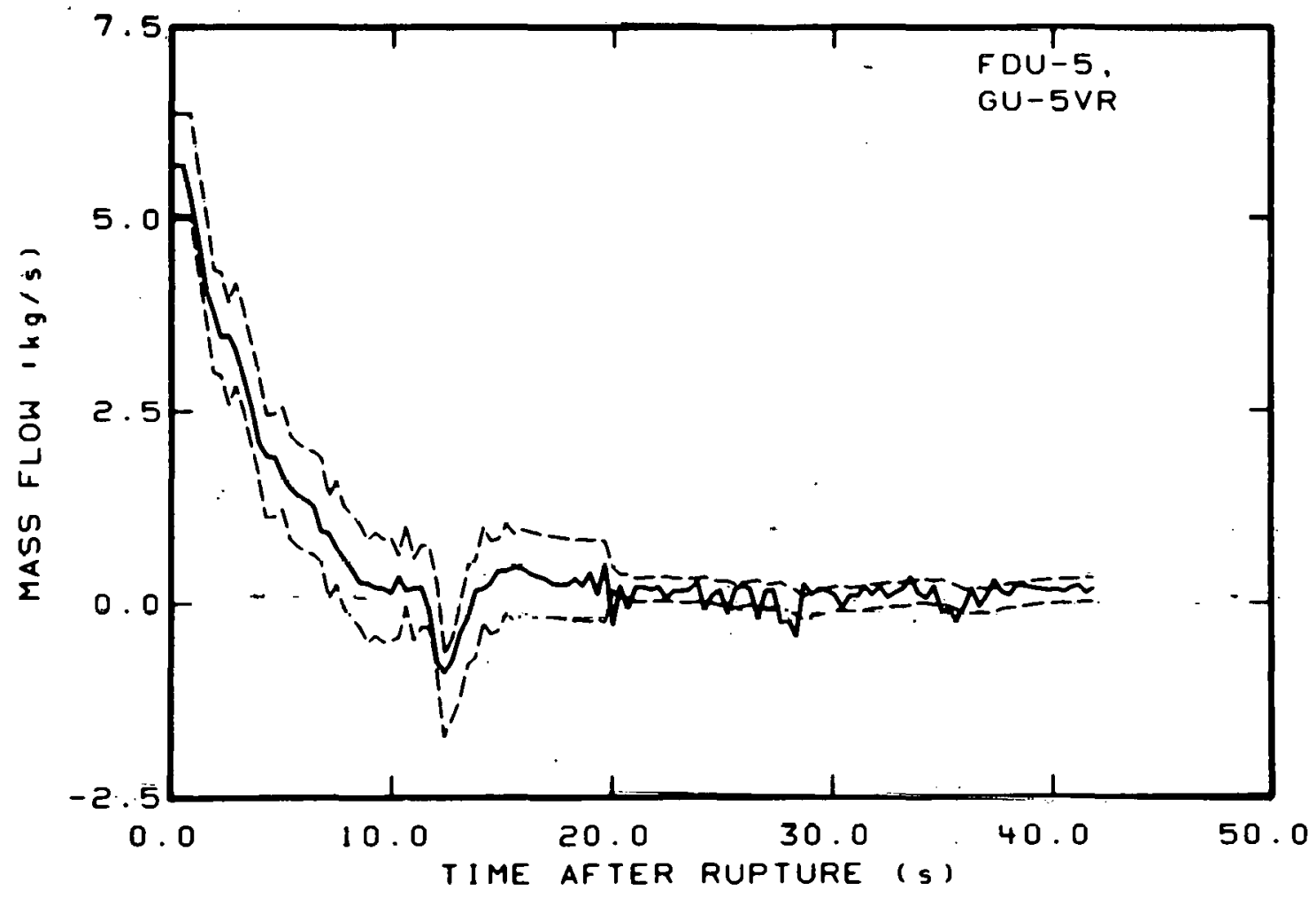

Fig. C-38 Mass flow in intact loop (FDU-5, GU-5VR). 


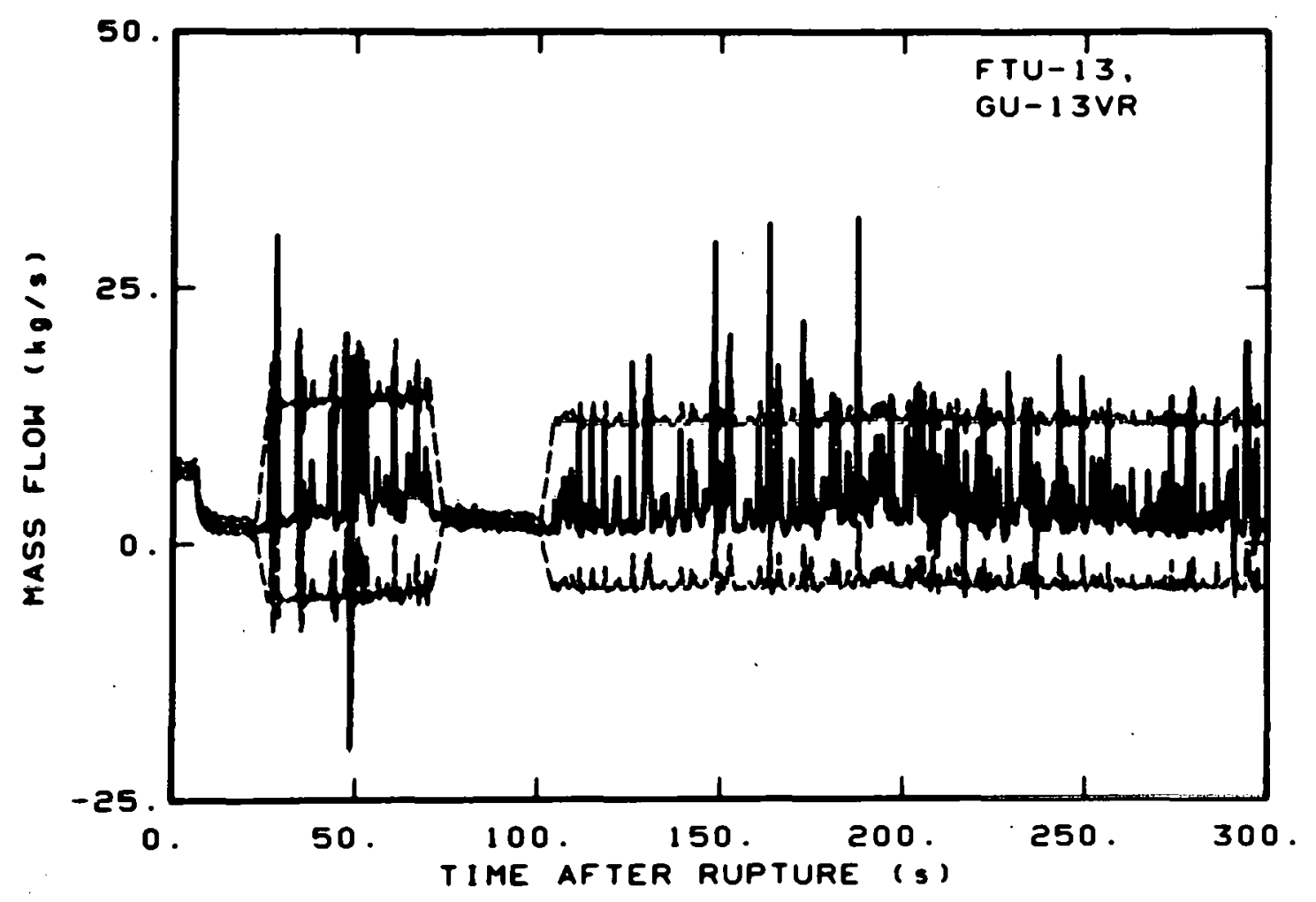

Fig. C-39 Mass flow in intact loop (FTU-13, GU-13VR).

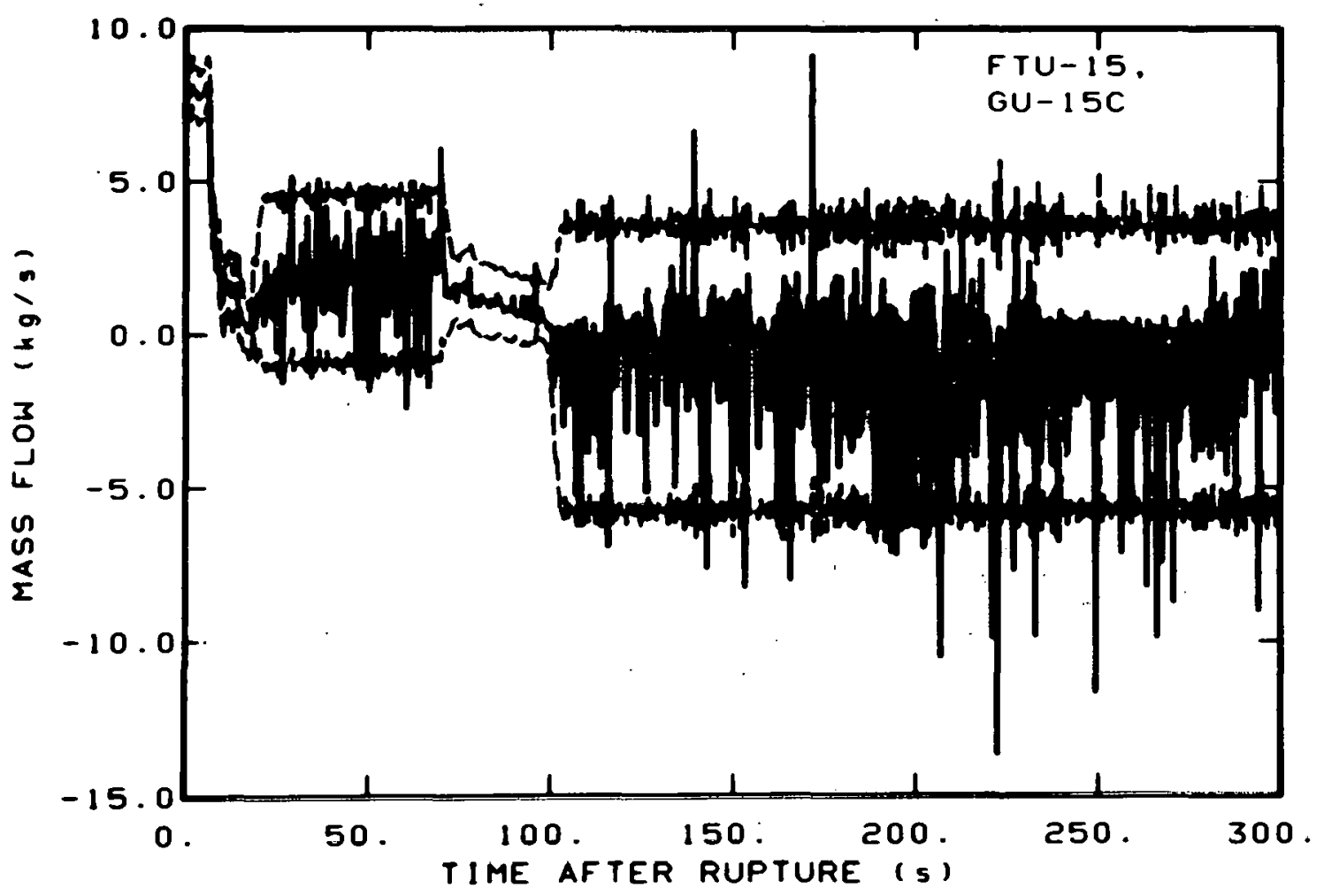

Fig. C-40 Mass flow in intact loop (FTU-15, GU-15C). 


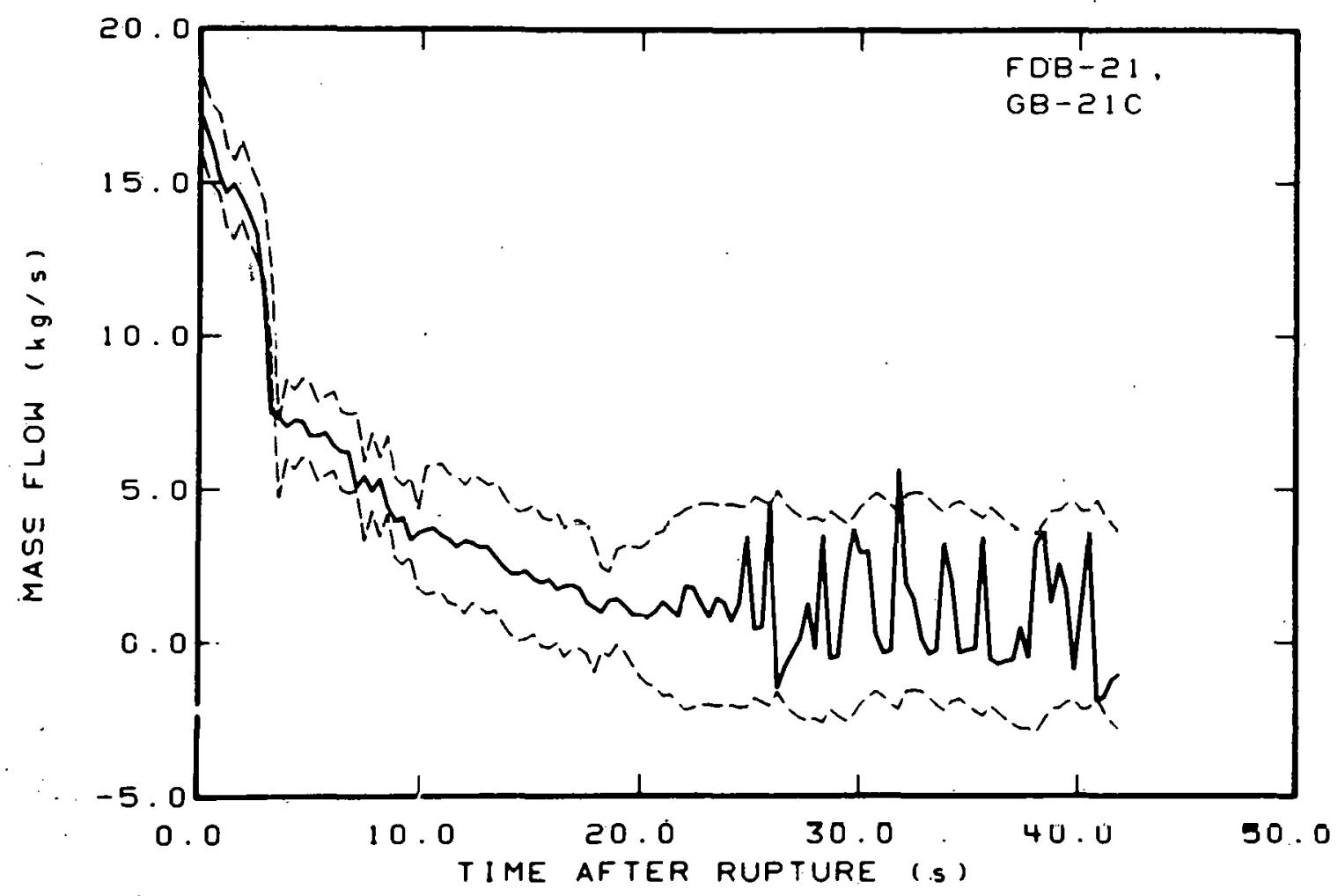

Fig. C-41 Mass flow in broken 1oop (FDB-21, GB-21C).

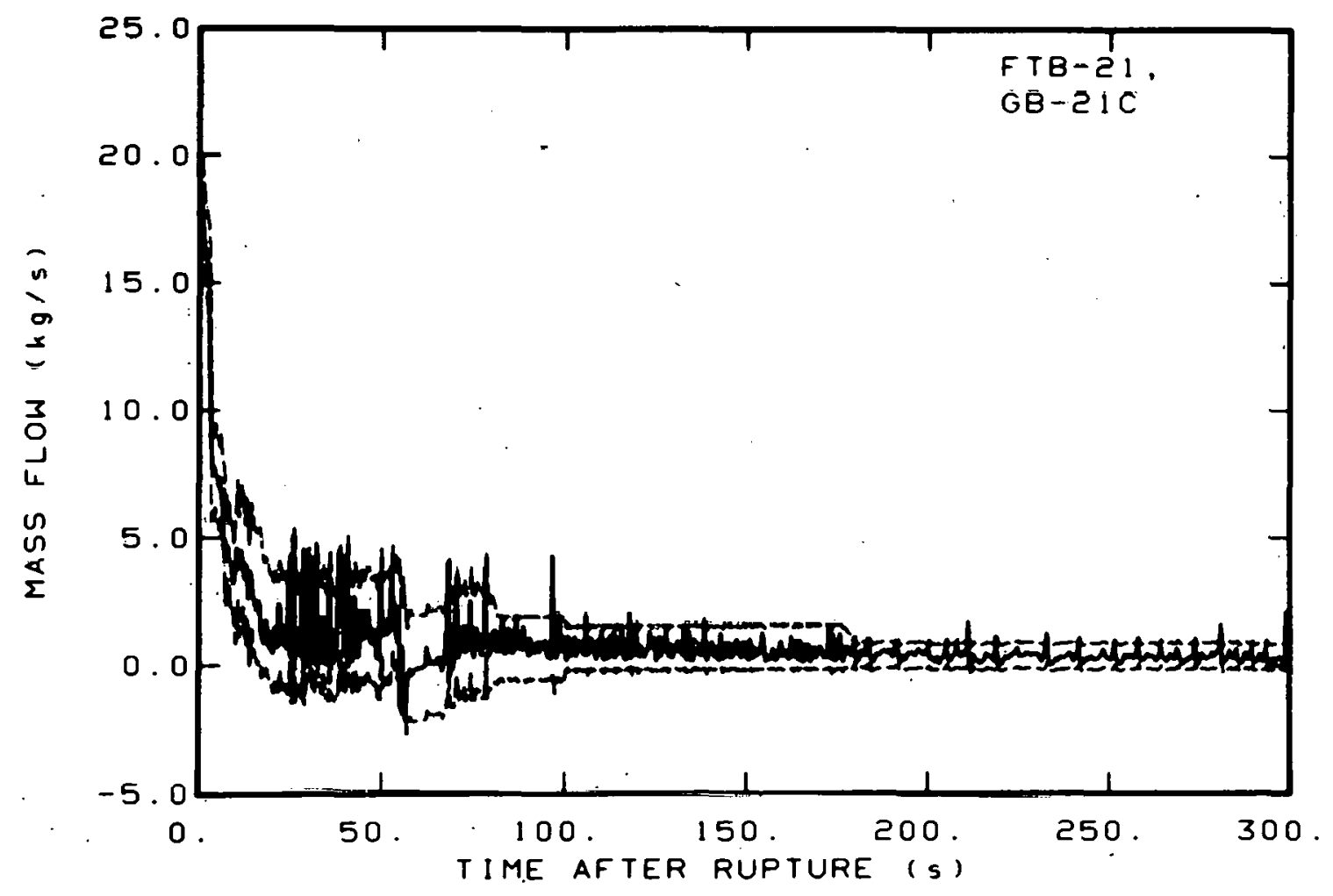

Fig. C-42 Mass flow in broken 1oop (FTB-21, GB-21C). 


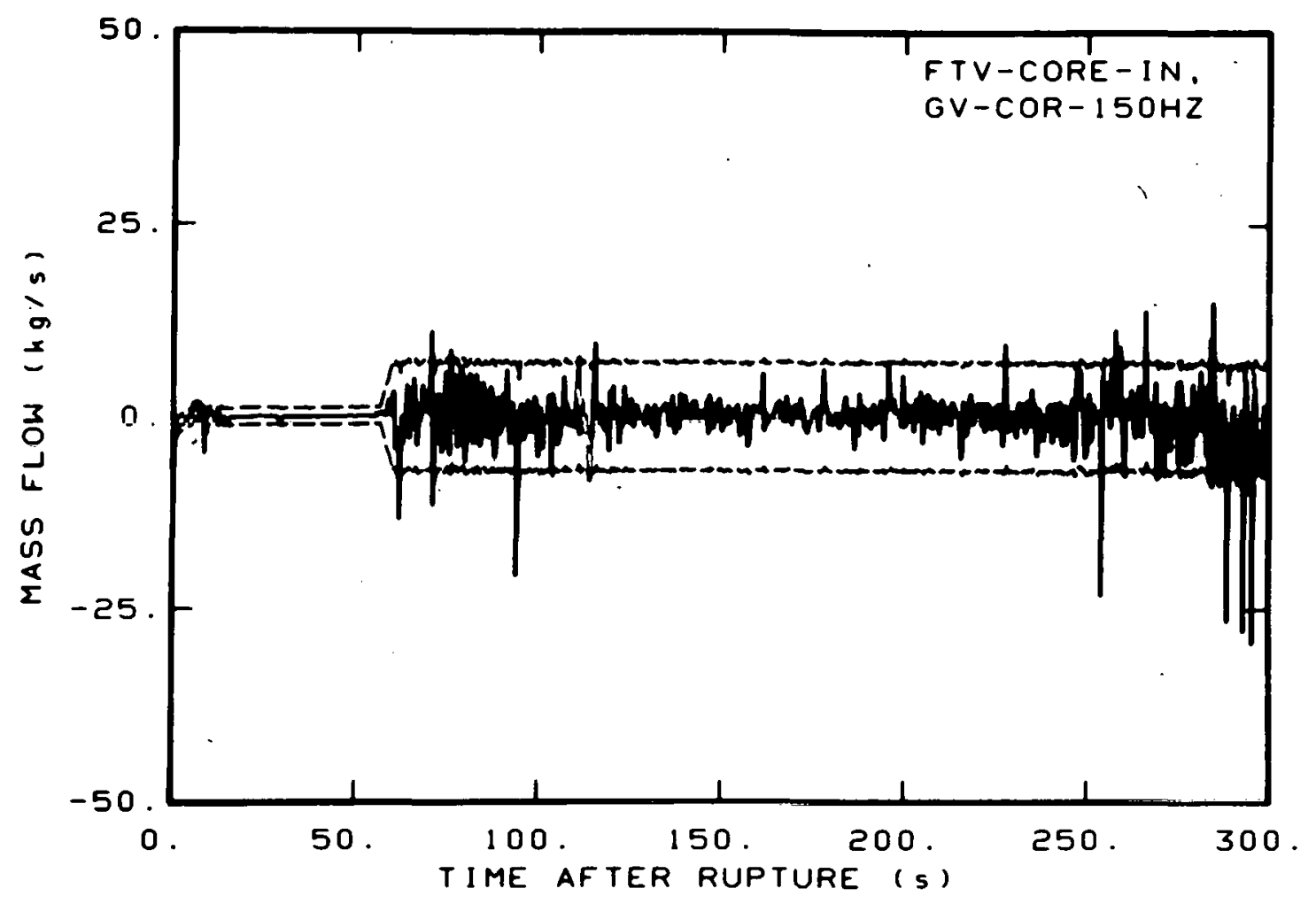

Fig. C-43 Mass flow in vessel (FTV-CORE-IN, GV-COR-150HZ):

Other errors in the data exist because of such factors as variability in installation procedures and techniques, calibration errors, variability in materials, and temperature and pressure sensitivities. These errors and the procedures for estimating them are discussed in Reference $\mathrm{C}-2$. They are referred to as engineering errors and the estimates are largely subjective. Because of the continuing effort to improve the accuracy of the measured data, such as through the use of better transducers, better signal conditioning and processing . equipment, and better calibration and installation techniques, the engineering errors for data from most of the transducer systems have changed from those published in Reference C-2. Table C-II provides a summary of engineering error values obtained from current analysis techniques as applied to the data presented herein.

In addition to the normal hardware and installation related sources of engineering error, a significant measurement uncertainty results when the current transducer systems are subjected to separated two-phase flow regimes during the course of the blowdown transient. Accordingly, for those data affected (fluid density, momentum flux, volumetric flow, and mass flow), which are presented in this appendix, a more extensive assessment was conducted for additional engineering error due to flow regime effects. Table C-III 1dentifies the data analyzed and the period in the blowdown process for which flow regime errors were included as a part of the total engineering error. The time of occurrence of separated two-phase flow and the resulting effect on the uncertainty of the data were evaluated by considering, on an individual basis, each detector output with reference to indications by other auxiliary measurements. 


\section{TABLE C-II}

GENER:AL MEASUREMENT ENGINEERING ERROR SOURCES AND ERROR VALUES (TEST S-06-4)

\begin{tabular}{|c|c|c|c|}
\hline $\begin{array}{c}\text { Measurement } \\
\text { Category }\end{array}$ & Error Sources & Error Value & Expected Error Value \\
\hline \multirow[t]{3}{*}{$\begin{array}{l}\text { Fluid } \\
\text { Temperature }\end{array}$} & $\begin{array}{l}\text { Changes in homogeneity of the } \\
\text { thermocouple wire due to cold } \\
\text { working }\end{array}$ & $\pm 1.11 \mathrm{~K}$ & \multirow{4}{*}{ $\pm 2.8 k^{[a]}$} \\
\hline & $\begin{array}{l}\text { Data interpretation from } \\
\text { standard reference tables }\end{array}$ & $\pm 1.11 \mathrm{~K}$ & \\
\hline & $\begin{array}{l}\text { General data acquisition } \\
\text { Frocessing }\end{array}$ & $\pm 2.50 \mathrm{~K}$ & \\
\hline & Termal aging of the thernocouples & $\pm 0.28 \mathrm{~K}$ & \\
\hline \multirow[t]{4}{*}{$\begin{array}{l}\text { Material } \\
\text { Temperature }\end{array}$} & $\begin{array}{l}\text { C.nanges in homogeneity of the } \\
\text { thermocouple wire due to cold } \\
\text { working }\end{array}$ & $\pm 1.11 k$ & \multirow{5}{*}{ $\pm 3.9 \mathrm{k}$} \\
\hline & Thermoccuple radial fosition & $\pm 2.78 \mathrm{~K}$ & \\
\hline & $\begin{array}{l}\text { Data interpretation from standard } \\
\text { reference tables }\end{array}$ & $\pm 1.11 \mathrm{~K}$ & \\
\hline & $\begin{array}{l}\text { General data acquisitior and } \\
\text { processing }\end{array}$ & $\pm 2.50 \mathrm{~K}$ & \\
\hline & -hermal aging of the thermocouples & $\pm 0.28 \mathrm{~K}$ & \\
\hline
\end{tabular}


TABLE C-II (continued)

\begin{tabular}{|c|c|c|c|}
\hline $\begin{array}{l}\text { Measurement } \\
\text { Category }\end{array}$ & Error Sources & Error Value & Expected Error Value \\
\hline \multirow[t]{4}{*}{ Pressure } & Entrance effects & $\begin{array}{l}+0.3 \% \text { of transducer } \\
\text { fult scale }\end{array}$ & $\cdot$ \\
\hline & Calibration & $\begin{array}{l}+0.26 \% \text { of transducer } \\
\text { full scale }\end{array}$ & . \\
\hline & Temperature sensitivity & $\begin{array}{l}+0.13 \% \text { of transducer } \\
\text { full scale }\end{array}$ & $\cdot$ \\
\hline & $\begin{array}{l}\text { General data acquisition and } \\
\text { processing }\end{array}$ & $\begin{array}{l}+0.1 \% \text { of system full } \\
\text { scale }\end{array}$ & \\
\hline \multirow[t]{6}{*}{$\begin{array}{l}\text { Differential } \\
\text { Pressure }\end{array}$} & Installation & $\begin{array}{l}+0.3 \% \text { of transducer } \\
\text { full scale }\end{array}$ & \\
\hline & $\begin{array}{l}\text { Calibration } \\
\text { Transducer ranges } \pm 4.96 \text { through } \\
\pm 199.26 \mathrm{kPa}\end{array}$ & $\begin{array}{l}+\left[(0.05)+(0.5 \mathrm{R} / \mathrm{FS})^{2}\right]^{1 / 2} \% \\
\text { of transducer full scale }\end{array}$ & \\
\hline & $\begin{array}{l}\text { Transducer ranges } \pm 344.74, \\
\pm 689.47, \pm 3447 \mathrm{kPa}\end{array}$ & $\begin{array}{l}+\left[(0.03)+(0.5 \mathrm{R} / \mathrm{FS})^{2}\right]^{1 / 2} \% \\
\text { of full scale }\end{array}$ & $\pm 2 \%$ of transducer \\
\hline & $\begin{array}{l}\text { Transducer ranges } \pm 6894, \\
\pm 10342 \mathrm{kPa}\end{array}$ & $\begin{array}{l}+\left[(0.02)+(0.5 \mathrm{R} / \mathrm{FS})^{2}\right]^{1 / 2} \% \\
\text { of full scale }\end{array}$ & . \\
\hline & & where & \\
\hline & & $\begin{aligned} R= & \text { transducer reading } \\
& (\mathrm{kPa}) \\
\mathrm{FS}= & \text { transducer range full } \\
& \text { scale }(\mathrm{kPa})\end{aligned}$ & \\
\hline
\end{tabular}


TAELE C-II (continued)

\begin{tabular}{|c|c|c|c|}
\hline $\begin{array}{l}\text { Measurement } \\
\text { Categary }\end{array}$ & Error Sources & Error Va-ue & Expected Error Value \\
\hline \multirow{3}{*}{$\begin{array}{l}\text { Differential } \\
\text { Pressure } \\
\text { (continued) }\end{array}$} & Temperature sensitivity & $\begin{array}{l}+0.5 \% \text { of transducer } \\
\text { full scale. }\end{array}$ & $\cdot$ \\
\hline & $\begin{array}{l}\text { General data acquisition arid } \\
\text { processing }\end{array}$ & $\begin{array}{l}+0.1 \% \text { of system ful: } \\
\text { scale }\end{array}$ & $\begin{array}{l} \pm 2 \% \text { of transducer } \\
\text { full scale }\end{array}$ \\
\hline & Air entrapment & $\pm 0.069 \mathrm{kPa}$ & \\
\hline \multirow{4}{*}{$\begin{array}{l}\text { Fluid Velocity } \\
\text { (point velacities } \\
\text { measured with } \\
\text { turboprobes) }\end{array}$} & Irstallation & $\begin{array}{l}+0.8 \% \text { of transducer } \\
\text { full scale }\end{array}$ & \\
\hline & Calibration & $\begin{array}{l}+5 \% \text { of transducer } \\
\text { fult scale }\end{array}$ & $\begin{array}{l}+[0.5825+ \\
\left.(0.008 R)^{2}\right]^{1 / 2}(\mathrm{~m} / \mathrm{s})\end{array}$ \\
\hline & $\begin{array}{l}\text { Data acquisition and processing } \\
\text { frequency conversion }\end{array}$ & $\begin{array}{l}+0.25 \% \text { of transducer } \\
\text { full scale }\end{array}$ & \\
\hline & General. & $\begin{array}{l}+0.1 \% \text { of system full } \\
\text { scale }\end{array}$ & $R=$ transducer read- \\
\hline Density & Calibration & $\pm 1.0 \%$ of reading $\left(\mathrm{kg} / \mathrm{m}^{3}\right)$ & \\
\hline$\therefore$ & Detector systen error & $\pm 2.1 \mathrm{~kg} / \mathrm{m}^{3}$ & \\
\hline$\cdots$ & $\begin{array}{l}\text { General tata acquisition and } \\
\text { processing }\end{array}$ & $\pm 1.6 \mathrm{~kg} / \mathrm{m}^{3}$ & $2-1$ \\
\hline
\end{tabular}


TABLE C-II (continued)

\begin{tabular}{|c|c|c|c|}
\hline $\begin{array}{l}\text { Measurement } \\
\text { Category }\end{array}$ & Error Sources & Error Value & Expected Error Value \\
\hline $\begin{array}{l}\text { Density } \\
\text { (continued) }\end{array}$ & $\cdot$ & $\begin{array}{l}\mathrm{Gr}[\mathrm{d}] \\
\text { where } \\
\mathrm{Gr}=\text { flow regime error } \\
\quad\left(\mathrm{kg} / \mathrm{m}^{2}\right)\end{array}$ & {$[\mathrm{d}]$} \\
\hline \multirow[t]{4}{*}{$\begin{array}{l}\text { Monentum Flux } \\
\text { (drag disc) }\end{array}$} & $\begin{array}{l}\text { Installation alignment and } \\
\text { velocity profile changes } \\
\text { from calibration conditions }\end{array}$ & {$[d]$} & $\cdot$ \\
\hline & Calibration & {$[\mathrm{e}]$} & {$[d]$} \\
\hline & $\begin{array}{l}\text { General data acquisition } \\
\text { and processing }\end{array}$ & $\begin{array}{l} \pm 0.1 \% \text { of system full } \\
\text { scale }\left(\mathrm{kg} / \mathrm{ms}^{2}\right)\end{array}$ & \\
\hline & Flow regimes & {$[d]$} & \\
\hline \multirow{5}{*}{$\begin{array}{l}\text { Volumetric Flow } \\
\text { (turbine flow- } \\
\text { meter) }\end{array}$} & Calibration instrument reading & $\begin{array}{l}+0.25 \% \text { of transducer } \\
\text { full scale }\end{array}$ & . \\
\hline & Calibration standards & $\pm 19.56 \times 10^{-2} \mathrm{\ell} / \mathrm{s}$ & \\
\hline & Velocity profile & $\pm 2.9 \%$ of reading & \\
\hline & $\begin{array}{l}\text { Frequency-to-voltage } \\
\text { conversion }\end{array}$ & $\begin{array}{l}+0.25 \% \text { of transducer } \\
\text { full scale }\end{array}$ & {$[d]$} \\
\hline & $\begin{array}{l}\text { General data acquisition } \\
\text { and processing }\end{array}$ & $\begin{array}{l}+0.1 \% \text { of system } \\
\text { ful1 scale }\end{array}$ & \\
\hline
\end{tabular}




\begin{tabular}{|c|c|c|c|}
\hline $\begin{array}{l}\text { Measurement } \\
\text { Category }\end{array}$ & Error Sources & Error Va:ue & Expected Error Value \\
\hline \multirow{2}{*}{$\begin{array}{l}\text { Volumetric Flow } \\
\text { (turbine flow- } \\
\text { meter) (continued) }\end{array}$} & Dead bands & $\begin{array}{l}+5 \% \text { of transducer } \\
\text { fuil scale }\end{array}$ & \multirow[t]{2}{*}[\mathrm{d}]{} \\
\hline & Flow regỉmes & [d] & \\
\hline $\begin{array}{l}\text { Mass Flow Rate } \\
\text { (from volumetric } \\
\text { flow and density } \\
\text { data) }\end{array}$ & $\begin{array}{l}\text { Combined results from } \\
\text { irdividual error sources } \\
\text { far volumetric flow and density } \\
\text { data }[f]\end{array}$ & [d] & [d] \\
\hline $\begin{array}{l}\text { Mass Flow Rate } \\
\text { (from momeritum } \\
\text { flux and density } \\
\text { data) }\end{array}$ & $\begin{array}{l}\text { Combined results from } \\
\text { individual error sources for } \\
\text { momentum flux and density data }[f]\end{array}$ & {$[d]$} & {$[d]$} \\
\hline \multirow{2}{*}{\multicolumn{4}{|c|}{$\begin{array}{l}\text { [a] This value is no longer valid after thermocouple dryout occurs. } \\
\text { [b] Value for transfucers with } 20684 \mathrm{kPa}=\text { ull-scale ranges. }\end{array}$}} \\
\hline & & & \\
\hline \multicolumn{4}{|c|}{$\begin{array}{l}\text { [c] Value is based on observed s:stem performance. It is more consersative than } \\
\text { statistical sumnation of the identi=ied engineering errors. }\end{array}$} \\
\hline \multicolumn{4}{|c|}{ [d] Error value is time and flow regime dependent. } \\
\hline \multicolumn{4}{|c|}{ [e] Dependent on transducer full-scale range reading. } \\
\hline \multicolumn{4}{|c|}{$\begin{array}{l}\text { [f] The general method for combining volumeiric low or momentum flux with density data to obtain mass flow } \\
\text {-rate and the resultingl errors in the daia. are explained in Reference } C-2 \text {. }\end{array}$} \\
\hline
\end{tabular}


TABLE C-III

TIME PERIODS WHEN FLOW REGIME ERRORS WERE APPLIED (TEST S-06-4).

\begin{tabular}{lcc}
$\begin{array}{c}\text { Transducer } \\
\text { Identification }\end{array}$ & $\begin{array}{c}\text { Time during which Flow Regime } \\
\text { Errors Were Applied }(\mathrm{s})\end{array}$ & Figure \\
\cline { 2 - 3 } FTU-1 & 1 to 20 & $\mathrm{C}-19$ \\
FTU-15 & 7 to 95 & $\mathrm{C}-20$ \\
GU-1C & 1 to 20 & $\mathrm{C}-28$ \\
GU-10VR & 8 to 20 & $\mathrm{C}-29$ \\
GU-15C & 7 to 95 & $\mathrm{C}-32$ \\
GB-23VR & 16 to 300 & $\mathrm{C}-33$ \\
FTU-1, GU-1C & 1 to 20 & $\mathrm{C}-37$ \\
FDU-5, GU-5VR & 1 to 20 & $\mathrm{C}-38$ \\
FTU-15, GU-15C & 7 to 95 & $\mathrm{C}-40$ \\
FDB-21, GB-21C & 7 to 300 & $\mathrm{C}-41$ \\
FTB-21, GB-21C & 7 to 300 & $\mathrm{C}-42$ \\
\hline
\end{tabular}

The gamma densitometer density measurement data are affected by two-phase separated flow regimes. The resulting transducer output is a measurement of the average attenuation of the gamma beam through the measured medium. The beam attenuation, in turn, is interpreted through physical relationship to be a measure of the average density along the beam path. When stratified type flow was considered present, the gamma beam attenuation was considered to be a result of a liquid layer and steam at system conditions. With this assumption and the system geometry, a void fraction was calculated and a new "effective" average density was calculated. The difference between the average density based on the assumption of homogeneous conditions and the average density for stratified conditions was considered to be the error.

Momentum flux measurement uncertainties for two-phase flow regimes present the must difficult engineering evaluation problems. The drag target and arm location, degree of flow stratification, transducer temperature sensitivity, and slip ratios all combine to produce possible flow regime errors ranging from a small fraction of the transducer output value to multiples of it. Therefore, the error values were obtained, where possible, through use of the observed discrepancies between the momentum flux and turbine flowmeter data in combinatinn with system pressure measuremente and the analysis of systcm fluid density measurements. 
The flow regime errors of the turbine flowmeter were estimated by calculating a void fraction and the cross-sectional liquid and steam flow areas for stratified flow. This calculation was accomplished using methods similar to those used to calculate the average density for stratified flows. A simple model was used to equate the forces on the turbine with the assumption of a known void fraction, stratified flow, known component densities, and slip ratio greater than unity. This process provided phase velocities. With the phase densities, velocities, and void fraction, a volumetric flow rate could be calculated. The difference between this value and the measured value was considered to be the error.

The overall standard deviation of a data point is taken as the root mean of the sum of the random error variation and the total engineering error variance; that is,

$$
\sigma_{0}^{2}=\sqrt{\sigma_{R}^{2}+\sigma_{E}^{2}}
$$

where

$\sigma_{\mathrm{O}}=$ overall standard deviation of a data point

$\sigma_{\mathrm{R}}=$ random error standard deviation

$\sigma_{\mathrm{E}}=$ engineering error standard deviation.

The error bands for the data are computed about the value given by the filled difference equation $y_{i}$ at time point, $i$; that is,

$$
\text { error band }=Y_{i} \pm 1.96 \sigma_{0}
$$

With due regard to the fact that $\sigma_{\mathrm{E}}$ has been estimated subjectively, the error band may be interpreted as an approximate $95 \%$ confidence interval within which any true value of the measured variable is consistent with the data.

On certain occasions, the symmetrical error band given by Equation (C-2) is not appropriate. On those occasions, asymmetrical error bands were computed. (That is, with the width being greater on one side of $y_{i}$ than on the other.)

Finally, the original data trace, along with its error band from Equation ( $\mathrm{C}-2)$, was input to a computer plot package. The resulting plot contained the actual data trace surrounded by an error band derived both from random error and engineering errur considerations. The indicated error bands after thermocouple dryout occurred for the fluid temperature measurements should be ignored. Error bands for these segments of the data were not obtained and bands only appear because of limitations in the plotting package. 


\section{REFERENCES}

C-1. G. E. P. Box and B. M. Jenkins, Time Series Analysis - Forecasting and Control, San Francisco: Holden-Day, 1970.

C-2. E. M. Feldman and S. A. Naff, Error Analysis for 1-1/2-Loop Semiscale System Isothermal Test Data, ANCR-1188 (May 1975). 
DISTRIBUTION RECORD FOR TREE-NUREG-1124

Internal Distribution

1 - Chicago Patent Group 9800 South Cass Avenue Argonne, Illinois 60439

2 - C. A. Benson Idaho Operations Office-DOE Idaho Falls, ID 83401

3 - R. J. Beers, ID

4 - P. E. Litteneker, ID

5 - R. E. Tiller, ID

6 - H. P. Pearson, Supervisor Information Management

7-16 - INEL Technical Library

17-36 - Authors

37-88 - Special Internal

External Distribution

89-90 - Saul Levine, Director Office of Nuclear Regulatory Research, NRC. Washington, D. C. 20555

91 - R. 1. Mattson

U. S. Nuclear Regulatory Commission, Div. of Systems Safety Ma i] Zone NL-5650 Washington, D. C. 20555

92 - V. Ste110 U. S. Nuclear Regulatory Commission, Div. Operating Reactor Mait Zone NRC-542 Washington, D. C. 20555

93-396 - Distribution under NRC-2, Water Reactor Safety Research Systems Engineering 